\title{
Steuerverfahren für selbstgeführte Stromrichter
}

Monograph

Author(s):

Jenni, Felix; Wüest, Dieter

Publication date:

1995

Permanent link:

https://doi.org/10.3929/ethz-a-001427314

Rights / license:

In Copyright - Non-Commercial Use Permitted 
Felix Jenni / Dieter Wüest

\section{Steuerverfahren fur selbstgeführte Stromrichter}

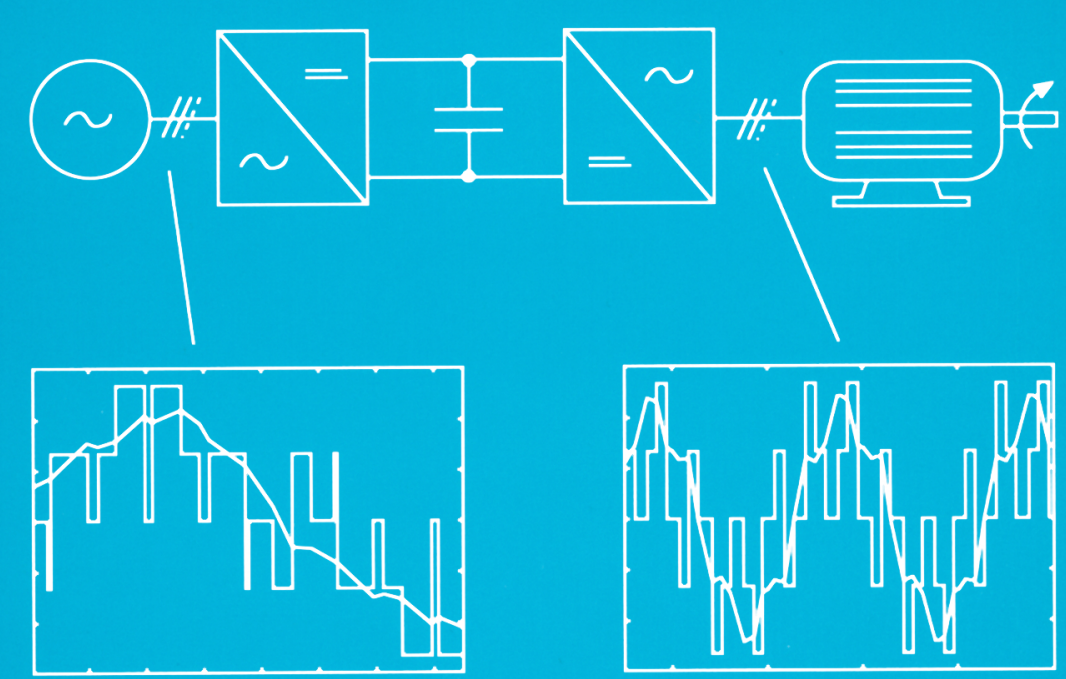


This work is licensed under a creative commons license 주

Die Deutsche Bibliothek - CIP-Einheitsaufnahme

\section{Jenni, Felix:}

Steuerverfahren für selbstgeführte Stromrichter / Felix Jenni;

Dieter Wüest. - Zürich: vdf, Hochschulverl. an der ETH

Zürich; Stuttgart: Teubner, 1995

ISBN 3-519-06176-7 (Teubner)

ISBN 978-3-7281-2141-7 (vdf)

DOI $10.3218 / 2141-\mathrm{X}$

\section{(C) 1995}

vdf Hochschulverlag AG an der ETH Zürich und

B.G. Teubner Stuttgart

www.vdf.ethz.ch 这

verlag@vdf.ethz.ch 


\section{Vorwort}

Mit der Problematik der Steuerung von Stromrichtern wird jedermann konfrontiert, der auf dem Gebiet der Leistungselektronik tätig ist. Verfahren für fremdgeführte Schaltungen sind in Fachbüchern ausführlich dokumentiert. Die Literatur über Steuerverfahren für selbstgeführte Stromrichter beschränkt sich dagegen weitgehend auf Dissertationen, Fachartikel und Konferenzbeiträge. Im Vordergrund stehen dabei in der Regel Teilprobleme und spezielle Anwendungen. Das Schwergewicht der meisten Artikel liegt dementsprechend auf spezifischen Details, während die grundlegenden Zusammenhänge nur knapp und nur auszugsweise behandelt werden. Eine umfassende Zusammenstellung der Steuerverfahren und ihrer Eigenschaften fehlt bis heute. Mit dem vorliegenden Werk soll diese Lücke geschlossen werden. Es kann als weiterführendes Lehrbuch sowie als Nachschlagewerk dienen. Der grösste Teil der beschriebenen Verfahren wurde detailliert nachvollzogen, soweit möglich analytisch behandelt und simuliert. Dies ermöglicht die repräsentativen quantitativen Gegenüberstellungen der einzelnen Verfahren. Wir hoffen mit unserem Beitrag den vielen schon in der Leistungselektronik tätigen und auch den zukünftigen Fachkolleginnen und -kollegen die Arbeit wesentlich zu erleichtern.

Unsere Arbeit war nur mit grosszügiger Hilfe von verschiedenen Seiten möglich. An erster Stelle danken wir der ETH Zürich und dem Lehrstuhl für Leistungselektronik und Messtechnik dafür, dass wir das vorliegende Buch im Rahmen unserer Tätigkeit als Oberassistenten bearbeiten konnten. Ein weiterer Dank geht an die konsultierten Fachleute, die uns bei der Festlegung des Inhalts beratend zur Seite standen. Sehr wertvoll war die Hilfe der Angehörigen des Lehrstuhls. Sie haben uns in interessanten Fachdiskussionen sowie mit dem Durcharbeiten von Entwürfen und der nachfolgenden konstruktiven Kritik sehr unterstützt. Die Aufgabe, das fertige Manuskript durchzusehen, hat freundlicherweise unser langjähriger Kollege Dr. Christian Stulz übernommen. Wir danken auch dem Aurel Stodola Fonds, der einen Beitrag an die Fertigstellung des Buches geleistet hat.

Zürich, im Frühjahr 1995

Felix Jenni Dieter Wüest 
4

Vorwort 


\section{Inhalt}

Verzeichnis der verwendeten Symbole

Teil I: Selbstgeführte Stromrichter als Bausteine von

Frequenzumrichtern

1 Einleitung

2 Funktion und Aufbau von modernen Frequenzumrichtern 20

2.1 Prinzip des Frequenzumrichters. . . . . . . . . . . . . . . . . . . 20

2.2 Anforderungen an einen Frequenzumrichter . . . . . . . . . . . . . . . 22

2.2.1 Anforderungen auf der Netzseite . . . . . . . . . . . . . . . . . 22

2.2.2 Anforderungen für die Speisung einer Drehstrommaschine . . . . . . . .23

2.2.3 Anforderungen für die Speisung einer allgemeinen AC-Last . . . . . . . .25

2.3 Netzseitiger Stromrichter . . . . . . . . . . . . . . . . . . . . 26

2.3.1 Netzseitiger Stromrichter für einen Spannungszwischenkreis . . . . . . . . . . 6

2.3.2 Netzseitiger Stromrichter für einen Stromzwischenkreis . . . . . . . . . . 28

2.4 Lastseitiger Stromrichter . . . . . . . . . . . . . . . . . . . . . . . . . . . . .29

2.4.1 Lastseitiger Stromrichter für einen Spannungszwischenkreis. . . . . . .29

2.4.2 Lastseitiger Stromrichter für einen Stromzwischenkreis . . . . . . . . . 30

2.5 Umrichterkonzepte und ihre Einsatzgebiete . . . . . . . . . . . . . . . . . . .

3 Leistungskreis von Frequenzumrichtern mit selbstgeführten Stromrichtern $\quad 33$

3.1 Schaltelemente der selbstgeführten Stromrichter . . . . . . . . . . . . . . . 33

3.1 .1 Idealisierte Schaltelemente . . . . . . . . . . . . . . . . . . . . . . . . . . . . . . . . . . .

3.1 .2 Reale Leistungshalbleiter . . . . . . . . . . . . . . . . . . . . . . 34

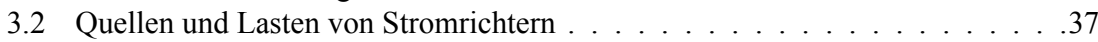

3.2 .1 Netze. . . . . . . . . . . . . . . . . . . . . . 37

3.2 .2 Drehstrommaschinen . . . . . . . . . . . . . . . . . . . . . . . . . . . . . . .

3.2.2.1 Asynchronmaschine . . . . . . . . . . . . . . . . . . . . . . . . . . . . .

3.2.2.2 Synchronmaschine . . . . . . . . . . . . . . . . . . . . . . . . . .

3.2.3 Transformatoren . . . . . . . . . . . . . . . . . . . . . 44

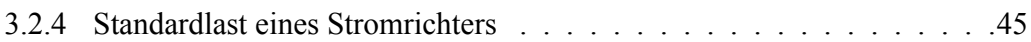

3.2 .5 Zwischenkreis . . . . . . . . . . . . . . . . . . 46

3.2.5.1 Spannungszwischenkreis . . . . . . . . . . . . . . . . . . . . . .

3.2.5.2 Stromzwischenkreis . . . . . . . . . . . . . . . . . . . . . . . . . . . . .

3.2 .6 Filter . . . . . . . . . . . . . . . . . . . . . . . 49

3.2.6.1 Filter für U-Stromrichter . . . . . . . . . . . . . . . . . . . . . . . . . . . . . . . . . .

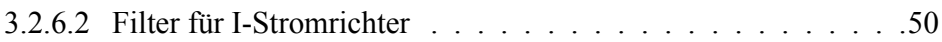

3.2 .7 Stromverdrängung . . . . . . . . . . . . . . . . . . . . . . . . . . . . . . . . .

3.3 Schaltungen von selbstgeführten Stromrichtern . . . . . . . . . . . . .53

3.3.1 Beschreibung durch Schaltfunktionen, kurzzeitige Mittelwerte und

Modulationsfunktionen . . . . . . . . . . . . . . . 53 
3.3.2 Zweistufige Stromrichter für Spannungszwischenkreis-Umrichter . . . .57

3.3.2.1 Einphasige Halbbrücke. . . . . . . . . . . . . . . . . . . . . . . . . . . . . . . . .

3.3.2.2 Einphasige Brücke . . . . . . . . . . . . . . . . . . . . . . . . . . . . .

3.3.2.3 Dreiphasige Brücke . . . . . . . . . . . . . . . . . . . . . . . .

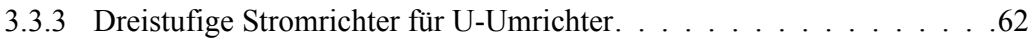

3.3.3.1 Einphasige Halbbrücke. . . . . . . . . . . . . . . . . . . 62 . . . . . . . . .

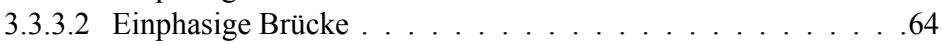

3.3.3.3 Dreiphasige Brücke . . . . . . . . . . . . . . 65

3.3.4 Verallgemeinerte Schaltungen für den U-Umrichter . . . . . . . . . . 66 . . . .

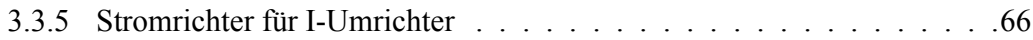

3.3.5.1 Einphasige Brücke . . . . . . . . . . . . . . . . . 66 . . . . . . . . . . . . . .

3.3.5.2 Dreiphasige Brücke . . . . . . . . . . . . . . 68

3.3.6 Überblick über die Kurvenformen von Spannungen und Strömen . . . .69

3.3.7 Dualität von Stromrichtern und Lastkreisen. . . . . . . . . . . . . . . 69

4 Regelkonzepte für selbstgeführte Stromrichter $\quad \mathbf{7 2}$

4.1 Regelung eines Umrichters mit Spannungszwischenkreis . . . . . . . . . . . 72

4.2 Wirk- und Blindstrom am selbstgeführten Stromrichter . . . . . . . . . . . . .73

4.3 Stromregelung. . . . . . . . . . . . . . . . . . . . . . . . . . . . . . . .

4.4 Beispiele für Regelungen von selbstgeführten Stromrichtern . . . . . . . . . . 79

4.4.1 Einfache Regelung eines Netzstromrichters. . . . . . . . . . . . . . . .79

4.4.2 Feldorientierte Regelung einer Asynchronmaschine . . . . . . . . . . . 80

4.4 .3 Speisung eines Inselnetzes. . . . . . . . . . . . . . . . . . . 80

4.5 Zusammenfassung der Anforderungen an den Modulator . . . . . . . . . . . .81

Teil II: Verfahren zur Pulserzeugung am Beispiel des zweistufigen Stromrichters für Spannungszwischenkreis $\quad 83$

5 Beschreibung von Stromrichtern und Pulsmustern 83

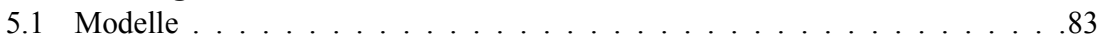

5.2 Definitionen und Kennwerte . . . . . . . . . . . . . . . . .85

5.2 .1 Stationärer Betrieb. . . . . . . . . . . . . . . . . .85

5.2.1.1 AC-Seite (Ausgangsseite) . . . . . . . . . . . . . .85

5.2.1.2 DC-Seite (Zwischenkreis) . . . . . . . . . . . . . . .88

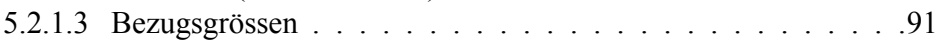

5.2.1.4 Drehmomentwelligkeit bei Drehstrommaschinen . . . . . . 91

5.2.2 Dynamische Betrachtungen . . . . . . . . . . . . . . . . 93

5.2.3 On-Line- und Off-Line-Modulation . . . . . . . . . . . . . . . . .93

5.3 Symmetrien . . . . . . . . . . . . . . . . . . . . . . .94

5.3 .1 Synchrone Steuerverfahren . . . . . . . . . . . . . . . . . .94

5.3.2 Symmetrien innerhalb der Periode . . . . . . . . . . . . . . . . . .94 . . . . . . . .

5.3 .3 Asynchrone Steuerverfahren . . . . . . . . . . . . . . . . 97

6 Grundfrequenzsteuerung 98

6.1 Halbbrücke . . . . . . . . . . . . . . . . . . . . . . . .98

6.2 Einphasige Brücke . . . . . . . . . . . . . . . . . . . . . . 99

6.3 Dreiphasige Brücke . . . . . . . . . . . . . . . . . . . . . 104

$\begin{array}{lll}7 \text { Trägerverfahren } & 109\end{array}$

7.1 Funktionsprinzip der Trägerverfahren . . . . . . . . . . . . . . . 109 
7.1.1 Erzeugen der Schaltsignale . . . . . . . . . . . . . . . . . . 109

7.1 .2 Trägersignale . . . . . . . . . . . . . . . . . 111

7.1 .3 Sollwertsignale . . . . . . . . . . . . . . . 114

7.1.3.1 Kontinuierliche Sollwertsignale . . . . . . . . . . . . . . 114

7.1.3.2 Abgetastete Sollwertsignale . . . . . . . . . . . . . 115

7.1.4 Beschreibung von Schaltfunktionen im Frequenzbereich. . . . . . . . 117

7.1.4.1 Modulationsfunktion und Modulationsgrad . . . . . . . . . . . 122

7.2 Halbbrücke . . . . . . . . . . . . . . . . . . . . . . . 122

7.2.1 Beschreibung von Spannungen und Strömen im Frequenzbereich . . . 122

7.2.2 Näherungsformeln für die Beschreibung von Spannungen und

Strömen . . . . . . . . . . . . . . . . . . . . . . . 124

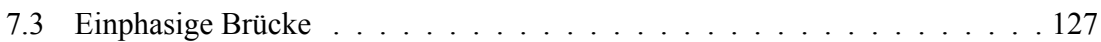

7.3.1 Sollwertsignale . . . . . . . . . . . . . . . . . 127

7.3.2 Trägersignale für einfache Trägerfrequenz in der Phasenspannung . . 127

7.3.3 Trägersignale für doppelte Trägerfrequenz in der Phasenspannung . . 129

7.3.4 Beschreibung von Spannungen und Strömen im Frequenzbereich . . . 129

7.3.5 Näherungsformeln für Spannungen und Ströme . . . . . . . . . . . . . . . . 132

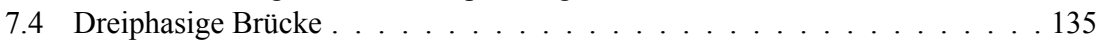

7.4.1 Trägersignale . . . . . . . . . . . . . . . . . . . . . . . . . . . . . . . . . . . . .

7.4 .2 Sollwertsignale . . . . . . . . . . . . . . . . 136

7.4.3 Beschreibung von Spannungen und Strömen im Frequenzbereich . . . 139

7.4.4 Näherungsformeln für die Beschreibung von Spannungen und

Strömen . . . . . . . . . . . . . . . . . 140

7.4.5 Drehmomentwelligkeit . . . . . . . . . . . . . . . . 149

8 Drehzeigermodulation $\quad \mathbf{1 5 2}$

8.1 Funktionsprinzip der Drehzeigermodulation . . . . . . . . . . . . . . . 152

8.2 Freiheitsgrade bei Drehzeigermodulatoren . . . . . . . . . . . . . . . 153

8.2.1 Aufbau eines Drehzeigers aus den Spannungszuständen des

Stromrichters . . . . . . . . . . . . . . . . . . . . 154

8.2.1.1 Nachbildung des Sollwertzeigers durch den kurzzeitigen

Mittelwert der Stromrichterspannung . . . . . . . . . . . . 155

8.2.2 Abfolge der Stromrichterzustände. . . . . . . . . . . . . . . . . 157

8.2.3 Einschaltdauer der Nullzustände . . . . . . . . . . . . . . . . . . 159

8.2.3.1 Beispiele von Aufteilungen der Einschaltdauer der

Nullzustände . . . . . . . . . . . . . . . . 160

8.2.3.2 Verzerrungsströme in Funktion der Aufteilung der

Nullzustände . . . . . . . . . . . . . . . . . . 164

8.3 Analogie zwischen Drehzeigermodulation und Trägerverfahren . . . . . . . . 165

8.4 Phasenspannungen, Verzerrungsströme und Drehmomentwelligkeit . . . . 166

8.4.1 Beschreibung im Frequenzbereich . . . . . . . . . . . . . . 166

8.4.2 Näherungsformeln für die Beschreibung von Spannungen und

Strömen . . . . . . . . . . . . . . . . 166

8.4.3 Drehmomentwelligkeit . . . . . . . . . . . . . 167

9 Vorausberechnete Pulsmuster $\quad \mathbf{1 6 8}$

9.1 Funktionsprinzip . . . . . . . . . . . . . . . . . . . . 168

9.2 Selektive Elimination von Harmonischen . . . . . . . . . . . . . . 171

9.2.1 Einphasige Brücke. . . . . . . . . . . . . . . . . . . 171

9.2.1.1 Berechnung der Schaltwinkel . . . . . . . . . . . . 171 
9.2.1.2 Resultate . . . . . . . . . . . . . . . . . . 174

9.2.1.3 Spezielle Aspekte. . . . . . . . . . . . . . . . . . . . 176

9.2.2 Dreiphasige Brücke . . . . . . . . . . . . . . . . . . 177

9.2.2.1 Berechnung der Schaltwinkel . . . . . . . . . . . . 177

9.2.2.2 Resultate . . . . . . . . . . . . . . . . . . . 179

9.2.2.3 Spezielle Aspekte. . . . . . . . . . . . . . . . . 181

9.3 Optimierte Pulsmuster . . . . . . . . . . . . . . . . . . . . . 182

9.3.1 Das Prinzip der Schaltwinkeloptimierung. . . . . . . . . . . . . . 182

9.3.2 Effektivwert des Verzerrungsstromes als Gütekriterium bei der einphasigen Brücke . . . . . . . . . . . . . . . . . 183

9.3.2.1 Berechnung des Gütekriteriums im Frequenzbereich . . . . . 183

9.3.2.2 Berechnung des Gütekriteriums im Zeitbereich . . . . . . . . 184

9.3.2.3 Resultate der Optimierung . . . . . . . . . . . . . . . . . 187

9.3.3 Minimaler Effektivwert des Verzerrungsstromes bei der dreiphasigen Brücke. . . . . . . . . . . . . . . . . . 188

9.3.3.1 Berechnung des Gütekriteriums im Frequenzbereich . . . . . 188

9.3.3.2 Resultate der Optimierung . . . . . . . . . . . . . . . 193

9.3.4 Optimierung nach weiteren Kriterien . . . . . . . . . . . . . . . . . 195

9.3.5 Dynamische Betrachtungen . . . . . . . . . . . . . . . . . . 199

9.3.5.1 Definition von transienten Zuständen im Modulator. . . . . . 199

9.3.5.2 Realisierung von Übergängen zwischen stationären Zuständen. . . . . . . . . . . . . 201

$\begin{array}{lr}10 \text { Phasenstromregler } & 204\end{array}$

10.1 Halbbrücke mit Zweipunktregler . . . . . . . . . . . . . . . . . . . . . . . 204

10.1.1 Funktionsprinzip. . . . . . . . . . . . . . . . . . 204

10.1.2 Schaltfrequenz und Modulationsfunktion . . . . . . . . . . . . . . 205

10.1.3 Phasenstrom . . . . . . . . . . . . . . . . . . . . 210

10.1.4 Dynamisches Verhalten . . . . . . . . . . . . . . . . . . . . . . . . . . . . . . . 11

10.2 Einphasige Brücke mit Dreipunktregler . . . . . . . . . . . . . . . . . . . . . . . 212

10.2.1 Funktionsprinzip. . . . . . . . . . . . . . . . . . . . . . . . . . . . . . . . . . . .

10.2.2 Schaltfrequenz. . . . . . . . . . . . . . . . . 212



10.2.4 Dynamisches Verhalten . . . . . . . . . . . . . . . . . 216

10.3 Dreiphasige Brücke mit Zweipunktreglern . . . . . . . . . . . . . . . . 217

10.3.1 Funktionsprinzip. . . . . . . . . . . . . . . . . . . 217

10.3.2 Schaltfrequenz und Verzerrungsstrom . . . . . . . . . . . . . . 218

10.3.3 Phasenströme und Zwischenkreisstrom . . . . . . . . . . . . . . . 221

10.3.4 Drehmomentwelligkeit . . . . . . . . . . . . . . . 221 . . . . 221

10.4 Phasenstromregler mit konstanter mittlerer Schaltfrequenz . . . . . . . . . 224

10.5 Diskrete Schaltzustandsänderung . . . . . . . . . . . . . . . . 225

11 Drehzeigerorientierte Stromregelung $\quad 228$

11.1 Prinzip der drehzeigerorientierten Stromregelung . . . . . . . . . . . . . 228

11.2 Stromzeiger-Komponentenregelung . . . . . . . . . . . . . . . . . . 229

11.3 Prädiktive Stromregelung . . . . . . . . . . . . . . . . . . . . . . . . . . . . . 238

11.3.1 Prädiktive Stromregelung mit Schalttabelle. . . . . . . . . . . . . 239

11.3.2 Prädiktive Stromregelung mit optimaler Auswahl des

Schaltzustandes . . . . . . . . . . . . . . . 245

11.3.3 Prädiktive Stromregelung nach dem Deadbeat-Prinzip . . . . . . . . . 247 
12 Spezielle Steuerverfahren für Drehstrommaschinen 251

12.1 Flussorientierte Stromregelung . . . . . . . . . . . . . . . . . 251

12.2 Direkte Fluss- und Drehmomentregelung. . . . . . . . . . . . . . . . . . 254

12.2.1 Regelung mit Schalttabelle . . . . . . . . . . . . . . . . . . . 254

12.2.2 Direkte Selbstregelung . . . . . . . . . . . . . . . . . . . . . . . . . . . . . . . . . . . . . . . . .

12.3 Deltamodulation. . . . . . . . . . . . . . . . . . . . . . 260

13 Vergleich der wichtigsten Steuerverfahren $\quad \mathbf{2 6 4}$

13.1 Klassierung der Verfahren . . . . . . . . . . . . . . . . . . . . . . . . . . . . . . . . . . . . . . . . . . .

13.2 Kennlinien. . . . . . . . . . . . . . . . . . . . 265

13.2.1 Einphasige Brücke. . . . . . . . . . . . . . . . . 265

13.2.2 Dreiphasige Brücke . . . . . . . . . . . . . . . . . . . . . . . . . . 266

13.2.2.1 Netzstromrichter . . . . . . . . . . . . . . . . . . . . . . . . . . . . . . . . . . . .

13.2.2.2 Antriebsstromrichter . . . . . . . . . . . . . . . . . . . . . . . . . . . . . . .

13.3 Spektra . . . . . . . . . . . . . . . . . . . 270

13.4 Frequenzbereiche . . . . . . . . . . . . . . . . . . . 270

13.5 Dynamik. . . . . . . . . . . . . . . . . . . . . 273

13.6 Anwendungsgebiete. . . . . . . . . . . . . . . . . . . . 274

Teil III: Anwendung der Steuerverfahren auf weitere Stromrichter und $\begin{array}{lr}\text { Stromrichtergruppen } & \mathbf{2 7 5}\end{array}$

14 Steuerverfahren für Stromzwischenkreis-Stromrichter $\mathbf{2 7 5}$

14.1 Einphasige Brücke . . . . . . . . . . . . . . . . . . . . . 276

14.2 Dreiphasige Brücke . . . . . . . . . . . . . . . . . . . 278

14.2.1 Grundfrequenzsteuerung . . . . . . . . . . . . . . . . 280

14.2.2 Einsatz von Pulsmustern für U-Stromrichter in I-Stromrichtern . . . . 281

14.2.3 Modifiziertes Trägerverfahren für I-Stromrichter . . . . . . . . . . . . 284

14.2.4 Weitere Steuerverfahren. . . . . . . . . . . . . . . . 285

15 Steuerverfahren für zusammengeschaltete Stromrichter $\mathbf{2 8 6}$

15.1 Serieschaltung von Stromrichtern. . . . . . . . . . . . . . . . . . . . . . . . . . . . . . . . . . . .

15.1.1 Schaltungen . . . . . . . . . . . . . . . . . . . . . . . . . . . . . . . . . . . . . . .

15.1.2 Grundfrequenzsteuerung . . . . . . . . . . . . . . . . 288

15.1.3 Trägerverfahren mit versetzter Taktung. . . . . . . . . . . . . . . . . 290

15.1.4 Drehzeigermodulation. . . . . . . . . . . . . . . . . . . . . . . . . . . . . . . . . . . . . .

15.1.5 Vorausberechnete Pulsmuster . . . . . . . . . . . . . . . . . . . . . . . . . . . . . . . . . . . . . . . .

15.1.6 Direkte Stromregelverfahren . . . . . . . . . . . . . . . . 295

15.1.7 Zwischenkreis . . . . . . . . . . . . . . . . . . . . 296

15.2 Parallelschaltung von Stromrichtern . . . . . . . . . . . . . . . . . . 297

15.2.1 Schaltungen . . . . . . . . . . . . . . . . . . . . . . . . . . . . . . . . . . . . . . . . . . . .

15.2.2 Steuerverfahren . . . . . . . . . . . . . . . . . . . 298

15.2.3 Kreisströme zwischen den Stromrichtern . . . . . . . . . . . . . . 299

15.2.4 Zwischenkreis . . . . . . . . . . . . . . . . . . . . . . . . . . . . . . . . . . . . .

15.3 Spezielle Transformatorschaltungen . . . . . . . . . . . . . . . . . . . . . . . . . . . . . . . . . . . .

15.3.1 Stern-/Dreieckschaltung . . . . . . . . . . . . . . . . . 300

15.3.2 Transformator mit Schwenkwicklungen . . . . . . . . . . . 302

16 Steuerverfahren für dreistufige Spannungszwischenkreis-Stromrichter $\quad \mathbf{3 0 4}$

16.1 Prinzip der Steuerung von dreistufigen Stromrichtern . . . . . . . . . . . . . 304 
16.2 Steuerverfahren . . . . . . . . . . . . . . . . . . 305

16.2.1 Grundfrequenzsteuerung . . . . . . . . . . . . . . . . 305

16.2.2 Trägerverfahren . . . . . . . . . . . . . . . . . . . . . . . . . . . . . . . . . . . .

16.2.3 Drehzeigermodulation. . . . . . . . . . . . . . . . . . . . . . . . . . . . . . . . . . . . . .

16.2.4 Weitere Steuerverfahren. . . . . . . . . . . . . . . . . . . . . . . . . . . . . . . . . . . . . . . .

16.2.5 Zwischenkreis . . . . . . . . . . . . . . . 313

Teil IV: Praktischer Einsatz von Steuerverfahren 315

17 Nichtidealitäten im Modulator und im Leistungskreis $\quad \mathbf{3 1 5}$

17.1 Modulator . . . . . . . . . . . . . . . . . . . 316

17.1.1 Quantisierungsfehler bei digitalen Modulatoren . . . . . . . . . . 316

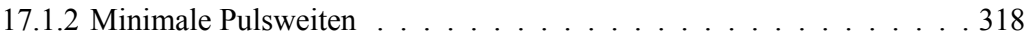

17.1.3 Unsymmetrien . . . . . . . . . . . . . . . . . . . . . . . . 319

17.2 Leistungshalbleiter . . . . . . . . . . . . . . . . . . . . . . . 319

17.2.1 Leitspannungsabfall . . . . . . . . . . . . . . . . . . . . 319

17.2.2 Schaltverzögerung und Steilheit der Spannungsflanken . . . . . . . . 321

17.3 Stromrichteraufbau . . . . . . . . . . . . . . . . . . . . . . . . . . . . . . . . . . . . . . . . . .

17.4 Last . . . . . . . . . . . . . . . . . . . . . . 326

17.4.1 Einfluss der geschalteten Spannungen auf die Last . . . . . . . . . . . 326

17.5 Welligkeit der Zwischenkreisspannung. . . . . . . . . . . . . . . . 326

18 Elektromagnetische Verträglichkeit $\quad 330$

18.1 Umgebung von selbstgeführten Stromrichtern . . . . . . . . . . . . . . . . . 330

18.1.1 Selbstgeführte Stromrichter als Störquellen. . . . . . . . . . . . . 330

18.1.2 Störwege. . . . . . . . . . . . . . . . . 331

18.1.3 Massnahmen zur elektromagnetischen Verträglichkeit . . . . . . . . . 332

18.2 Normen und Vorschriften . . . . . . . . . . . . . . . . . . . . . . . 333

$\begin{array}{ll}19 \text { Implementierung von Modulatoren } & 337\end{array}$

19.1 Schaltungen . . . . . . . . . . . . . . . . . . . . . . . . . . . . . . . . . . . . . . . .

19.1.1 Analoge Schaltungen . . . . . . . . . . . . . . . . . . 337 . . . . . . . . . . . . . .

19.1.2 Digitale Schaltungen. . . . . . . . . . . . . . . . . . . 338

19.1.3 Modulatoren mit logischen Schaltwerken. . . . . . . . . . . . . . . 339

19.1.4 Implementierungen auf einem Mikroprozessor . . . . . . . . . . . . . 341

19.2 Integrierte Bausteine . . . . . . . . . . . . . . . . . . . . . . . . . . . . . . . . . . . . . . . . . . . . . . .

19.3 Praktische Probleme. . . . . . . . . . . . . . . . . . . . 345

Teil V: Anhänge 349

A Literatur $\quad 349$

$\begin{array}{ll}\text { B Verwendete Grundlagen } & \mathbf{3 5 7}\end{array}$

B.1 Das Dreiphasensystem . . . . . . . . . . . . . . . . . . . . . 357

B.1.1 Spannungen und Ströme. . . . . . . . . . . . . . . . . . . 357

B.1.2 Beschreibung von Dreiphasensystemen mittels Zeigern . . . . . . . . 359

B.2 Fourierreihe . . . . . . . . . . . . . . . . . 361

B.2.1 Periodische Signale . . . . . . . . . . . . . . . . . . . . . . . . . . . . . . . . . . . . .

B.2.2 Nicht exakt periodische Signale . . . . . . . . . . . . 363

$\begin{array}{ll}\text { C Sachverzeichnis } & 365\end{array}$ 


\section{Verzeichnis der verwendeten Symbole}

\begin{tabular}{|c|c|c|c|}
\hline \multicolumn{2}{|c|}{$\begin{array}{l}\text { Darstellung von Spannungen und } \\
\text { Strömen }\end{array}$} & $F$ & $\begin{array}{l}\text { allgemeine mathematische Funk- } \\
\text { tion }\end{array}$ \\
\hline$i, u$ & Momentanwerte & $f$ & $\begin{array}{l}\text { Freiheitsgrade bei vorausberech- } \\
\text { neten Pulsmustern }\end{array}$ \\
\hline$\hat{\imath}, \hat{u}$ & Amplituden (Scheitelwerte) & I & Frequenz \\
\hline $\bar{i}, u$ & kurzzeitige Mittelwerte & $f_{\text {inst }}$ & momentane Schaltfrequenz \\
\hline$I, U$ & lineare Mittelwerte & $f_{L}$ & Frequenz im Lastkreis \\
\hline$I_{\text {eff }}, U_{\text {eff }}$ & $\begin{array}{l}\text { Effektivwerte (quadratische Mit- } \\
\text { telwerte) }\end{array}$ & $f_{N}$ & $\begin{array}{l}\text { Netzfrequenz } \\
\text { Resonanzfrequenz }\end{array}$ \\
\hline$I_{d}, U_{d}$ & $\begin{array}{l}\text { lineare Mittelwerte von Gleich- } \\
\text { grössen (im Zwischenkreis) }\end{array}$ & $\begin{array}{l}\text { Res } \\
f_{S} \\
f_{T}\end{array}$ & $\begin{array}{l}\text { mittlere Schaltfrequenz } \\
\text { Trägerfrequenz }\end{array}$ \\
\hline$i_{1}, u_{1}$ & $\begin{array}{l}\text { Momentanwerte der Grund- } \\
\text { schwingungen }\end{array}$ & $f_{\text {Tast }}$ & $\begin{array}{l}\text { Abtastfrequenz } \\
\text { Grundfrequenz }\end{array}$ \\
\hline$\hat{\imath}_{1}, \hat{u}_{1}$ & $\begin{array}{l}\text { Amplituden der Grundschwin- } \\
\text { gungen }\end{array}$ & $\frac{G}{g}$ & $\begin{array}{l}\text { komplexe Übertragungsfunktion } \\
\text { Gewichtungsfunktion }\end{array}$ \\
\hline$i_{v}, u_{v}$ & $\begin{array}{l}\text { Momentanwerte der } v \text {-ten Harmo- } \\
\text { nischen }\end{array}$ & $\begin{array}{l}I_{B} \\
I_{\delta}\end{array}$ & $\begin{array}{l}\text { Bezugswert für die Ströme } \\
\text { Toleranzband für den Strom }\end{array}$ \\
\hline$\hat{\imath}_{v}, \hat{u}_{v}$ & $\begin{array}{l}\text { Amplitude der v-ten Harmo- } \\
\text { nischen }\end{array}$ & $i$ & $\begin{array}{l}\text { Strom } \\
\text { Laufvariable }\end{array}$ \\
\hline$\underline{\hat{\imath}}, \underline{\hat{u}}$ & $\begin{array}{l}\text { komplexe Darstellung von Wech- } \\
\text { selgrössen }\end{array}$ & $i_{A / B}$ & $\begin{array}{l}\text { Ausgangsströme in den Brücken- } \\
\text { zweigen } A \text { bzw. } B\end{array}$ \\
\hline$\underline{i}, \underline{u}$ & Drehzeiger (Momentanwerte) & $i_{C}$ & Kondensatorstrom \\
\hline$i_{\alpha / \beta}, u_{\alpha / \beta}$ & Komponenten der Drehzeiger & $i_{d}$ & Zwischenkreisstrom \\
\hline $\begin{array}{l}i, u \\
i_{d / q}, u_{d / q}\end{array}$ & $\begin{array}{l}\text { Festzeiger (Momentanwerte) } \\
\text { Komponenten der Festzeiger }\end{array}$ & $i_{d+/ d 0 / d-}$ & $\begin{array}{l}\text { Ströme in den Zwischenkreislei- } \\
\text { tern }\end{array}$ \\
\hline & & $i_{k}$ & Kurzschlussstrom \\
\hline Formel- & nd Schaltplanzeichen & & $\begin{array}{l}\text { Laststrom } \\
\text { Netzstrom }\end{array}$ \\
\hline$A$ & Amplitude & $i_{R / S}$ & Strom im Rotor bzw. Stator bei \\
\hline$a, b, c$ & $\begin{array}{l}\text { Projektionsachsen der Phasen } U, V \\
\text { bzw. } W \text { in der Drehzeiger-Ebene }\end{array}$ & $i_{\text {Soll }}$ & $\begin{array}{l}\text { Drehstrommaschinen } \\
\text { Sollwert des Stromes }\end{array}$ \\
\hline$a_{v}$ & Fourierkoeffizient der Cosinus- & $i_{V Z}$ & Verzerrungsstrom \\
\hline$b_{v}$ & $\begin{array}{l}\text { Schwingung } \\
\text { Fourierkoeffizient der Sinus- }\end{array}$ & $i_{U / V}$ & $\begin{array}{l}\text { Ausgangsstrome in den Brucken- } \\
\text { zweigen } U, V \text { bzw. } W\end{array}$ \\
\hline$C$ & $\begin{array}{l}\text { Schwingung } \\
\text { Kapazität }\end{array}$ & $\underline{i}_{S t r}$ & $\begin{array}{l}\text { Drehzeiger der Stromrichter-Pha- } \\
\text { senströme }\end{array}$ \\
\hline$C_{d}$ & Zwischenkreiskapazität & $j$ & komplexer Operator \\
\hline$C_{F}$ & Filterkapazität & $j$ & Laufvariable \\
\hline$d, q$ & Achsen in der Festzeigerebene & $K$ & Konstante \\
\hline$e$ & Gegenspannung & $\begin{array}{l}k \\
k_{i}\end{array}$ & $\begin{array}{l}\text { Laufvariable } \\
\text { Klirrfaktor des Stromes }\end{array}$ \\
\hline
\end{tabular}




\begin{tabular}{|c|c|c|c|}
\hline$k_{u}$ & Klirrfaktor der Spannung & \multirow[t]{3}{*}{ SI...SVI } & \multirow[t]{2}{*}{$\begin{array}{l}\pi / 3 \text {-Sektoren in der Drehzeigere- } \\
\text { bene, gegenüber } S 1 \ldots S 6 \text { um } \pi / 6 \text { ge- }\end{array}$} \\
\hline$L$ & Induktivität & & \\
\hline$L_{d}$ & Zwischenkreisinduktivität & & dreht \\
\hline$L_{k}$ & Kurzschlussinduktivität im Last- & \multirow{3}{*}{$\begin{array}{l}s \\
s_{A / B}\end{array}$} & Schaltfunktion \\
\hline & kreis & & Schaltfunktion der Umschalter in \\
\hline$L_{F}$ & Filterinduktivität & & den Brückenzweigen $A$ bzw. $B$ \\
\hline$L_{h}$ & Hauptinduktivität & \multirow[t]{2}{*}{$s_{A+/ A-}$} & Schaltfunktion der Schalter im \\
\hline$L_{\sigma}$ & Streuinduktivität & & Brückenzweig $A$ \\
\hline$l$ & $\begin{array}{l}\text { Anzahl Lösungen bei vorausbe- } \\
\text { rechneten Pulsmustern }\end{array}$ & $s_{U / V / W}$ & $\begin{array}{l}\text { Schaltfunktion der Umschalter in } \\
\text { den Brückenzweigen } U, V \text { bzw. } W\end{array}$ \\
\hline$m$ & Modulationsfunktion & $T$ & Periodendauer \\
\hline$M$ & Modulationsgrad & $T_{R}$ & Rotorzeitkonstante \\
\hline \multirow[t]{2}{*}{$M_{B}$} & Bezugswert für das elektrische & $T_{T}$ & Periode der Trägerfrequenz \\
\hline & Drehmoment & $T_{\text {Tast }}$ & Periode des Abtastfrequenz \\
\hline$M_{e l}$ & elektrisches Drehmoment & $T_{1}$ & Periode der Grundschwingung \\
\hline \multirow[t]{2}{*}{$M_{e l, V Z}$} & \multirow{2}{*}{$\begin{array}{l}\text { Welligkeit des elektrischen Dreh- } \\
\text { momentes (im Leerlauf) }\end{array}$} & $t$ & Zeit \\
\hline & & $t_{d}$ & Verzögerungszeit (Delay Time) \\
\hline$M_{m e c h}$ & mechanisches Drehmoment & $t_{E}$ & Einschaltzeit \\
\hline$n$ & Laufnummer der Trägerbänder & $t_{f}$ & Abfallzeit (Fall Time) \\
\hline \multirow[t]{2}{*}{$P$} & \multirow{2}{*}{$\begin{array}{l}\text { zeitlicher Mittelwert der Leistung, } \\
\text { Wirkleistung }\end{array}$} & $t_{r}$ & Anstiegszeit (Rise Time) \\
\hline & & $t_{T o t}$ & Totzeit \\
\hline$P_{d}$ & Leistung im Zwischenkreis & \multirow[t]{2}{*}{$t_{0} \ldots t_{7}$} & Einschaltzeiten der Stromrichter- \\
\hline$P_{L}$ & Wirkleistung im Lastkreis & & zustände ${ }^{0} Z \ldots{ }^{7} Z$ \\
\hline$P_{N}^{L}$ & Wirkleistung im Netz & \multirow{4}{*}{$\begin{array}{l}U_{B} \\
{ }^{0} \underline{U} . .{ }^{7} \underline{U}\end{array}$} & Bezugswert für Spannungen \\
\hline$Q$ & Blindleistung & & Diskrete Spannungszustände am \\
\hline$Q_{L}$ & Blindleistung im Lastkreis & & Stromrichter in Drehzeigerdarstel- \\
\hline$Q_{N}$ & Blindleistung im Netz & & lung \\
\hline$q$ & Schaltzahl & $u$ & Spannung \\
\hline$R$ & ohmscher Widerstand & \multirow{2}{*}{$u_{A 0 / B 0}$} & Mittelpunktspannungen bei ein- \\
\hline$R_{k}$ & ohmscher Anteil der Kurzschlus- & & phasigen Schaltungen \\
\hline \multirow{3}{*}{$R_{N}$} & \multirow{3}{*}{$\begin{array}{l}\text { simpedanz im Lastkreis } \\
\text { ohmscher Anteil der Netzimpe- } \\
\text { danz }\end{array}$} & \multirow[t]{2}{*}{$u_{A B}$} & Phasenspannung bei der einpha- \\
\hline & & & sigen Brückens \\
\hline & & $u_{d}$ & Zwischenkreisspannung \\
\hline \multirow[t]{2}{*}{$R_{R / S}$} & Rotor- und Statorwiderstand bei & $u_{G}$ & Gleichtaktspannung \\
\hline & Drehstrommaschinen & $u_{L}$ & Lastspannung \\
\hline \multirow[t]{2}{*}{$R_{1 / 2}$} & \multirow{2}{*}{$\begin{array}{l}\text { Wicklungswiderstände von Trans- } \\
\text { formatoren }\end{array}$} & $u_{N}$ & Netzspannung \\
\hline & & $u_{N 0}$ & Sternpunkt(-Nullpunkt)-Spannung \\
\hline$S_{A / B}$ & $\begin{array}{l}\text { Umschalter im Brückenzweig } A \\
\text { bzw. } B\end{array}$ & $u_{R / S}$ & $\begin{array}{l}\text { Spannungen im Rotor bzw. Stator } \\
\text { bei Drehstrommaschinen }\end{array}$ \\
\hline \multirow{3}{*}{$\begin{array}{l}S_{A+/ A-} \\
S_{U / V / W}\end{array}$} & Schalter im Brückenzweig $A$ & \multirow[t]{2}{*}{$u_{U 0 / V 0 / W 0}$} & Mittelpunktspannungen bei der \\
\hline & Umschalter im Brückenzweig $U, V$ & & dreiphasigen Brückenschaltung \\
\hline & bzw. $W$ & \multirow[t]{2}{*}{$u_{U / V / W}$} & Phasenspannungen bei der drei- \\
\hline$S$ & Scheinleistung & & phasigen Brückenschaltung \\
\hline & Scheinleistung im Lastkreis & \multirow[t]{2}{*}{$u_{U V / V W / W U}$} & verkettete Spannungen bei der \\
\hline & Scheinleistung im Netz & & dreiphasigen Brückenschaltung \\
\hline \multirow[t]{3}{*}{$S 1 \ldots S 6$} & $\pi / 3$-Sektoren in der Drehzeigere- & $u_{\text {Soll }}$ & Sollwert der Spannung \\
\hline & bene, begrenzt durch ${ }^{1} \underline{U} . .{ }^{6} \underline{U}$ & $\underline{u}_{S t r}$ & $\begin{array}{l}\text { Drehzeiger der Stromrichter-Mit- } \\
\text { telpunkt- und Phasenspannungen }\end{array}$ \\
\hline & & $W$ & Energie \\
\hline
\end{tabular}




\begin{tabular}{|c|c|c|c|}
\hline$X$ & Reaktanz & $B$ & Bezugswert \\
\hline$x$ & allgemeines Signal & $d$ & Gleichgrössen (Zwischenkreis) \\
\hline$x$ & Hilfsgrösse & $d, q$ & $d$ - bzw. $q$-Komponente von Fest- \\
\hline$x_{T}$ & Trägersignal & & zeigern \\
\hline$x_{\text {Soll }}$ & Sollwertsignal & $F$ & Filter \\
\hline$Z$ & Impedanz & $G$ & Gleichtaktkomponente \\
\hline$Z$ & Zielfunktion bei Optimierungen & Ist & Istwert \\
\hline${ }^{0} Z \ldots{ }^{7} Z$ & $\begin{array}{l}\text { Diskrete Stromrichterzustände } \\
\text { (entsprechend }{ }^{0} \underline{U} . .{ }^{7} \underline{U} \text { ) }\end{array}$ & $\begin{array}{l}\text { inst } \\
k\end{array}$ & $\begin{array}{l}\text { Momentanwert } \\
\text { Kurzschluss }\end{array}$ \\
\hline$z_{S t r}$ & $\begin{array}{l}\text { Variable für den Stromrichter-Zu- } \\
\text { stand }\end{array}$ & $\begin{array}{l}L \\
M \\
\max \end{array}$ & $\begin{array}{l}\text { Lastkreis } \\
\text { Moment } \\
\text { Maximalwert }\end{array}$ \\
\hline$\alpha$ & Steuerwinkel & $\min$ & Minimalwert \\
\hline$\alpha, \beta$ & Achsen in der Drehzeigerebene & $N$ & Netz \\
\hline$\alpha, \beta$ & Schaltwinkel & $N$ & Sternpunkt \\
\hline$\gamma$ & Phasenwinkel des Trägersignals & $n$ & Nennwert \\
\hline$\Delta$ & Abweichung, Differenz & $n$ & Laufnummer der Trägerbänder \\
\hline$\mu$ & Laufnummer der Seitenlinien bei & $R$ & Rotor \\
\hline & Trägerverfahren & $S$ & Stator \\
\hline$v$ & Ordnungszahl der Harmonischen & $S$ & Schalter \\
\hline$\varphi$ & Phasenwinkel & Soll & Sollwert \\
\hline$\varphi_{e}$ & Phasenwinkel der Gegenspannung & Str & Stromrichter \\
\hline$\varphi_{m}$ & $\begin{array}{l}\text { Phasenwinkel der Modulations- } \\
\text { funktion }\end{array}$ & $\begin{array}{l}T \\
\text { Tast }\end{array}$ & $\begin{array}{l}\text { Träger } \\
\text { Abtastung }\end{array}$ \\
\hline$\varphi_{u / i}$ & $\begin{array}{l}\text { Phasenwinkel der Spannung bzw. } \\
\text { des Stromes }\end{array}$ & $U, V, W$ & $\begin{array}{l}\text { Brückenzweig in dreiphasigen } \\
\text { Schaltungen }\end{array}$ \\
\hline$\varphi_{\text {Soll }}$ & Phasenwinkel des Sollwertes & $V$ & Verlust \\
\hline$\varphi_{v}$ & $\begin{array}{l}\text { Phasenwinkel der } v \text {-ten Harmo- } \\
\text { nischen }\end{array}$ & $V Z$ & Verzerrungsanteil \\
\hline$\varphi_{1}$ & $\begin{array}{l}\text { Phasenwinkel der Grundschwin- } \\
\text { gung }\end{array}$ & 0 & $\begin{array}{l}\text { Mittelpunkt des Zwischenkreises } \\
\text { (Nullpunkt) }\end{array}$ \\
\hline $\begin{array}{l}\Psi_{R / S} \\
\omega\end{array}$ & $\begin{array}{l}\text { Rotor- bzw. Statorflussverkettung } \\
\text { Kreisfrequenz }\end{array}$ & $(v=) 1$ & Grundschwingung \\
\hline $\begin{array}{l}\omega_{B} \\
\omega_{\text {mech }}\end{array}$ & $\begin{array}{l}\text { Bezugswert für Kreisfrequenz } \\
\text { Kreisfrequenz der Welle bei Dreh- }\end{array}$ & $\alpha, \beta$ & $\begin{array}{l}\alpha \text { - bzw. } \beta \text {-Komponente von Dreh- } \\
\text { zeigern }\end{array}$ \\
\hline & strommaschinen & $\mu$ & Laufnummer der Seitenlinien bei \\
\hline$\omega_{R / S}$ & Rotor- bzw. Statorkreisfrequenz & & Trägerverfahren \\
\hline$\omega_{T}$ & Träger-Kreisfrequenz & $v$ & Ordnungszahl von Harmonischen \\
\hline$\omega_{1}$ & $\begin{array}{l}\text { Kreisfrequenz der Grundschwin- } \\
\text { gung }\end{array}$ & $\Psi$ & Fluss \\
\hline
\end{tabular}

Allgemeine Begriffe und Abkürzungen

Häufig verwendete Indices

$\begin{array}{ll}a, b, c & \begin{array}{l}\text { Auf die Achse } a, b \text { bzw. } c \text { bezoge- } \\ \text { ne Komponente von Drehzeigern }\end{array} \\ a, b, c & \text { Teilstromrichter } \\ A, B & \text { Brückenzweig in einphasigen } \\ & \text { Schaltungen }\end{array}$

AC Wechselgrösse

ASM Asynchronmaschine

DC Gleichgrösse

SM Synchronmaschine

$x$, Ersatzgrösse für $x$

$\underline{x}^{*} \quad$ konjugiert-komplexer Zeiger zu $\underline{x}$ 


\section{Teil I}

\section{Selbstgeführte Stromrichter als Bausteine von Frequenzum- richtern}

\section{Einleitung}

Die grundlegende Aufgabe von leistungselektronischen Schaltungen, als Teil eines Gesamtsystems, ist die Umformung von elektrischer Energie bei gleichzeitiger Steuerung des Energieflusses. Die Spannungen und Ströme können dabei als Wechselgrössen einer bestimmten Frequenz (AC-Grössen) oder als Gleichgrössen (DC-Grössen) vorliegen. Die sich ergebenden Varianten der Umformung sind schematisch in Bild 1.1 dargestellt. Als Hauptklassen können die AC-DC-Wandler (die DC-AC-Wandler sind dort mit eingeschlossen), die AC-AC-Wandler sowie die DC-DC-Wandler betrachtet werden.

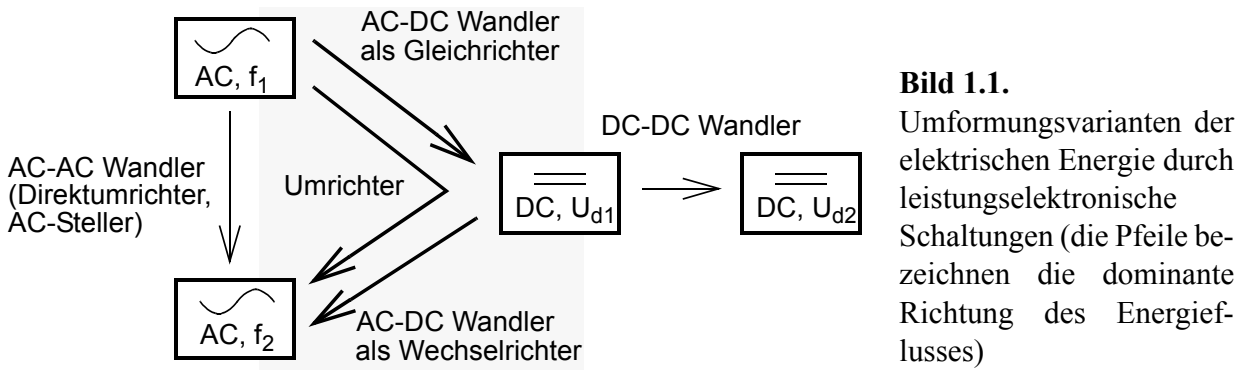

Das vorliegende Buch beschränkt sich auf die Behandlung der $A C$-DC-Wandler. Sie werden gemäss DIN 41750 abhängig von der dominanten Energieflussrichtung auch als Gleich- oder Wechselrichter bezeichnet: findet sie von AC nach DC statt, so liegt ein Gleichrichter vor, während bei umgekehrtem Energiefluss von einem Wechselrichter gesprochen wird. In diesem Buch wird für beide Wandler mehrheitlich der gemeinsame Oberbegriff Stromrichter verwendet.

Die (Frequenz-)Umrichter sind aus einem Gleich- und einem Wechselrichter aufgebaut und nehmen eine Wandlung von AC über DC wieder zu AC vor. Sie werden häufig auch 
als Zwischenkreisumrichter bezeichnet, um sie eindeutig von den Direktumrichtern, die in der Klassierung nach Bild $1.1 \mathrm{zu}$ den direkten AC-AC-Wandlern gehören, zu unterscheiden. Sie weisen gegenüber den direkten AC-AC-Wandlern erhebliche Vorteile auf. Ihre wichtige Rolle in der Praxis führt dazu, dass sie als eigener Wandlertyp betrachtet werden.

Umrichter haben in den letzten Jahren stetig an Bedeutung gewonnen. Der Grund dafür ist vor allem die Ablösung der herkömmlichen Gleichstromantriebe durch umrichtergespeiste Drehstromantriebe in praktisch allen Bereichen der elektrischen Antriebstechnik. Die Drehstrommotoren sind kostengünstiger, wartungsfreundlicher und kompakter als Gleichstrommotoren. Es finden aber auch andere typische Umrichteranwendungen, z.B. unterbrechungsfreie Stromversorgungen, statische Kupplungen zwischen Versorgungsnetzen, getaktete Leistungsverstärker usw. eine immer grössere Verbreitung. Die AC-DC Wandler als selbständige Schaltungen haben neben ihren traditionellen Einsatzgebieten als Gleichrichter, z.B. in Gleichstromantrieben, in Elektrolyseanlagen und für die Ladung von Akkumulatoren, neue Aufgaben als Wechselrichter erhalten: Gleichstrombahnen mit Wechselstrommotoren, batteriegespeiste elektrische Fahrzeuge mit Wechselstrommotoren, photovoltaische Energieerzeugung u.a.

Die traditionellen Umrichter sind aus fremdgeführten Gleich- und Wechselrichterschaltungen (mit Dioden und Thyristoren) aufgebaut und weisen einen Gleichstromzwischenkreis auf. Sie werden deshalb auch als I-Umrichter bezeichnet. Diese Technik ist bis heute in allen Anwendungsgebieten verbreitet. Die verhältnismässig ungünstigen Kurvenformen der Spannungen und Ströme am Ein- und Ausgang des Umrichters, sowie der vom eingestellten Betriebspunkt abhängige Blindleistungsbedarf, haben jedoch dazu geführt, dass die fremdgeführten Schaltungen in zunehmendem Masse durch selbstgeführte Schaltungen (mit abschaltbaren Leistungshalbleitern) ersetzt werden. Diese ermöglichen einerseits die Erzeugung von erheblich besseren Kurvenformen und andererseits eine beliebige Einstellung der Blindleistung auf beiden Seiten des Umrichters. Damit ist es im Gegensatz zu einer fremdgeführten Schaltung ohne Zusatzaufwand möglich, eine Asynchronmaschine zu speisen. Während der Ablösungsprozess von fremdgeführt zu selbstgeführt auf der Lastseite mehrheitlich vollzogen ist, geschieht er auf der Netzseite mit etwas Verzögerung. Die bestehenden Vorschriften bezüglich Verzerrungsanteil der aus dem Netz bezogenen Ströme (Netzrückwirkungen) erlauben nach wie vor die Verwendung der konventionellen Gleichrichterschaltungen. Diese sind deutlich kostengünstiger als selbstgeführte. Die geplante Verschärfung der entsprechenden Normen wird jedoch auch hier in nächster Zukunft zu einem vermehrten Einsatz der selbstgeführten Schaltungen führen.

Innerhalb der Umrichter mit selbstgeführten Schaltungen haben sich solche mit Spannungszwischenkreis (U-Umrichter) weitgehend durchgesetzt. Eine relativ grosse Kapazität im Gleichspannungszwischenkreis sorgt für eine gut geglättete Gleichspannung. Die selbstgeführten Stromrichter erzeugen daraus auf der AC-Seite geschaltete Spannungen. Die weitaus grösste Verbreitung haben die ein- und dreiphasigen Brückenschaltungen gemäss Bild 1.2, die aus 4 bzw. 6 abschaltbaren Leistungshalbleitern und den dazu antiparallelen Dioden aufgebaut sind. Sie stellen die einfachsten Topologien dar, die eine gleichmässige und hohe Ausnutzung aller Leistungshalbleiter ermöglichen. 

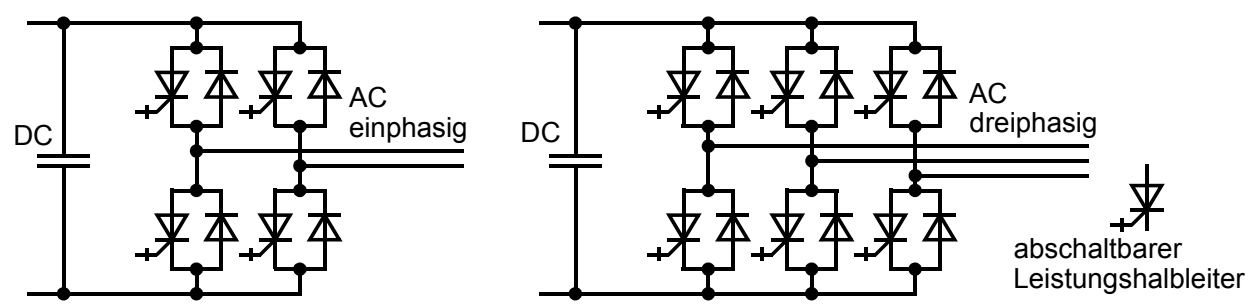

Bild 1.2. Ein- und dreiphasige Brücke mit abschaltbaren Leistungshalbleitern für Spannungszwischenkreis (U-Stromrichter)

U-Umrichter mit selbstgeführten Brückenschaltungen auf einer oder gar auf beiden Seiten sind heute die Standardlösungen für fast alle Umrichteranwendungsgebiete von wenigen Watt bis zu mehreren Megawatt Leistung.

In speziellen Fällen kommen auch andere selbstgeführte Stromrichterschaltungen zum Einsatz. Vor allem im Bereich sehr grosser Leistungen über 1MW reichen die zweistufigen Brückenschaltungen nach Bild 1.2 häufig nicht mehr aus, weil keine Leistungshalbleiter mit den erforderlichen Grenzdaten existieren. An ihre Stelle treten dreistufige oder sogar höherstufige Brückenschaltungen, die mit gleichen Halbleitern auf dem doppelten Spannungsniveau arbeiten können. Im weiteren werden auch Stromrichteranordnungen gebaut, die einer Serie- oder Parallelschaltung von zwei oder mehreren Brücken entsprechen.

Auch I-Umrichter lassen sich mit einem oder zwei selbstgeführten Stromrichtern realisieren. In den wenigen Anwendungen dieser Variante sind die Stromrichter stets einfache Brückenschaltungen.

Es ist die Aufgabe des Umrichters, an seinen Ein- und -Ausgängen die Spannungen und Ströme auf diejenigen Werte einzustellen, welche durch die Anwendung und den gewünschten Betriebspunkt bestimmt sind. In den meisten Fällen sind dies stationär betrachtet sinusförmige Verläufe mit bestimmten Amplituden, Phasenlagen und Frequenzen. Wegen der schaltenden Arbeitsweise der Stromrichter ist es nicht möglich, diese Kurvenformen exakt nachzubilden. Vielmehr ergeben die Schalthandlungen neben den angestrebten Verläufen (Grundschwingungen) Verzerrungen in den Spannungen und Ströme. Diese verursachen zusätzliche Verluste in Umrichtern, Netzen und Lasten und ergeben Verzerrungsblindleistung, welche eine grössere Dimensionierung aller Schaltungskomponenten nach sich zieht. Zusätzlich ergeben sich weitere, anwendungsspezifische Beeinflussungen der Last. Wichtige Beispiele dafür sind die Drehmomentwelligkeit in umrichtergespeisten Antrieben oder die Verzerrung der Spannung in einem Netz durch den verzerrten Strom eines Stromrichters.

Bei gegebenen Schaltungen und Betriebspunkten ist die Art und Grösse der Verzerrungen allein durch die eingesetzten Steuerverfahren für die Stromrichter (häufig auch als Modulationsverfahren oder Taktverfahren bezeichnet) bestimmt. Eine geschickte Steuerung ermöglicht die Optimierung der Kurvenformen und beeinflusst damit die erwähnten Unzulänglichkeiten massgeblich. Es ergeben sich dabei jedoch z.T. einander widersprechende Randbedingungen für das Steuerverfahren. Das wichtigste Beispiel ist die Schalt- 
frequenz: ihre Erhöhung würde in den meisten Fällen eine Reduktion der unerwünschten Phänomene mit sich bringen. Da aber die Schaltverluste des Stromrichters proportional zur Schaltfrequenz ansteigen, kann diese Eigenschaft nur beschränkt ausgenutzt werden. Für die sehr unterschiedlichen Anwendungsgebiete und Leistungsbereiche der Umrichter ergeben sich völlig verschiedene optimale Steuerverfahren, die stets Kompromisse zwischen verschiedenen Optimierungszielen darstellen.

Die zentrale Bedeutung der Steuerverfahren und die Problematik ihrer Optimierung hat in den letzten Jahren eine grosse Zahl von entsprechenden Fachartikeln entstehen lassen. Die meisten von ihnen stellen ausgewählte Steuerverfahren vor und optimieren diese für bestimmte Anwendungen. Daneben gibt es auch Übersichtsartikel, welche verschiedene Verfahren miteinander vergleichen. Die Beschreibung der einzelnen Verfahren fällt dort allerdings stets sehr knapp aus. Eine Auswahl von Übersichtsartikeln ist im Literaturverzeichnis (Anhang A) in einer separaten Liste aufgeführt.

Dieses Buch stellt die Steuerverfahren für selbstgeführte Stromrichter in den Mittelpunkt. Es gibt eine Übersicht über die Grundtypen von Verfahren und beschreibt ihre wichtigsten Varianten ausführlich. Die Wahl von einheitlichen Schaltungen und Betriebszuständen sowie die Definition von Kennwerten ermöglicht direkte Vergleiche zwischen den Verfahren. Neben den Standardverfahren wird ausserdem eine ganze Reihe von abgewandelten Lösungen und weiteren speziellen Steuerverfahren kurz behandelt. Dabei muss die Auswahl allerdings auf die wichtigsten der zahlreichen in der Literatur vorgeschlagenen Varianten beschränkt bleiben.

$\mathrm{Zu}$ allen behandelten Verfahren wird auf entsprechende Fachartikel verwiesen. Sie sollen in erster Linie zur Vertiefung der behandelten Themen dienen. Zum Teil beschreiben sie auch weitere verwandte Verfahren, auf die in diesem Buch nicht eingegangen wird. Die angegebenen Quellen stellen eine beschränkte Auswahl aus der riesigen Anzahl von Fachpublikationen dar. Verweise auf Dissertationen, die das Thema Steuerverfahren behandeln, werden bewusst keine gemacht, da diese in der Regel nur schwer zugänglich sind.

Das Buch ist in 5 Teile gegliedert, welche je einen weitgehend in sich abgeschlossenen Themenkreis behandeln:

- Teil I gibt eine Einführung in die Thematik der Umrichter. Die relevanten Schaltungen und ihre Umgebungen, d.h. Quellen, Zwischenkreise und Lasten, werden vorgestellt und modelliert. Im weiteren wird die Grundstruktur der Steuerung und Regelung der Stromrichter besprochen und die Funktion der Steuerverfahren darin aufgezeigt. Das Schwergewicht der Einführung liegt vor allem auf den für die Besprechung von Steuerverfahren benötigten Zusammenhänge. Für die Aneignung von Grundlagenkenntnissen über Umrichter und selbstgeführte Stromrichter sei auf die zahlreichen neueren Lehrbücher der Leistungselektronik verwiesen, welche jeweils am Ende der Kapitel erwähnt sind.

- Im Teil II werden die Grundtypen von Steuerverfahren am Beispiel der Standardschaltungen, den zweistufigen selbstgeführten Stromrichtern für U-Umrichter, vorgestellt. Jedem Grundtyp ist ein Kapitel gewidmet. Die Behandlung besteht aus Beschreibungen, analytischen Berechnungen und z.T. Simulationen. Der Teil II wird eingeleitet 
durch die Definition von einheitlichen Schaltungsmodellen und Kennwerten und wird abgeschlossen durch ein zusammenfassendes Kapitel mit dem Vergleich der Verfahren.

- Im Teil III wird die Übertragung der Steuerverfahren aus Teil II auf andere Stromrichtertopologien beschrieben. Zur Sprache kommen I-Stromrichter, 3-stufige U-Stromrichter sowie kombinierte U-Stromrichter.

- Teil IV wendet sich schliesslich der praktischen Realisierung der Steuerungen zu. Zuerst werden dabei die nichtidealen Phänomene beschrieben, welche bei der Erzeugung und Umsetzung der Schaltvorgänge im Stromrichter entstehen. Weiter wird der wichtige Aspekt der elektromagnetischen Verträglichkeit (EMV) kurz beleuchtet. Den Abschluss bildet eine Übersicht über die aktuellen Varianten zur Implementierung von Steuerverfahren (Modulatoren).

- Teil $V$ enthält die verschiedenen Anhänge. Sie umfassen eine kurze Zusammenstellung von wichtigen verwendeten Grundlagen der Elektrotechnik, ein Literaturverzeichnis sowie ein Sachverzeichnis. 


\section{Funktion und Aufbau von modernen Frequenzumrichtern}

\subsection{Prinzip des Frequenzumrichters}

Innerhalb eines Stromversorgungssystems findet der Energieaustausch zwischen den angeschlossenen Verbrauchern und Erzeugern mittels Wechselspannung und -strom einer bestimmten Frequenz ( $50 \mathrm{~Hz}$ oder $60 \mathrm{~Hz}$ ) bzw. in speziellen Fällen auch mittels DC-Grössen statt. Bei vielen Anwendungen wird die Energie bei einer anderen festen oder einer variablen Frequenz benötigt. Es kann sich dabei sowohl um reine Energieverbraucher oder -erzeuger handeln, als auch um Systeme, welche abhängig von der Betriebsart sowohl Energie aus dem Netz beziehen oder dorthin zurückspeisen. Im folgenden werden alle diese Verbraucher oder Erzeuger unter dem Oberbegriff Last zusammengefasst. Die entsprechenden Umrichter-Last-Systeme lassen sich in die folgenden Hauptgruppen unterteilen:

- drehzahlvariable Drehstromantriebe mit Synchron- oder Asynchronmaschinen für verschiedenste Anwendungen

- Versorgung von Bord- und Inselnetzen, unterbrechungsfreie Stromversorgungen

- Energietransfer in ein anderes Versorgungsnetz oder in ein Bahnnetz

- Versorgung von allgemeinen AC-Lasten, z.B. von Lautsprechern durch einen geschalteten Leistungsverstärker oder die Lichtbogenstrecke von Schweissanlagen

- Einspeisung nicht konventionell erzeugter Energie (aus Sonne, Wind, etc.) ins Netz

- kurzzeitige Speicherung von Energie oder Blindleistungskompensation mit Hilfe eines DC-Kreises mit induktivem oder kapazitivem Energiespeicher.

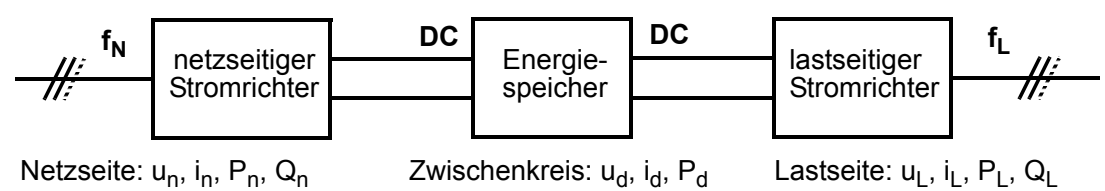

Bild 2.1. Prinzipieller Aufbau eines Frequenzumrichters

Ein Frequenzumrichter, im folgenden nur noch Umrichter genannt, mit dem in Bild 2.1 dargestellten prinzipiellen Aufbau, erlaubt die Umsetzung von elektrischer Energie in AC-Form von einer Frequenz in eine andere. Er besteht aus 3 Grundbausteinen: 
- Der netzseitige Stromrichter stellt das Bindeglied zwischen dem speisenden Netz und dem Zwischenkreis dar (AC-DC). Er ist je nach Ausführung der Schaltung auch ein Stellglied zur Steuerung des Energieflusses.

- Der lastseitige Stromrichter stellt die von der Last geforderten Betriebsverhältnisse ein, d. h. Frequenz, Spannung und Strom. Auch er repräsentiert ein Stellglied für den Energiefluss.

- Der Zwischenkreis ist für die Zwischenspeicherung der Energie verantwortlich. Dies geschieht entweder als Gleichspannung (Spannungszwischenkreis- bzw. U-Umrichter) oder als Gleichstrom (Stromzwischenkreis- bzw. I-Umrichter). Die beiden Varianten sind in Bild 2.2 dargestellt. Als Speicher dient im einen Fall eine Kapazität und im anderen eine Induktivität. Diese sind so bemessen, dass die Spannung $u_{d}$ beim U-Umrichter bzw. der Strom $i_{d}$ beim I-Umrichter stark geglättet sind und als Gleichgrössen betrachtet werden können. Dadurch werden die beiden Stromrichter soweit entkoppelt, dass sie sich unabhängig voneinander steuern lassen.

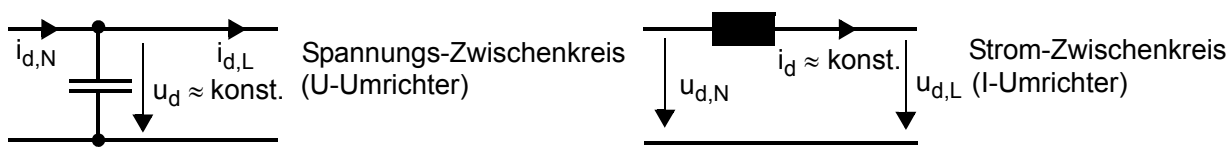

Bild 2.2. Zwischenkreis bei U- und I-Umrichtern

Für die netz- und lastseitigen Stromrichter gibt es eine Vielzahl von möglichen Ausführungen. Insbesondere stehen fremdgeführte und selbstgeführte Schaltungen zur Auswahl. Fremdgeführt heisst, dass die Kommutierung der Ströme im Stromrichter von aussen, z.B. durch die Netzspannung, unterstützt werden muss. Die eingesetzten Leistungshalbleiter sind in diesem Fall Thyristoren oder Dioden. Selbstgeführt bedeutet dagegen, dass der Stromrichter die Spannungen bzw. Ströme ohne äussere Unterstützung umschalten kann. Dazu müssen abschaltbare Halbleiter (Kapitel 3.1) verwendet werden. In den Kapiteln 2.3 und 2.4 wird näher auf die Stromrichterschaltungen eingegangen.

Die Speichergrösse des Zwischenkreises ist begrenzt und wird aus Kosten- und Platzgründen möglichst klein gehalten. Aus dieser Tatsache kann eine Vorschrift zur Steuerung des gesamten Umrichters abgeleitet werden: zumindest im zeitlichen Mittel müssen die netz- und lastseitigen Wirkleistungen übereinstimmen, da sich sonst die mittlere Spannung bzw. der mittlere Strom im Zwischenkreis ändert:

$$
P_{N}=P_{d}=P_{L}
$$

Die anderen Parameter am Ein- und Ausgang des Umrichters lassen sich dagegen im Prinzip unabhängig voneinander einstellen. Dies gilt insbesondere auch für die Blindleistungen $Q_{N}$ und $Q_{L}$. In einem DC-Kreis tritt definitionsgemäss keine Blindleistung auf. Die Stromrichter können beide als unabhängige Blindleistungsquellen bzw. -senken wirken.

Wie bereits in Bild 2.1 angedeutet, können sowohl die Netz- als auch die Lastseite einoder mehrphasig ausgeführt sein. Im weiteren existiert eine Vielzahl von Anwendungen, in denen eine DC-Quelle eine AC-Last oder eine AC-Quelle eine DC-Last speisen. In diesen Fällen liegt ein einzelner AC-DC-Wandler vor, der auch als reduzierter Frequenzum- 
richter betrachtet werden kann. Die Tabelle 2.1 gibt einen Überblick über die verschiedenen Umrichtervarianten und nennt gleichzeitig je ein typisches Anwendungsbeispiel.

Tabelle 2.1. Übersicht über die verschiedenen Umrichtertypen

\begin{tabular}{|c|c|c|c|}
\hline \multicolumn{3}{|c|}{ Umrichtertypen } & typisches Beispiel einer Anwendung \\
\hline $\mathrm{f}_{\mathrm{N}} / /$ & $\begin{array}{l}\text { Frequenz- } \\
\text { umrichter }\end{array}$ & $\|^{f_{L}}$ & $\begin{array}{l}\text { einphasige unterbrechungsfreie } \\
\text { Stromversorgung }\end{array}$ \\
\hline $\mathrm{f}_{\mathrm{N}} / /$ & $\begin{array}{l}\text { Frequenz- } \\
\text { umrichter }\end{array}$ & $\|^{f_{L}}$ & $\begin{array}{l}\text { Kupplung zwischen einem einphasigen Bahn- } \\
\text { netz und einem dreiphasigen Versorgungsnetz }\end{array}$ \\
\hline $\mathrm{f}_{\mathrm{N}} / / /$ & $\begin{array}{l}\text { Frequenz- } \\
\text { umrichter }\end{array}$ & $\|^{f_{L}}$ & Schweissanlage \\
\hline $\mathrm{f}_{\mathrm{N}} / / /$ & $\begin{array}{l}\text { Frequenz- } \\
\text { umrichter }\end{array}$ & $\|^{f_{L}}$ & drehzahlvariabler Drehstromantrieb \\
\hline $\mathrm{f}_{\mathrm{N}} /{ }_{i}$ & Stromrichter & DC & Gleichstromantrieb \\
\hline DC & Stromrichter & $H: i^{f_{L}}$ & photovoltaische Energieerzeugung \\
\hline
\end{tabular}

\subsection{Anforderungen an einen Frequenzumrichter}

Die Anforderungen an einen Frequenzumrichter lassen sich sinnvoll in solche für die Netz- und solche für die Lastseite aufteilen. Auf der Lastseite sind dabei einige grundsätzlich verschiedene Lastkategorien zu unterscheiden. Die Strukturierung der folgenden Abschnitte ist entsprechend gewählt.

\subsubsection{Anforderungen auf der Netzseite}

Bild 2.3 zeigt den netzseitigen Teil des Frequenzumrichters. In der Regel ist der Leistungsfluss im Zwischenkreis durch die Last bestimmt, so dass dem netzseitigen Stromrichter die Aufgabe zukommt, diese Leistung aus dem Netz zu beziehen oder ins Netz einzuspeisen. Als Regelgrössen werden dabei nicht die Leistung selbst, sondern beim U-Umrichter der Mittelwert der Spannung $u_{d}$ und beim I-Umrichter der Mittelwert des Stromes $i_{d}$ im Zwischenkreis verwendet. Diese Grössen werden auf vorgegebene Werte, die konstant oder abhängig vom Betriebspunkt des lastseitigen Stromrichters sein können, geregelt. Im Netz sind die Frequenz und die Spannung näherungsweise konstant. Die Anfor-

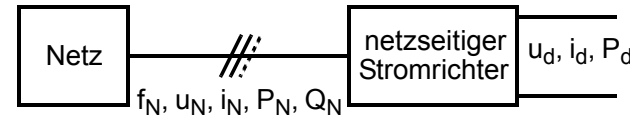

Bild 2.3.

Netzseite des Frequenzumrichters 
derungen an den Stromrichter beziehen sich deshalb primär auf den Strom und auf die Leistung:

1. Der Netzstrom soll möglichst sinusförmig sein, damit überwiegend Grundschwingungsleistung fliesst. Unter der Annahme, dass die Netzspannung selbst ebenfalls weitgehend sinusförmig ist, verursachen alle von der Grundschwingung verschiedenen Anteile im Strom Verzerrungsblindleistung und sind deshalb unerwünscht.

2. In der Regel soll die mit dem Netz ausgetauschte Blindleistung minimal sein. Dazu muss die Grundschwingung des Stromes möglichst dieselbe Phasenlage wie die Netzspannung aufweisen. Eine Ausnahme bildet der spezielle Fall, dass der netzseitige Stromrichter nebenbei noch die Funktion eines Blindleistungskompensators für das Netz übernehmen muss.

3. Die Amplitude der Grundschwingung des Phasenstromes soll so eingestellt sein, dass die benötigte Leistung aus dem Netz bezogen wird.

Bei Anwendungen, in denen der Umrichter die Kupplung zwischen zwei Versorgungsnetzen darstellt, gelten diese Anforderungen auf beiden Seiten.

\subsubsection{Anforderungen für die Speisung einer Drehstrommaschine}

Werden Drehstrommaschinen direkt ans Netz geschaltet, so lässt sich ihre Drehzahl nicht oder zumindest nur mit grossen Einschränkungen variieren. Mit Hilfe eines Stromrichters nach Bild 2.4, der eine freie Einstellung der Statorfrequenz ermöglicht, kann dagegen ein Antrieb mit beliebig variabler Drehzahl realisiert werden. Als Maschinen kommen meis-

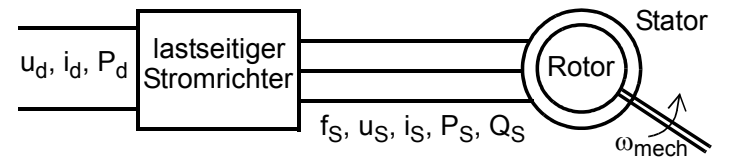

Bild 2.4.

Umrichtergespeiste

Drehstrommaschine

tens Asynchronmaschinen mit Kurzschlussläufer (ASM) und Synchronmaschinen (SM) zum Einsatz. Für diese beiden Typen sind in Bild 2.5 die charakteristischen statischen Drehmoment-Drehzahl Kennlinienfelder dargestellt. Dabei dient die am Stator eingespeiste Frequenz $f_{S}$ bzw. Kreisfrequenz $\omega_{S}$ als Kurvenparameter. Mit einer frei einstellbaren Statorfrequenz lässt sich ein beliebiger Betriebspunkt innerhalb der gekennzeichneten Flächen einstellen. Bei der SM ist die Drehzahl (bei einem zweipoligen Motor) mit der Statorfrequenz identisch. Je nach Vorzeichen des elektrischen Drehmomentes ist bei der ASM in jedem beliebigen Arbeitspunkt $P$ die Drehzahl um den Betrag der Rotorkreisfrequenz $\omega_{R}$ grösser oder kleiner als die Statorkreisfrequenz (Schlupf).

Im gesamten Grunddrehzahlbereich $\left(\left|\omega_{\text {mech }}\right| \leq \omega_{\text {mech, }}\right)$ wird mit vollem Fluss gefahren, so dass sich motorisch und generatorisch stets das maximale Drehmoment erreichen lässt. Für Drehzahlen oberhalb der Nenndrehzahl muss der Fluss reduziert werden, da die Statorspannung höchstens der maximal verfügbare Stromrichterspannung entsprechen kann. Diese Massnahme wird als Feldschwächung bezeichnet. Das erreichbare Drehmoment nimmt dabei proportional mit dem Fluss ab. Bild 2.6 zeigt die für beide Maschinentypen 

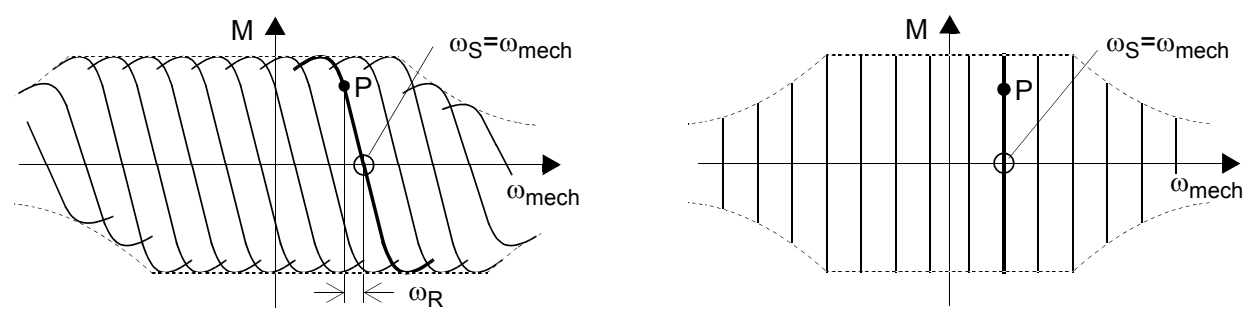

Bild 2.5. Drehmoment-Drehzahl-Kennlinienfeld zweipoliger Maschinen ( $p=1)$,

$\omega_{\text {mech }}=2 \pi n, n$ : Drehzahl in Umdrehungen pro Sekunde,

links: ASM mit Kurzschlussläufer, rechts: SM

gültige typische Spannungs-Frequenz-Kennlinie und den zugehörigen Verlauf der Flussverkettung $\Psi_{S}$. Bei einer permanent erregten SM ist der Feldschwächbereich stark eingeschränkt.

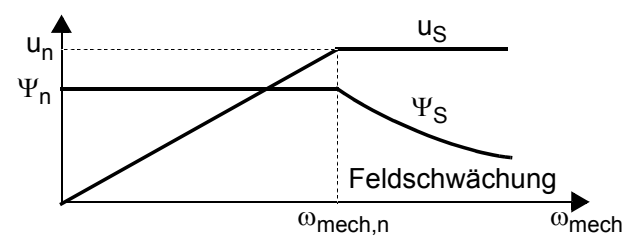

Bild 2.6.

Statorspannung und Flussverkettung in Funktion der Drehzahl bei einem Drehstrommotor (Statorwiderstand vernachlässigt)

Aufgrund der Eigenschaften der Maschinen können die Anforderungen an den Frequenzumrichter wie folgt formuliert werden:

1. Der lastseitige Stromrichter ist das Stellglied für die Regelung der Maschine. Ohne zusätzliche Regelung verhält sich ein U-Stromrichter am Ausgang wie eine einstellbare Spannungsquelle und ein I-Stromrichter wie eine Stromquelle. Durch die Steuerung bzw. Regelung kann das Stromrichterverhalten dem Motor angepasst werden. Der Zwischenkreis muss eine selbständig geregelte DC-Quelle darstellen. Soll die Maschine auch abgebremst werden können, so muss ein Energieaustausch in beiden Richtungen möglich sein, d.h. es muss auch Energie in den Zwischenkreis zurückgespeist werden können.

2. Die Ausgangsfrequenz soll in einem bestimmten Bereich (meist von null bis zu einem vorgegebenen Maximalwert) stetig variiert werden können. Der Maximalwert liegt gewöhnlich unterhalb von $200 \mathrm{~Hz}$, kann bei speziellen Anwendungen jedoch bis einige $\mathrm{kHz}$ betragen. Zusätzlich muss die Phasenfolge umkehrbar sein (Drehrichtung).

3. Die Statorgrössen sollen ein symmetrisches Dreiphasensystem mit möglichst sinusförmigen Strömen bilden. Jede Abweichung von der idealen Form verursacht Drehmomentpulsationen und zusätzliche Verluste.

4. Je nachdem, ob ein U- oder ein I-Umrichter eingesetzt wird, muss die Statorspannung oder der Statorstrom in der Amplitude stetig von null, oder einem Wert nahe bei null, bis zum Maximalwert eingestellt werden können. 
5. Die Wirk- und Blindleistung am Ausgang wird durch die Maschine, bzw. deren Regelung bestimmt. Eine ASM benötigt für den Feldaufbau stets induktive Blindleistung, während bei einer umrichtergespeisten SM die Blindleistung null sein kann.

6. Die geforderte Dynamik des Antriebes gibt die Geschwindigkeit vor, mit welcher der lastseitige Stromrichter in der Lage sein muss, seine Ausgangsgrössen zu verstellen.

Bekanntlich werden auch andere Maschinentypen wie z.B. bürstenlose Gleichstrom-, Reluktanz- und Schrittmotoren mit Stromrichtern gespeist. Dabei sind die Idealverläufe von Spannungen und Strömen z.T. nicht sinusförmig. Auf solche Motoren, sowie auf nichtsinusförmige Spannungs- und Strom-Sollverläufe wird in diesem Buch nicht weiter eingegangen.

\subsubsection{Anforderungen für die Speisung einer allgemeinen AC-Last}

Unter den Begriff 'Speisung einer AC-Last' fallen die Speisungen jeglicher Art von Inselnetzen, die unterbrechungsfreien Stromversorgungen, aber auch eine Reihe spezieller Anwendungen wie zum Beispiel geschaltete Leistungsverstärker für Audio-Anwendungen oder für Rundsteuerungen. Der Umrichter kann dabei, wie in Bild 2.7 dargestellt, nur ein Stromrichter (AC-DC-Wandler) oder ein vollständiger Umrichter sein, je nachdem ob die Speisung aus einer AC- oder DC-Quelle erfolgt. Die Phasenzahl auf der Lastseite kann grundsätzlich beliebig sein, üblich sind aber ein- und dreiphasige Ausführungen.

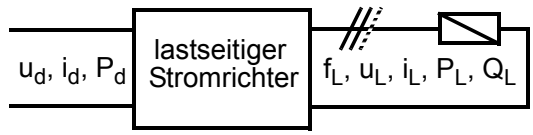

Bild 2.7.

Speisung einer AC-Last durch einen Umrichter

Für die sehr unterschiedlichen Einsatzgebiete lassen sich eine Reihe von gemeinsamen Anforderungen formulieren. Dabei wird, wie bei den Drehstromantrieben, der Zwischenkreis als selbständig geregelte DC-Quelle betrachtet:

1. Der lastseitige Stromrichter soll sich an seinem Ausgang wie eine einstellbare Spannungsquelle verhalten. Anwendungen, die eine Stromquellenspeisung erfordern, sind selten.

2. Die Ausgangsspannung soll möglichst gut dem vorgegebenen Sollwert entsprechen. Dieser kann je nach Anwendung sehr unterschiedlich aussehen. In den meisten Fällen ist ein sinusförmiger Verlauf gefordert. Als Kennwerte dienen dann die Frequenz und die Amplitude, welche beide als konstant oder variabel vorgegeben werden können. Der Stellbereich reicht im Extremfall bei der Spannung von null bis zu einem durch den Umrichter gegebenen Maximalwert und bei der Frequenz von null bis zu mehreren kHz. Der Sollwert kann aber auch einen beliebigen Verlauf aufweisen, z.B. bei einem Leistungsverstärker. Seine Bandbreite kann dabei bis einige $10 \mathrm{kHz}$ betragen.

3. Die Verzerrungen der Ausgangsgrössen sollen möglichst klein sein.

4. Der Umrichter muss in der Lage sein, für die vorgesehene Last den entsprechenden Strom, bzw. die entsprechende Wirk, Blind- und Verzerrungsblindleistung (durch Ver- 
zerrungsanteile in den Phasenströmen verursachte Blindleistung), zu liefern. Speziell zu beachten sind dabei nichtlineare Lasten, wie zum Beispiel Netzteile von elektronischen Geräten, die vom Umrichter zu versorgen sind. In den meisten Fällen ist zusätzlich Kurzschlussfestigkeit gefordert, die sich in der Regel durch eine schnelle Regelung oder eine spezielle Überstromschutzvorrichtung erreichen lässt.

\subsection{Netzseitiger Stromrichter}

Die im Kapitel 2.2 beschriebenen Anforderungen werden einerseits durch die Auswahl von geeigneten Stromrichterschaltungen und andererseits durch eine angepasste Steuerung und Regelung erreicht. Im folgenden werden zuerst die heute aktuellen Schaltungen betrachtet. Wesentlich ist dabei vor allem die Unterscheidung von ungesteuerten und fremdgeführten Stromrichtern auf der einen Seite und von selbstgeführten Stromrichtern auf der anderen.

Die besprochenen Schaltungen sind jeweils die Grundausführungen einer ganzen Klasse von Schaltungen, welche alle ähnliche Eigenschaften zeigen. Ein Beispiel dafür ist der sogenannte 6-pulsige Gleichrichter (Schaltung 1 in Tabelle 2.2). Zwei bzw. vier derartige Gleichrichter lassen sich in bekannter Weise zu einer 12- bzw. 24-pulsigen Schaltung erweitern. Diese erweiterten Varianten zeigen zwar ein verbessertes, vom Prinzip her aber gleichartiges Verhalten. Im Falle der selbstgeführten Stromrichter werden solche erweiterten Schaltungen später noch näher betrachtet, wobei dann die Steuerverfahren im Vordergrund stehen werden.

\subsubsection{Netzseitiger Stromrichter für einen Spannungszwischenkreis}

Tabelle 2.2 zeigt die 4 Grundausführungen eines netzseitigen Stromrichters für einen Spannungszwischenkreis zusammen mit Hinweisen auf ihre wesentlichen Eigenschaften. Die schwarz ausgezogenen Teile in den Schemata sind die einphasigen Schaltungen und die grauen Teile sind die Ergänzungen zu den entsprechenden dreiphasigen Ausführungen. Die Schaltungen 1 bis 3 sind fremd- bzw. netzgeführt, die Schaltung 4 ist selbstgeführt. Sie unterscheiden sich ganz wesentlich in bezug auf Aufwand und Eigenschaften, wobei mit aufsteigender Nummer der Schaltung der Aufwand steigt, dafür aber die Eigenschaften besser werden:

Schaltung 1: Näherungsweise ergeben die ungesteuerten Gleichrichterbrücken eine konstante Gleichspannung, die dem Spitzenwert $\hat{u}_{N}$ der Eingangsspannung entspricht (bei dreiphasigen Schaltungen dem Spitzenwert der verketteten Spannungen). Genau betrachtet weist die Gleichspannung eine von der Netzfrequenz abhängige Welligkeit auf und ihr Mittelwert sinkt bei zunehmender Belastung leicht ab. Der Netzstrom besteht aus kurzen hohen Pulsen, was eine grosse Verzerrungsblindleistung zur Folge hat. Die Stromform lässt sich mit Hilfe der gestrichelt eingezeichneten Glättungsdrossel verbessern, sie wird aber bestenfalls rechteckförmig. Die aufgenommene Grundschwingungsblindleistung $\left(Q_{N}\right)$ ist sehr klein, da die Stromgrundschwingung in jedem Betriebsfall 
Tabelle 2.2. Schaltungen für den netzseitigen Stromrichter mit Spannungszwischenkreis

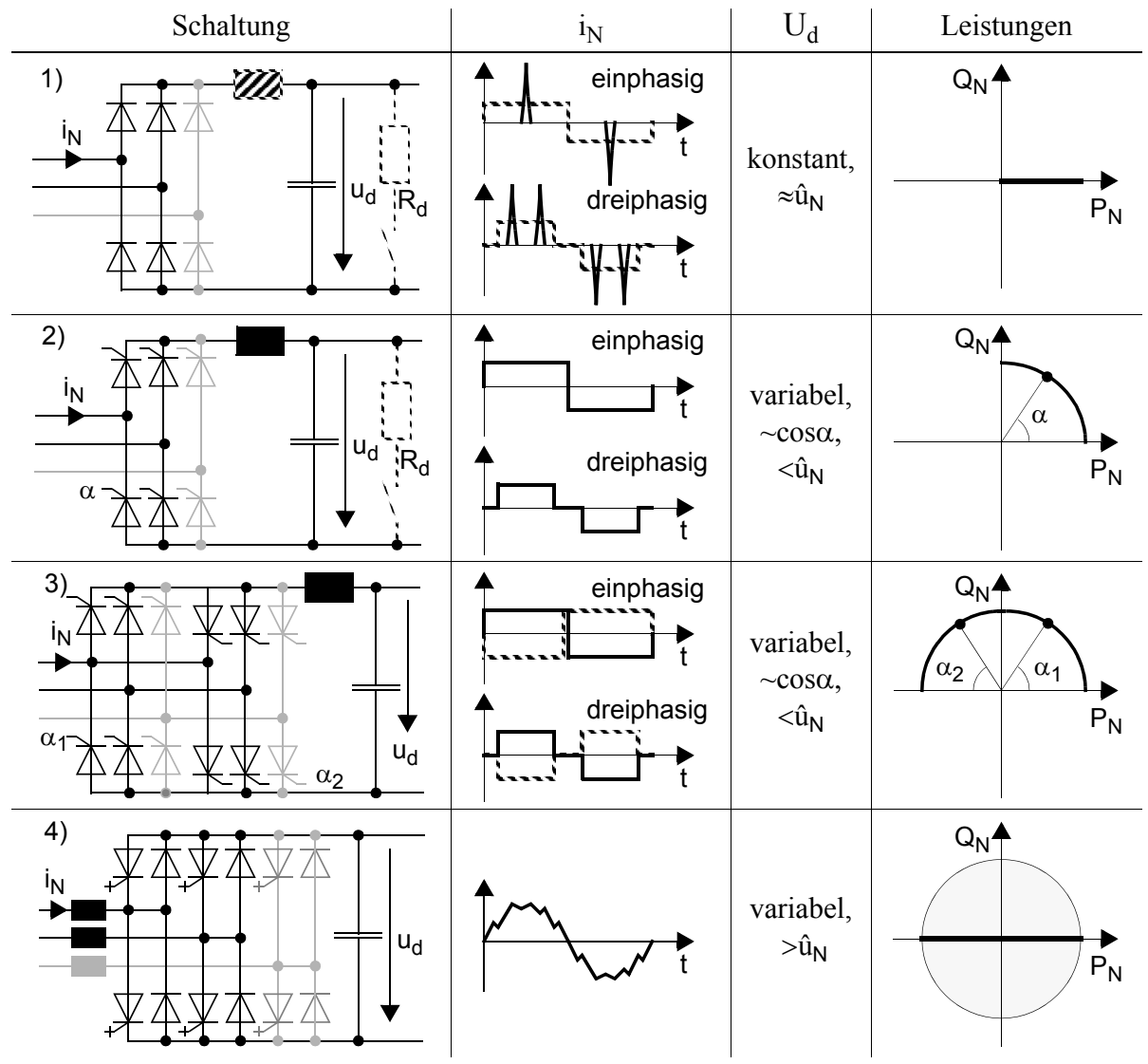

näherungsweise in Phase mit der Netzspannung ist. Eine Rückspeisung von Energie ins Netz ist nicht möglich. Soll die Last Energie in den Zwischenkreis zurückspeisen können, so ist ein Widerstand $R_{d}$ im Zwischenkreis erforderlich, der bei Bedarf zugeschaltet wird und die aufzunehmende Energie in Wärme umsetzt.

Ohne Glättungsdrossel ist die Schaltung wegen des ungünstigen Netzstromes nur für sehr kleine Leistungen zulässig.

Schaltung 2: Gesteuerte Gleichrichter können im Gegensatz zu ungesteuerten nur mit der Glättungsdrossel im Zwischenkreis betrieben werden. Die Netzströme sind idealisiert ebenfalls rechteckförmig. Mit Hilfe des Steuerwinkels $\alpha$ lässt sich der Mittelwert der Zwischenkreisspannung $U_{d}$ von null bis zum Maximalwert stetig variieren. Das Verhältnis von Wirk- zu Blindleistung im Netz ist ebenfalls durch den Steuerwinkel bestimmt. Die entsprechende Ortskurve ist ein Viertelkreis, d.h. die Blindleistung ist stets induktiv. Der Radius des Viertelkreises ist durch die Amplitude des Zwischenkreisstromes gegeben, die sich lastabhängig einstellt. Da die Zwischenkreisspannung beim UUmrichter nicht negativ werden soll, bleibt das Problem der Energierückspeisung bei dieser Schaltung bestehen. 
Schaltung 3: Umkehrgleichrichter verhalten sich grundsätzlich gleich wie die Schaltungen 2. Durch Hinzufügen eines entgegengesetzt gepolten gesteuerten Gleichrichters wird jedoch die Umkehr der Energieflussrichtung möglich. Je nach Vorzeichen des benötigten Laststromes ist eine der beiden Thyristorbrücken aktiv. Die Ortskurve wird auf einen Halbkreis erweitert, wobei die Blindleistung weiterhin stets induktiv ist. Diese Schaltungen sind geeignet für Anwendungen, welche häufig und viel Energie ins Netz zurückspeisen.

Schaltung 4: Selbstgeführte Stromrichter als aufwendigste der 4 Grundschaltungen erlauben einerseits eine gute Annäherung des Netzstromes an die Sinusform und andererseits eine flexible Steuerung der Leistung. Die Wirkleistung ist wie bei den Schaltungen 2 und 3 dadurch gegeben, dass die Zwischenkreisspannung auf einen gewünschten Wert geregelt wird bzw. der Leistungsbedarf der Last gedeckt sein muss. Für den Leistungsfluss sind ohne weiteres beide Richtungen möglich. Die Blindleistung kann auf einen beliebigen Wert eingestellt werden. Die Beschränkung auf den dargestellten Kreis ergibt sich daraus, dass der Stromrichter-Strom auf einen Maximalwert begrenzt ist. In der Regel wird die Blindleistung auf null gehalten. Damit die beliebige Vorgabe von Wirk- und Blindleistung möglich ist, muss allerdings die Zwischenkreisspannung immer leicht grösser sein als der Spitzenwert der Netzspannung.

Die Auswahl der geeigneten Schaltung für eine bestimmte Anwendung erfolgt aufgrund der spezifischen Anforderungen an den netzseitigen Stromrichter: Regelbarkeit der Zwischenkreisspannung, Kurvenform des Netzstromes, netzseitige Blindleistung sowie Fähigkeit zur Umkehr der Energieflussrichtung. Aus Kostengründen wird stets eine möglichst einfache Schaltung eingesetzt.

\subsubsection{Netzseitiger Stromrichter für einen Stromzwischenkreis}

Im Gegensatz zum U-Umrichter gibt es für den I-Umrichter nur zwei grundsätzliche Stromrichtervarianten für die Netzseite, nämlich eine fremd- und eine selbstgeführte. Sie sind in Tabelle 2.3 dargestellt. Beide Schaltungen erlauben die Regelung des Zwischenkreisstromes auf einen beliebigen positiven Wert. Daneben unterscheiden sie sich jedoch deutlich:

Schaltung 5: Im Vergleich zur Schaltung 2 fehlt beim fremdgeführten Stromrichter hier nur der Glättungskondensator der den Stromzwischenkreis in einen Spannungszwischenkreis umwandeln würde. Das Verhalten auf der Netzseite ist jedoch weitgehend gleich. Ein wichtiger Unterschied ist die Möglichkeit zur Umkehr der Energieflussrichtung. Da beim I-Umrichter dazu die Spannung im Zwischenkreis invertiert werden muss, während der Strom sein Vorzeichen beibehält, kann dies mit dem Stromrichter für eine Stromrichtung ohne weiteres erreicht werden.

Schaltung 6: Selbstgeführte Stromrichter für einen Stromzwischenkreis zeigen vom Netz her gesehen ähnliche Eigenschaften wie die Schaltung 4, obwohl die Topologie eine andere ist. Mit dieser Schaltung ist eine flexible Leistungssteuerung möglich und der Netzstrom kann gut an die Sinusform angenähert werden. Da die Innenimpedanz jedes Netzes induktiv ist, benötigt die Schaltung eingangsseitig zusätzlich Kondensatoren, die bei den Schaltvorgängen im Stromrichter als Freilaufzweige dienen. 
Tabelle 2.3. Schaltungen für den netzseitigen Stromrichter mit Stromzwischenkreis

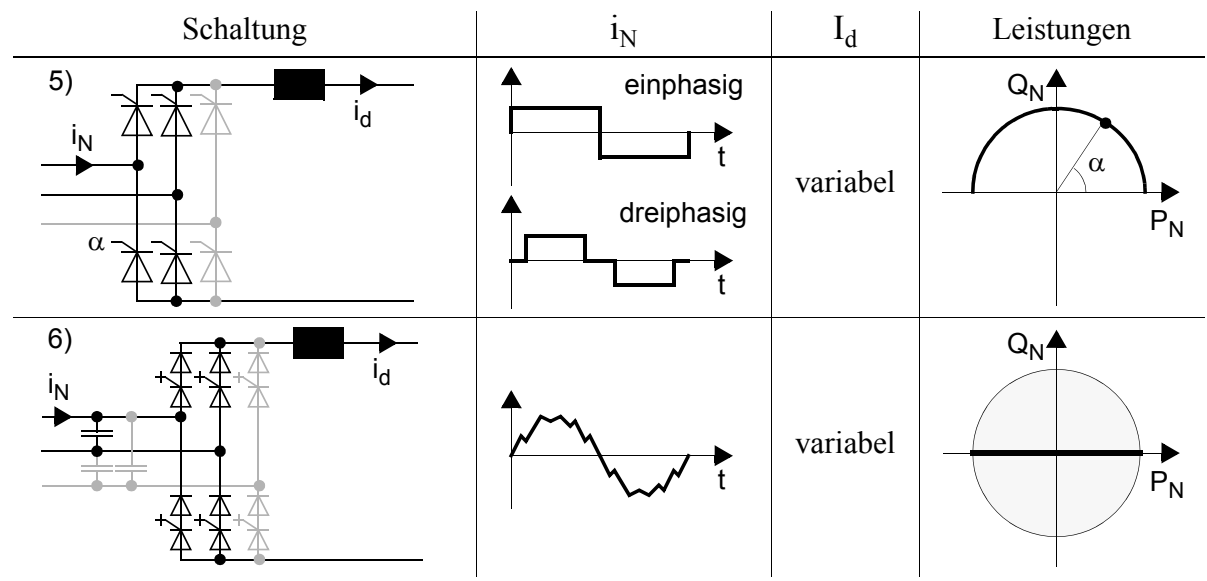

In der Schaltung 6 sind die Leistungshalbleiter in Störfällen, z.B. bei plötzlichem Lastunterbruch), grossen Spannungsbelastungen ausgesetzt. Die Beherrschung dieses Problems erfordert zusätzlichen Aufwand in Form von Schutzbeschaltungen bei der Realisierung des Stromrichters. Nicht zuletzt deswegen hat der selbstgeführte I-Stromrichter bis heute kaum praktische Bedeutung erlangt. Die Verwendung der Schaltung 5 ist die Regel.

\subsection{Lastseitiger Stromrichter}

\subsubsection{Lastseitiger Stromrichter für einen Spannungszwischenkreis}

Auf der Lastseite des U-Umrichters steht nur eine einzige Schaltung zur Auswahl: der selbstgeführte Stromrichter in Tabelle 2.4. Er ist prinzipiell identisch mit der Schaltung 4 für die Netzseite. In rein gesteuerter Ausführung verhält er sich für die Last wie eine Spannungsquelle. Der Verlauf der geschalteten Ausgangsspannung ist durch das Steuerverfahren bestimmt. Der Strom in der Last kann sich frei einstellen. Der Stromrichter ist also in der Lage, nach Bedarf Wirk- und Blindleistung abzugeben bzw. aufzunehmen. Die Kurvenform des Laststromes hängt von der Spannung und von der Impedanz der Last ab.

Tabelle 2.4. Schaltung für den lastseitigen Stromrichter mit Spannungszwischenkreis

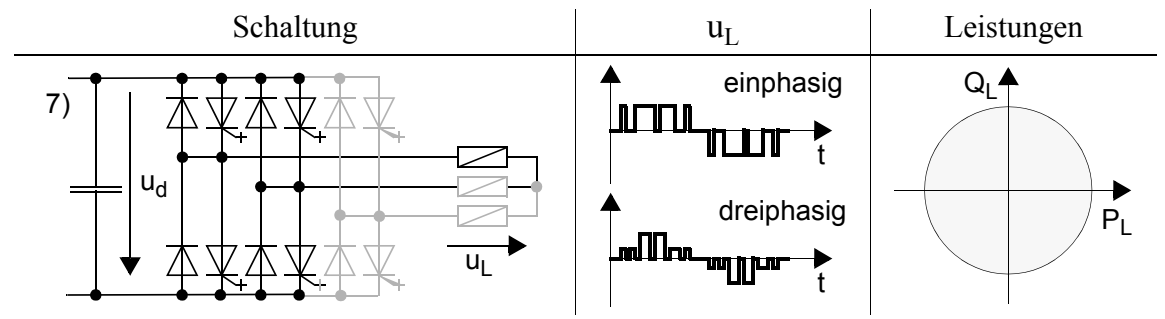


Durch die Anwendung eines geeigneten Steuerverfahrens für den Stromrichter kann sie der Sinusform gut angenähert werden. Dies ist ein Hinweis darauf, welche zentrale Bedeutung dem Steuerverfahren zukommt.

Der U-Umrichter mit einem selbstgeführten Stromrichter auf der Lastseite ist heute sowohl über alle Anwendungsgebiete, wie auch über fast alle Leistungsklassen hinweg der am meisten eingesetzte Frequenzumrichter. Bei der Leistung gibt es eine obere Grenze, über der die Realisierung mit den erhältlichen abschaltbaren Leistungshalbleitern nicht mehr möglich ist.

\subsubsection{Lastseitiger Stromrichter für einen Stromzwischenkreis}

Bei einem Stromzwischenkreis stehen auch auf der Lastseite zwei Schaltungsvarianten zur Auswahl. Sie sind in Tabelle 2.5 dargestellt und lassen sich wie folgt charakterisieren:

Tabelle 2.5. Schaltung für den lastseitigen Stromrichter mit Stromzwischenkreis

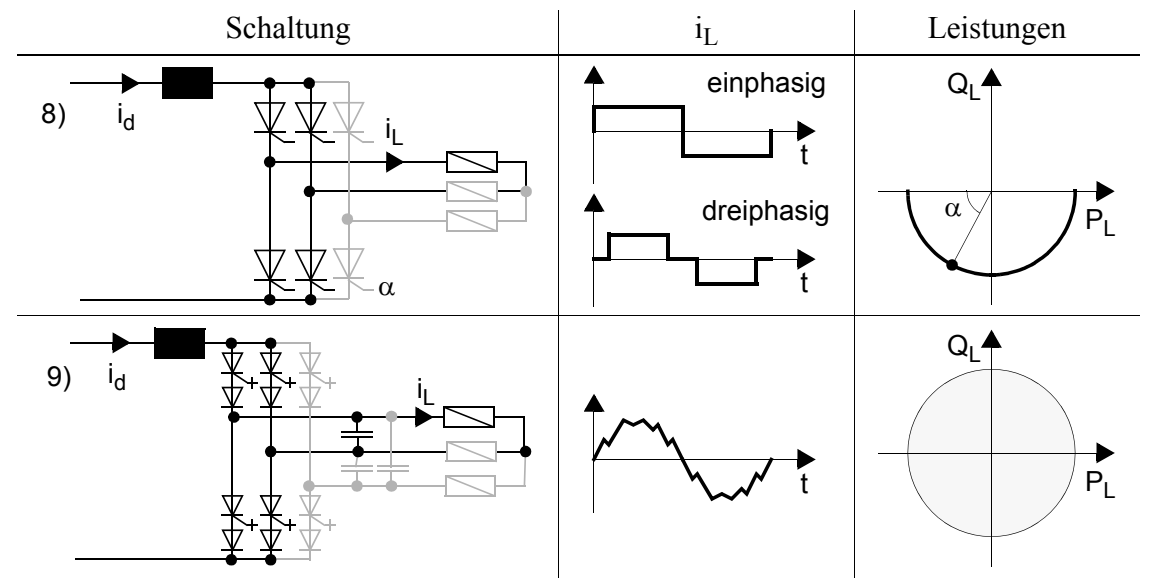

Schaltung 8: Lastgeführte Stromrichter verhalten sich gleich wie gesteuerte Stromrichter auf der Netzseite. Sie können grundsätzlich nur Lasten speisen, welche imstande sind, die benötigte induktive Blindleistung zu liefern. Beispiele sind SM oder ein Netz, nicht aber ASM. Der an die Last abgegebene Strom ist rechteckförmig und seine Amplitude entspricht demjenigen des Zwischenkreisstromes. Die abgegebene Wirkleistung kann über den Steuerwinkel variiert werden. Da aber in der Regel die entsprechende Änderung der Blindleistung nicht erwünscht ist, erfolgt die Steuerung gewöhnlich über eine Anpassung des Zwischenkreisstromes.

Schaltung 9: Die selbstgeführten Stromrichter können auch beim I-Umrichter einen Laststrom erzeugen, der nahezu sinusförmig ist. Dabei hängt die sich ergebende Kurvenform stark vom gewählten Steuerverfahren ab. Die Amplitude ist auch bei konstantem Zwischenkreisstrom frei einstellbar und die Wirk- und Blindleistung kann in sehr weiten Bereichen vorgegeben werden. 
Lastgeführte I-Umrichter haben eine grosse Bedeutung im Bereich grosser Leistungen, wo die geforderte Strom- und Spannungsbelastung der Schaltelemente praktisch nur die Verwendung von Thyristoren erlaubt. Zudem fällt dort auch der Kostenaspekt stark ins Gewicht, so dass die einfachen fremdgeführten Schaltungen bevorzugt werden. Selbstgeführte Stromrichter auf der Lastseite haben bisher kaum Verbreitung gefunden.

\subsection{Umrichterkonzepte und ihre Einsatzgebiete}

Die Übersicht in Tabelle 2.6 zeigt einerseits, wie die einzelnen Typen von netzseitigen und lastseitigen Stromrichtern kombiniert werden und andererseits, wo die Einsatzschwerpunkte dieser Umrichter liegen. Es ist anzumerken, dass es sich um Standardlösungen auf dem aktuellen Stand der Technik handelt und dass in der Praxis durchaus weitere Varianten zu finden sind. In der Übersicht berücksichtigt sind nur U- und I-Umrichter mit DC-Zwischenkreis nach dem in Bild 2.1 gezeigten Prinzip, sowie reduzierte Ausführungen die nur aus dem netz- oder den lastseitigen Stromrichter bestehen.

Tabelle 2.6. Umrichtertopologien für verschiedene Einsatzgebiete und Leistungsklassen

\begin{tabular}{|c|c|c|c|}
\hline Anwendung & $<10 \mathrm{kVA}^{\mathrm{a}}$ & 10kVA...100kVA & $>100 \mathrm{kVA}$ \\
\hline $\begin{array}{c}\text { Antriebe } \\
\text { Werkzeugmaschinen } \\
\text { Stell- und Positionierantriebe } \\
\text { Pumpen, Lüfter, Stofförderung } \\
\text { Fahrzeuge, Traktion } \\
\text { Spezialantriebe }\end{array}$ & $\begin{array}{l}\text { UU } 1 / 7^{\mathrm{b}} \\
\text { UU } 1 / 7 \\
\text { UU } 1 / 7 \\
\text { UU } 7^{\mathrm{c}} \\
\text { UU } 1 / 7\end{array}$ & $\begin{array}{c}\text { UU } 1 / 7 \\
\text { UU } 2 / 73 / 7 \\
\text { UU } 1 / 72 / 7 \\
\text { UU } 7 \\
\text { UU } 1 / 72 / 73 / 74 / 7\end{array}$ & $\begin{array}{c}\text { UU } 2 / 7, \text { IU } 5 / 85 / 9 \\
\text { UU } 4 / 7 \\
\text { UU } 2 / 7 \text { 3/7 } 4 / 7 \text {, IU } 5 / 85 / 9\end{array}$ \\
\hline $\begin{array}{c}\text { Netzanwendungen } \\
\text { Einspeisung von Energie } \\
\text { Aktivfilter } \\
\text { netzfreundliche Gleichrichter } \\
\text { Netzkupplungen, HGÜ } \\
\text { Blindleistungskompensatoren }\end{array}$ & $\begin{array}{l}\text { UU } 7 \\
\text { UU } 7 \\
\text { UU } 7\end{array}$ & $\begin{array}{l}\text { UU 7, IU8 } \\
\text { UU } 7 \\
\text { UU } 7 \\
\text { UU } 7\end{array}$ & $\begin{array}{c}\text { UU } 7 \text {, IU } 8 \\
\text { UU } 7 \\
\text { UU } 3 / 77 / 7, \text { IU } 8 / 8 \\
\text { UU } 7\end{array}$ \\
\hline $\begin{array}{c}\text { Versorgung von AC-Lasten } \\
\text { USV } \\
\text { Inselnetze, Bordnetze } \\
\text { Schweissmaschinen } \\
\text { Leistungsverstärker }\end{array}$ & $\begin{array}{l}\text { UU } 2 / 7 \\
\text { UU } 7 \\
\text { UU } 1 / 7\end{array}$ & $\begin{array}{l}\text { UU } 2 / 7 \\
\text { UU } 7 \\
\text { UU } 1 / 7 \\
\text { UU } 1 / 7\end{array}$ & $\begin{array}{l}\text { UU } 2 / 7 \\
\text { UU } 7 \\
\text { UU } 1 / 7\end{array}$ \\
\hline \multicolumn{4}{|c|}{$\begin{array}{l}\text { a. Nennleistung des Umrichters } \\
\text { b. UU: U-Umrichter, IU: I-Umrichter, 1/7: Umrichter bestehend aus den Schaltungen } 1 \text { und } 7 \\
\text { c. UU 7: nur ein Stromrichter mit DC-Speisung } \\
\text { d. HGÜ: Hochspannungs-Gleichstrom-Energieübertragung }\end{array}$} \\
\hline
\end{tabular}

Es zeigt sich deutlich, dass bei den Anwendungen der U-Umrichter dominiert und im unteren Leistungsbereich sogar fast ausschliesslich zur Anwendung gelangt. Steht die 
Lastseite im Vordergrund, wie das vor allem bei Antrieben der Fall ist, kommen netzseitig schwergewichtig einfache ungesteuerte oder allenfalls fremdgeführte Gleichrichter zum Einsatz. Die zunehmend schärfer werdenden Vorschriften an die Stromform (Kapitel 18) lassen aber in Zukunft auch auf der Netzseite einen starken Trend zu selbstgeführten Schaltungen erwarten. Dieser Aspekt ist unter der Rubrik netzfreundliche Gleichrichter berücksichtigt. Bei Netzanwendungen dagegen, wo der Netzstromrichter die zentrale Rolle innerhalb des Systems spielt, ist dieser fast ausnahmslos als selbstgeführte Schaltung ausgeführt. Lastseitig ist beim U-Umrichter die Verwendung eines selbstgeführten Stromrichters vorgegeben. Das äusserst breite Anwendungsfeld, quer durch alle Einsatzgebiet und durch alle Leistungsklassen, ist der Grund für die sehr grosse Bedeutung dieser Schaltung.

Der I-Umrichter dagegen ist, wie bereits erwähnt wurde, praktisch nur im Bereich der grossen Leistungen zu finden. Dabei kommen fast ausschliesslich die beidseitig fremdgeführten Varianten zur Anwendung. Der selbstgeführte I-Stromrichter hat wenig Bedeutung.

Literatur zu Kapitel 2: [Mey1], [Heu1], [Lap1], [Mic1], [Hag1], [Bro1] 


\section{Leistungskreis von Frequenzumrichtern mit selbstgeführten Stromrichtern}

\subsection{Schaltelemente der selbstgeführten Stromrichter}

\subsubsection{Idealisierte Schaltelemente}

Die Schlüsselelemente eines Stromrichters sind die Leistungshalbleiter, welche als schnelle statische Schalter eingesetzt werden. Die folgenden Betrachtungen konzentrieren sich auf die Halbleiter in selbstgeführten Stromrichtern. Bild 3.1 zeigt die entsprechenden 3 Halbleitertypen als Schaltsymbole und ihre idealisierten Kennlinien. Sie repräsentieren zwei verschiedene Schalter, einer rückwärts leitend, der andere rückwärts sperrend, sowie eine gewöhnliche Diode. Die idealisierte Betrachtung bedeutet, dass der Spannungsabfall im leitenden Zustand und der Leckstrom im sperrenden Zustand als null angenommen werden. Die Schalter werden über ein logisches Schaltsignal $s$ gesteuert: der Wert $s=1$ bezeichnet den eingeschalteten und $s=0$ den ausgeschalteten Zustand. Das Schaltsignal $s(t)$ in Funktion der Zeit wird als Schaltfunktion bezeichnet. In selbstgeführten Schaltungen müssen die Schaltelemente die Fähigkeit besitzen, den Strom in Vorwärtsrichtung $\left(i_{S}>0\right)$ abschalten zu können. Halbleiter mit dieser Fähigkeit werden $a b$ schaltbar genannt.

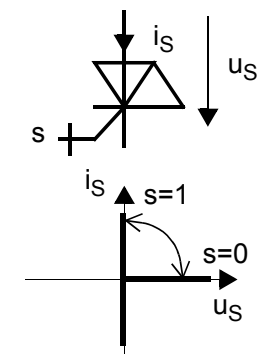

Schalter rückwärts leitend

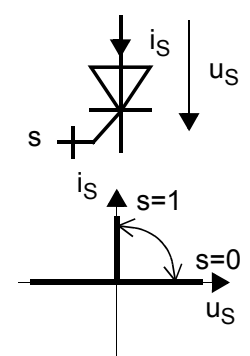

Schalter rückwärts sperrend

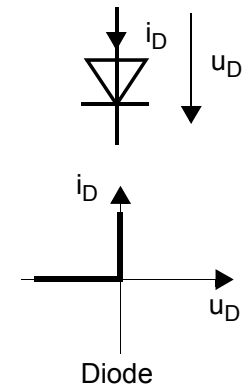

Bild 3.1.

Idealisierte Leistungshalbleiter für einen selbstgeführten Stromrichter

Die idealisierte Betrachtung beinhaltet im weiteren, dass die Übergänge zwischen dem ein- und dem ausgeschalteten Zustand, wie in Bild 3.2 links dargestellt, ohne Verzögerung und mit unendlich steilen Schaltflanken ausgeführt werden können. Der Schalter ist damit gänzlich verlustlos, sowohl in bezug auf Leit- wie auf Schaltverluste. 

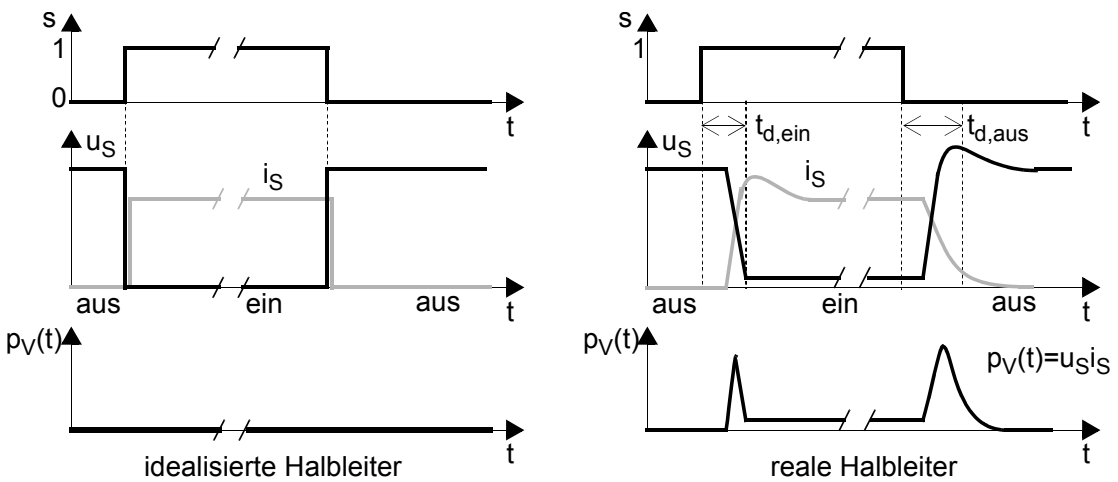

Bild 3.2. Ein- und Ausschaltverhalten von Leistungshalbleitern

\subsubsection{Reale Leistungshalbleiter}

Die Tabelle 3.1 beschreibt die 4 am meisten eingesetzten abschaltbaren Halbleitertypen. In Bild 3.3 sind zusätzlich die Einsatzbereiche dieser Halbleiter bezüglich der beiden wichtigsten Parameter, Nennleistung des Stromrichters und Schaltfrequenz, dargestellt. Dazu ist anzumerken, dass die Leistungshalbleiter einer raschen Entwicklung unterliegen und die Grenzdaten schnell ändern.

Tabelle 3.1. Abschaltbare Leistungshalbleiter

\begin{tabular}{|c|c|c|c|c|c|}
\hline Schaltsymbol & Grenzdaten & $\begin{array}{l}\text { Schaltver- } \\
\text { zögerungen } \\
t_{d \text {,ein }} u . t_{d, \text { aus }}\end{array}$ & $\begin{array}{l}\text { minimale } \\
\text { Pulsweite }\end{array}$ & $\begin{array}{l}\text { Leitspan- } \\
\text { nungsab- } \\
\text { fall }\end{array}$ & $\begin{array}{l}\text { Aufwand für Be- } \\
\text { schaltung und } \\
\text { Ansteuerung }\end{array}$ \\
\hline $\begin{array}{c}\text { MOSFET } \\
\text { Feldeffekttransistor } \\
\text { Mons }\end{array}$ & $\begin{array}{c}\mathrm{u}_{\mathrm{DS}}=1000 \mathrm{~V} / \\
\mathrm{i}_{\mathrm{D}}=15 \mathrm{~A} \\
\text { oder } \\
\mathrm{u}_{\mathrm{DS}}=50 \mathrm{~V} / \\
\mathrm{i}_{\mathrm{D}}=250 \mathrm{~A}\end{array}$ & $0.05 \ldots 0.5 \mu \mathrm{s}$ & $0.5 \ldots 2 \mu \mathrm{s}$ & $\begin{array}{c}1 \ldots 10 \mathrm{~V} \\
(\leq 1 \% \text { von } \\
\left.\mathrm{u}_{\mathrm{DS}, \max }\right)\end{array}$ & klein \\
\hline $\begin{array}{l}\text { IGBT, Insulated Gate } \\
\text { Bipolar Transistor }\end{array}$ & $\begin{array}{c}\mathrm{u}_{\mathrm{CE}}=1600 \mathrm{~V} \\
\mathrm{i}_{\mathrm{C}}=1200 \mathrm{~A}\end{array}$ & $0.4 \ldots 1.2 \mu \mathrm{s}$ & $2 \ldots .5 \mu \mathrm{s}$ & $2 \ldots 4 \mathrm{~V}$ & klein \\
\hline Bipolartransistor & $\begin{array}{c}\mathrm{u}_{\mathrm{CE}}=1200 \mathrm{~V} \\
\mathrm{i}_{\mathrm{C}}=300 \mathrm{~A}\end{array}$ & $2 \ldots 15 \mu \mathrm{s}$ & $5 \ldots 50 \mu \mathrm{s}$ & $1 \ldots 2 \mathrm{~V}$ & gross \\
\hline Gate-Turn-Off Thyristor & $\begin{array}{c}\mathrm{u}_{\mathrm{AK}}=4500 \mathrm{~V} \\
\mathrm{i}_{\mathrm{A}}=3000 \mathrm{~A}\end{array}$ & $10 \ldots 40 \mu \mathrm{s}$ & $50 \ldots 200 \mu \mathrm{s}$ & $2 \ldots 3 \mathrm{~V}$ & sehr gross \\
\hline
\end{tabular}




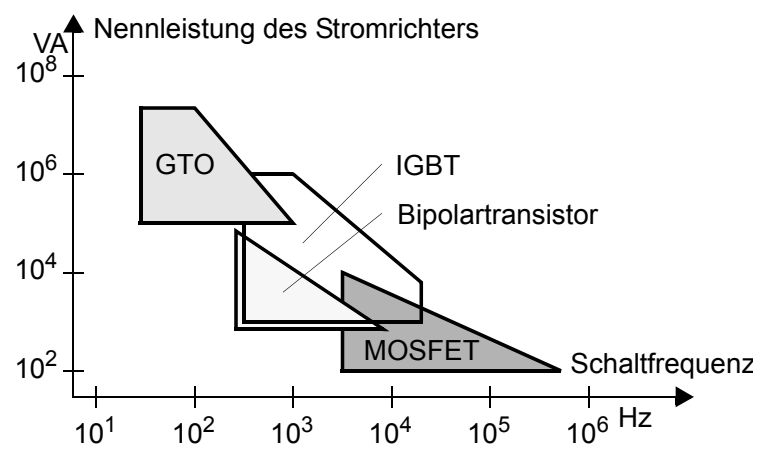

Bild 3.3.

Einsatzbereiche von abschaltbaren Halbleitern

Es ist ersichtlich, dass sich die Bereiche stark unterscheiden: der MOSFET als schnellstes Element ist geeignet für hohe Schaltfrequenzen bis gegen 1MHz. Er ist aber nur in Stromrichtern bis zu einigen kVA Leistung und eher niedriger Spannung einsetzbar. Der verhältnismässig langsame GTO kommt in Anlagen sehr grosser Leistung zum Zuge, darf dafür aber nur mit wenigen $100 \mathrm{~Hz}$ geschaltet werden. Der Bipolartransistor und der IGBT liegen in bezug auf beide Parameter zwischen MOSFET und GTO. Der IGBT mit seinem erweiterten Arbeitsbereich (Safe Operating Area, SOA) löst dabei den älteren Bipolartransistor sukzessive ab. Bei der Betrachtung der Steuerverfahren für die Stromrichter spielt die zulässige Schaltfrequenz für die Halbleiter eine zentrale Rolle. Sie beeinflusst massgeblich, wie gut die Kurvenformen der Spannungen und Ströme am Stromrichter ihren idealen Sollwerten angenähert werden können.

Reales Schaltverhalten: Die Einschränkung der Schaltfrequenz hängt bei allen Halbleitern damit zusammen, dass sie nicht in der Lage sind, verzögerungsfrei und vor allem beliebig schnell zu schalten. Bild 3.2 rechts zeigt die typischen Verläufe von Spannung und Strom in einem realen Schalter. Einerseits sind die Schaltflanken gegenüber dem Steuersignal verzögert und andererseits weisen sie eine endliche Steilheit auf. Die typischen Verzögerungszeiten $t_{d, \text { ein }}$ bzw. $t_{d \text {, aus }}$ beim Ein- und Ausschalten der verschiedenen Halbleiter sind der Tabelle $3.1 \mathrm{zu}$ entnehmen. Beim Schalten entstehen kurzzeitig erhebliche Verluste im Halbleiter. Die mittlere Verlustleistung im realen Schalter setzt sich zusammen aus den Durchlassverlusten, welche im eingeschalteten Zustand auftreten und den Schaltverlusten. Letztere sind proportional zur Schaltfrequenz.

Der GTO und der Bipolartransistor müssen zusätzlich mit einem Entlastungsnetzwerk versehen werden, das die Belastung des Elementes beim Schalten reduziert und einen Teil der Verluste übernimmt. Diese Netzwerke sind im Vergleich zum Halbleiter voluminös und teuer. Sie sind funktionell als fester Bestandteils des Schalters zu betrachten.

Die hohen Verlustspitzen, die in den Schaltmomenten im Halbleiter auftreten, schränken nicht nur die mittlere Schaltfrequenz ein, sondern bewirken auch, dass zwischen den einzelnen Schalthandlungen ein minimales Zeitintervall liegen muss. Nur so kann sich der Halbleiter thermisch genügend regenerieren. Derartige Effekte können mit Hilfe von thermischen Ersatzmodellen studiert werden. Die sich ergebenden minimalen Pulsweiten, das heisst die Zeitintervalle zwischen zwei Schaltvorgängen, sind ebenfalls in Tabelle 3.1 an- 
gegeben. Diese Zeiten stellen weitere Randbedingungen für die Steuerverfahren der Stromrichter dar.

Ansteuerung: Ein weiterer wichtiger Aspekt für den Einsatz eines Halbleiters ist sein Bedarf an Ansteuerelektronik. Unabhängig vom Typ des verwendeten Leistungshalbleiters hat die Ansteuerung typischerweise die in Bild 3.4 als Blockdiagramm dargestellten Funktionen zu erfüllen. Da die Halbleiter in der Regel auf unterschiedlichen Potentialen liegen, muss die Schnittstelle zur übergeordneten Steuerelektronik potentialgetrennt sein. In Frage kommen dafür induktive Übertrager, Optokoppler oder Lichtleiter. Die Stromversorgung der Ansteuerung muss entweder ebenfalls potentialgetrennt erfolgen, oder sie muss dem Leistungskreis direkt beim Schalter entnommen werden. In den meisten Fällen wird der Strom im Halbleiter überwacht. Bei Überstrom schaltet die Ansteuerung selbständig aus und gibt eine Fehlermeldung an die übergeordnete Steuerung weiter. Die Ansteuerschaltungen für die verschiedenen Halbleitertypen unterscheiden sich vor allem in ihrer Endstufe. Diese hat die Aufgabe aus dem logischen Steuerbefehl ein $(s=1)$ oder aus $(s=0)$ die entsprechende Gatespannung $u_{G}$ den Gatestrom $i_{G}$ oder den Basisstrom $i_{B} \mathrm{zu}$ erzeugen. Sie bestimmt im wesentlichen auch den Leistungsbedarf der gesamten Ansteuerschaltung. Die Steuerleistung ist für die spannungsgesteuerten MOSFETs und IGBTs wesentlich kleiner als für die stromgesteuerten Bipolartransistoren und GTOs. Zu beachten ist vor allem die sehr grosse Steuerleistung der GTOs beim Abschalten.

Der Entwicklungstrend bei den Ansteuerungen geht in Richtung Standardisierung und Integration. Es sind MOSFET- und IGBT-Schaltermodule erhältlich, welche ihre Ansteuerung inklusive Potentialtrennung bereits im Modul integriert haben. Andererseits gibt es eine Reihe von speziellen Bausteinen für die einfache Realisierung von externen Ansteuerungen.

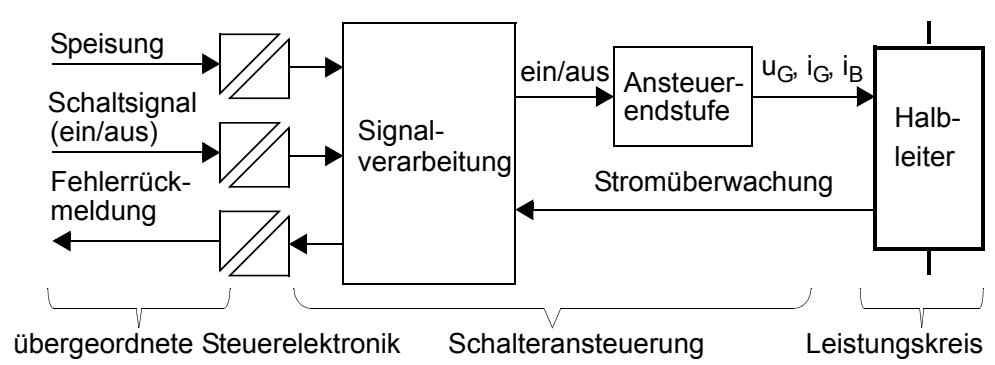
Bild 3.4.
Blockdiagramm einer Ansteue- rung für Leis- tungshalbleiter

Die Funktionen der idealisierten Schalter in Bild 3.1 lassen sich nicht in allen Fällen allein durch einen der 4 verschiedenen Halbleiter realisieren. Nur der MOSFET ist durch seine interne Diode selbst rückwärts leitend. Bei den GTOs sind einige Typen mit einer zusätzlichen auf der Siliziumscheibe mitintegrierten antiparallelen Diode erhältlich. Die anderen Elemente müssen zur Realisierung eines rückwärtsleitenden Schalters extern mit einer antiparallelen Diode beschaltet werden. Ungünstiger verhält es sich mit dem rückwärts sperrenden Schalter. Es gibt nur vereinzelte GTOs in einer symmetrisch sperrenden Version. Bei allen anderen Halbleiter muss eine Diode in Serie geschaltet werden, was den gesamten Spannungsabfall im leitenden Zustand und damit die Durchlassverluste dras- 
tisch erhöht. Dies ist ein schwerwiegender Nachteil für die I-Umrichter, da dort rückwärtssperrende Schaltelemente benötigt werden.

$\mathrm{Zu}$ den Dioden als weitere Leistungshalbleiter soll hier nur eine generelle Bemerkung gemacht werden: beim Einsatz in einem selbstgeführten Stromrichter ist vor allem eine sehr kleine Sperrverzugszeit notwendig, da sonst durch die schnellen Schaltvorgänge unzulässig grosse Rückwärtsströme entstehen. Es gibt Diodentypen (Fast-Recovery- und Soft-Recovery-Diodes), die speziell für diesen Einsatz entwickelt worden sind.

Literatur zu Kapitel 3.1: [Hag1], [Mic1], [Mül1]

\subsection{Quellen und Lasten von Stromrichtern}

\subsubsection{Netze}

Ein Versorgungsnetz, sei es ein Inselnetz oder ein Verbundnetz, ist in der Regel ein räumlich verteiltes System bestehend aus Generatoren, Leitungen und Lasten. Für Betrachtungen im Zusammenhang mit Stromrichtern genügt meistens eine sehr einfache Modellierung, bestehend aus einer Wechselspannungsquelle $e_{N}$ und einer ohmsch-induktiven Innenimpedanz $R_{N}$ und $L_{N}$ pro Phase nach Bild 3.5. Von besonderer Bedeutung im Zusammenhang mit Stromrichtern ist die Frage, welche Rückwirkungen die nichtsinusförmigen Phasenströme auf das Netz haben. Dazu kann die Maschengleichung (3.1) betrachtet werden:
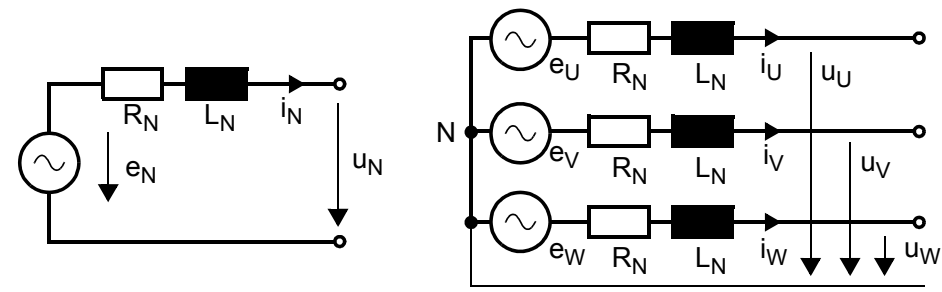

\section{Bild 3.5.}

Ersatzschaltungen für ein Versorgungsnetz, links: einphasig, rechts: dreiphasig

$$
u_{N}=e_{N}-R_{N} i_{N}-L_{N} \frac{d i_{N}}{d t}
$$

Es ist leicht zu erkennen, dass die Spannung $u_{N}$ vom bezogenen Strom $i_{N}$ abhängt. Weicht dieser von der Sinusform ab, so ergibt sich eine entsprechende Verzerrung für $u_{N}$. Sie ist unerwünscht, weil sie bei anderen Verbrauchern im gleichen Netz zu Funktionsstörungen und zusätzlichen Verlusten führen kann. Es existieren deshalb Normen für die maximal zulässigen Strom- und Spannungsverzerrungen im Netz (Kapitel 18). Da diese Verzerrungsanteile stets im Frequenzbereich oberhalb der Netzfrequenz liegen, fällt vor allem der Spannungsabfall über der Induktivität ins Gewicht. Für die meisten Betrachtungen zu diesem Themenkreis kann deshalb der ohmsche Anteil $R_{N}$ vernachlässigt werden. 
Die Ersatzquellenspannung $e_{N}$ selbst ist im allgemeinen ebenfalls nicht exakt sinusförmig. Sie repräsentiert die Verzerrungen, welche durch andere Verbraucher im Netz verursacht werden. Dank der erwähnten Normen ist die Abweichung von der Sinusform jedoch in der Regel klein.

Ein verzerrter Netzstrom ist auch deshalb unerwünscht, weil er in Leitungen und Transformatoren zusätzliche Verluste verursacht.

\subsubsection{Drehstrommaschinen}

Die ausführliche Herleitung der Gleichungen für die Asynchron- und die Synchronmaschine (ASM und SM) würde an dieser Stelle zu weit führen. Dazu wird auf die entsprechende Literatur (z.B. [Büh1], [Kle1], [Leo1], [Mey2], [Spä1]) verwiesen. Es werden nur die wesentlichen Eigenschaften kurz betrachtet, welche im Hinblick auf die Besprechung von Steuerverfahren für stromrichtergespeiste Antriebe wichtig sind. Dies sind einerseits die Modellierung der Maschinen als Last eines Stromrichters und andererseits die Auswirkungen der nichtsinusförmigen Speisung auf das Verhalten der Maschinen.

\subsubsection{Asynchronmaschine}

Bild 3.6 zeigt den prinzipiellen Aufbau einer ASM. Im Stator wie im Rotor befinden sich 3 um jeweils $2 \pi / 3$ gegeneinander versetzte Wicklungen. Handelt es sich um eine Maschine mit Kurzschlussläufer, wie das bei Umrichterantrieben üblich ist, so sind die Rotorwicklungen, die nur aus den Stäben des Rotorkäfigs bestehen, kurzgeschlossen.

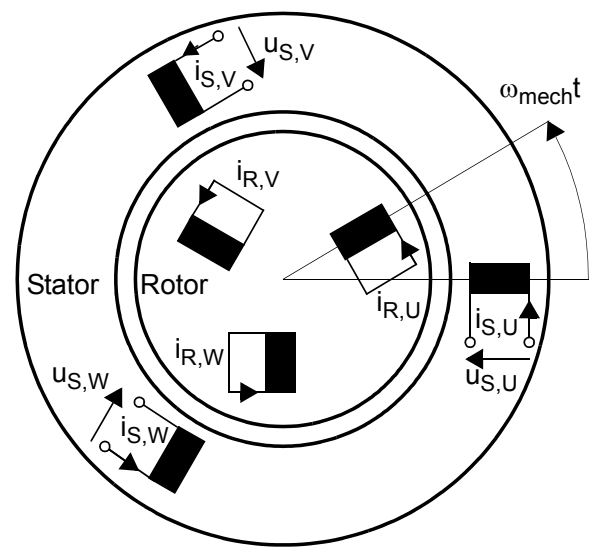

Bild 3.6.

Schematische Darstellung einer zweipoligen Asynchronmaschine mit Kurzschlussläufer $(p=1)$

Die Kreisfrequenzen der Stator- und Rotorgrössen $\omega_{S}$ bzw. $\omega_{R}$ sowie der Welle $\omega_{\text {mech }}$ sind gemäss (3.2) miteinander gekoppelt. Dieser Zusammenhang ist auch im Kennlinienfeld in Bild 2.5 links erkennbar.

$$
\omega_{S}=p \omega_{\text {mech }}+\omega_{R}, \quad p \text { : Polpaarzahl }
$$


Es ist allgemein üblich, Drehstrommaschinen mit Hilfe von Drehzeigern (in $\alpha \beta$-Darstellung, Anhang B.1.2) zu beschreiben. Alle Spannungen, Ströme und Flussverkettungen in der Maschine lassen sich in Zeiger überführen. Der dynamische Betriebszustand der ASM kann auf diese Weise durch 6 Grundgleichungen beschrieben werden. In der statorfesten Darstellung nehmen sie die allgemein bekannte Form (3.3) bis (3.8) an:

$$
\begin{aligned}
& \underline{u}_{S}=R_{S_{-S}}+\frac{d \underline{\Psi}_{S}}{d t} \\
& 0=R_{R_{-R}}+\frac{d \underline{\Psi}_{R}}{d t}-j p \omega_{\text {mech }} \underline{\Psi}_{R} \\
& \underline{\Psi}_{S}=L_{S} i_{-S}+L_{h \cdot R} i_{-R} \\
& \underline{\Psi}_{R}=L_{h_{-S}}+L_{R_{-R}} \\
& M_{e l}=\frac{3 p}{2} \operatorname{Im}\left(\underline{\Psi}_{S}{ }^{*} i_{-S}\right)=\frac{3 p}{2} \frac{L_{h}}{L_{R}} \operatorname{Im}\left(\underline{\Psi}_{R}{ }^{*} i_{-S}\right) \\
& \omega_{m e c h}=\frac{1}{J} \int\left(M_{e l}-M_{\text {Last }}\right) d t
\end{aligned}
$$

mit $\quad \underline{\Psi}_{S}, \underline{\Psi}_{R} \quad$ Stator- bzw. Rotorflussverkettung

$L_{S}, L_{R} \quad$ Induktivität der Stator- bzw. Rotorwicklung

$L_{h} \quad$ Kopplungsinduktivität (Hauptinduktivität)

$R_{S}, R_{R} \quad$ Stator- bzw. Rotorwicklungswiderstand

$M_{\text {el }}, M_{\text {Last }} \quad$ elektrisches bzw. Last-Drehmoment

$J \quad$ Massenträgheitsmoment

Alle weiteren Betrachtungen in diesem Buch gehen von einer zweipoligen Maschine aus, so dass $p=1$ wird und aus den Gleichungen verschwindet.

Für die Untersuchung von Modulationsverfahren ist in erster Linie der stationäre Betrieb von Bedeutung, wobei die Maschine jedoch mit nichtsinusförmigen Grössen betrieben wird. Alle Spannungs-, Strom- und Flussverkettungszeiger weisen dann eine konstante mittlere Kreisfrequenz $\omega_{S}$ auf. Wegen der nichtsinusförmigen Speisung sind jedoch die Amplituden und Kreisfrequenzen der Zeiger mit einer Welligkeit behaftet. Das Drehmoment und die Drehzahl der ASM sind im Mittel konstant, weisen aber ebenfalls eine Welligkeit auf. Bei der Drehzahl ist diese wegen der verhältnismässig grossen mechanischen Zeitkonstante in der Regel vernachlässigbar.

Für die Bestimmung der Ersatzschaltung der ASM als Last eines Stromrichters wird (3.3) mit Hilfe der Gleichungen (3.5) und (3.6) umgeformt und auf die Form (3.9) gebracht:

$$
\underline{u}_{S}=R_{S} i_{-S}+\left(L_{S}-\frac{L_{h}^{2}}{L_{R}}\right) \frac{d i_{S}}{d t}+\frac{L_{h}}{L_{R}} \frac{d \underline{\Psi}_{R}}{d t}
$$


Die Statorspannung setzt sich aus 3 Termen zusammen. Die ersten beiden sind abhängig vom Statorstrom. Der dritte ist abhängig von der Rotorflussverkettung, deren Ableitung sich mit (3.4) und (3.6) in die Form (3.10) bringen lässt:

$$
\frac{d \underline{\Psi}_{R}}{d t}=j \omega_{m e c h} \underline{\Psi}_{R}-\frac{R_{R}}{L_{R}} \underline{\Psi}_{R}+\frac{R_{R} L_{h}}{L_{R}} i_{S}
$$

Wie im Zeigerdiagramm in Bild 3.7 dargestellt ist, kann der Statorstrom $\underline{i}_{S}$ in die Komponenten $i^{\prime}{ }_{S}$ und $i^{\prime \prime}{ }_{S}$ zerlegt werden, wobei $i^{\prime}{ }_{S}$ parallel und $i^{\prime \prime}{ }_{S}$ senkrecht zu $\underline{\Psi}_{R}$ liegt. Da-

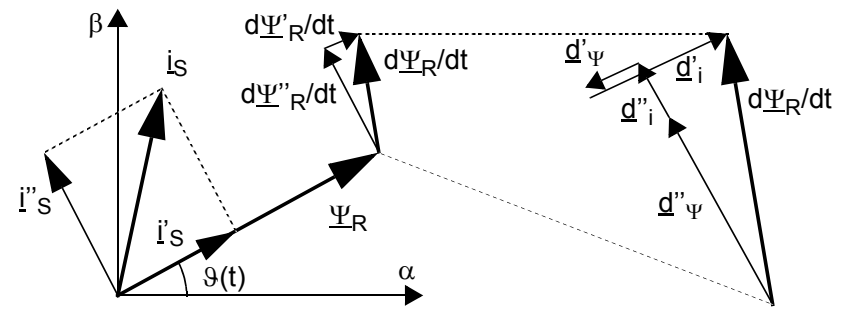

\section{Bild 3.7.}

Zeigerdiagramme zur Gleichung (3.11), links: Aufspaltung des Statorstromes $\underline{i}_{S}$ in die Komponenten $i^{\prime}{ }_{S}$ und $i "{ }_{S}$ rechts: Aufspaltung der Ableitung der Rotorflussverkettung

mit lässt sich (3.10) in die Form (3.11) bringen und anschliessend in die zwei Differentialgleichungen (3.12) und (3.13) aufspalten:

$$
\begin{aligned}
& \frac{d \underline{\Psi} R}{d t}=\underbrace{j \omega_{\text {mech }} \underline{\Psi}_{R}}_{\underline{d}^{\prime \prime} \Psi}-\underbrace{\frac{R_{R}}{L_{R}} \underline{\Psi}_{R}}_{\underline{d}^{\prime} \Psi}+\underbrace{\frac{R_{R} L_{h}}{L_{R}} i_{S}}_{\underline{d}_{i}}+\underbrace{\frac{R_{R} L_{h}}{L_{R}} i^{\prime \prime}{ }_{S}}_{\underline{d}_{i}{ }_{i}} \\
& \frac{d \underline{\Psi}_{R}^{\prime}}{d t}=-\underline{d}^{\prime} \Psi+\underline{d}_{-i}=-\frac{R_{R}}{L_{R}} \Psi_{R}+\frac{R_{R} L_{h}}{L_{R}} i_{S}{ }_{S}=\frac{R_{R}}{L_{R}}\left(L_{h} i_{-}^{\prime}{ }_{S}-\underline{\Psi}_{R}\right) \\
& \frac{d \underline{\Psi}^{\prime \prime}}{d t}=\underline{d "}^{\prime \prime}+\underline{d}^{\prime \prime}{ }_{i}=j \omega_{\text {mech }} \underline{\Psi}_{R}+\frac{L_{h} R_{R}}{L_{R}} \underline{i}_{S}
\end{aligned}
$$

Zur Interpretation dieser Gleichungen werden $\underline{\Psi}_{R}$ und $d \underline{\Psi}_{R} / d t$ in die Polarkoordinatendarstellung (3.14) und (3.15) gebracht:

$$
\begin{gathered}
\underline{\Psi}_{R}=\left|\underline{\Psi}_{R}\right| e^{j \vartheta} \\
\Rightarrow \quad \frac{d \underline{\Psi}_{R}}{d t}=\underbrace{\frac{d\left|\underline{\Psi}_{R}\right|}{d t} e^{j \vartheta}}_{\frac{d \underline{\Psi}_{R}^{\prime}}{d t}}+\underbrace{j \frac{d \vartheta}{d t}\left|\underline{\Psi}_{R}\right| e^{j \vartheta}}_{\frac{d \underline{\Psi}^{\prime \prime} R}{d t}}
\end{gathered}
$$


Die beiden Summanden in (3.15) entsprechen den Komponenten $d \underline{\Psi}^{\prime}{ }_{R} / d t$ und $d \underline{\Psi}{ }_{R} / d t$. Durch einen Vergleich der Beträge kommt man schliesslich auf die Gleichungen (3.16) für die Ableitung des Betrages und (3.17) für die Rotationsbewegung von $\underline{\Psi}_{R}$ :

$$
\begin{aligned}
& \frac{d\left|\underline{\Psi}_{R}\right|}{d t}=\frac{R_{R}}{L_{R}}\left|L_{h} i_{-}{ }^{\prime}-\underline{\Psi}_{R}\right| \\
& \frac{d \vartheta}{d t}\left|\underline{\Psi}_{R}\right|=\omega_{\text {mech }}\left|\underline{\Psi}_{R}\right|+\frac{R_{R} L_{h}}{L_{R}}\left|i_{S}\right| \Rightarrow \frac{d \vartheta}{d t}=\omega_{\text {mech }}+\frac{R_{R}}{L_{R}} \frac{L_{h}\left|i^{\prime \prime} S\right|}{\left|\underline{\Psi}_{R}\right|}
\end{aligned}
$$

Im stationären Zustand und bei rein sinusförmiger Speisung der Maschine ist die Rotorflussverkettung ein Zeiger konstanter Länge mit der Winkelgeschwindigkeit $\omega_{S}$. Es gilt dann:

$$
\begin{gathered}
\underline{\Psi}_{R}=\left|\underline{\Psi}_{R}\right| e^{j \omega_{S} t}, \quad \frac{d\left|\underline{\Psi}_{R}\right|}{d t}=0, \quad \frac{d \vartheta}{d t}=\omega_{S} \\
\Rightarrow \quad \frac{d \underline{\Psi}_{R}}{d t}=j \omega_{S}\left|\underline{\Psi}_{R}\right| e^{j \omega_{S} t}
\end{gathered}
$$

Wird die ASM mit nichtsinusförmigen Grössen gespeist, so ist $\underline{i}_{S}$ ein Zeiger, dessen Länge und Winkelgeschwindigkeit eine Welligkeit aufweisen. Diese wirkt sich grundsätzlich auch in den Gleichungen (3.16) und (3.17) aus. Der Einfluss wird aber in beiden Gleichungen durch die Rotorzeitkonstante $T_{R}=L_{R} / R_{R}$ abgeschwächt. Sie ist bei allen ASM derart gross, dass die Rotorflussverkettung im Normalfall nur eine vernachlässigbar kleine Welligkeit in Betrag und Winkelgeschwindigkeit erhält. Damit darf die Gleichung (3.18) auch für nichtsinusförmige Speisung der Maschine als gute Näherung verwendet werden.

Aufgrund dieser Tatsache kann nun (3.9) in die Form (3.19) gebracht werden. Die ASM im stationären Zustand lässt sich durch die Ersatzschaltung in Bild 3.8 modellieren.

$$
\underline{u}_{S}=R_{S_{-S}}+L_{\sigma} \frac{d \underline{i}_{S}}{d t}+\underline{e}
$$$$
\text { mit } L_{\sigma}=L_{S}-\frac{L_{h}^{2}}{L_{R}},
$$$$
\underline{e}=\frac{L_{h}}{L_{R}} \frac{d \underline{\Psi}_{R}}{d t} \approx j \omega_{S} \frac{L_{h}}{L_{R}} \underline{\Psi}_{R} \approx j \omega_{S} \frac{L_{h}}{L_{R}}\left|\underline{\Psi}_{R}\right| e^{j \omega_{S} t}
$$

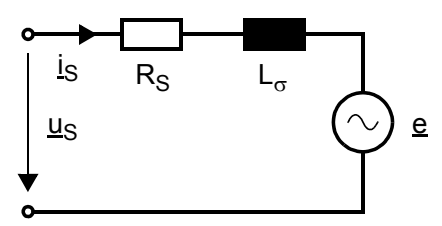

Bild 3.8.

Stationäres Ersatzschaltbild der ASM

Die Amplitude der auftretenden Gegenspannung $\underline{\underline{e}}$ wird im Grunddrehzahlbereich, wo in der Regel mit konstantem Fluss gefahren wird, proportional zur Statorkreisfrequenz grös- 
ser. Im Feldschwächbereich bleibt sie konstant, da der Fluss dort mit steigender Frequenz reduziert wird. Für die Beschreibung der ASM mit Phasengrössen gilt dieselbe Ersatzschaltung für jede Phase. Die Gegenspannungen sind in diesem Fall symmetrisch und verlaufen sinusförmig.

Mit Hilfe der Aufspaltung des Statorstromes $\underline{i}_{S}$ in die Komponenten $i^{\prime}{ }_{S}$ und $i^{\prime \prime}{ }_{S}$ gemäss Bild 3.7 lässt sich auch die Gleichung (3.7) in eine skalare Form (3.20) bringen:

$$
M_{e l}=\frac{3}{2} \frac{L_{h}}{L_{R}}\left|\underline{\Psi}_{R}\right|\left|i^{\prime \prime} S\right|
$$

Daraus wird deutlich, dass nur der zur Rotorflussverkettung senkrechte Anteil des Statorstromes $i^{\prime \prime}{ }_{S}$ zur Drehmomentbildung beiträgt. Bei nichtsinusförmiger Speisung gehen sowohl der Mittelwert als auch die Welligkeit von $i^{\prime \prime}{ }_{S}$ direkt proportional in das Drehmoment ein. Weiter ist aus (3.12) ersichtlich, dass nur der zu $\underline{\Psi}_{R}$ parallele Anteil von $\underline{i}_{S}$, nämlich $i_{S}^{\prime}$, für die Flussbildung verantwortlich ist. Die Welligkeit von $i^{\prime}{ }_{S}$ wirkt sich dabei, wie bereits gesagt, wegen der grossen Rotorzeitkonstanten praktisch nicht aus.

Das Drehmoment lässt sich auch anhand der Ersatzschaltung nach Bild 3.8 direkt bestimmen. Setzt man (3.19) in (3.7) ein, so erhält man nach geeigneter Umformung:

$$
M_{e l}=\frac{3}{2} \frac{1}{\omega_{S}} \operatorname{Re}\left(\underline{e}^{*} i_{S}\right)=\frac{3}{2} \frac{1}{\omega_{S}} \operatorname{Re}\left(\underline{e}_{-}{ }_{S}^{*}\right)
$$

Diese Gleichung lässt sich geometrisch interpretieren: nur der jeweils zur Gegenspannung $\underline{e}$ parallele Anteil des Stromes $\underline{i}_{S}$ trägt zur Drehmomentbildung bei.

Neben der Welligkeit des Drehmomentes, welche je nach Anwendung sehr störend sein kann, hat die nichtsinusförmige Speisung der ASM noch weitere unangenehme Auswirkungen:

- Die Verzerrungsanteile in den Maschinenströmen verursachen zusätzliche Verluste in den Wicklungen. Dieser Effekt wird unter Umständen durch die Stromverdrängung (Kapitel 3.2.7) noch verstärkt.

- Im weiteren bilden sich neben der Drehmomentwelligkeit weitere Kräfte aus, welche einzelne Teile der Maschine in Vibration versetzen. Dies äussert sich in erster Linie durch störende Geräusche in der Form eines Pfeifens oder Rauschens. Sie sind im Bereich der besten akustischen Wahrnehmung, d.h. um $1 \mathrm{kHz}$ besonders unerwünscht.

Alle diese Phänomene unterstreichen die Notwendigkeit, die Verzerrungen in den Maschinenströmen generell klein zu halten.

\subsubsection{Synchronmaschine}

Bild 3.9 zeigt den prinzipiellen Aufbau einer SM. Im Stator befindet sich wie bei der ASM ein dreiphasiges Wicklungssystem. Der Rotor besteht aus einem rotierenden Magneten. Ob das Feld dabei durch einen Permanentmagneten oder durch eine stromdurchflossene Spule erzeugt wird, spielt für die folgenden Betrachtungen keine Rolle. Im Gegensatz zur ASM laufen das Drehfeld und der Rotor stets mit derselben Kreisfrequenz um. Es gilt deshalb die Beziehung (3.22): 


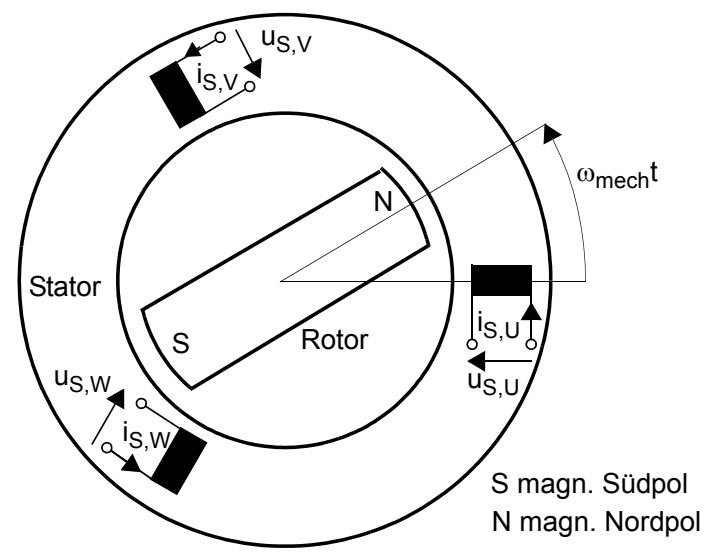

$\omega_{S}=p \omega_{\text {mech }}, \quad p:$ Polpaarzahl
Bild 3.9.

Schematische Darstellung einer zweipoligen Synchronmaschine $(p=1)$

Auch hier ist es gebräuchlich, die Maschine mittels Drehzeigern zu beschreiben. Der Betriebszustand der SM ist auf diese Weise durch 4 dynamische Gleichungen bestimmt:

$$
\begin{aligned}
& \underline{u}_{-S}=R_{S} i_{-S}+\frac{d \underline{\Psi}_{S}}{d t} \\
& \underline{\Psi}_{S}=L_{S_{-S}} \underline{i}+\underline{\Psi}_{0} \\
& M_{e l}=\frac{3 p}{2} \operatorname{Im}\left(\underline{\Psi}_{S}{ }^{*} i_{S}\right)=\frac{3 p}{2} \operatorname{Im}\left(\underline{\Psi}_{0}{ }^{*} i_{-S}\right) \\
& \omega_{\text {mech }}=\frac{1}{J} \int\left(M_{e l}-M_{\text {Last }}\right) d t
\end{aligned}
$$

$\begin{array}{lll}\operatorname{mit} & \underline{\Psi}_{S} & \text { Statorflussverkettung } \\ & \underline{\Psi}_{0} & \text { durch das Rotorfeld hervorgerufene Flussverkettung } \\ & L_{S} & \text { Induktivität der Statorwicklung } \\ R_{S} & \text { Statorwicklungswiderstand } \\ M_{e l}, M_{\text {Last }} & \text { elektrisches und Last-Drehmoment } \\ J & \text { Massenträgheitsmoment }\end{array}$

Wie schon bei der ASM wird auch bei der SM im folgenden stets $p=1$ angenommen.

Es wird nur der stationäre Betrieb betrachtet. Setzt man (3.24) in (3.23) ein, so ergibt sich die Beziehung für die Statorspannung und den Statorstrom:

$$
\underline{u}_{S}=R_{S} i_{-S}+L_{S} \frac{d i_{S}}{d t}+\frac{d \underline{\Psi}_{0}}{d t}
$$

Unter der Annahme, dass wegen der verhältnismässig grossen mechanischen Zeitkonstante die Drehzahl auch bei nichtsinusförmiger Speisung keine Welligkeit aufweist, gilt: 


$$
\underline{\Psi}_{0}=\left|\underline{\Psi}_{0}\right| e^{j \omega_{S} t} \quad \Rightarrow \quad \frac{d \underline{\Psi}_{0}}{d t}=j \omega_{S}\left|\underline{\Psi}_{0}\right| e^{j \omega_{S} t}=\underline{u}_{-p}
$$

Die Spannung $\underline{u}_{p}$ wird Polradspannung genannt. Sie ist ein Drehzeiger mit Kreisfrequenz $\omega_{S}$ und einer zur Drehzahl proportionalen Länge. Die SM kann deshalb durch die Ersatzschaltung in Bild 3.10 modelliert werden, also im Prinzip gleich wie die ASM. Die wirksame Induktivität im Ersatzschaltbild ist allerdings grösser, da bei der SM die volle statorseitige Induktivität $L_{S}$ darin eingeht, während es bei der ASM nur die gesamte Streuinduktivität ist. Damit können sich die Stromverzerrungen etwas weniger gut ausbilden. Als Gegenspannung wirkt die Polradspannung $\underline{u}_{p}$. Sie ergibt in den Statorwicklungen sinusförmige Spannungen mit einer zur Drehzahl proportionalen Amplitude.

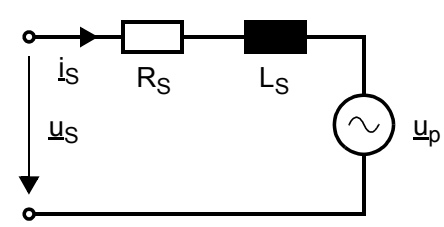

Bild 3.10.

Stationäres Ersatzschaltbild der SM

Im weiteren ist aus (3.25) ersichtlich, dass das Drehmoment aus dem Produkt von $\underline{\Psi}_{0}$ und der dazu senkrechten Komponente des Statorstromes $\underline{i}_{S}$ gebildet wird. Die zum Fluss parallele Komponente geht nicht in das Drehmoment ein. Dies gilt auch für den Verzerrungsanteil im Statorstrom: nur dessen zur Polradspannung senkrechte Komponente verursacht eine Welligkeit des Drehmomentes.

Die nichtsinusförmige Speisung der SM hat grundsätzlich dieselben unerwünschten Auswirkungen wie bei der ASM: Drehmomentwelligkeit, zusätzliche Verluste und störende Geräusche.

\subsubsection{Transformatoren}

Bei vielen Anwendungen wird auf der Netzseite, wie in Bild 3.11 dargestellt, ein Transformator vor den Umrichter geschaltet. Dieser kann verschiedene Aufgaben erfüllen:

1. Potentialtrennung vom Netz. Damit kann nach Wunsch ein beliebiger Punkt des Umrichters bzw. der Lastseite geerdet werden. Diese Option ist vor allem in bezug auf die Sicherheits- und Schutzaspekte einer Anlage wichtig.

2. Anpassung des Spannungsniveaus an die Bedürfnisse der Last und des Umrichters (vor allem der Leistungshalbleiter).

3. Mit Hilfe von Transformatoren mit mehreren Wicklungen lassen sich durch geeignete Schaltungstopologien Netzströme mit verbesserter Kurvenformen erreichen. Ein Beispiel dafür sind 12- oder höherpulsige Gleichrichterschaltungen (Kapitel 16).

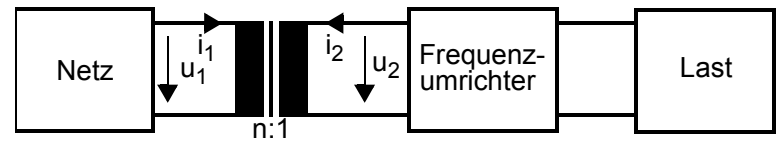

Bild 3.11.

Frequenzumrichter mit Transformator 
Bild 3.12 zeigt das allgemein verwendete sogenannte T-Ersatzschaltbild eines einphasigen bzw. einer Phase eines dreiphasigen Transformators. Unter der Annahme, dass sich
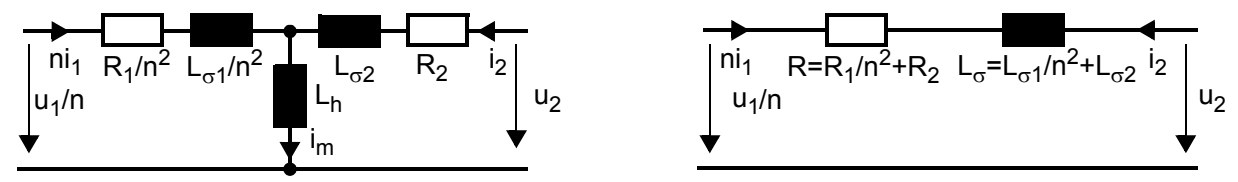

Bild 3.12. Ersatzschaltung für eine Phase eines Transformators,

links: T-Ersatzschaltung, rechts: vereinfachte Schaltung für $L_{h} \rightarrow \infty$

der Umrichter auf der Sekundärseite befindet, ist die Ersatzschaltung auf diese Seite bezogen. Die Hauptinduktivität $L_{h}$ ist normalerweise deutlich grösser als die Streuinduktivitäten $L_{\sigma 1}$ und $L_{\sigma 2}$, so dass der Magnetisierungsstrom $i_{m}$ gegenüber den Strömen $n i_{1}$ und $i_{2}$ im belasteten Zustand sehr klein wird. Vor allem bei Transformatoren mittlerer und grosser Leistung liegt $i_{m}$ im Bereich von unter $2 \%$ des Transformator-Nennstromes. Für Betrachtungen, bei denen der Umrichter im Mittelpunkt steht, kann $i_{m}$ deshalb in der Regel vernachlässigt werden. Damit gilt das vereinfachte Ersatzschaltbild in Bild 3.12 rechts. Vom Umrichter aus gesehen wirkt der Transformator dann einfach als zusätzliche Zuleitungsimpedanz, die zur Innenimpedanz des Netzes zu addieren ist.

$\mathrm{Zu}$ beachten ist, dass der Hauptfluss auf einem geschlossen Pfad im stark nichtlinearen Eisen verläuft. Sobald das Kernmaterial gesättigt ist, steigt der Magnetisierungsstrom $i_{m}$ sehr stark an, so dass das vereinfachte Ersatzschaltbild nicht mehr gültig ist. Der Transformator ist jedoch so ausgelegt, dass dies im Normalbetrieb nicht geschieht. Es ist jedoch äusserst wichtig, dass die Eingangsströme des Umrichters keine Gleichanteile aufweisen. Diese würden über die Hauptinduktivität fliessen und sehr rasch zur Sättigung des Eisens führen. Das Vermeiden solcher Gleichanteile gehört in den Aufgabenbereich des Steuerverfahrens für den netzseitigen Stromrichter.

\subsubsection{Standardlast eines Stromrichters}

Für die Untersuchung von Steuerverfahren für selbstgeführte Stromrichter ist es sinnvoll, eine Standardlast in ein- und dreiphasiger Ausführung gemäss Bild 3.13 einzuführen. Wie gezeigt wurde, entspricht sie sowohl der Ersatzschaltung des Netzes als auch derjenigen von Drehstrommaschinen.
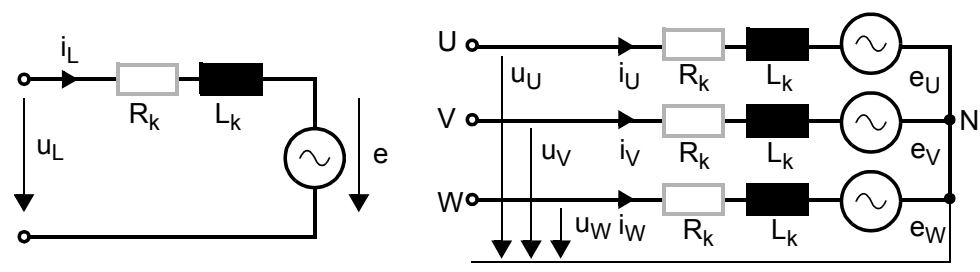

Bild 3.13.

Standardlast eines Stromrichters, links: einphasig, rechts: dreiphasig 
Es wird angenommen, dass die Gegenspannungsquellen $e$ bzw. $e_{U}, e_{V}$ und $e_{W}$ sinusförmige Spannungen mit variabler Amplitude und Frequenz abgeben. Die dreiphasige Ersatzschaltung wird stets als symmetrisch angenommen. Auf diese Weise sind alle in der Schaltung auftretenden Verzerrungen und Unsymmetrien auf den Stromrichter bzw. sein Steuerverfahren zurückzuführen.

Als weitere Vereinfachung wird der Widerstand $R_{k}$ als null angenommen. Die Vereinfachung ist erlaubt, wenn man sich in erster Linie für die Verzerrungsanteile in den Spannungen und Strömen interessiert, die in einem deutlich höheren Frequenzbereich liegen als die Grundfrequenz. Dort ist der induktive Anteil der Impedanz $L_{k}$ dominant. Mit der Vernachlässigung des ohmschen Anteiles im Lastkreis werden die berechneten Verzerrungen des Stromes gegenüber der Realität höchstens etwas zu gross. Nicht zulässig ist die Vereinfachung dagegen für Betrachtungen, welche die Regelung des Umrichters betreffen. Insbesondere im Bereich sehr niedriger Grundfrequenz, zum Beispiel im Anlaufbereich eines Antriebes, wird $R_{k}$ gegenüber $L_{k}$ dominant. Generell ist bei Anlagen mittlerer und grosser Leistung die Vernachlässigung von $R_{k}$ eher zulässig, da dort bereits bei der Nennfrequenz des Umrichters das Verhältnis zwischen induktivem und ohmschem Anteil der Impedanz 10 und mehr beträgt.

Als Standardlast wird eine Sternschaltung verwendet. Dadurch sind die Lastspannungen identisch mit den Phasenspannungen $u_{U}, u_{V}, u_{W}$ und die Lastströme identisch mit den Phasenströmen $i_{U}, i_{V}, i_{W}$. Es ist jedoch für den Stromrichter unbedeutend, ob die Last in Stern oder in Dreieck geschaltet ist.

\subsubsection{Zwischenkreis}

\subsubsection{Spannungszwischenkreis}

Die Struktur der beiden Zwischenkreistypen für U-Umrichter und I-Umrichter mit einer Kapazität bzw. einer Induktivität als Speicher wurde bereits in Bild 2.2 vorgestellt. Weiter wurde in Kapitel 2.1 gesagt, dass die beiden Stromrichter eines Umrichters bis auf die Regelung der mittleren Wirkleistung unabhängig voneinander betrachtet werden können. Im folgenden soll diese Aussage präzisiert werden.


Bild 3.14. Varianten für die Modellierung des Zwischenkreises eines U-Umrichters, links: reale Schaltung, mitte: Ersatz des netzseitigen Stromrichters durch ideale Gleichstromquelle, rechts: Ersatz der Kapazität durch eine ideale Gleichspannungsquelle

Bild 3.14 zeigt die Möglichkeiten der Modellierung für einen Zwischenkreises im U-Umrichter. In der realen Schaltung kann die Zwischenkreisspannung nach der Differentialgleichung (3.29) berechnet werden: 


$$
u_{d}=\frac{1}{C_{d}} \int\left(i_{d, N}-i_{d, L}\right) d t
$$

Beide Stromrichter verursachen im Zwischenkreis mit Welligkeiten behaftete Gleichströme. Diese lassen sich allgemein wie folgt beschreiben:

$$
i_{d, N}=I_{d, N}+i_{d, N, V Z}, \quad i_{d, L}=I_{d, L}+i_{d, L, V Z}
$$

Die Anteile $I_{d, N}$ und $I_{d, L}$ bezeichnen die beiden zeitlichen Mittelwerte und die Anteile $i_{d, N, V Z}$ und $i_{d, L, V Z}$ beschreiben die Welligkeit der Ströme. Aus der Forderung nach einer ausgeglichenen Leistungsbilanz geht die Beziehung (3.31) hervor:

$$
I_{d, N}=I_{d, L}=I_{d}
$$

Geht man davon aus, dass sich beide Stromrichter im stationären Betrieb befinden, so ist der Mittelwert der Zwischenkreisspannung konstant. Sie weist aber ebenfalls eine Welligkeit $u_{d, V Z}$ auf, die sich wie folgt berechnen lässt:

$$
u_{d, V Z}=\frac{1}{C_{d}} \int i_{C} d t=\frac{1}{C_{d}} \int\left(i_{d, N, V Z}-i_{d, L, V Z}\right) d t
$$

Diese Spannungswelligkeit ist dafür verantwortlich, dass eine zusätzliche Kopplung zwischen den beiden Umrichterseiten entsteht. Sie wirkt sowohl auf der Netz-, als auch auf der Lastseite auf die Spannungen und Ströme ein. Eine exakte analytische Beschreibung des Umrichters ist dementsprechend nur in Form eines Differentialgleichungssystems möglich, das beide Umrichterseiten umfasst.

Häufig möchte man allerdings den Einfluss einer Umrichterseite auf den Zwischenkreis für sich allein betrachten. Dazu kann das vereinfachte Modell in Bild 3.14 mitte verwendet werden. Es ersetzt einen der beiden Stromrichter durch eine ideale einstellbare Gleichstromquelle, welche zu jeder Zeit den benötigten Mittelwert $I_{d}$ liefert. Die Welligkeit der Zwischenkreisspannung beschränkt sich dann auf den durch den verbleibenden Stromrichter verursachten Anteil:

$$
u_{d, V Z}=-\frac{1}{C_{d}} \int_{d, L, V Z} d t
$$

Von Bedeutung ist neben den stationären Betrachtungen auch der Fall, dass die Zwischenkreisspannung auf einen anderen Mittelwert eingestellt werden soll. Dies wird dadurch erreicht, dass die beiden Mittelwerte $I_{d, N}$ und $I_{d, L}$ während einer gewissen Zeit ungleich gross gemacht werden, so dass ein Ladestrom für die Kapazität entsteht. Die entsprechende Regelung wird gewöhnlich auf der Netzseite des Umrichters vorgenommen.

Ist die Kapazität gross genug, so dass die Welligkeit der Spannung vernachlässigt werden darf, so verhält sich der Zwischenkreis wie eine Gleichspannungsquelle. In diesem Fall, der in Bild 3.14 rechts dargestellt ist, sind die beiden Umrichterseiten vollständig entkoppelt. Der Zwischenkreis ist in der Lage, die Welligkeit beider Gleichströme aufzunehmen, ohne dass dies Auswirkungen auf die Spannung hat. $\mathrm{Zu}$ beachten ist allerdings, dass dieses Modell für dynamische Betrachtungen ungeeignet ist: es tritt auch dann keine Spannungsänderung auf, wenn die Mittelwerte der beiden Ströme nicht übereinstimmen. 
Die Modellierung des Zwischenkreiskondensators als ideale Kapazität ist für Systembetrachtungen ausreichend. Nur für Detailuntersuchungen auf der Schaltungsebene sind unter Umständen parasitäre Effekte zu berücksichtigen. In erster Linie betrifft dies die seriellen Induktivitäten in der Kapazität selbst und in den Zuleitungen. Sie können in den Schaltmomenten am Stromrichter zu Spannungsspitzen führen.

\subsubsection{Stromzwischenkreis}

Die Verhältnisse im Stromzwischenkreis sind weitgehend dual zu denjenigen im Spannungszwischenkreis. Dementsprechend ergeben sich die Modellierungsvarianten nach Bild 3.15. In der realen Schaltung stellt sich der Strom nach der Differentialgleichung (3.34) ein. Weiter lassen sich die beiden Spannungen gemäss (3.35) in ihre Mittelwerte und ihre Welligkeiten aufspalten. Damit die Leistungsbilanz ausgeglichen ist, muss (3.36) gelten. Und schliesslich ergibt sich die Welligkeit des Zwischenkreisstromes mit (3.37).
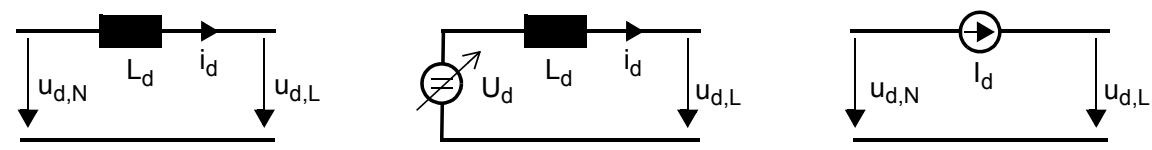

Bild 3.15. Varianten für die Modellierung des Zwischenkreis eines I-Umrichters, links: reale Schaltung,

mitte: Ersatz des netzseitigen Stromrichters durch eine ideale Gleichspannungsquelle, rechts: Ersatz der Induktivität durch eine ideale Gleichstromquelle

$$
\begin{aligned}
& i_{d}=\frac{1}{L_{d}} \int_{d, N}\left(u_{d, L}\right) d t \\
& u_{d, N}=U_{d, N}+u_{d, N, V Z}, \quad u_{d, L}=U_{d, L}+u_{d, L, V Z} \\
& U_{d, N}=U_{d, L}=U_{d} \\
& i_{d, V Z}=\frac{1}{L_{d}} \int\left(u_{d, N, V Z}-u_{d, L, V Z}\right) d t
\end{aligned}
$$

Diese Welligkeit verursacht auch hier eine Kopplung zwischen den beiden Umrichterseiten, indem sie beidseits auf die Ausgangsgrössen einwirkt.

Der Einfluss eines Stromrichters allein auf den Zwischenkreis lässt sich anhand des Modells in Bild 3.15 mitte untersuchen. Dort ist der netzseitige Stromrichter durch eine ideale Gleichspannungsquelle $U_{d}$ ersetzt. Die Gleichung (3.37) vereinfacht sich damit zu:

$$
i_{d, V Z}=-\frac{1}{L_{d}} \int_{d, L, V Z} d t
$$

Soll der Zwischenkreisstrom geändert werden, so sind die Mittelwerte der beiden Spannungen an der Induktivität $u_{d, A}$ und $u_{d, B}$ vorübergehend ungleich gross zu machen. Die entstehende Spannungsdifferenz ergibt die gewünschte Stromänderung. Wie beim U-Umrichter erfolgt die entsprechende Regelung über den Netzstromrichter. 
Die vollständige Entkopplung der beiden Stromrichter wird mit dem Modell in Bild 3.15 rechts erreicht. Unter der Annahme, die Induktivität sei so gross, dass die Welligkeit des Stromes vernachlässigbar wird, kann der Zwischenkreis durch eine Stromquelle modelliert werden. Auch hier ist anzumerken, dass dieses Modell für dynamische Betrachtungen nicht geeignet ist.

Im Gegensatz zum Spannungszwischenkreis ist die Betrachtung der Zwischenkreisdrossel als reine Induktivität nicht immer genügend. Vor allem bei Umrichtern kleiner Leistung ist die Güte solcher Drosseln relativ klein, so dass der Wicklungswiderstand für Systemuntersuchungen nicht vernachlässigt werden darf. Zusätzlich wird generell ein anderes Konzept bei der Regelung des Umrichters angewandt: während bei U-Umrichtern die Zwischenkreisspannung meistens konstant auf dem Maximalwert gehalten wird, wird bei I-Umrichtern der Zwischenkreisstrom variiert. Um die deutlich grösseren Verluste im Zwischenkreis und in den Leistungshalbleitern zu reduzieren, wird er stets auf einem möglichst kleinen Wert gehalten.

\subsubsection{Filter}

\subsubsection{Filter für U-Stromrichter}

U-Stromrichter stellen für ihre Ausgangsseite Spannungsquellen dar, die jedoch nichtsinusförmige Kurvenformen abgeben. Die Phasenströme stellen sich entsprechend der Konfiguration des Lastkreises ein. Geht man von der in Kapitel 3.2.4 definierten Standardlast aus, so wirkt die Lastinduktivität $L_{k}$ dabei als Filter erster Ordnung.

In gewissen Anwendungen ist gefordert, dass die Spannung und der Strom an der Last besonders kleine Verzerrungen aufweisen. In diesem Fall wird die Verwendung eines Filters nach Bild 3.16 erforderlich. Es besteht aus den zusätzlichen Komponenten $L_{F}$ und $C_{F}$, so dass zusammen mit der bereits vorhandenen Lastinduktivität $L_{k}$ ein Filter 3. Ordnung entsteht. Von Bedeutung sind in erster Linie die 3 Übertragungsfunktionen (3.39) bis (3.41), welche die Grössen im Lastkreis in Funktion der eingestellten Ausgangsspannung $u_{A B}$ am Stromrichter beschreiben. Sie gelten unter der Annahme, dass die Gegenspannung $e$ rein sinusförmig verläuft und die Kreisfrequenz $\omega_{1}$ aufweist. Sie stellt damit für jede andere Kreisfrequenz einen Kurzschluss dar.

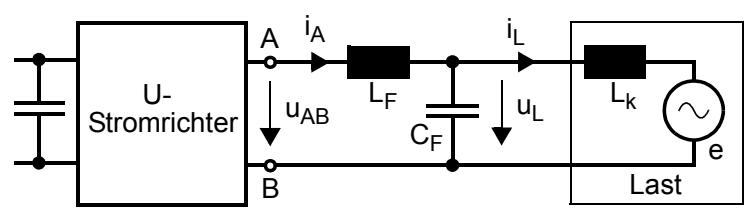

$$
\underline{G}_{1}=\frac{\hat{i}_{A}}{\underline{\hat{u}}_{A B}}=\frac{1-\omega^{2} L_{k} C_{F}}{j \omega\left(L_{k}+L_{F}\right)-j \omega^{3} L_{k} L_{F} C_{F}}, \quad \omega \neq \omega_{1}
$$

Bild 3.16.

U-Stromrichter mit Ausgangsfilter und Standardlast 


$$
\begin{array}{ll}
\underline{G}_{2}=\frac{\hat{\underline{u}}_{L}}{\underline{\hat{u}}_{A B}}=\frac{j \omega L_{k}}{j \omega\left(L_{k}+L_{F}\right)-j \omega^{3} L_{k} L_{F} C_{F}}, & \omega \neq \omega_{1} \\
\underline{G}_{3}=\frac{\hat{\hat{i}}_{L}}{\underline{\hat{u}}_{A B}}=\frac{1}{j \omega\left(L_{k}+L_{F}\right)-j \omega^{3} L_{k} L_{F} C_{F}}, & \omega \neq \omega_{1}
\end{array}
$$
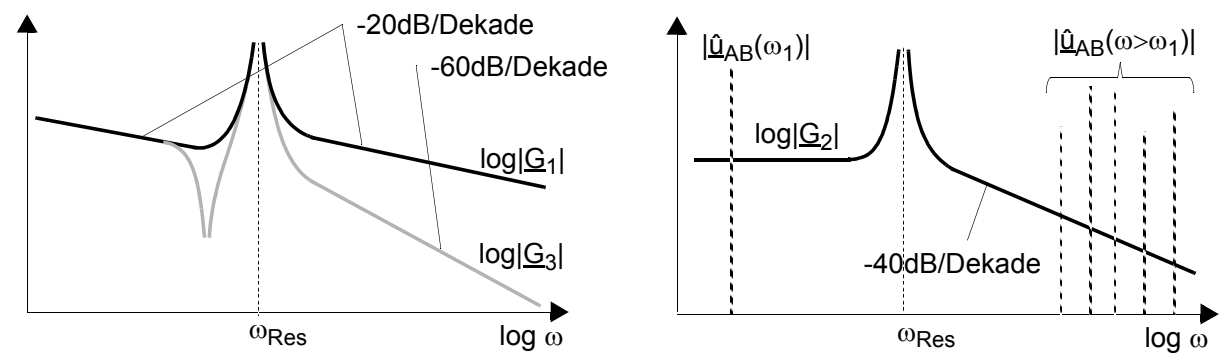

Bild 3.17. Graphische Darstellung der Filterübertragungsfunktionen $\underline{G}_{1}=\underline{\hat{t}}_{A} / \underline{\hat{u}}_{A B}, \underline{G}_{2}=\underline{\hat{u}}_{L} / \underline{\hat{u}}_{A B}$ und $\underline{G}_{3}=\underline{\hat{\imath}}_{L} / \hat{\underline{u}}_{A B}$

In Bild 3.17 sind die Beträge der Übertragungsfunktionen (Amplitudengänge) graphisch dargestellt. Dabei tritt deutlich in Erscheinung, dass das Filter ein sehr ausgeprägtes Resonanzverhalten zeigt. Die entsprechende Resonanzkreisfrequenz $\omega_{R e s}$ ist durch die Filterelemente bestimmt:

$$
\omega_{\text {Res }}=\sqrt{\frac{L_{k}+L_{F}}{L_{k} L_{F} C_{F}}}
$$

Oberhalb von $\omega_{\text {Res }}$ dämpft das Filter die Lastspannung mit $40 \mathrm{~dB}$ und den Laststrom mit $60 \mathrm{~dB}$ pro Dekade. Um diese Charakteristik optimal zu nutzen, ist dafür zu sorgen, dass wie in Bild 3.17 rechts angedeutet der Arbeitsfrequenzbereich (Grundfrequenzbereich) des Umrichters unterhalb und die Verzerrungsanteile in den Ausgangsgrössen möglichst weit oberhalb der Resonanzfrequenz liegen. Auf diese Weise beeinflusst das Filter die normale Funktion des Umrichters praktisch nicht, hält jedoch die Verzerrungen von der Last fern. Für die Regelung des Umrichters kann die Filterresonanz allerdings ein nicht zu unterschätzendes Problem darstellen. Die Dämpfung im Leistungskreis ist vor allem bei Anwendungen mittlerer und grosser Leistung sehr klein. Schnelle Regelvorgänge oder auch Störungen im Netz können leicht Oszillationen verursachen.

Wichtige Anwendungsgebiete für derartige Filter sind selbstgeführte Stromrichter am Netz, die Speisung von Inselnetzen oder geschaltete Leistungsverstärker. Dabei werden auch andere Filtertopologien verwendet.

\subsubsection{Filter für I-Stromrichter}

Beim I-Stromrichter ist bereits in der Grundschaltung ein Filter 2. Ordnung vorhanden. Wie bereits in Kapitel 2 erklärt wurde, sind am Ausgang des Stromrichters Kondensatoren 
als Freilaufpfade nötig. Sie wirken gleichzeitig als Filter. Bild 3.18 zeigt die Lastseite eines I-Umrichters. Die Übertragungsfunktionen (3.43) und (3.44) beschreiben dann die Lastgrössen in Funktion des eingestellten Ausgangsstromes. Die entsprechenden Amplitudengänge sind in Bild 3.19 wiederum graphisch dargestellt.

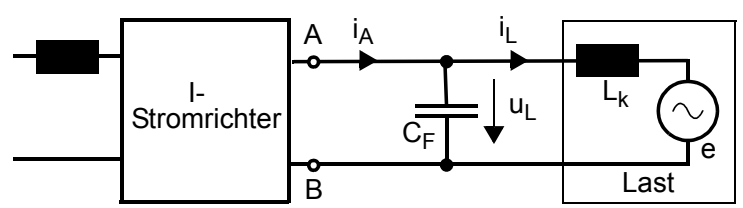

$\underline{G}_{4}=\frac{\hat{u}_{L}}{\hat{i}_{A}}=\frac{j \omega L_{k}}{1-\omega^{2} L_{k} C_{F}}, \quad \omega \neq \omega_{1}$

$\underline{G}_{5}=\frac{\hat{i}_{L}}{\hat{i}_{A}}=\frac{1}{1-\omega^{2} L_{k} C_{F}}, \quad \omega \neq \omega_{1}$
Bild 3.18.

I-Stromrichter mit Ausgangsfilter und Standardlast
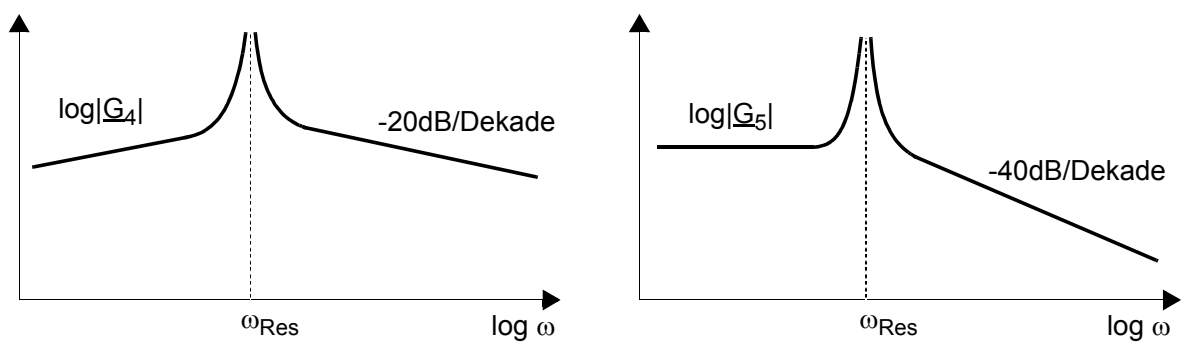

Bild 3.19. Graphische Darstellung der Filterübertragungsfunktionen $\underline{G}_{4}=\underline{\hat{u}}_{L} / \underline{\hat{t}}_{A}, \underline{G}_{5}=\underline{\hat{\imath}}_{L} / \underline{\hat{t}}_{A}$

Auch dieses Filter weist eine Resonanzstelle auf. Die Resonanzkreisfrequenz $\omega_{\text {Res }}$ ergibt sich gemäss (3.45). Da die Filterkapazität gegenüber derjenigen beim U-Stromrichter in der Regel grösser ist, wird $\omega_{\text {Res }}$ entsprechend kleiner.

$$
\omega_{\text {Res }}=\frac{1}{\sqrt{L_{k} C_{F}}}
$$

Oberhalb von $\omega_{\text {Res }}$ wirkt das Filter für die Lastspannung mit $20 \mathrm{~dB}$ und für den Laststrom mit 40dB pro Dekade. Der Stromrichter sollte so betrieben werden, dass die Arbeitsfrequenz unterhalb der Resonanzfrequenz und die Verzerrungen möglichst weit oberhalb davon liegen.

Da bereits die Grundschaltung eines I-Stromrichters ein Resonanzverhalten zeigt, stellt deren Beherrschung eines der zentralen Probleme für die Regelung dar. Beim Betrieb einer ASM ergibt die Hauptinduktivität der Maschine zusammen mit der Filterkapazität 
eine weitere Resonanzstelle. Diese liegt üblicherweise in der Nähe von $\omega_{1}$. Ohne geeignete Regelung kann sie zur Instabilität führen [Jen1].

\subsubsection{Stromverdrängung}

Alle bisherigen Betrachtungen wurden unter der Annahme gemacht, dass die auftretenden ohmschen Widerstände unabhängig von der Frequenz konstant sind. Dieser Ansatz ist nicht immer zulässig. Der Effekt der Stromverdrängung (Skineffekt) sorgt dafür, dass sich der Strom in einem Leiter mit zunehmender Frequenz ungleichmässig auf den Querschnitt verteilt. Es ergibt sich damit ein grösserer wirksamer Widerstand. Bild 3.20 zeigt qualitativ die Stromdichte $j$ in einem zylindrischen Leiter bei Belastung mit Wechselstrom. Sie
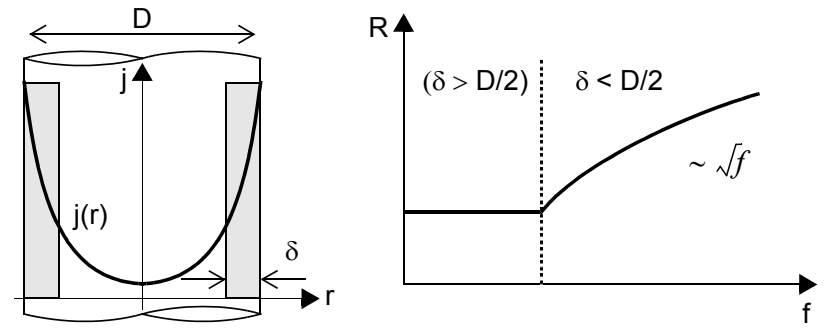

Bild 3.20.
Stromverdrängung in einem ge-
raden zylindrischen Leiter,
links: Stromdichte $j$ als Funkti-
on des Radius und Eindringtie-
fe $\delta$,
rechts: wirksamer Widerstand
als Funktion der Frequenz

ist an der Aussenseite des Leiters am grössten. Näherungsweise kann angenommen werden, der ganze Strom fliesse gleichmässig verteilt mit der Oberflächenstromdichte in einem Rohr mit der Wanddicke $\delta$ (entsprechend der im Bild grau eingezeichneten Flächen). $\delta$ wird als Eindringtiefe bezeichnet und lässt sich nach der Formel (3.46) bestimmen:

$$
\delta=\sqrt{\frac{\rho}{\pi \mu_{0} \mu_{r} f}} \quad \text { für } \quad \delta<\frac{D}{2}
$$

mit $\rho \quad$ spezifischer Widerstand des Leiters $\left(\rho_{\text {Kupfer }}=1.75 \times 10^{-8} \Omega \mathrm{m}\right)$

$\mu_{r} \quad$ magnetische Permeabilität des Leiters $\left(\mu_{r, \text { Kupfer }}=1\right)$

$\mu_{0} \quad$ magnetische Feldkonstante $\left(4 \pi \times 10^{-7} \mathrm{VsA}^{-1} \mathrm{~m}^{-1}\right)$

$f \quad$ Frequenz des angelegten Wechselstromes

Die Formel ist verwendbar, sofern die Eindringtiefe kleiner als der Leiterradius $D / 2$ ist. Andernfalls wird mit gleichmässig über die gesamte Fläche verteiltem Strom gerechnet $(\delta=D / 2)$. Der wirksame Widerstand ist umgekehrt proportional zur genutzten Fläche und damit für kleine Eindringtiefen auch zu $\delta$. Sein Verlauf als Funktion der Frequenz ist in Bild 3.20 rechts dargestellt. Als Beispiel sind nachfolgend die Eindringtiefen für einen Kupferleiter bei 3 verschiedenen Frequenzen angegeben:

$$
\delta_{\text {Kupfer }}(50 \mathrm{~Hz})=9.4 \mathrm{~mm}, \delta_{\text {Kupfer }}(500 \mathrm{~Hz})=3.0 \mathrm{~mm}, \delta_{\text {Kupfer }}(5 \mathrm{kHz})=0.94 \mathrm{~mm}
$$


Es wird deutlich, dass die Stromverdrängung schon bei sehr tiefen Frequenzen spürbar wird, wenn der Querschnitt des Leiter gross ist. Sie ist deshalb bei Anlagen hoher Leistung besonders zu beachten. Bei Leiterdurchmessern von mehreren Millimetern wird die Widerstandserhöhung bereits ab einigen $100 \mathrm{~Hz}$ wirksam.

Bei Asynchronmaschinen für Direktanlauf am Netz (mit 'Stromverdrängungs-Rotor') wird die Stromverdrängung gezielt dazu ausgenützt, um beim Betrieb am Netz das Anfahrmoment zu vergrössern [Fis1]. Sie ist deshalb bereits im Bereich der Netzfrequenz stark wirksam und fällt für höherfrequente Verzerrungsanteile im Rotorstrom entsprechend noch deutlicher ins Gewicht.

In Wicklungen (Drosseln, Transformatoren, Maschinen) bewirkt die gegenseitige Beeinflussung der einzelnen Windungen eine stärkere Stromverdrängung und damit einen grösseren Anstieg des wirksamen Widerstandes als in einem einzelnen Leiter. Dieses Phänomen wird als 'Proximity Effect' bezeichnet ([Sto1], [Dow1]). Gleichzeitig nimmt die Induktivität der Spule mit steigender Frequenz ab. Im Frequenzbereich, der für Betrachtungen an selbstgeführten Stromrichtern von Bedeutung ist, bleibt trotzdem normalerweise der induktive Anteil in der Impedanz klar dominant. Vor allem für Verlustbetrachtungen ist aber die Zunahme des Widerstandes durch Stromverdrängung zu beachten.

Literatur zu Kapitel 3.2: [Bro1], [Büh1], [Büh2], [Dow1], [Fis1], [Hag1], [Heu1], [Jen1], [Kle1], [Lap1], [Leo1], [Mey1], [Mey2], [Mic1], [Sto1], [Spä1]

\subsection{Schaltungen von selbstgeführten Stromrichtern}

Nachdem einerseits die Bausteine und andererseits das Umfeld der selbstgeführten Stromrichter besprochen worden sind, sollen im folgenden die verschiedenen Schaltungstopologien für die Stromrichter selbst vorgestellt werden. Dies geschieht auf der Basis der in Kapitel 3.1.1 eingeführten idealisierten Schaltelemente. Im Vordergrund steht dabei das Verhalten der Schaltungen an ihren Ein- und Ausgängen. Dazu werden zwei Beschreibungen benützt: die exakten Transformationsgleichungen für Ströme und Spannungen mittels Schaltfunktionen und die zeitkontinuierlichen Transformationsgleichungen mittels Modulationsfunktionen.

\subsubsection{Beschreibung durch Schaltfunktionen, kurzzeitige Mittelwerte und Modulationsfunktionen}

Die Beschreibungsarten für Stromrichter werden am Beispiel eines Brückenzweiges (auch als Zweipaar oder Phasenglied bezeichnet) für einen U-Stromrichter vorgestellt. Betrachtet wird die Schaltung in Bild 3.21. Die Last liegt zwischen den Mittelpunkten des Zweiges und des Zwischenkreises. Die Zwischenkreisspannung $u_{d}$ und der Ausgangsstrom $i_{A}$ sind zumindest kurzzeitig geglättete Grössen, die keine Sprünge ausführen können. Dafür sorgen die im Bild angedeuteten Kapazitäten und Induktivitäten, die in der äusseren Beschaltung eines U-Stromrichters stets vorhanden sind. Die beiden rückwärts- 

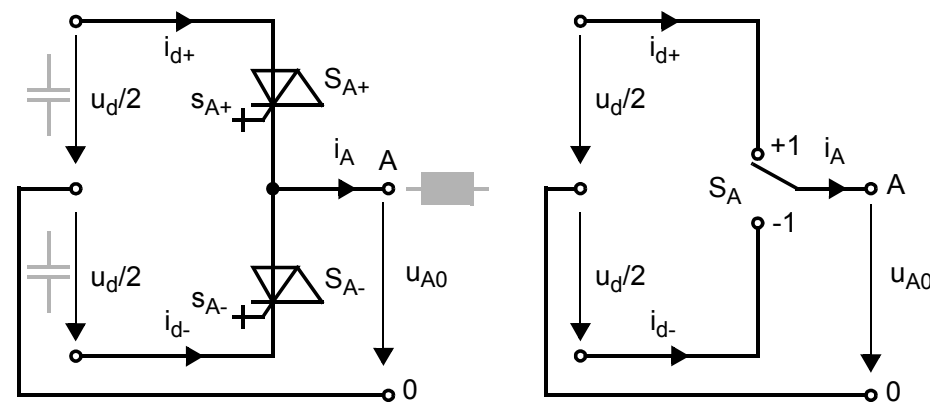

Bild 3.21.

Brückenzweig für zweistufige U-Stromrichter, links: Schaltung mit idealisierten Elementen, rechts: Ersatzschaltung mit Umschalter

leitenden Schaltelemente bilden zusammen den Brückenzweig, der sich theoretisch in 4 verschiedenen Zuständen befinden kann:

1. $s_{A+}=1$ und $s_{A=}=0$ : der Strom $i_{A}$ fliesst unabhängig von seinem Vorzeichen im oberen Schaltelement $S_{A^{+}}$und die Ausgangsspannung $u_{A 0}$ beträgt $u_{d} / 2$.

2. $s_{A+}=0$ und $s_{A_{-}}=1$ : der Strom $i_{A}$ fliesst unabhängig von seinem Vorzeichen im unteren Schaltelement $S_{A}$ und die Ausgangsspannung $u_{A 0}$ beträgt $-u_{d} / 2$.

3. $s_{A+}=1$ und $s_{A-}=1$ : dieser Zustand würde den Zwischenkreis kurzschliessen und ist deshalb verboten.

4. $s_{A+}=0$ und $s_{A-}=0$ : der Strom $i_{A}$ fliesst je nach seinem Vorzeichen im oberen oder im unteren Schaltelement. Die Ausgangsspannung ist demzufolge abhängig von der Stromrichtung. Da der U-Stromrichter am Ausgang das Verhalten einer Spannungsquelle aufweisen soll, ist diese Situation unerwünscht. Der Schaltzustand wird üblicherweise nur als Ruhezustand der Schaltung benutzt.

Im Betrieb werden nur die ersten beiden Zustände verwendet. Es ist also jederzeit eines der beiden Schaltelemente eingeschaltet und das andere ausgeschaltet (d.h. $s_{A_{+}}+s_{A_{-}}=1$ ). Unter diesen Voraussetzungen lassen sich mit den Schaltfunktionen $s_{A^{+}}$und $s_{A_{-}}$die diskreten Transformationsgleichungen gemäss (3.47) und (3.48) formulieren.

$$
\begin{aligned}
& u_{A 0}=\left(s_{A+}-s_{A-}\right) \frac{u_{d}}{2} \\
& i_{d+}=s_{A+} i_{A}, \quad i_{d-}=s_{A-} i_{A}
\end{aligned}
$$

Die Ausgangsspannung (Mittelpunktspannung) $u_{A 0}$ ist eine Funktion der Schalterzustände und der Zwischenkreisspannung. Ihr Wert kann sich sprungartig ändern. Die Stellmöglichkeit beschränkt sich jedoch auf die zwei Schaltniveaus $+u_{d} / 2$ und $-u_{d} / 2$. Beim Strom ist die Wirkung umgekehrt: je nach Stellung der Schalter wird der Phasenstrom auf einen der beiden Leiter des Zwischenkreises geschaltet. Die Verläufe der Zwischenkreisströme weisen demzufolge Sprünge auf.

Die Spannungsbelastung der Schaltelemente im ausgeschalteten Zustand beträgt $u_{d}$. Ihre Spannungsfestigkeit ist deshalb auf den maximalen Wert der Zwischenkreisspannung auszulegen, wobei zusätzlich die zu erwartende Abschaltüberspannung (durch Streuinduktivitäten bedingt) und eine Reserve einzurechnen sind. 
Aufgrund der Transformationsgleichungen kann die Ersatzschaltung in Bild 3.21 rechts gebildet werden. Die beiden Schaltelemente sind dort durch einen einzigen Umschalter ersetzt. Seine Stellung wird durch die Schaltfunktion $s_{A}$ bestimmt, welche die Werte +1 und -1 annehmen kann. Der Zusammenhang mit den Schaltfunktionen der beteiligten Schaltelemente $s_{A+}$ und $s_{A \text { - }}$ ergibt sich wie folgt:

$$
s_{A}=s_{A+}-s_{A-}, \quad s_{A+}=\frac{1+s_{A}}{2}, \quad s_{A-}=\frac{1-s_{A}}{2}
$$

Die Transformationsgleichungen lassen sich neu in der Form von (3.50) und (3.51) darstellen:

$$
\begin{aligned}
& u_{A 0}=s_{A} \frac{u_{d}}{2} \\
& i_{d+}=\frac{1+s_{A}}{2} i_{A}, \quad i_{d^{-}}=\frac{1-s_{A}}{2} i_{A}
\end{aligned}
$$

Die Ersatzschaltung als Umschalter, zusammen mit den entsprechenden Gleichungen, bildet die Grundlage für alle systembezogenen Betrachtungen am Stromrichter. Mittels Schaltfunktionen wird der ideal schaltende Stromrichter exakt beschrieben. Die Eigenschaften des verwendeten Steuerverfahrens können anhand von ihnen untersucht werden.

Kurzzeitiger Mittelwert: Bei der Steuerung des Stromrichters geht es darum, die Ausgangsspannung $u_{A 0}$ möglichst gut einem vorgegebenen kontinuierlichen Sollwert $u_{A 0 \text {,Soll }}$ folgen zu lassen. Wegen der geschalteten Charakteristik von $u_{A 0}$ kann dies jedoch, wie in Bild 3.22 dargestellt, nur für den kurzzeitigen Mittelwert $\bar{u}_{A 0}$ erreicht werden. Er ent-


Bild 3.22. Nachbildung des Sollwertes $u_{A 0 \text {,Soll }}$ durch den kurzzeitigen Mittelwert $\bar{u}_{A 0}$ links: mit niedriger Schaltfrequenz, rechts: mit höherer Schaltfrequenz

spricht dem niederfrequenten Anteil in $u_{A 0}$, der sich ergibt, wenn jeweils über einen vollständigen Taktzyklus (Taktintervall) $T_{T}$ gemittelt wird:

$$
\bar{u}_{A 0}(t)=\frac{1}{T_{T}} \int_{t-T_{T} / 2}^{t+T_{T} / 2} u_{A 0}(\tau) d \tau
$$

Die Länge des Zeitintervalls $T_{T}$ hängt von der Schaltfrequenz ab. Im Bild 3.22 ist ersichtlich, dass es mit grösserer Schaltfrequenz kleiner wird. Die Nachbildung des Sollwertes ist dadurch besser. 
Kurzzeitige Mittelwerte können von allen am Stromrichter auftretenden Grössen, d.h. Schaltfunktionen, Spannungen und Strömen gebildet werden. In den meisten Anwendungen sind die Sollwerte sinusförmig. Die kurzzeitigen Mittelwerte entsprechen dann näherungsweise den Grundschwingungen der verschiedenen Grössen.

Zeitkontinuierliche Beschreibung mit der Modulationsfunktion $m_{A}(t)$ : Die exakte Beschreibung des Stromrichters mittels Schaltfunktionen ist unhandlich. Für viele Untersuchungen genügt es, das Verhalten der Schaltung für die kurzzeitigen Mittelwerte bzw. die Grundschwingung zu betrachten. Dies gilt beispielsweise für Regelungen und für Leistungsbetrachtungen (Wirk- und Blindleistungen).

Die zeitkontinuierliche Beschreibung basiert darauf, dass in den diskreten Gleichungen (3.50) und (3.51) die Momentanwerte durch die entsprechenden kurzzeitigen Mittelwerte ersetzt werden. Gleichzeitig wird angenommen, dass sich die Zwischenkreisspannung $u_{d}$ wegen der guten Glättung kurzzeitig nicht stark ändert, so dass sie bei der Bildung des kurzzeitigen Mittelwertes eine Konstante darstellt. Dasselbe gilt für den Phasenstrom $i_{A}$. Es resultieren damit die Gleichungen (3.53) und (3.54):

$$
\begin{aligned}
& \bar{u}_{A 0}=\overline{\left(s_{A} \frac{u_{d}}{2}\right)}=\bar{s}_{A} \frac{u_{d}}{2} \\
& \dot{i}_{d+}=\overline{\left(\frac{1+s_{A}}{2} i_{A}\right)}=\frac{1+\bar{s}_{A}}{2} i_{A}, \quad \bar{i}_{d-}=\overline{\left(\frac{1-s_{A}}{2} i_{A}\right)}=\frac{1-\bar{s}_{A}}{2} i_{A}
\end{aligned}
$$

Die Grösse $\bar{s}_{A}$ wird dabei als Modulationsfunktion $m_{A}$ bezeichnet. Sie verläuft proportional zum Sollwert $u_{A 0 \text {,Soll }}$. Damit nehmen die zeitkontinuierlichen Gleichungen des Stromrichters die folgende Form an:

$$
\begin{aligned}
& \bar{u}_{A 0}=m_{A} \frac{u_{d}}{2} \\
& \bar{i}_{d+}=\frac{1+m_{A}}{2} i_{A}, \quad i_{d-}=\frac{1-m_{A}}{2} i_{A}
\end{aligned}
$$

In vielen Anwendungen verläuft der Sollwert $u_{A 0 \text {,Soll }}$ sinusförmig, so dass die zeitkontinuierlichen Gleichungen gilt:

$$
\begin{aligned}
& m_{A}=M_{A} \sin \left(\omega_{1} t+\varphi_{m}\right), \quad M_{A} \text { Modulationsgrad } \\
& \bar{u}_{A 0}=M_{A} \frac{u_{d}}{2} \sin \left(\omega_{1} t+\varphi_{m}\right) \\
& \bar{i}_{d+}=\left[1+M_{A} \sin \left(\omega_{1} t+\varphi_{m}\right)\right] \frac{i_{A}}{2}, \quad \bar{i}_{d-}=\left[1-M_{A} \sin \left(\omega_{1} t+\varphi_{m}\right)\right] \frac{i_{A}}{2}
\end{aligned}
$$

Die zeitkontinuierliche Beschreibung entspricht in diesem Fall einer GrundschwingungsBetrachtung. Unter der Annahme, dass sich auch $i_{A}$ sinusförmig einstellt, enthalten die Ströme auf der DC-Seite einen Gleichanteil und pulsieren zusätzlich mit den Kreisfrequenzen $\omega_{1}$ und $2 \omega_{1}$ (Kapitel 5.2). 


\subsubsection{Zweistufige Stromrichter für Spannungszwischenkreis-Umrichter}

Die zweistufigen Schaltungen sind die Standardtopologien für U-Stromrichter. Die Bezeichnung zweistufig kommt daher, dass der Stromrichter die Ausgangsspannung zwischen zwei diskreten Niveaus umschalten kann.

\subsubsection{Einphasige Halbbrücke}

Die Basis für alle weiteren Schaltungen ist die einphasige Halbbrücke, entsprechend einem einzelnen Brückenzweig nach Bild 3.21. Dabei muss der Mittelpunkt des Zwischenkreises existieren und zugänglich sein. Da dies in der Praxis meistens nicht der Fall ist, kommt die Schaltung in dieser Form kaum zur Anwendung. Ihre in Kapitel 3.3.1 beschriebene Funktion und die zugehörigen Gleichungen sind jedoch für das Verständnis der weiteren Schaltungen von grosser Bedeutung.

\subsubsection{Einphasige Brücke}

Die Brücke gemäss Bild 3.23 ist die gebräuchliche Schaltung für alle einphasigen Anwendungen. Sie besteht aus zwei Brückenzweigen, zwischen welche die Last geschaltet wird. Der Mittelpunkt des Zwischenkreises ist nicht angeschlossen.
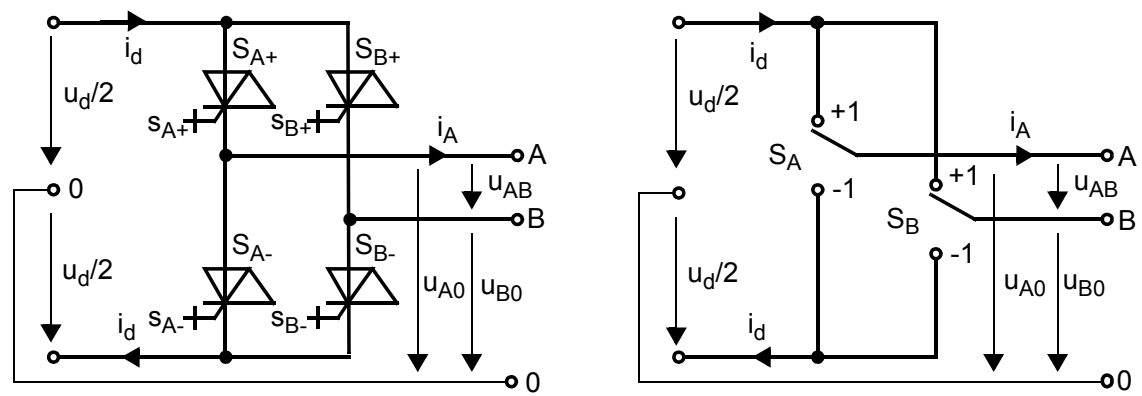

Bild 3.23. Einphasige zweistufige Brücke für U-Stromrichter, links: Schaltung mit idealisierten Elementen, rechts: Ersatzschaltung mit Umschaltern

Beide Brückenzweige werden so betrieben, wie in Kapitel 3.3.1 erklärt wurde: es ist immer eines der Schaltelemente eingeschaltet und das andere ausgeschaltet. Die Schaltung lässt sich deshalb, wie in Bild 3.23 rechts dargestellt, mit Hilfe von zwei Umschaltern modellieren. Die Transformationsgleichungen haben die Form:

$$
\begin{aligned}
& u_{A B}=\left(s_{A}-s_{B}\right) \frac{u_{d}}{2} \\
& i_{d}=\left(s_{A}-s_{B}\right) \frac{i_{A}}{2}
\end{aligned}
$$

Die Ausgangsspannung (Phasenspannung) kann bei der Brückenschaltung 3 Schaltniveaus annehmen, nämlich $+u_{d}, 0$ und $-u_{d}$. Stehen beide Umschalter in derselben Position 
(beide +1 oder beide -1 ), so ist die Last im Stromrichter kurzgeschlossen. Dementsprechend ist dann auch der Zwischenkreisstrom null. Sonst fliesst der Phasenstrom in positiver oder negativer Richtung über den Zwischenkreis, wobei die Ströme in beiden Zwischenkreisleitern stets entgegengesetzt gleich sind.

Zeitkontinuierliche Beschreibung: Diese kann einfach durch Einsetzen der Modulationsfunktionen in den Gleichungen (3.80) und (3.81) abgeleitet werden:

$$
\begin{aligned}
& \bar{u}_{A B}=\left(m_{A}-m_{B}\right) \frac{u_{d}}{2} \\
& i_{d}=\left(m_{A}-m_{B}\right) \frac{i_{A}}{2}
\end{aligned}
$$

Normalerweise werden die beiden Modulationsfunktionen entgegengesetzt gleich gewählt. Für diesen Fall vereinfacht sich die zeitkontinuierliche Beschreibung zu:

$$
\begin{aligned}
& u_{A B}=m u_{d} \quad \text { mit } m=m_{A}=-m_{B} \\
& \dot{i}_{d}=m i_{A}
\end{aligned}
$$

Für eine sinusförmige Modulationsfunktion gelten in diesem Fall:

$$
\begin{aligned}
& m=M \sin \left(\omega_{1} t+\varphi_{m}\right) \quad \Rightarrow \quad u_{A B}=M u_{d} \sin \left(\omega_{1} t+\varphi_{m}\right) \\
& \dot{i}_{d}=M i_{A} \sin \left(\omega_{1} t+\varphi_{m}\right)
\end{aligned}
$$

\subsubsection{Dreiphasige Brücke}

Die Schaltung in Bild 3.24 ist die Standardschaltung für dreiphasige Anwendungen. Sie besteht aus 3 Brückenzweigen, welche je eine Phase des Lastkreises speisen. Die Laststromkreise sind auf diese Weise geschlossen, so dass der Mittelpunkt des Zwischenkreises nicht benötigt wird. Dies gilt sowohl für eine in Stern wie für eine in Dreieck geschaltete Last. Selbst wenn der Mittelpunkt 0 und der Laststernpunkt $N$ zugänglich sind, werden diese nicht miteinander verbunden. Sonst würde die Schaltung zu 3 unabhängigen Brückenzweigen mit gemeinsamem Zwischenkreis degenerieren.

Die auf den Mittelpunkt 0 bezogenen Mittelpunktspannungen $u_{U 0}, u_{V 0}, u_{W 0}$ sind Funktionen der Stellung ihres jeweiligen Umschalters:

$$
u_{U 0}=s_{U} \frac{u_{d}}{2}, \quad u_{V 0}=s_{V} \frac{u_{d}}{2}, \quad u_{W 0}=s_{W} \frac{u_{d}}{2}
$$

Die verketteten Spannungen $u_{U b} u_{V W}, u_{W U}$ (im Bild 3.24 nicht bezeichnet) sind ebenfalls durch die Schaltfunktionen der beiden beteiligten Brückenzweige bestimmt:

$$
u_{U V}=\left(s_{U}-s_{V}\right) \frac{u_{d}}{2}, \quad u_{V W}=\left(s_{V}-s_{W}\right) \frac{u_{d}}{2}, \quad u_{W U}=\left(s_{W}-s_{U}\right) \frac{u_{d}}{2}
$$



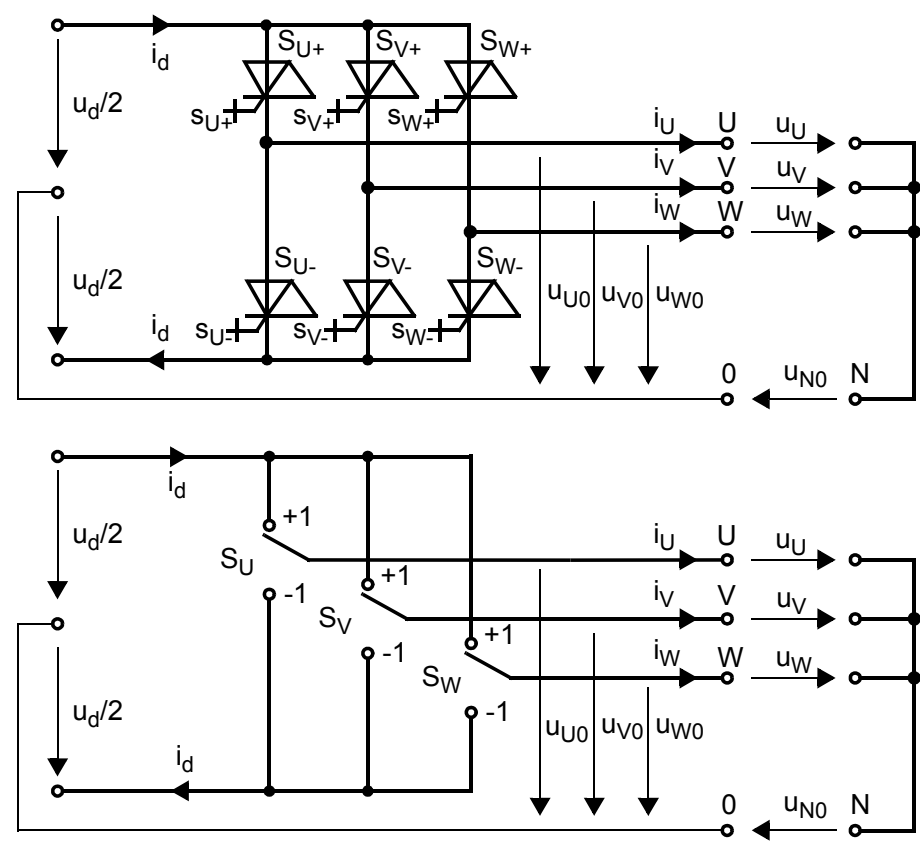

Bild 3.24.

Dreiphasige zweistufige Brücke für UStromrichter, oben: Schaltung mit idealisierten Elementen, unten: Ersatzschaltung mit Umschaltern

Sie können damit, wie die Ausgangsspannung der einphasigen Brückenschaltung, die 3 Niveaus $+u_{d}, 0$ und $-u_{d}$ annehmen.

Da der Mittelpunkt 0 nicht angeschlossen ist, fliesst in beiden Leitern des Zwischenkreises derselbe Strom $i_{d}$ :

$$
i_{d}=\frac{s_{U}+1}{2} i_{U}+\frac{s_{V}+1}{2} i_{V}+\frac{s_{W}+1}{2} i_{W}
$$

Die Knotenregel besagt, dass die Summe der 3 Phasenströme null ist. Damit kann (3.70) umgeformt werden:

$$
i_{U}+i_{V}+i_{W}=0 \quad \Rightarrow \quad i_{d}=\frac{1}{2}\left(s_{U} i_{U}+s_{V} i_{V}+s_{W} i_{W}\right)
$$

Ist die Last in Dreieck geschaltet, so liegen die bereits bestimmten verketteten Spannungen über ihr an. Ist sie in Stern geschaltet, so sind es die Phasenspannungen $u_{U}, u_{V}$ $u_{W}$ :

$$
u_{U}=u_{U 0}-u_{N 0}, \quad u_{V}=u_{V 0}-u_{N 0}, \quad u_{W}=u_{W 0}-u_{N 0}
$$

Die Sternpunktspannung $u_{N 0}$ stellt sich abhängig von der Art der Last ein. Für den üblichen Fall einer symmetrischen Last ohne Gleichtaktkomponente (Anhang B) gilt jedoch (3.73), was eingesetzt in (3.72) für die Phasenspannungen (3.74) ergibt:

$$
u_{N 0}=\left(s_{U}+s_{V}+s_{W}\right) \frac{u_{d}}{6}
$$




$$
u_{U}=\left(s_{U}-\frac{s_{V}}{2}-\frac{s_{W}}{2}\right) \frac{u_{d}}{3}, \quad u_{V}=\left(s_{V}-\frac{s_{U}}{2}-\frac{s_{W}}{2}\right) \frac{u_{d}}{3}, \quad u_{W}=\left(s_{W}-\frac{s_{U}}{2}-\frac{s_{V}}{2}\right) \frac{u_{d}}{3}
$$

Die möglichen Kombinationen der Schalterstellungen von $S_{U}, S_{V}$ und $S_{W}$ des Stromrichters ergeben die 8 verschiedenen Stromrichterzustände ${ }^{0} Z$ bis ${ }^{7} Z$. Sie sind zusammen mit den entsprechenden Spannungen in der Tabelle 3.2 zusammengestellt. Es zeigt sich, dass

\begin{tabular}{c|c|c|c|c|c|c|c|c|c|c} 
Zustand & $\mathrm{s}_{\mathrm{U}}$ & $\mathrm{s}_{\mathrm{V}}$ & $\mathrm{s}_{\mathrm{W}}$ & $\mathrm{u}_{\mathrm{U} 0}$ & $\mathrm{u}_{\mathrm{V} 0}$ & $\mathrm{u}_{\mathrm{W} 0}$ & $\mathrm{u}_{\mathrm{U}}$ & $\mathrm{u}_{\mathrm{V}}$ & $\mathrm{u}_{\mathrm{W}}$ & $\mathrm{u}_{\mathrm{N} 0}$ \\
\hline${ }^{0} \mathrm{Z}$ & -1 & -1 & -1 & $-\mathrm{u}_{\mathrm{d}} / 2$ & $-\mathrm{u}_{\mathrm{d}} / 2$ & $-\mathrm{u}_{\mathrm{d}} / 2$ & 0 & 0 & 0 & $-\mathrm{u}_{\mathrm{d}} / 2$ \\
\hline${ }^{1} \mathrm{Z}$ & 1 & -1 & -1 & $\mathrm{u}_{\mathrm{d}} / 2$ & $-\mathrm{u}_{\mathrm{d}} / 2$ & $-\mathrm{u}_{\mathrm{d}} / 2$ & $2 \mathrm{u}_{\mathrm{d}} / 3$ & $-\mathrm{u}_{\mathrm{d}} / 3$ & $-\mathrm{u}_{\mathrm{d}} / 3$ & $-\mathrm{u}_{\mathrm{d}} / 6$ \\
\hline${ }^{2} \mathrm{Z}$ & 1 & 1 & -1 & $\mathrm{u}_{\mathrm{d}} / 2$ & $\mathrm{u}_{\mathrm{d}} / 2$ & $-\mathrm{u}_{\mathrm{d}} / 2$ & $\mathrm{u}_{\mathrm{d}} / 3$ & $\mathrm{u}_{\mathrm{d}} / 3$ & $-2 \mathrm{u}_{\mathrm{d}} / 3$ & $\mathrm{u}_{\mathrm{d}} / 6$ \\
\hline${ }^{3} \mathrm{Z}$ & -1 & 1 & -1 & $-\mathrm{u}_{\mathrm{d}} / 2$ & $\mathrm{u}_{\mathrm{d}} / 2$ & $-\mathrm{u}_{\mathrm{d}} / 2$ & $-\mathrm{u}_{\mathrm{d}} / 3$ & $2 \mathrm{u}_{\mathrm{d}} / 3$ & $-\mathrm{u}_{\mathrm{d}} / 3$ & $-\mathrm{u}_{\mathrm{d}} / 6$ \\
\hline${ }^{4} \mathrm{Z}$ & -1 & 1 & 1 & $-\mathrm{u}_{\mathrm{d}} / 2$ & $\mathrm{u}_{\mathrm{d}} / 2$ & $\mathrm{u}_{\mathrm{d}} / 2$ & $-2 \mathrm{u}_{\mathrm{d}} / 3$ & $\mathrm{u}_{\mathrm{d}} / 3$ & $\mathrm{u}_{\mathrm{d}} / 3$ & $\mathrm{u}_{\mathrm{d}} / 6$ \\
\hline${ }^{5} \mathrm{Z}$ & -1 & -1 & 1 & $-\mathrm{u}_{\mathrm{d}} / 2$ & $-\mathrm{u}_{\mathrm{d}} / 2$ & $\mathrm{u}_{\mathrm{d}} / 2$ & $-\mathrm{u}_{\mathrm{d}} / 3$ & $-\mathrm{u}_{\mathrm{d}} / 3$ & $2 \mathrm{u}_{\mathrm{d}} / 3$ & $-\mathrm{u}_{\mathrm{d}} / 6$ \\
\hline${ }^{6} \mathrm{Z}$ & 1 & -1 & 1 & $\mathrm{u}_{\mathrm{d}} / 2$ & $-\mathrm{u}_{\mathrm{d}} / 2$ & $\mathrm{u}_{\mathrm{d}} / 2$ & $\mathrm{u}_{\mathrm{d}} / 3$ & $-2 \mathrm{u}_{\mathrm{d}} / 3$ & $\mathrm{u}_{\mathrm{d}} / 3$ & $\mathrm{u}_{\mathrm{d}} / 6$ \\
\hline${ }^{7} \mathrm{Z}$ & 1 & 1 & 1 & $\mathrm{u}_{\mathrm{d}} / 2$ & $\mathrm{u}_{\mathrm{d}} / 2$ & $\mathrm{u}_{\mathrm{d}} / 2$ & 0 & 0 & 0 & $\mathrm{u}_{\mathrm{d}} / 2$
\end{tabular}

Tabelle 3.2.

Schaltzustände und Spannungen des dreiphasigen Stromrichters

bei den Mittelpunktspannungen zwei, bei den Phasenspannungen 5 und bei der Sternpunktspannung 4 Schaltniveaus auftreten. Die 3 Phasen können über den Stromrichter kurzgeschlossen werden, indem alle Umschalter in dieselbe Stellung (alle +1 oder alle -1) gebracht werden.

Die Zustände ${ }^{0} Z$ und ${ }^{7} Z$ ergeben Phasenspannungen von null und werden deshalb als Nullzustände bezeichnet. Im Gegensatz dazu wird für ${ }^{1} Z$ bis ${ }^{6} Z$ die Bezeichnung spannungsbildende Zustände verwendet.

Zeitkontinuierliche Beschreibung: Auch hier lassen sich die Spannungen und der Zwischenkreisstrom vereinfacht durch zeitkontinuierliche Funktionen beschreiben. Dazu müssen in den Transformationsgleichungen nur die Schaltfunktionen $s_{U}, s_{V}$ und $s_{W}$ durch die Modulationsfunktionen $m_{U}, m_{V}$ und $m_{W}$ ersetzt werden:

$$
\begin{aligned}
& \bar{u}_{U 0}=m_{U} \frac{u_{d}}{2}, \quad \bar{u}_{V 0}=m_{V} \frac{u_{d}}{2}, \quad \bar{u}_{W 0}=m_{W} \frac{u_{d}}{2} \\
& \bar{u}_{N 0}=\left(m_{U}+m_{V}+m_{W}\right) \frac{u_{d}}{6} \\
& \bar{i}_{d}=\frac{1}{2}\left(m_{U} i_{U}+m_{V} i_{V}+m_{W} i_{W}\right)
\end{aligned}
$$

Üblicherweise sind die Modulationsfunktionen symmetrisch, so dass gilt: 


$$
m_{U}(t)=m(t), \quad m_{V}(t)=m\left(t-\frac{T_{1}}{3}\right), \quad m_{W}(t)=m\left(t-\frac{2 T_{1}}{3}\right)
$$

Ist zusätzlich auch die Last symmetrisch, so wird $\bar{i}_{d}$ zu einem reinen Gleichstrom ohne Pulsationsanteil (Kapitel 5.2).

Beschreibung des Stromrichters mittels Drehzeigern: Die geschalteten Mittelpunktund Phasenspannungen eines dreiphasigen Stromrichters können auch als Drehzeiger $\underline{u}_{S t r}$ in der $\alpha \beta$-Ebene dargestellt werden (Anhang B.1.2). Dabei resultieren als Ortskurve der Zeigerspitze 8 diskrete Werte, entsprechend den diskreten Stromrichterspannungen. Der Drehzeiger $\underline{u}_{S t r}$ beschreibt gleichzeitig die Mittelpunkt- und die Phasenspannungen, da die eventuell in der Mittelpunktspannung vorhandenen Gleichtaktkomponenten (identisch mit $u_{N O}$ ) nicht in den Zeiger eingehen. Die 8 Stromrichterzustände mit den entsprechenden Komponenten von $\underline{u}_{S t r}$ und der zugehörigen Sternpunktspannung sind in Tabelle 3.3 zusammengestellt.

\begin{tabular}{c|c|c|c|c} 
Zustand & Zeiger $\underline{\mathrm{u}}_{\mathrm{Str}}$ & $\mathrm{u}_{\mathrm{Str}, \alpha}$ & $\mathrm{u}_{\mathrm{Str}, \beta}$ & $\underline{\mathrm{u}_{\mathrm{Str}}}$ \\
\hline${ }^{0} \mathrm{Z}$ & ${ }^{0} \underline{\mathrm{U}}$ & 0 & 0 & 0 \\
\hline${ }^{1} \mathrm{Z}$ & ${ }^{1} \underline{\mathrm{U}}$ & $2 \mathrm{u}_{\mathrm{d}} / 3$ & 0 & $2 \mathrm{u}_{\mathrm{d}} / 3$ \\
\hline${ }^{2} \mathrm{Z}$ & ${ }^{2} \underline{\mathrm{U}}$ & $\mathrm{u}_{\mathrm{d}} / 3$ & $\mathrm{u}_{\mathrm{d}} / \sqrt{3}$ & $2 \mathrm{u}_{\mathrm{d}} / 3$ \\
\hline${ }^{3} \mathrm{Z}$ & ${ }^{3} \underline{\mathrm{U}}$ & $-\mathrm{u}_{\mathrm{d}} / 3$ & $\mathrm{u}_{\mathrm{d}} / \sqrt{3}$ & $2 \mathrm{u}_{\mathrm{d}} / 3$ \\
\hline${ }^{4} \mathrm{Z}$ & ${ }^{4} \underline{\mathrm{U}}$ & $-2 \mathrm{u}_{\mathrm{d}} / 3$ & 0 & $2 \mathrm{u}_{\mathrm{d}} / 3$ \\
\hline${ }^{5} \mathrm{Z}$ & ${ }^{5} \underline{\mathrm{U}}$ & $-\mathrm{u}_{\mathrm{d}} / 3$ & $-\mathrm{u}_{\mathrm{d}} / \sqrt{3}$ & $2 \mathrm{u}_{\mathrm{d}} / 3$ \\
\hline${ }^{6} \mathrm{Z}$ & ${ }^{6} \underline{\mathrm{U}}$ & $\mathrm{u}_{\mathrm{d}} / 3$ & $-\mathrm{u}_{\mathrm{d}} / \sqrt{3}$ & $2 \mathrm{u}_{\mathrm{d}} / 3$ \\
\hline${ }^{7} \mathrm{Z}$ & ${ }^{7} \underline{\mathrm{U}}$ & 0 & 0 & 0
\end{tabular}

Tabelle 3.3.

Drehzeiger $\underline{u}_{S t r}, \alpha$ - und $\beta$-Komponenten und Betrag von $\underline{u}_{S t r}$

Bild 3.25. zeigt die Lage der Zeiger in der $\alpha \beta$-Ebene. Die Spitzen der 6 spannungsbildenden Zeiger liegen gleichmässig verteilt (Winkelschritt $\pi / 3$ ) auf einem Kreis mit dem Radius $2 u_{d} / 3$. Sie definieren damit die $6 \pi / 3$-Sektoren $S 1$ bis $S 6$. Die Nullspannungs-Zeiger ${ }^{0} \underline{U}$ und ${ }^{7} \underline{U}$ haben die Länge null. Die zugehörige Sternpunktspannung kann der Tabelle 3.2 entnommen werden.

\subsubsection{Dreistufige Stromrichter für U-Umrichter}

\subsubsection{Einphasige Halbbrücke}

Der dreistufige U-Stromrichter ist im Gegensatz zum zweistufigen in der Lage, in den Mittelpunktspannungen 3 Spannungsniveaus einzustellen. Die entsprechende Grundschaltung, die einphasige Halbbrücke, bestehend aus einem einzelnen Brückenzweig, ist in Bild 3.26 links dargestellt. Der Brückenzweig besteht hier aus 4 rückwärtsleitenden 


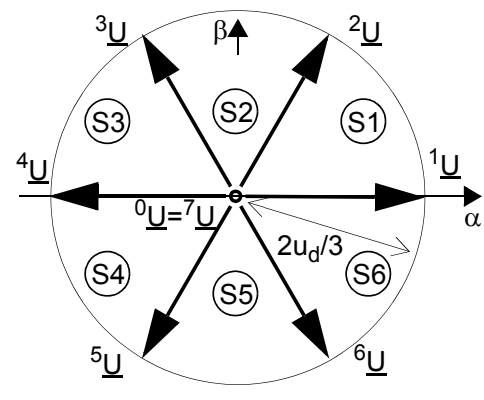

Bild 3.25.

Spannungszustände des Stromrichters in Drehzeigerdarstellung

Schaltelementen. Diese lassen sich so betreiben, dass keines von ihnen mit mehr als der halben Zwischenkreisspannung $u_{d} / 2$ belastet wird. Dazu muss aber der Mittelpunkt des Zwischenkreises zugänglich sein. Er wird nicht nur als Anschlussklemme für die Last benützt, sondern vor allem auch über die beiden zusätzlichen Dioden $D_{+}$und $D_{-}$mit dem Brückenzweig verbunden. Die Tabelle 3.4 beschreibt die 3 im Betrieb verwendeten Kombinationen von Schaltzuständen der Schaltelemente. Alle restlichen Kombinationen würden entweder eine grössere Spannungsbelastung über einem der Schaltelemente ergeben, den Zwischenkreis kurzschliessen oder die Ausgangsspannung nicht eindeutig definieren. Der zusätzliche Zustand, in dem alle Schaltelemente ausgeschaltet sind, stellt die Ruhestellung des Stromrichters dar. Er wird jedoch im Betrieb nicht verwendet.
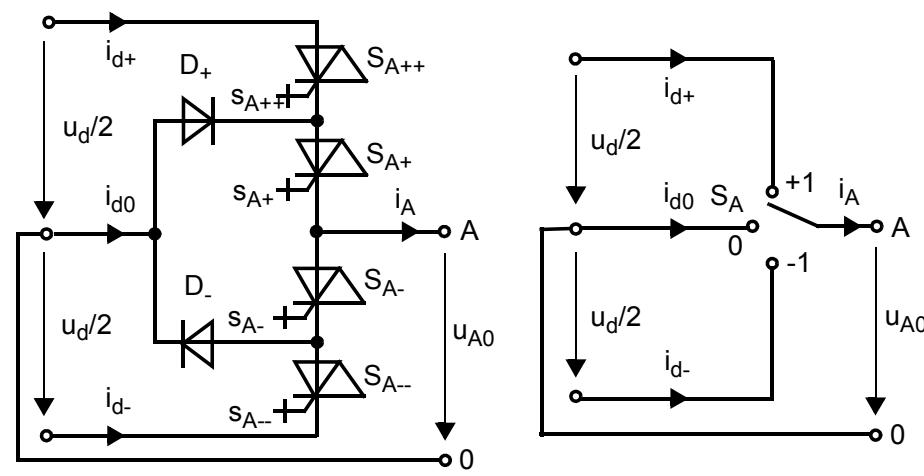

Bild 3.26.

Einphasige dreistufige Halbbrücke für UStromrichter, links: Schaltung mit idealisierten Elementen, rechts: Ersatzschaltung mit Umschaltern

\begin{tabular}{c|c|c|c|c}
$\mathrm{s}_{\mathrm{A}++}$ & $\mathrm{s}_{\mathrm{A}+}$ & $\mathrm{s}_{\mathrm{A}-}$ & $\mathrm{s}_{\mathrm{A}--}$ & $\mathrm{u}_{\mathrm{A}}$ \\
\hline 1 & 1 & 0 & 0 & $\mathrm{u}_{\mathrm{d}} / 2$ \\
\hline 0 & 1 & 1 & 0 & 0 \\
\hline 0 & 0 & 1 & 1 & $-\mathrm{u}_{\mathrm{d}} / 2$
\end{tabular}

\section{Tabelle 3.4.}

Schaltzustände im Normalbetrieb des dreistufigen Brückenzweiges

Aufgrund der beschriebenen Funktion lässt sich auch der dreistufige Stromrichter, wie in Bild 3.26 rechts, durch eine Ersatzschaltung mit einem Umschalter darstellen. Dieser hat 3 Stellungen und kann die Last mit allen 3 Anschlüssen des Zwischenkreises verbinden. Die Schaltfunktion $s_{A}$ des Umschalters $S_{A}$ nimmt dementsprechend die Werte $+1,0$ 
und -1 an. Die Transformationsgleichung (3.78) für die Ausgangsspannung ist gleich wie beim zweistufigen Stromrichter. Die Spannung kann aber jetzt 3 Schaltniveaus aufweisen, nämlich $+u_{d} / 2,0$ und $-u_{d} / 2$. Die Gleichungen für die Zwischenkreisströme in (3.79) sind dagegen anders, da beim dreistufigen Stromrichter 3 Leiter zur Verfügung stehen.

$$
\begin{aligned}
& u_{A}=s_{A} \frac{u_{d}}{2} \\
& i_{d+}=s_{A} \frac{s_{A}+1}{2} i_{A}, \quad i_{d 0}=\left(1-s_{A}^{2}\right) i_{A}, \quad i_{d-}=s_{A} \frac{s_{A}-1}{2} i_{A}
\end{aligned}
$$

Der dreistufige Stromrichter weist gegenüber dem normalen zweistufigen im wesentlichen zwei Vorteile auf:

1. Da jeder Schalter nur mit der halben Zwischenkreisspannung belastet wird, lässt sich mit vorgegebenen Halbleitern ein Stromrichter für die doppelte Nennspannung und damit auch für die doppelte Leistung realisieren.

2. Das zusätzliche Spannungsniveau am Ausgang erlaubt eine bessere Anpassung der Kurvenform an den vorgegebenen Sollwert. Die Verzerrungen in den Ausgangsgrössen lassen sich dadurch verkleinern.

Die Vorteile werden mit einem grösseren Aufwand an Halbleitern und den dazugehörigen Ansteuerungen und Beschaltungen bezahlt. Dazu kommen die erhöhten Leitverluste, da sich stets zwei Halbleiterelemente im Stromkreis befinden. Eine genaue Analyse der Schaltung zeigt auch, dass die Halbleiter sehr unterschiedlich belastet sind und dass sich diese Unsymmetrie betriebsabhängig ändert.

Zeitkontinuierliche Beschreibung: Die Modulationsfunktionen der dreistufigen Schaltungen sehen gleich aus wie diejenigen der zweistufigen. Das zusätzliche Schaltniveau erlaubt zwar verbesserte Steuerverfahren, die kurzzeitigen Mittelwerte sind jedoch identisch. In gewohnter Weise erhält man deshalb die zeitkontinuierliche Beschreibung durch Ersetzen der Schaltfunktionen $s_{U}, s_{V}$ und $s_{W}$ in den Transformationsgleichungen durch die Modulationsfunktionen $m_{U}, m_{V}$ und $m_{W}$. Wie bei den Transformationsgleichungen stimmen generell die zeitkontinuierlichen Spannungsgleichungen für zwei- und dreistufige Stromrichter überein, während die Stromgleichungen wegen der unterschiedlichen Anzahl Leiter im Zwischenkreis voneinander abweichen.

Speisung des Zwischenkreises: Bei allen dreistufigen Schaltungen muss der Mittelpunkt des Zwischenkreises zugänglich sein. In Bild 3.27 sind verschiedene Varianten gezeigt, die diese Bedingung erfüllen. Befindet sich ein zweistufiger Stromrichter auf der Netzseite des Umrichters (Bild 3.27. oben), so ist der Mittelpunkt des Zwischenkreises nur virtuell definiert. Damit die beiden Kondensatoren dieselbe Spannung beibehalten, muss für den zeitlichen Mittelwert des Stromes $I_{d 0}=0$ gelten. Dies bedeutet gleichzeitig, dass beide Stufen des Zwischenkreises dieselbe Leistung übertragen. Damit die Spannungsaufteilung mit Sicherheit immer symmetrisch ist, muss sie durch geeignete Massnahmen im Steuerverfahren des lastseitigen Stromrichters aktiv geregelt werden. 


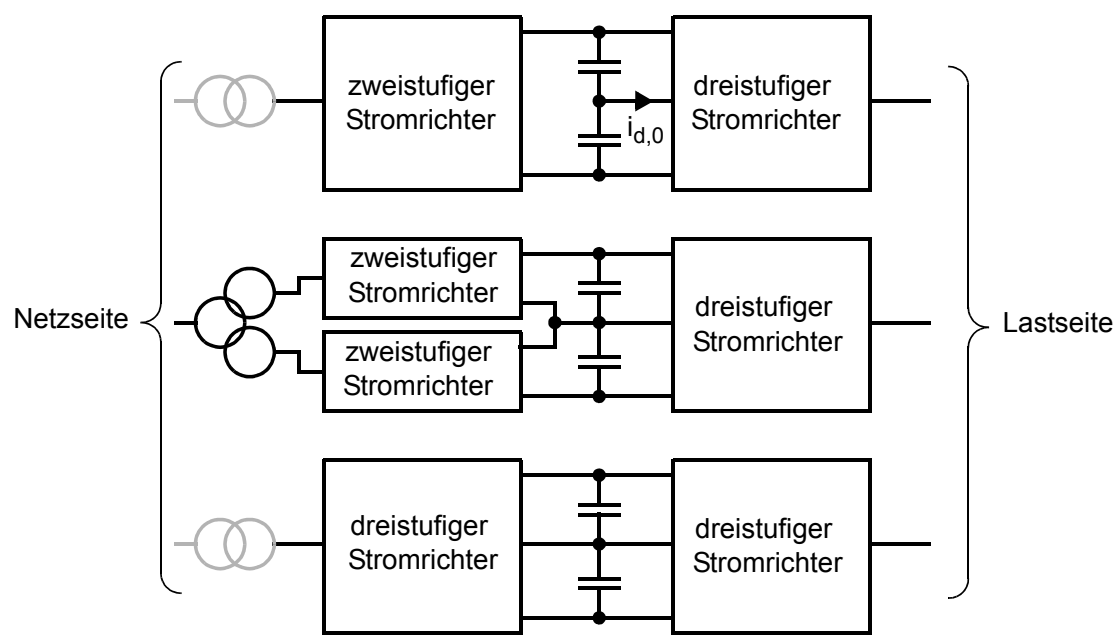

Bild 3.27. Dreistufiger U-Umrichter, oben: virtueller Mittelpunkt, mitte: getrennte Speisung der beiden Zwischenkreishälften, unten: beidseitig dreistufiger Stromrichter

Die Speisung des Zwischenkreises mittels zwei getrennten Stromrichtern (Bild 3.27 mitte) erlaubt die Symmetrierung von der Netzseite her. Es ist im Prinzip sogar eine beliebig unsymmetrische Belastung des Zwischenkreises erlaubt, was allerdings keine praktische Bedeutung hat. Während bei den beiden anderen Varianten der Transformator auf der Netzseite fakultativ ist, wird er hier unbedingt benötigt, damit die Ausgänge der netzseitigen Stromrichter in Serie geschaltet werden können.

Der beidseitig dreistufige Umrichter (Bild 3.27 unten) ergibt netz- und lastseitig dieselben Verhältnisse bezüglich Schaltniveaus und Kurvenformen. Auch hier ist eine Regelung der Spannungsaufteilung notwendig. Sie kann wahlweise durch einen der beiden Stromrichter vorgenommen werden.

\subsubsection{Einphasige Brücke}

Die dreistufige Brücke besteht aus zwei Brückenzweigen. In Bild 3.28 ist das entsprechende Ersatzschaltbild mit Umschaltern dargestellt. Die Transformationsgleichungen lauten wie folgt:

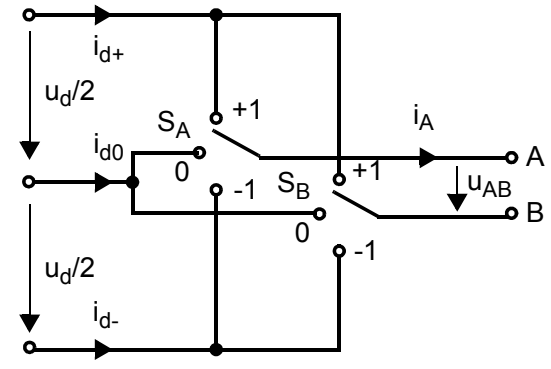

Bild 3.28.

Einphasige dreistufige Brücke für U-Stromrichter, Ersatzschaltung mit Umschaltern 


$$
\begin{aligned}
& u_{A B}=\left(s_{A}-s_{B}\right) \frac{u_{d}}{2} \\
& i_{d+}=\left(s_{A} \frac{s_{A}+1}{2}-s_{B} \frac{s_{B}+1}{2}\right) i_{A}, \quad i_{d 0}=\left(s_{B}^{2}-s_{A}^{2}\right) i_{A}, \\
& i_{d-}=\left(s_{A} \frac{s_{A}-1}{2}-s_{B} \frac{s_{B}-1}{2}\right) i_{A}
\end{aligned}
$$

Die Ausgangsspannung $u_{A B}$ kann dank der jeweils 3 Umschalterstellungen an beiden Lastanschlüssen 5 Niveaus annehmen: $\pm u_{d}, \pm u_{d} / 2$ und 0 .

\subsubsection{Dreiphasige Brücke}

Die dreiphasige Brücke besteht aus 3 dreistufigen Brückenzweigen. Bild 3.29 zeigt direkt das Ersatzschaltbild mit Umschaltern. Die Transformationsgleichungen lauten wie folgt:

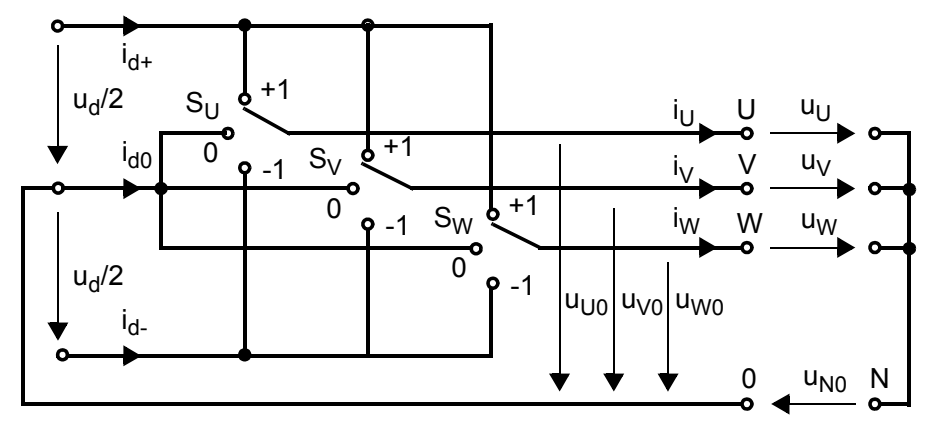

$$
\begin{aligned}
& u_{U 0}=s_{U} \frac{u_{d}}{2}, \quad u_{V 0}=s_{V} \frac{u_{d}}{2}, \quad u_{W 0}=s_{W} \frac{u_{d}}{2} \\
& i_{d^{+}}=s_{U} \frac{s_{U}+1}{2} i_{U}+s_{V} \frac{s_{V}+1}{2} i_{V}+s_{W} \frac{s_{W}+1}{2} i_{W}, \quad i_{d 0}=-s_{U}^{2} i_{U}-s_{V}^{2} i_{V}-s_{W}^{2} i_{W}, \\
& i_{d-}=s_{U} \frac{s_{U}-1}{2} i_{U}+s_{V} \frac{s_{V}-1}{2} i_{V}+s_{W} \frac{s_{W}-1}{2} i_{W}
\end{aligned}
$$

Die Spannungsgleichungen sind gleich wie bei der zweistufigen Schaltung. Die Anzahl der möglichen Spannungsniveaus am Ausgang ist aber deutlich grösser:

Mittelpunktspannungen $u_{U 0}, u_{V 0}, u_{W 0}: 3$ Schaltniveaus, nämlich $\pm u_{d} / 2,0$

Verkettete Spannungen $u_{U V}, u_{V W}, u_{W U}: 5$ Schaltniveaus, nämlich $\pm u_{d}, \pm u_{d} / 2,0$

Und bei symmetrischer Belastung gilt zusätzlich auch:

Phasenspannungen $u_{U}, u_{V}, u_{W^{:}} 9$ Schaltniveaus, nämlich $\pm 2 u_{d} / 3, \pm u_{d} / 2, \pm u_{d} / 3, \pm u_{d} / 6,0$

Sternpunktspannung $u_{N 0}$ : 7 Schaltniveaus, nämlich $\pm u_{d} / 2, \pm u_{d} / 3, \pm u_{d} / 6,0$ 
Beschreibung mittels Drehzeigern: Auch die dreistufige Brückenschaltung kann durch Drehzeiger beschrieben werden. Die bezeichneten Punkte in Bild 3.30 sind die 19 diskreten Positionen, welche die Spitze des Spannungszeigers $\underline{u}_{S t r}$ annehmen kann. Die zugehörigen Stellungen der 3 Brückenzweige sind jeweils angegeben. Die 6 Zeiger auf dem inneren Sechseck lassen sich durch je zwei verschiedene Kombinationen erzeugen und der Nullspannungszeiger sogar durch 3.

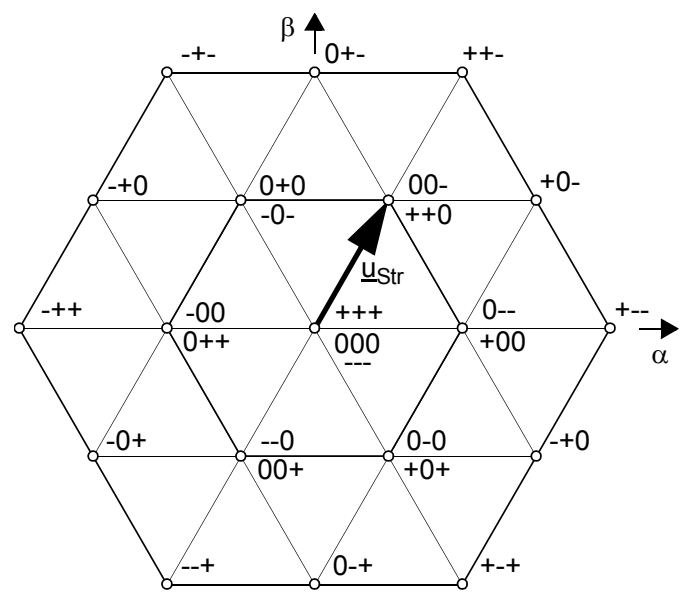

Bild 3.30.

Spannungszustände des dreistufigen Stromrichters in Drehzeigerdarstellung, Beispiel für $\underline{u}_{S t r}$ : 00-: $s_{U}=0, s_{V}=0, s_{W}=-1$, $++0: s_{U}=+1, s_{V}=+1, s_{W}=0$,

\subsubsection{Verallgemeinerte Schaltungen für den U-Umrichter}

Bisher wurden ein- und dreiphasige Schaltungen betrachtet. Durch Hinzufügen weiterer Brückenzweige lässt sich prinzipiell eine Last mit beliebiger Phasenzahl speisen. Eine Anwendung dafür stellt zum Beispiel der sogenannte bürstenlose Gleichstrommotor dar. Desgleichen kann auch die Stufenzahl der Schaltung beliebig erhöht werden, was jedoch in der Praxis bisher nicht gemacht wird. Beide Erweiterungen bringen eine Zunahme der Anzahl Schaltniveaus für die Spannungen über der Last und damit eine verbesserte Nachbildung der angestrebten Kurvenform. Zusätzlich erlauben sie eine Steigerung der gesamten Stromrichterleistung für gegebene Halbleiter. Die Beschreibung dieser Schaltungen kann in analoger Weise zu den bisher betrachteten vorgenommen werden.

\subsubsection{Stromrichter für I-Umrichter}

Die selbstgeführten Schaltungsvarianten für den I-Stromrichter sind weniger zahlreich als für den U-Stromrichter. In der Praxis werden nur ein- und dreiphasige Brücken eingesetzt.

\subsubsection{Einphasige Brücke}

Bild 3.31 zeigt die einphasige Brücke für den I-Stromrichter. Sie unterscheidet sich vom U-Stromrichter nur im Typ der Schaltelemente, welche hier rückwärts sperrend sein müssen. Sie werden jedoch nach anderen Kriterien geschaltet. Im Gegensatz zum U-Stromrichter, wo die Schaltelemente eines Brückenzweiges eine Einheit bilden, sind es beim I- 

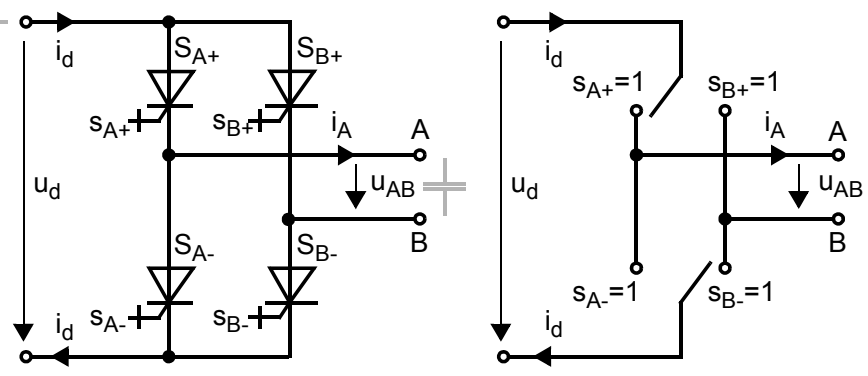

Bild 3.31.

Einphasige Brücke für I-Stromrichter, links: Schaltung mit idealisierten Elementen, rechts Ersatzschaltung mit Umschaltern

Stromrichter je die beiden oberen $\left(s_{A_{+}} / s_{B_{+}}\right)$und unteren $\left(s_{A_{-}} / s_{B-}\right)$ Elemente. Der Strom im Zwischenkreis $i_{d}$ und die ausgangsseitige Spannung $u_{A B}$ sind bedingt durch die im Bild angedeutete äussere Beschaltung des Stromrichters geglättet und können keine Sprünge ausführen. Daraus ergeben sich die folgenden Bedingungen für die beiden oberen Schaltelemente:

1. $s_{A+}=1$ und $s_{B+}=0$ : der Strom $i_{d}$ aus dem positiven Leiter des Zwischenkreises wird auf die Klemme $A$ geleitet.

2. $s_{A+}=0$ und $s_{B+}=1$ : der Strom $i_{d}$ aus dem positiven Leiter des Zwischenkreises wird auf die Klemme $B$ geleitet.

3. $s_{A+}=0$ und $s_{B+}=0$ : dieser Zustand würde den Zwischenkreisstrom unterbrechen und ist deshalb verboten.

4. $s_{A+}=1$ und $s_{B+}=1$ : der Strom $i_{d}$ aus dem positiven Leiter des Zwischenkreises fliesst je nach Vorzeichen der Spannung $u_{A B}$ entweder zu der Klemme $A$ oder $B$. Da der I-Stromrichter am Ausgang das Verhalten einer Stromquelle aufweisen soll, ist diese Situation unerwünscht.

Damit ergeben nur die erste und zweite Schaltkombination sinnvolle Betriebszustände. Sie geben den beiden Schaltern $s_{A^{+}}$und $s_{B^{+}}$das Verhalten eines Umschalters wie er in der Ersatzschaltung in Bild 3.31 rechts dargestellt ist. Dasselbe gilt für die beiden unteren Schalter $s_{A-}$ und $s_{B-}$. Die Schaltfunktionen der beiden Schalterpaare müssen die folgenden Bedingungen einhalten:

$$
s_{A^{+}}+s_{B+}=1, \quad s_{A-}+s_{B-}=1
$$

Es ist dabei durchaus erlaubt, dass der Zwischenkreisstrom über einen der Brückenzweige kurzgeschlossen wird und nicht über die Last fliesst.

Die Transformationsgleichungen für den Phasenstrom und die Zwischenkreisspannung haben die folgende Form:

$$
\begin{aligned}
& i_{A}=\left(s_{A^{+}}-s_{A^{-}}\right) i_{d}=-\left(s_{B+}-s_{B-}\right) i_{d} \\
& u_{d}=\left(s_{A^{+}}-s_{A^{-}}\right) u_{A B}=-\left(s_{B^{+}}-s_{B_{-}}\right) u_{A B}
\end{aligned}
$$

Der Stromrichter verhält sich am Ausgang wie eine Stromquelle, wobei der Strom $i_{A}$ die drei Schaltniveaus $i_{d}, 0$ und $-i_{d}$ aufweisen kann. Die Eigenschaften sind dual zu denjenigen der einphasigen Brücke mit Spannungszwischenkreis: der Strom beim I-Stromrichter entspricht der Ausgangsspannung beim U-Stromrichter. Der geschaltete Verlauf der Zwi- 
schenkreisspannung beim I-Stromrichter entspricht dem Zwischenkreisstrom beim UStromrichter.

Zeitkontinuierliche Beschreibung: Analog zu den U-Stromrichtern können hier die verknüpften Schaltfunktionen $\left(s_{A+}-s_{A-}\right)$ und $\left(s_{B+}-s_{B-}\right)$ in den Transformationsgleichungen durch kontinuierliche Modulationsfunktionen $m_{A}$ und $m_{B}$ ersetzt werden. Dadurch erhält man die zeitkontinuierlichen Gleichungen für den Stromrichter:

$$
\begin{aligned}
& \dot{i}_{A}=m_{A} i_{d}=-m_{B} i_{d} \\
& u_{d}=m_{A} u_{A B}=-m_{B} u_{A B}
\end{aligned}
$$

\subsubsection{Dreiphasige Brücke}

Die dreiphasige Brücke für den I-Stromrichter ist in Bild 3.32 links dargestellt. Sie besteht aus 3 Brückenzweigen mit je zwei rückwärts sperrenden Schaltelementen. Wie die ein-


Bild 3.32. Dreiphasige Brücke für I-Stromrichter, links: Schaltung mit idealisierten Elementen, rechts: Ersatzschaltung mit Umschaltern

phasige Schaltung wird sie so betrieben, dass beide Anschlüsse des Zwischenkreises jeweils mit einer Phase verbunden sind. Dies entspricht der Ersatzschaltung in Bild 3.32 rechts. Für die Schaltfunktionen in den beiden Brückenhälften gilt die Bedingung:

$$
s_{U+}+s_{V+}+s_{W+}=1, \quad s_{U-}+s_{V_{-}}+s_{W-}=1
$$

Die entsprechenden Transformationsgleichungen lauten:

$$
\begin{aligned}
i_{U}= & \left(s_{U^{+}}-s_{U_{-}}\right) i_{d}, \quad i_{V}=\left(s_{V+}-s_{V-}\right) i_{d}, \quad i_{W}=\left(s_{W+}-s_{W-}\right) i_{d} \\
u_{d}= & \left(s_{U^{+}} s_{V_{-}}-s_{U_{-}} s_{V+}\right) u_{U V}+\left(s_{V+} s_{W-}-s_{V-} s_{W+}\right) u_{V W}+ \\
& +\left(s_{W+} s_{U_{-}}-s_{W_{-}} s_{U+}\right) u_{W U}
\end{aligned}
$$

Die Phasenströme weisen wie bei der einphasigen Schaltung die Schaltniveaus $i_{d}, 0$ und $-i_{d}$ auf, analog zu den verketteten Spannungen beim U-Stromrichter. Der Zwischenkreis sieht jederzeit eine der verketteten Spannungen oder aber einen Kurzschluss über einen 
der 3 Brückenzweige. Durch Einsetzen von (3.89) und der Maschengleichung $u_{U V}+u_{V W}+u_{W U}=0$ in (3.91) ergibt für $u_{d}$ in Funktion der verketteten Spannungen:

$$
u_{d}=\left(s_{U^{+}}-s_{U_{-}}\right) u_{U V^{-}}\left(s_{W+}-s_{W-}\right) u_{V W}
$$

Ersetzt man in (3.92) die verketteten Spannungen durch die Phasenspannungen (mit $\left.u_{U V}=u_{U}-u_{V}, u_{V W}=u_{V}-u_{W}, u_{W U}=u_{W}-u_{U}\right)$ erhält man:

$$
u_{d}=\left(s_{U_{+}}-s_{U_{-}}\right) u_{U^{+}}\left(s_{V+}-s_{V_{-}}\right) u_{V}+\left(s_{W+}-s_{W_{-}}\right) u_{W}
$$

Zeitkontinuierliche Beschreibung: Auch bei der Dreiphasenbrücke werden die verknüpften Schaltfunktionen $\left(s_{U+}-s_{U-}\right),\left(s_{V+}-s_{V-}\right)$ und $\left(s_{W+}-s_{W-}\right)$ in den Transformationsgleichungen durch die Modulationsfunktionen $m_{U}, m_{V}$ und $m_{W}$ ersetzt. Damit ergibt sich:

$$
\begin{aligned}
& i_{U}=m_{U} i_{d}, \quad i_{V}=m_{V} i_{d}, \quad i_{W}=m_{W} i_{d} \\
& u_{d}=m_{U} u_{U}+m_{V} u_{V}+m_{W} u_{W}
\end{aligned}
$$

Zusätzlich kann aus (3.89) abgeleitet werden, dass die Summe $m_{U}+m_{V}+m_{W}$ stets null sein muss.

\subsection{6 Überblick über die Kurvenformen von Spannungen und Strömen}

Die Tabellen 3.5 und 3.6 geben einen Überblick über die charakteristischen Kurvenformen der besprochenen Schaltungen. Die Schaltfrequenz pro Schaltelement ist überall gleich gross. Es wird die idealisierte Annahme getroffen, dass die Zwischenkreise ideal geglättete Grössen aufweisen. Die Modulationsfunktionen $m(t)$ sind überall sinusförmig angenommen.

Beim U-Stromrichter ist die unterschiedliche Qualität der Nachbildung der Kurvenform leicht zu erkennen. Je grösser die Anzahl der zur Verfügung stehenden Schaltniveaus ist, desto besser lässt sich die Sinusform modellieren. Generell ergeben die Brückenschaltungen bessere Kurvenformen als die Halbbrücken und die dreistufigen Schaltungen bessere als die zweistufigen.

Beim I-Stromrichter dagegen ist die Kurvenform des Ausgangsstromes in den gezeigten Schaltungen prinzipiell gleichartig. Sie entspricht derjenigen der verketteten Spannung am U-Stromrichter.

Innerhalb der Möglichkeiten, welche die Schaltung selbst bietet, ist die Aufgabe der Steuerverfahren, eine optimale Qualität der Kurvenformen zu gewährleisten.

\subsubsection{Dualität von Stromrichtern und Lastkreisen}

In den vorangegangenen Ausführungen wurde an mehreren Stellen der Ausdruck dual gebraucht. Es existieren Regeln, mit denen eine elektrische Schaltung in eine zu ihr duale Schaltung umgewandelt werden kann. Beispiele solcher Regeln sind:

- Spannungsquellen werden zu Stromquellen

- Induktivitäten werden zu Kapazitäten

- parallele Anordnungen werden zu seriellen Anordnungen 
Tabelle 3.5. Charakteristische Kurvenformen für die Ausgangsspannungen beim U-Stromrichter, gestrichelt: Grundschwingung bzw. Modulationsfunktion

\begin{tabular}{|c|c|c|c|}
\hline Schaltung & $\mathrm{u}_{\mathrm{A} 0}$ bzw. $\mathrm{u}_{\mathrm{U} 0}$ & $\mathrm{u}_{\mathrm{AB}}$ bzw. $\mathrm{u}_{\mathrm{UV}}$ & $\mathrm{u}_{\mathrm{U}(\mathrm{N})}$ \\
\hline $\begin{array}{c}\text { einphasige Halbbrücke, } \\
\text { zweistufig }\end{array}$ & & & - \\
\hline $\begin{array}{c}\text { einphasige Brücke, } \\
\text { zweistufig }\end{array}$ & & & - \\
\hline $\begin{array}{l}\text { dreiphasige Brücke, } \\
\text { zweistufig }\end{array}$ & & & \\
\hline $\begin{array}{c}\text { einphasige Halbbrücke, } \\
\text { dreistufig }\end{array}$ & 用证 & & - \\
\hline $\begin{array}{l}\text { einphasige Brücke, } \\
\text { dreistufig }\end{array}$ & 咁 & & - \\
\hline $\begin{array}{l}\text { dreiphasige Brücke, } \\
\text { dreistufig }\end{array}$ &  & . & 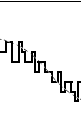 \\
\hline
\end{tabular}

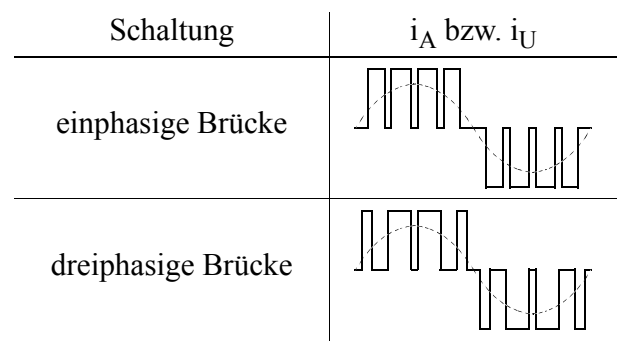

Tabelle 3.6.

Charakteristische Kurvenformen für die Ausgangsströme beim I-Stromrichter, gestrichelt: Grundschwingung bzw. Modulationsfunktion

- Sternschaltungen werden zu Dreieckschaltungen

- rückwärtsleitende Schalter werden zu rückwärtssperrenden

Wandelt man nach diesen Regeln einen einphasigen selbstgeführten U-Stromrichter um, so erhält man die entsprechende Schaltung für den I-Stromrichter (Bild 3.33.). Eine der wesentlichen Eigenschaften der Dualität ist, dass die Spannungen in der Schaltung den Strömen in der dualen Schaltung entsprechen und umgekehrt. So wird der idealisiert als Gleichspannungsquelle modellierte Zwischenkreis beim U-Stromrichter zu einer Gleichstromquelle beim I-Stromrichter. Wie bereits in den Tabellen 3.5 und 3.6 ersichtlich war, geht die geschaltete Ausgangsspannung $u_{A B}$ des U-Stromrichters über in den geschalteten Ausgangsstrom $i_{A}$ des I-Stromrichters. Die beiden dualen Grössen können ge- 

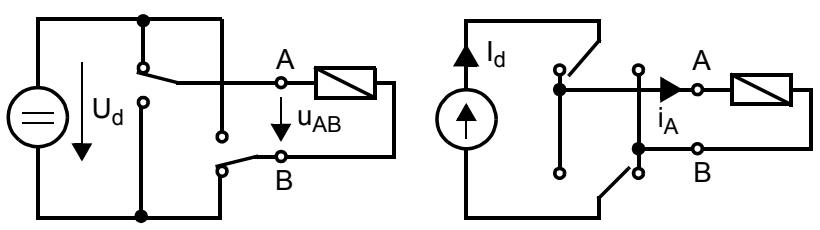

Bild 3.33.

Duale Schaltungen am Beispiel der einphasigen Brücke, links: U-Stromrichter, rechts: I-Stromrichter

nau dieselbe Kurvenform aufweisen. Diese Eigenschaft wird bei der Behandlung von Steuerverfahren für den I-Stromrichter (Kapitel 14) eine wichtige Rolle spielen. Verfahren für U-Stromrichter können prinzipiell in solche für den I-Stromrichter umgesetzt werden.

Wird eine Stromrichterschaltung durch ihre duale Schaltung ersetzt, so muss auch der Lastkreis in seine duale Form übergehen. Es wurde bereits gezeigt, dass praktisch alle in der Praxis vorkommenden Stromrichterlasten der Ersatzschaltung in Bild 3.34 links entsprechen. Zu ihrer Speisung ist der U-Stromrichter ideal geeignet. Lasten nach der dualen Ersatzschaltung gemäss Bild 3.34 mitte, die für einen I-Stromrichter ideal wären, kommen dagegen praktisch nicht vor. Soll in der Praxis ein I-Stromrichter verwendet werden, so muss deshalb der Last durch Parallelschalten einer Kapazität, wie in Bild 3.34 rechts, zuerst das duale Verhalten gegeben werden. Dies bedeutet einerseits einen zusätzlichen Schaltungsaufwand und andererseits wird ein Resonanzkreis geschaffen, der bei der Steuerung des Stromrichters berücksichtigt werden muss.
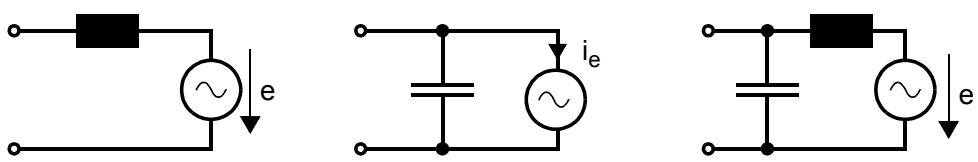

Bild 3.34. Lasttypen für U- und I-Stromrichter, links: Standardlast für einen U-Stromrichter, mitte: duale Schaltung für einen I-Stromrichter, rechts: angepasste Standardlast für einen I-Stromrichter

Es muss an dieser Stelle betont werden, dass nicht zu jeder Schaltung eine duale Variante existiert. So ist die dreiphasige I-Stromrichterbrücke nicht exakt dual zur Brückenschaltung mit Spannungszwischenkreis. Darauf wird in Kapitel 14 näher eingegangen.

Literatur zu Kapitel 3.3: [Hag1], [Heu1], [Mey1], [Mic1] 


\section{Regelkonzepte für selbstgeführte Stromrichter}

Dieses Kapitel beschränkt sich ausschliesslich auf die Regelung von U-Umrichtern. Viele der grundsätzlichen Betrachtungen lassen sich aber auf I-Umrichter übertragen.

\subsection{Regelung eines Umrichters mit Spannungszwischenkreis}

Bild 4.1 zeigt die am meisten verwendete prinzipielle Regelstruktur für einen U-Umrichter. Wie bereits in Kapitel 2 erwähnt, werden die Netz- und die Lastseite getrennt geregelt, wobei die im Zwischenkreis fliessende Leistung eine Kopplung der beiden Seiten ergibt. Diese Aufteilung ergibt eine übersichtliche Regelstruktur und erlaubt zudem eine stufenweise Inbetriebnahme des Umrichters.

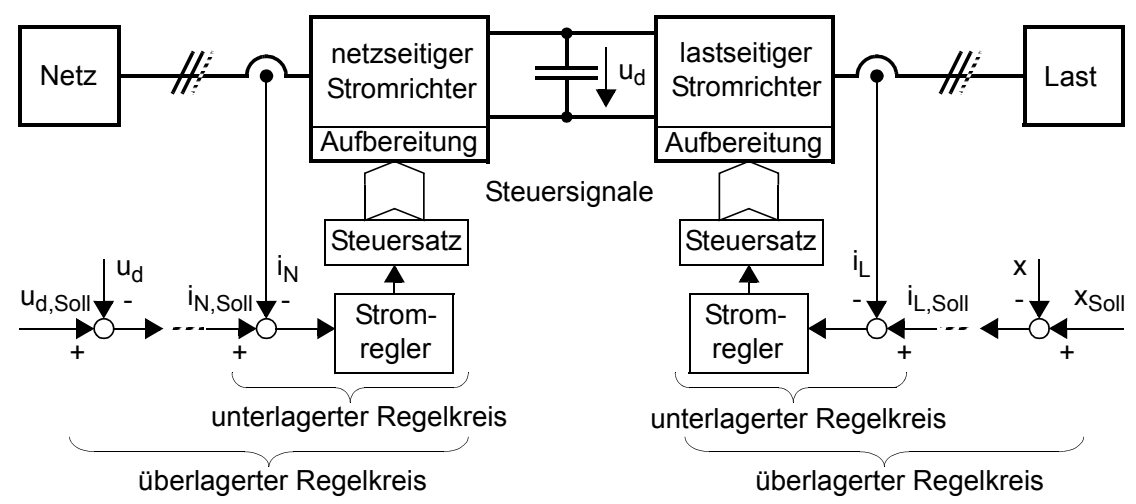

Bild 4.1. Standard-Regelstruktur für einen U-Umrichter

Bei beiden Stromrichtern werden in einem schnellen unterlagerten Regelkreis die ausgangsseitigen Ströme geregelt. Diese sind damit jederzeit unter Kontrolle und können auf einen Maximalwert begrenzt werden. Der Umrichter ist auf diese Weise gegen Überlastung geschützt. Für die überlagerte Regelung verhält sich der stromgeregelte Stromrichter wie eine einstellbare Stromquelle.

Die einzige Möglichkeit zum Eingriff in den Leistungskreis stellen die Steuersignale für die einzelnen Schaltelemente der beiden Stromrichter dar. Die Stellgrössen müssen deshalb im Steuersatz in die entsprechenden Ein- und Ausschaltbefehle (Schaltfunktionen) umgesetzt werden. Bei einem selbstgeführten Stromrichter wird der Steuersatz als (Puls- 
weiten-) Modulator bezeichnet. Die Ansteuerelektronik der Leistungshalbleiter, wo die Aufbereitung der Steuersignale in die eigentlichen Steuerspannungen bzw. -ströme geschieht (Kapitel 3.1), wird hier als Bestandteil des Stromrichters betrachtet.

Die lastseitige Regelung hat primär die Aufgabe, die Last in den gewünschten Betriebszustand zu bringen. Bei einem Antrieb zum Beispiel kann die Regelgrösse das Drehmoment, die Drehzahl oder den Drehwinkel der Welle sein. Bei der Speisung eines unabhängigen Netzes ist es die Spannung an der Last, d.h. am Ausgang des Filters (Beispiel Kapitel 4.4.3). In Bild 4.1 steht $x$ stellvertretend für eine dieser Grössen. Die gesamte Regelstruktur kann sehr komplex sein. Ihre Aufteilung in einen unterlagerten Regelkreis für die Ströme und eine überlagerte Regelung für die lastspezifischen Regelgrössen ist aber meistens vorzufinden.

Die Lastseite legt die Leistung im Zwischenkreis fest. Es ist deshalb die primäre Aufgabe der netzseitigen Regelung, diese Leistung zur Verfügung zu stellen. Dies geschieht, indem die Zwischenkreisspannung $u_{d}$ als Regelgrösse verwendet wird. Bei den meisten Anwendungen wird sie auf einen konstanten Wert geregelt. Es ist jedoch auch möglich, dass ihr Wert in Abhängigkeit vom Betriebszustand der Last variieren soll. In diesem Fall ist ihr Sollwert nicht unabhängig, sondern wird von der Lastseite her vorgegeben. Im Falle eines selbstgeführten Stromrichters kann auch die netzseitige Regelstruktur komplex werden, weil dann, wie in Kapitel 2.3 erwähnt, auch die Blindleistung beliebig einstellbar ist.

Für die Auslegung der Regelung werden normalerweise die in Kapitel 3.3 definierten zeitkontinuierlichen Transformationsgleichungen der Stromrichter verwendet. Es werden also Grundschwingungsbetrachtungen gemacht und der Einfluss der stets vorhandenen Verzerrungsanteile in den Spannungen und Strömen auf die Regelkreise wird nicht berücksichtigt. Er muss unter Umständen aber separat zusätzlich abgeklärt werden. Eine Abweichung von diesem Prinzip stellen die Steuerverfahren zur direkten Stromregelung, die in den Kapiteln 10 und 11 behandelt werden, dar. Sie entsprechen dem unterlagerten Regelkreis, arbeiten aber mit dem exakten Stromverlauf, d.h. mit Berücksichtigung der Verzerrungen.

In vielen Fällen kann das Systemverhalten durch Vorsteuerungen bzw. Störgrössenaufschaltungen stark verbessert werden. Ein Beispiel dafür ist die Aufschaltung des lastseitigen Zwischenkreisstromes auf den netzseitigen Stromregelkreis. Damit wird dessen Reaktionsgeschwindigkeit auf Laständerungen markant erhöht.

\subsection{Wirk- und Blindstrom am selbstgeführten Stromrichter}

Bei den meisten Anwendungen ist es von Bedeutung, den Wirk- und Blindstrom und damit die Wirk- und Blindleistung in der Last getrennt regeln zu können. Am Beispiel der Standardlast nach Kapitel 3.2.4 wird gezeigt, wie diese Entkopplung vollzogen werden kann. Betrachtet werden die Leistungen in der Gegenspannungsquelle $e$, da diese in den meisten Anwendungen die grösste Bedeutung haben.

In der zeitkontinuierlichen Betrachtungsweise gilt für Grundschwingungsbetrachtungen die Ersatzschaltung in Bild 4.2 für den Lastkreis. 

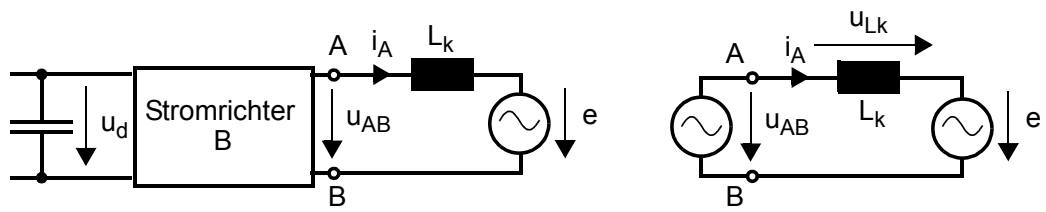

Bild 4.2. Selbstgeführter Stromrichter mit Standardlast, links: einphasige Schaltung, rechts: Ersatzschaltung mit Spannungsquellen

Wirkstrom: Bild 4.3 links zeigt das Wechselgrössen-Zeigerdiagramm für die Ersatzschaltung nach Bild 4.2 für den Fall, dass die Gegenspannungsquelle eine reine Wirkleistung abgibt oder aufnimmt. Der Zeiger $\underline{\underline{\hat{t}}}_{A}$ liegt dann parallel bzw. antiparallel zu $\underline{\hat{e}}$ und die

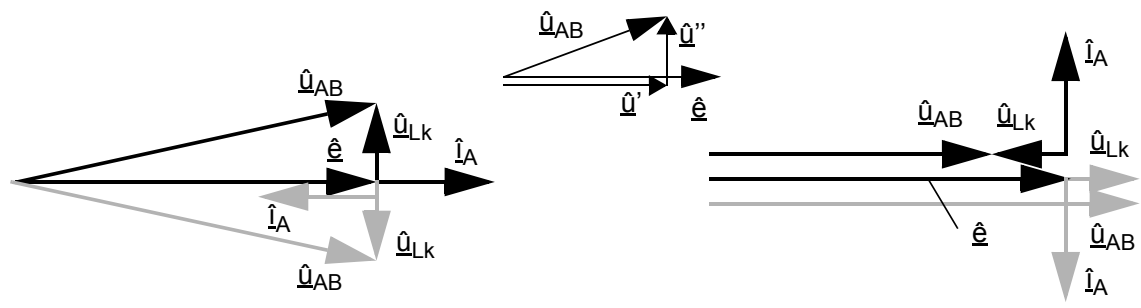

Bild 4.3. Zeigerdiagramme für den Lastkreis, oben mitte: Aufspaltung von $\underline{\hat{u}}_{A B}$ in die Komponenten $\hat{u}^{\prime}$ und $\hat{u}^{\prime \prime}$,

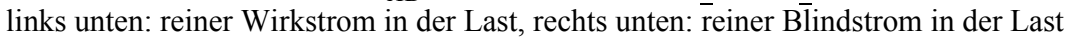

Spannung über der Induktivität $\underline{\hat{~}}_{L k}$ steht senkrecht zu den beiden Zeigern. Ist die Stromrichterspannung $\underline{\hat{x}}_{A B}$ voreilend bezüglich der Gegenspannung $\underline{\hat{e}}$, so nimmt die Gegenspannungsquelle Leistung auf (schwarze Zeiger im Bild), ist sie nacheilend, entspricht dies einer Leistungsabgabe (graue Zeiger). Die Amplitude $\hat{\imath}_{A}$ des Stromes $\underline{\underline{\underline{t}}}_{A}$ ist gemäss (4.1) durch die Spannung über der Induktivität $\underline{\hat{u}}_{L k}$ bestimmt und der Betrag der Leistung in der Gegenspannungsquelle ergibt sich zu:

$$
\begin{aligned}
& \hat{i}_{A}=\frac{\hat{u}_{L k}}{\omega_{1} L_{k}} \\
& P=\frac{\hat{e} \hat{i}_{A}}{2}=\frac{\hat{e} \hat{u}_{L k}}{2 \omega_{1} L_{k}}
\end{aligned}
$$

Spaltet man die Stromrichterspannung $\underline{\hat{\underline{u}}}_{A B}$ in die zu $\underline{\hat{e}}$ parallele Komponente $\hat{u}^{\prime}$ und die zu $\underline{\hat{e}}$ senkrechte Komponente $\hat{u}^{\prime \prime}$ auf, so gilt für diese Komponenten die Beziehung:

$$
\hat{u}_{A B}=\hat{u}^{\prime}+\hat{u}^{\prime \prime} \quad \text { mit } \quad \hat{u}^{\prime}=\hat{e}, \quad \hat{u}^{\prime \prime}=\hat{u}_{L k}
$$

Damit gilt die folgende Aussage: in der Gegenspannungsquelle fliesst eine reine Wirkleistung, wenn die Amplituden $\hat{u}^{\prime}$ und $\hat{e}$ übereinstimmen. Die Amplitude des Stromes und die Wirkleistung sind dann proportional zur Amplitude $\hat{u}^{\prime \prime}$. 
In der gewählten Betriebsart liefert der Stromrichter neben der Wirkleistung zusätzlich die Blindleistung für die Induktivität.

Blindstrom: Bild 4.3 rechts zeigt das Zeigerdiagramm des Lastkreises unter der Voraussetzung, dass die Gegenspannungsquelle eine reine Blindleistung abgibt oder aufnimmt. Die Zeiger $\underline{\hat{e}}$ und $\underline{\hat{t}}_{A}$ stehen senkrecht aufeinander. Dazu müssen $\underline{\hat{e}}$ und $\underline{\hat{v}}_{L k}$ parallel oder antiparallel liegen. Die Amplitude des Stromes ist wiederum durch (4.1) bestimmt und die Blindleistung in der Gegenspannungsquelle ergibt sich analog zu (4.2):

$$
Q=\frac{\hat{e} \hat{i}_{A}}{2}=\frac{\hat{e} \hat{u}_{L k}}{2 \omega_{1} L_{k}}
$$

Für die Amplituden der Komponenten $\hat{u}^{\prime}$ und $\underline{\hat{u}}^{\prime \prime}$ gilt hier:

$$
\hat{u}^{\prime}=\hat{e} \pm \hat{u}_{L k}, \quad \hat{u}^{\prime \prime}=0
$$

Eine reine Blindleistung in der Gegenspannungsquelle stellt sich also ein, wenn die Ausgangsspannung $\underline{\hat{u}}_{A B}$ und die Gegenspannung $\underline{\hat{e}}$ in Phase zueinander liegen. Die Amplitude des Ausgangsstromes ist dann proportional zur Differenz der beiden Spannungsamplituden. Dabei gibt die Gegenspannungsquelle Blindleistung ab, das heisst verhält sich wie eine Kapazität, wenn $\hat{u}_{A B}$ kleiner ist als $\hat{e}$ (schwarze Zeiger im Bild) und nimmt Blindleistung auf, das heisst, verhält sich wie eine Induktivität, wenn $\hat{u}_{A B}$ grösser ist als $\hat{e}$ (graue Zeiger).

Steuerung des Wirk- und Blindstromes: Entsprechend der vorangegangenen Betrachtungen lassen sich bei gegebener Gegenspannung der Wirk- und Blindstrom am Ausgang des Stromrichters mit Hilfe der Aufspaltung von $\underline{\hat{u}}_{A B}$ in die Komponenten $\hat{u}^{\prime}$ und $\hat{u}^{\prime \prime}$ unabhängig voneinander steuern: der Wirkstrom ist proportional zu $\hat{u}^{\prime \prime}$ und der Blind $\bar{d}$ strom ist proportional zur Differenz $\left|\hat{u}^{\prime}-\hat{e}\right|$.

Die Entkopplung ist jedoch nur gegeben, weil bei der Definition der Standardlast der ohmsche Anteil der Lastimpedanz null gesetzt wurde. Dies ist für Grundschwingungsbetrachtungen nicht immer zulässig. Vor allem bei Antrieben im Anlaufbereich, wenn $\omega_{1}$ sehr klein ist, wird der ohmsche Spannungsabfall gegenüber dem induktiven spürbar oder sogar dominant. Die Wirk- und Blindstromsteuerung erhält dadurch eine frequenzabhängige gegenseitige Kopplung. Diese lässt sich bei der Regelung allerdings relativ leicht berücksichtigen (Kopplungsnetzwerke, [Föl1]).

\subsection{Stromregelung}

Die Beschreibung des Systems und die Implementierung der Regelung kann in verschiedenen Darstellungsarten erfolgen. Dabei sind einphasige und dreiphasige Schaltungen verschieden zu behandeln. 
Einphasige Regelungen: Bild 4.4 zeigt die zwei Stromregelstrukturen, welche für eine einphasige Regelung üblich sind. In beiden Fällen liefert der Ausgang des Stromreglers den Sollwert für die Ausgangsspannung am Stromrichter. Dieser wird dann im Modulator in die entsprechenden Schaltfunktionen umgesetzt.

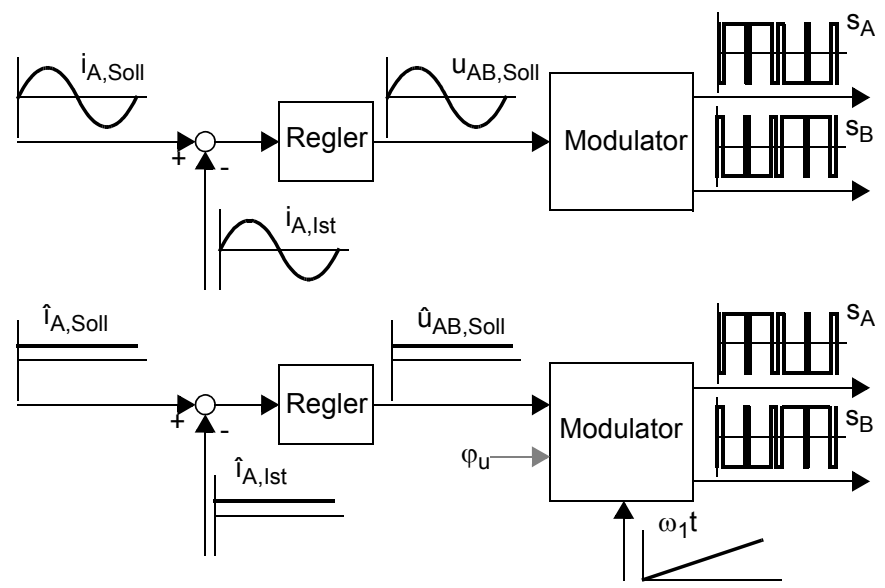

Bild 4.4.

Einphasige Stromregelstrukturen, oben: mit Zeitfunktionen, unten: mit Amplituden (und Phasen)

Arbeitet die Regelung mit Zeitfunktionen (Bild 4.4 oben), so kann der Sollwert im Prinzip einen beliebigen zeitlichen Verlauf aufweisen (z.B. ein Audiosignal für einen geschalteten Leistungsverstärker). Bei den meisten Anwendungen ist er jedoch im stationären Zustand sinusförmig. Am Eingang des Modulators liegt dann ebenfalls eine sinusförmige Zeitfunktion an. Wird ein konventioneller PI-Regler (Proportional-Integral-Regler) verwendet, so ergibt sich eine stationäre Regelabweichung. Der Strom-Istwert ist gegenüber seinem Sollwert stets leicht verzögert. Der Grund dafür ist, dass der Regler bei der Grundkreisfrequenz $\omega_{1}$ nur eine begrenzte Verstärkung aufweisen kann.

Es ist zu beachten, dass der Stromistwert in der Praxis wegen der geschalteten Spannung am Stromrichter stets Verzerrungen aufweist. Diese können unter Umständen die Regelung ungünstig beeinflussen. Aus diesem Grund wird der Istwert in der Regel leicht gefiltert. Anders verhält es sich bei Phasenstromreglern (Kapitel 10, auch Toleranzbandregler genannt). Diese nicht linearen Verfahren basieren auf der Rückkopplung des verzerrten Stromistwertes.

In der Struktur nach Bild 4.4 unten werden die Amplituden von Soll- und Istwert des Stromes auf den Regler geführt. Ein sinusförmiger Verlauf ist implizit vorausgesetzt. Der Modulator erhält als Eingangsgrösse deshalb nur die Amplitude der einzustellenden Spannung. Zusätzlich wird die gewünschte Grundfrequenz $\omega_{1}$, in Form eines zeitabhängigen Referenzwinkels $\omega_{1} t$ zugeführt. Diese Grösse kann aus einem freilaufenden oder einem geregelten Oszillator (zum Beispiel für eine Netzanwendung aus einem netzsynchronisierten Phasenregelkreis d.h. PLL) stammen. Unter Umständen soll auch der Phasenwinkel $\varphi_{u}$ der Ausgangsspannung gegenüber dem Referenzwinkel variiert werden. In diesem Fall ist er als weitere Eingangsgrösse für den Modulator vorzugeben. Dazu ist gegebenfalls ein separater Regelkreis notwendig. 
Die kompliziertere Struktur der Amplituden-Regelung hat den Vorteil, dass der Regler im stationären Fall Gleichgrössen zu verarbeiten hat. Dasselbe gilt für einen allfälligen Regelkreis für die Phase $\varphi_{u}$. Damit lässt sich der Strom mit PI-Reglern prinzipiell stationär exakt, d.h. ohne ständige Regelabweichung, dem Sollwert nachzuführen. Wie gut die Nachführung in der Praxis ist, hängt von der Qualität des Modulators ab, der die Umsetzung des Spannungssollwertes aus Amplitude und Phase in Zeitfunktionen zu übernehmen hat.

Dreiphasige Regelungen: Bei dreiphasigen Regelungen stehen neben der Darstellung der Grössen als Zeitfunktionen auch die Dreh- und Festzeigerdarstellung zur Auswahl. Die letztere eignet sich besonders für komplexe Regelalgorithmen. Beispiel dafür sind die feldorientierte Regelung einer Maschine oder die getrennte Wirk- und Blindleistungsregelung eines Stromrichters am Netz.

Bild 4.5 zeigt die Stromregler für die verschiedenen Beschreibungsarten. Die Regelung der Phasenströme (Bild 4.5 oben) bedingt 3 getrennte Regelkreise. Der Modulator setzt die 3 Spannungssollwerte, die als Zeitfunktionen anliegen, in Steuersignale um. Wie beim entsprechenden einphasigen Regler sind auch hier die Phasenströme gegenüber dem Sollwert stets leicht verzögert. Da schaltungsbedingt die Summe der Phasenströme null ist, d.h. nur zwei Freiheitsgrade existieren, beeinflussen sich die 3 Regelkreise gegenseitig. Bei der Auslegung ist darauf zu achten, dass dadurch keine Instabilitäten entstehen. Weiter sind die verzerrten Stromistwerte für eine optimales Verhalten der Regelung normalerweise leicht zu filtern. Es gibt daneben allerdings auch für dreiphasige Schaltungen nichtlineare Regelverfahren (Kapitel 11 und 12), die auf der Basis der verzerrten Ströme arbeiten.

Erfolgt die Regelung mit Zeigern, so entspricht dies jeweils zwei separaten Regelkreisen für die Zeigerkomponenten. Im Falle der Drehzeiger (Bild 4.5 mitte) sind die verarbeiteten Grössen weiterhin Zeitfunktionen, das heisst im stationären Betrieb sinusförmig. Das Problem der ständigen Regelabweichungen bleibt bestehen. Dies ändert sich, wenn die Regelung mit Festzeigern (Bild 4.5 unten) arbeitet. Hier sind die Grössen im stationären Fall Gleichgrössen, so dass die Regler die Sollwerte exakt einstellen können. Dafür muss dem Modulator als zusätzliche Information die Grundfrequenz in Form eines zeitabhängigen Referenzwinkels $\omega_{1} t$ zugeführt werden. Dieser entspricht der Lage des rotierenden dq-Koordinatensystems in Bezug auf das ruhende $\alpha \beta$-System. Wie im letzten Abschnitt in Kapitel 4.2 erklärt wurde, erfolgt die Steuerung der Stromzeigerkomponenten im Falle der idealisierten Standardlast jeweils über die orthogonale Komponente der Stromrichterspannung. Dies entspricht der im Bild angedeuteten Auskreuzung der Sollwertkomponenten. In der Praxis weist die Lastimpedanz stets einen mehr oder weniger grossen ohmschen Anteil auf. Dadurch ergibt sich wie bereits erwähnt eine frequenzabhängige Kopplung der beiden Regelkreise. Sie führt dazu, dass die Regelstruktur auch anders aussehen kann: z.T. werden Kopplungsnetzwerke eingesetzt, welche aus 4 frequenzabhängigen Übertragungsfunktionen bestehen oder es ist sogar eine direkte Aufschaltung der Reglerausgänge ohne Auskreuzung der Komponenten möglich (gestrichelte Linien im Bild). 



Bild 4.5. Dreiphasige Stromreglerstrukturen, oben: mit Phasengrössen, mitte: mit Drehzeigern, unten: mit Festzeigern

In Bild 4.5 ist zu erkennen, dass in allen 3 Fällen gleichartige Steuersignale für die Brückenzweige des Stromrichters erzeugt werden müssen. Der Modulator führt deshalb bei den Zeigerregelungen zusätzlich die Rücktransformation in Phasengrössen durch. 


\subsection{Beispiele für Regelungen von selbstgeführten Stromrichtern}

Die Vielfalt an verschiedenen Regelstrukturen für Lasten, die mit einem selbstgeführten Stromrichter gespeist werden, ist äusserst gross. Selbst innerhalb der einzelnen Anwendungsgebiete existieren häufig mehrere Varianten (z.B. die verschiedenen feldorientierten Regelverfahren für Drehstrommaschinen). Im folgenden werden 3 ausgesuchte Beispiele von vollständigen Regelstrukturen vorgestellt. Sie sollen vor allem die Rolle des Modulators innerhalb der Regelung aufzeigen. Es werden nur die Grundstrukturen, ohne Zusatzfunktionen wie Strombegrenzung, Feldschwächung etc. dargestellt.

\subsubsection{Einfache Regelung eines Netzstromrichters}

Bild 4.6 zeigt ein verhältnismässig einfaches Regelungsprinzip für einen einphasigen selbstgeführten Stromrichter am Netz. Es entspricht der Konfiguration mit einem unterlagerten Regelkreis für den Netzstrom $i_{N}$ und dem überlagerten Regelkreis für die eigentliche Regelgrösse, die Zwischenkreisspannung $u_{d}$. Der überlagerte Regler gibt die Amplitude des Stromes $\hat{\imath}_{N \text {,Soll }}$ vor. Die Multiplikation mit der zurückgeführten, normierten Netzspannung ergibt einen Stromsollwert $i_{N, \text { Soll }}$, der einen sinusförmigen Verlauf aufweist und in Phase mit der Netzspannung liegt. Auf diese Weise ist sichergestellt, dass der Stromrichter keine Blindleistung bezieht. Der unterlagerte Stromregler arbeitet nach dem Prinzip in Bild 4.4 oben, also mit Zeitfunktionen.

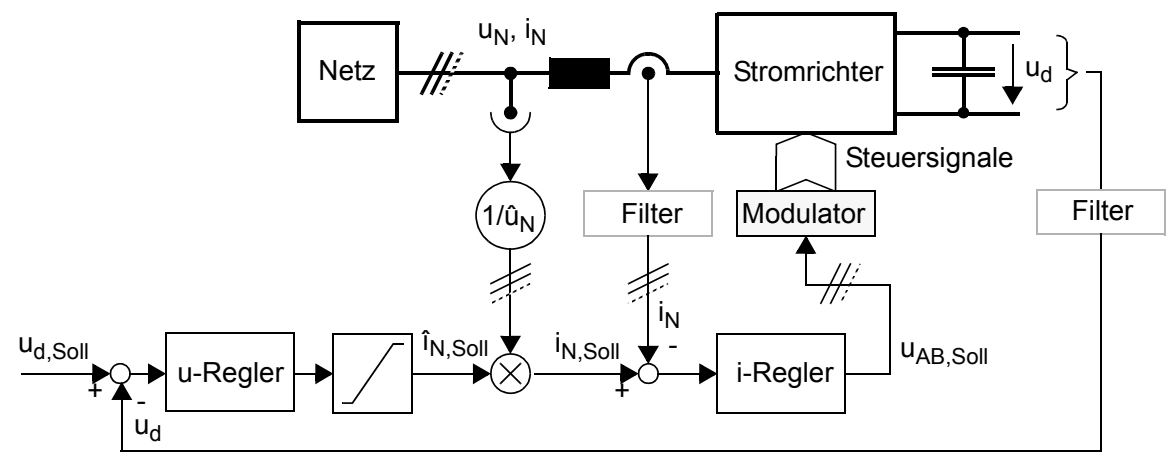

Bild 4.6. Beispiel für die Regelung eines selbstgeführten Stromrichter am Netz

Da die Regelung für die zeitkontinuierlichen Stromrichtergrössen ausgelegt ist, müssen die verzerrten Messgrössen unter Umständen gefiltert werden. Die Filterung wird jedoch möglichst schwach ausgelegt, damit sie die Dynamik der Regelung nicht allzu stark einschränkt.

Bei dreiphasigen Schaltungen muss der Stromregelkreis, wie im Bild angedeutet, dreifach, für jede Phase getrennt implementiert werden. Der Modulator muss dementsprechend 3 Sollwerte verarbeiten können. 


\subsubsection{Feldorientierte Regelung einer Asynchronmaschine}

Bild 4.7 zeigt das häufig angewendete Regelverfahren für eine ASM mit Feldorientierung auf die Rotorflussverkettung $\underline{\Psi}_{R}$. Es arbeitet mit Festzeigern in einem rotierenden, auf $\underline{\Psi}_{R}$ ausgerichteten $d q$-Koordinatensystem. Dabei liegt $\underline{\Psi}_{R}$ liegt stets auf der $d$-Achse. Zwei parallele überlagerte Regelkreise stellen den Betrag $\left|\underline{\Psi}_{R}\right|$ und die Drehzahl $\omega_{\text {mech }}$ der Maschine ein. Der Flussregler ergibt direkt den Sollwert für die flussbildende Komponente des Statorstromes $i_{S \text {, d,Soll }}$. Der Ausgang des Drehzahlreglers entspricht dagegen dem einzustellenden Drehmoment $M_{\text {el,Soll }}$. Erst nach der Division durch $\left|\underline{\Psi}_{R}\right|$ liegt der Sollwert für die momentbildende Stromkomponente $i_{S, q, \text { Soll }}$ vor. Diese Beziehungen lassen sich aus den Gleichungen der ASM in Kapitel 3.2.2.1 ableiten.

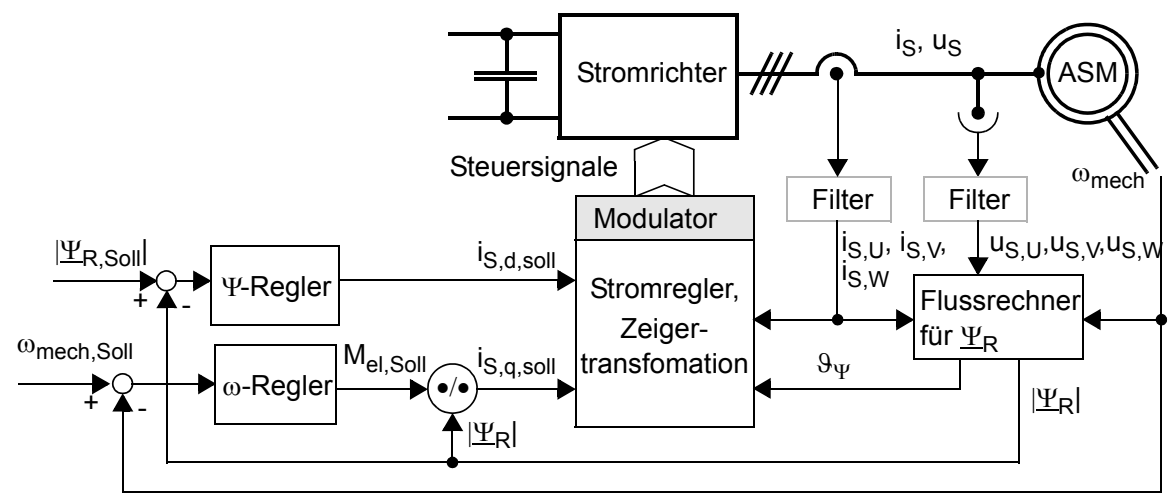

Bild 4.7. Beispiel für die feldorientierte Regelung einer ASM

Der unterlagerte Stromregelkreis zusammen mit dem Modulator kann in allen 3 Formen nach Bild 4.5 ausgeführt sein. Je nach dem gewählten Reglertyp sind entweder die Solloder die Istwerte des Statorstromes oder beide in die benötigte Darstellungsform zu transformieren. In jedem Fall wird dazu der Referenzwinkel $\vartheta_{\Psi}(t)$ des rotierenden, auf $\underline{\Psi}_{R}$ orientierten $d q$-Koordinatensystems gebraucht: entweder zur Zeigertransformation oder im Modulator als Referenzwinkel.

Die Grössen $\left|\underline{\Psi}_{R}\right|$ und $\vartheta_{\Psi}$ der Rotorflussverkettung werden im Flussrechner aus den Statorspannungen und -strömen sowie der Drehzahl nachgebildet.

\subsubsection{Speisung eines Inselnetzes}

Die Struktur der Regelung eines Inselnetzes nach Bild 4.8 ist sehr einfach: Regelgrösse ist die Spannung an der Last $u_{L}$. Ihr Sollwert muss im gezeigten Beispiel als Zeitfunktion vorliegen. Am Reglerausgang ergibt sich direkt der Sollwert für die Stromrichterspannung. In dieser einfachsten Form existiert kein Stromregelkreis. Der Laststrom soll sich frei einstellen können. Für den Schutz gegen Überlastung bzw. zur Sicherstellung der Kurzschlussfestigkeit ist allerdings eine Stromüberwachung notwendig. Sie ist in Bild 4.8 nicht eingezeichnet. 


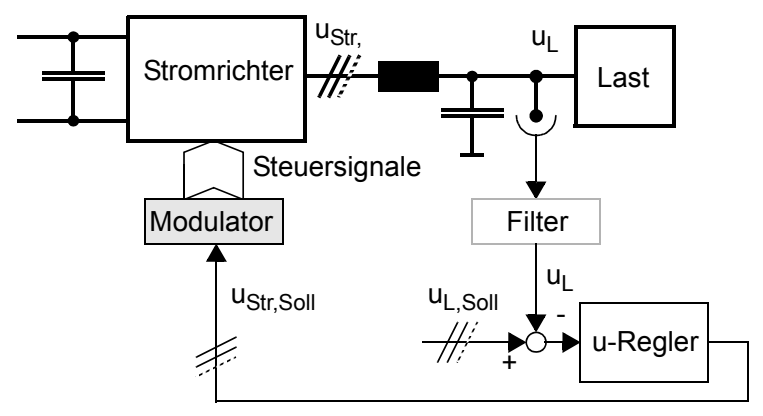

Bild 4.8.

Beispiel für die Regelung eines Inselnetzes

Es muss erwähnt werden, dass der Lastkreis zusammen mit dem Filter eine Resonanzstelle aufweist. Diese muss durch die Regelung beherrscht werden.

\subsection{Zusammenfassung der Anforderungen an den Modulator}

Im Sinne einer Zusammenfassung der Kapitel 4.3 und 4.4 werden nachfolgend die allgemeinen Anforderungen an den Modulator für einen selbstgeführten Stromrichter formuliert:

1. Der Modulator ist Teil eines Regelkreises, meistens für den Phasenstrom im Stromrichter. Seine Aufgabe ist die Umsetzung des Sollwertes für die Ausgangsspannung (in zeitkontinuierlicher Form, d.h. durch ihre Grundschwingung vorgegeben) in die Schaltfunktionen für die einzelnen Schaltelemente.

2. Bei einphasigen Schaltungen ist eine Sollwertspannung, bei dreiphasigen Schaltungen sind zwei bzw. 3 Sollwertspannungen zu verarbeiten.

3. Der kurzzeitige Mittelwert der Ausgangsspannung des Stromrichters soll präzis und mit minimaler Verzögerung dem Sollwert nachgeführt werden. Der Modulator soll die Regeldynamik des gesamten Systems möglichst wenig einschränken.

4. Sind die Sollwerte durch Zeitfunktionen oder durch Drehzeiger beschrieben, so entspricht der Modulator im mathematischen Modell des Regelkreises einem gewöhnlichen Proportionalglied.

5. Erfolgt die Vorgabe mittels Drehzeiger, so wirkt der Modulator im Regelkreis als Rücktransformator auf Phasengrössen.

6. Erfolgt die Vorgabe mittels Amplitude und Phase bzw. mittels Festzeiger, so wirkt der Modulator im Regelkreis als Rücktransformator auf Zeitfunktionen. Dazu muss ihm zusätzlich ein zeitabhängiger Referenzwinkel vorgegeben werden.

7. Der Modulator beeinflusst massgeblich die Art und Grösse der Verzerrungen in den Ausgangsgrössen. Eine optimale Modulation ergibt bei einer gegebenen Schaltfrequenz minimale Verzerrungen.

Literatur zu Kapitel 4: [Bro1], [Büh2], [Fö11], [Heu1], [Kle1], [Leo1], [Mey2], [Spä1] 


\section{Teil II}

\section{Verfahren zur Pulserzeugung am Beispiel des zweistufigen Stromrichters für Spannungszwischenkreis}

\section{Beschreibung von Stromrichtern und Pulsmustern}

In diesem Kapitel werden die Werkzeuge bereitgestellt, die anschliessend zur Beschreibung der verschiedenen Steuerverfahren benötigt werden. Es handelt sich dabei um Kennwerte von Pulsmustern sowie von Spannungen und Strömen am Stromrichter. Zusätzlich werden wichtige grundsätzliche Eigenschaften dieser Grössen diskutiert.

\subsection{Modelle}

Ausgangspunkt für die Behandlung der Steuerverfahren sind die in Bild 5.1 dargestellten idealisierten Ersatzschaltungen der zweistufigen Stromrichter und ihrer Umgebung. Die Stromrichter sind dabei mit Umschaltern modelliert. Sie werden von einem ideal geglätteten Zwischenkreis gespeist und die Last entspricht der in Kapitel 3.2.4 definierten Standardlast.

Die idealisierten Modelle sind für die Beschreibung und den Vergleich von Steuerverfahren besonders geeignet, weil sich bei gegebenen Schaltfunktionen des Stromrichters alle Spannungen und Ströme auf einfache Weise berechnen lassen. Am Beispiel der Halbbrücke soll dies kurz veranschaulicht werden:

1. Gegeben ist die Schaltfunktion $s_{A}$.

2. Die Phasenspannung ist durch die Transformationsgleichung des Stromrichters bestimmt:

$$
u_{A 0}=s_{A} \frac{U_{d}}{2}
$$

Die Charakteristik von Schaltfunktionen und der entstehenden Spannungen und Ströme lässt sich für stationäre Betrachtungen besonders gut mit Fourierreihen beschreiben 




Bild 5.1.

Vereinfachte Modelle der zweistufigen U-Stromrichter, oben links: Halbbrücke, oben rechts: einphasige Brücke unten: dreiphasige Brücke

(Anhang B.2). Ist die Fourierreihe (5.2) der (DC-freien) Schaltfunktion $s_{A}$ bekannt, so kann (5.1) gemäss (5.3) auf jede einzelne Frequenzkomponente angewendet werden:

$$
\begin{aligned}
& s_{A}(t)= \sum_{v=1}^{\infty}\left[a_{s, v} \cos \left(v \omega_{1} t\right)+b_{s, v} \sin \left(v \omega_{1} t\right)\right] \\
& \Rightarrow \quad a_{u, v}=a_{s, v} \frac{U_{d}}{2}, \quad b_{u, v}=b_{s, v} \frac{U_{d}}{2}, \hat{u}_{A 0, v}=\sqrt{a_{u, v}^{2}+b_{u, v}^{2}}
\end{aligned}
$$

3. Für die gegebene Last lässt sich anschliessend mit der Differentialgleichung (5.4) der Phasenstrom bestimmen.

$$
i_{A}(t)=\frac{1}{L_{k}} \int\left(u_{A 0}-e\right) d t
$$

Auch die Berechnung des Phasenstromes kann mit den Fourierkoeffizienten durchgeführt werden. Dabei wird die Gegenspannung $e$ gemäss (5.5) als rein sinusförmig angenommen, so dass sie nur die Grundschwingung beeinflusst. Alle anderen Frequenzkomponenten sehen als Last nur die Induktivität $L_{k}$. Weiter ist zu beachten, dass die Stromkomponenten eine Phasenverschiebung von $\pi / 2$ erhalten, was einem Vertauschen der Sinus- und Cosinuskoeffizienten mit Umkehr des Vorzeichens für die neuen Cosinuskoeffizienten entspricht. Aufgrund dieser Überlegungen resultieren die Beziehungen (5.6) für die Grundschwingung und (5.7) für die übrigen Harmonischen.

$$
\begin{aligned}
& e(t)=\hat{e} \sin \left(\omega_{1} t+\varphi_{e}\right)=a_{e, v=1} \cos \left(\omega_{1} t\right)+b_{e, v=1} \sin \left(\omega_{1} t\right) \\
& a_{i, v=1}=-\frac{1}{\omega_{1} L_{k}}\left(b_{u, v=1}-b_{e, v=1}\right), b_{i, v=1}=\frac{1}{\omega_{1} L_{k}}\left(a_{u, v=1}-a_{e, v=1}\right) \\
& \hat{i}_{A, v=1}=\sqrt{a_{i, v=1}^{2}+b_{i, v=1}^{2}}
\end{aligned}
$$




$$
\begin{aligned}
& a_{i, v}=-\frac{1}{v \omega_{1} L_{k}} b_{u, v}, \quad b_{i, v}=\frac{1}{v \omega_{1} L_{k}} a_{u, v}, \\
& \hat{i}_{A, v}=\sqrt{a_{i, v}^{2}+b_{i, v}^{2}}=\frac{\hat{u}_{A 0, v}}{v \omega_{1} L_{k}}, \quad v>1
\end{aligned}
$$

Damit sind bereits alle Grössen der AC-Seite bestimmt. Im Falle einer komplexeren Last, zum Beispiel einem Ausgangsfilter, können weitere Ströme und Spannungen auf ähnliche Art berechnet werden.

4. Schliesslich ergeben sich die Zwischenkreisströme aus dem Phasenstrom und der Schaltfunktion:

$$
i_{d+}=\frac{1+s_{A}}{2} i_{A}, \quad i_{d-}=\frac{1-s_{A}}{2} i_{A}
$$

Es ist zu beachten: nur weil angenommen wird, dass diese Ströme keine Veränderung der Zwischenkreisspannung bewirken, kann in der beschriebenen Art vorgegangen werden. Ansonsten müsste diese Veränderung in (5.1) berücksichtigt werden, was ein gekoppeltes System von Differentialgleichungen zur Folge hätte.

Die vereinfachte Berechnung der Spannungen und Ströme am Stromrichter ist bei allen idealisierten Schaltungen auf dieselbe Weise möglich.

\subsection{Definitionen und Kennwerte}

\subsubsection{Stationärer Betrieb}

Viele wichtige Kennwerte von Pulsmustern und Stromrichtergrössen beziehen sich auf den stationären Betrieb. Sie stellen eine Grundlage für quantitative Vergleiche von Steuerverfahren dar.

\subsubsection{AC-Seite (Ausgangsseite)}

Die Grössen auf der AC-Seite des Stromrichters lassen sich gemäss dem Beispiel in Bild 5.2 in die Komponenten Grundschwingung und Verzerrungsanteil aufspalten.

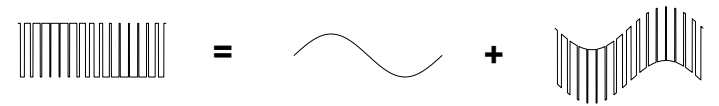

Gesamtsignal $\mathrm{u}_{\mathrm{A} 0}$
Grundschwingung $\mathrm{u}_{\mathrm{A} 0, v=1}$
Verzerrungsanteil $\mathrm{u}_{\mathrm{A} 0, \mathrm{VZ}}$
Bild 5.2.

Aufspaltung einer Grösse auf der ACSeite des Stromrichters in Komponenten (Beispiel Halbbrücke)

Grundschwingung (im Beispiel $u_{A 0, v=1}$ ): Die Grundschwingung ist der eigentliche Nutzanteil der Grösse. Sie entspricht der zeitkontinuierlichen Beschreibung des Stromrichters (Kapitel 3.3) und lässt sich durch die Modulationsfunktion beschreiben: 


$$
u_{A 0, v=1}=m \frac{U_{d}}{2}, \quad m=M \sin \left(\omega_{1} t+\varphi_{m}\right), \quad M=\frac{\hat{u}_{A 0, v=1}}{U_{d} / 2}
$$

Dabei wird $M$ als Modulationsgrad bezeichnet. Er beschreibt das Verhältnis zwischen der Amplitude der Ausgangsspannungs-Grundschwingung und der halben Zwischenkreisspannung. Wie in den folgenden Kapiteln gezeigt wird, lässt sich durch geeignete Methoden bei allen Schaltungen ein maximaler Modulationsgrad von $M_{\max }=4 / \pi$ erreichen. Damit kann die Amplitude der Ausgangsspannungs-Grundschwingung grösser werden als ihr maximaler Momentanwert. In der Literatur kommt teilweise auch der Aussteuerungsgrad $A$ zur Anwendung, der dann im Gegensatz zu $M$ stets im Intervall $[0,1]$ liegt:

$$
A=\frac{\pi}{4} M
$$

Die Frequenz der Grundschwingung heisst Grundfrequenz $f_{1}$ und die entsprechende Periode Grundperiode $T_{1}=1 / f_{1}$. Häufig werden Frequenzen auf $f_{1}$ normiert, so dass die Grundfrequenz den bezogenen Wert eins erhält.

Um eine einheitliche Darstellung der Steuerverfahren zu erhalten, wird in der Folge die Phasenlage der Grundschwingung wo möglich so gewählt, dass sich für die Ausgangsspannung des Stromrichters eine reine Sinusfunktion ergibt. Der Phasenwinkel $\varphi_{m}$ wird dann null.

In speziellen Fällen (z.B. bei einem geschalteten Verstärker) kann anstelle einer Grundschwingung ein beliebiger Kurvenverlauf am Ausgang verlangt sein. Die Modulationsfunktion $m$ ist dann nicht sinusförmig. Anstatt von der Grundschwingung muss dann von einem Nutzsignal gesprochen werden.

Verzerrungsanteil oder Modulationsverzerrung (im Beispiel $u_{A, V Z}$ ): Bei sinusförmiger Modulation umfasst der Verzerrungsanteil alle Frequenzkomponenten neben der Grundschwingung. Sie lassen sich durch verschiedene Kenngrössen beschreiben:

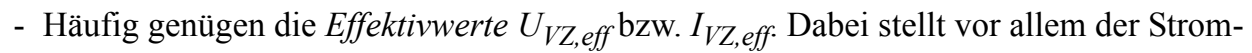
effektivwert ein Mass für die zusätzliche Belastung der Schaltungskomponenten durch die Modulationsverzerrung dar.

- Von Interesse ist auch der Spitzenwert $\hat{\imath}_{V Z}$. Er ist ein Mass für die maximale Abweichung des Phasenstromes von der Grundschwingung.

Der Verlauf und die Kennwerte der Verzerrungsanteile stellen wesentliche Vergleichskriterien für die Steuerverfahren dar. Grundsätzlich sollten sie bei allen Anwendungen möglichst klein sein. In den folgenden Kapiteln wird häufig der Betriebsfall betrachtet, bei dem die Phasenströme nur aus Verzerrungsanteilen bestehen. Die Grundschwingung der Phasenspannung und die Gegenspannung stimmen dort überein, so dass die Grundschwingungen der Ströme null werden. Die Verzerrungsanteile hängen bei vielen Steuerverfahren stark vom eingestellten Modulationsgrad $M$ ab, so dass ihre Kennwerte als Funktion davon darzustellen sind. 
Gesamtsignal (im Beispiel $u_{A 0}$ ): Das Gesamtsignal ist diejenige Grösse, welches für die Dimensionierung des Umrichters und der Last von Bedeutung ist:

- Die Effektivwerte $U_{\text {eff }}$ bzw. $I_{\text {eff }}$ sind massgebend für die Dauerbelastung der einzelnen Schaltungskomponenten.

- Die Scheitelwerte $\hat{u}$ bzw. $\hat{\imath}$ beschreiben die Spitzenbelastungen der Komponenten.

- Der Klirrfaktor $k$ gemäss (5.11) stellt ein Mass für die Abweichung des Gesamtsignals von der Sinusform dar.

$$
k=\frac{U_{V Z, e f f}}{U_{e f f}}=\sqrt{\frac{U_{e f f}^{2}-\hat{u}_{v=1}^{2} / 2}{U_{e f f}^{2}}}
$$

Schaltfrequenz $f_{S}$ und Schaltzahl $q$ : Die Schaltfrequenz $f_{S}$ stellt einen der wichtigsten Parameter eines Steuerverfahrens dar. Sie wird pro Schalter angegeben und ist die Anzahl Schaltzyklen pro Zeiteinheit, bestehend aus je einem Ein- und einem Ausschaltvorgang.

Die Schaltzahl $q$ bringt die Schaltfrequenz in Bezug zur Grundfrequenz. Sie bezeichnet die Anzahl Schaltzyklen pro Grundperiode:

$$
q=\frac{f_{s}}{f_{1}}
$$

Die Schaltzahl bezieht sich wie die Schaltfrequenz auf die einzelnen Schalter. Diese erzeugen je $2 q$ Schaltflanken pro Grundperiode. Da die Schalter in den Stromrichtern zu verschiedenen Zeiten schalten, ergeben sich in den meisten Spannungen und Strömen mehr als $2 q$ Schaltvorgänge (Kommutierungsvorgänge). Die Tabelle 5.2 gibt eine entsprechende Übersicht.

Tabelle 5.1. Anzahl Schaltvorgänge in Abhängigkeit der Schaltzahl $q$ und dem Schaltungstyp

\begin{tabular}{l|c|c|c}
\multicolumn{1}{c|}{ Anzahl Schaltvorgänge in } & Halbbrücke & einphasige Brücke & dreiphasige Brücke \\
\hline Mittelpunktspannung $\mathrm{u}_{\mathrm{A} 0}$ bzw. $\mathrm{u}_{\mathrm{U} 0}$ & $2 \mathrm{q}$ & $2 \mathrm{q}$ & $2 \mathrm{q}$ \\
\hline Phasenspannung $\mathrm{u}_{\mathrm{AB}}$ bzw. $\mathrm{u}_{\mathrm{U}}$ & - & $4 \mathrm{q}$ & $6 \mathrm{q}$ \\
\hline verkettete Spannung $\mathrm{u}_{\mathrm{UV}}$ & - & - & $4 \mathrm{q}$ \\
\hline Ausgangsstrom $\mathrm{i}_{\mathrm{A}}$ bzw. $\mathrm{i}_{\mathrm{U}}$ & $2 \mathrm{q}$ & $4 \mathrm{q}$ & $6 \mathrm{q}$ \\
\hline Zwischenkreistrom $\mathrm{i}_{\mathrm{d}}$ bzw. $\mathrm{i}_{\mathrm{d}+}$ & $2 \mathrm{q}$ & $4 \mathrm{q}$ & $6 \mathrm{q}$
\end{tabular}

Fourierreihe: Besonders anschaulich ist, wie bereits erwähnt, die Beschreibung der Grössen als Fourierreihen. Dies vor allem, weil dadurch die Auftrennung in Grundschwingung und Verzerrungsanteil sehr leicht ersichtlich wird und weil sich auch die frequenzmässige Zusammensetzung des Verzerrungsanteils direkt zeigt. Das Frequenzspektrum wird in der Regel durch Linienspektra für Amplituden und Phasen dargestellt. Bei der Betrachtung von Steuerverfahren interessiert vor allem das Amplitudenspektrum (Beispiel in Bild 5.3). 


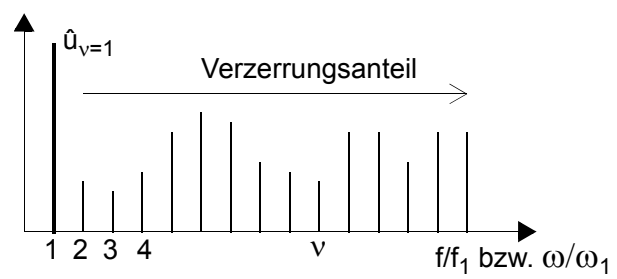

Bild 5.3.

Beispiel für das Amplitudenspektrum einer Spannung

Sind die Fourierkoeffizienten einer Wechselgrösse bekannt, so lassen sich daraus ihre Effektivwerte berechnen:

$$
U_{\text {eff }}=\sqrt{\sum_{v=1}^{\infty} \frac{\hat{u}_{v}^{2}}{2},} \quad U_{V Z, \text { eff }}=\sqrt{\sum_{v=2}^{\infty} \frac{\hat{u}_{v}^{2}}{2}} .
$$

\subsubsection{DC-Seite (Zwischenkreis)}

Auch die DC-seitigen Grössen des Stromrichters lassen sich in Komponenten aufspalten. Dies betrifft vor allem den Zwischenkreisstrom $i_{d}$, aber auch die Spannung $u_{d}$, sobald diese nicht mehr als ideal geglättet angenommen wird. Zur Herleitung der Komponenten wird die Transformationsgleichung für $i_{d+}$ der einphasigen Halbbrücke betrachtet:

$$
i_{d+}=\frac{1+s_{A}}{2} i_{A}
$$

Spaltet man die Schaltfunktion und den Phasenstrom gemäss (5.15) und (5.16) in ihre Grundschwingungen und ihre Verzerrungsanteile auf und setzt diese Gleichungen in (5.14) ein, so erhält man nach geeigneter Umformung (5.17). Bild 5.4 illustriert die Aufspaltung von $i_{d+}$ gemäss (5.17) in seine Komponenten.

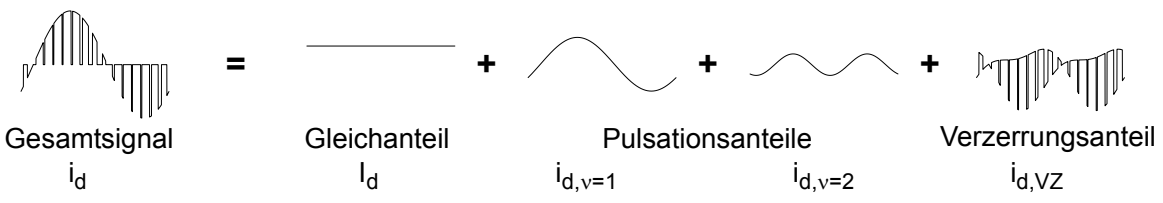

Bild 5.4. Aufspaltung des Zwischenkreisstromes in Komponenten, Beispiel Halbbrücke

$$
\begin{aligned}
& s_{A}(t)=M \sin \left(\omega_{1} t\right)+s_{A, V Z} \\
& i_{A}(t)=\hat{i}_{A, v=1} \sin \left(\omega_{1} t+\varphi_{i}\right)+i_{A, V Z} \\
& i_{d+}=\frac{M \hat{i}_{A, v=1}}{4} \cos \varphi_{i}+\frac{\hat{i}_{A, v}=1}{2} \sin \left(\omega_{1} t+\varphi_{i}\right)-\frac{M \hat{i}_{A, v}=1}{4} \cos \left(2 \omega_{1} t+\varphi_{i}\right)+
\end{aligned}
$$




$$
+\frac{\hat{i}_{A, v=1}}{2} \sin \left(\omega_{1} t+\varphi_{i}\right) s_{A, V Z}+\frac{M}{2} \sin \left(\omega_{1} t\right) i_{A, V Z}+\frac{s_{A, V Z} i_{A, V Z}}{2}+\frac{{ }^{i} A, V Z}{2}
$$

Gleichanteil (im Beispiel $\left.I_{d}\right)$ :

$$
I_{d}=\frac{M \hat{i}_{A, v}=1}{4} \cos \varphi_{i}
$$

Er bestimmt die Wirkleistung, welche durch den Stromrichter übertragen wird, und ist damit der eigentliche Nutzanteil. Sein Betrag hängt vom Modulationsgrad, von der Amplitude des Phasenstromes und vor allem auch von dessen Phasenlage ab. Der Gleichanteil kann auch aus der Wirkleistungsbilanz (5.19) bestimmt werden:

$$
U_{d} I_{d}=\frac{\hat{u}_{A 0, v=1} \hat{i}_{A, v}=1}{2} \cos \varphi_{i}=\frac{M}{4} U_{d} \hat{i}_{A, v}=1 \cos \varphi_{i}
$$

Pulsationsanteile (im Beispiel $i_{d, v=1}, i_{d, v=2}$ ):

$$
i_{d, v=1}=\frac{\hat{i}_{A, v=1}}{2} \sin \left(\omega_{1} t+\varphi_{i}\right), i_{d, v=2}=-\frac{M \hat{i}_{A, v}=1}{4} \cos \left(2 \omega_{1} t+\varphi_{i}\right)
$$

Bei der Halbbrücke pulsiert der Zwischenkreisstrom sowohl mit der einfachen als auch mit der doppelten Grundfrequenz. Der Anteil $i_{d, v=1}$ wird verursacht, weil die beiden Zwischenkreisleiter abwechselnd je während einer halben Periode der Grundschwingung stärker belastet sind. Der Anteil $i_{d, v=2}$ dagegen entsteht, weil die momentane Ausgangsleistung des Stromrichters mit $2 \omega_{1}$ pulsiert. Da der Stromrichter selbst keine Energiespeicher aufweist, erscheint diese Pulsation auch im Zwischenkreis und muss von den Gleichspannungsquellen aufgenommen werden.

Die verschiedenen Schaltungen unterscheiden sich bezüglich Pulsationsanteil wesentlich. Bei der einphasigen Brücke ist der Pulsationsanteil $i_{d, v=2}$ ebenfalls vorhanden, da die Leistung auf der AC-Seite pulsiert. Der Zwischenkreisstrom fliesst aber stets über beide Leiter gleichzeitig, so dass $i_{d, v=1}$ nicht auftritt. Die dreiphasige Brücke schliesslich weist bei symmetrischem Betrieb auf der AC-Seite über alle 3 Phasen gesehen keine Leistungspulsation auf. Deshalb entsteht im Zwischenkreis überhaupt kein Pulsationsanteil. Die Tabelle 5.2 zeigt eine Übersicht der auftretenden Gleich- und Pulsationsanteile in den verschiedenen Schaltungen.

Werden die Stromrichter in zeitkontinuierlicher Form beschrieben, umfasst dies den Gleichanteil und die Pulsationsanteile. Diese werden deshalb nicht dem Verzerrungsanteil zugerechnet.

Verzerrungsanteil (im Beispiel $i_{d, V Z}$ ):

$$
i_{d, V Z}=\frac{\hat{i}_{A, v=1}}{2} \sin \left(\omega_{1} t+\varphi_{i}\right) s_{A, V Z}+\frac{M}{2} \sin \left(\omega_{1} t\right) i_{A, V Z}+\frac{s_{A, V Z} i_{A, V Z}}{2}+\frac{i_{A, V Z}}{2}
$$


Tabelle 5.2. Gleich- und Pulsationsanteile im Zwischenkreisstrom bei symmetrischem Betrieb

\begin{tabular}{c|c|c|c} 
& Halbbrücke $\left(f \ddot{r} \mathrm{i}_{\mathrm{d}+}\right)$ & einphasige Brücke & dreiphasige Brücke \\
\hline $\begin{array}{c}\text { Gleichanteil } \\
\mathrm{I}_{\mathrm{d}}\end{array}$ & $\frac{M \hat{i}_{A, v}=1}{4} \cos \varphi_{i}$ & $\frac{M \hat{i}_{A, v}=1}{2} \cos \varphi_{i}$ & $\frac{3 M \hat{i}_{U, v}=1}{4} \cos \varphi_{i}$ \\
\hline $\begin{array}{c}\text { Pulsationsanteil } \\
\mathrm{i}_{\mathrm{d}, v=1}\end{array}$ & $\frac{\hat{i}_{A, v=1} \sin \left(\omega_{1} t+\varphi_{i}\right)}{2}$ & - & - \\
\hline $\begin{array}{c}\text { Pulsationsanteil } \\
\mathrm{i}_{\mathrm{d}, v=2}\end{array}$ & $-\frac{M \hat{i}_{A, v=1}}{4} \cos \left(2 \omega_{1} t+\varphi_{i}\right)$ & $-\frac{M \hat{i}_{A, v=1}}{2} \cos \left(2 \omega_{1} t+\varphi_{i}\right)$ & -
\end{tabular}

Wie bei den Ausgangsgrössen führen auch im Zwischenkreis die Schaltvorgänge zu einer Verzerrung der idealen Kurvenverläufe. Sie setzt sich aus allen Kreuzprodukten, in denen die Verzerrungsanteile von $s_{A}$ und $i_{A}$ enthalten sind, zusammen. Prinzipiell können dabei auch kleine zusätzliche Gleichanteile und Anteile mit Kreisfrequenz $\omega_{1}$ oder $2 \omega_{1}$ entstehen. Diese werden ihrer Entstehung wegen trotzdem dem Verzerrungsanteil zugerechnet. Als Kennwert wird häufig der Effektivwert $I_{d, V Z \text {,eff }}$ angegeben. Der Verzerrungsanteil ist wie derjenige auf der AC-Seite ein wichtiges Merkmal eines Steuerverfahrens.

Auch der Zwischenkreisstrom wird häufig als Fourierreihe mit der Grundfrequenz $f_{1}$ dargestellt. Bild 5.3 zeigt ein entsprechendes Beispiel. Im Gegensatz zu den Ausgangsgrössen, welche möglichst mittelwertfrei sein sollten, tritt hier natürlich eine DC-Komponente ungleich null auf.

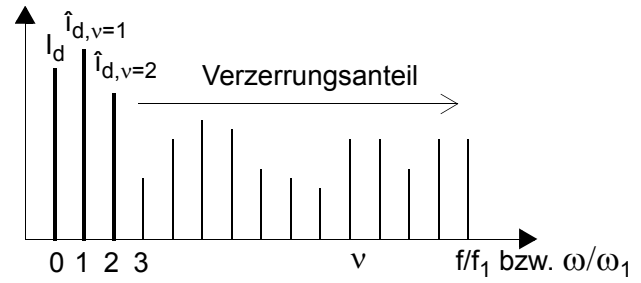

Bild 5.5.

Beispiel eines Amplitudenspektrums des Zwischenkreisstromes der Halbbrücke

Im Falle eines realen Zwischenkreises mit endlich grossem Glättungskondensator nach Bild 5.6 entsteht eine Spannungswelligkeit $u_{d, V Z}$. Sie kann wie folgt berechnet werden:

$$
u_{d, V Z}(t)=\frac{1}{C_{d}} \int_{C} d t, \quad i_{C}=I_{d}-i_{d}
$$

Daraus wird deutlich, dass vor allem die niederfrequenten Anteile von $i_{C}$ eine grosse Welligkeit verursachen. Ist ein Pulsationsanteil vorhanden, so fällt dieser am stärksten ins Gewicht. Der Effektivwert des Stromes $I_{C, \text { eff }}$ ist ein Mass für die Belastung des Kondensators. 


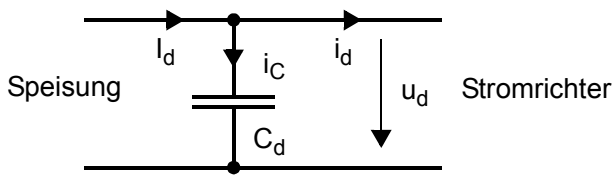

Bild 5.6. Realer Zwischenkreis

\subsubsection{Bezugsgrössen}

Es ist allgemein üblich, Spannungen und Ströme auf schaltungsspezifische Parameter zu beziehen. Auf diese Weise wird der Vergleich zwischen Schaltungen mit verschiedener Dimensionierung möglich. Die Tabelle 5.3 gibt einen Überblick über die gewählten Bezugsgrössen. Die Bezugsspannung $U_{B}$ ist jeweils so gewählt, dass sich für alle Schaltungen eine einheitliche Definition des Modulationsgrades ergibt. Für den Bezugswert der Ströme $I_{B}$ ist $U_{B}$ durch die Impedanz $\omega_{1} L_{k}$ zu dividieren. Physikalisch gesehen ist dies die Amplitude der Stromgrundschwingung für $M=1$ bei Gegenspannung null.

\begin{tabular}{|c|c|c|c|c|}
\hline & Halbbrücke & einphasige Brücke & dreiphasige Brücke & \multirow{5}{*}{$\begin{array}{l}\text { Tabelle 5.3. } \\
\text { Bezugsgrössen } \\
\text { und Modulati- } \\
\text { onsgrad in ver- } \\
\text { schiedenen } \\
\text { Schaltungen }\end{array}$} \\
\hline $\begin{array}{l}\text { Bezugsspan- } \\
\text { nung } U_{B}\end{array}$ & $\frac{U_{d}}{2}$ & $U_{d}$ & $\frac{U_{d}}{2}$ & \\
\hline $\begin{array}{c}\text { Bezugsstrom } \\
\mathrm{I}_{\mathrm{B}}\end{array}$ & $\frac{U_{B}}{\omega_{1} L_{k}}=\frac{U_{d}}{2 \omega_{1} L_{k}}$ & $\frac{U_{B}}{\omega_{1} L_{k}}=\frac{U_{d}}{\omega_{1} L_{k}}$ & $\frac{U_{B}}{\omega_{1} L_{k}}=\frac{U_{d}}{2 \omega_{1} L_{k}}$ & \\
\hline $\begin{array}{c}\text { Bezugsreaktanz } \\
\mathrm{X}_{\mathrm{B}}\end{array}$ & $\frac{U_{B}}{\hat{i}_{A, n}}=\frac{U_{d}}{2 \hat{i}_{A, n}}$ & $\frac{U_{B}}{\hat{i}_{A, n}}=\frac{U_{d}}{\hat{i}_{A, n}}$ & $\frac{U_{B}}{\hat{i}_{U, n}}=\frac{U_{d}}{2 \hat{i}_{U, n}}$ & \\
\hline $\begin{array}{l}\text { Modulations- } \\
\operatorname{grad} \mathrm{M}\end{array}$ & $\frac{\hat{u}_{A 0, v=1}}{U_{B}}$ & $\frac{\hat{u}_{A 0, v=1}}{U_{B}}$ & $\frac{\hat{u}_{U 0, v=1}}{U_{B}}=\frac{\hat{u}_{U, v=1}}{U_{B}}$ & \\
\hline
\end{tabular}

In der Praxis existiert stets ein Nennstrom $\hat{\imath}_{A, n}$ bzw. $\hat{\imath}_{U, n}$, auf den die einzelnen Komponenten dimensioniert sind. Mit seiner Hilfe lässt sich die Bezugsreaktanz $X_{B}$ definieren. In den folgenden Kapiteln werden die Verzerrungsströme stets auf $I_{B}$ bezogen. Sie liegen damit in einer von der Schaltung und der Grundfrequenz unabhängigen Form vor. Bei graphischen Darstellungen von Strömen ist es dagegen teilweise notwendig, für $\omega_{1} L_{k}$ einen konkreten Wert anzunehmen. In diesen Fällen wird jeweils $\omega_{1} L_{k}=0.2 X_{B}$ gewählt, was einem Verhältnis von $I_{B}$ zu $\hat{\imath}_{A, n}$ von 5 entspricht.

\subsubsection{Drehmomentwelligkeit bei Drehstrommaschinen}

Bei stromrichtergespeisten Antrieben ist die Drehmomentwelligkeit, verursacht durch die nichtsinusförmigen Ströme von grosser Bedeutung. Sie gilt als weiterer Kennwert eines Steuerverfahrens für dreiphasige Stromrichter. Dabei interessiert in den meisten Fällen vor allem der auftretende Spitzenwert, d.h. die maximale Abweichung vom eingestellten mittleren Drehmoment. 
In Kapitel 3.2.2 wurde gezeigt, dass die mit Drehzeigern formulierte Gleichung (5.23) die Berechnung des Drehmoments in der Maschine mit Hilfe der Parameter ihrer Ersatzschaltung erlaubt. Bei einer stromrichtergespeisten Maschine gilt damit:

$$
M_{e l}(t)=\frac{3}{2} \frac{1}{\omega_{S}} \operatorname{Re}\left(e_{-}^{*} i_{S}\right)=\frac{3}{2} \frac{1}{\omega_{1}} \operatorname{Re}\left(e_{-}^{*} i_{S t r}\right)=\frac{3}{2} \frac{1}{\omega_{1}}\left(e_{\alpha} i_{S t r, \alpha}+e_{\beta} i_{S t r, \beta}\right)
$$

Die Drehmomentwelligkeit $M_{e l, V Z}$ lässt sich als Differenz des mittleren Momentes von dessen Momentanwert definieren:

$$
M_{e l, V Z}=M_{e l}-\bar{M}_{e l}=\frac{3}{2} \frac{1}{\omega_{1}} \operatorname{Re}\left(\underline{e}_{-}^{*} i_{S t r, V Z}\right)=\frac{3}{2} \frac{1}{\omega_{1}}\left(e_{\alpha} i_{S t r, V Z, \alpha}+e_{\beta} i_{S t r, V Z, \beta}\right)
$$

Geht man von gegebener Gegenspannung $\underline{e}$ und Grundkreisfrequenz $\omega_{1}$ aus, so hängt die Drehmomentwelligkeit einerseits vom Verlauf des Verzerrungsstromes und andererseits von dessen Phasenlage gegenüber $\underline{e}$ ab. Diese Phasenlage ist abhängig vom Arbeitspunkt der Maschine, vor allem vom eingestellten mittleren Drehmoment. Der Grund dafür liegt darin, dass der momentbildende Grundschwingungsstrom (d.h. der Wirkstrom, Kapitel 4.2) in erster Linie über die Phasenverschiebung zwischen der Stromrichterspannung und der Gegenspannung eingestellt wird.

Soll der Einfluss verschiedener Steuerverfahren auf die Drehmomentwelligkeit miteinander verglichen werden, so muss dies bei genau definierten Betriebsbedingungen geschehen. Speziell geeignet dazu ist der Leerlauf: die Stromgrundschwingung besteht dort nur aus dem allfälligen Magnetisierungsstrom (bei einer ASM), der einen sehr kleinen Spannungsabfall über $L_{k}$ verursacht. Näherungsweise gilt die Beziehung:

$$
\bar{M}_{e l}=0 \Rightarrow \quad \underline{e}=\underline{u}_{S t r, v=1}, \quad M_{e l, V Z}=\frac{3}{2} \frac{1}{\omega_{1}} \operatorname{Re}\left(\underline{u}_{-S t r, v}=1{ }^{*} \underline{-}_{S t r, V Z}\right)
$$

Bei den Betrachtungen in den folgenden Kapiteln wird zudem vorausgesetzt, dass die Maschine stets mit einem konstanten Fluss (Nennfluss) betrieben wird. Die Amplitude $|\underline{e}|$ ist dann proportional zu $\omega_{1}$. Dadurch ergibt sich im Leerlauf, wie in Bild 5.7 dargestellt, auch eine Proportionalität zwischen $\left|\underline{u}_{S t r, v=1}\right|$ bzw. dem Modulationsgrad $M$ und $\omega_{1}$. Die Kreisfrequenz, die sich beim Modulationsgrad $M=1$ einstellt, wird dabei als Bezugskreisfrequenz $\omega_{B}$ gewählt.

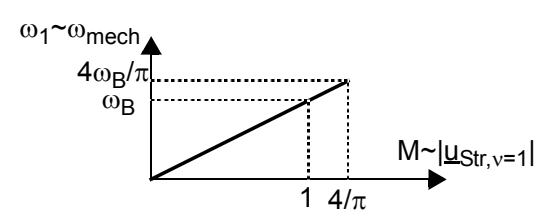

Bild 5.7.

Leerlaufkennlinie für Drehstrommaschinen

Damit kann gesagt werden, dass die Drehmomentwelligkeit im Leerlauf in der voll magnetisierten Maschine eine Funktion des am Stromrichter eingestellten Modulationsgrades $M$ und dem dabei verursachten Verzerrungsstrom darstellt. Sowohl die Gegenspannung $\underline{e}$ als auch die Grundkreisfrequenz $\omega_{1}$ sind durch $M$ bestimmt. 
Die Bezugsgrösse für die Drehmomentwelligkeit $M_{B}$ wird wie folgt gewählt:

$$
M_{B}=\frac{\left.U_{B} I_{B}\right|_{\omega_{B}}}{\omega_{B}}=\frac{U_{B}^{2}}{\omega_{B}^{2} L_{k}}
$$

\subsubsection{Dynamische Betrachtungen}

Auch wenn die stationären Eigenschaften eines Steuerverfahrens bei der Beschreibung im Vordergrund stehen, interessieren auch Fragen, welche dynamische Vorgänge verschiedener Art betreffen:

- Wie schnell und in welchem Bereich können die Parameter der Grundschwingung, das heisst $M, f_{1}$ sowie die Winkellage $\varphi_{m}$ der Grundschwingung variiert werden?

- Entstehen durch die Veränderung dieser Parameter vorübergehende zusätzliche Verzerrungen, zum Beispiel Gleichanteile in den Phasenströmen des Stromrichters?

- Wie verhält sich der Stromrichter, wenn neben der Grundschwingung auch Parameter des Steuerverfahrens, zum Beispiel die Schaltfrequenz, ändern?

Die aufgeworfenen Fragen lassen sich in der Regel nicht in geschlossener Form mathematisch behandeln, sondern müssen anhand von Simulationen und Prinzipbeschreibungen untersucht werden. Die verschiedenen Steuerverfahren zeigen in bezug auf das dynamische Verhalten jedoch erhebliche Unterschiede. Am besten lassen sich die Vorgänge anhand von Schrittantworten untersuchen. Dabei wird dem Modulator z.B. eine schrittartige Änderung des Modulationsgrades vorgegeben und sein Verhalten darauf beobachtet.

\subsubsection{On-Line- und Off-Line-Modulation}

Die Art und Weise, wie die Schaltsignale aus den gegebenen Sollwerten erzeugt werden, stellt die Eigenheit jedes Steuerverfahrens dar. Dabei können zwei prinzipiell verschiedene Varianten unterschieden werden:

On-Line: Mit Hilfe der Sollwerte und allfälliger Hilfssignale werden die Schaltsignale fortlaufend generiert. Dies kann mit einer analogen oder digitalen Schaltung, oder auch mit einem Rechner erfolgen. Die Komplexität des Algorithmus ist wegen der Echtzeitimplementation begrenzt. Dagegen kann grundsätzlich schnell und einfach auf sich verändernde Sollwerte reagiert werden bzw. der Modulator kann nahezu beliebigen Sollwertverläufen folgen. Die Eingangssignale von On-Line-Modulatoren sind Zeitfunktionen, d.h. Phasengrössen oder Drehzeiger.

Off-Line: Die Abfolge der Schaltsignale ist vorausberechnet und in einer Tabelle abgelegt. Eine digitale Schaltung oder ein Rechner liest die Tabelle fortlaufend aus und gibt die Steuersignale an den Stromrichter weiter. Die Pulsmuster können dabei nach einem beliebig komplexen Algorithmus bestimmt worden sein. Für Sollwertvariationen können entweder verschiedene Tabellen bereitgestellt werden oder der Rechner rechnet die abge- 
speicherten Daten entsprechend um. In beiden Fällen können sich Probleme mit der Dynamik ergeben: grundsätzlich sind nur stationäre Betriebsfälle definiert. Dynamische Übergänge erfolgen prinzipiell durch direktes Wechseln zwischen stationär definierten Pulsmustern. Die Eingangssignale von Off-Line-Modulatoren sind Amplituden- und Phasensollwerte bzw. Festzeiger.

\subsection{Symmetrien}

\subsubsection{Synchrone Steuerverfahren}

Ein Steuerverfahren heisst synchron, falls jede Grundperiode seiner Schaltfunktionen und damit jede Grundperiode der Ausgangsspannungen am Stromrichter identisch aussieht.

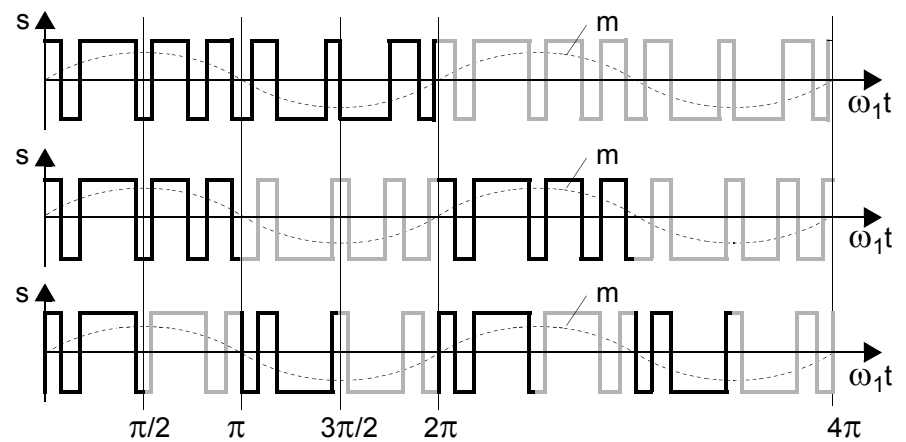

Bild 5.8.

Beispiele für eine synchrone Schaltfunktionen, $q=7$,

oben: ohne Symmetrie innerhalb der Periode, mitte: halbperiodensymmetrisch, unten: viertelperiodensymmetrisch

In Bild 5.8 sind Beispiele synchroner Schaltfunktionen dargestellt. Bei synchronen Verfahren ist es sinnvoll, die Zeitachse durch eine Winkelachse $\omega_{1} t$ zu ersetzen, so dass eine Grundperiode auf $2 \pi$ abgebildet wird. Es gelten die folgenden Aussagen:

- Die Schaltfrequenz $f_{s}$ ist ein ganzzahliges Vielfaches der Grundfrequenz $f_{1}$.

- Die Schaltzahl $q$ ist ganzzahlig.

- Die Schaltfunktionen sowie die Spannungen und Ströme auf der Ausgangsseite des Stromrichters weisen ein Frequenzspektrum von der Art auf, wie es in Bild 5.3 dargestellt wurde. Es treten die Grundschwingung und ihre ganzzahligen Oberschwingungen auf. Ein Gleichanteil wäre prinzipiell möglich, wird jedoch in der Regel vermieden. Im allgemeinen Fall, wenn wie in Bild 5.8 oben keine Symmetrie innerhalb der Periode vorliegt, haben die Gleichungen für die Fourierkoeffizienten die gewohnte Form. Für die Schaltfunktionen gilt:

$$
a_{s, 0}=\frac{1}{2 \pi} \int_{0}^{2 \pi} s\left(\omega_{1} t\right) d\left(\omega_{1} t\right), \quad a_{s, v}=\frac{1}{\pi} \int_{0}^{2 \pi}\left[s\left(\omega_{1} t\right) \cos \left(v \omega_{1} t\right)\right] d\left(\omega_{1} t\right),
$$




$$
b_{s, v}=\frac{1}{\pi} \int_{0}^{2 \pi}\left[s\left(\omega_{1} t\right) \sin \left(v \omega_{1} t\right)\right] d\left(\omega_{1} t\right) \quad v=1,2,3 \ldots
$$

\subsubsection{Symmetrien innerhalb der Periode}

Synchrone Grössen können zusätzlich innerhalb der Periode Symmetrien aufweisen. Diese vereinfachen die Berechnung der Fourierreihe.

Halbperiodensymmetrie: Die Schaltfunktion in Bild 5.8 mitte setzt sich aus zwei identischen Halbperioden zusammen, wobei die zweite Halbperiode gegenüber der ersten invertiert ist. Sie ist deshalb halbperiodensymmetrisch. Die Fourierkoeffizienten können hier durch eine Integration über eine Halbperiode berechnet werden:

$$
\begin{aligned}
a_{s, v} & =\frac{2}{\pi} \int_{0}^{\pi}\left[s\left(\omega_{1} t\right) \cos \left(v \omega_{1} t\right)\right] d\left(\omega_{1} t\right), \\
b_{s, v} & =\frac{2}{\pi} \int_{0}^{\pi}\left[s\left(\omega_{1} t\right) \sin \left(v \omega_{1} t\right)\right] d\left(\omega_{1} t\right), \quad v=1,3,5, \ldots
\end{aligned}
$$

Es kann gezeigt werden, dass in diesem Fall nur noch ungerade Harmonische auftreten. Dementsprechend ist auch kein Gleichanteil vorhanden.

In der Schaltfunktion eines Brückenzweiges lässt sich Halbperiodensymmetrie nur mit ungerader Schaltzahl $q$ erreichen.

Viertelperiodensymmetrie: Eine zusätzliche Axialsymmetrie innerhalb der Halbperiode mit den Symmetrieachsen bei $\pi / 2$ und bei $3 \pi / 2$, wie in Bild 5.8 unten, reduziert das Integrationsintervall für die Fourierkoeffizienten auf eine Viertelperiode:

$$
a_{s, v}=0, \quad b_{s, v}=\frac{4}{\pi} \int_{0}^{\pi / 2}\left[s\left(\omega_{1} t\right) \sin \left(v \omega_{1} t\right)\right] d\left(\omega_{1} t\right), \quad v=1,3,5, \ldots
$$

Alle Teilschwingungen weisen hier entweder dieselbe Phasenlage wie die Grundschwingung auf oder verlaufen gegenphasig zu ihr. In den gezeigten Beispielen, in denen die Grundschwingung eine reine Sinusfunktion ist, werden alle Cosinuskoeffizienten $a_{v}$ null.

Die Schaltfunktionen von Brückenzweigen können nur viertelperiodensymmetrisch sein, wenn ihre Schaltzahl $q$ ungerade ist und wenn sie je eine Schaltflanke bei $\omega_{1} t=0$ und $\omega_{1} t=\pi$ aufweisen.

Zeigen alle Schaltfunktionen eines dreiphasigen Stromrichters dieselbe Symmetrie, so setzt sich diese in den Ausgangsspannungen des Stromrichters fort. Bei der einphasigen Brücke ist dies dagegen nicht der Fall: die Phasenspannung $u_{A B}$ kann, wie das Beispiel in Bild 5.9 zeigt, stets auf verschiedene Arten gebildet werden. Sie kann dabei halb- oder viertelperiodensymmetrisch sein, ohne dass die Schaltfunktionen der beiden Brücken- 
zweige selbst eine Symmetrie innerhalb der Periode aufweisen. Zudem lassen sich alle Symmetrien auch mit geradzahligen Schaltzahlen $q$ erreichen. Ähnliche Phänomene wer-

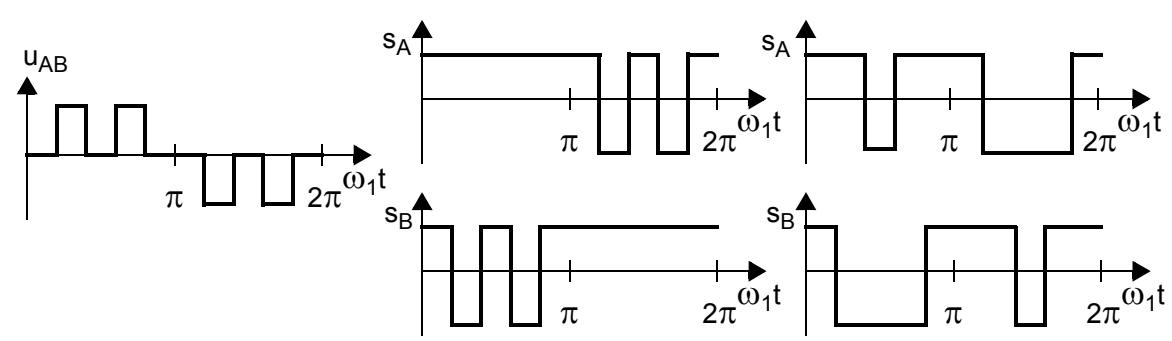

Bild 5.9. Beispiel einer viertelperiodensymmetrischen Phasenspannung bei der einphasigen Brücke, $q=2$, links: Phasenspannung, rechts: Schaltfunktionen der Brückenzweige (zwei verschiedene Varianten)

den dann auch bei der Zusammenschaltung von mehreren Stromrichtern (Kapitel 15) und bei den dreistufigen Stromrichtern (Kapitel 16) in Erscheinung treten.

Im Falle der vorgegeben Last ohne ohmschen Anteil bleibt die Symmetrie der Ausgangsspannungen auch in den Phasenströmen vollständig erhalten. Ist dagegen ein ohmscher Anteil vorhanden, so ergibt sich eine unterschiedliche Phasenverschiebung für die einzelnen Frequenzkomponenten der Phasenströme. Eine allfällige Viertelperiodensymmetrie geht damit verloren. Die Halbperiodensymmetrie bleibt dagegen erhalten.

Die Erfahrung zeigt, dass die Nachbildung eines sinusförmigen Sollwertes mit minimalem Klirrfaktor für eine gegebene Schaltzahl stets viertelperiodensymmetrische Kurvenverläufe ergibt, sofern Viertelperiodensymmetrie überhaupt möglich ist.

Drehmomentwelligkeit bei Viertelperiodensymmetrie: Wird eine ASM mit 3 gleichen, viertelperiodensymmetrischen Spannungen gespeist, so lassen sich die Harmonischen der Drehmomentwelligkeit im Leerlauf leicht aus dem Spektrum der Spannungen ableiten. Dieses ist für alle Phasen abgesehen von den Phasenverschiebungen identisch und besteht aus ungeraden Harmonischen mit Ausnahme aller Vielfachen der dritten (Anhang B.1.1). Zur Herleitung wird vorerst in (5.25) der Verzerrungsstrom als Summe seiner Harmonischen ausgedrückt:

$$
M_{e l, V Z}=\frac{3}{2} \frac{1}{\omega_{1}} \operatorname{Re}\left(\underline{u}_{S t r, v=1}{ }^{*} \sum_{v=5,7,11,13, \ldots}^{\infty} i_{\text {Str }, v}\right)
$$

Wie bereits in (5.7) ersichtlich ist, können aus den Frequenzkomponenten der Phasenspannungen die entsprechenden Phasenstromkomponenten berechnet werden. Unter Annahme einer rein induktiven Lastimpedanz sehen die Zeitfunktionen der Oberschwingungen in der Phase $U$ wie folgt aus:

$$
u_{U, v}=\hat{u}_{v} \sin \left(v \omega_{1} t\right), \quad v=5,7,11,13, \ldots
$$




$$
\Rightarrow \quad i_{U, v>1}=-\hat{i}_{v} \cos \left(v \omega_{1} t\right)=-\frac{\hat{u}_{v}}{v \omega_{1} L_{k}} \cos \left(v \omega_{1} t\right)
$$

Diese Stromkomponenten lassen sich zusammen mit denjenigen der anderen Phasen in die einzelnen Zeiger $\underline{\underline{i}}_{S t r, v}$ transformieren und in (5.30) einsetzen. Dasselbe gilt für die Spannungsgrundschwingungen. Nach geeigneter Umformung ergibt sich auf diese Weise:

$$
\begin{aligned}
& M_{e l, V Z}=-\frac{3}{2} \frac{\hat{u}_{v=1}}{\omega_{1}} \sum_{v=5,7,11,13, \ldots}^{\infty} K_{v} \hat{i}_{v} \sin \left[\left(v+K_{v}\right) \omega_{1} t\right] \\
& \text { mit } \quad K_{v}=\left\{\begin{array}{cc}
1 & \text { für } v=5,11,17, \ldots \\
-1 & \text { für } v=7,13,19, \ldots
\end{array}\right.
\end{aligned}
$$

Diese Gleichung kann umgeformt werden zu:

$$
\begin{gathered}
M_{e l, V Z}=-\frac{3}{2} \frac{\hat{u}_{v=1}}{\omega_{1}} \sum_{\substack{v=6,12,18, \ldots \\
\infty}}^{\infty}\left(\hat{i}_{v-1}-\hat{i}_{v+1}\right) \sin \left(v \omega_{1} t\right) \\
=-\frac{3}{2} \frac{\hat{u}_{v=1}}{\omega_{1}^{2} L_{k}} \sum_{\substack{k \\
v}, 12, \ldots}\left(\frac{\hat{u}_{v-1}}{v-1}-\frac{\hat{u}_{v+1}}{v+1}\right) \sin \left(v \omega_{1} t\right)
\end{gathered}
$$

In der Drehmomentwelligkeit treten nur die Vielfachen der 6. Harmonischen auf. Jede wird dabei durch die beiden benachbarten Harmonischen in den Phasenströmen gebildet. Obschon (5.33) nur für den Leerlauf gilt, ist diese Aussage auch für beliebige Arbeitspunkte richtig.

\subsubsection{Asynchrone Steuerverfahren}

Nicht jedes Steuerverfahren erzeugt synchrone Schaltfunktionen und Ausgangsspannungen. Bild 5.10 zeigt einen sogenannt asynchronen Verlauf. Auch hier lässt sich eine Aufteilung in eine Grundschwingung und einen Verzerrungsanteil vornehmen. Dabei unterscheidet sich die Grundschwingung gegenüber einem synchronen Verlauf nicht. Es ist allein der Verzerrungsanteil, welcher aperiodisch ist. In diesem Fall besteht das Frequenzspektrum nicht mehr nur aus den ganzzahligen Harmonischen der Grundschwingung. $\mathrm{Zu}$ seiner näherungsweisen Beschreibung kann eine Fourierreihe für eine Sequenz von mehreren Grundperioden gebildet werden. Es entsteht ein verdichtetes Linienspektrum (Anhang B.2). Dabei treten dann vor allem auch Zwischen- (Inter-) und Subharmonische auf. Wie im synchronen Fall kann zur Berechnung von Spannungen und Strömen das Überlagerungsprinzip für die vorhandenen Frequenzkomponenten angewandt werden. Auch die Effektivwerte lassen sich analog zu (5.13) aus den einzelnen Frequenzkomponenten berechnen.

Typische asynchrone Steuerverfahren sind einerseits alle rückgekoppelten Verfahren (Kapitel 10, 11 und 12) und andererseits die asynchronen Trägerverfahren (Kapitel 7) und Drehzeigermodulationen (Kapitel 8). 


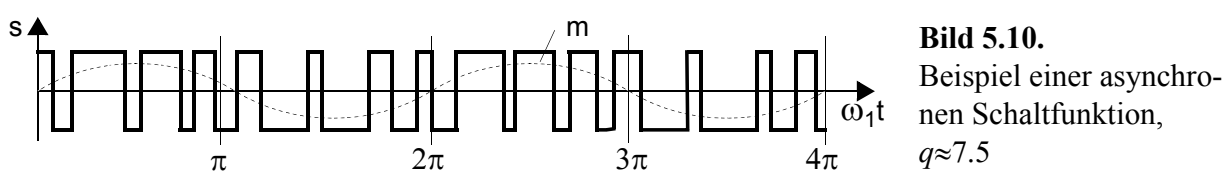




\section{Grundfrequenzsteuerung}

Die einfachsten Steuerverfahren für selbstgeführte Stromrichter sind Grundfrequenzsteuerungen. Diese ergeben Schaltfunktionen, die pro Grundperiode nur zwei Schaltvorgänge aufweisen. Die Schaltfrequenz entspricht der eingestellten Grundfrequenz und ist damit die kleinstmögliche. Grundfrequenzsteuerungen sind synchrone Verfahren, d.h. im stationären Betrieb sind die Spannungsverläufe in jeder Periode identisch. Sie ermöglichen bei allen Schaltungen den maximalen Modulationsgrad $M=4 / \pi$ und damit die beste Ausnutzung der Zwischenkreisspannung.

\subsection{Halbbrücke}

Bild 6.1 zeigt eine Halbbrücke mit der für die Grundfrequenzsteuerung typischen Mittelpunktspannung $u_{A 0}$. Sind die beiden Zeitintervalle $T_{+}$und $T_{-}$ungleich gross, so weist $u_{A 0}$ eine DC-Komponente auf. Dieser Betrieb würde einem Gleichspannungssteller (DC-DCWandler) entsprechen. Beim Einsatz der Schaltung als Wechselrichter soll $u_{A 0}$ eine Wechselspannung der Frequenz $f_{1}=1 / T_{1}$ nachbilden und möglichst keine DC-Komponente enthalten. Aus diesem Grund ist $T_{+}=T_{-}$zu wählen. Die Amplitude der Ausgangsspannung kann dabei nicht variiert werden.
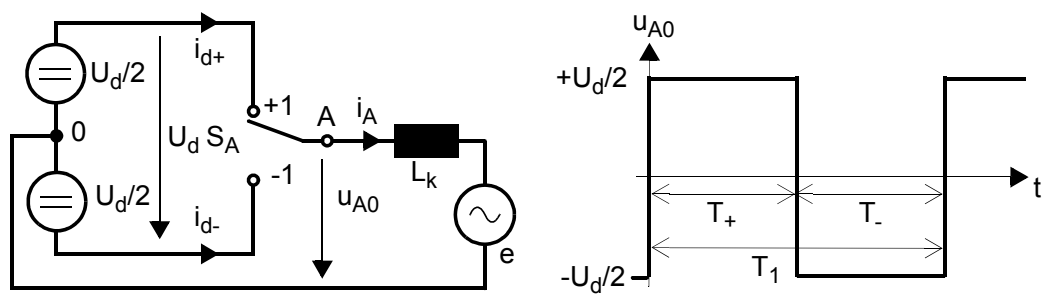

Bild 6.1. Links: Halbbrücke, rechts: Phasenspannung bei Grundfrequenzsteuerung

Phasenspannung: Die Fourierreihe, die Spannungsgrundschwingung, der Modulationsgrad, der Spannungseffektivwert und der Spannungsklirrfaktor für die in Bild 6.1 rechts dargestellte Mittelpunktspannung sind wie folgt gegeben:

$$
u_{A 0}=\frac{4}{\pi} \frac{U_{d}}{2}\left[\sin \left(\omega_{1} t\right)+\frac{1}{3} \sin \left(3 \omega_{1} t\right)+\frac{1}{5} \sin \left(5 \omega_{1} t\right) \ldots\right]
$$




$$
\begin{aligned}
& u_{A 0}=\frac{4}{\pi} \frac{U_{d}}{2}\left(\sum_{v=1,3,5 \ldots}^{\infty} \frac{1}{v} \sin \left(v \omega_{1} t\right)\right) \\
& \hat{u}_{A 0, v=1}=\frac{4}{\pi} \frac{U_{d}}{2}, \quad U_{A 0, v=1, \text { eff }}=\frac{1}{\sqrt{2}} \frac{4}{\pi} \frac{U_{d}}{2}, \quad M=\frac{4}{\pi} \\
& U_{A 0, \text { eff }}=\frac{U_{d}}{2}, \quad k_{u}=\frac{\sqrt{U_{A 0, \text { eff }}^{2}-U_{A 0, v}^{2}=1, \text { eff }}}{U_{A 0, \text { eff }}}=\sqrt{1-\frac{8}{\pi^{2}}}=0.435
\end{aligned}
$$

Verzerrungsstrom: Die Berechnung des Verzerrungsstromes kann von der nachfolgend beschriebenen einphasigen Brücke übernommen werden, wenn in (6.14) die Spannung $U_{d}$ durch $U_{d} / 2$ ersetzt und der Steuerwinkel $\alpha=0$ gewählt wird:

$$
I_{A, V Z, \text { eff }}=\frac{U_{d} / 2}{\omega_{1} L_{k}} \sqrt{-\frac{8}{\pi^{2}}+\frac{\pi^{2}}{12}}=0.109 \frac{U_{d} / 2}{\omega_{1} L_{k}}
$$

\subsection{Einphasige Brücke}

Bild 6.2. zeigt die einphasige Brücke, bestehend aus zwei Brückenzweigen, und eine mit Grundfrequenzsteuerung erzeugte Phasenspannung. Wie bei der Halbbrücke soll zuerst der Mittelwert dieser Spannung betrachtet werden. Er lässt sich wie folgt berechnen:
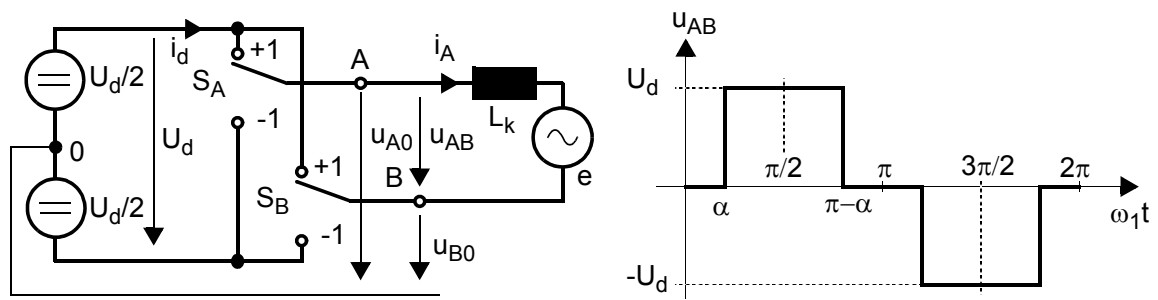

Bild 6.2. Links: Einphasige Brücke mit Grundfrequenzsteuerung, rechts: Phasenspannung

$$
\bar{U}_{A B}=\frac{U_{d}}{2} \frac{1}{T_{1}} \int_{0}^{T_{1}}\left(s_{A}-s_{B}\right) d t=\frac{U_{d}}{2}\left(\frac{1}{T_{1}} \int_{0}^{T_{1}} s_{A} d t-\frac{1}{T_{1}} \int_{0}^{T_{1}} s_{B} d t\right)=\frac{U_{d}}{2}\left(\bar{s}_{A}-\bar{s}_{B}\right)
$$

Bei einem Wechselrichter ist normalerweise verlangt, dass der Mittelwert der Spannung $u_{A B}$ gleich null ist. Dazu müssen die Mittelwerte $\bar{s}_{A}$ und $\bar{s}_{B}$ identisch sein. Ist dies nicht der Fall, so arbeitet die Schaltung als Gleichspannungsteller (Vierquadrantensteller). Es kann weiter gezeigt werden, dass die Nachbildung für einen gegebenen Modulationsgrad 
mit dem kleinsten Klirrfaktor erfolgt, wenn die Phasenspannung zusätzlich viertelperiodensymmetrisch ist (entsprechend dem Beispiel in Bild 6.2 rechts). Im folgenden wird nur dieser Fall betrachtet.

Bei einer viertelperiodensymmetrischen Phasenspannung verbleibt der Steuerwinkel $\alpha$ als freier Parameter. Er kann zur Einstellung des Modulationsgrades verwendet werden. Die Steuerung kann dabei auf zwei verschiedene Arten erfolgen:

1. Mit zwei symmetrischen, rechteckförmigen Schaltfunktionen für die beiden Brückenzweige (Bild 6.3 links), die um $\pi$-2 $\alpha$ gegeneinander phasenverschoben sind. In diesem Fall enthalten die beiden Schaltfunktionen selbst auch keine Gleichkomponenten.

2. Mit zwei Schaltfunktionen mit identischen Gleichkomponenten (Bild 6.3 rechts), die um $\pi$ gegeneinander verschoben sind. Das Zeitintervall $T_{+}$für beide Schaltfunktionen beträgt dabei $\pi-2 \alpha$.
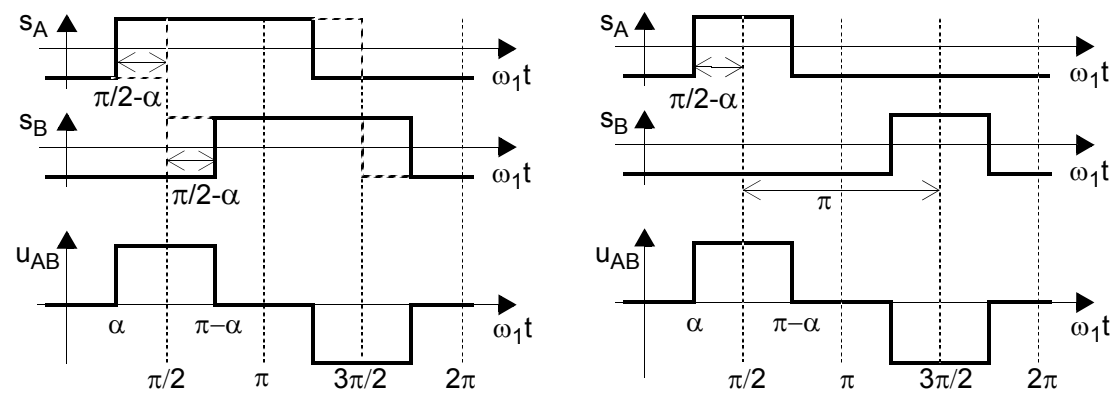

Bild 6.3. Schaltfunktionen und Phasenspannung bei der einphasigen Brücke, links: mit um $\pi / 2-\alpha$ aus der Nullage verschobenen, symmetrischen Schaltfunktionen, rechts: mit unsymmetrischen, um $\pi$ gegeneinander verschobenen Schaltfunktionen

Phasenspannung: Für die Phasenspannung $u_{A B}$ lassen sich die Fourierreihe, die Amplitude der Grundschwingung, der Effektivwert der Grundschwingung, der Gesamteffektivwert und der Klirrfaktor direkt in Funktion des Steuerwinkels $\alpha$ beschreiben:

$$
\begin{gathered}
u_{A B}=\frac{4}{\pi} U_{d}\left[\cos (\alpha) \sin \left(\omega_{1} t\right)+\frac{1}{3} \cos (3 \alpha) \sin \left(3 \omega_{1} t\right)+\frac{1}{5} \cos (5 \alpha) \ldots\right] \\
=\frac{4}{\pi} U_{d} \sum_{v=1,3,5 \ldots}^{\infty} \frac{1}{v} \cos (v \alpha) \sin \left(v \omega_{1} t\right) \\
\hat{u}_{A B, v=1}=\frac{4}{\pi} U_{d} \cos \alpha, \quad U_{A B, v}=1, \text { eff }=\frac{1}{\sqrt{2}} \frac{4}{\pi} U_{d} \cos \alpha, \quad M=\frac{4}{\pi} \cos \alpha \\
U_{A B, \text { eff }}=U_{d} \sqrt{1-2 \alpha / \pi}, \quad k_{u}=\frac{\sqrt{U_{A B, e f f}^{2}-U_{A B, v}^{2}=1, \text { eff }}}{U_{A B, \text { eff }}}=\sqrt{1-\frac{8 \cos ^{2} \alpha}{\pi(\pi-2 \alpha)}}
\end{gathered}
$$


Aus den Formeln ist ersichtlich, dass alle Grössen mit Ausnahme des Klirrfaktors ihre Maxima bei der Vollaussteuerung, d.h. $\alpha=0$ erreichen:

$$
\begin{aligned}
& \hat{u}_{A B, v=1, \text { max }}=\frac{4}{\pi} U_{d}=1.27 U_{d}, \quad M_{\text {max }}=\frac{4}{\pi} \\
& U_{A B, v=1, \text { eff, } \text { max }}=\frac{4}{\pi} \frac{1}{\sqrt{2}} U_{d}=0.90 U_{d}, \quad U_{A B, \text { eff }, \text { max }}=U_{d}
\end{aligned}
$$

Die Verläufe von $U_{A B, v=1, \text { eff, }} U_{A B \text {,eff }}$ und $k_{u}$ in Funktion von $\alpha$ sind in Bild 6.4 dargestellt. Der Klirrfaktor zeigt ein Minimum bei $\alpha=0.405$ und steigt danach bei kleiner Aussteuerung $(\alpha \rightarrow \pi / 2)$ bis auf eins an. Weiter zeigt Bild 6.5 die Amplituden der Spannungsgrundschwingung sowie der 3. und 5. Harmonischen in Funktion von $\alpha$.
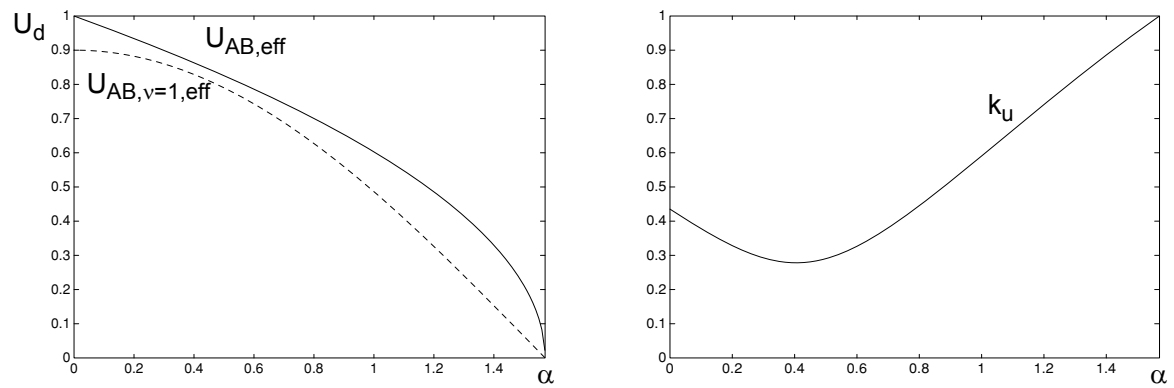

Bild 6.4. Links: Effektivwerte $U_{A B, v=1, \text { eff }}$ und $U_{A B \text {, eff }}$ in Funktion des Steuerwinkels $\alpha$, rechts: Klirrfaktor der Phasenspannung in Funktion von $\alpha$

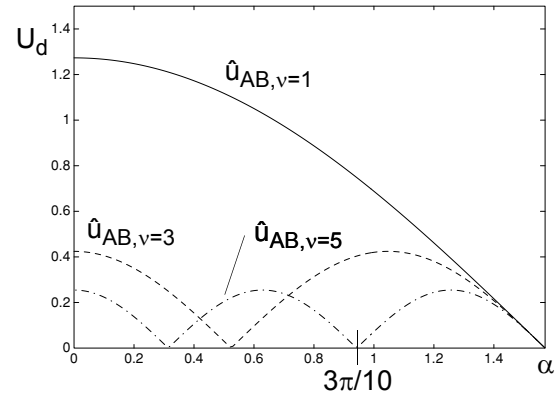

Bild 6.5.

Amplituden der Harmonischen $\hat{u}_{A B, v=1}$, $\hat{u}_{A B, v=3}$ und $\hat{u}_{A B, v=5}$ in Funktion des Steuerwinkels $\alpha$

Verzerrungsstrom: Neben den Spannungen sind die auftretenden Ströme von grossem Interesse. Bild 6.6 zeigt neben der Phasenspannung den Verzerrungsanteil des Phasenstromes für zwei verschiedene Steuerwinkel: im Bild oben für Vollaussteuerung $\alpha=0$ und im Bild unten für $\alpha=3 \pi / 10$, wo die 5. Harmonische gerade vollständig eliminiert ist (in Bild 6.5 bezeichnet). In den ebenfalls dargestellten Spektra zeigt sich deutlich, wie die Harmonischen des Phasenstromes durch die Lastinduktivität mit zunehmender Ordnung stärker gedämpft werden. Für die Vollausteuerung verläuft der Abfall der einzelnen Spannungs- 

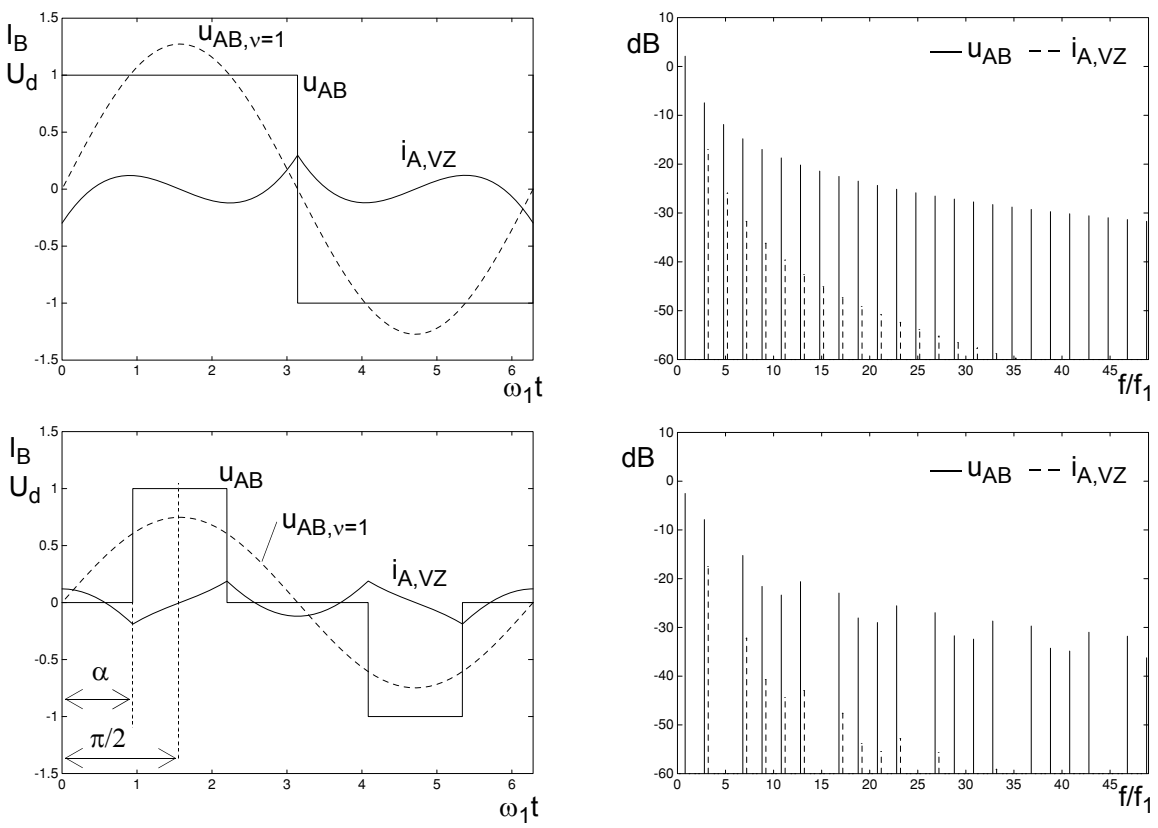

Bild 6.6. Phasenspannung, Spannungsgrundschwingung, Verzerrungsstrom und entsprechende Spektra, 0dB: $U_{d}$ bzw. $I_{B}$, oben: für $\alpha=0$, unten: für $\alpha=3 \pi / 10$

amplituden mit $1 / v$ und derjenige der Stromamplituden mit $1 / v^{2}$. Die Amplituden der Harmonischen des Phasenstromes können in bekannter Weise aus denjenigen der Phasenspannung bestimmt werden:

$$
\hat{i}_{A, v}=\frac{\hat{u}_{A B, v}}{v \omega_{1} L_{k}}, \quad v=3,5,7 \ldots
$$

Der Effektivwert des gesamten Verzerrungsstromes $I_{A, V Z \text {,eff }}$ entspricht der geometrischen Summe der Effektivwerte aller Stromoberschwingungen $I_{A, v, \text { eff }}$ Unter Berücksichtigung der Eigenschaft, dass der Verzerrungsstrom nur durch die Phasenspannung bestimmt und unabhängig von der Gegenspannung ist, lässt sich die Berechnung aber einfacher vornehmen. Es wird dazu der Kurzschlussstromes $i_{A, k}$ verwendet. Dieser fliesst, wenn die Gegenspannung als null angenommen wird. Da die Phasenspannung einen geschalteten Verlauf aufweist, ist er stets stückweise linear. Bei Grundfrequenzsteuerung

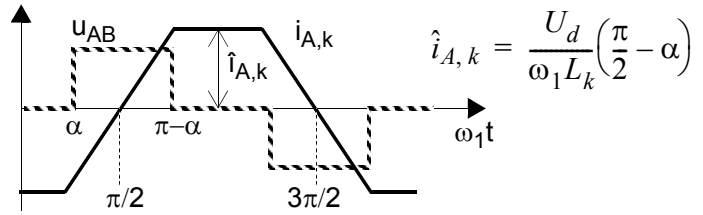

\section{Bild 6.7.}

Kurzschlussstrom der einphasigen Brücke 
nimmt er den in Bild 6.7 dargestellten trapezförmigen Verlauf an. Sein Effektivwert lässt sich dadurch relativ leicht aus dem zeitlichen Verlauf berechnen. Das Resultat lautet:

$$
I_{A, k, \text { eff }}=\frac{U_{d}}{\omega_{1} L_{k}} \sqrt{\frac{4}{3 \pi} \alpha^{3}-\alpha^{2}+\frac{\pi^{2}}{12}}
$$

Der Verzerrungsanteil im Phasenstrom ergibt sich aus $i_{A, k}$ nach Abzug der Grundschwingung gemäss (6.12). Damit gilt für die Effektivwerte (6.13) und mit Hilfe von (6.11) kann $i_{A, V Z, \text { eff }}$ schliesslich nach (6.14) als Funktion von $\alpha$ dargestellt werden:

$$
\begin{aligned}
& i_{A, V Z}=i_{A, k, V Z}=i_{k}-i_{k, v=1}, \\
& I_{A, V Z, \text { eff }}^{2}=I_{A, k, \text { eff }}^{2}-I_{A, \text { eff }, v=1}^{2}, \quad I_{A, \text { eff }, v=1}^{2}=\frac{1}{2}\left(\frac{4 \cos \alpha}{\pi} \frac{U_{d}}{\omega_{1} L_{k}}\right)^{2} \\
& I_{A, V Z, \text { eff }}=\frac{U_{d}}{\omega_{1} L_{k}} \sqrt{\frac{4}{3 \pi} \alpha^{3}-\alpha^{2}-8\left(\frac{\cos \alpha}{\pi}\right)^{2}+\frac{\pi^{2}}{12}}
\end{aligned}
$$

Bild 6.8 zeigt den Verlauf von $i_{A, V Z \text {,eff }}$ zusammen mit den Anteilen der 3. und 5. Harmonischen in Funktion von $\alpha$. Es zeigt sich, dass der Verzerrungsstrom vor allem durch den Anteil der 3. Harmonischen bestimmt wird. In der Nähe der ersten Nullstelle dieser Harmonischen hat der gesamte Verzerrungsstrom ein erstes, sehr ausgeprägtes Minimum (bei $\alpha=0.497)$. Beim maximalen Steuerwinkel $(\alpha=\pi / 2)$, wo die Phasenspannung dauernd auf null bleibt, wird auch der Verzerrungsstrom null.

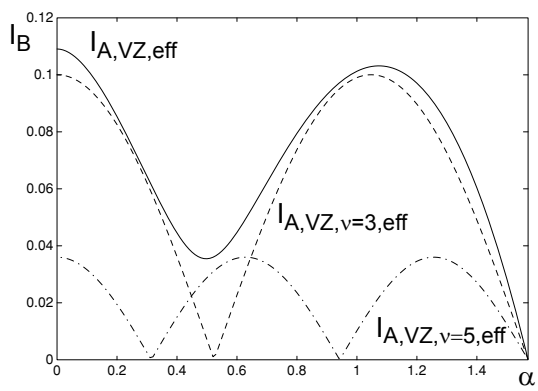

Bild 6.8.

Effektivwerte des gesamten Verzerrungsstromes sowie seiner 3. und 5. Harmonischen

Der Spitzenwert des Verzerrungsstromes tritt viermal pro Periode auf, z.B. an der Stelle $\pi-\alpha$ (Bild 6.6). Da andererseits bei $\pi / 2$ stets ein Nulldurchgang liegt, kann seine Berechnung durch das folgende Integral vorgenommen werden:

$$
\hat{i}_{A, V Z}=\frac{1}{\omega_{1} L_{k}} \int_{\pi / 2}^{\pi-\alpha}\left(u_{A B}-u_{A B, v=1}\right) d\left(\omega_{1} t\right)=\left(\frac{\pi}{2}-\alpha-\frac{4}{\pi} \cos ^{2} \alpha\right) \frac{U_{d}}{\omega_{1} L_{k}}
$$


Zwischenkreisstrom: Der Zwischenkreisstrom $i_{d}$ kann mit der Transformationsgleichung der einphasigen Brücke aus dem Phasenstrom bestimmt werden:

$$
i_{d}=\frac{i_{A}}{2}\left(s_{A}-s_{B}\right)
$$

Bild 6.9. zeigt den Zwischenkreisstrom für einen nacheilenden Laststrom (in bezug auf die Phasenspannung). Sein Mittelwert $I_{d}$ ist durch den mittleren Leistungsfluss bestimmt. Im gewählten Beispiel fliesst die Energie vom Zwischenkreis in die Last. Im Spektrum von $i_{d}$ treten nur geradzahlige Harmonische auf. Die ersten beiden Linien sind die DCKomponente $(f=0)$ und der Pulsationsanteil bei der zweifachen Grundfrequenz $\left(f / f_{1}=2\right)$. Die restlichen Harmonischen gehören zum Verzerrungsanteil, wobei ein Teil davon auch auf $f / f_{1}=2$ zuliegen kommt und sich dort dem Pulsationsanteil überlagert.
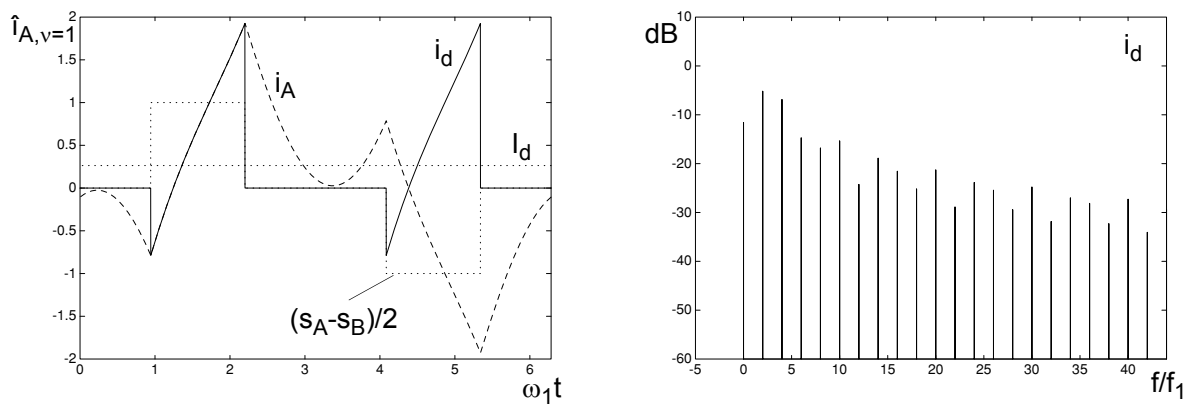

Bild 6.9. Links: Zwischenkreisstrom sowie Phasenstrom und Schaltfunktion, $\hat{\imath}_{A, v=1}=0.2 I_{B}$, rechts: Spektrum des Zwischenkreisstromes, $0 \mathrm{~dB}: \hat{i}_{A, v}=1$

\subsection{Dreiphasige Brücke}

Die Grundfrequenzsteuerung im Dreiphasensystem wird anhand der Schaltung in Bild 6.10 diskutiert. An der Last soll ein symmetrisches Spannungssystem erzeugt werden. Dazu müssen auch die 3 Mittelpunktspannungen $u_{U 0}, u_{V 0}$ und $u_{W 0}$ symmetrisch sein, d.h identische, aber um $\pm 2 \pi / 3$ gegeneinander phasenverschobene Verläufe aufweisen. Im Gegensatz zur einphasigen Brücke dürfen hier deshalb die Phasenlagen der Schaltfunktionen in den 3 Brückenzweigen relativ zueinander nicht variiert werden.

Bei Grundfrequenzsteuerung wird jeder Brückenzweig je während einer Halbperiode auf $+U_{d} / 2$ und auf $-U_{d} / 2$ geschaltet. Die entstehenden Mittelpunkt- und Phasenspannung sowie die Sternpunktspannung sind in Bild 6.11 dargestellt. Sie sind alle gleichspannungsfrei. An der Last treten 4 Spannungsniveaus auf: $\pm 2 U_{d} / 3$ und $\pm U_{d} / 3$. Der Modulationsgrad entspricht dem maximal erreichbaren Wert $4 / \pi$ und kann nicht variiert werden.

Mittelpunkt- und Phasenspannungen: Bild 6.12 zeigt die zu diesem Schaltmuster gehörenden Spektra der Mittelpunkt- und Phasenspannungen. Sie unterscheiden sich nur in 


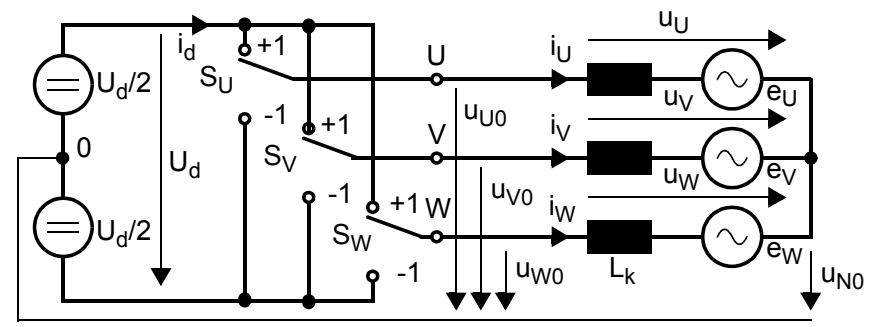

Bild 6.10.

Dreiphasige Brücke
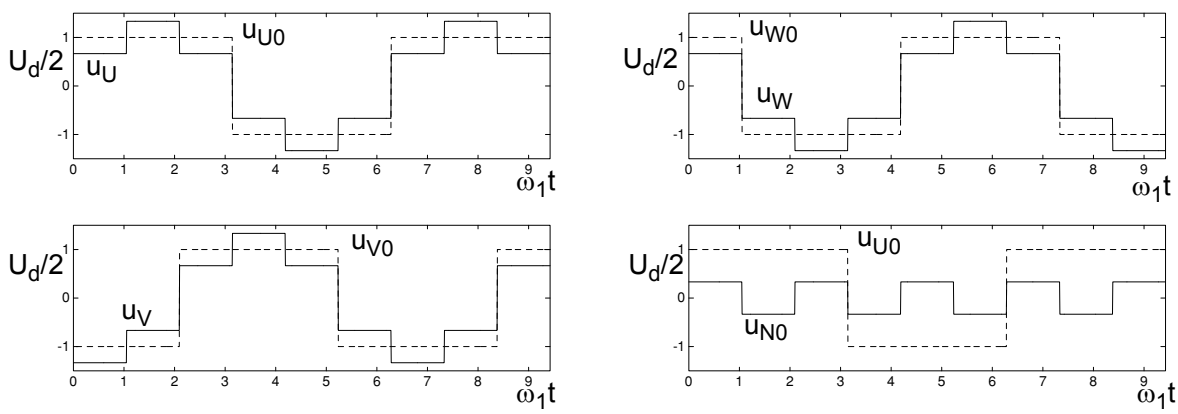

Bild 6.11. Grundfrequenzsteuerung bei der dreiphasigen Brückenschaltung, links oben und unten sowie rechts oben: Mittelpunkt- und Phasenspannungen rechts unten: eine Mittelpunktspannung und Sternpunktspannung

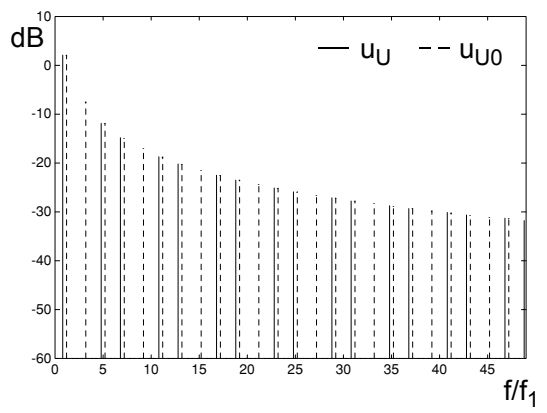

Bild 6.12.

Spektra der Mittelpunkt- und der Phasenspannung, 0dB: $U_{d} / 2$

der 3. Harmonischen und deren Vielfachen voneinander. Diese sind Gleichtaktkomponenten und treten nur im Spektrum der Mittelpunktspannung auf. Die Fourierreihe der Phasenspannung in der Phase $U$ erhält die folgende Form:

$$
u_{U}(t)=\frac{4}{\pi} \frac{U_{d}}{2} \quad \sum^{\infty} \quad \frac{1}{v} \sin \left(v \omega_{1} t\right)
$$

$1,5,7,11,13, \ldots$

Die Harmonischen in den Phasen $V$ und $W$ sind jeweils um $\pm 2 v \pi / 3$ verschoben. Dabei bilden diejenigen der Ordnungen 1, 7, 11, 19, 23, 31 usw. Systeme mit Phasenfolge $U / V / W$ und diejenigen der Ordnungen 5, 13, 17, 25 usw. solche mit umgekehrter Phasenfolge 
$U / W / V$. Die Amplituden, die Effektivwerte, der Modulationsgrad und der Klirrfaktor sind für alle 3 Phasen identisch. Einfache Rechnungen führen auf die folgenden Werte:

$$
\begin{aligned}
& \hat{u}_{U, v=1}=\frac{4}{\pi} \frac{U_{d}}{2}=1.273 \frac{U_{d}}{2}, \quad U_{U, v=1, \text { eff }}=\frac{1}{\sqrt{2}} \frac{4}{\pi} \frac{U_{d}}{2}=0.900 \frac{U_{d}}{2}, \quad M=\frac{4}{\pi} \\
& U_{U, \text { eff }}=\sqrt{\frac{8}{9}} \frac{U_{d}}{2}=0.943 \frac{U_{d}}{2}, \quad k_{u}=\frac{\sqrt{U_{U, \text { eff }}^{2}-U_{U, v}^{2}=1, \text { eff }}}{U_{U, \text { eff }}}=\sqrt{1-\frac{9}{\pi^{2}}}=0.297
\end{aligned}
$$

Der Vergleich mit der einphasigen Grundfrequenzsteuerung bei $\alpha=0$ zeigt, dass hier der Klirrfaktor wesentlich kleiner ist. Der Grund dafür ist das Fehlen der 3. Harmonischen und deren Vielfachen in der Phasenspannung. Der Klirrfaktor der Spannung entspricht etwa dem Minimum beim einphasigen Stromrichter (Bild 6.4).

Verzerrungsstrom: Die Verzerrungsanteile in den Phasenströmen sind alle abgesehen von den Phasenverschiebungen identisch. Ihr Verlauf in der Phase $U$ und das entspre-
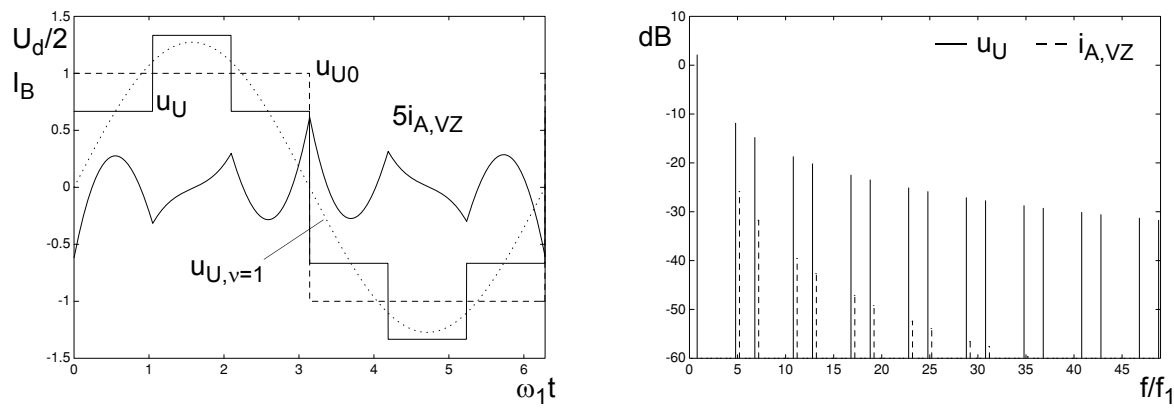

Bild 6.13. Links: Mittelpunkt- und Phasenspannung, Spannungsgrundschwingung und Verzerrungsstrom (Faktor 5) in der Phase $U$, rechts: entsprechende Spektra, 0dB: $U_{d} / 2$ bzw. $I_{B}$

chende Spektrum sind in Bild 6.13 dargestellt. Der Effektivwert kann über die Fourierreihe bestimmt werden. Die numerische Auswertung ergibt:

$$
I_{U, V Z, \text { eff }}=\frac{1}{\sqrt{2}} \sqrt{\sum_{v=5,7,11 \ldots} \hat{i}_{U, v}^{2}}=0.0417 \frac{U_{d} / 2}{\omega_{1} L_{k}}
$$

Der Spitzenwert $\hat{\imath}_{U, V Z}$ lässt sich auf gleiche Weise wie bei der einphasigen Brücke berechnen. Es resultiert:

$$
\hat{i}_{U, V Z}=\frac{2 U_{d}}{\omega_{1} L_{k}}\left(\frac{\pi}{9}-\frac{1}{\pi}\right)=0.123 \frac{U_{d} / 2}{\omega_{1} L_{k}}
$$


Zwischenkreisstrom: Der Zwischenkreisstrom lässt sich mit der entsprechenden Transformationsgleichung aus den 3 Phasenströmen berechnen:

$$
i_{d}=\frac{1}{2}\left(i_{U} s_{U}+i_{V} s_{V}+i_{W} s_{W}\right)
$$

Bild 6.14 zeigt seinen Verlauf und sein Spektrum für das Beispiel von nacheilenden Phasenströmen (in bezug auf die Ausgangsspannungen). Die DC-Komponente $I_{d}$ ist durch die Wirkleistung bestimmt. Die auftretenden Harmonischen sind Vielfache der sechsten. Sie repräsentieren den Verzerrungsanteil von $i_{d}$. Ein Pulsationsanteil $\left(f / f_{1}=2\right)$ fehlt bei symmetrischer Belastung des Stromrichters.
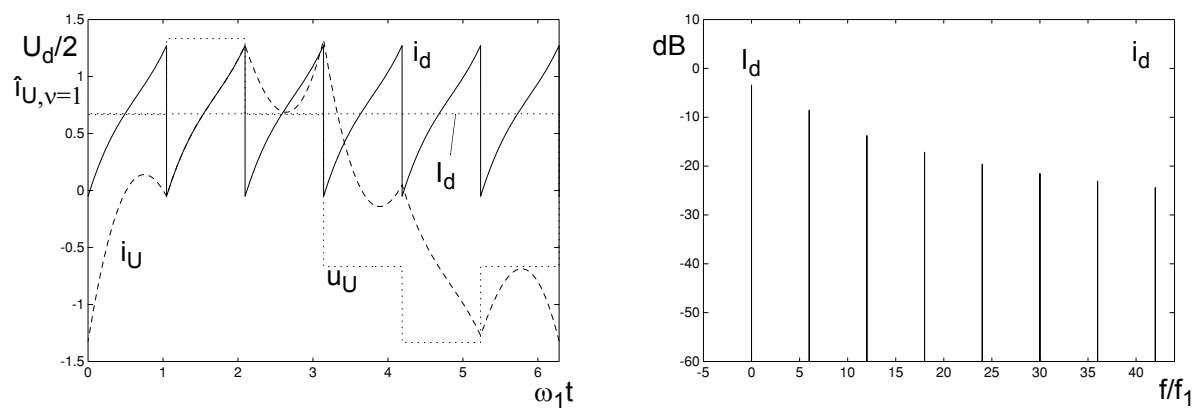

Bild 6.14. Links: Zwischenkreisstrom sowie Phasenspannung und -strom der Phase $U$, $\hat{\imath}_{U / V / W, v=1}=0.2 I_{B}$, rechts: Spektrum des Zwischenkreisstromes, 0dB: $\hat{\imath}_{U / V / W, v=1}$

Drehmomentwelligkeit: Gemäss Kapitel 5.2.1.4 kann aus dem Spektrum der Phasenspannungen direkt die Drehmomentwelligkeit in einem Antrieb im Leerlauf bestimmt werden. Setzt man die Fourierkoeffizienten aus (6.17) in (5.33) ein, so erhält man:

$$
\begin{aligned}
M_{e l, V Z} & \left.=-\frac{3}{2}\left(\frac{4}{\pi} \frac{U_{d}}{2}\right)^{2} \frac{1}{\omega_{1}^{2} L_{k}} \sum_{v=6,12,18, \ldots}^{\infty}\left[\frac{1}{(v-1)^{2}}-\frac{1}{(v+1)^{2}}\right] \sin \left(v \omega_{1} t\right)\right\} \\
& =\frac{6 U_{d}^{2}}{\pi^{2} \omega_{1}^{2} L_{k}}\left[\left(\frac{1}{5^{2}}-\frac{1}{7^{2}}\right) \sin \left(6 \omega_{1} t\right)+\left(\frac{1}{11^{2}}-\frac{1}{13^{2}}\right) \sin \left(12 \omega_{1} t\right)+\ldots\right]
\end{aligned}
$$

Es ist ersichtlich, dass sich die Wirkung der beiden Stromharmonischen, die im Drehmoment auf dieselbe Frequenz fallen, teilweise kompensiert. Aus diesem Grund wird die Drehmomentwelligkeit bei Grundfrequenzsteuerung trotz der stark verzerrten Ströme im Vergleich mit anderen Steuerverfahren verhältnismässig klein. Dies ändert sich allerdings leicht, wenn die Lastimpedanz einen merklichen ohmschen Anteil aufweist $\left(R_{k} \neq 0\right)$. Da die Stromharmonischen dann unterschiedliche Phasenlagen erhalten, ist die gegenseitige Kompensation im Drehmoment weniger gut.

Bild 6.15 zeigt den zeitlichen Verlauf der Drehmomentwelligkeit. Der Spitzenwert entspricht näherungsweise der Amplitude der niedrigsten Teilschwingung bei $6 \omega_{1}$ : 


$$
\hat{M}_{e l, V Z} \approx \frac{6 U_{d}^{2}}{\pi^{2} \omega_{1}^{2} L_{k}}\left(\frac{1}{5^{2}}-\frac{1}{7^{2}}\right)=0.294 M_{B}
$$

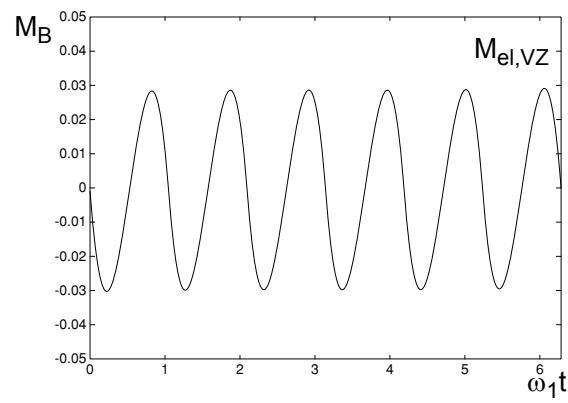

Bild 6.15.

Drehmomentwelligkeit im Leerlauf bei Grundfrequenzsteuerung, $\omega_{1}=(4 / \pi) \omega_{B}$

Variation der Grundschwingungsamplitude: Wird das Einschaltverhältnis $T_{+} / T_{\text {- }}$ der Mittelpunktspannungen nicht eins gewählt, so kann die Amplitude der Phasenspannung variiert werden (Bild 6.16 links). In den Mittelpunktspannungen treten dabei Gleichkomponenten auf. Sofern diese in allen Phasen gleich gross sind, wirken sie als Gleichtaktkomponenten und fehlen in den Phasenspannungen. Im Vergleich zur symmetrischen Steuerung treten in den Spektra jedoch viele zusätzliche Harmonische auf, insbesondere auch geradzahlige. Die Amplituden der Harmonischen sind im Verhältnis zur Grundschwingung auch sehr gross, d.h. der Klirrfaktor ist gross. Diese Art der Amplitudenvariation hat deshalb kaum praktische Bedeutung.
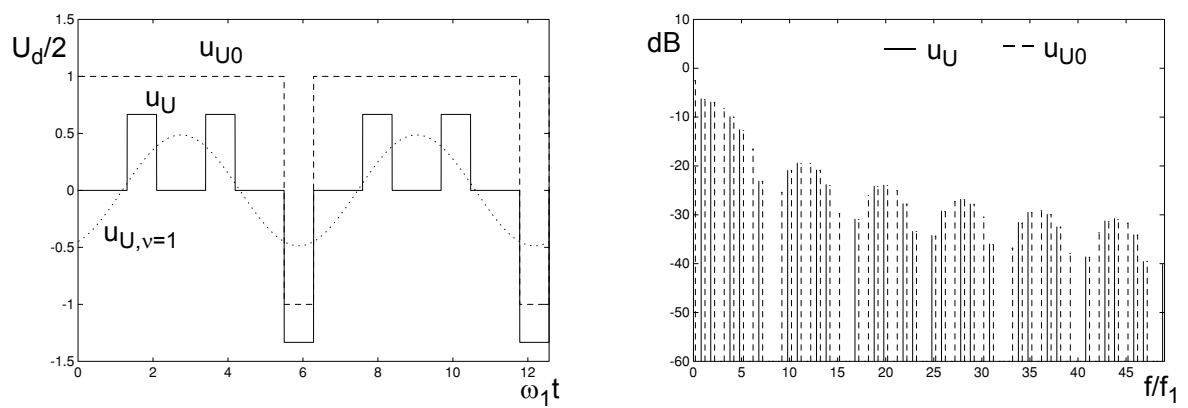

Bild 6.16. Links: Mittelpunkt- und Phasenspannung sowie Grundschwingung der Phasenspannung bei unsymmetrischer Steuerung, $T_{+} / T_{-}=7$, rechts: entsprechende Spektra, $0 \mathrm{~dB}: U_{d} / 2$

Literatur zu Kapitel 6: [Hag1], [Mey1] 


\section{Trägerverfahren}

Trägerverfahren sind die klassischen Pulsweitenmodulationsverfahren. Sie werden heute sehr häufig verwendet, da sie einfach implementiert werden können und gute Resultate liefern. Die Bezeichnung 'Trägerverfahren' stammt ursprünglich aus der Nachrichtentechnik: bei der Amplitudenmodulation wird die Amplitude eines hochfrequenten, sinusförmigen Trägersignals proportional zu einem Sollwertsignal (Sprache, Musik) moduliert. Analog dazu wird bei der Pulsweitenmodulation die Breite von Rechteckpulsen in Abhängigkeit eines Sollwertsignales variiert.

Für Trägerverfahren wird auch die Bezeichnung Unterschwingungsverfahren verwendet.

\subsection{Funktionsprinzip der Trägerverfahren}

\subsubsection{Erzeugen der Schaltsignale}

Das Funktionsprinzip der Trägerverfahren lässt sich einfach am Beispiel der Halbbrücke in Bild 7.1 zeigen: ein Trägersignal, im Bild das Sägezahnsignal $x_{T}$, wird mit dem Sollwertsignal $x_{A \text {,Soll }}$ verglichen. Ist das Sollwertsignal grösser als das Trägersignal, so wird die erzeugte Schaltfunktion $s_{A}=+1$ und andernfalls $s_{A}=-1$.
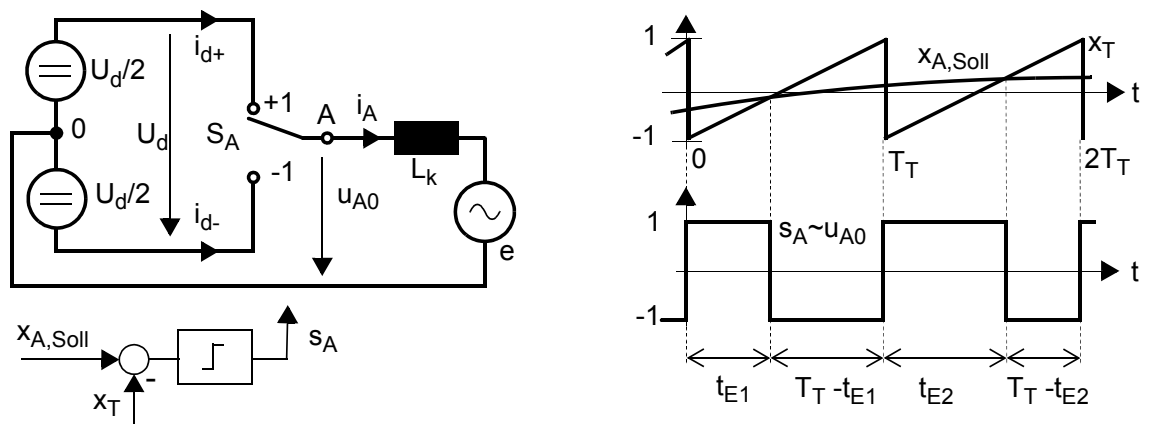

Bild 7.1. Funktionsprinzip der Trägerverfahren

Um einfache Verhältnisse zu erhalten, wird nachfolgend die Amplitude des Trägersignals immer eins gewählt. Bei unverzerrter Modulation darf die Amplitude des Sollwertsignales höchstens gleich derjenigen des Trägersignals sein: 


$$
-\hat{x}_{T} \leq x_{A, \text { Soll }} \leq \hat{x}_{T}, \quad \hat{x}_{T}=1 \quad \Rightarrow \quad-1 \leq x_{A, \text { Soll }} \leq 1
$$

Der Schaltzeitpunkt $t_{E}$ innerhalb der Trägerperiode $T_{T}$ ist durch (7.2) bestimmt:

$$
t_{E}=\left[1+x_{A, \text { Soll }}\left(t_{E}\right)\right] \frac{T_{T}}{2}
$$

Da $x_{A \text {,Soll }}$ oft eine nicht triviale Funktion von $t_{E}$ ist, kann die Gleichung in den meisten Fällen nicht analytisch nach $t_{E}$ aufgelöst werden.

Für die folgende qualitative Betrachtung wird angenommen, dass $x_{A \text {, Soll }}$ innerhalb einer Trägerperiode $T_{T}$ konstant ist. Nach (7.2) ist dann $t_{E}$, abgesehen von einer Konstanten, proportional zum Sollwert $x_{A, \text { Soll }}$. Damit lässt sich der kurzzeitige Mittelwert der Schaltfunktion $s_{A}$ und daraus derjenige der Phasenspannung $u_{A 0}$ über eine Trägerperiode $T_{T}$ berechnen:

$$
\bar{s}_{A}=\frac{1}{T_{T}} \int_{0}^{T_{T}} s_{A} d t=\frac{2 t_{E}-T_{T}}{T_{T}}=x_{A, \text { Soll }}, \quad \bar{u}_{A 0}=\frac{U_{d}}{2} x_{A, \text { Soll }}
$$

Der kurzzeitige Mittelwert $\bar{u}_{A 0}$ der Phasenspannung $u_{A 0}$ kann mit diesem Verfahren proportional zum Sollwert $x_{A \text {,Soll }}$ geführt werden. Die Übereinstimmung ist umso besser, je weniger der Sollwert innerhalb der Trägerperiode ändert.

Anstelle des gezeichneten sägezahnförmigen Trägersignals können auch andere Verläufe verwendet werden. Sehr häufig wird z.B. ein symmetrisches Dreiecksignal eingesetzt.

Anstelle des Sollwertes $x_{A, \text { Soll }}$ kann auch der Sollwert $u_{A 0, \text { Soll }}$ für die Mittelpunktspannung vorgegeben werden. Zwischen den beiden Grössen gilt die Beziehung:

$$
u_{A 0, \text { Soll }}=\frac{U_{d}}{2} x_{A, \text { Soll }}
$$

Synchrone- und asynchrone Modulation: Als Sollwert für die Modulation werde ein sinusförmiges Signal mit der Periodendauer $T_{1}$ verwendet. Bei Trägerverfahren kann die Schaltzahl $q=T_{1} / T_{T}=f_{T} / f_{1}$ (Verhältnis von Träger- zu Grundfrequenz) grundsätzlich beliebig sein. Ist die Schaltzahl ganzzahlig, so ergibt sich eine synchrone Modulation, andernfalls wird die Modulationsart als asynchron bezeichnet. Die beiden Fälle sind in Bild 7.2 illustriert.

In allen Bildern in diesem Kapitel sind synchrone Modulationen dargestellt. Bei den Simulationen lassen sich die Spektra dadurch mittels Fourierreihen einfach und exakt bestimmen.

Spannungsspektra bei Trägerverfahren: Die Spektra bei Trägerverfahren haben immer den gleichen Aufbau. Dieser soll am Beispiel in Bild 7.3 diskutiert werden: im Basisband (Frequenzbereich des Sollwertsignals) liegen die Spektrallinien des Sollwertsignales. Bei einem sinusförmigen Sollwert ist dies eine einzelne Spektrallinie bei $f_{1}$. Um die Vielfachen der Trägerfrequenz $f_{T}$ treten die Trägerbänder auf; das erste um die einfache Trägerfrequenz $f_{T}$ das zweite um die doppelte Trägerfrequenz $2 f_{T}$ und so fort. Jedes dieser Trägerbänder lässt sich in eine Spektrallinie bei der $n$-fachen Trägerfrequenz sowie in ein un- 

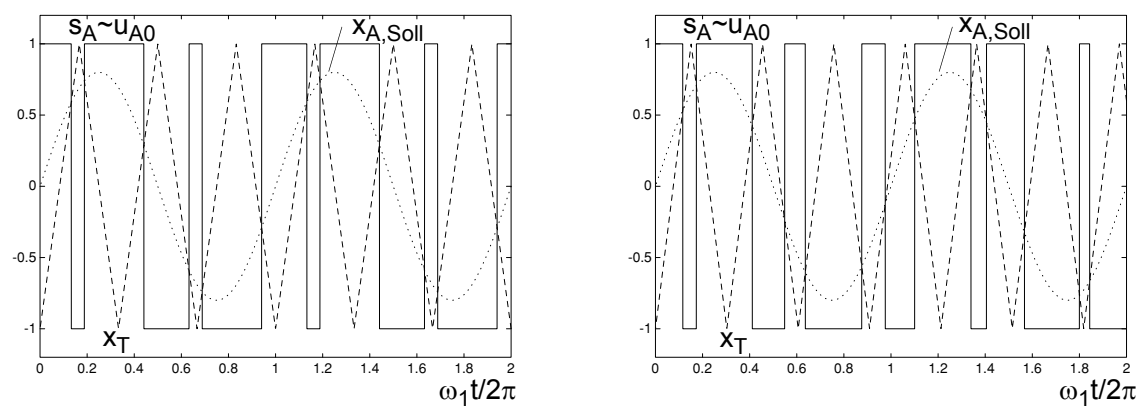

Bild 7.2. Links: Sollwert und Trägersignal bei synchroner Modulation, $q=3, \hat{x}_{A, \text { Soll }_{l}}=0.8$, rechts: Sollwert und Trägersignal bei asynchroner Modulation, $q=3.3, x_{A, \text { Soll }}=0.8$

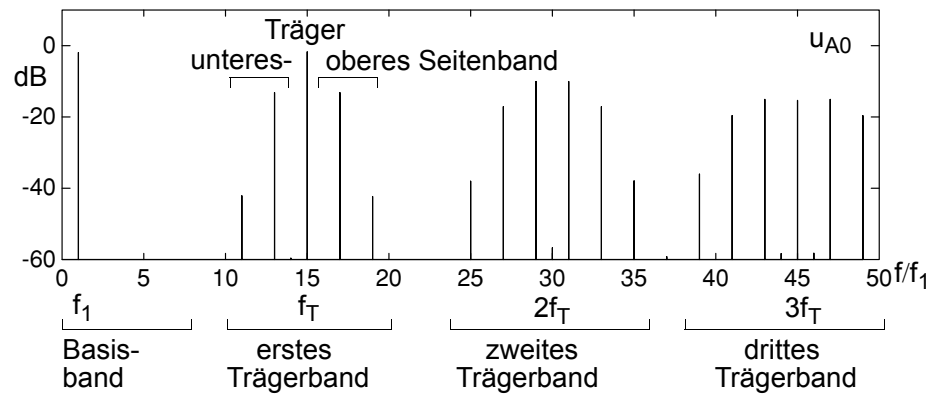

Bild 7.3.

Spannungsspektrum bei Trägerverfahren, $q=f_{T} / f_{1}=15$, 0dB: $U_{d} / 2$

teres- bzw. ein oberes Seitenband zerlegen. Welche Spektrallinien mit welcher Amplitude auftreten, ist von der Art der Modulation abhängig. Insbesondere treten die Vielfachen des Trägersignals selbst oft nicht auf. Es können aber auch ganze Trägerbänder fehlen.

\subsubsection{Trägersignale}

Als Träger kommen vor allem Sägezahn- und Dreiecksignale zum Einsatz. Prinzipiell sind aber auch andere Verläufe denkbar, sofern sie die Bedingung erfüllen, dass der resultierende kurzzeitige Mittelwert der Schaltfunktion proportional zum Sollwert ist. Als Beispiel dafür sei ein asymmetrisches Dreiecksignal genannt. Vier mögliche Signalverläufe sind in Bild 7.4 dargestellt:

Sägezahnsignal für Rückflankenmodulation: Die Vorderflanke (ansteigende Flanke) der Schaltfunktion ist fest und die Position der Rückflanke (abfallende Flanke) wird moduliert.

Sägezahnsignal für Vorderflankenmodulation: Hier wird die Position der Vorderflanke der Schaltfunktion moduliert und die Rückflanke bleibt fest.

Dreiecksignal für symmetrische Modulation: Bei dieser Modulationsart werden die Positionen beider Flanken der Schaltfunktion moduliert. Ändert der Sollwert innerhalb einer Trägerperiode nur wenig, so sind die beiden Schaltflanken näherungsweise symmetrisch zu den Scheitelpunkten des Dreiecksignals. 

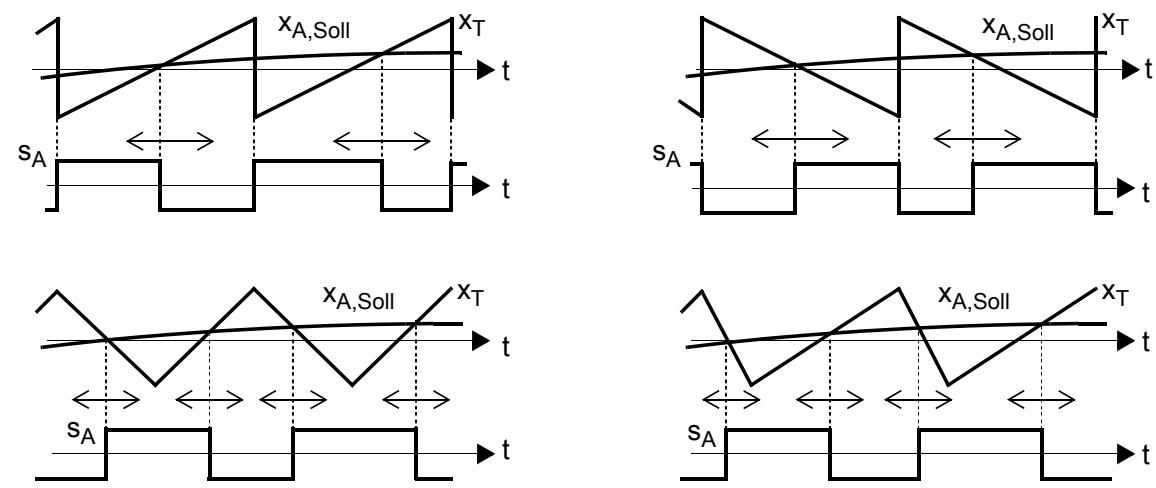

Bild 7.4. Trägersignale und Schaltfunktionen bei Trägerverfahren, oben links: Sägezahnsignal für Rück-, oben rechts: für Vorderflankenmodulation (ansteigende Flanke der Schaltfunktion=Vorderflanke, abfallende=Rückflanke), unten links: Dreiecksignal für symmetrische Modulation, unten rechts: allgemeines, asymmetrisches Trägersignal

Asymmetrisches Dreiecksignal: Beide Schaltflanken werden moduliert. Die Positionen bezüglich des Scheitelpunktes sind aber nicht symmetrisch.

Alle vorgestellten Trägersignale erfüllen die Forderung, dass der kurzzeitige Mittelwert, und damit die Grundschwingung der Ausgangsspannung, sehr gut mit dem Sollwert übereinstimmt. Die Amplitudenspektra der Spannungen (Bild 7.5) für Vorder- und Rückflankenmodulation sind identisch. Sie unterscheiden sich aber von denen mit symmetrischer und asymmetrischer Modulation. Symmetrische Trägersignale haben die Eigenschaft, dass die Seitenbänder aus wenigen Spektrallinien mit schnell abnehmenden Amplituden bestehen, die um die Trägerfrequenz konzentriert sind. Interessant ist, dass die Spannungs-Effektivwerte bei allen vier Trägerformen identisch sind, obwohl die Spektra verschieden sind. Diese Eigenschaft wird in Kapitel 7.2.2 hergeleitet.

Frequenzmodulation des Trägersignals: Bei verschiedenen Anwendungen sind die konzentriert auftretenden Spektrallinien unerwünscht. Sie wirken sich akustisch als Geräusch mit festen Frequenzkomponenten (Pfeifen) aus. Durch Variation der Trägerfrequenz können die Spektra 'verschmiert' werden. Für die Frequenzmodulation werden zwei Verfahren eingesetzt:

- Das Trägersignal wird frequenzmoduliert. In Bild 7.6 ist das für eine zur Grundfrequenz synchrone Modulationsfrequenz illustriert. Das ursprüngliche Trägersignal ist ein symmetrisches Dreieck mit einem Spektrum wie in Bild 7.5 unten links. Für die im Beispiel verwendete Frequenzvariation gilt:

$$
\omega_{T}=\omega_{T 0}\left[1+0.2 \cos \left(2 \omega_{1} t\right)\right]
$$

Im Spektrum treten dadurch weitere Spektrallinien auf. Die dominanten Amplituden werden aber geringfügig kleiner als bei unmoduliertem Träger. 

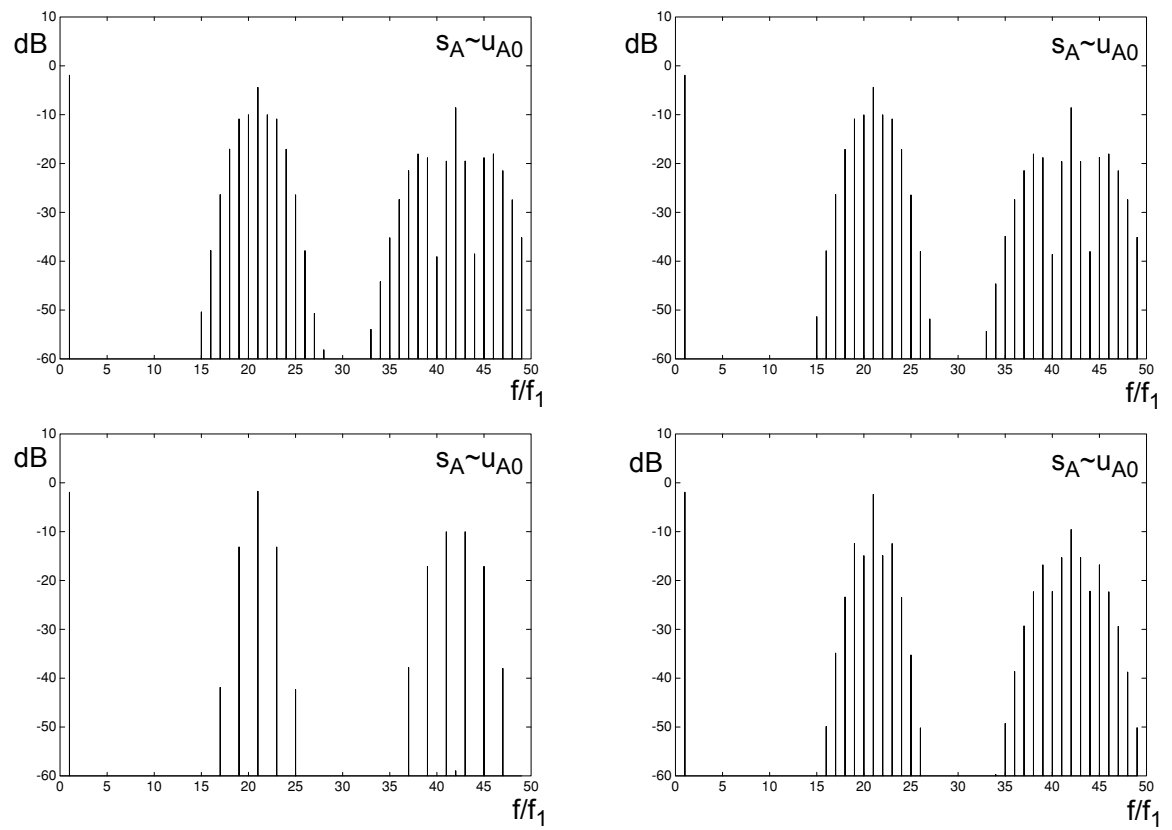

Bild 7.5. Spektra für verschiedene Trägersignale, $q=f_{T} / f_{1}=21,0 \mathrm{~dB}: U_{d} / 2$, oben links: Rückflankenmodulation, oben rechts: Vorderflankenmodulation, unten links: symmetrische Modulation, unten rechts: asymmetrische Modulation

- Die Trägerfrequenz kann durch Einfügen von Rauschen stochastisch variiert werden. Im resultierenden Spektrum verschwinden die diskreten Frequenzlinien zugunsten eines breitbandigen 'Rauschens'.

Mit der geschickten Wahl von Amplitude und Frequenz der Trägerfrequenzvariation können zusätzlich die Stromverzerrungen oder bei Antrieben auch die Drehmomentwelligkeit reduziert werden.

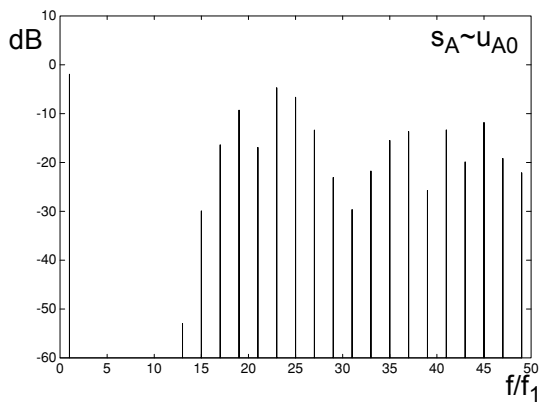

Bild 7.6.

Spektrum bei symmetrischer Modulation und synchroner Variation der Trägerfrequenz gemäss (7.5), $q=21,0 \mathrm{~dB}: U_{d} / 2$ 


\subsubsection{Sollwertsignale}

\subsubsection{Kontinuierliche Sollwertsignale}

Die wichtigste Sollwertsignalform für selbstgeführte Stromrichter ist der sinusförmige Verlauf gemäss (7.6), wie ihn die beiden Kurven in Bild 7.7 links zeigen.



Bild 7.7. Sollwertverläufe für die Pulsweitenmodulation, von links nach rechts: Sinusform, $\hat{x}_{\text {Soll }} \approx 0.8$, Maximalaussteuerung mit $\hat{x}_{\text {Soll }}=1$, Übersteuerung mit $\hat{x}_{\text {Soll }} \approx 1.2$, Rechteckform entsprechend $\hat{x}_{\text {Soll }} \rightarrow \infty$

$$
x_{\text {Soll }}=\hat{x}_{\text {Soll }} \sin \left(\omega_{1} t\right)
$$

Damit sich in jeder Trägerperiode Schnittpunkte von Sollwert- und Trägersignal ergeben, muss für den Sollwert $\hat{x}_{\text {Soll }} \leq 1$ erfüllt sein. Wird der Sollwert zu gross gewählt, so wird der Modulator übersteuert. Er funktioniert dann zwar weiter, aber die entstehenden Ausgangssignale entsprechen nicht mehr einem sinusförmigen Sollwert, sondern den Verläufen mit Begrenzung der Amplitude auf $\hat{x}_{\text {Soll }}=1$. Bei sehr starker Übersteuerung entspricht der wirksame Sollwert-Verlauf schliesslich einem Rechtecksignal. Die Grundschwingungsamplitude $\hat{x}_{\text {Soll, } v=1}$ ist in Bild 7.8 illustriert. Bis zu $\hat{x}_{\text {Soll }}=1$ entspricht sie $\hat{x}_{\text {Soll }}$ und darüber wird sie durch (7.7) beschrieben:

$$
\hat{x}_{\text {Soll }, v=1}=\frac{2}{\pi}\left[\hat{x}_{\text {Soll }} \operatorname{asin}\left(\frac{1}{\hat{x}_{\text {Soll }}}\right)+\sqrt{1-\frac{1}{\hat{x}^{2} \text { Soll }}}\right], \quad \hat{x}_{\text {Soll }}>1
$$

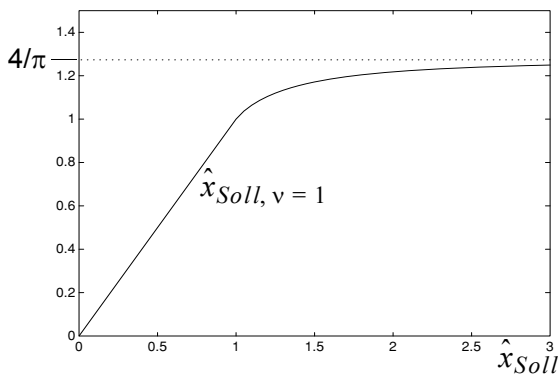

Bild 7.8.

Amplitude der Sollwertgrundschwingung im linearen und im übersteuerten Bereich

Im Bereich der Übersteuerung treten zusätzliche ungerade Oberschwingungen auf. Ist der Sollwert $x_{\text {Soll }}$ ein reines Rechtecksignal (entsprechend einer sehr starken Übersteuerung 
des Modulators), so resultiert die grösstmögliche Grundschwingungsamplitude entsprechend der Grundfrequenzsteuerung:

$$
\begin{aligned}
& x_{\text {Soll }}=\frac{4}{\pi}\left[\sin \left(\omega_{1} t\right)+\frac{1}{3} \sin \left(3 \omega_{1} t\right)+\frac{1}{5} \sin \left(5 \omega_{1} t\right)+\ldots\right], \\
& \hat{x}_{\text {Soll }, v=1}=\frac{4}{\pi}=1.273
\end{aligned}
$$

Weitere Sollwertverläufe: Neben den besprochenen Sollwerten sind beliebige Verläufe denkbar. Ein Beispiel ist die Verwendung eines pulsweitenmodulierten Stromrichters als Audio-Leistungsverstärker. Dabei ist der Sollwert ein Sprach- oder Musiksignal mit $\left|\hat{x}_{\text {Soll }}\right| \leq 1$.

In dreiphasigen Anwendungen werden die Sollwertsignale zum Teil gezielt modifiziert, um höhere Modulationsgrade zu erreichen (Kapitel 7.4.2).

Nachfolgend werden die Amplituden der Sollwerte immer so gewählt, dass die Signale nicht verzerrt werden, d.h. $\hat{x}_{\text {Soll }} \leq 1$.

\subsubsection{Abgetastete Sollwertsignale}

Für die Bestimmung der Schnittpunkte von Träger- und Sollwertsignal (Schaltzeitpunkte) sind verschiedene Verfahren gebräuchlich, die sich in der Erfassung (sampling) des Sollwertsignals unterscheiden. Mangels deutscher Bezeichnungen dafür werden die gebräuchlichen englischen verwendet. Welches Verfahren verwendet wird, ist abhängig von der Implementierung des Modulators.

Natural sampling: Bei der Beschreibung des Funktionsprinzips der Trägerverfahren wurden die Schaltzeitpunkte aus den kontinuierlichen Sollwert- und Trägersignalen bestimmt. Dieses Verfahren wird als natural sampling bezeichnet. Es lässt sich einfach in analoger Schaltungstechnik realisieren. Die mathematische Bestimmung der Schaltzeitpunkte ist dabei wie schon erwähnt nicht in geschlossener Form möglich, da sich transzendente Gleichungen ergeben. Insbesondere für die Implementierung auf Rechnern werden deshalb die nachfolgenden Näherungen verwendet.

Regular sampling: Wird, wie in Bild 7.9 dargestellt, das Sollwertsignal $x_{\text {Soll }}$ in regelmässigen Abständen abgetastet und festgehalten (sample-hold), so spricht man von uniform oder regular sampling. Die Schaltfunktion $s$ wird dann aus den Schnittpunkten des abgetasteten Signals $x^{*}$ Soll mit dem Trägersignal $x_{T}$ berechnet. Verwendet werden zwei Arten von Abtastung, das single edge sampling und das double edge sampling. Bei dem in Bild 7.9 links dargestellten single edge sampling wird der Sollwert nur im Zeitpunkt eines Scheitelwertes des Trägersignals abgetastet. Für die Abtastfrequenz gilt $f_{\text {Tast }}=f_{T}$. Entsprechend der Darstellung rechts im Bild wird beim double edge sampling bei beiden Scheitelwerten des Trägersignals abgetastet und es gilt $f_{\text {Tast }}=2 f_{T}$. Es ist leicht einzusehen, dass bei gleicher Trägerfrequenz der Sollwert so besser nachgebildet wird.

Durch die Abtastung wird die ursprüngliche Funktion verzerrt. Für die resultierenden Frequenzkomponenten einer abgetasteten Sinusfunktion $x_{\operatorname{Soll}}=\hat{x}_{\operatorname{Soll}} \operatorname{Sin}\left(\omega_{1} t\right)$ gilt: 

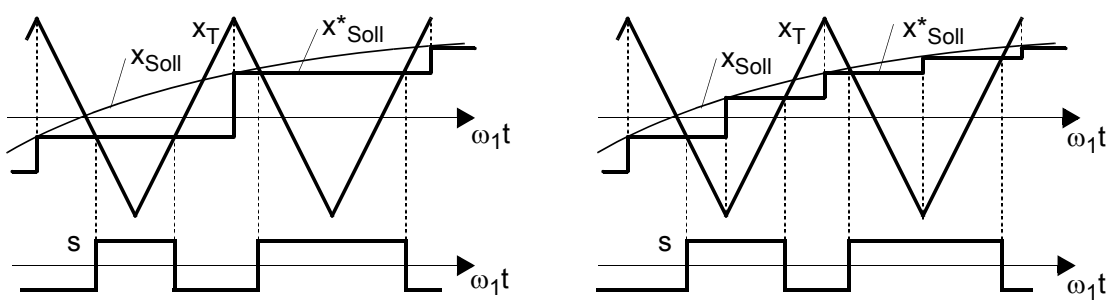

Bild 7.9. Regular sampling, links: single edge sampling, rechts: double edge sampling

$$
\hat{x}_{\text {Soll }}(f)=\frac{\hat{x}_{\text {Soll }}}{\sqrt{2} \pi\left(f / f_{\text {Tast }}\right)} \sqrt{1-\cos \left(2 \pi f / f_{\text {Tast }}\right)}, \quad \varphi^{*}(f)=-\pi\left(f / f_{\text {Tast }}\right),
$$

für $\quad f=n f_{\text {Tast }} \mp f_{1}, \quad n=0,1,2 \ldots$

Die Gleichungen zeigen, dass die Amplituden der Spektrallinien mit steigender Frequenz abnehmen. Es treten Seitenlinien um die Vielfachen der Abtastfrequenz $f_{\text {Tast }}$ auf. Alle Frequenzkomponenten erhalten eine Phasendrehung $\varphi(f)$ gegenüber der ursprünglichen Funktion. Die Grundschwingung $x^{*}{ }_{S o l l, v=1}$ des abgetasteten Sollwertes ist gegenüber dem echten Sollwert leicht abgeschwächt und um eine halbe Abtastperiode $\left(T_{\text {Tast }} / 2\right)$ nacheilend. Damit wird zwangsläufig auch die Grundschwingung der Stromrichterausgangsspannung gegenüber dem echten Sollwert um diese Zeit nacheilend. Die besprochenen Eigenschaften lassen sich anhand von Bild 7.10 für single und double edge sampling verdeutlichen: die Signalverläufe zeigen Trägersignal $x_{T}$, Sollwertsignal $x_{\text {Soll }}$, dessen abgetasteter Verlauf $x^{*}$ Soll und die Grundschwingung $x^{*}{ }_{\text {Soll,v=1 }}$ links für single und rechts für double edge sampling.
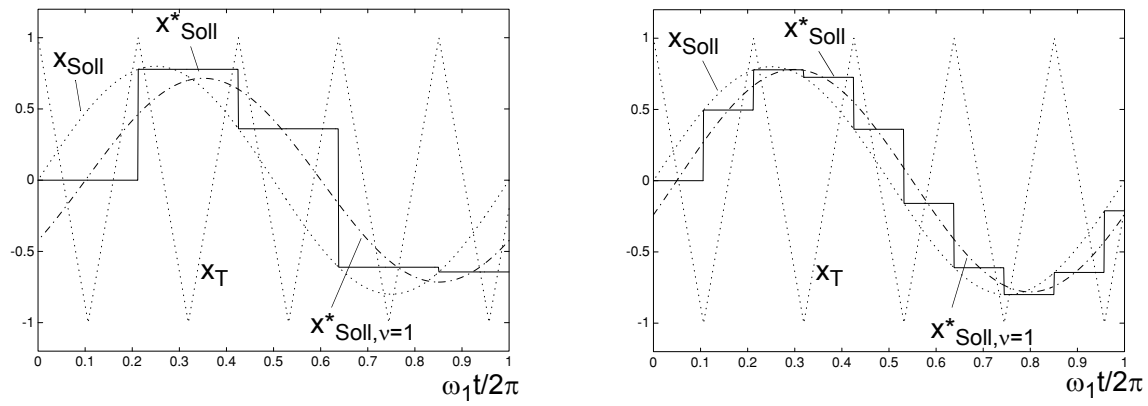

Bild 7.10. Signalverläufe bei abgetasteten Sollwerten, $f_{\text {Tast }} / f_{1}=4.7$, links: single edge sampling, rechts: double edge sampling

Bild 7.11 links zeigt die Spektra von $x^{*}$ Soll für die beiden Arten der Abtastung. Neben der gewünschten Grundfrequenz $f_{1}$ treten die unerwünschten Seitenbänder der Abtastfrequenz $n f_{T} \pm f_{1}$ bei single edge sampling und $2 n f_{T} \pm f_{1}$ bei double edge sampling auf. Für die Amplituden der Spektrallinien von $x^{*}$ Soll in Funktion des Frequenzverhältnisses $f / f_{\text {Tast }}$ 
kann die Hüllkurve in Bild 7.11 rechts angegeben werden. Daraus lässt sich ablesen, dass bei sehr kleinen Verhältnissen von $f_{1} / f_{\text {Tast }}$ (häufige Abtastung) die Grundschwingung sehr wenig abgeschwächt wird. Die höherfrequenten Komponenten liegen in diesem Fall nahe bei $f=n f_{\text {Tast }}$. Ihre Amplituden werden sehr klein, da bei $f=n f_{\text {Tast }}$ die Hüllkurve Nullstellen aufweist. Wird das Verhältnis $f_{1} / f_{\text {Tast }}$ grösser, so wird die Grundschwingung stärker abgeschwächt und umgekehrt die höherfrequenten Anteile grösser.
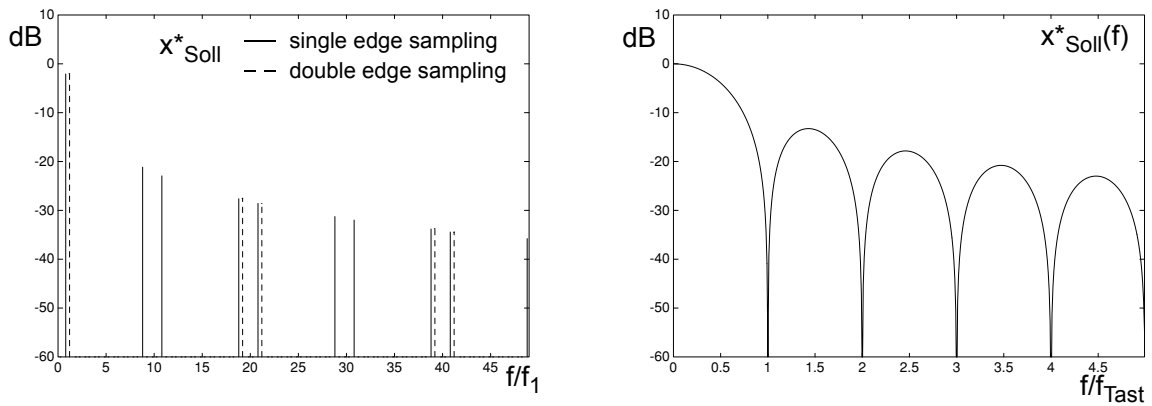

Bild 7.11. Links: Spektra der abgetasteten Signalverläufe, $f_{\text {Tast }} / f_{1}=10, \hat{x}_{\text {Soll }}=0.8,0 \mathrm{~dB}: 1$, rechts: Hüllkurve für Amplituden der Spektrallinien

\subsubsection{Beschreibung von Schaltfunktionen im Frequenzbereich}

Die Beschreibung einer mittels eines Trägerverfahrens erzeugten Schaltfunktion $s$ wird nachfolgend in allgemeiner Form, d.h. für Trägersignale mit asymmetrischen Flanken eingeführt. Die Resultate werden anschliessend aber nur für dreieckförmige Trägersignale weiter diskutiert.

Für die Herleitung der pulsweitenmodulierten Schaltfunktion $s$ wird von der Fourierreihe eines allgemeinen, periodischen Schaltsignals gemäss der Darstellung in Bild 7.12 ausgegangen. Das Signal lässt sich mit (7.10) als Fourierreihe beschreiben. $s_{m}$ ist dabei eine Gleichkomponente und $s_{V Z}$ erfasst die Summe der Harmonischen.

$$
\begin{aligned}
& s=s_{m}+s_{V Z}, \\
& s_{m}=\frac{a+b}{2}-1, \\
& s_{V Z}=\frac{2}{\pi} \sum_{n=1}^{\infty} \frac{1}{n}\left\{\left[\sin \left(n a \frac{\pi}{2}\right)+\sin \left(n b \frac{\pi}{2}\right)\right] \cos \left[n\left(\omega_{T} t+\gamma\right)\right]\right. \\
& \left.\quad+\left[\cos \left(n a \frac{\pi}{2}\right)-\cos \left(n b \frac{\pi}{2}\right)\right] \sin \left[n\left(\omega_{T} t+\gamma\right)\right]\right\}
\end{aligned}
$$

Die Flanken des Schaltsignals werden jetzt entsprechend dem in Bild 7.13 dargestellten Trägerverfahren moduliert. Zusätzlich gelten die nachfolgenden Beziehungen:

- Die Sollwertfunktion $x_{\text {Soll }}$ ist ein cosinusförmiges Signal mit der Kreisfrequenz $\omega_{1}$ und der Phasenlage $\varphi_{\mathrm{X}}$ : 


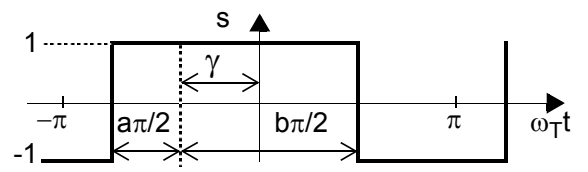

Bild 7.12.

Allgemeines Schaltsignal $s$

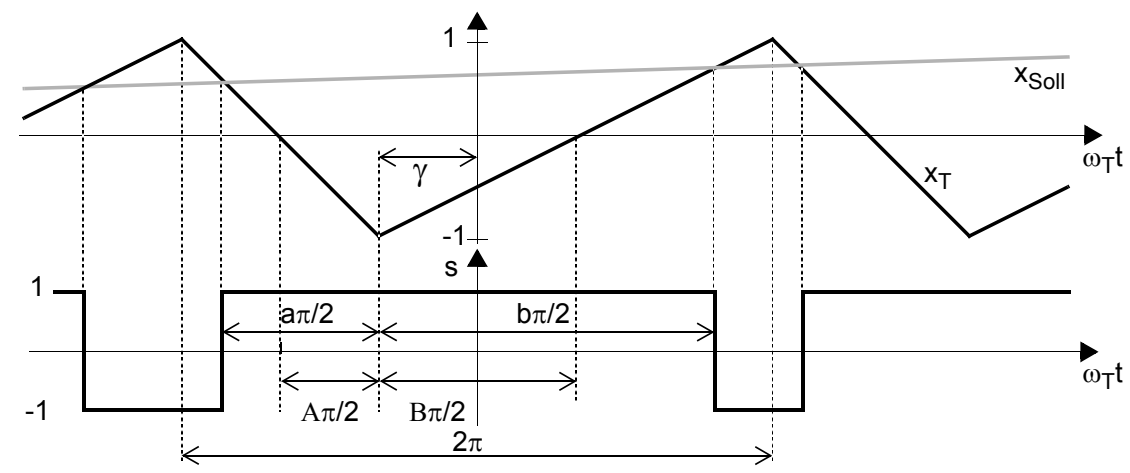

Bild 7.13. Pulsweitenmoduliertes Schaltsignal

$$
x_{\text {Soll }}=\hat{x}_{\text {Soll }} \cos \left(\omega_{1} t+\varphi_{x}\right) .
$$

So nehmen die resultierenden Formeln eine übersichtliche Form an. Sinus-Sollwerte können einfach mit dem Winkel $\varphi_{x}=-\pi / 2$ berücksichtigt werden.

- Es gilt $A \pi / 2+B \pi / 2=\pi$, und damit $A+B=2$. Damit kann mit der Grösse $A$ allein die Symmetrie des Dreiecksignals beschrieben werden: $A=0$ bzw. $A=2$ ergeben je ein sägezahnförmiges und $A=1$ ein dreieckförmiges Trägersignal.

- Für die beiden Schaltwinkel $a \pi / 2$ und $b \pi / 2$ resultieren in Funktion des Sollwertes:

$$
\begin{aligned}
a \frac{\pi}{2} & =A \frac{\pi}{2}\left[1+\hat{x}_{\text {Soll }} \cos \left(\omega_{1} t+\varphi_{x}\right)\right] \\
b \frac{\pi}{2} & =B \frac{\pi}{2}\left[1+\hat{x}_{\text {Soll }} \cos \left(\omega_{1} t+\varphi_{x}\right)\right]=(2-A) \frac{\pi}{2}\left[1+\hat{x}_{\text {Soll }} \cos \left(\omega_{1} t+\varphi_{x}\right)\right]
\end{aligned}
$$

Die Gleichungen in (7.12) können in (7.10) eingesetzt werden. Dabei treten unter anderem verschachtelte Produkte von Sinus- und Cosinusfunktionen auf, die sich mit den allgemein gültigen Beziehungen in (7.13) als unendliche Summen mit Besselfunktionen erster Ordnung darstellen lassen:

$$
\begin{aligned}
& \sin (\alpha \cos \beta)=\sum_{\substack{\mu=0 \\
\infty}}^{\infty} k_{\mu} \sin \left(\mu \frac{\pi}{2}\right) J_{\mu}(\alpha) \cos (\mu \beta), \\
& \cos (\alpha \cos \beta)=\sum_{\mu=0} k_{\mu} \cos \left(\mu \frac{\pi}{2}\right) J_{\mu}(\alpha) \cos (\mu \beta), \\
& \mu=0,1,2,3 \ldots, \quad k_{\mu}= \begin{cases}1 & \text { für } \mu=0 \\
2 & \text { für } \mu=1,2,3, \ldots\end{cases}
\end{aligned}
$$


Die Verläufe der Besselfunktionen 0. bis 7. Ordnung $\left(J_{0}\right.$ bis $\left.J_{7}\right)$ zeigt Bild 7.14. Die Funktionen höherer Ordnung sind bei kleinen Werten von $\alpha$ sehr klein und die Maximalwerte der einzelnen Verläufe nehmen mit steigender Ordnungszahl $\mu$ langsam ab.

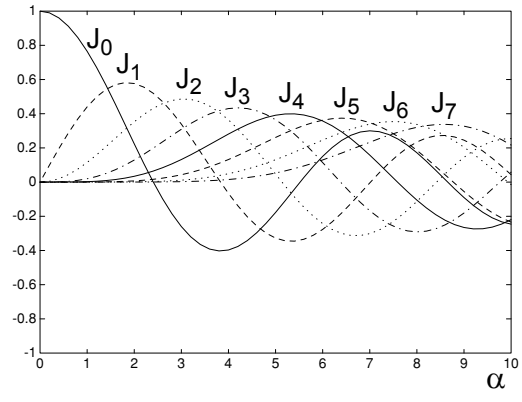

Bild 7.14.

Besselfunktionen $J_{\mu}(\alpha)$ für $\mu=0 \ldots 7$

Unter Verwendung der Beziehungen (7.12) und (7.13) in (7.10) resultieren nach längerer Zwischenrechnung (7.14) und (7.15).

$$
\begin{aligned}
& s_{m}=\hat{x}_{\text {Soll }} \cos \left(\omega_{1} t+\varphi_{x}\right) \\
& s_{V Z}=\sum_{n=1 \mu=0}^{\infty} \sum_{n \mu} c_{n \mu}\left\{\sin \left[\left(n \omega_{T} t+\mu \omega_{1} t\right)+\left(n \gamma+\mu \varphi_{x}\right)+(n A+\mu) \frac{\pi}{2}\right]\right. \\
& \left.\quad+\sin \left[\left(n \omega_{T} t-\mu \omega_{1} t\right)+\left(n \gamma-\mu \varphi_{x}\right)+(n A+\mu) \frac{\pi}{2}\right]\right\}, \\
& c_{n \mu}=\frac{k_{\mu}}{n \pi}\left\{J_{\mu}\left(n \frac{\pi}{2} \hat{x}_{\text {Soll }} A\right)-\cos [\pi(n+\mu)] J_{\mu}\left[n \frac{\pi}{2} \hat{x}_{\text {Soll }}(2-A)\right]\right\}
\end{aligned}
$$

$s_{m}$ ist eine Wechselgrösse und entspricht direkt dem Sollwert $x_{\text {Soll }} . s_{V Z}$ in (7.15) beschreibt den Verzerrungsanteil von $s$. Die Frequenzkomponenten von $s_{V Z}$ sind aber nur noch dann Harmonische von $\omega_{1}$, wenn $\omega_{T}$ ein geradzahliges Vielfaches von $\omega_{1}$ ist.

Die Gleichung (7.15) zeigt die Frequenzen, Phasenlagen und Amplituden der höherfrequenten Spektrallinien. Es treten immer paarweise die Summe und die Differenz $n \omega_{T^{ \pm}}$ $\mu \omega_{1}$ auf. $n \omega_{T}$ bezeichnet die Vielfachen der Trägerfrequenz, d.h. die Trägerbänder und $\mu \omega_{1}$ die jeweiligen Seitenbänder. Die Phasenlagen der einzelnen Schwingungen ist von $n$, $\mu, \gamma, \varphi_{x}$ und $A$ abhängig. Der Betrag von $c_{n, \mu}$ entspricht den Amplituden jedes Schwingungspaares. Diese nehmen mit $1 / n$ ab. Die Werte der Besselfunktionen werden mit steigender Ordnung $\mu$ und grösser werdendem Argument ebenfalls kleiner. Damit sind nur die Teilschwingungen mit kleinen Werten von $n$ und $\mu$ dominant. Es sei betont, dass die Amplituden der einzelnen Schwingungen unabhängig vom Verhältnis $\omega_{T} / \omega_{1}$ sind.

Im allgemeinen Fall treten sämtliche Kombinationen von $n \omega_{\mathrm{T}} \pm \mu \omega_{1}$ auf. Für ein unsymmetrisches Trägersignal mit $\omega_{T}=10 \omega_{1}(q=10)$ wird dies in Bild 7.15 illustriert.

Entsprechend der praktischen Bedeutung wird nachfolgend die Beschreibung der Schaltfunktion $s$ für symmetrische Trägersignale $x_{T}$ weiter betrachtet. 


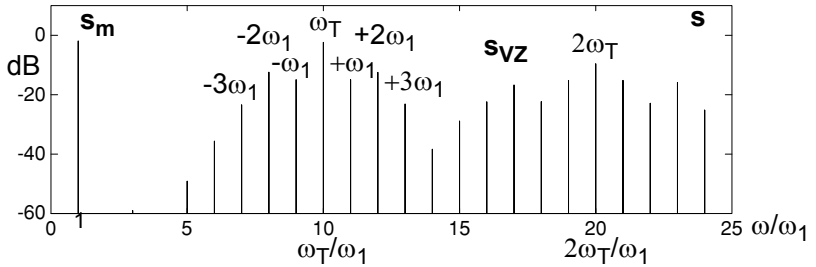

Bild 7.15.

Spektrum der Schaltfunktion $s$ bei unsymmetrischem Träger, $\hat{x}_{\text {Soll }}=0.8,0 \mathrm{~dB}: 1$

Symmetrisches Trägersignal: Bei dieser Signalform ist $A=1$. Für alle geradzahligen Kombinationen von $n+\mu$ wird deshalb $c_{n \mu}=0$, d.h. die Hälfte aller höherfrequenten Spektrallinien fällt weg. Diese Eigenschaft kann für die weitere Vereinfachung von (7.15) ausgenutzt werden. Für symmetrische Trägersignale $(n+\mu=$ ungerade) resultieren so (7.16) und (7.17). Das entsprechende Spektrum illustriert Bild 7.16.

$$
\begin{aligned}
& s_{m}=\hat{x}_{\text {Soll }} \cos \left(\omega_{1} t+\varphi_{x}\right) \\
& s_{V Z}=\sum_{n=1 \mu=0}^{\infty} \sum_{n \mu}^{\infty} c_{n}\left\{\cos \left[\left(n \omega_{T} t+\mu \omega_{1} t\right)+\left(n \gamma+\mu \varphi_{x}\right)\right]\right. \\
& c_{n \mu}=\frac{2 k_{\mu}}{n \pi} \sin \left[(n+\mu) \frac{\pi}{2}\right] J_{\mu}\left(n \frac{\pi}{2} \hat{x}_{\text {Soll }}\right), \quad n+\mu \text { : ungerade }
\end{aligned}
$$



Bild 7.16.

Spektrum der Schaltfunktion $s$ bei symmetrischem Träger, $\hat{x}_{\text {Soll }}=0.8,0 \mathrm{~dB}: 1$

Im ersten Trägerband treten als Seitenbänder die geraden Vielfachen der Grundfrequenz auf, als Seitenbänder im zweiten Trägerband die ungeraden und so fort. Das Argument der Besselfunktion ist $n(\pi / 2) \hat{x}_{\text {Soll }}$. Im ersten Trägerband gilt $n=1$ und damit wird das Argument der Funktion immer $\leq \pi / 2$. Die Betrachtung der Besselfunktionen in Bild 7.14 zeigt, dass für diesen Fall nur die Funktionen niedriger Ordnung ( $\mu$ klein) einen signifikanten Beitrag liefern. Das bedeutet, dass die Seitenbänder um die einfache Trägerfrequenz sehr schnell abklingen. $\operatorname{Im} n$. Trägerband werden die Argumente der Besselfunktionen laufend grösser. Damit treten die Frequenzen höherer Ordnung stark in Erscheinung, was einem langsameren Abklingen der Seitenbänder entspricht. Die Trägerbänder verlaufen deshalb mit zunehmender Ordnung ineinander. Ein Beispiel ist die Frequenzlinie bei $\omega_{T} / \omega_{1}=24$ in Bild 7.16. Sie gehört bereits zum 3. Trägerband und wird gebildet durch $3 \omega_{T}-6 \omega_{1}$.

Mittels der Koeffizienten $c_{n \mu}$ können die Amplituden der einzelnen Teilschwingungen von $s$ in Funktion der Sollwertamplitude $\hat{x}_{\text {Soll }}$ berechnet werden. Für das erste Trägerband 
$(n=1)$ sind sie in Bild 7.17 dargestellt. Ein interessanter Spezialfall ist der Sollwert $x_{\text {Soll }}=0$. Das Argument der Besselfunktionen wird null und damit hat $J_{0}(0)$ den Wert eins während alle anderen Funktionen null sind. Da auch die Grösse $s_{m}=0$ wird, resultiert für $s=s_{V Z}$ (7.18). Wie zu erwarten war, entspricht der Ausdruck einem Rechtecksignal der Kreisfrequenz $\omega_{T}$ und der Amplitude eins.

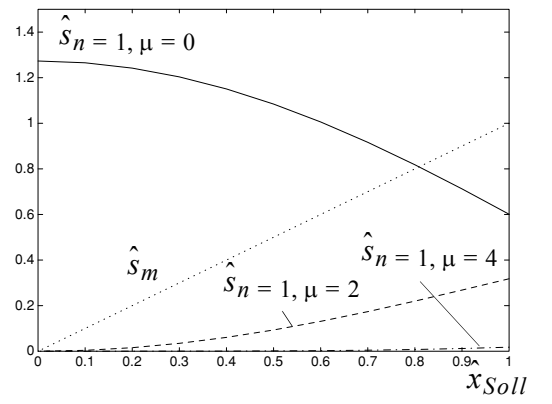

Bild 7.17.

Grundschwingungsamplitude $\hat{s}_{m}$ und Amplituden der Teilschwingungen der Schaltfunktion $\hat{s}_{n, \mu}$ im ersten Trägerband $(n=1)$

$$
x_{\text {Soll }}=0 \quad \Rightarrow \quad s=s_{V Z}=\frac{4}{\pi} \sum_{n=1}^{\infty} \frac{1}{n} \sin \left(n \frac{\pi}{2}\right) \cos \left(n \omega_{T} t+n \gamma\right)
$$

Bei kleinen Verhältnissen von Träger- zu Grundfrequenz können die Seitenbänder der einfachen Trägerfrequenz stören. Sie können sich als niederfrequente Schwebungen oder auch als Subharmonische bemerkbar machen.

Synchrone Modulation mit Drei- und Fünffachtaktung: In Spezialfällen von ganzzahligen Verhältnissen von Träger- zu Grundfrequenz kann eine Seitenlinie auch mit der Grundschwingung zusammenfallen. Dies führt vor allem bei $\omega_{T} / \omega_{1}=3$ und 5 zu einer nennenswerten Abweichung der Grundschwingung von $s_{m}$ gegenüber dem Sollwert.

Nichtsinusförmige Sollwerte: Bei verschiedenen Steuerverfahren treten periodische aber nichtsinusförmige Sollwerte auf. Es stellt sich deshalb die Frage, ob die resultierenden Schaltfunktionen ebenfalls im Frequenzbereich beschrieben werden können. Periodische Sollwertverläufe können mittels Fourierzerlegung in eine Summe von harmonischen Schwingungen zerlegt werden. Für die Schaltwinkel gelten so analog zu (7.12):

$$
\begin{aligned}
a \frac{\pi}{2} & =A \frac{\pi}{2}\left[1+\hat{x}_{\text {Soll }} \cos \left(\omega_{x} t+\varphi_{x}\right)+\hat{y}_{\text {Soll }} \cos \left(\omega_{y} t+\varphi_{y}\right)+\ldots\right], \\
b \frac{\pi}{2} & =(2-A) \frac{\pi}{2}\left[1+\hat{x}_{\text {Soll }} \cos \left(\omega_{x} t+\varphi_{x}\right)+\hat{y}_{\text {Soll }} \cos \left(\omega_{y} t+\varphi_{y}\right)+\ldots\right],
\end{aligned}
$$

Mit diesen Funktionen kann $s_{m}$ bestimmt werden. Die Gleichung (7.20) zeigt, dass $s_{m}$ auch hier genau die Sollwertverläufe wiedergibt.

$$
s_{m}=\frac{a+b}{2}-1=\hat{x}_{\text {Soll }} \cos \left(\omega_{x} t+\varphi_{x}\right)+\hat{y}_{\text {Soll }} \cos \left(\omega_{y} t+\varphi_{y}\right)+\ldots
$$


Die Beschreibung der Verzerrungsanteile mit $s_{V Z}$ wird sehr aufwendig. Die Lösungen enthalten Produkte von unendlichen Summen, deren Darstellung sehr unübersichtlich wird.

Die vorgestellten Eigenschaften von $s_{m}$ und $s_{V Z}$ gelten für beliebige Sollwerte, d.h. auch für nicht periodische Verläufe.

\subsubsection{Modulationsfunktion und Modulationsgrad}

Die Funktion $s_{m}$ entspricht direkt dem Sollwert $x_{\text {Soll }}$, bzw. dem auf $U_{B}$ bezogenen Spannungssollwert $u_{\text {Soll }}$. Durch Multiplikation mit der Bezugsspannung $U_{B}$ der entsprechenden Schaltung erhält man die Ausgangsspannung. (Der Spezialfall, dass diese durch Seitenlinien der Trägerbänder verfälscht wird, ist dabei ausgenommen.)

Für Sollwerte mit sinusförmigem Verlauf gilt zwischen Modulationsfunktion $m, s_{m}$, $x_{\text {Soll }}$ und $u_{\text {Soll }}$ :

$$
m=s_{m}=x_{\text {Soll }}=\frac{u_{\text {Soll }}}{U_{B}}, \quad M=\hat{s}_{m}=\hat{x}_{\text {Soll }}=\frac{\hat{u}_{\text {Soll }}}{U_{B}}
$$

Der Sollwert $x_{\text {Soll }}$ kann also direkt durch die Modulationsfunktion $m$ und der Scheitelwert $\hat{x}_{\text {Soll }}$ durch den Modulationsgrad $M$ ersetzt werden. (Bei Übersteuerung muss statt dem Sollwert $x_{\text {Soll }}$ die Grundschwingung von $x_{\text {Soll }}^{*}$ eingesetzt werden.)

Literatur zu Kapitel 7.1: [Bow2], [Bow3], [Boy1], [Boy2], [Fuk1], [Hou1], [Gra1], [Ric1], [Ste1], [Ste2]

\subsection{Halbbrücke}

\subsubsection{Beschreibung von Spannungen und Strömen im Frequenzbereich}

Betrachtet wird Bild 7.1 links. Die Schaltfunktion $s_{A}$ sei mit einem symmetrischen Dreieck-Trägersignal erzeugt worden. Für das Frequenzspektrum gelten so (7.16) und (7.17).

Mittelpunktspannung: Bei gegebenem Spektrum der Schaltfunktion $s_{A}$ ergibt sich durch Multiplikation mit der Bezugsspannung $U_{d} / 2$ direkt das Spektrum der Spannung (7.22). Das Spektrum der Mittelpunktspannung $u_{A 0}$ unterscheidet sich von demjenigen der Schaltfunktion in Bild 7.16 nur in der Bezugsgrösse.

$$
u_{A 0}=s_{A} \frac{U_{d}}{2}
$$

Verzerrungsstrom: Bei bekannten Spannungsverzerrungen lässt sich der Verzerrungsstrom $i_{A, V Z}$ bestimmen. Für die Standardlast gilt allgemein (7.23). Mit der Anwendung dieser Gleichung auf jede Frequenzkomponente resultiert für $i_{A, V Z}(7.24)$ und für dessen Effektivwert (7.25). 


$$
\begin{aligned}
& i_{A, v}=\frac{1}{L_{k}} \int \hat{u}_{A 0, v} \cos \left(\omega_{v} t+\varphi_{v}\right) d t=\frac{\hat{u}_{A 0, v}}{\omega_{v} L_{k}} \sin \left(\omega_{v} t+\varphi_{v}\right) \\
& i_{A, V Z}=\sum_{n=1 \mu=0}^{\infty} \sum_{\left(n \omega_{T}+\mu \omega_{1}\right) L_{k}}^{\infty} \sin \left[\left(n \omega_{T} t+\mu \omega_{1} t\right)+\left(n \gamma+\mu \varphi_{x}\right)\right] \\
& +\frac{\hat{u}_{A 0, n, \mu}}{\left(n \omega_{T}-\mu \omega_{1}\right) L_{k}} \sin \left[\left(n \omega_{T} t-\mu \omega_{1} t\right)+\left(n \gamma-\mu \varphi_{x}\right)\right] \\
& I_{A, V Z, \text { eff }}^{2}=\frac{1}{2} \sum_{n=1 \mu=0}^{\infty} \sum_{i \mu, \mu}^{\infty} \\
& =\frac{1}{2} \sum_{n=1 \mu=0}^{\infty} \sum_{\mu=}^{\infty}\left\{\left[\frac{\hat{u}_{A 0, n, \mu}}{\left(n \omega_{T}+\mu \omega_{1}\right) L_{k}}\right]^{2}+\left[\frac{\hat{u}_{A 0, n, \mu}}{\left(n \omega_{T}-\mu \omega_{1}\right) L_{k}}\right]^{2}\right\}
\end{aligned}
$$

Die Berechnung der Summen in (7.24) und (7.25) für die Bestimmung des Verzerrungsstromes wird relativ aufwendig. Signifikante Beiträge liefern aber nur die Terme für tiefe Frequenzen. Gute Resultate erreicht man schon, wenn nur über $n=1$ summiert wird (erstes Trägerband). In Kapitel 7.2.2 wird eine noch einfachere Näherung für die Ermittlung des Verzerrungsstromes vorgestellt. Deshalb wird die Formel (7.25) nicht weiter verwendet.

Zwischenkreisströme: Für die Ströme in den Zwischenkreisleitern gilt:

$$
i_{d+}=i_{A} \frac{1+s_{A}}{2}, \quad i_{d-}=i_{A} \frac{1-s_{A}}{2}
$$

Für einen gegenüber dem Spannungs-Sollwert um $\pi / 4$ nacheilenden Strom sind der zeitliche Verlauf und der Mittelwert des Zwischenkreisstromes $i_{d+}$ in Bild 7.18 links dargestellt. Das Bild rechts zeigt das Spektrum des Zwischenkreisstromes. Für die Analyse der auftretenden Frequenzlinien kann die Gleichung für $i_{d+}$ in (7.26) mit (7.27) angenähert werden. Dabei werden die normalerweise kleinen Beiträge, gebildet aus dem Produkt der Stromverzerrungen und der Schaltfunktion, vernachlässigt:

$$
i_{d+}=\left(i_{A, v=1}+i_{A, V Z}\right) \frac{1+s_{A}}{2} \approx \frac{1}{2}\left(i_{A}+i_{A, v=1} s_{m}+i_{A, v=1} s_{V Z}\right)
$$

Der erste Summand, der Strom $i_{A}$, besteht aus den gleichen Frequenzkomponenten wie die Spannung $u_{A 0}$ (Bild 7.5 unten links). Durch die Multiplikation von harmonischen Schwingungen entstehen neue Frequenzen, bestehend aus der Summe und der Differenz der ursprünglichen Frequenzen. Das Produkt aus den Grundschwingungen von Phasenstrom und Schaltfunktion $i_{A, v=1} s_{m}$ ergibt die Gleichstromkomponente und den Pulsationsanteil bei der doppelten Grundfrequenz $2 f_{1}$. Die Amplitude der Gleichstromkomponente ist zusätzlich vom Phasenwinkel zwischen Schaltfunktion und Phasenstrom abhängig (Kapitel 5.2.1.2). Der 3. Summand $i_{A, v=1} s_{V Z}$ generiert aus jeder Frequenzlinie von $s_{V Z}$ 
zwei neue Frequenzen, die um $\pm f_{1}$ gegenüber der ursprünglichen Frequenzkomponente verschoben sind. Zum Teil fallen zwei dieser neuen Komponenten zu einer einzigen Frequenzlinie zusammen. Der Zwischenkreisstrom weist so dieselben Trägerbänder auf, wie die Schaltfunktion allerdings mit anderen Frequenzlinien innerhalb der Bänder.
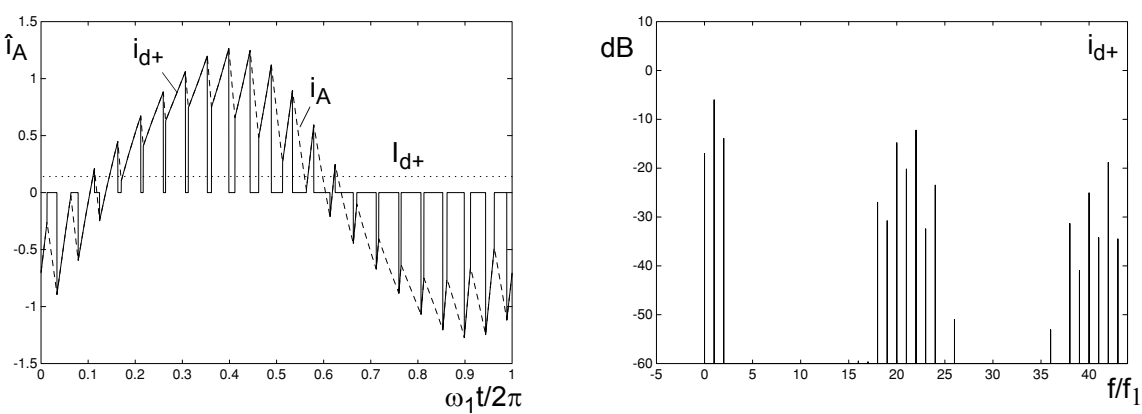

Bild 7.18. Links: zeitlicher Verlauf und Mittelwert des Zwischenkreisstromes sowie Phasenstrom, $M=0.8, q=21, \hat{\imath}_{\mathrm{A}}=0.2 I_{B}$, rechts: Spektrum von $i_{d+}, 0 \mathrm{~dB}: \hat{\imath}_{\mathrm{A}}$

\subsubsection{Näherungsformeln für die Beschreibung von Spannungen und Strömen}

Die Effektivwerte der Mittelpunktspannung und des Verzerrungsstromes können durch Näherungsformeln beschrieben werden. Diese sind schon bei relativ kleinen Schaltzahlen $q$ sehr genau (Bild 7.20). Die Resultate haben eine einfachere Form, als sie bei der Berechnung im Frequenzbereich erhalten würden. Für die Vorstellung der Berechnung werden die Spannungs- und Stromverläufe in Bild 7.19 links betrachtet.
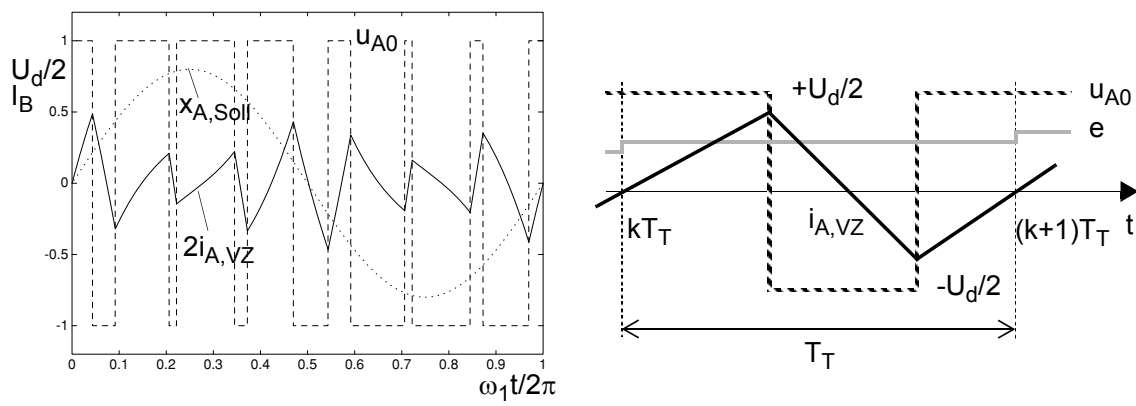

Bild 7.19. Links: Sollwert, Mittelpunktspannung und Verzerrungsstrom, $q=7, M=0.8$, rechts: Darstellung einer Trägerperiode für die Berechnung des Verzerrungsstromes

Kurzzeitiger Spannungsmittelwert: Entsprechend dem Ziel der Modulation ist diese in (7.3) berechnete Grösse in guter Näherung proportional zum Sollwert $x_{A, \text { Soll }}$ :

$$
\bar{u}_{A 0} \approx \frac{U_{d}}{2} x_{A, \text { Soll }}
$$


Spannungs-Effektivwert: Mit (7.29) lässt sich bei der einphasigen Halbbrücke der Spannungseffektivwert exakt bestimmen. Er ist innerhalb jeder Trägerperiode unabhängig von allen Betriebsparametern (insbesondere auch von $f_{T}$ ) konstant, da der Betrag der Mittelpunktspannung jederzeit $U_{d} / 2$ beträgt:

$$
U_{A 0, e f f}=\sqrt{\frac{1}{T} \int_{0}^{T} u_{A 0}^{2}(t) d t}=\frac{U_{d}}{2}
$$

Effektivwert der Spannungsgrundschwingung: Wie die Berechnung im Frequenzbereich zeigt, ist die Spannungsgrundschwingung $u_{A 0, v=1}$ proportional zum Sollwert $x_{A, \text { Soll }}$ bzw. zur Modulationsfunktion $m$. Damit gilt für den Effektivwert:

$$
U_{A 0, v=1, \text { eff }}=\frac{1}{\sqrt{2}} \frac{U_{d}}{2} \hat{x}_{A, \text { Soll }}=\frac{1}{\sqrt{2}} \frac{U_{d}}{2} M
$$

Spannungs-Klirrfaktor: Aus den Effektivwerten der Spannung und ihrer Grundschwingung lässt sich der Klirrfaktor $k_{u}$ mit (7.31) bestimmen. Er ist unabhängig von der Schaltzahl q. In Abhängigkeit des Modulationsgrades liegt er im Bereich $0.707 \leq k_{u} \leq 1$, mit dem Maximum bei $M=0$ :

$$
k_{u}=\frac{\sqrt{U_{A 0, e f f}^{2}-U_{A 0, v=1, e f f}^{2}}}{U_{A 0, e f f}}=\frac{\sqrt{\left(U_{d} / 2\right)^{2}\left(1-M^{2} / 2\right)}}{U_{d} / 2}=\sqrt{1-\frac{M^{2}}{2}}
$$

Effektivwert des Verzerrungsstromes: Der Verzerrungsstrom lässt sich auch aus dem zeitlichen Verlauf der Phasenspannung bestimmen. Dazu werden, wie in Bild 7.19 rechts dargestellt, die Spannungen und der Verzerrungsstrom innerhalb einer Trägerperiode betrachtet. Für die Berechnung werden die folgenden, näherungsweise erfüllten Annahmen getroffen:

1. Die sinusförmig verlaufende Gegenspannung $e(t)$ sei innerhalb einer Trägerperiode $T_{T}$ konstant und gleich dem Spannungssollwert. Damit wird $i_{A, v=1}=0$ und $u_{A, v=1}=e$.

2. Der Verzerrungsstrom $i_{A, V Z}$ ist zu Beginn und am Ende einer Trägerperiode null.

3. Minimum und Maximum von $i_{A, V Z}$ liegen symmetrisch zur Nullinie.

Die Bedingungen 2 und 3 werden vom realen Verzerrungsstrom nur näherungsweise eingehalten. Sie sind auch von der Form des Trägersignals abhängig. Die auftretenden kleinen Abweichungen haben aber sehr wenig Einfluss auf das Resultat. Damit kann der Bei$\operatorname{trag} \Delta I_{A, V Z, \text { eff }}$ der $k$. Trägerperiode zum Effektivwert bestimmt werden:

$$
\begin{aligned}
T_{T} \Delta I_{A, V Z, \text { eff }}^{2}\left(k T_{T}\right)= & \int_{k T_{T}}^{(k+1) T_{T}} i_{A, V Z}^{2} d t=\frac{1}{L_{k}^{2}} \int_{k T_{T}}^{(k+1) T_{T}}\left(u_{A 0}-u_{A 0, \text { Soll }}\right)^{2} d t \\
& =\left(\frac{T_{T} U_{d}}{2 L_{k}}\right)^{2} \frac{T_{T}}{48}\left\{1-\left[M \sin \left(k \frac{2 \pi}{q}\right)\right]^{2}\right\}^{2}
\end{aligned}
$$


Den gesamten Verzerrungsstrom erhält man durch Summation der Teilbeiträge über eine Grundschwingungsperiode $T_{1}=q T_{T}$ :

$$
T_{1} I_{A, V Z, \text { eff }}^{2}=\sum_{k=1}^{q} T_{T} \Delta I_{A, V Z, \text { eff }}^{2}\left(k T_{T}\right)
$$

Ist das Verhältnis von Träger- zu Grundfrequenz gross, so kann die Summation durch eine Integration ersetzt werden. Nach einer Zwischenrechnung resultiert:

$$
I_{A, V Z, \text { eff }} \approx \frac{1}{\sqrt{48}} \frac{U_{d}}{2} \frac{T_{T}}{L_{k}} \sqrt{\frac{3}{8} M^{4}-M^{2}+1}
$$

Der Effektivwert des Verzerrungsstromes ist proportional zur Trägerperiodendauer, d.h. er nimmt mit steigender Trägerfrequenz ab. Sein Maximum erreicht er bei $M=0$.

Spitzenwert des Verzerrungsstromes: Der Spitzenwert $\hat{l}_{A, V Z}$ des dreieckförmig verlaufenden Verzerrungsstromes wird, unabhängig vom Modulationsgrad, beim Nulldurchgang der Spannungsgrundschwingung maximal und erreicht den Wert:

$$
\hat{i}_{A, V Z} \approx \frac{U_{d}}{2} \frac{T_{T}}{4 L_{k}}
$$

Die Vergleiche der exakten Werte von Spannungen, Klirrfaktor und Strömen mit den vorgestellten Näherungen sind für ein Dreieck-Trägersignal in Bild 7.20 dargestellt. Es zeigt sich eine gute Übereinstimmung, die mit steigendem Verhältnis von Träger zu Grundfrequenz immer besser wird.
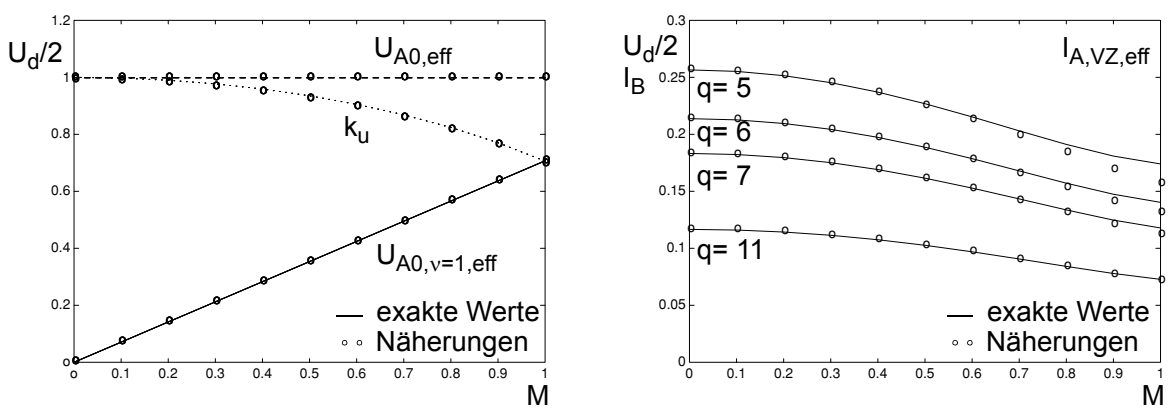

Bild 7.20. Links: Effektivwert der Phasenspannung, ihrer Grundschwingung und Klirrfaktor für $q=T_{1} / T_{T} \geq 5$, rechts: Effektivwerte des Verzerrungsstromes für verschiedene Schaltzahlen 


\subsection{Einphasige Brücke}

Betrachtet wird die Schaltung in Bild 7.21. Die Phasenspannung $u_{A B}$ setzt sich aus den Mittelpunktspannungen $u_{A 0}$ und $u_{B 0}$ der beiden Brückenzweige zusammen. Beide Zweige werden mit einem Trägerverfahren gesteuert. Dabei können die beiden Schalter völlig unabhängig voneinander, oder aber in geschickt gewählter Abhängigkeit gesteuert werden. Die nachfolgenden Betrachtungen zeigen, wie die Steuerung optimal gewählt werden kann.
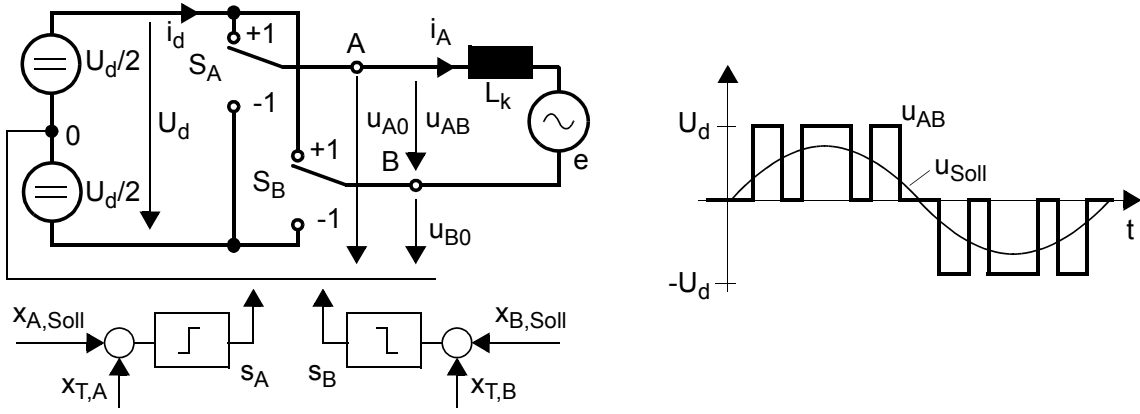

Bild 7.21. Trägerverfahren für die einphasige Brücke

\subsubsection{Sollwertsignale}

Das Ziel der Modulation ist ein optimaler Verlauf der Lastspannung $u_{A B}$. Diese ist durch die beiden Mittelpunktspannungen $u_{A 0}$ und $u_{B 0}$ bestimmt:

$$
u_{A B}=u_{A 0}-u_{B 0}, \quad u_{A B}=u_{A 0}-u_{B 0}
$$

Für die Grundschwingung ist der kurzzeitige Mittelwert $u_{A B}$ massgebend. Er soll dem Sollwert $u_{A B, S o l l}$ folgen. Dieser lässt sich aus beliebigen Kombinationen der Spannungen $u_{A 0, \text { Soll }}$ und $u_{B 0, \text { Soll }}$ erzeugen. Üblicherweise wird eine symmetrische Aufteilung des Spannungssollwertes auf die beiden Zweige gewählt (7.37). Der Grund dafür wird bei der nachfolgenden Diskussion der möglichen Träger ersichtlich: es resultieren am ehesten symmetrische Verhältnisse und damit geringe Verzerrungen innerhalb einer Trägerperiode.

$$
u_{A 0, \text { Soll }}=\frac{u_{A B, \text { Soll }}}{2} \sim x_{A, \text { Soll }}, \quad u_{B 0, \text { Soll }}=-\frac{u_{A B, \text { Soll }}}{2} \sim x_{B, \text { Soll }}
$$

\subsubsection{Trägersignale für einfache Trägerfrequenz in der Phasenspannung}

Die Phasenspannung soll minimale Verzerrungen enthalten. Bei gegebener Grundschwingung heisst das, der Spannungseffektivwert soll möglichst klein sein. Mit einfachen Überlegungen lässt sich zeigen, wie die Spannungen $u_{A 0}$ und $u_{B 0}$ verlaufen müssen, damit der 
Effektivwert von $u_{A B}$ minimal wird. Betrachtet werden die beiden Ausschnitte aus den Lastspannungsverläufen in Bild 7.22 oben, die den gleichen Kurzzeitmittelwert $\bar{u}_{A B}$ ergeben. Die Amplituden der resultierenden Grundschwingungen werden so in beiden Fällen identisch. Solange die Phasenspannung ungleich null, d.h. $+U_{d}$ oder $-U_{d}$ ist, ergibt sie einen Beitrag zum gesamten Spannungseffektivwert. Damit die Verzerrungen klein sind, muss an der Last während möglichst kurzer Zeit eine Spannung ungleich null auftreten.
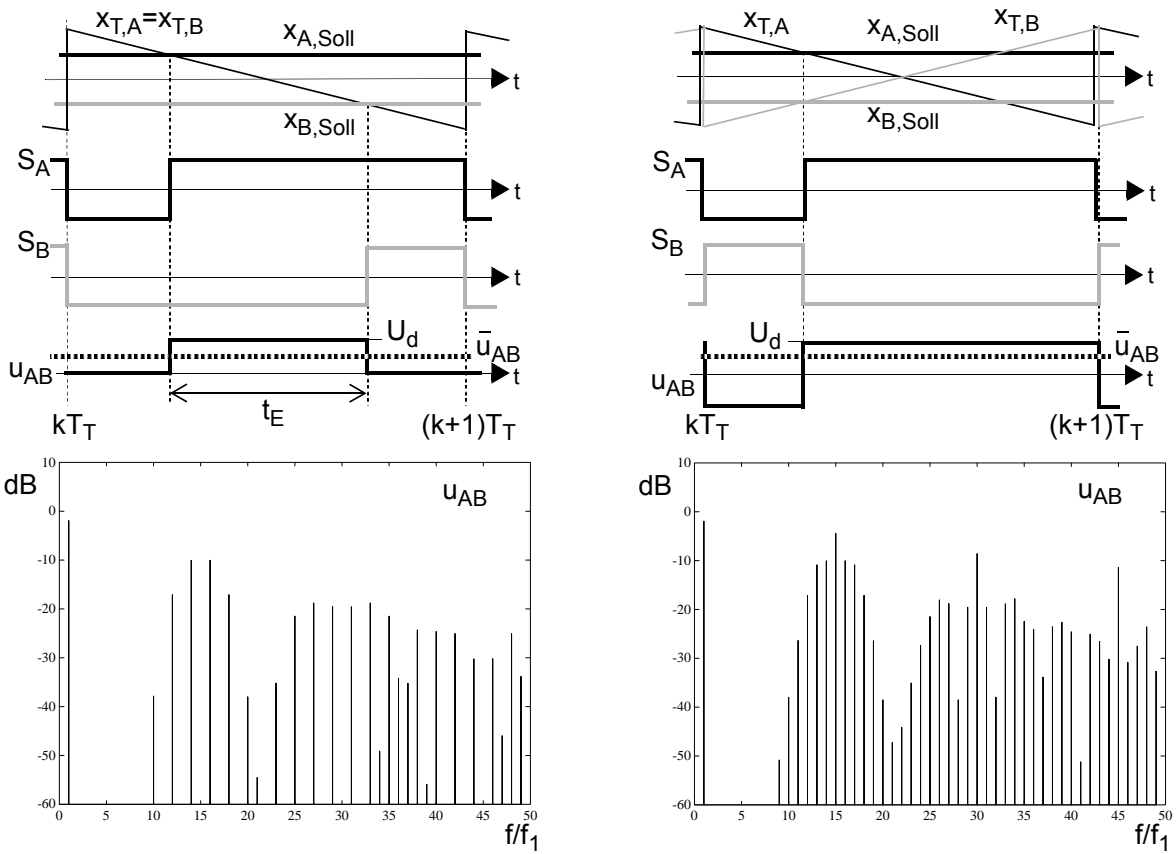

Bild 7.22. Oben: Ausschnitt aus Sollwert-, Träger- und Schaltsignalen sowie der Phasenspannung bei einem Umschaltvorgang in der Phasenspannung pro Trägerperiode, oben links: gleiches Sägezahnsignal als Träger für beide Brückenzweige, oben rechts: zwei invertierte Sägezahnsignale als Träger, unten: entsprechende Spannungsspektra der Phasenspannung, $q=15,0 \mathrm{~dB}: U_{d}$

Bild 7.22 links: Der Verlauf der Phasenspannung ist bezüglich der Einschaltdauer optimal. Kürzer kann $t_{E}$ nicht mehr werden, da sonst der benötigte Kurzzeitmittelwert nicht erreicht wird. Der Beitrag zum gesamten Spannungseffektivwert ist durch (7.38) gegeben. Die Gleichung zeigt die Abhängigkeit des Spannungseffektivwertes vom Sollwert.

$$
T_{T} \Delta U_{A B, \text { eff }}^{2}=U_{d}^{2} t_{E}=U_{d}^{2} T_{T} \frac{x_{A 0, \text { Soll }}-x_{B 0, \text { Soll }}}{2} \leq U_{d}^{2} T_{T}
$$

Bild 7.22 rechts: Der resultierende Beitrag zum Effektivwert ist bei diesem Phasenspannungsverlauf in jeder Trägerperiode unabhängig von den Sollwerten. Er entspricht dem 
maximal möglichen Wert. So resultiert für den Effektivwert (7.39). Selbstverständlich werden dabei auch die Stromverzerrungen gross.

$$
T_{T} \Delta U_{A B, \text { eff }}^{2}=U_{d}^{2} T_{T}=\text { konstant } \quad \Rightarrow \quad U_{A B, \text { eff }}=U_{d}
$$

Die Betrachtungen verdeutlichen eine einfache Regel für die Brückenschaltung: sollen die Spannungsverzerrungen klein bleiben, dürfen innerhalb einer Trägerperiode an der Last nur eine Spannungspolarität und spannungslose Abschnitte auftreten, wie das auch in Bild 7.21 rechts skizziert ist. Während der positiven Halbwelle des Sollwertes ist die Polarität plus und entsprechend während der negativen minus. Diese Regel wird von allen praktisch verwendeten Verfahren eingehalten.

Bei beiden Kurvenverläufen in Bild 7.22 besteht die Phasenspannung nur aus einem Spannungspuls pro Trägerperiode. Damit ist im Spannungsspektrum das erste Trägerband dominant, wie die entsprechenden Spektra in Bild 7.22 unten bestätigen.

\subsubsection{Trägersignale für doppelte Trägerfrequenz in der Phasenspannung}

Bild 7.23 zeigt zwei Möglichkeiten, wie die Phasenspannung $u_{A B}$ aus zwei Spannungspulsen pro Trägerperiode aufgebaut werden kann. Bei dem im linken Bild dargestellten Verfahren werden dazu zwei Sägezahnsignale verwendet. Das zweite ist gegenüber dem ersten invertiert und um eine halbe Trägerperiode versetzt. Im rechten Bild ist ein Dreiecksignal eingesetzt. Die entsprechenden Spektra im Bild unten zeigen, dass für beide Verfahren alle Amplituden im ersten Trägerband null sind. Für die Last wird erst die doppelte Trägerfrequenz wirksam, was sich sehr positiv auf den Verzerrungsstrom auswirkt. Es ist zu beachten, dass das erste Trägerband in den Mittelpunktspannungen nach wie vor auftritt. Es verschwindet aber in der Phasenspannung, weil die entsprechenden Frequenzkomponenten in beiden Teilsignalen gleichphasig sind und sich deshalb kompensieren.

In erster Näherung sind beide Verfahren gleichwertig. Die exakte Berechnung ergibt minimal kleinere Stromverzerrungen bei der Verwendung von zwei versetzten Sägezahnsignalen. Bei $q=7$ beträgt der Unterschied ca. $1 \%$ und bei grösseren Frequenzverhältnissen immer weniger. Ein Vorteil des dreieckförmigem Trägersignals ist dagegen die Konzentration auf weniger Frequenzlinien.

In praktischen Anwendungen werden bei einphasigen Brückenschaltungen fast ausschliesslich dreieckförmige Träger verwendet. Diese Modulation lässt sich auch mit nur einem Sollwert und dafür zwei entgegengesetzten Trägersignalen durchführen. Von den so erzeugten Schaltsignalen muss anschliessend eines noch invertiert werden. Bild 7.24 verdeutlicht dieses Prinzip.

Alle erwähnten Varianten zur Erzeugung von zwei Spannungspulsen innerhalb einer Trägerperiode werden als Trägerverfahren mit versetzter Taktung bezeichnet.

\subsubsection{Beschreibung von Spannungen und Strömen im Frequenzbereich}

Analog zur Halbbrücke interessieren die verschiedenen auftretenden Spannungen, Ströme und deren Frequenzen an der Last. Diese Grössen lassen sich auch hier für beliebige Trä- 

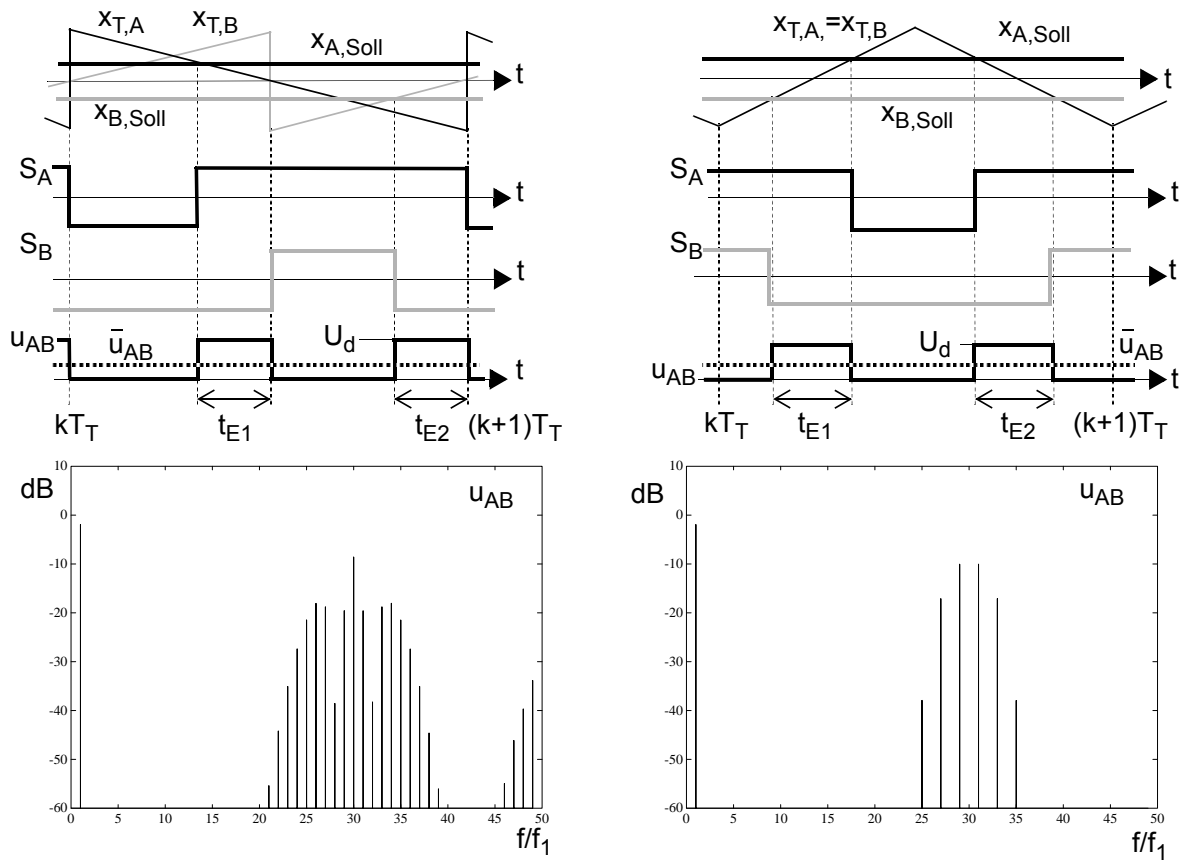

Bild 7.23. Oben: Ausschnitt aus Sollwert-, Träger- und Schaltsignalen sowie der Phasenspannung bei zwei Pulsen bzw. 4 Schaltvorgängen pro Trägerperiode in der Phasenspannung, oben links: zwei gespiegelte und versetzte Sägezahnsignale als Träger, oben rechts: ein Dreiecksignal als Träger für beide Sollwertsignale, unten: entsprechende Spannungsspektra der Phasenspannung, $q=15$, 0dB: $U_{d}$

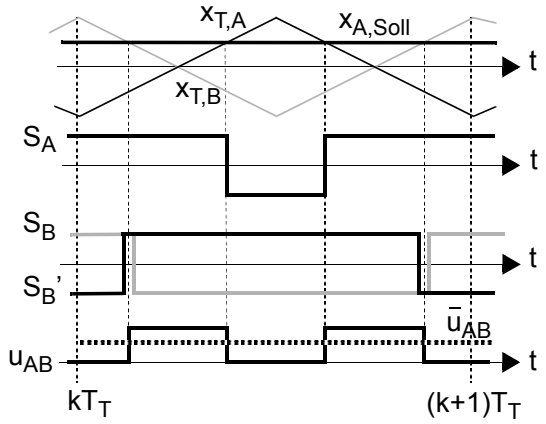

Bild 7.24.

Erzeugung der Schaltsignale mit zwei entgegengesetzten Dreieck-Trägersignalen

gersignale berechnen. Entsprechend ihrer praktischen Bedeutung werden nachfolgend wieder die symmetrischen, d.h. dreieckförmigen Trägersignale betrachtet.

Für die Modulation wird das sinusförmige Sollwertsignal $u_{A B, \text { Soll }}$ für die Phasenspannung in zwei entgegengesetzt gleich grosse Sollwertsignale für die beiden Brückenzweige aufgeteilt. Der Maximalwert von $u_{A B \text {,Soll }}$ darf die doppelte Amplitude der Sollwerte für die Mittelpunktspannungen $u_{A 0 / B 0, \text { Soll }}$ erreichen: 


$$
\begin{aligned}
& u_{A B, \text { Soll }}=U_{d} M \cos \left(\omega_{1} t+\varphi_{x}\right) \\
& u_{A 0, \text { Soll }}=\frac{u_{A B, \text { Soll }}}{2}=\frac{U_{d}}{2} M \cos \left(\omega_{1} t+\varphi_{x}\right)=-u_{B 0, \text { Soll }}, \\
& x_{A, \text { Soll }}=\frac{u_{A 0, \text { Soll }}}{U_{d} / 2}=\operatorname{Mcos}\left(\omega_{1} t+\varphi_{x}\right)=-x_{B, \text { Soll }}
\end{aligned}
$$

Sind die beiden Schaltfunktionen $s_{A}$ und $s_{B}$ im Frequenzbereich bestimmt, so lässt sich mit (7.40) auch die Phasenspannung im Frequenzbereich angeben.

Entsprechend den Gleichungen (7.16) und (7.17) lauten die Gleichungen für die Schaltfunktionen $s_{A}$ und $s_{B}$ :

$$
\begin{aligned}
& s_{A}=s_{A, m}+s_{A, V Z}, \\
& s_{A, m}=\operatorname{Mcos}\left(\omega_{1} t+\varphi_{x}\right), \\
& s_{A, V Z}=\sum_{n=1 \mu=0}^{\infty} \sum_{n \mu} c_{1}\left\{\cos \left[\left(n \omega_{T} t+\mu \omega_{1} t\right)+\left(n \gamma+\mu \varphi_{x}\right)\right]\right. \\
& \left.s_{B}=s_{B, m}+s_{B, V Z}, \quad \cos \left[\left(n \omega_{T} t-\mu \omega_{1} t\right)+\left(n \gamma-\mu \varphi_{x}\right)\right]\right\} \\
& s_{B, m}=M \cos \left(\omega_{1} t+\varphi_{x}+\pi\right)=-s_{A, m}, \\
& s_{B, V Z}=\sum_{n=1 \mu=0}^{\infty} \sum_{n \mu}\left\{\cos \left[\left(n \omega_{T} t+\mu \omega_{1} t\right)+\left(n \gamma+\mu\left\{\varphi_{x}+\pi\right\}\right)\right]\right. \\
& \left.c_{n \mu}=\frac{2 k_{\mu}}{n \pi} \sin \left[(n+\mu) \frac{\pi}{2}\right] J_{\mu}\left(n \frac{\pi}{2} M\right), \quad \cos \left[\left(n \omega_{T} t-\mu \omega_{1} t\right)+\left(n \gamma-\mu\left\{\varphi_{x}+\pi\right\}\right)\right]\right\} \\
& k_{\mu}=\left\{\begin{array}{l}
1 \text { für } \mu=0 \\
2 \text { für } \mu=1,2,3, \ldots
\end{array}\right.
\end{aligned}
$$

Aus den beiden Schaltfunktionen lässt sich direkt die Phasenspannung bestimmen:

$$
\begin{aligned}
& u_{A B}=\left(s_{A}-s_{B}\right) \frac{U_{d}}{2}=\left(s_{A, m}-s_{B, m}\right) \frac{U_{d}}{2}+\left(s_{A, V Z}-s_{B, V Z}\right) \frac{U_{d}}{2} \\
& u_{A B, m}=U_{d} M \cos \left(\omega_{1} t+\varphi_{x}\right), \\
& u_{A B, V Z}=U_{d} \sum_{n=2,4, \ldots \mu=1,3, \ldots \quad}^{\infty} \sum_{n \mu}\left\{\cos \left[\left(n \omega_{T} t+\mu \omega_{1} t\right)+\left(n \gamma+\mu\left\{\varphi_{x}+\pi\right\}\right)\right]\right. \\
& \left.+\cos \left[\left(n \omega_{T} t-\mu \omega_{1} t\right)+\left(n \gamma-\mu\left\{\varphi_{x}+\pi\right\}\right)\right]\right\}
\end{aligned}
$$

Die Gleichungen zeigen die schon erwähnten Eigenschaften nochmals auf: 
- Die Amplitude der Spannungsgrundschwingung $u_{A B, m}$ entspricht der doppelten Sollwert-Amplitude der Mittelpunktspannungen.

- Alle ungeraden Trägerbänder $(n=1,3,5 \ldots)$ fallen weg, da dort die Amplituden $C_{n \mu}=0$ werden. Insbesondere tritt das erste Trägerband $(n=1)$ nicht auf.

Zwischenkreisstrom: Wie in Kapitel 3.3.2.2 hergeleitet, ist der Strom durch den Phasenstrom und die beiden Schaltfunktionen bestimmt:

$$
i_{d}=\frac{i_{A}}{2}\left(s_{A}-s_{B}\right)
$$

Für einen gegenüber dem Spannungssollwert um $\pi / 4$ nacheilenden Phasenstrom sind in Bild 7.25 der zeitliche Verlauf und das Spektrum des Zwischenkreisstromes dargestellt. Das Spektrum der zugehörigen Phasenspannung zeigt Bild 7.23 unten rechts. Unter Vernachlässigung der Produkte des Verzerrungsstromes $i_{A, V Z}$ und der Schaltfunktionen $s_{A}$ und $s_{B}$ gilt:

$$
i_{d} \approx i_{A, v=1}\left(s_{A, m}-s_{B, m}\right)+i_{A, v=1}\left(s_{A, V Z}-s_{B, V Z}\right)
$$

Der erste Term der Gleichung beschreibt die Gleichstromkomponente und den Pulsationsanteil mit der doppelten Grundfrequenz. Die Amplitude der Gleichstromkomponente ist zusätzlich vom Phasenwinkel zwischen Schaltfunktion und Phasenstrom abhängig. Im ersten Trägerband treten die Frequenzen von $\left(s_{A, V Z} s_{B, V Z}\right) \pm f_{1}$ auf. Zum Teil fallen auch hier zwei dieser neuen Komponenten zu einer einzigen Frequenzlinie zusammen.


Bild 7.25. Links: zeitlicher Verlauf und Mittelwert von Zwischenkreis- und Phasenstrom, $M=0.8$, $q=15, \hat{\imath}_{\mathrm{A}}=0.2 I_{B}$, rechts: Spektrum von $i_{d}, 0 \mathrm{~dB}: \hat{i}_{A}$

\subsubsection{Näherungsformeln für Spannungen und Ströme}

Auch für die Brückenschaltung lassen sich gute und einfache Näherungen für Spannungen und Ströme angeben. Für die Berechnungen wird Bild 7.26 betrachtet.

Kurzzeitiger Spannungsmittelwert: Entsprechend dem Ziel der Modulation ist diese Grösse in guter Näherung proportional zum Sollwert $u_{A B, S o l l}$ : 


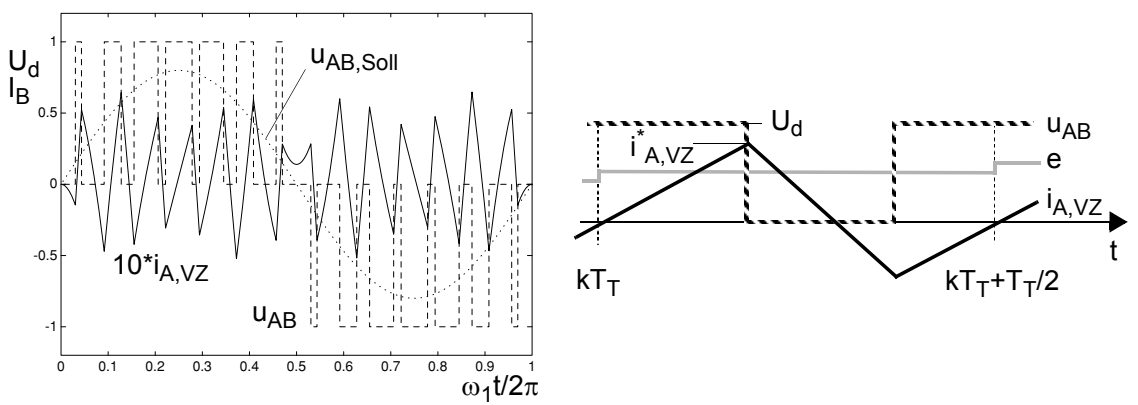

Bild 7.26. Links: Spannungssollwert, Phasenspannung und Verzerrungsstrom, $q=7, M=0.8$, rechts: Darstellung einer halben Trägerperiode für die Berechnung des Verzerrungsstromes

$$
\bar{u}_{A B} \approx u_{A B, \text { Soll }}=\frac{U_{d}}{2}\left(x_{A, \text { Soll }}-x_{B, \text { Soll }}\right)=m U_{d}
$$

Effektivwert der Phasenspannung: Für die Bestimmung des Spannungseffektivwertes ist die Zeit $t_{E}$ massgebend, in der die Lastspannung ungleich null ist. Pro Trägerperiode wird $t_{E}$ näherungsweise durch (7.49) beschrieben:

$$
t_{E} \approx T_{T} \frac{u_{A B, \text { Soll }}}{U_{d}}=T_{T} M \cos \left(\omega t+\varphi_{x}\right)
$$

Damit lässt sich der Beitrag der k. Trägerperiode zum Spannungseffektivwert bestimmen:

$$
T_{T} \Delta U_{A B, e f f}^{2}\left(k T_{T}\right)=U_{d}^{2} t_{E}\left(k T_{T}\right)=U_{d}^{2} T_{T} M \cos \left(\omega k T_{T}\right)
$$

Durch Aufsummieren der Teilbeiträge und Mitteln über eine Grundschwingungsperiode kann der Effektivwert der Lastspannung bestimmt werden. Wird die Summation durch eine Integration ersetzt, so erhält man für den Spannungseffektivwert:

$$
\begin{aligned}
& U_{A B, \text { eff }}=\sqrt{\frac{1}{q T_{T}} \sum_{k=1}^{q} U_{d}^{2} t_{E}\left(k T_{T}\right) \approx U_{d} \sqrt{\frac{2}{\pi} M},} \\
& U_{A B, \text { eff }, \text { max }}=0.798 U_{d} \quad \text { bei } M=1
\end{aligned}
$$

Effektivwert der Spannungsgrundschwingung: Die Grundschwingung ist in guter Näherung proportional zum Sollwert $u_{A B \text {,Soll }}$ und für den Effektivwert gilt somit:

$$
U_{A B, v=1, \text { eff }} \approx \frac{1}{\sqrt{2}} u_{A B, \text { Soll }}=M \frac{U_{d}}{\sqrt{2}}
$$

Spannungs-Klirrfaktor: Mit den Effektivwerten von $u_{A B}$ und $u_{A B, v=1}$ resultiert: 


$$
k_{u}=\frac{\sqrt{U_{A B, e f f}^{2}-U_{A B, v=1, e f f}^{2}}}{U_{A B, e f f}} \approx \sqrt{1-\frac{\pi}{4} M}
$$

In Abhängigkeit des Modulationsgrades liegt der Klirrfaktor im Bereich $0.463 \leq k_{u} \leq 1$ mit dem Maximum bei $M=0$.

Effektivwert des Verzerrungsstromes: Der Verzerrungsstrom wird, wie bei der Halbbrücke, aus dem zeitlichen Verlauf der Lastspannung bestimmt. Das Vorgehen für die Berechnung und die dazu getroffenen Voraussetzungen sind identisch. Wie in Bild 7.26 rechts dargestellt, sind aber die treibenden Spannungen für den Stromaufbau $U_{d^{-}} e$ und für den Stromabbau $e$. Für die Gegenspannung gilt $e=u_{A B, \text { Soll }}$. Für den Verzerrungsstrom ergibt sich auf diese Weise:

$$
I_{A, V Z, \text { eff }} \approx \frac{1}{\sqrt{48}} \frac{U_{d} T}{L_{k}} \sqrt{\frac{3}{8} M^{4}-\frac{8}{3 \pi} M^{3}+\frac{1}{2} M^{2}}
$$

Die Näherungsformel für $I_{A, V Z \text {,eff }}$ stimmt umso genauer, je grösser die Schaltzahl $q$ ist. Der Verzerrungsstrom erreicht sein Maximum bei $M=0.617$.

Bild 7.27. zeigt die Vergleiche der exakten Werte mit den vorgestellten Näherungen für ein Dreieck-Trägersignal. Insbesondere bei den Verzerrungsströmen im Bild rechts zeigt sich die gute Übereinstimmung zwischen den exakten Werten aus den Simulationen und den Näherungsformeln deutlich. Sie wird mit steigender Schaltzahl immer besser.
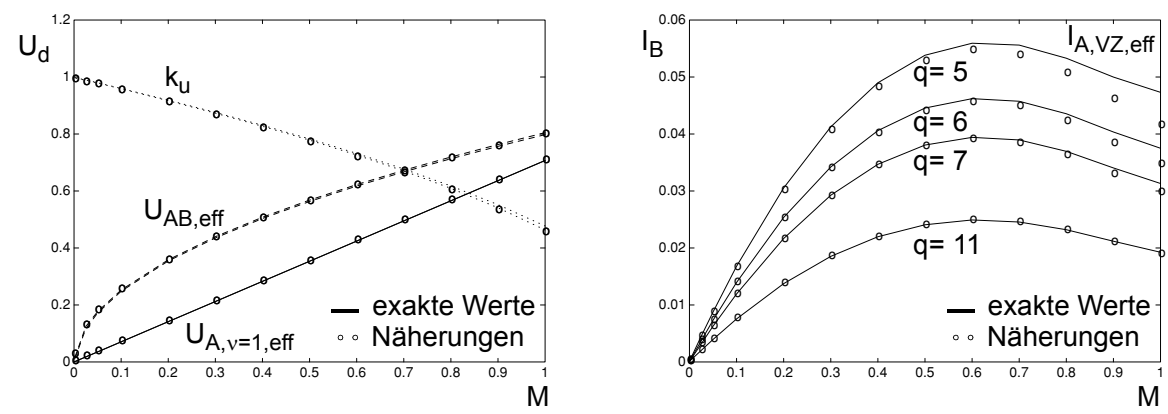

Bild 7.27. Links: Spannungen und Klirrfaktor bei der Brückenschaltung für $q=5$ und $q=11$ rechts: Verzerrungsströme für verschiedene Schaltzahlen

Spitzenwerte des Verzerrungsstromes: Bei $u_{A B, \text { Soll }}=M U_{d} \cos \left(\omega_{1} t+\varphi_{x}\right)$ gilt für den lokalen Spitzenwert $\hat{l}_{A, V Z}^{*}$ in Funktion des Winkels und des Modulationsgrades:

$$
\hat{i}_{A, V Z}^{*}=\frac{1}{4} M \frac{U_{d} T_{T}}{L_{k}}\left[\cos \left(\omega_{1} t+\varphi_{x}\right)-M \cos ^{2}\left(\omega_{1} t+\varphi_{x}\right)\right]
$$


Aus (7.55) lässt sich herleiten, dass die Maximalwerte von $\hat{\imath}_{A, V Z}^{*}$ im Bereich $0 \leq M \leq 0.5 \mathrm{ab}-$ hängig vom Modulationsgrad sind. Für $M \geq 0.5$ bleiben sie konstant:

$\hat{i}_{A, V Z} \approx \frac{1}{4} \frac{U_{d} T_{T}}{L_{k}} M(1-M)$ für $M \leq 0.5, \quad \hat{i}_{A, V Z} \approx \frac{1}{16} \frac{U_{d} T_{T}}{L_{k}} \quad$ für $M>0.5$

\subsection{Dreiphasige Brücke}

Betrachtet wird die dreiphasige Brückenschaltung in Bild 7.28. Jeder Brückenzweig wird für sich mit einem Trägerverfahren gesteuert.
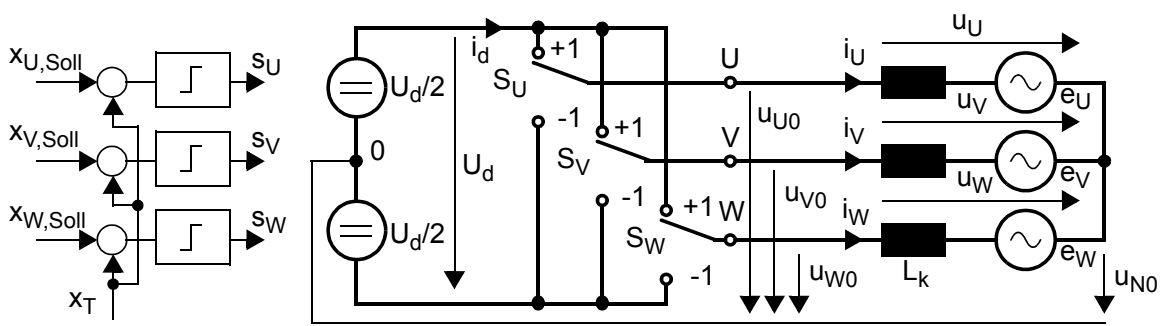

Bild 7.28. Trägerverfahren für die dreiphasige Brückenschaltung

\subsubsection{Trägersignale}

Im Prinzip können für alle 3 Brückenzweige verschiedene Trägersignale gewählt werden. Identische Amplitudenspektra ergeben sich bei dreiphasigen Stromrichtern aber nur, wenn für alle 3 Brückenzweige die gleiche Signalform verwendet wird. Die Trägerfrequenz und deren Vielfache sind in diesem Fall Gleichtaktkomponenten, d.h. sie sind nur in den Mittelpunkt- und Sternpunktspannungen vorhanden und fallen in den Phasenspannungen weg. Wie bei den einphasigen Schaltungen resultieren bei symmetrischen Trägersignalen die besten Verhältnisse bezüglich der auftretenden Spektrallinien und Verzerrungsströme. In der Praxis wird fast ausschliesslich die symmetrische Dreiecksform verwendet. Die nachfolgenden Betrachtungen werden deshalb auf diesen Fall beschränkt.

Die Schaltzahl $q$, d.h. das Verhältnis von Träger- zu Sollwertfrequenz kann beliebig sein. Im allgemeinen Fall ist die Modulation asynchron.

Synchrone Trägersignale: Interessant ist der Spezialfall, in dem die Schaltzahl $q=3 k$ $(k=1,2,3 \ldots)$ beträgt, d.h das Verhältnis von Träger- zu Sollwertfrequenz ein ganzzahliges Vielfaches von 3 ist. In diesem Fall werden bei symmetrischen Sollwerten auch die 3 resultierenden Schaltfunktionen exakt symmetrisch. Zusätzlich zu den identischen Amplitudenspektra werden in diesem Fall die Phasenspektra der 3 Mittelpunktspannungen bezüglich ihrer jeweiligen Sollwerte identisch. Das bedeutet, dass alle Harmonischen mit den Kreisfrequenzen $3 k \omega_{1}$ gleichphasig und damit Gleichtaktsignale sind. Diese Fre- 
quenzanteile treten deshalb nur in den Mittelpunkt- und Sternpunktspannungen auf. Sie fehlen in den Phasenspannungen vollständig. Diese Eigenschaft ist für eine synchrone Modulation mit dreieckförmigem Trägersignal und einer Schaltzahl von $q=21$ in Bild 7.29 rechts dargestellt.

Die besprochenen Eigenschaften lassen sich anhand der Beschreibung der Schaltfunktionen im Frequenzbereich herleiten.

\subsubsection{Sollwertsignale}

Für die nachfolgende Betrachtung der Sollwertsignale wird vorausgesetzt, dass die Grundschwingung der Mittelpunktspannung an jedem Brückenzweig sehr gut dem Sollwert entspricht (d.h. keine synchrone Drei- oder Fünffachtaktung).

Als Sollwerte können verschiedene Verläufe verwendet werden. Üblicherweise sollen die Phasenspannungen ein symmetrisches Dreiphasensystem bilden, bestehend aus 3 um je eine Drittelperiode versetzten, identischen sinusförmigen Signalen. Diese lassen sich einfach erzeugen, indem als Sollwerte für die Mittelpunktspannungen 3 symmetrische Funktionen verwendet werden:

$$
\begin{aligned}
& u_{U 0 / V 0 / W 0, \text { Soll }}=x_{U 0 / V 0 / W 0, \text { Soll }} \frac{U_{d}}{2}=M \frac{U}{2} \sin \left(\omega_{1} t+\Delta \varphi_{U / V / W}\right) \\
& \Delta \varphi_{U / V / W}=0,-2 \pi / 3,-4 \pi / 3
\end{aligned}
$$

Der Modulationsgrad $M$ darf dabei maximal eins werden, da sonst, wie in Kapitel 7.1.3 beschrieben, der Modulator übersteuert wird und dadurch niederfrequente Verzerrungen entstehen. Die Summe der 3 Sollwerte ist null. Wie im Anhang B.1.1 gezeigt, enthält die Sternpunktspannung keinen Grundschwingungsanteil und die Mittelpunkt- und Phasenspannungen sind identisch entsprechend den Verläufen in Bild 7.29.
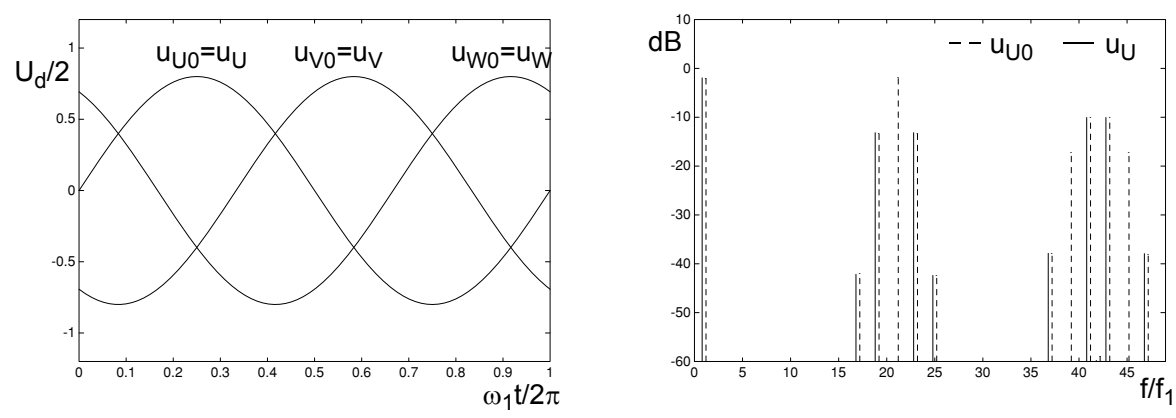

Bild 7.29. Links: niederfrequente Anteile der Mittelpunkt- und Phasenspannungen bei sinusförmigen Sollwerten, $M=0.8$, rechts: Spektra der geschalteten Phasen- und Mittelpunktspannung, $q=21,0 \mathrm{~dB}: U_{d} / 2$

Vergrösserung der maximalen Sollwertamplitude durch Injektion von Harmonischen: Die Eigenschaft, dass Gleichtaktkomponenten der Mittelpunktspannungen in 
den Phasenspannungen nicht mehr auftreten, kann zur Vergrösserung der Sollwertamplituden und damit zur erweiterten Aussteuerung des Stromrichters ausgenutzt werden. Dazu wird allen 3 Sollwerten für die Brückenzweige das Gleichtaktsignal $u_{G, \text { Soll }}$ mit der dreifachen Grundfrequenz zugefügt (injiziert). Es ist im einfachsten Fall ein Sinussignal gemäss (7.58). Die resultierenden niederfrequenten Anteile (Spannungen im Basisband) von Mittelpunkt-, Sternpunkt- und Phasenspannung zeigt Bild 7.30 links. Im gleichen Bild rechts sind die Spektra von Phasen- und Mittelpunktspannung dargestellt. Es zeigt sich, dass bei gleichem Modulationsgrad die Amplitudenverteilung der Spektrallinien in
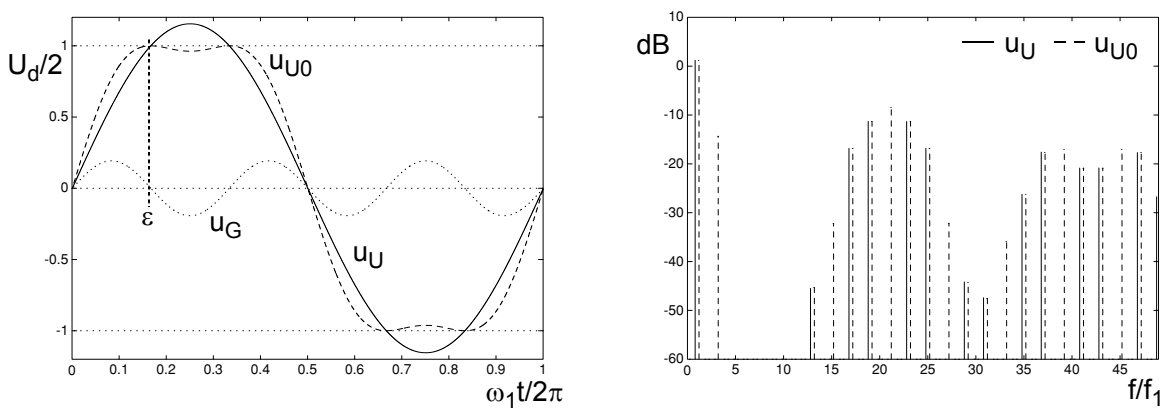

Bild 7.30. Links: niederfrequente Anteile von Mittelpunkt, Sternpunkt- und Phasenspannung bei Injektion einer 3. Harmonischen, $A_{I}=1 / 6, M=1.155$, rechts: zugehörige Spannungsspektra der geschalteten Mittelpunkt- und Phasenspannung für $q=21,0 \mathrm{~dB}: U_{d} / 2$

den Trägerbändern so ist, dass die gesamten Verzerrungen von Spannungen und Strömen kleiner sind als bei rein sinusförmigen Sollwerten.

$$
u_{G, \text { Soll }}=A_{I} M \frac{U_{d}}{2} \sin \left(3 \omega_{1} t\right)
$$

Die Amplitude der injizierten Harmonischen kann nach verschiedenen Gesichtspunkten gewählt werden. Meistens wird sie so optimiert, dass der zulässige Modulationsgrad maximal wird. Da die Gleichtaktspannung aber auch die Strom- und Spannungsverzerrungen beeinflusst, kann auch eine Optimierung auf minimale Verzerrungen vorgenommen werden. Wird die Amplitude der 3. Harmonischen für maximale Aussteuerung optimiert, so liegen die auftretenden Verzerrungen sehr nahe bei den Werten, wie sie mit einer Optimierung auf minimale Verzerrungen erreicht werden. Weiter kann die Amplitude abhängig vom Modulationsgrad der Grundschwingung variiert werden. Der Einfachheit halber wird aber üblicherweise ein festes Amplitudenverhältnis von 3. Harmonischer zur Grundschwingung verwendet, d.h. $A_{I}$ bleibt konstant.

Amplitude der 3. Harmonischen für maximale Aussteuerung: Die Amplitude $A_{I}$ in (7.58) kann mit Hilfe einer einfachen Überlegung bestimmt werden: beim Winkel $\varepsilon=\pi / 3$ kann die Amplitude der Mittelpunktspannung $u_{U 0, \text { Soll }}$ mit der 3. Harmonischen nicht be- 
einflusst werden, da diese dort null ist (Bild 7.30). Deshalb wird $A_{I}$ so gewählt, dass die Mittelpunktspannung bei $\pi / 3$ maximal ist (Tangente horizontal) und es resultiert:

$$
A_{I}=\frac{1}{6}=16.7 \%
$$

Der Modulationsgrad $M$ kann jetzt soweit vergrössert werden, bis die Sollwerte für die Mittelpunktspannungen bei $\varepsilon=\pi / 3$ den Wert $U_{d} / 2$ erreichen. Man erhält so den grösstmöglichen Modulationsgrad ohne niederfrequente Verzerrungen in den Phasenspannungen, der sich bei dreiphasigen Stromrichtern erreichen lässt:

$$
M_{\text {max }}=2 / \sqrt{3}=1.155
$$

Amplitude der 3. Harmonischen für minimale Stromverzerrungen: Die 3. Harmonische kann auch für minimale Stromverzerrungen optimiert werden. Die Bestimmung der optimalen Amplitude wird später in Kapitel 7.4.4 bei der Berechnung des Verzerrungsstromes vorgestellt. Man erhält dort $A_{I}=1 / 4=25 \%$. Der maximale Modulationsgrad wird unwesentlich kleiner, nämlich $M_{\max } \approx 1.122$ ([Kol1]).

Dreieckförmige Gleichtaktspannung: Als Gleichtaktspannung kann anstelle einer einzelnen Harmonischen auch eine Summe von Vielfachen der 3. Harmonischen verwendet werden. Eine solche Summe ist beispielsweise ein dreieckförmiges Gleichtaktsignal wie in Bild 7.31 dargestellt. Die Amplitude des Dreiecksignals für maximale Aussteuerung wird in der gleichen Art wie bei der Injektion von nur einer 3. Harmonischen bestimmt. Sie beträgt $A_{I}=A_{D r}=1 / 4$. Die enthaltene 3. Harmonische hat dabei eine Amplitude von $A_{3} \approx 1 / 5$. Der maximale Modulationsgrad beträgt auch hier wieder $M_{\max }=2 / \sqrt{3}=1.155$. Diese Form der Gleichtaktspannung tritt näherungsweise bei der meistverwendeten Variante der Drehzeigermodulation auf (Kapitel 8).

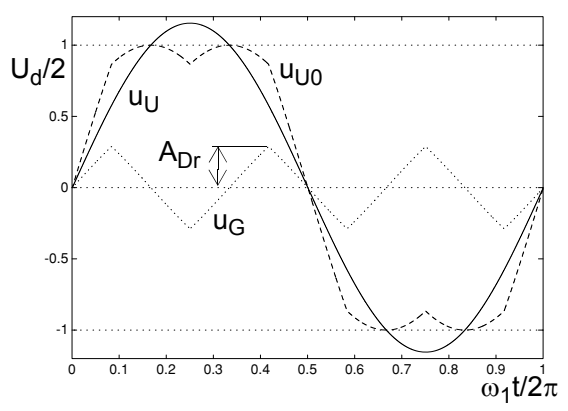

Bild 7.31.

Niederfrequente Anteile von Mittelpunkt-, Sternpunkt- und Phasenspannung bei Injektion eines Dreiecksignals, $M=1.155$

Bei Trägerverfahren wird normalerweise nur die einfachste Form, die Injektion einer Harmonischen, eingesetzt, da andere Signalverläufe keine Vergrösserung des Modulationsgrades mehr erlauben und auch bei den Verzerrungen keine Verbesserungen ergeben.

Bei allen vorgestellten Sollwerten mit einer Gleichtaktspannung ungleich null treten in den Schaltfunktionen zusätzliche Spektrallinien auf. Dies sind im Basisband der Mittel- 
punktspannung die injizierten Frequenzen. In den Trägerbändern sind weitere dominante Spektrallinien enthalten, die breiter um die Trägerfrequenzen verteilt sind als bei rein sinusförmigen Sollwerten. Diese Eigenschaften sind in Bild 7.30 rechts gut sichtbar.

Diskontinuierliche Sollwerte: Mit Hilfe geeigneter Gleichtaktsignale können die Sollwerte auch so verändert werden, dass in jeder Phase in bestimmten Ausschnitten Maximalaussteuerung auftritt. In diesen Zeitabschnitten wird dann der betreffende Brückenzweig nicht geschaltet, was zu einer reduzierten mittleren Schaltfrequenz und damit zu kleineren Schaltverlusten führt. Diese Verfahren werden vor allem bei Drehzeigermodulationen verwendet und deshalb in Kapitel 8.2.3 genauer betrachtet.

\subsubsection{Beschreibung von Spannungen und Strömen im Frequenzbereich}

Wie in Kapitel 3.3.2.3 gezeigt, lässt sich die Phasenspannung der dreiphasigen Brücke aus Mittelpunkt- und Sternpunktspannung zusammensetzen:

$$
u_{U / V / W}=u_{U 0 / V 0 / W 0}-u_{N 0}, \quad u_{N 0}=\frac{1}{3}\left(u_{U 0}+u_{V 0}+u_{W 0}\right)
$$

Die Gleichung (7.61) zeigt nochmals die bekannte Eigenschaft, dass für $u_{N 0}=0$ die Mittelpunkt- gleich den Phasenspannungen sind.

Für alle 3 Brückenzweige werde das gleiche, symmetrische Trägersignal verwendet. Die Sollwerte $x_{U \text {,Soll }}, x_{V, \text { Soll }}$ und $x_{W, \text { Soll }}$ unterscheiden sich nur durch ihre relativen Phasenlagen $\Delta \varphi_{U / V / W}=0,-2 \pi / 3$ und $-4 \pi / 3$. Sie werden wieder als Cosinusfunktionen angesetzt. Damit ergeben sich analog zu (7.16), (7.17) und (7.43) für die 3 Schaltfunktionen die Gleichungen (7.62) und (7.63). Multipliziert mit $U_{d} / 2$ entsprechen diese direkt den 3 Mittelpunktspannungen $u_{U 0}, u_{V 0}$ und $u_{W 0}$.

$$
\begin{aligned}
& x_{U / V / W, \text { Soll }}=M \cos \left(\omega_{1} t+\varphi_{x}+\Delta \varphi_{U / V / W}\right) \\
& s_{U / V / W, m}=M \cos \left(\omega_{1} t+\varphi_{x}+\Delta \varphi_{U / V / W}\right), \\
& s_{U / V / W, V Z}=\sum_{n=1 \mu=0}^{\infty} \sum_{n \mu} c_{n}\left\{\cos \left[\left(n \omega_{T} t+\mu \omega_{1} t\right)+\left(n \gamma+\mu\left\{\varphi_{x}+\Delta \varphi_{U / V / W}\right\}\right)\right]\right. \\
& c_{n \mu}=\frac{2 k_{\mu}}{n \pi} \sin \left[(n+\mu) \frac{\pi}{2}\right] J_{\mu}\left(n \frac{\pi}{2} M\right), \quad k_{\mu}=\left\{\begin{array}{l}
1 \\
\text { für } \mu=0 \\
2 \text { für } \mu=1,2,3, \ldots
\end{array}\right.
\end{aligned}
$$

Die Gleichungen zeigen, dass die Amplituden der einzelnen Frequenzkomponenten in allen 3 Phasen immer gleich sind und nur ihre jeweilige Phasenlage ändert. Weiter lassen sich die folgenden Eigenschaften daraus herleiten:

- Für die grundfrequenten Anteile der Schaltfunktionen gilt (7.64). Daraus folgt, dass die Grundschwingungen der Phasenspannungen direkt denjenigen der Mittelpunktspannungen entsprechen:

$$
s_{U, m}+s_{V, m}+s_{W, m}=0 \quad \Rightarrow \quad u_{U / V / W, v=1}=u_{U 0 / V 0 / W 0, v=1}
$$


- Alle in den Brückenzweigen auftretenden Vielfachen des Trägers $(n=1,3,5 \ldots$ und $\mu=0)$ sind in den 3 Phasen gleichphasig und fallen deshalb in den Phasenspannungen weg. Sie treten aber in der Sternpunktspannung $u_{N 0}$ auf.

- Für alle Vielfachen von $\mu=3(\mu=3,6,9, \ldots)$ werden die Anteile ebenfalls gleichphasig und fallen in den Phasenspannungen weg. Sie sind aber in der Sternpunktspannung enthalten.

- Für alle Frequenzen, bei denen die Sternpunktspannung $u_{N 0}=0$ ist, sind die entsprechenden Anteile in Phasen- und Mittelpunktspannung identisch.

Die besprochenen Eigenschaften sind z.B. im Spektrum in Bild 7.29 ersichtlich.

Die vorgestellte Berechnung der auftretenden Frequenzkomponenten gilt für Spannungssollwerte, die nur aus einer Grundschwingung bestehen. Wie in Kapitel 7.1.4 besprochen, lässt sie sich grundsätzlich auch auf Sollwerte, die höherfrequente Harmonische enthalten (Injektion von 3. Harmonischen) anwenden. Die Resultate werden aber sehr unübersichtlich. Deshalb wird darauf nicht weiter eingegangen.

Verzerrungsstrom: Ist das Spektrum der Phasenspannung bekannt, so kann daraus entsprechend den Gleichungen (7.23), (7.24) und (7.25) der Verzerrungsstrom in dieser Phase bestimmt werden. Da nachfolgend eine Näherung vorgestellt wird, soll auf die exakte Rechnung an dieser Stelle nicht weiter eingegangen werden.

Zwischenkreisstrom $i_{d}$ : Für den Zwischenkreisstrom gilt:

$$
i_{d}=\frac{1}{2}\left(i_{U^{S}}{ }_{U}+i_{V S_{V}}+i_{W^{S}}{ }_{W}\right)
$$

In Bild 7.32 sind der zeitliche Verlauf und das Spektrum des Zwischenkreisstromes $i_{d}$ für einen gegenüber dem sinusförmigen Spannungssollwert um $\pi / 4$ nacheilenden Phasenstrom dargestellt. Das zugehörige Spektrum der Phasenspannung entspricht demjenigen in Bild 7.29 rechts. Die Analyse der auftretenden Frequenzen kann in der gleichen Art vorgenommen werden, wie bei den einphasigen Schaltungen. Der Zwischenkreisstrom ist die Summe von 3 Teilströmen von der Art des Zwischenkreisstromes der Halbbrücke. Allerdings kompensieren sich bei symmetrischer Steuerung und Belastung aus Symmetriegründen eine ganze Reihe von Frequenzanteilen: einerseits treten dadurch keine Pulsationsanteile auf und andererseits bestehen die einzelnen Trägerbänder aus weniger Frequenzlinien. Bei synchroner Modulation liegen diese, wie im gezeigten Beispiel, alle auf Vielfachen der 6. Harmonischen.

\subsubsection{Näherungsformeln für die Beschreibung von Spannungen und Strömen}

Auch im dreiphasigen Fall können die auftretenden Spannungen und Ströme mit Näherungsformeln erfasst werden. Die Verhältnisse sind aber wesentlich unübersichtlicher als in einphasigen Schaltungen. Mit der nachfolgenden Überlegung lassen sie sich aber etwas vereinfachen: 

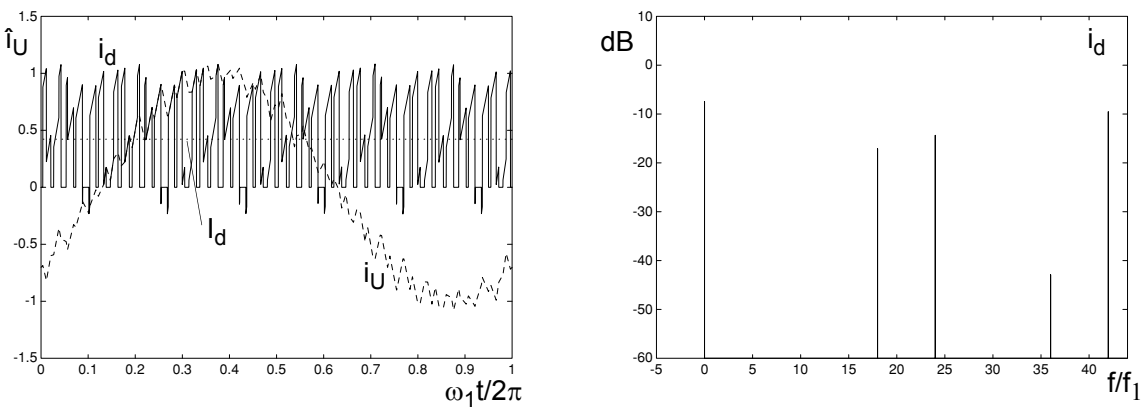

Bild 7.32. Links: zeitlicher Verlauf und Mittelwert des Zwischenkreisstromes sowie Strom der Phase $U, M=0.8, q=21, \hat{\imath}_{U / V / W}=0.2 I_{B}$, rechts: Spektrum von $i_{d}, 0 \mathrm{~dB}: \hat{\imath}_{U / V / W}$

- Die Spannungs- und Stromverläufe im symmetrischen Dreiphasensystem wiederholen sich im Winkelabstand von $\pi / 3$ mit alternierenden Vorzeichen und wechselnder Zuordnung zu den 3 Phasen.

- Die Summe der quadrierten Effektivwerte in allen 3 Phasen, gebildet über einen Winkel von $\pi / 3$, ist für jeden Sektor (Winkelausschnitt der Länge $\pi / 3$ ) identisch und es gilt für die Grösse $X$ (Spannung oder Strom):

$$
X_{e f f}^{2}=\left(X_{U, e f f}^{2}+X_{V, e f f}^{2}+X_{W, e f f}^{2}\right) / 3
$$

Es genügt also, die gewünschten Effektivwerte in einem beliebigen Winkelauschnitt zu berechnen. Dabei bewährt sich aber auch hier, den Ausschnitt so anzusetzen, dass einer der 3 Sollwerte eine reine Cosinusfunktion ist, da sich dann die übersichtlichsten Verhältnisse ergeben. Mit einem cosinusförmigen Verlauf in der Phase $U$ und einer beliebigen Gleichtaktkomponente $u_{G}$ gilt für die 3 Sollwertverläufe:

$$
\begin{aligned}
& u_{U 0 / V 0 / W 0, \text { Soll }}=\frac{U_{d}}{2} x_{U / V / W, \text { Soll }}=M \frac{U_{d}}{2} \cos \left(\omega_{1} t+\Delta \varphi_{U / V / W}\right)+u_{G} \\
& \Delta \varphi_{U / V / W}=0,-2 \pi / 3,-4 \pi / 3
\end{aligned}
$$

Bild 7.33 zeigt die Phasenspannung und den resultierenden Verzerrungsstrom der Phase $U$ für einen Sollwert ohne Gleichtaktkomponente. Nachfolgend wird von allen 3 Phasen nur ein Ausschnitt der Länge $\pi / 3$ benötigt, wie in Bild 7.34 dargestellt.

Die folgenden Berechnungen von Spannungen und Strömen basieren auf einer Modulation mit abgetasteten Sollwerten. Um die zeitkontinuierlichen Verläufe gut zu erfassen ohne die Rechnung unnötig zu komplizieren wird zweimal pro Trägerperiode abgetastet, d.h. mit der Frequenz $f_{\text {Tast }}=2 f_{T}$ bzw. der Abtastperiode $T_{\text {Tast }}=T_{T} / 2$. Abgetastet wird in jedem Scheitelpunkt des Trägers entsprechend dem double edge sampling in Kapitel 7.1.3.2. Die Resultate sind grundsätzlich nicht vom Abtastverfahren abhängig. Die Anzahl der Abtastungen beeinflusst aber die Genauigkeit der Resultate.

Vorerst wird angenommen, dass pro $\pi / 3$ Ausschnitt eine ganze Zahl von Abtastperioden enthalten sind. Damit ist die einfache Summation über eine Periode möglich. Später kann diese Voraussetzung wieder fallengelassen werden. 


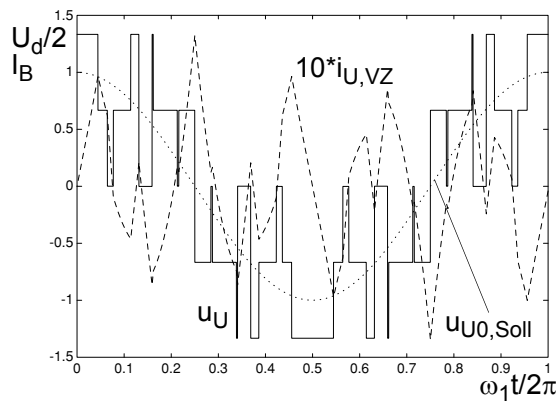

Bild 7.33.

Spannungssollwert, Phasenspannung und Verzerrungsstrom bei der dreiphasigen Brücke, $q=7, M=0.8$

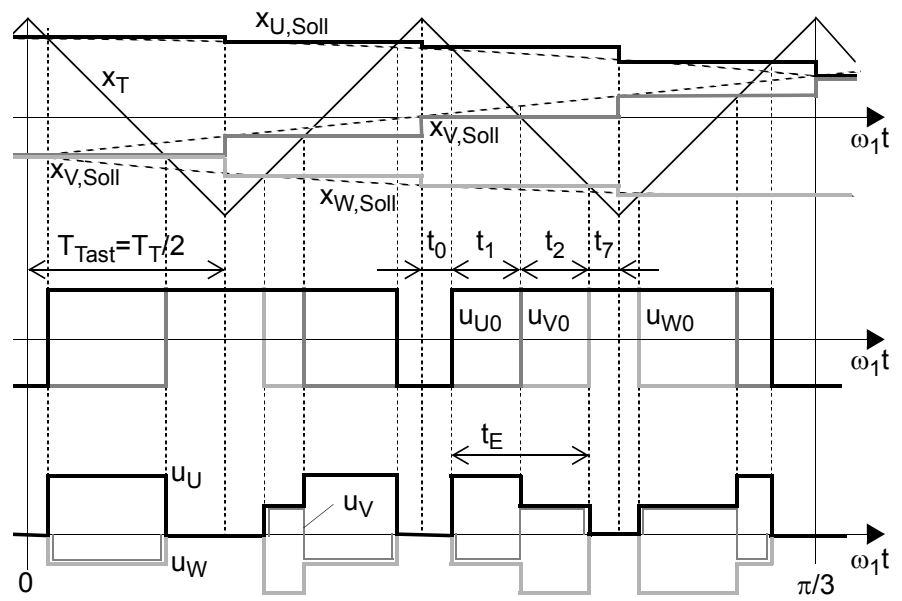

Bild 7.34.

Oben: Sollwert- und Trägersignale im Winkelausschnitt $[0, \pi / 3]$, mitte: Mittelpunktspannungen, unten: Phasenspannungen

Kurzzeitige Spannungsmittelwerte: Entsprechend dem Ziel der Modulation sind die kurzzeitigen Mittelwerte der Mittelpunktspannungen in guter Näherung proportional zu den Sollwerten. Die Übereinstimmung wird umso genauer, je höher die Trägerfrequenz ist:

$$
\bar{u}_{U 0 / V 0 / W 0} \approx u_{U 0 / V 0 / W 0, \text { Soll }}=\frac{U_{d}}{2} x_{U / V / W, \text { Soll }}
$$

Die Phasenspannungen $u_{U / V / W}$ entsprechen den Mittelpunktspannungen ohne Gleichtaktkomponente.

Phasenspannungs-Effektivwert: Allgemein gilt für den quadratischen Mittelwert einer abgetasteten Spannung mit dem Abtastintervall $T_{\text {Tast }}=T_{1} / 2 q$ :

$$
U_{\text {eff }}^{2}=\frac{1}{T_{1}} \sum_{k=1}^{L q} u_{k}^{2} T_{\text {Tast }}=\frac{T_{\text {Tast }}}{2 q T_{\text {Tast }}} \sum_{k=1}^{\angle q} u_{k}^{2}=\frac{1}{2 q} \sum_{k=1}^{\angle q} u_{k}^{2}
$$

Für die Berechnung der Phasenspannung werden die Sollwertverläufe nach (7.67) im Winkelbereich $[0, \pi / 3]$ abgetastet. In jedem Zustand der dreiphasigen Brücke in dem die 
Phasenspannungen ungleich null sind, treten an den Lasten betragsmässig nur zwei verschiedene Spannungsamplituden auf: einmal $2 U_{d} / 3$ und zweimal $U_{d} / 3$. Die Gesamtdauer dieser Zustände pro Abtastintervall wird mit $t_{E}$ bezeichnet (Bild 7.34). Für die Effektivwertbildung werden die Spannungen quadriert, d.h. die Vorzeichen spielen keine Rolle. Der Beitrag zum quadratischen Mittelwert der Summe der 3 Spannungen ist damit immer gleich gross:

$$
\begin{aligned}
& T_{\text {Tast }}\left(\Delta U_{U, \text { eff }}^{2}+\Delta U_{V, \text { eff }}^{2}\right.\left.\Delta U_{W, \text { eff }}^{2}\right)=\int_{0}^{t_{E}} u_{U}^{2} d t+\int_{0}^{t_{E}} u_{V}^{2} d t+\int_{0}^{t_{E}} u_{W}^{2} d t \\
&=\left[\left(\frac{2}{3} U_{d}\right)^{2}+\left(\frac{1}{3} U_{d}\right)^{2}+\left(\frac{1}{3} U_{d}\right)^{2}\right] t_{E}=\frac{2}{3} t_{E} U_{d}^{2}
\end{aligned}
$$

Die Gleichung zeigt, dass die Summe der quadrierten Effektivwerte der Phasenspannungen proportional zu der Zeit ist, in der die Ausgangsspannungen des Stromrichters ungleich null sind. Damit der Verzerrungsanteil der Phasenspannungen möglichst klein ist, muss der Stromrichter für eine vorgegebene Grundschwingungsamplitude also möglichst kurz in diesen Zuständen verbleiben.

Für die weitere Berechnung müssen die Beiträge in (7.70) über eine Sechstelperiode summiert und gemittelt werden. Mit $N=2 q / 6$ gilt:

$$
3 U_{\text {eff }}^{2}=U_{U, \text { eff }}^{2}+U_{V, \text { eff }}^{2}+U_{W, \text { eff }}^{2}=\frac{2}{3} U_{d}^{2} \frac{1}{N T_{\text {Tast }} \sum_{N}} t_{E}=\frac{2}{3} U_{d}^{2} \frac{1}{N} \sum_{N} \frac{t_{E}}{T_{\text {Tast }}}
$$

Da die Effektivwerte in allen 3 Phasen gleich sind, kann die Gleichung nach dem Effektivwert pro Phase aufgelöst werden:

$$
U_{U / V / W, \text { eff }}=\sqrt{\frac{1}{3} \frac{2}{3} U_{d}^{2} \frac{1}{N} \sum_{N} \frac{t_{E}}{T_{\text {Tast }}}}=\frac{\sqrt{2}}{3} U_{d} \sqrt{\frac{1}{N} \sum_{N} \frac{t_{E}}{T_{\text {Tast }}}}
$$

Für die Bestimmung der Spannungseffektivwerte muss jetzt noch die Summe der gemittelten Einschaltzeiten $t_{E} / T_{\text {Tast }}$ bestimmt werden. Die Einschaltzeit $t_{E}$ lässt sich ausgehend von Bild 7.34 berechnen:

$$
t_{E}=t_{1}+t_{2}=T_{\text {Tast }} \frac{\left(u_{U 0, \text { Soll }}-u_{W 0, \text { Soll }}\right)}{U_{d}}=\frac{\sqrt{3}}{2} M T_{\text {Tast }} \sin \left(\omega_{1} t+\frac{\pi}{3}\right)
$$

Wird die Länge der Abtastintervalle klein gewählt, so kann die Summation durch ein Integral ersetzt werden:

$$
\frac{1}{N} \sum \frac{t_{E}}{T_{\text {Tast }}} \approx \frac{\sqrt{3}}{2} M\left[\frac{1}{\pi / 3} \int_{0}^{\pi / 3} \sin \left(\omega_{1} t+\frac{\pi}{3}\right) d\left(\omega_{1} t\right)\right]=\frac{3 \sqrt{3}}{2 \pi} M
$$

Die Näherung für die Summe in (7.74) kann nun in (7.72) eingesetzt werden und es resultiert für den Spannungseffektivwert in jeder Phase: 


$$
U_{U / V / W, \text { eff }} \approx U_{d} \sqrt{\frac{M}{\sqrt{3} \pi}}
$$

Die Gleichung zeigt, dass bei genügend kleinen Tastintervallen der Effektivwert unabhängig von der Schaltzahl ist. Für den maximal möglichen Modulationsgrad im Dreiphasensystem, $M=2 / \sqrt{3}$, erhält man den maximalen Effektivwert:

$$
U_{U / V / W, \text { eff, } \text { max }} \approx U_{d} \sqrt{\frac{2}{3 \pi}}=0.461 U_{d}
$$

Wie die Berechnung zeigt, ist der Effektivwert der Phasenspannungen unabhängig von der Gleichtaktspannung. Diese setzt nur den jeweiligen maximalen Modulationsgrad fest.

Effektivwert der Spannungsgrundschwingung: Der Effektivwert der Phasenspannungs-Grundschwingung entspricht in sehr guter Näherung dem Effektivwert der Sollwertspannungen ohne Gleichtaktkomponente:

$$
U_{v=1, \text { eff }}=\frac{1}{\sqrt{2}} \frac{U_{d}}{2} M
$$

Spannungs-Klirrfaktor: Aus den Effektivwerten $U_{\text {eff }}$ und $U_{v=1, \text { eff }}$ kann der Spannungsklirrfaktor berechnet werden:

$$
k_{u}=\frac{\sqrt{U_{e f f}^{2}-U_{v 1, e f f}^{2}}}{U_{e f f}} \approx \sqrt{1-M \frac{\sqrt{3} \pi}{8}}
$$

Der Klirrfaktor liegt im Bereich $0.463 \leq k_{u} \leq 1$, mit einem Minimum beim maximalen Modulationsgrad $M=2 / \sqrt{3}=1.155$ und einem Maximum bei $M=0$.

Bild 7.35 zeigt die Verläufe der exakten simulierten und der mittels Näherungsformeln erhaltenen Effektivwerte sowie der zugehörigen Klirrfaktoren für zwei verschiedene Gleichtaktspannungen. Die Rechnungen wurden für verschiedene Verhältnisse von Träger- zu Grundfrequenz durchgeführt. Die Vergleiche zeigen, dass die Näherungsrechnung unabhängig von der Gleichtaktspannung bei Verhältnissen von $T_{\text {Tast }} / T_{1}>10$, bzw. Schaltzahlen $q>5$ sehr gute Resultate ergibt, die mit wachsender Abtastfrequenz schnell noch besser werden. Bei abgetasteten Sollwerten ist die Übereinstimmung sogar noch etwas besser als beim dargestellten natural sampling. Dies ist leicht vorstellbar, da die Rechnung ja auf abgetasteten Grössen basiert.

Effektivwert des Verzerrungsstromes: Betrachtet werden die Phasenspannungen und -ströme innerhalb eines Abtastintervalles $T_{\text {Tast }}$ in Bild 7.36. Der Winkelausschnitt entspricht etwa dem zweitletzten Abtastintervall in Bild 7.34.

Für die Berechnung des Verzerrungsstromes wird die Last gemäss Bild 7.28 durch die Induktivitäten $L_{k}$ und die Gegenspannungen $e_{U}, e_{V}$ und $e_{W}$ beschrieben. Für die Berechnung werden zusätzlich folgende Annahmen getroffen: 

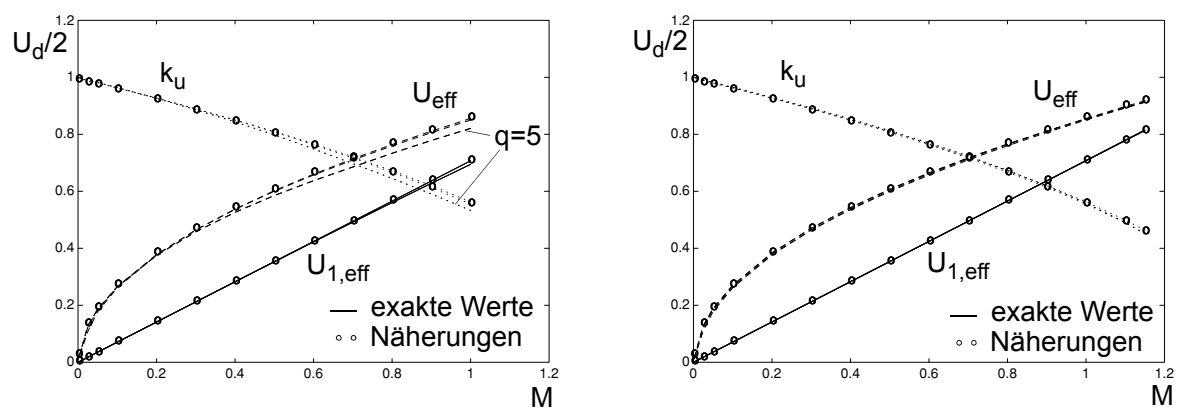

Bild 7.35. Phasenspannung, Spannungsgrundschwingung und zugehöriger Klirrfaktor für zeitkontinuierliche Sollwerte und Schaltzahlen von $q=5,11,21$, links: Gleichtaktspannung null, rechts: $16.7 \%$ 3. Harmonische
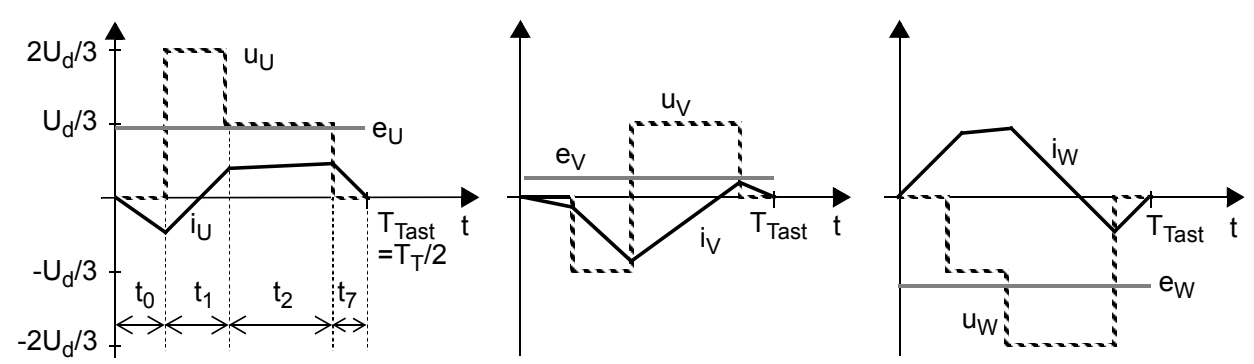

Bild 7.36. Phasenspannungen und -ströme innerhalb eines Abtastintervalles

- Die Sollwerte $u_{U 0, \text { Soll }}, u_{V 0, \text { Soll }}$ und $u_{W 0, \text { Soll }}$ werden am Anfang jedes Abtastintervalls abgetastet.

- Die Gegenspannungen sind innerhalb einer Abtastperiode $T_{\text {Tast }}\left(=T_{T} / 2\right)$ konstant. Sie entsprechen in Betrag und Phase den Spannungssollwerten ohne Gleichtaktkomponenten $e_{U / V / W}=u_{U / V / W, \text { Soll }}$. Somit tritt kein Grundschwingungsstrom auf.

- Zu Beginn und am Ende jedes Abtastintervalles ist der Verzerrungsstrom null.

Mit den getroffenen Annahmen können jetzt die Stromverläufe (7.79) innerhalb eines Abtastintervalles und damit die Beiträge $\Delta I^{2}$ VZ,eff aller 3 Phasen zum Effektivwert (7.80) des Stromes berechnet werden.

$$
\begin{aligned}
& i_{U / V / W}=\frac{1}{L_{k}} \int_{0}^{T_{T} / 2}\left(u_{U / V / W}-e_{U / V / W}\right) d t \\
& 3 \Delta I_{U / V / W, V Z, \text { eff }}^{2} \frac{T_{T}}{2}=\int_{0}^{T_{T} / 2} i_{U}^{2} d t+\int_{0}^{T_{T} / 2} i_{V}^{2} d t+\int_{0}^{T_{T} / 2} i_{W}^{2} d t
\end{aligned}
$$


Obwohl die Stromverläufe innerhalb eines Abtastintervalls stückweise linear sind, wird die analytische Berechnung aufwendig. Deshalb werden nur noch interessante Teilresultate betrachtet. Für den Verlauf des 'kurzzeitigen Effektivwertes' gilt (7.81).

Für die weitere Auswertung von (7.81) werden jetzt die Zeiten $t_{0}$, $t_{1}$ und $t_{2}$ benötigt. Diese lassen sich mit Hilfe der abgetasteten Spannungsverläufe in Bild 7.34 einfach bestimmen. Alle 3 Zeiten sind Funktionen des Winkels $\omega_{1} t$ und des Modulationsgrades. $t_{0}$ ist zusätzlich von der Gleichtaktspannung abhängig (7.82).

$$
\begin{aligned}
& 3 \Delta I_{U / V / W, V Z, \text { eff }}^{2}\left(\omega_{1} t\right)=\left(\frac{U_{d}}{L_{k}}\right)^{2}\left\{\frac{M^{2}}{8}\left[\left(\frac{T_{T}}{2}\right)^{2}-3 \frac{T_{T}}{2}\left(t_{0}+t_{1}\right)+3\left(t_{0}+t_{1}\right)^{2}\right]\right. \\
& \quad-\frac{M}{6\left(T_{T} / 2\right)}\left[\cos \left(\omega_{1} t\right)\left(2 t_{1}^{3}+3 t_{0} t_{1}^{2}\right)+\cos \left(\frac{\pi}{3}-\omega_{1} t\right) t_{2}^{2}\left(3 \frac{T_{T}}{2}-3 t_{0}-3 t_{1}-t_{2}\right)\right] \\
& \left.+\frac{2}{9\left(T_{T^{\prime}}\right)}\left[t_{1}^{3}+t_{2}^{3}\right]\right\}
\end{aligned}
$$

\section{Gleichtaktspannung null:}

$$
t_{0}=\frac{1}{2} \frac{T_{T}}{2}\left[1-M \cos \left(\omega_{1} t\right)\right]
$$

Gleichtaktspannung für $t_{0}=t_{7}$ :

$$
t_{0}=\frac{1}{2}\left(\frac{T_{T}}{2}-t_{1}-t_{2}\right)=\frac{1}{2} \frac{T_{T}}{2}\left[1-M \frac{\sqrt{3}}{2} \sin \left(\omega_{1} t+\pi / 3\right)\right]
$$

Diese Gleichtaktspannung entspricht der symmetrischen Verteilung der Nullzustände bei der Drehzeigermodulation, die in Kapitel 8.2.3 betrachtet wird. Ihr Verlauf ist näherungsweise dreieckförmig mit der Kreisfrequenz $3 \omega_{1}$ und der Amplitude $\hat{u}_{G}=(M / 4)\left(U_{d} / 2\right)$.

Gleichtaktspannung bei Injektion einer 3. Harmonischen: Wird die Amplitude der injizierten Harmonischen so gewählt, dass die Stromverzerrungen minimal sind, resultiert (7.84) und bei einer Amplitude für maximale Aussteuerung (7.85).

$$
\begin{array}{ll}
\hat{u}_{G, \text { Soll }}=\frac{M}{4} \frac{U_{d}}{2}, & t_{0}=\frac{1}{2} \frac{T_{T}}{2}\left\{1+M\left[\frac{1}{4} \cos \left(3 \omega_{1} t\right)-\cos \left(\omega_{1} t\right)\right]\right\} \\
\hat{u}_{G, \text { Soll }}=\frac{M}{6} \frac{U_{d}}{2}, & t_{0}=\frac{1}{2} \frac{T_{T}}{2}\left\{1+M\left[\frac{1}{6} \cos \left(3 \omega_{1} t\right)-\cos \left(\omega_{1} t\right)\right]\right\}
\end{array}
$$

Für die Zeiten $t_{1}$ und $t_{2}$ gelten (7.86) und (7.87):

$$
t_{1}=\frac{T_{T}}{2} \frac{\left(u_{U 0, \text { Soll }}-u_{V 0, \text { Soll }}\right)}{U_{d}}=\frac{T_{T}}{2} M \frac{\sqrt{3}}{2} \sin \left(\pi / 3-\omega_{1} t\right)
$$




$$
t_{2}=\frac{T_{T}}{2} \frac{\left(u_{V 0, \text { Soll }^{-}} u_{W 0, \text { Soll }}\right)}{U_{d}}=\frac{T_{T}}{2} M \frac{\sqrt{3}}{2} \sin \left(\omega_{1} t\right)
$$

Die in (7.82) bis (7.87) beschriebenen Zeiten können nun in (7.81) eingesetzt werden. Analog zur Berechnung des Spannungseffektivwertes kann durch Aufsummieren und Mitteln der Beiträge zum Stromeffektivwert der mittlere Stromeffektivwert bestimmt werden. Da sich die Verhältnisse immer nach $\pi / 3$ wiederholen, genügt es über diesen Winkelbereich zu mitteln. Der Winkelschritt beträgt $\Delta \omega_{1} t=2 \pi T_{\text {Tast }} / T_{1}=2 \pi /(6 N)$. Für den Effektivwert in einer Phase gilt:

$$
\begin{aligned}
3 I_{U / V / W, V Z, \text { eff }}^{2} & =\frac{1}{N} \sum_{\substack{k=1 \\
N}}^{N} 3 \Delta I_{V Z, \text { eff }}^{2}\left(k \Delta \omega_{1} t\right) \\
I_{U / V / W, V Z, \text { eff }}^{2} & =\frac{1}{N} \sum_{k=1}^{N} \Delta I_{V Z, \text { eff }}^{2}\left(k \Delta \omega_{1} t\right)=\frac{1}{\pi / 3} \sum_{k=1}^{N} \Delta I_{V Z, \text { eff }}^{2}\left(\frac{k}{N} \frac{\pi}{3}\right)\left(\Delta \omega_{1} t\right)
\end{aligned}
$$

Bei sehr kleinen Winkelschritten $\Delta \omega_{1} t$, d.h. grossen Verhältnissen von Abtast- zu Grundfrequenz, kann in (7.89) die Summation durch eine Integration ersetzt werden:

$$
I_{U / V / W, V Z, \text { eff }}^{2}=\frac{1}{\pi / 3} \int_{0}^{\pi / 3} \Delta I_{V Z, \text { eff }}^{2}\left(\omega_{1} t\right) d\left(\omega_{1} t\right)
$$

Mit dem Integral (7.90) kann nun der Stromeffektivwert in Funktion des Modulationsgrades für die verschiedenen Gleichtaktspannungen berechnet werden. Nach einer Zwischenrechnung resultiert:

$$
I_{U / V / W, V Z, \text { eff }} \approx \frac{U_{d} M}{4 L_{k} \sqrt{3}} \frac{T_{T}}{2} \sqrt{0.5-0.735 M+K_{G} M^{2}}
$$

Abhängig von den Gleichtaktspannungen beträgt der Faktor $K_{G}$ :

- Gleichtaktspannung $\mathrm{u}_{G}=0: K_{G}=0.375$.

- Gleichtaktspannung für $t_{0}=t_{7}: K_{G}=0.330$.

- Gleichtaktspannung mit 16.7\% 3. Harmonischer: $K_{G}=0.334$

- Gleichtaktspannung mit 25\% 3. Harmonischer: $K_{G}=0.328$

Die Gleichung zeigt, dass wie zu erwarten war, der Verzerrungsstrom mit steigendem Verhältnis von Grund- zu Trägerfrequenz, d.h. steigender Schaltzahl, abnimmt. Bild 7.37 zeigt die Stromeffektivwerte für verschiedene Gleichtaktspannungen.

Bei einer Gleichtaktspannung von null kann der Modulationsgrad $M$ maximal eins werden. Der Vergleich der angenäherten mit den simulierten exakten Werten in Bild 7.37 zeigt, dass bei steigendem Verhältnis von Träger- zu Grundschwingungsfrequenz die Übereinstimmung schnell sehr gut wird. Bei den abgetasteten Sollwerten ist die Genauigkeit zwischen Rechnung und Simulation noch besser, da für die Rechnung ja von abgetasteten Sollwerten ausgegangen wurde. 

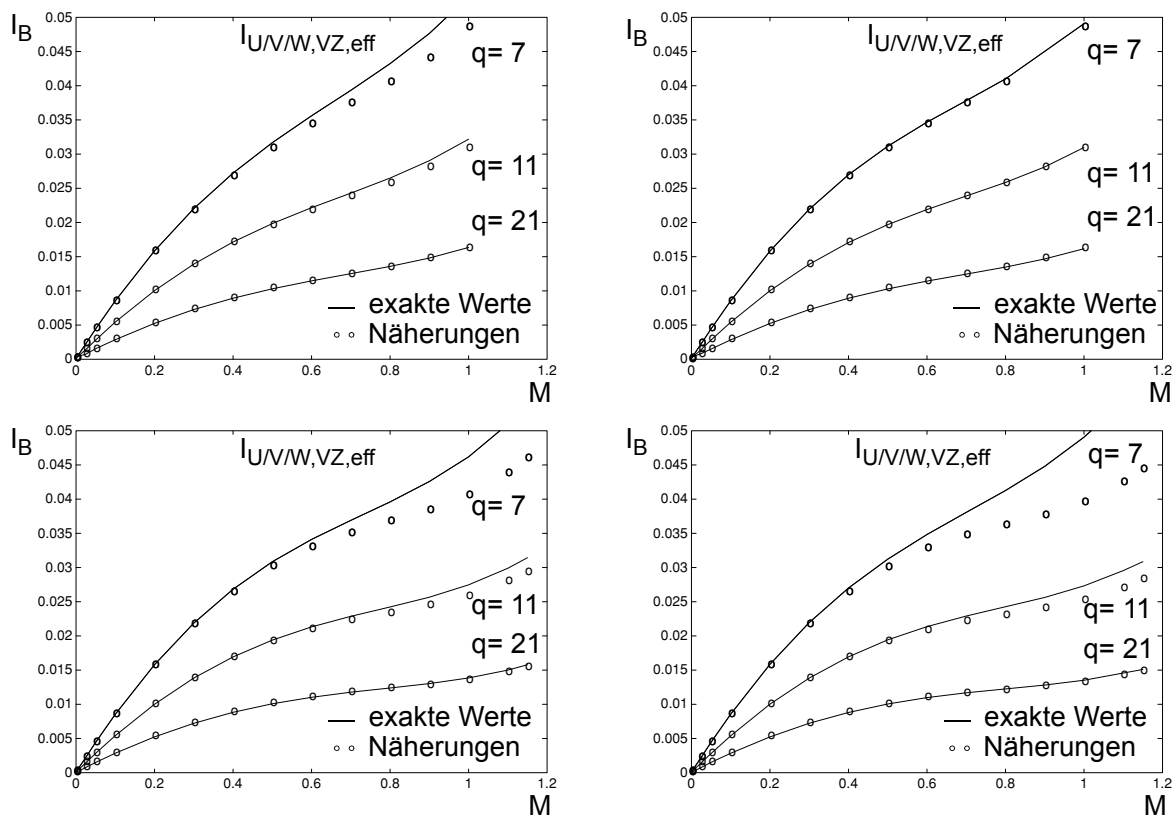

Bild 7.37. Effektivwerte der Verzerrungsströme für oben links: zeitkontinuierliche Sollwerte und $u_{G}=0$, oben rechts: abgetastete Sollwerte und $u_{G}=0$, unten links: zeitkontinuierliche Sollwerte und Injektion von $16.7 \% 3$. Harmonischer, unten rechts: zeitkontinuierliche Sollwerte und Injektion von 25\% 3. Harmonischer

Bei der Injektion von 3. Harmonischen zeigt sich, dass die Verzerrungsströme kleiner werden als bei einer Gleichtaktspannung von null. Es wird aber auch deutlich, dass sich die Verzerrungen mit $25 \% 3$. Harmonischen gegenüber $16.7 \%$ nur sehr geringfügig verkleinern lassen. Die Verzerrungsströme für abgetastete Sollwertsignale entsprechen mit sehr guter Genauigkeit den mittels Näherungsrechnung erhaltenen Werten.

Spitzenwert des Verzerrungsstromes: Der Spitzenwert des Verzerrungsstromes tritt beim Nulldurchgang der Phasenspannung auf (Bild 7.33). Für die Phasenspannung und den Verzerrungsstrom in diesem Punkt gelten näherungsweise die Verläufe in Bild 7.38.

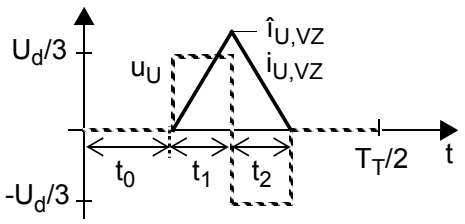

Bild 7.38.

Phasenspannung und Verzerrungsstrom beim Nulldurchgang der Spannung

Unter der Annahme, dass die Gegenspannung in diesem Punkt innerhalb einer Abtastpe- 
riode $T_{T} / 2$ konstant gleich null ist, fällt die Phasenspannung nur über der Induktivität $L_{k}$ ab. Wird weiter angenommen, dass der Verzerrungsstrom zu Beginn des positiven Spannungspulses null ist, so gilt:

$$
\hat{i}_{U, V Z}=\frac{U_{d}}{3} \frac{1}{L_{k}} t_{1} \quad \text { und } \quad t_{1}=t_{2}=\frac{\sqrt{3}}{2} \frac{1}{2} \frac{T_{T}}{2} M
$$

Das Auflösen von (7.92) ergibt für den Spitzenwert des Verzerrungsstromes in Funktion des Modulationsgrades $M$ :

$$
\hat{i}_{U / V / W, V Z}=\frac{1}{8 \sqrt{3}} \frac{U_{d} T_{T} M}{L_{k}}
$$

Diese Gleichung gilt in guter Näherung und ist unabhängig von der Gleichtaktspannung. Den jeweiligen Maximalwert erhält man durch Einsetzen des grössten Modulationsgrades.

Gleichtaktspannung für minimalen Verzerrungsstrom: Aus Gleichung (7.81) lässt sich die Zeit $t_{0}$ bestimmen, welche den minimalen Verzerrungsstrom ergibt. Dazu wird die Gleichung nach $t_{0}$ abgeleitet und gleich null gesetzt:

$$
\frac{\partial}{\partial t_{0}}\left[3 \Delta I_{U / V / W, V Z, e f f}^{2}\left(\omega_{1} t\right)\right]=0
$$

Die Auflösung von (7.94) ergibt nach längerer Zwischenrechnung für $t_{0}$ :

$$
t_{0}=\frac{T_{T} / 2}{2}\left\{1+M\left[\frac{1}{4} \cos \left(3 \omega_{1} t\right)-\cos \left(\omega_{1} t\right)\right]\right\}
$$

Aus $t_{0}$ in Funktion des Winkels $\omega_{1} t$ kann die entsprechende Gleichtaktspannung $u_{G}$ ermittelt werden:

$$
u_{G}\left(\omega_{1} t\right)=-\frac{M}{4} \frac{U_{d}}{2} \cos \left(3 \omega_{1} t\right)
$$

Wie schon früher ohne Beweis erwähnt, besteht die optimale Gleichtaktspannung für minimalen Verzerrungsstrom aus einer reinen 3. Harmonischen mit einer Amplitude von $25 \%$ der Grundschwingung.

\subsubsection{Drehmomentwelligkeit}

Für die Drehmomentwelligkeit im Leerlauf in einem Antrieb kann von der in Kapitel 5.2.1.4 eingeführten Drehzeigergleichung (7.97) ausgegangen werden:

$$
M_{e l, V Z}=\frac{3}{2 \omega_{1}} \operatorname{Re}\left(\underline{u}_{-S t r, v=1}{ }^{*} i_{-S t r, V Z}\right)
$$

Die Spannungen und Ströme in Zeigerdarstellung können mittels der Transformationen in Anhang B.1.2 aus den Phasengrössen bestimmt werden: 


$$
\begin{aligned}
{ }_{-S t r, V Z}=\frac{1}{L_{k}} \int\left(\underline{u}_{-S t r}-\underline{u}_{S t r, v=1}\right) d t & \\
\Rightarrow \quad M_{e l, V Z}= & \frac{3}{2 \omega_{1} L_{k}} \operatorname{Re}\left[\underline{u}_{S t r, v=1} * \int\left(\underline{u}_{-S t r}-\underline{u}_{S t r, v=1}\right) d t\right] \\
= & \frac{3}{2 \omega_{1} L_{k}}\left[u_{S t r, v=1, \alpha} \int\left(u_{S t r, \alpha}-u_{S t r, v=1, \alpha}\right) d t\right. \\
& \left.\quad+u_{S t r, v=1, \beta} \int\left(u_{S t r, \beta}-u_{S t r, v=1, \beta}\right) d t\right]
\end{aligned}
$$

Die Drehmomentverläufe für zwei verschiedene Gleichtaktspannungen sind in Bild 7.39 dargestellt. Die Verläufe und Spitzenwerte sind leicht von der Phasenlage des Trägersignals bezüglich der Grundschwingungen und stark von der Gleichtaktspannung abhängig.
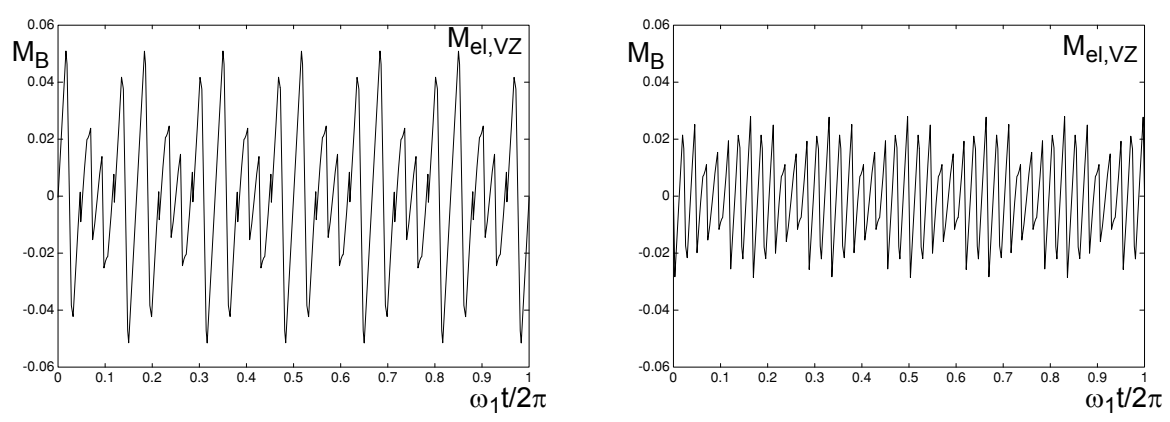

Bild 7.39. Drehmomentwelligkeit im Leerlauf bei $q=21, \omega_{1}=\omega_{B}$ und $M=1$, links: Gleichtaktspannung null, rechts: Injektion von $25 \% 3$. Harmonischen

Bei der Drehmomentwelligkeit interessiert vor allem der Spitzenwert. Dieser kann mit einer weiteren Näherungsrechnung bestimmt werden. Dazu werden dieselben Annahmen getroffen wie bereits bei der Berechnung des Effektivwertes für die Phasenströme. Insbesondere wird der Zeiger $\underline{u}_{S t r, v=1}$ innerhalb eines Taktintervalles $T_{T}$ als konstant angenommen. Bild 7.40 links zeigt den entsprechenden typischen Drehmomentverlauf während eines solchen Taktintervalles. Der Spitzenwert wird darin am Ende von $t_{1}$ erreicht. Er wird u.a. dann am grössten, wenn der Zeiger $\underline{U}_{S t r, v=1}$ auf der reellen Achse liegt. Dann ist $t_{1}$ maximal und $t_{2}=0$. Durch die Symmetrie des Dreiphasensystems tritt dieser Maximalwert sechsmal pro Periode in Abständen von $\pi / 3$ auf.

Die Berechnung des Spitzenwertes kann vorgenommen werden, in dem mit Hilfe der Zeiten $t_{0}, t_{1}$ und $t_{7}$ aus (7.82) bis (7.87) die Integralgleichung (7.98) für $\omega_{1} t=0$ gelöst wird. Es ergibt sich die folgende Formel: 


$$
\hat{M}_{e l, V Z} \approx \frac{3 T_{T}}{8 \omega_{1} L_{k}}\left(\frac{U_{d}}{2}\right)^{2} M^{2}\left[\frac{3}{2}\left(\frac{4}{3}-M\right)-\frac{4 t_{0}\left(\omega_{1} t=0\right)}{T_{T}}\right]
$$

$t_{0}$ ist abhängig von der Art der Gleichtaktspannung. Die Resultate für verschiedene Gleichtaktspannungen sind in Bild 7.40 rechts dargestellt. Die Trägerfrequenz ist dabei
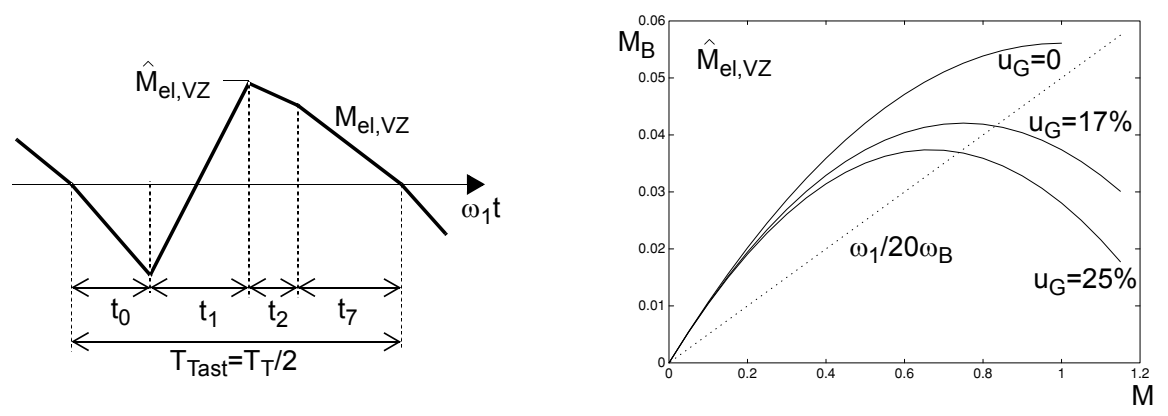

Bild 7.40. Links: Verläufe für die Berechnung der Drehmomentwelligkeit, rechts: Spitzenwert der Drehmomentpulsation für Gleichtaktspannungen von $u_{G}=0 \%$, $16.7 \%$ und $25 \% 3$. Harmonischer, $q=21$ bei $M=1$

(zusätzlich ist der bezogene Verlauf von $\omega_{1}$ aufgetragen)

konstant und die Grundfrequenz wird, wie bei einem Antrieb üblich, proportional zum Modulationsgrad variiert. Die Kurven beschreiben den maximal möglichen Spitzenwert. Bei niedriger Schaltzahl wird dieser jedoch nicht regelmässig erreicht, weil sich dann die Spannungsgrundschwinungen innerhalb eines Taktintervalles leicht ändern. Bei asynchroner Modulation tritt der berechnete Spitzenwert deshalb nur sporadisch auf, nämlich wenn das Taktintervall bezüglich der Grundschwingung gerade besonders ungünstig zu liegen kommt. Bei synchroner Modulation dagegen kann je nach Phasenlage des Trägersignals dauernd ein leicht kleinerer Spitzenwert resultieren.

Interessant ist die Tatsache, dass die Spitzenwerte der Drehmomentwelligkeit bei den gleichen Gleichtaktspannungen minimal werden, wie die Verzerrungsströme. Wird die Gleichtaktspannung so gewählt, dass sich $t_{0}=t_{7}$ ergibt, so hat die Drehmomentpulsation den gleichen Verlauf wie bei $25 \% 3$. Harmonischer.

Literatur zu Kapitel 7.4: [Boo1], [Bos1], [Bow4], [Boy1] [Broe1], [Dep1], [Kli1], [Kol1], [Kol2], [Schö1], [Ste1], [Ste2], [Ric1], [Trz1], [Wei1] 


\section{Drehzeigermodulation}

Die Drehzeigermodulation ist heute wohl das wichtigste und gebräuchlichste On-Line Modulationsverfahren für dreiphasige Brückenschaltungen. Sie ist eng mit den Trägerverfahren für die gleichen Schaltungen verwandt. Es kann gezeigt werden, dass die Drehzeigermodulation einer Trägermodulation mit abgetasteten Sollwerten entspricht. Wie der Name andeutet, werden die Schaltfunktionen mit Hilfe von Drehzeigern erzeugt. Für die Beschreibung des Verfahrens werden die Drehzeigerdarstellung in Anhang B.1.2 und die Darstellung der Stromrichtergrössen mittels Zeigern in Kapitel 3.3.2.3 benötigt. Die betrachtete Schaltung und die Drehzeiger der zugehörigen geschalteten Mittelpunkt- bzw. Phasenspannungen in der $\alpha \beta$-Ebene zeigt Bild 8.1. Da jede dreiphasige Brückenschaltung nur die 8 diskreten Zustände ${ }^{0} Z \ldots{ }^{7} Z$ einnehmen kann, sind die Momentanwerte von Mittelpunkt- und Phasenspannungen und damit die entsprechenden 8 Spannungszeiger ${ }^{0} \underline{U}$ bis ${ }^{7} \underline{U}$ bestimmt.Von den Zeigern sind sechs ungleich null und liegen paarweise mit umgekehrtem Vorzeichen auf den 3 um $2 \pi / 3$ versetzten Achsen $a, b, c$. Die zwei Zeiger ${ }^{0} \underline{U}$ und ${ }^{7} \underline{U}$ haben die Länge null. Zwischen den Spannungszeigern ${ }^{1} \underline{U} . . .6{ }^{6} \underline{U}$ liegen die Sektoren $S 1 \ldots S 6$. Die Länge der transformierten Zeiger ${ }^{1} \underline{U} . . .6 \underline{U}$ beträgt je $2 U_{d} / 3$.
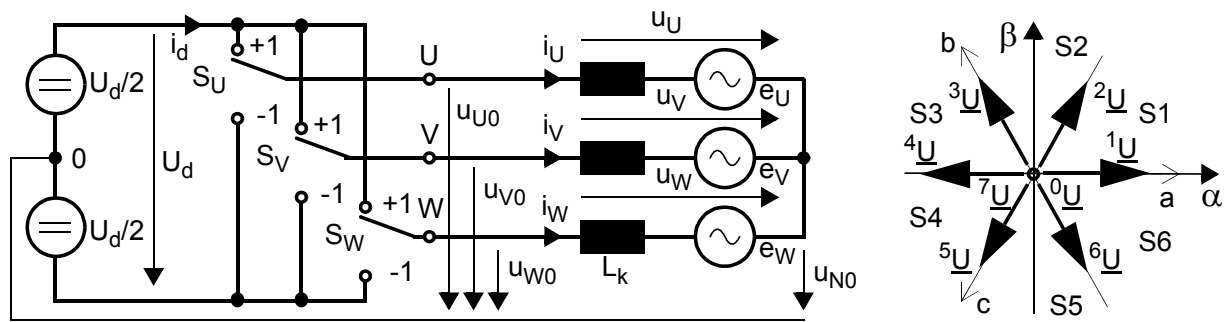

Bild 8.1. Links: dreiphasige Brücke im Zustand ${ }^{7} Z$, rechts: Spannungszeiger ${ }^{0} U . .{ }^{7} U$

\subsection{Funktionsprinzip der Drehzeigermodulation}

Analog wie bei den Phasengrössen in Kapitel 3.3.1 können kurzzeitige Mittelwerte von Drehzeigern definiert werden. Die Mittelung erfolgt dabei über eine Abtastperiode $T_{\text {Tast }}$. Der kurzzeitige Mittelwert des Spannungszeigers $\underline{u}_{\text {Str }}$ wird damit wie folgt gebildet:

$$
\bar{u}_{-S t r}=\frac{1}{T_{\text {Tast }}} \int_{t}^{t+T_{\text {Tast }}} \underline{u}_{-S t r} d t=\frac{1}{T_{\text {Tast }}} \sum_{k}^{k} \underline{U} t_{k}, \quad \sum_{k} t_{k}=t_{\text {Tast }}
$$


${ }^{k} \underline{U}$ sind die während der Zeit $t_{k}$ auftretenden Spannungszeiger. Für den in Bild 8.2 links dargestellten Zeiger $\underline{u}_{\text {Str }}$ gilt so:

$$
\underline{\bar{u}}_{-S t r}={ }^{1} \underline{u}+{ }^{2} \underline{u}, \quad \text { mit } \quad{ }^{1} \underline{u}=\frac{t_{1}}{T_{\text {Tast }}} \underline{1}, \quad{ }^{2} \underline{u}=\frac{t_{2}}{T_{\text {Tast }}}{ }^{2} \underline{U}
$$

Ziel der Drehzeigermodulation ist nun, den Zeiger $u_{S t r}$ so zu erzeugen, dass er dem Sollwert $\underline{u}_{\text {Soll }}$ möglichst gut entspricht. Den prinzipiellen Ablauf zeigt Bild 8.2 rechts: $\underline{u}_{S o l l}$ wird in festen Zeitabständen $T_{\text {Tast }}$ abgetastet und dann mit den möglichen diskreten Stromrichterspannungen nachgebildet. Für die Bildung von $u_{S t r}$ in einer Abtastperiode werden in der Praxis nur zwei der spannungsbildenden Zeiger ${ }^{-1} \underline{U} . .{ }^{6} \underline{U}$ verwendet. Deren Gewicht wird durch ihre Einschaltdauer variiert. Während der verbleibenden Zeit einer Abtastperiode werden einer oder beide Nullspannungszeiger ${ }^{0} \underline{U}$ bzw. ${ }^{7} \underline{U}$ angelegt. Der Betrag von $\underline{u}_{S t r}$ ist durch die Maximalaussteuerung begrenzt.

Je grösser die Abtastfrequenz, desto kleiner werden der Verzerrungsstrom und die Drehmomentwelligkeit. Das Verhältnis von Abtast- zu Grundschwingungsperiode $T_{\text {Tast }}$ t $T_{1}$ kann beliebig sein, d.h. das Verfahren ist vom Prinzip her asynchron.
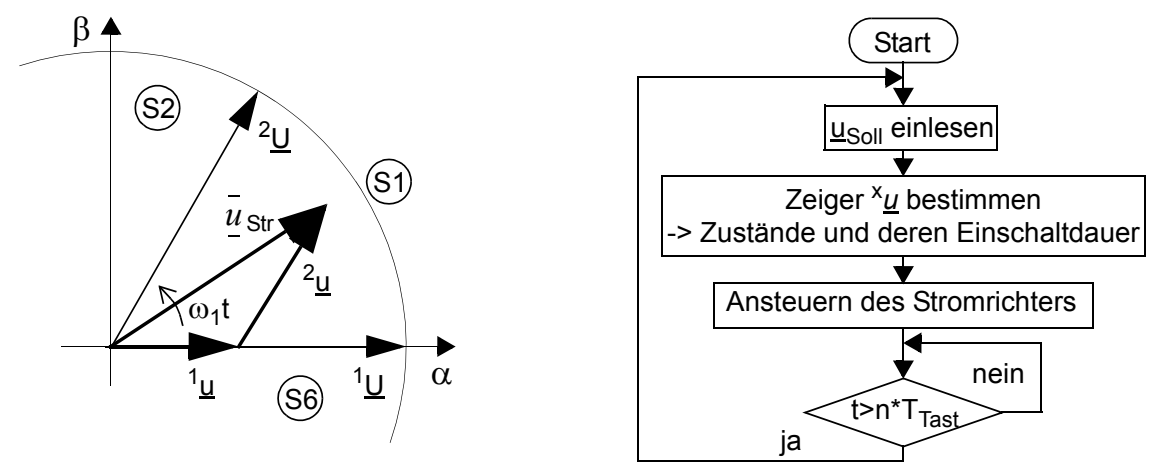

Bild 8.2. Links: Bildung des Spannungszeigers $\underline{u}_{S t r}$ aus den Zeigern ${ }^{1} \underline{U}$ und ${ }^{2} \underline{U}$, rechts: Ablauf der Drehzeigermodulation ( $n$ : ganze Zahl)

\subsection{Freiheitsgrade bei Drehzeigermodulatoren}

Bei der Drehzeigermodulation existieren 3 Freiheitsgrade:

- Der gewünschte Zeiger $\underline{u}_{\text {Str }}$ lässt sich aus verschiedenen Kombinationen der Zeiger ${ }^{1} \underline{U} . .{ }^{6} \underline{U}$ zusammensetzen.

- Die Abfolge der benötigten Zustände kann verschieden gewählt werden.

- Spannung null an der Last kann mit einem oder beiden Nullspannungszeigern ${ }^{0} \underline{U}$ oder ${ }^{7} \underline{U}$ erzeugt werden.

Die nachfolgenden Betrachtungen zeigen, dass das Ausnutzen dieser Freiheitsgrade zum Teil sehr eindeutige Optima ergibt. 


\subsubsection{Aufbau eines Drehzeigers aus den Spannungszuständen des Stromrichters}

Bild 8.3. links zeigt, wie ein beliebiger Spannungszeiger $\underline{u}=|\underline{u}| e^{j \omega_{1} t}$ aus den 3 Zeigern $\underline{u}_{a}$, $\underline{u}_{b}$ und $\underline{u}_{c}$, die parallel zu den Achsen $a, b, c$ liegen, zusammengesetzt werden kann. Diese 3 Zeiger wiederum lassen sich mit den diskreten Zeigern ${ }^{1} \underline{U}$ bis ${ }^{6} \underline{U}$ realisieren: positive $\underline{u}_{a}$ werden mittels ${ }^{1} \underline{U}$, negative mittels ${ }^{4} \underline{U}\left(\underline{u}_{b}\right.$ mittels ${ }^{5} \underline{U}$ bzw. ${ }^{2} \underline{U}$ und $\underline{u}_{c}$ mittels ${ }^{3} \underline{U}$ bzw. $\left.{ }^{6} \underline{U}\right)$ erzeugt. Ihre jeweilige Länge wird durch das Verhältnis Einschaltdauer der entsprechenden Spannungszeiger zu Abtastperiode eingestellt. In der restlichen Zeit des Abtastintervalles, in der keiner der Spannungszeiger ${ }^{1} \underline{U} . .{ }^{6} \underline{U}$ aktiv ist, muss einer der beiden Nullspannungszeiger ${ }^{0} \underline{U}$ bzw. ${ }^{7} \underline{U}$ eingeschaltet werden.
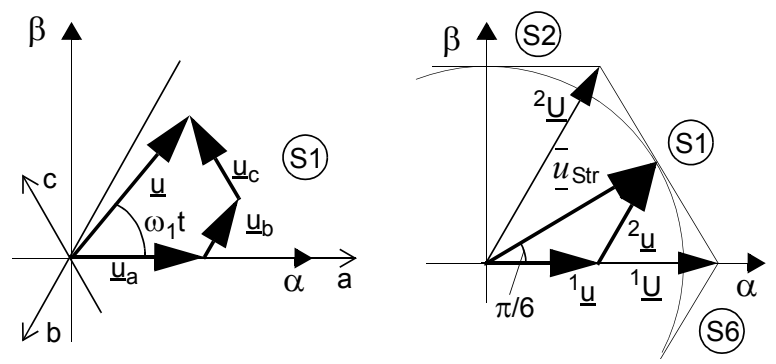

Bild 8.3.

Links: Zerlegung eines Zeigers $\underline{u}$ in Zeiger parallel zu den Achsen $a, b, c$ rechts: Bestimmung der maximalen Länge von $\underline{u}$

Für die Beziehungen zwischen $\underline{u}$ und den Teilzeigern $\underline{u}_{a}, \underline{u}_{b}$ und $\underline{u}_{c}$ gelten:

$$
\left|\underline{u}_{-b}\right|=-\frac{2}{\sqrt{3}}|\underline{u}| \sin \left(\omega_{1} t+\frac{\pi}{3}\right)+\left|\underline{u}_{-a}\right|, \quad\left|\underline{u}_{-c}\right|=\frac{2}{\sqrt{3}}|\underline{u}| \sin \left(\omega_{1} t-\frac{\pi}{3}\right)+\left|\underline{u}_{-a}\right|
$$

Die 3 Teilspannungen sind durch (8.3) überbestimmt. Es kann noch eine weitere Bedingung eingeführt werden: für die maximale Ausnutzung des Stromrichters muss $|u| \mid$ möglichst gross gemacht werden können. Dabei kann die Einschaltdauer aller Teilzeiger zusammen aber höchstens gleich der Dauer einer Abtastperiode $T_{\text {Tast }}$ sein. Deshalb muss für jeden Zeiger $\underline{u}$ die algebraische Summe der Längen (Einschaltdauern) der 3 Teilspannungen $\underline{u}_{a}, \underline{u}_{b}$ und $\underline{u}_{c}$ möglichst klein sein. Bei der Berechnung der Phasenspannungen zeigt sich, dass mit der gleichen Bedingung der Effektivwert und der Klirrfaktor der Spannung minimal werden (siehe auch Kapitel 7.4.4). Es kann also folgende Zusatzbedingung formuliert werden:

$$
\begin{aligned}
& \left|u_{-a}\right|+\left|u_{-b}\right|+\left|u_{-c}\right|=\min . \quad \Rightarrow \\
& \left|u_{-a}\right|+\left|-\frac{2}{\sqrt{3}}\right| u_{-}\left|\sin \left(\omega_{1} t+\frac{\pi}{3}\right)+\right| u_{-a}||+\left|\frac{2}{\sqrt{3}}\right| \underline{-}\left|\sin \left(\omega_{1} t-\frac{\pi}{3}\right)+\right| u_{-a}||=\min .
\end{aligned}
$$

Eine längere Zwischenrechnung ergibt, dass der Zeiger $\underline{u}$ für minimale Einschaltzeit immer nur mit den beiden direkt benachbarten Spannungszeigern gebildet werden muss, d.h. im Sektor $S 1$ aus ${ }^{1} \underline{U}$ und ${ }^{2} \underline{U}$, im Sektor $S 2$ aus ${ }^{2} \underline{U}$ und ${ }^{3} \underline{U}$ und so fort. Im Sektor $S 1$ wird damit $\underline{u}_{c}=0$ und mit $\underline{u}_{a}={ }^{1} \underline{u}$ und $\underline{u}_{b}={ }^{2} \underline{u}$ resultieren aus (8.3): 


$$
\begin{aligned}
& \left|\underline{-}_{-a}\right|=\left|{ }^{1} \underline{u}\right|=\left|{ }^{1} \underline{U}\right| \frac{t_{1}}{T_{\text {Tast }}}=\frac{2}{\sqrt{3}}|\underline{u}| \sin \left(\frac{\pi}{3}-\omega_{1} t\right), \\
& \left|\underline{u}_{b}\right|=\left|{ }^{2} \underline{u}\right|=\left|{ }^{2} \underline{U}\right| \frac{t_{2}}{T_{\text {Tast }}}=\frac{2}{\sqrt{3}}|\underline{u}| \sin \left(\omega_{1} t\right)
\end{aligned}
$$

Aus (8.5) lässt sich jetzt direkt die Einschaltdauer der einzelnen Zeiger bestimmen:

$$
\begin{aligned}
& t_{1}=\frac{\left|{ }^{1} \underline{u}\right|}{\left|{ }^{1} \underline{U}\right|} T_{\text {Tast }}=\sqrt{3} T_{\text {Tast }} \frac{|\underline{u}|}{U_{d}} \sin \left(\frac{\pi}{3}-\omega_{1} t\right), \\
& t_{2}=\frac{\left|{ }^{2} \underline{u}\right|}{\left|{ }^{2} \underline{U}\right|} T_{\text {Tast }}=\sqrt{3} T_{\text {Tast }} \frac{|\underline{u}|}{U_{d}} \sin \left(\omega_{1} t\right)
\end{aligned}
$$

Mit dieser Zerlegung kann ein beliebiger Spannungszeiger $\underline{u}_{S t r}$ im Sektor $S 1$ aufgebaut werden.

Sind die Zeiten $t_{1}$ und $t_{2}$ gegeben, so ist auch die Summe der Restzeit innerhalb der Abtastperiode bestimmt:

$$
t_{0}+t_{7}=T_{\text {Tast }}-\left(t_{1}+t_{2}\right)
$$

\subsubsection{Nachbildung des Sollwertzeigers durch den kurzzeitigen Mittelwert der Stromrichterspannung}

Betrachtet wird Bild 8.3. rechts. Der Zeiger $u_{\text {Str }}$ beschreibt 3 symmetrische, sinusförmige Phasenspannungen. Die Zeigerlänge entspricht den Amplituden der 3 Spannungen. Die Spitze des Spannungszeigers bewegt sich in Funktion des Drehwinkels mit der Kreisfrequenz $\omega_{1}=2 \pi f_{1}$ auf einem Kreis.

Die maximal mögliche Zeigerlänge ist durch die Summe der Einschaltzeiten aller benötigten spannungsbildenden Zeiger ${ }^{1} \underline{U} . .{ }^{6} \underline{U}$ pro Abtastperiode bestimmt. Mit (8.6) lässt sich zeigen, dass die Summe von $t_{1}+t_{2}$ bei $\omega_{1} t=\pi / 6$ maximal wird $\left(t_{1}=t_{2}\right)$. Die maximale Zeigerlänge ist also durch diesen Punkt bestimmt. Wenn $t_{1}+t_{2}$ den Wert $T_{\text {Tast }}$ erreicht, gilt für die Länge des Zeigers $\underline{u}_{S t r}$ :

$$
\left|\bar{u}_{S t r, \max }\right|=\frac{2}{\sqrt{3}} \frac{U_{d}}{2}
$$

Die Amplituden der diesem Zeiger entsprechenden sinusförmigen Phasenspannungen sind damit um den Faktor $2 / \sqrt{3}$ grösser als $U_{d} / 2$.

Modulationsgrad $M$ : Der Modulationsgrad ist auch hier als Verhältnis der Amplitude der Phasenspannung zur halben Zwischenkreisspannung definiert:

$$
M=\frac{\hat{u}_{U / V / W}}{U_{d} / 2}=\frac{\left|\bar{u}_{S t r}\right|}{U_{d} / 2}=\frac{4}{3} \frac{\left|\underline{u}_{S t r}\right|}{|x \cdot U|}
$$


Einsetzen von (8.8) in (8.9) ergibt bei sinusförmigen Phasenspannungen einen maximalen Modulationsgrad von $M_{\max }=2 / \sqrt{3}=1.155$.

Die Einschaltzeiten $t_{1}$ und $t_{2}$ lassen sich mit (8.6) und (8.9) in Funktion des Modulationsgrades ausdrücken:

$$
t_{1}=\frac{\sqrt{3}}{2} T_{\text {Tast }} M \sin \left(\frac{\pi}{3}-\omega_{1} t\right), \quad t_{2}=\frac{\sqrt{3}}{2} T_{\text {Tast }} M \sin \left(\omega_{1} t\right)
$$

Zeigerlängen in einem beliebigen Sektor: Bis dahin wurde immer nur der Sektor $S 1$, d.h. der Winkelbereich $[0, \pi / 3]$ betrachtet. Liegt der Zeiger $\underline{u}_{S o l l}$ in einem anderen Sektor, so wird er ebenfalls in die Komponenten parallel zu den beiden direkt benachbarten Zeigern ${ }^{1} \underline{U} . .{ }^{6} \underline{U}$ zerlegt. Wird nur der relative Winkel $\varepsilon$ innerhalb des Sektors betrachtet, ist die Zerlegung identisch zu der im Sektor $S 1$. Die effektive Berechnung kann so immer auf den Bereich $0 \leq \varepsilon \leq \pi / 3$ reduziert werden. Dazu wird $\omega_{1} t$ so oft um $\pi / 3$ reduziert bis der Rest im Sektor $S 1$ liegt. Mit dem Winkel $\varepsilon$ können dann die gewünschten Grössen berechnet und anschliessend wieder dem entsprechenden Sektor zugeordnet werden. Dazu wird noch die Information benötigt, in welchem Sektor $\underline{u}_{S o l l}$ liegt. Für einen Zeiger im Sektor $S 3$ gilt für ${ }^{3} \underline{u}$ und ${ }^{4} \underline{u}$ so beispielsweise:

$$
\begin{aligned}
& \left|{ }_{-}^{3} \underset{-}{u}\left(\omega_{1} t\right)\right|=\left|{ }^{1} \underset{-}{u}(\varepsilon)\right|=\frac{2}{\sqrt{3}}\left|{ }_{-S o l l}\right| \sin \left(\frac{\pi}{3}-\varepsilon\right), \\
& \left|{ }^{4} \underset{\underline{u}}{u}\left(\omega_{1} t\right)\right|=\left|{ }^{2} \underline{u}(\varepsilon)\right|=\frac{2}{\sqrt{3}}\left|{ }_{-S o l l}\right| \sin (\varepsilon) \quad \text { mit } \quad \varepsilon=\left(\omega_{1} t-2 \frac{\pi}{3}\right)
\end{aligned}
$$

Die Zuordnung der im Sektor $S 1$ berechneten Zeiger $\left.\right|^{1} \underline{u}(\varepsilon) \mid$ und $\left.\right|^{2} \underline{u}(\varepsilon) \mid$ zu den Spannungszeigern ${ }^{1} \underline{U} . .{ }^{6} \underline{U}$ in Funktion des Sektors in dem $\underline{u}_{\text {Soll }}$ liegt zeigt Tabelle 8.2. Die gleichen Zuordnungen gelten auch für die Einschaltzeiten der einzelnen Stromrichterzustände.

\begin{tabular}{c|c|c|c|c|c|c} 
Sektor & $\left|{ }^{1} \underline{\mathrm{u}}\left(\omega_{1} \mathrm{t}\right)\right|$ & $\left.\right|^{2} \underline{\mathrm{u}}\left(\omega_{1} \mathrm{t}\right) \mid$ & $\left.\right|^{3} \underline{\mathrm{u}}\left(\omega_{1} \mathrm{t}\right) \mid$ & $\left.\right|^{4} \underline{\mathrm{u}}\left(\omega_{1} \mathrm{t}\right) \mid$ & $\left|{ }^{5} \underline{\mathrm{u}}\left(\omega_{1} \mathrm{t}\right)\right|$ & $\left|{ }^{6} \underline{\mathrm{u}}\left(\omega_{1} \mathrm{t}\right)\right|$ \\
\hline $\mathrm{S} 1$ & $\left.\right|^{1} \underline{\mathrm{u}}(\varepsilon) \mid$ & $\left.\right|^{2} \underline{\mathrm{u}}(\varepsilon) \mid$ & & & & \\
\hline $\mathrm{S} 2$ & & $\left.\right|^{1} \underline{\mathrm{u}}(\varepsilon) \mid$ & $\left.\right|^{2} \underline{\mathrm{u}}(\varepsilon) \mid$ & & & \\
\hline $\mathrm{S} 3$ & & & $\left.\right|^{1} \underline{\mathrm{u}}(\varepsilon) \mid$ & $\left.\right|^{2} \underline{\mathrm{u}}(\varepsilon) \mid$ & & \\
\hline $\mathrm{S} 4$ & & & & $\left.\right|^{1} \underline{\mathrm{u}}(\varepsilon) \mid$ & $\left.\right|^{2} \underline{\mathrm{u}}(\varepsilon) \mid$ & \\
\hline $\mathrm{S} 5$ & & & & & $\left.\right|^{1} \underline{\mathrm{u}}(\varepsilon) \mid$ & $\left.\right|^{2} \underline{\mathrm{u}}(\varepsilon) \mid$ \\
\hline S6 & $\left.\right|^{2} \underline{\mathrm{u}}(\varepsilon) \mid$ & & & & & $\left.\right|^{1} \underline{\mathrm{u}}(\varepsilon) \mid$
\end{tabular}

Tabelle 8.1.

Zerlegung des Zeigers $\underline{u}_{\text {Soll }}$ in die direkt benachbarten Spannungszeiger

Ist $\underline{u}_{\text {Soll }}$ durch seine $\alpha$ - und $\beta$-Komponenten gegeben, so sind die Zeiger ${ }^{x} \underline{u}$ durch lineare Kombinationen dieser Komponenten bestimmt, d.h es treten keine trigonometrischen Funktionen mehr auf. Diese Beziehungen zeigt die Tabelle 8.2. Für die Zerlegung selbst muss aber vorgängig bestimmt werden, in welchem Sektor der Zeiger $\underline{u}_{\text {Soll }}$ liegt. 
Tabelle 8.2. Zerlegung der $\alpha$ - und $\beta$-Komponenten von $\underline{u}_{\text {Soll }}\left(=u_{\alpha}+j u_{\beta}\right)$ in die direkt benachbarten Spannungszeiger

\begin{tabular}{|c|c|c|c|c|c|c|}
\hline Sektor & $\left|{ }^{1} \underline{u}\right|$ & $\left.\right|^{2} \underline{\mathrm{u}} \mid$ & $\left.\right|^{3} \underline{\mathrm{u}} \mid$ & $\left|{ }^{4} \underline{u}\right|$ & $\left|{ }^{5} \underline{\mathrm{u}}\right|$ & $\left|{ }^{6} \underline{\mathrm{u}}\right|$ \\
\hline $\mathrm{S} 1$ & $\mathrm{u}_{\alpha}-\mathrm{u}_{\beta} / \sqrt{3}$ & $2 u_{\beta} / \sqrt{3}$ & & & & \\
\hline S2 & & $\mathrm{u}_{\alpha}+\mathrm{u}_{\beta} / \sqrt{3}$ & $\begin{array}{c}-\mathrm{u}_{\alpha}+\mathrm{u}_{\beta} / \\
\sqrt{3}\end{array}$ & & & \\
\hline S3 & & & $2 u_{\beta} / \sqrt{3}$ & $\begin{array}{c}-\mathrm{u}_{\alpha}-\mathrm{u}_{\beta} / \\
\sqrt{3}\end{array}$ & & \\
\hline S4 & & & & $\begin{array}{c}-\mathrm{u}_{\alpha}+\mathrm{u}_{\beta} / \\
\sqrt{3}\end{array}$ & $-2 u_{\beta} / \sqrt{3}$ & \\
\hline S5 & & & & & $\begin{array}{c}-\mathrm{u}_{\alpha}-\mathrm{u}_{\beta} / \\
\sqrt{3}\end{array}$ & $\mathrm{u}_{\alpha}-\mathrm{u}_{\beta} / \sqrt{3}$ \\
\hline S6 & $\mathrm{u}_{\alpha}+\mathrm{u}_{\beta} / \sqrt{3}$ & & & & & $-2 u_{\beta} / \sqrt{3}$ \\
\hline
\end{tabular}

\subsubsection{Abfolge der Stromrichterzustände}

Beim Wechsel von einem Schaltzustand auf den nächsten können im Stromrichter ein, zwei oder 3 Schaltvorgänge stattfinden. Die Schaltzustände und die möglichen Zustandsübergänge sind in Bild 8.4 links, in der gleichen räumlichen Anordnung wie die entsprechenden Spannungszeiger in der $\alpha \beta$-Ebene gezeichnet. Zusätzlich sind die zwei Nullzustände ${ }^{0} Z$ und ${ }^{7} Z$ separiert aufgetragen. Jeder Pfeil bedeutet, dass ein Brückenzweig schaltet. Wird von einem Zustand auf den nächsten gewechselt, ergibt das nur einen Schaltvorgang im Stromrichter. Zwei Schaltvorgänge erfolgen beim Überspringen des direkt benachbarten und 3 beim Wechsel in den inversen Schaltzustand (z.B. ${ }^{1} Z \rightarrow{ }^{4} Z$ ).
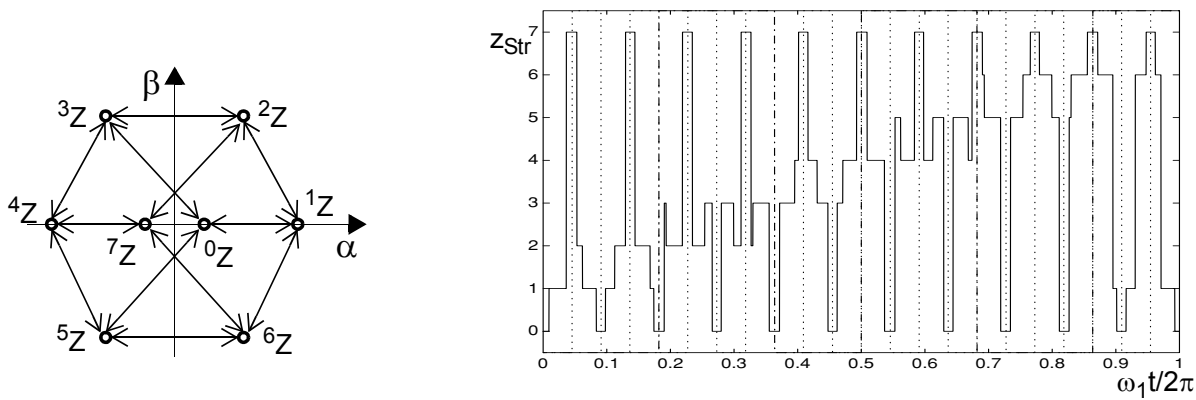

Bild 8.4. Links: Übergänge zwischen den 8 Schaltzuständen, rechts: Stromrichterzustände während einer Grundschwingungsperiode, $T_{\text {Tast }} / T_{1}=22$, punktierte Linien: Abtastintervalle, strichpunktiert: Sektorgrenzen 
Unter Berücksichtigung des Zieles, den Stromrichter mit einer möglichst niedrigen Schaltfrequenz zu betreiben, sind die Zustandsübergänge mit nur einem Schaltvorgang zu bevorzugen.

Sind die innerhalb einer Taktperiode benötigten Spannungszustände bestimmt, so stellt sich die Frage, in welcher Folge sie optimal geschaltet werden. Für die folgende Betrachtung wird davon ausgegangen, dass der Stromrichter pro Taktperiode zwei spannungsbildende Zustände $\left({ }^{1} Z \ldots{ }^{6} Z\right)$ und mindestens einen Nullzustand einnehmen muss.

Am Ende der Taktperiode $T_{\text {Tast }}$ wird bei allen betrachteten Anordnungen die Ablaufreihenfolge umgekehrt. Damit wird erreicht, dass an dieser Stelle nicht geschaltet wird.

In Bild 8.5 sind 3 mögliche Zustandssequenzen im ersten Sektor dargestellt:

\section{Sequenz 1:}

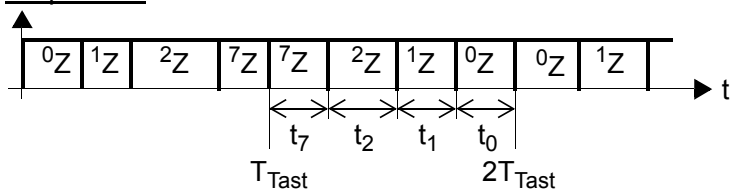

Sequenz 2:
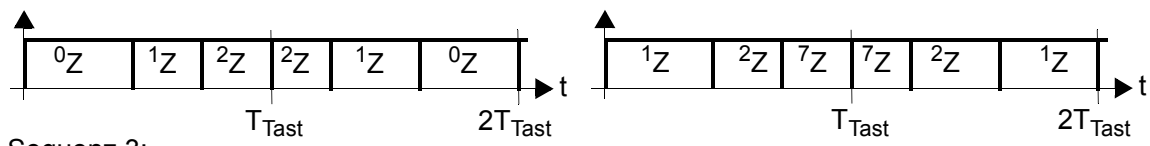

Sequenz 3:

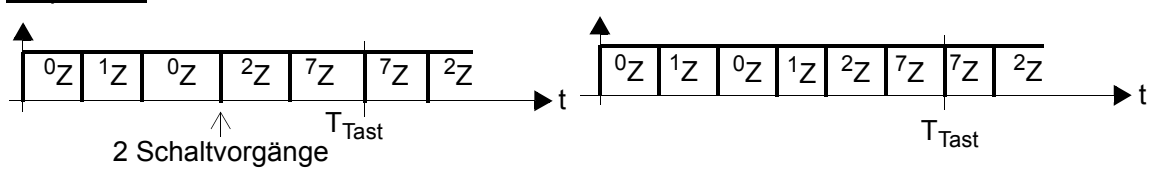

Bild 8.5. Mögliche Sequenzen der Stromrichterzustände

Sequenz 1, 3 Schaltvorgänge pro Abtastperiode: Bei der obersten Sequenz in Bild 8.5 werden beide Nullzustände ${ }^{0} Z$ und ${ }^{7} Z$ verwendet. Bei allen 3 Zustandswechseln erfolgt jeweils nur ein Schaltvorgang. Pro Abtastintervall schalten dabei alle 3 Brückenzweige je einmal. Die Zustandsabfolgen über eine ganze Grundschwingungsperiode für diesen Fall zeigt Bild 8.4 rechts. Diese Abfolge entspricht der Standardvariante der Drehzeigermodulation, wie sie in den meisten Anwendungen eingesetzt wird. Aus diesem Grunde soll sie als Referenz für die nachfolgenden Pulsmuster verwendet werden. Die Anzahl Schaltvorgänge pro Taktperiode wird mit 100\% angesetzt.

Sequenz 2, zwei Schaltvorgänge pro Abtastperiode: Bei dieser Sequenz wird nur ein Nullzustand verwendet $\left({ }^{0} Z\right.$ in der linken und ${ }^{7} Z$ in der rechten Sequenz). Im Vergleich zur Standardvariante ergibt das $67 \%$ der Schaltvorgänge pro Abtastperiode. Für dieselbe mittlere Schaltfrequenz kann deshalb die Abtastperiode um 33\% verkürzt, bzw. die Taktfrequenz um 50\% erhöht werden. Diese Sequenz stellt einen Grenzfall der ersten dar, in dem die Dauer des einen Nullzustandes null ist. Das Nichtverwenden eines Nullzustandes bedeutet, dass ein Zweig des Stromrichters in der betrachteten Abtastperiode nicht schaltet. 
Sequenz 3, 4 und mehr Schaltvorgänge pro Abtastperiode: In Bild 8.5 unten sind zwei Abläufe vorgestellt, bei denen jeweils ein spannungsbildender Zustand auf zwei Zeitabschnitte verteilt wird. Dadurch resultiert eine höhere Schaltfrequenz. Solche Ansätze bieten keinerlei Vorteile und werden deshalb nicht eingesetzt.

Die nachfolgenden Betrachtungen zeigen, dass von den 3 betrachteten Sequenzen in einem grossen Aussteuerbereich die erste und in einem kleineren Bereich die zweite minimale Verzerrungsströme ergeben.

Zuordnung der Zeiten $t_{0}$ und $t_{7}$ zu den Nullzuständen in beliebigen Sektoren: Die im Sektor $S 1$ berechneten Einschaltzeiten $t_{0}$ und $t_{7}$ für die Zustände ${ }^{0} Z$ und ${ }^{7} Z$ müssen in den Sektoren $S 2, S 4$ und $S 6$ noch vertauscht werden. $t_{0}$ entspricht dann der Einschaltdauer des Zustandes ${ }^{7} Z$ und $t_{7}$ der von ${ }^{0} Z$. Dieses Vertauschen ist selbstverständlich nur nötig wenn $t_{0}$ und $t_{7}$ verschieden sind.

\subsubsection{Einschaltdauer der Nullzustände}

Nachdem für ein Abtastintervall die benötigten spannungsbildenden Zustände mit ihrer Einschaltdauer und Abfolge bestimmt sind, ist der letzte Freiheitsgrad jetzt noch die Aufteilung der Restzeit auf die Zustände ${ }^{0} Z$ und ${ }^{7} Z$ :

$$
t_{0}+t_{7}=T_{\text {Tast }}-t_{1}-t_{2}
$$

Diese Aufteilung beeinflusst die kurzzeitigen Mittelwerte der Spannungen in Zeigerdarstellung nicht. Sie äussert sich aber in der Sternpunktspannung $u_{N 0}$. Wie von den Trägerverfahren her bekannt ist, können damit der maximale Modulationsgrad und die Spannungs- und Stromverzerrungen beeinflusst werden.

Aus Tabelle 3.2 lässt sich direkt der kurzzeitige Mittelwert der Sternpunktspannung herleiten: im ersten Sektor gilt dafür (8.13). Mit (8.12) kann $t_{7}$ eliminiert werden und die Gleichung für die Sternpunktspannung vereinfacht sich zu (8.14). Der kurzzeitige Mittelwert ist jetzt durch die Zeiten $t_{0}, t_{1}$ und $t_{2}$ bestimmt. Die Einschaltzeiten $t_{1}$ und $t_{2}$ sind gemäss (8.10) durch den Modulationsgrad $M$ und den Winkel $\omega_{1} t$ bestimmt. Umgekehrt kann für eine beliebig vorgegebene Spannung $u_{N 0}$ mit (8.15) die Zeit $t_{0}$ berechnet werden. Die Betrachtungen zeigen, dass die zeitliche Aufteilung der Nullzustände fest mit $u_{N 0}$ verknüpft sind.

$$
\begin{aligned}
& \bar{u}_{N 0}=\frac{U_{d}}{2} \frac{1}{T_{\text {Tast }}}\left(-t_{0}-\frac{t_{1}}{3}+\frac{t_{2}}{3}+t_{7}\right) \\
& \bar{u}_{N 0}=\frac{U_{d}}{2} \frac{1}{T_{\text {Tast }}}\left(T_{\text {Tast }}-2 t_{0}-\frac{4}{3} t_{1}-\frac{2}{3} t_{2}\right) \\
& t_{0}=\left(\frac{1}{2}-\frac{\bar{u}_{N 0}}{U_{d}}\right) T_{\text {Tast }}-\frac{2}{3} t_{1}-\frac{1}{3} t_{2}
\end{aligned}
$$


Damit die niederfrequenten Anteile der Mittelpunktspannungen nach wie vor symmetrisch bleiben, muss der kurzzeitige Mittelwert der Sternpunktspannung aus einer Gleichspannung oder beliebigen Vielfachen der 3. Harmonischen bestehen (Anhang B.1.1). Die Wechselgrösse mit der niedrigsten möglichen Frequenz ist damit die 3. Harmonische. Ein Sektor beschreibt in diesem Fall immer mindestens eine Halbperiode der Sternpunktspannung.

\subsubsection{Beispiele von Aufteilungen der Einschaltdauer der Nullzustände}

In praktischen Anwendungen werden die folgenden Aufteilungen der Nullzustände eingesetzt:

- Aufteilung so, dass die Sternpunktspannung $u_{N 0}=0$ ist.

- $t_{0}=t_{7}$ : diese einfache Aufteilung wird bei der Standardvariante der Drehzeigermodulation verwendet.

- Nur ein Nullzustand pro Abtastintervall, d.h. $t_{0}=0$ oder $t_{7}=0$. Damit lassen sich im Bereich hoher Aussteuerung kleinere Stromverzerrungen erreichen.

Die Spektra all dieser Verfahren sind identisch mit denjenigen von regular sampled Trägerverfahren mit gleicher Sternpunktspannung und gleicher Schaltzahl. Von den Spektra der natural sampled Verfahren, wie sie in Kapitel 7.4.2 betrachtet wurden, weichen sie nur wenig ab. Die in der Mittelpunktspannung auftretenden Vielfachen der 3. Harmonischen fallen in der Phasenspannung wieder weg und fehlen entsprechend auch in den Zeigern.

Nullzustände für $u_{N 0}=0$ : Dies bedeutet, das die niederfrequenten Anteile der Mittelpunktspannungen aller 3 Brückenzweige rein sinusförmig sind. Dazu wird in (8.15) $u_{N 0}=0$ gesetzt und es resultieren die Zeiten:

$$
\begin{aligned}
t_{0}=\frac{1}{2} T_{\text {Tast }}-\frac{2}{3} t_{1}-\frac{1}{3} t_{2}=\frac{T_{\text {Tast }}}{2}\left[1-M \cos \left(\omega_{1} t\right)\right], \\
t_{7}=T_{\text {Tast }}-t_{0}-t_{1}-t_{2}=\frac{T_{\text {Tast }}}{2}\left[1-M \sin \left(\omega_{1} t+\frac{\pi}{6}\right)\right]
\end{aligned}
$$

Damit die beiden Zeiten in jedem Fall positiv sind, gilt für den maximalen Modulations$\operatorname{grad} M=1$, d.h. es kann nicht die ganze Abtastperiode $T_{\text {Tast }}$ für die spannungsbildenden Zustände verwendet werden. Diese Eigenschaft ist von den Trägerverfahren her bekannt. Die resultierenden Mittelpunkt-, Phasen- und Sternpunktspannung nach dem Ausfiltern der durch den Schaltbetrieb erzeugten höherfrequenten Frequenzkomponenten zeigt Bild 8.6 links. Die entsprechenden Spannungsspektra der ungefilterten Mittelpunkt- und Phasenspannungen sind im gleichen Bild rechts für eine Schaltzahl von $q=21\left(T_{\text {Tast }} / T_{1}=42\right)$ dargestellt.

Gleiche Dauer beider Nullzustände, $t_{0}=t_{7}$ : Bei dieser Verteilung heben sich die Beiträge der beiden Nullzustände zur Sternpunktspannung auf. Mit Einsetzen von $t_{1}$ und $t_{2}$ folgt: 

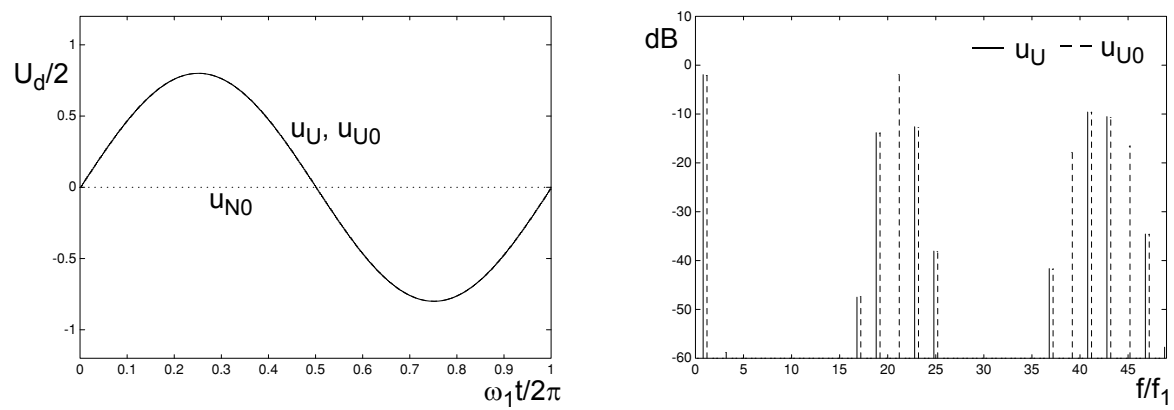

Bild 8.6. Links: Mittelpunkt-, Phasen- und Sternpunktspannung ohne Schaltanteile für eine Sternpunktspannung von null und $M=0.8$, rechts: Spannungsspektra der geschalteten Spannungen, $T_{\text {Tast }} / T_{1}=42,0 \mathrm{~dB}: U_{d} / 2$

$$
\bar{u}_{N 0}=\frac{U_{d}}{2} \frac{1}{T_{\text {Tast }}}\left(-\frac{t_{1}}{3}+\frac{t_{2}}{3}\right)=\frac{U_{d}}{4} M \sin \left(\omega_{1} t-\frac{\pi}{6}\right)
$$

In den nachfolgenden Sektoren wiederholt sich dieser Spannungsverlauf mit abwechselndem Vorzeichen. Die resultierende Spannung entspricht näherungsweise einem Dreiecksignal mit der Amplitude $U_{d} / 8$. Die Einschaltzeiten $t_{0}$ und $t_{7}$ dürfen bei maximaler Aussteuerung null werden und damit $t_{1}+t_{2}=T_{\text {Tast }}$. Auf diese Weise lässt sich der maximal mögliche Modulationsgrad von $M=2 / \sqrt{3}=1.155$ erreichen. Bild 8.7 zeigt die Spannungen ohne Schaltanteile und die Spektra der geschalteten Mittelpunkt- und Phasenspannungen. Im Vergleich zum ersten Verfahren weisen die Trägerbänder der Spektra mehr dominante Spektrallinien und diese kleinere Amplituden auf.
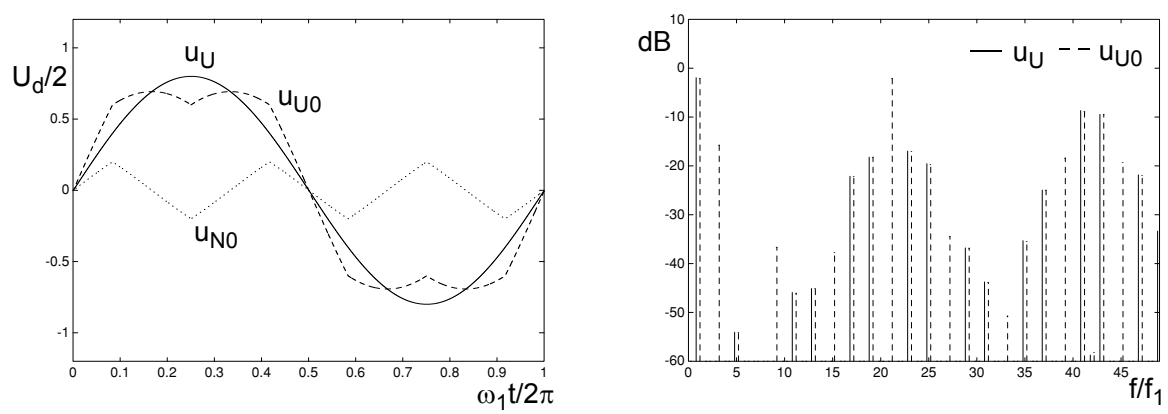

Bild 8.7. Links: Mittelpunkt-, Phasen- und Sternpunktspannung ohne Schaltanteile bei gleich langen Nullzuständen, $M=0.8$, rechts: Spannungsspektra der geschalteten Spannungen, $T_{\text {Tast }} / T_{1}=42,0 \mathrm{~dB}: U_{d} / 2$

Nur ein Nullzustand pro Abtastperiode, $t_{0}=0$ oder $t_{7}=0$ : Bei dieser Aufteilung wird pro Abtastintervall ein Nullzustand gar nicht verwendet. So erfolgen pro Intervall nur noch zwei statt 3 Schaltvorgänge, d.h. die resultierende Schaltfrequenz wird kleiner. Es 
stellt sich nun die Frage, welcher Nullzustand verwendet werden soll. Dafür existieren verschiedene Ansätze, die alle ähnliche Resultate ergeben. Davon werden 3 betrachtet:

Variante 1: Die Nullzustände werden wie folgt aufgeteilt:

$$
\begin{array}{llll}
0 \leq \varepsilon<\frac{\pi}{6} & \Rightarrow & t_{0}=0, & t_{7}=T_{\text {Tast }}-t_{1}-t_{2}, \\
\frac{\pi}{6} \leq \varepsilon<\frac{\pi}{3} & \Rightarrow & t_{7}=0, & t_{0}=T_{\text {Tast }}-t_{1}-t_{2}
\end{array}
$$

Die Spannungen $u_{U 0}, u_{U}$ und $u_{N 0}$ ohne Schaltanteile sind für diese Aufteilung in Bild 8.8 links dargestellt. Obwohl die Mittelpunkt- und die Sternpunktspannung stark verzerrt sind, verläuft die Phasenspannung wieder sinusförmig. Jede Mittelpunktspannung hat während einer Sechstelperiode den Wert $+U_{d} / 2$ und während einem gleich langen Winkelausschnitt den Wert $-U_{d} / 2$. Wie der abgebildete Verlauf von $u_{U 0}$ in Bild 8.8 rechts bestätigt, wird in diesen Zeitabschnitten der jeweilige Brückenzweig nicht geschaltet. Aus diesem Grund werden solche Modulationsverfahren auch als diskontinuierlich bezeichnet.

In Bild 8.8 rechts ist zusätzlich ein Stromverlauf für eine annähernd ohmsche Last abgebildet. Die Schaltverluste in den Halbleitern sind proportional zum Produkt von Strom und Spannung in den Schaltzeitpunkten. Wird der Stromrichter im Bereich des Strommaximums nicht geschaltet, so lassen sich diese Verluste am stärksten reduzieren. Aus diesem Grund werden die Bereiche mit Vollaussteuerung oft so gelegt, dass sie mit den Strommaxima zusammenfallen.
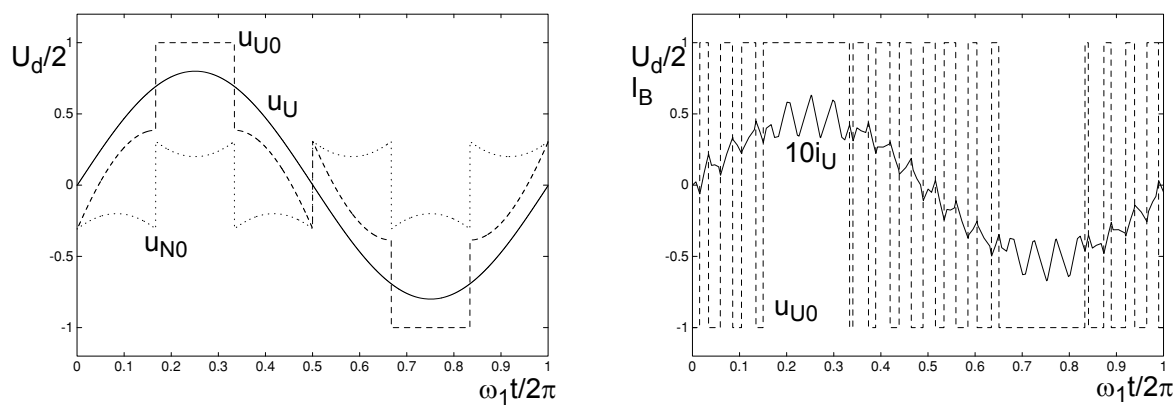

Bild 8.8. Nur ein Nullzustand pro Abtastperiode nach Variante $1, M=0.8, T_{\text {Tast }} / T_{1}=42$, links: Mittelpunkt-, Phasen- und Sternpunktspannung ohne Schaltanteile, rechts: geschaltete Mittelpunktspannung und Phasenstrom, $\hat{\imath}_{U, v=1}=0.05 I_{B}$

Die Spektra der Mittelpunkt- und Phasenspannung zeigt Bild 8.9. Die Trägerbänder sind noch breiter als bei der Verwendung von zwei Nullzuständen gleicher Dauer (Bild 8.7).

Mit den gleichen Überlegungen wie bei zwei Nullzuständen gleicher Länge lässt sich zeigen, dass auch bei diesen Verfahren der maximale Modulationsgrad $M=2 / \sqrt{3}=1.155$ beträgt. 


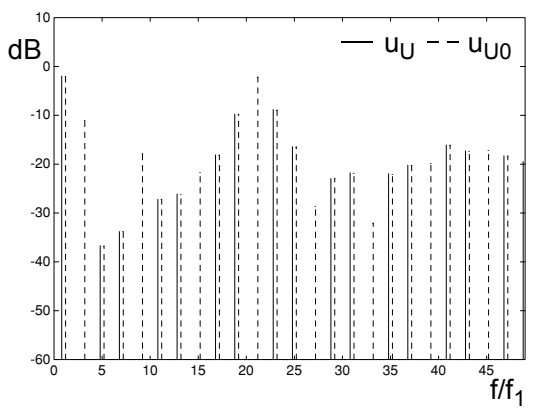

Bild 8.9.

Spektra bei einem Nullzustand pro Abtastperiode nach Variante 1, $M=0.8, T_{\text {Tast }} / T_{1}=42,0 \mathrm{~dB}: U_{d} / 2$

Variante 2: Das Zeitintervall, in dem ein Brückenzweig nicht geschaltet wird, kann innerhalb gewisser Grenzen verschoben werden um das Schalten im Bereich des Stromscheitelwertes zu vermeiden. Dadurch können die Schaltverluste reduziert werden. Bild 8.10 illustriert dies für einen Lastwinkel zwischen Strom und Spannung von ca. $\pi / 4$. In diesem Fall wird in $S 1$ der Zustand ${ }^{0} Z$ gar nie verwendet, d.h. $t_{0}=0$. Für die Dauer von ${ }^{7} Z$ gilt: $t_{7}=T_{\text {Tast }}{ }^{-} t_{1}-t_{2}$.

Die Grenzen des Bereichs, in dem das Schalten in einem Brückenzweig verhindert werden kann, liegen bezüglich dem Sollwertmaximum bei $\pm \pi / 3$. Das Beispiel zu Variante 2 und die nachfolgende Variante 3 stossen bereits an diese Grenzen.
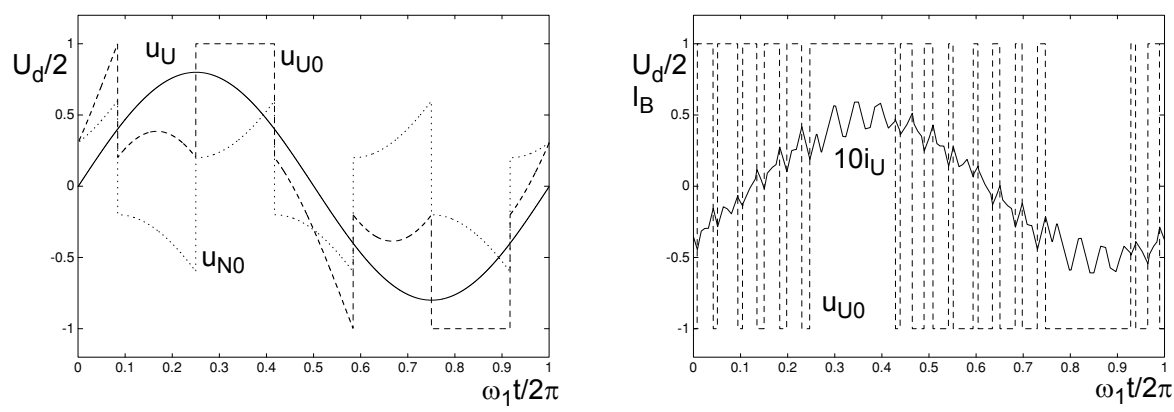

Bild 8.10. Nur ein Nullzustand pro Abtastperiode nach Variante $2, M=0.8, T_{\text {Tast }} / T_{1}=42$, links: Mittelpunkt-, Phasen- und Sternpunktspannung ohne Schaltanteile, rechts: effektive Mittelpunktspannung und Phasenstrom, $\hat{l}_{U, v=1}=0.05 I_{B}$

Variante 3: Hier wird, wie in Bild 8.11 dargestellt, während 4 Zeitabschnitten pro Periode nicht geschaltet. Die Verteilung der Nullzustände entspricht dabei derjenigen nach (8.18) wenn $t_{0}$ und $t_{7}$ vertauscht werden. Die Anordnung ist interessant, weil sie sehr kleine Verzerrungsströme ergibt.

Die vorgestellten diskontinuierlichen Sollwertverläufe können selbstverständlich auch mit gewöhnlichen Trägerverfahren implementiert werden. Dabei wird aber die Generierung der Sollwerte aufwendiger als bei der Drehzeigermodulation. 


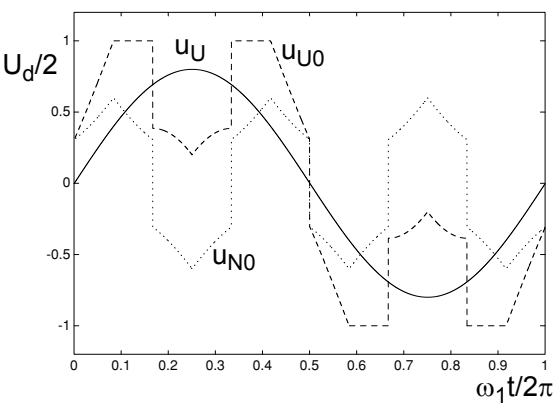

Bild 8.11.

Nur ein Nullzustand pro Abtastperiode nach Variante 3, $M=0.8$, Mittelpunkt-, Phasen- und Sternpunktspannung ohne Schaltanteile

\subsubsection{Verzerrungsströme in Funktion der Aufteilung der Nullzustände}

Für die besprochenen Aufteilungen der Nullzustände sind die berechneten Verzerrungsströme in Bild 8.12 dargestellt. Der Verlauf a beschreibt den Effektivwert des Verzerrungsstromes für eine Sternpunktspannung von null. Dieser Verlauf entspricht dem einer regular sampled symmetrischen PWM. Kurve b gilt für die Standardvariante der Drehzeigermodulation mit zwei gleich langen Nullzuständen, $t_{0}=t_{7}$. Die Verläufe $c_{1}, c_{2}, c_{3}$ und $\mathrm{d}_{1}, \mathrm{~d}_{2}, \mathrm{~d}_{3}$ gelten für diskontinuierliche Verfahren, bei denen ein Nullzustand jeweils nicht verwendet wird. Die Länge der Abtastintervalle ist bei den Kurven a, b und c identisch und die Schaltzahl beträgt:

$$
q=\frac{1}{2} \frac{T_{1}}{T_{\text {Tast }}}=21
$$

Wird nur ein Nullzustand pro Abtastintervall verwendet, so sinkt die Anzahl Schaltvorgänge auf zwei Drittel ab. Der Verzerrungsstrom wird dabei grösser. Bei den mit $d$ gekennzeichneten Verzerrungsströmen ist die Länge des Abtastintervalls soweit verkürzt, dass die resultierende Schaltzahl $q$ wieder 21 beträgt. Dabei wird der Vorteil dieser Nullzustandsverteilungen ersichtlich: oberhalb eines Modulationsgrades von $\mathrm{M} \approx 0.8 \ldots 0.9$ ergeben diese Verfahren bei einer vorgegebenen Anzahl Schaltvorgängen die kleinsten Verzerrungsströme.

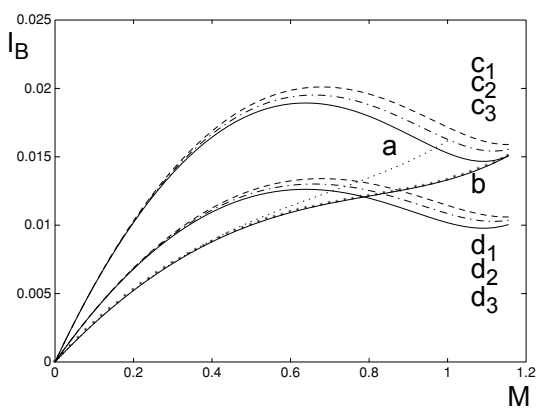

Bild 8.12.

Effektivwerte der Verzerrungsströme bei verschiedenen Aufteilungen der Nullzustände,

$T_{\text {Tast }} / T_{1}=42$,

a: Sternpunktspannung null,

b: $t_{0}=t_{7}$, entsprechend der Standardvariante der Drehzeigermodulation,

c: nur ein Nullzustand $\left(t_{0}=0\right.$ oder $\left.t_{7}=0\right), \mathrm{c}_{1}$ : Variante $1, \mathrm{c}_{2}$ : Variante 2, $\mathrm{c}_{3}$ : Variante 3 ,

d: wie c, aber $T_{\text {Tast }} / T_{1}=63, \mathrm{~d}_{1}$ : Variante $1, \mathrm{~d}_{2}:$ Variante $2, \mathrm{~d}_{3}$ : Variante 3 


\subsection{Analogie zwischen Drehzeigermodulation und Trägerverfahren}

Die vorgestellte Drehzeigermodulation liefert die identischen Schaltmuster, wie sie mit-

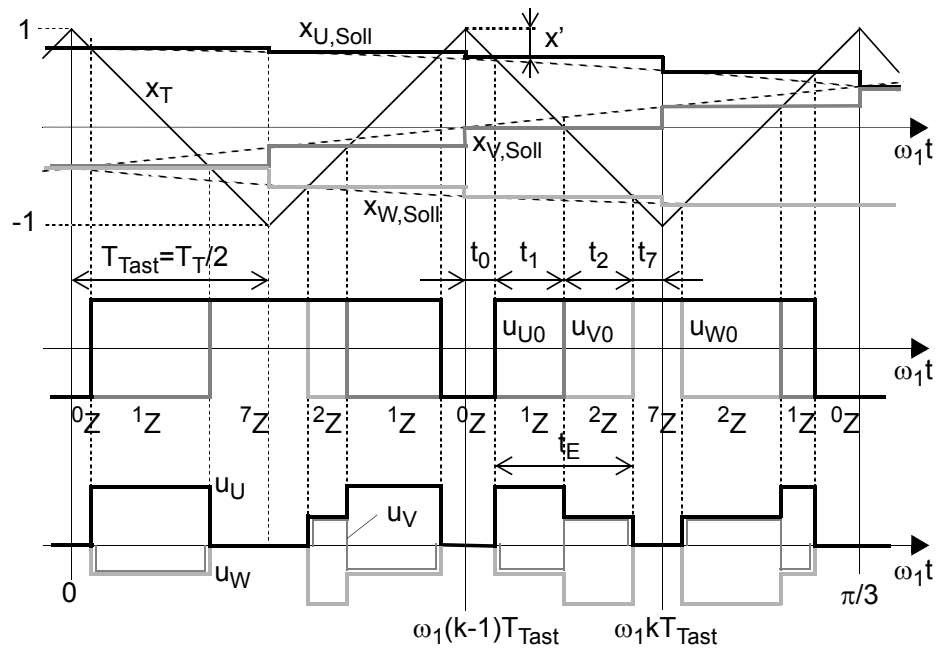

Bild 8.13. Beziehung zwischen Drehzeigermodulation und Trägerverfahren. oben: kontinuierliche und abgetastete Sollwerte sowie Trägersignal, mitte: Schaltmuster der 3 Mittelpunktspannungen, unten: Phasenspannungen

tels einem double edge, regular sampled Trägerverfahren und entsprechenden Spannungssollwerten erzeugt werden. Dies lässt sich anhand der Signalverläufe innerhalb einer Sechstelperiode in Bild 8.13 zeigen. Die Phasenlagen der Sollwerte sind so gewählt, dass die Phase $U$ einen cosinusförmigen Verlauf hat. Der Ausschnitt entspricht so dem Sektor $S 1$ in der Drehzeigerdarstellung. Zur Zeit $t=0$ liegt der Sollwertzeiger, gebildet aus den Sollwerten für die Phasen $U, V$ und $W$, auf der reellen $\alpha$-Achse.

Für die Erzeugung der Stromrichter-Ausgangsspannung nach dem Trägerverfahren werden die in Bild 8.13 oben dargestellten Sollwerte und das Dreieck-Trägersignal verwendet. Bei jedem Scheitelwert des Dreiecks werden die Sollwerte abgetastet und danach festgehalten. Die Schnittpunkte der abgetasteten Sollwerte mit dem Trägersignal ergeben die Schaltfunktionen $s_{U}, s_{V}$ und $s_{W}$ und multipliziert mit $U_{d} / 2$ die bekannten Verläufe der Mittelpunktspannungen $u_{U 0}, u_{V 0}$ und $u_{W 0}$ des Stromrichters. Diese 3 Spannungen sind in jedem Zeitabschnitt durch die im Bild angeschriebenen Stromrichterzustände bestimmt.

Mit Hilfe von Bild 8.13 lassen sich mittels einfacher geometrischer Überlegungen die Beziehungen zwischen den abgetasteten Sollwerten und den entsprechenden Einschaltzeiten der Stromrichterzustände herleiten. Die Amplitude des Dreiecksignals sei eins und die Sollwerte immer kleiner oder gleich eins. Damit gelten:

$$
\frac{t_{0}}{T_{\text {Tast }}}=\frac{1}{2}\left[1-x^{\prime}\left(\omega_{1} k T_{\text {Tast }}\right)\right]=\frac{1}{2}\left[1-M \cos \left(\omega_{1} k T_{\text {Tast }}\right)\right]
$$




$$
\begin{aligned}
& \frac{t_{1}}{T_{\text {Tast }}}=\frac{1}{2}\left[x_{U, \text { Soll }}\left(\omega_{1} k T_{\text {Tast }}\right)-x_{V, \text { Soll }}\left(\omega_{1} k T_{\text {Tast }}\right)\right]=\frac{\sqrt{3}}{2} M \sin \left(\frac{\pi}{3}-\omega_{1} k T_{\text {Tast }}\right) \\
& \frac{t_{2}}{T_{\text {Tast }}}=\frac{1}{2}\left[x_{V, \text { Soll }}\left(\omega_{1} k T_{\text {Tast }}\right)-x_{W, \text { Soll }}\left(\omega_{1} k T_{\text {Tast }}\right)\right]=\frac{\sqrt{3}}{2} M \sin \left(\omega_{1} k T_{\text {Tast }}\right)
\end{aligned}
$$

Die Resultate der Gleichungen sind identisch mit den berechneten Zeiten für die Drehzeigermodulation bei einer Sternpunktspannung von null (8.10) und (8.16). Der Vergleich bestätigt, dass regular sampled Träger- und Drehzeigerverfahren die gleichen Pulsmuster liefern.

\subsection{Phasenspannungen, Verzerrungsströme und Drehmomentwelligkeit}

\subsubsection{Beschreibung im Frequenzbereich}

Wie bei den Trägerverfahren in Kapitel 7.4.3 ist auch hier die analytische Beschreibung der Phasenspannung als Summe von Teilschwingungen grundsätzlich möglich. Die resultierenden Ausdrücke werden dabei aber sehr unübersichtlich. Die nachfolgende näherungsweise Beschreibung im Zeitbereich dagegen ergibt einfache Formeln.

\subsubsection{Näherungsformeln für die Beschreibung von Spannungen und Strömen}

Dank der sehr engen Verwandtschaft mit den Trägerverfahren können sämtliche analytischen Berechnungen von Spannungen, Strömen und Drehmomentwelligkeit vollständig aus Kapitel 7.4.4 übernommen werden. Einzig für die dort nicht behandelten diskontinuierlichen Verfahren kommt neu (8.22) dazu. Es sei an dieser Stelle nochmals betont, dass es sich dabei um sehr gute Näherungen handelt, die bei Schaltzahlen $q>10$ praktisch exakt werden. Insbesondere gilt bei den Drehzeigermodulationen, dass die Sollwerte abgetastet sind. Die Näherungsformeln stimmen deshalb hier sogar noch besser als bei den natural sampled Trägerverfahren.

Verzerrungsstrom bei der Standardvariante der Drehzeigermodulation: Zum Vergleich mit (8.22) wird die Formel (7.91) für $t_{0}=t_{7}$ nochmals angegeben:

$$
I_{U / V / W, V Z, \text { eff }}=\frac{U_{d} M}{4 \sqrt{3} L_{k}} \frac{T_{T}}{2} \sqrt{0.5-0.735 M+0.33 M^{2}}, \quad T_{T}=2 T_{\text {Tast }}
$$

Verzerrungsstrom bei den diskontinuierlichen Verfahren: Bei nur einem Nullzustand pro Abtastintervall gilt: 


$$
I_{U / V / W, V Z, e f f}=\frac{U_{d} M}{4 \sqrt{3} L_{k}} \frac{T_{T}}{2} \sqrt{2-K_{G 1} M+K_{G 2} M^{2}}, \quad T_{T}=2 T_{\text {Tast }}
$$

Für die Konstanten $K_{G 1}$ und $K_{G 2}$ gilt in Abhängigkeit der Nullzustandsaufteilung innerhalb eines Sektors:

- Variante 1 (Bild 8.8): $K_{G 1}=3.122, K_{G 2}=1.280$

- Variante 2 (Bild 8.10): $K_{G 1}=3.216, K_{G 2}=1.385$

- Variante 3 (Bild 8.11): $K_{G 1}=3.310, K_{G 2}=1.435$

\subsubsection{Drehmomentwelligkeit}

Für die Standardvariante der Drehzeigermodulation mit $t_{0}=t_{7}$ gelten die Herleitung und Formel in Kapitel 7.4.5. Der Scheitelwert der Welligkeit ist identisch mit demjenigen von Trägerverfahren mit Injektion von $25 \% 3$. Harmonischen.

Bei den diskontinuierlichen Verfahren muss die Drehmomentwelligkeit für jeden Fall neu bestimmt werden.

Literatur zu Kapitel 8: [Broe1], [Broe2], [Boy1], [Fuk1], [Han1], [Hol6], [Jen2], [Kol1], [Kol2], [Kol3], [Kol4], [Kol5], [Mur1], [Oga1], [Web1], [Wei1] 


\section{Vorausberechnete Pulsmuster}

\subsection{Funktionsprinzip}

Bei einem On-Line Steuerverfahren werden die Schaltwinkel fortlaufend bestimmt. Im Gegensatz dazu werden sie bei einem Off-Line Verfahren für verschiedene Parameter vorausberechnet und als Pulsmuster für ganze Perioden in einer Tabelle abgelegt. Im Betrieb braucht der Modulator dann nur das den Eingangsgrössen entsprechende Pulsmuster auszugeben. Das Bild 9.1 zeigt den grundsätzlichen Aufbau eines solchen Modulators. Als Eingangsgrössen der Kontrolleinheit werden der Modulationsgrad $M$, die Grundkreisfrequenz $\omega_{1}$ und die Phasenlage $\varphi_{u}$ der einzustellenden Ausgangsspannung vorgegeben. Weiter braucht der Modulator zur Erzeugung der Schaltfunktionen zusätzlich eine Zeitbasis in der Form eines Zählers. Dieser kann sich innerhalb oder ausserhalb des Modulators befinden und ist je nach Anwendung freilaufend oder geführt. Ein Beispiel einer externen, geführten Zeitbasis ist der Modulator für einen Netzstromrichter. Dort muss $\omega_{1}$ auf die Netzkreisfrequenz synchronisiert sein. Dies ist nur realisierbar, wenn die Netzperiode ein ganzzahliges Vielfaches des Zeitintervalls des Zählers ist. Dazu wird der Zähler mit einem PLL (Phase-Locked-Loop) auf das Netz synchronisiert.

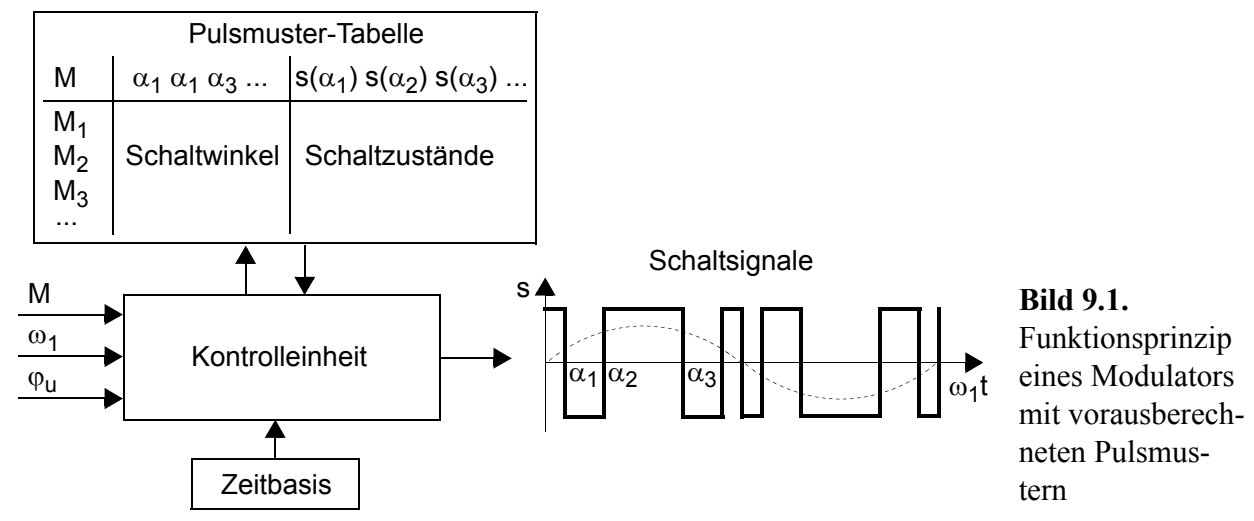

In der Tabelle sind für alle benötigten Modulationsgrade die Schaltwinkel bezüglich der Grundperiode und die zugehörigen Schaltzustände abgelegt. Die Kontrolleinheit sorgt dafür, dass die richtigen Schaltwinkel aus der Tabelle ausgelesen werden. Sie werden aufgrund der vorgegebenen Grundfrequenz in Schaltzeiten umgerechnet und mit Hilfe der Zeitbasis zur richtigen Zeit ausgegeben. Sobald die ganze Periode des Pulsmusters abgearbeitet ist, wird eine neue Periode gestartet. Das Steuerverfahren ist synchron. 
Dasselbe Pulsmuster kann, wie das Beispiel in Bild 9.2 zeigt, für verschiedene Grundfrequenzen verwendet werden. Es ist allerdings $\mathrm{zu}$ beachten, dass dabei die absolute Schaltfrequenz variiert. Sie nimmt proportional mit der Grundfrequenz zu. Deshalb kann häufig nicht der gesamte Betriebsbereich durch Pulsmuster mit derselben Schaltzahl abgedeckt werden. Dies soll am Beispiel eines Modulators für eine ASM mit Spannungsführung nach dem Spannungs-Frequenz-Kennlinien Verfahren gemäss Bild 9.3 erläutert werden: damit die Schaltfrequenz $f_{S}$ im Bereich zwischen den vorgegebenen Maximal-
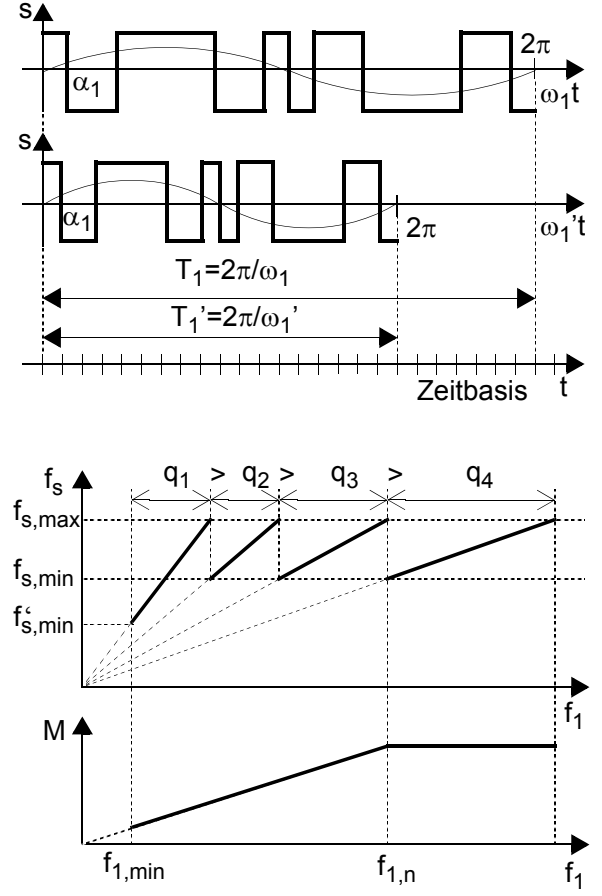

Bild 9.2.

Gleiches Pulsmuster für zwei verschiedene Grundkreisfrequenzen
Bild 9.3.

Modulator für eine ASM mit Spannungsführung nach dem Spannungs-Frequenz-Kennlinien Verfahren, Schaltfrequenz und Modulationsgrad in Funktion der Grundfrequenz

und Minimalwerten $f_{s, \max }$ und $f_{s, \min }$ bleibt, muss bei zunehmender Grundfrequenz mehrmals auf eine kleinere Schaltzahl gewechselt werden. Unterhalb der Nennfrequenz $f_{1, n}$ verhält sich der Modulationsgrad proportional zur Grundfrequenz, so dass für jeden Punkt auf der Kennlinie ein separates Pulsmuster vorhanden sein muss. Dabei weisen die Muster je nach Schaltzahl eine unterschiedliche Anzahl Schaltwinkel auf (im Bild $q_{1}, q_{2}$ und $q_{3}$ ). Im Bereich der Feldschwächung bleibt $M$ konstant und es kann ein einziges Pulsmuster mit der Schaltzahl $q_{4}$ verwendet werden. Speziell zu beachten ist der Anfahrbereich $\left(f_{1}\right.$ sehr klein). Wenn sich die Grundfrequenz gegen null bewegt, so muss die Schaltzahl $q$ immer häufiger erhöht werden um die Schaltfrequenz oberhalb von $f_{s, \min } \mathrm{zu}$ halten. Dabei kann $q$ beliebig gross werden. Abhängig von der Speicherkapazität für die Pulsmuster ergibt sich deshalb eine untere Grenze $f_{1, \text { min }}$ für die Verwendung eines Off-Line Verfahrens. Wenn man, wie im Bild 9.3 angedeutet, in diesem Bereich zusätzlich eine kleinere Schaltfrequenz $f_{S, \min }$ zulässt, verschiebt $\operatorname{sich} f_{1, \min }$ etwas weiter hinunter. Die verbleibende Lü- 
cke bis auf null kann jedoch nur geschlossen werden, wenn man dort auf ein asynchrones Steuerverfahren umstellt.

Die Forderung nach einer Beschränkung der Schaltfrequenz innerhalb mehr oder weniger engen Grenzen ist sinnvoll, weil damit einerseits der Verzerrungsanteil in den Phasenströmen und andererseits die Schaltverluste im Stromrichter im gesamten Betriebsbereich in der gleichen Grössenordnung bleiben. Dasselbe gilt für die Drehmomentwelligkeit bei einem Antrieb.

Alle Parameter lassen sich nur quantisiert einstellen: die Auflösung des Modulationsgrades ist durch die Anzahl der gespeicherten Pulsmuster begrenzt und die Grundkreisfrequenz, die Phasenlage des Pulsmusters sowie die Position der Schaltwinkel sind durch die Auflösung der Zeitbasis quantisiert.

In der Regel erfüllt ein Mikroprozessor die Funktion der Kontrolleinheit (Kapitel 19). Die Ausgabe der Schaltereignisse kann dabei mittels Interrupts realisiert werden. Diese Lösung ist eher schwerfällig und beansprucht viel Rechenleistung. Besser ist eine Auslagerung aus dem eigentlichen Rechner in eine Ausgabelogik (z.B. programmierbarer Logikbaustein oder ASIC). Dort wird einfach die Schaltzeit und der Schaltzustand abgelegt. Bei Übereinstimmung der Schaltzeit mit der Zeitbasis erfolgt die Ausgabe automatisch. Die Ausgabe muss dabei auch für diejenigen Schaltwinkel korrekt erfolgen, welche sehr nahe beieinander liegen. Die entsprechenden kritischen Sequenzen sind durch den Charakter der Pulsmuster gegeben, wobei jedoch die zeitlichen Abstände vor allem auch mit zunehmender Grundfrequenz kleiner werden. Damit die Rechengeschwindigkeit nicht auf diesen Grenzfall ausgelegt sein muss, drängt sich der Einsatz einer Ausgabelogik auf, die mehrere Schaltereignisse speichern und verarbeiten kann. Das Nachladen von weiteren Schaltereignissen braucht dann nur mit einer Repetitionsrate zu erfolgen, die dem mittleren Abstand zwischen den Schaltzeiten entspricht.

Im Vordergrund des Kapitels 9 stehen die Berechnung der Pulsmuster und ihre stationären Eigenschaften. Der stationäre Betrieb des Modulators ist einfach realisierbar, da nur ein einziges Pulsmuster zyklisch auszugeben ist. Die Reaktion auf Änderungen der Eingangsparameter $\mathrm{M}, \omega_{1}$ und $\varphi_{u}$ stellt dagegen an die Kontrolleinheit sehr grosse Ansprüche. Auf diese Problematik wird in Kapitel 9.3.5 speziell eingegangen.

Die abgespeicherten Schaltwinkel können nach beliebigen Kriterien berechnet worden sein. Den einfachsten Fall eines Modulators mit vorausberechneten Pulsmustern stellt die Grundfrequenzsteuerung dar. Sie ergibt für einen dreiphasigen Stromrichter eine Tabelle mit nur einem Muster und 6 Schaltwinkeln. Im allgemeinen handelt es sich aber um Pulsmuster mit niedriger Schaltzahl, welche On-Line nicht implementierbar sind, weil die Schaltwinkel einer ganzen Periode das Resultat einer globalen Optimierung darstellen. Da bei der eigentlichen Berechnung der Schaltwinkel keine Echtzeitprobleme zu bewältigen sind, können die Algorithmen fast beliebig komplex sein.

Dreiphasige Pulsmuster: Das Prinzipschema in Bild 9.1 bezieht sich auf einen einphasigen Modulator. Es sieht für dreiphasige Schaltungen jedoch grundsätzlich gleich aus. Normalerweise ist davon auszugehen, dass wie in Bild 9.2 die Schaltfunktionen für die 3 Brückenzweige abgesehen von der Phasenverschiebung identisch sind. Es herrschen dann vollständig symmetrische Verhältnisse und die Phasenspannungen und -ströme sind frei von allen Vielfachen der 3. Harmonischen (Anhang B.1). Die Berechnung der Schaltwin- 


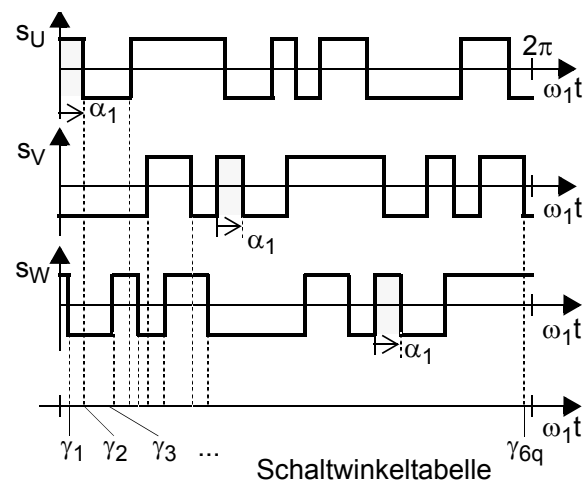

Bild 9.4.

Dreiphasiges, symmetrisches Pulsmuster und zugehörige sortierte Schaltwinkeltabelle

kel kann anhand einer einzelnen Schaltfunktion erfolgen. Der Modulator dagegen betrachtet das Ganze als ein Pulsmuster, bestehend aus den sortierten Schaltwinkeln $\gamma_{1}$ bis $\gamma_{6 q}$ und jeweils 3 Schaltzuständen $s_{U}, s_{V}$ und $s_{W}$. Die abgespeicherten Tabellen sind entsprechend grösser.

\subsection{Selektive Elimination von Harmonischen}

\subsubsection{Einphasige Brücke}

\subsubsection{Berechnung der Schaltwinkel}

Es wird direkt die Phasenspannung der einphasigen Brücke (Schaltung gemäss Bild 5.1) betrachtet. Bild 9.5 zeigt die allgemeine Form eines viertelperiodensymmetrischen Verlaufes. Wenn die Schaltfunktionen der Brückenzweige $s_{A}$ und $s_{B}$ beide die Schaltzahl $q$ aufweisen, ergibt das für die Phasenspannung $4 q$ Schaltwinkel pro Grundperiode. Wegen der Symmetrie lässt sie sich jedoch durch die $q$ Schaltwinkel $\alpha_{1}$ bis $\alpha_{q}$ einer Viertelperiode vollständig beschreiben. Gemäss Kapitel 5.3.2 können auch die Fourierkoeffizienten aus dem Verlauf einer Viertelperiode berechnet werden und es treten in der gewählten Darstellung nur Sinuskoeffizienten auf:

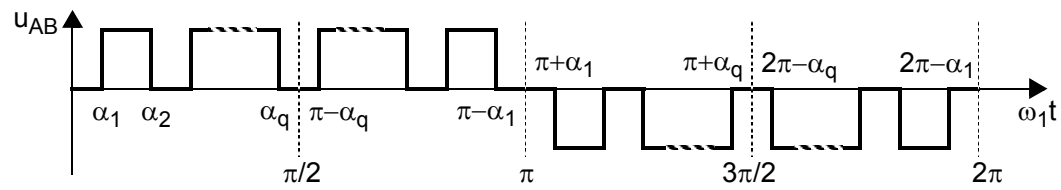

Bild 9.5. Viertelperiodensymmetrische Phasenspannung einer einphasigen Brücke

$$
b_{v}=\frac{4}{\pi} \int_{0}^{\pi / 2}\left[u_{A B} \sin \left(v \omega_{1} t\right)\right] d\left(\omega_{1} t\right), \quad v=1,3,5, \ldots
$$




$$
b_{v}=\frac{4}{\pi}\left[\int_{\alpha_{1}}^{\alpha_{2}} U_{d} \sin \left(v \omega_{1} t\right) d\left(\omega_{1} t\right)+\ldots+\int_{\alpha_{q-1}}^{\alpha_{q}} U_{d} \sin \left(v \omega_{1} t\right) d\left(\omega_{1} t\right)\right]
$$

Die Integrale in (9.1) lassen sich leicht ausrechnen, so dass sich die Fourierkoeffizienten in Funktion der Schaltwinkel ergeben:

$$
\begin{gathered}
b_{v}=\frac{4 U_{d}}{v \pi}\left\{\left[\cos \left(v \alpha_{1}\right)-\cos \left(v \alpha_{2}\right)\right]+\ldots+\left[\cos \left(v \alpha_{q-1}\right)-\cos \left(v \alpha_{q}\right)\right]\right\} \\
b_{v}=\frac{4 U_{d}}{v \pi} \sum_{i=1}^{q}-1^{(i+1)} \cos \left(v \alpha_{i}\right), \quad \hat{u}_{A B, v}=\left|b_{v}\right|, \quad v=1,3,5, \ldots
\end{gathered}
$$

In der gewählten Darstellung ist der Koeffizient $b_{1}$ für die Grundschwingung stets positiv und proportional zum Modulationsgrad.

Ausgehend von (9.2) können die Schaltwinkel so gewählt werden, dass die Harmonischen ein gewünschtes Verhalten zeigen. Beim Prinzip der selektiven Elimination von Harmonischen bedeutet dies, dass eine Reihe von Harmonischen vollständig verschwinden soll. Üblicherweise werden diejenigen der niedrigsten Ordnungen ausgewählt, da diese im Phasenstrom die grössten Verzerrungsanteile verursachen. Eine weitere Bedingung ist, dass die Amplitude der Grundschwingung dem Sollwert $\hat{u}_{A B \text {, Soll }}$ entsprechen soll. Diese Forderungen lassen sich im Gleichungssystem (9.3) zusammenfassen und direkt auf die Fourierkoeffizienten übertragen:

$$
\begin{aligned}
& \hat{u}_{A B, v=1}-\hat{u}_{A B, \text { Soll }}=0 \\
& \left.\Rightarrow \quad \begin{array}{c}
b_{1}-M U_{d}=0 \\
b_{3}=0 \\
b_{5}=0 \\
\cdots \\
b_{2 q-1}=0
\end{array}\right\} q \text { Gleichungen } \\
& \hat{u}_{A B, v=3}=0 \\
& \left.\Rightarrow \quad \begin{array}{c}
b_{1}-M U_{d}=0 \\
b_{3}=0 \\
b_{5}=0 \\
\cdots \\
b_{2 q-1}=0
\end{array}\right\} q \text { Gleichungen } \\
& \hat{u}_{A B, v=5}=0 \\
& \left.\Rightarrow \quad \begin{array}{c}
b_{1}-M U_{d}=0 \\
b_{3}=0 \\
b_{5}=0 \\
\cdots \\
b_{2 q-1}=0
\end{array}\right\} q \text { Gleichungen } \\
& \left.\Rightarrow \quad \begin{array}{c}
b_{1}-M U_{d}=0 \\
b_{3}=0 \\
b_{5}=0 \\
\cdots \\
b_{2 q-1}=0
\end{array}\right\} q \text { Gleichungen } \\
& \hat{u}_{A B, v}=2 q-1=0
\end{aligned}
$$

Unter Verwendung von (9.2) und nach dem Vereinfachen der Gleichungen ergibt sich schliesslich das Gleichungssystem (9.4), das als Ausgangspunkt für die Berechnung der Schaltwinkel dient:

$$
\begin{aligned}
& \left.\begin{array}{l}
\left\{\sum_{i=1}(-1)^{i+1} \cos \left(\alpha_{i}\right)\right\}-\frac{\pi}{4} M=0 \\
\sum_{i=1}^{q}(-1)^{i+1} \cos \left(3 \alpha_{i}\right)=0 \\
\ldots
\end{array}\right\} \mid\left[\begin{array}{c}
F_{1}\left(\alpha_{1} \ldots \alpha_{q}\right) \\
\ldots \\
\ldots \\
F_{q}\left(\alpha_{1} \ldots \alpha_{q}\right)
\end{array}\right]=F\left(\alpha_{1} \ldots \alpha_{q}\right)=0 \\
& \sum(-1)^{i+1} \cos \left([2 q-1] \alpha_{i}\right)=0
\end{aligned}
$$


Mit Hilfe der $q$ Schaltwinkel können $q$ Gleichungen aufgestellt und gelöst werden. Neben der Festlegung des Modulationsgrades sind $q$-1 Harmonische eliminierbar.

Auflösung des Gleichungssystems: Das transzendente Gleichungssystem (9.4) lässt sich nur mit numerischen Methoden auflösen. Es wird an dieser Stelle eine kurze Beschreibung des Lösungsweges gegeben. Mehr über die Lösungsmethoden sind in Fachbüchern für Ingenieurmathematik zu finden, z.B. [Dir1], [Ham1].

Verwendet wird meistens das Newton-Verfahren. Es basiert auf einer Linearisierung des Funktionsvektors $\underline{F}\left(\alpha_{1} \ldots \alpha_{q}\right)$ für einen gegebenen Satz Anfangswinkel $\alpha_{1,0}$ bis $\alpha_{q, 0}$ :

$$
\underline{F}\left(\alpha_{1} \ldots \alpha_{q}\right) \approx \underline{F}\left(\alpha_{1,0} \ldots \alpha_{q, 0}\right)+\operatorname{gradF}\left(\alpha_{1,0} \ldots \alpha_{q, 0}\right) \Delta \underline{\alpha}
$$

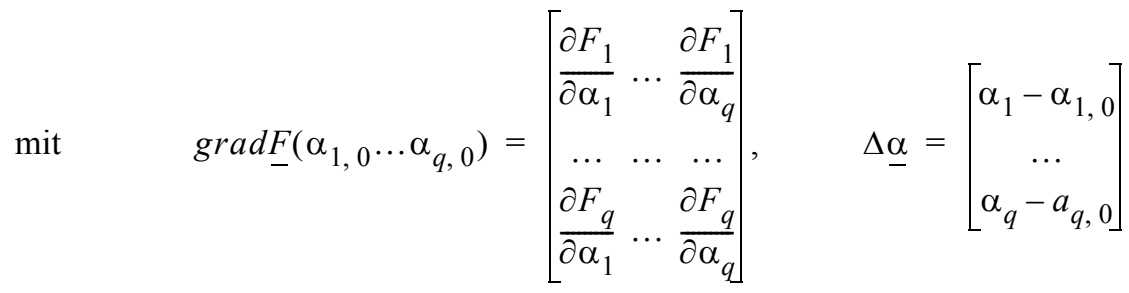

Der Gradient $\operatorname{gradF}\left(\alpha_{1,0} \ldots \alpha_{q, 0}\right)$ beschreibt die partiellen Ableitungen der zu eliminierenden Harmonischen nach den einzelnen Schaltwinkeln. Er lässt sich beim vorliegenden Problem analytisch berechnen. Anstelle des nichtlinearen Gleichungssystems (9.4) tritt so das lineare System (9.6), welches mit den Methoden der linearen Algebra gelöst werden kann:

$$
\begin{aligned}
& \underline{F}\left(\alpha_{1,0} \ldots \alpha_{q, 0}\right)+\operatorname{gradF}\left(\alpha_{1,0} \ldots \alpha_{q, 0}\right) \Delta \underline{\alpha}=0 \\
& \left.\quad \Rightarrow\left[\begin{array}{c}
\alpha_{1,1} \\
\ldots \\
\alpha_{q, 1}
\end{array}\right]=-\operatorname{grad}_{-}\left(\alpha_{1,0} \ldots \alpha_{q, 0}\right)\right]^{-1} \underline{F}\left(\alpha_{1,0} \ldots \alpha_{q, 0}\right)+\left[\begin{array}{c}
\alpha_{1,0} \\
\ldots \\
\alpha_{q, 0}
\end{array}\right]
\end{aligned}
$$

Allerdings sind die Lösungswinkel $\alpha_{1,1}$ bis $\alpha_{q, 1}$ aus (9.7) noch nicht die Lösungen des ursprünglichen Systems (9.4). Sie liegen jedoch näher bei diesen als die Anfangswinkel $\alpha_{1,0}$ bis $\alpha_{q, 0}$. Die richtigen Lösungen lassen sich bestimmen, indem iterativ jeweils die Lösungswinkel aus (9.7) wieder als Anfangswinkel in (9.6) eingesetzt werden. Unterscheiden sich schliesslich die beiden Winkelsätze um weniger als die verlangte Genauigkeit, so ist das endgültige Pulsmuster gefunden. Am Beispiel einer einzelnen Gleichung $F(x)=0$ mit einer Variablen ist das Newton-Verfahren in Bild 9.6 graphisch illustriert. Die beschriebene Berechnung der Schaltwinkel erfolgt auf dieselbe Weise, jedoch in einem durch die $q$ Schaltwinkel aufgespannten $q$-dimensionalen Raum.

Während der gesamten Prozedur müssen die Schaltwinkel jederzeit im Intervall $[0, \pi / 2]$ bleiben und dürfen ihre Reihenfolge nicht verändern. Andernfalls würde das Pulsmuster seine physikalische Realität verlieren. Diese Randbedingungen lassen sich gemäss (9.8) als Ungleichungen formulieren: 


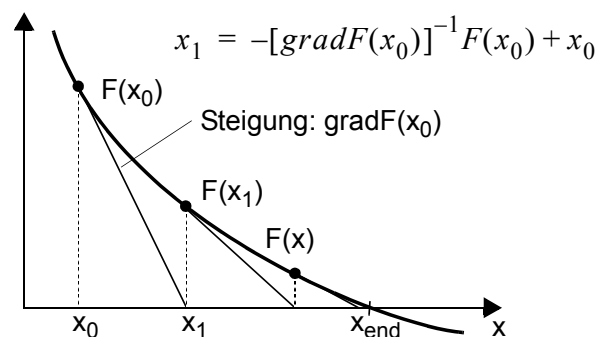

$0 \leq \alpha_{1} \leq \alpha_{2} \leq \ldots \leq \alpha_{q} \leq \frac{\pi}{2}$
Bild 9.6.

Newton-Verfahren zur Lösung nichtlinearer Gleichungssysteme, Beispiel einer Gleichung mit einer Variablen $F(x)=0$

Das beschriebene Verfahren konvergiert nur, falls bereits die ersten Anfangswinkel nahe genug bei den endgültigen Lösungen liegen. Diese Forderung stellt ein grosses Problem dar und seine Beherrschung erfordert einige Erfahrung.

\subsubsection{Resultate}

In [Kat1] konnte gezeigt werden, dass das Gleichungssystem (9.4) höchstens eine Lösung hat. Sie existiert im Bereich zwischen $M=0$ und einem maximalen Modulationsgrad $M_{\text {max }}$, der von der Schaltzahl $q$ abhängt. Bild 9.7 zeigt die Lösungen für die Schaltzahlen $q=4$ und $q=21$. Dargestellt sind die Schaltwinkel in Abhängigkeit des Modulationsgrades und als Beispiel je die zeitlichen Verläufe und Spektra der Phasenspannung und des Verzerrungsanteils im Phasenstrom für den Modulationsgrad $M=0.8$. Die Schaltwinkel verändern sich in einem weiten Bereich nahezu linear mit dem Modulationsgrad. Erst in der Nähe der maximalen Aussteuerung wird der Verlauf merklich nichtlinear. Die maximal erreichbare Aussteuerung liegt generell leicht über eins, also höher als bei den Trägerverfahren. Sie beträgt $M_{\max } \approx 1.07$ bei $q=2$ und sinkt mit steigender Schaltzahl rasch gegen $M_{\max }=1$. Dieser Trend ist auch in Bild 9.7 zu erkennen. Bei den zeitlichen Verläufen lässt sich feststellen, dass der Verzerrungsstrom für die grössere Schaltzahl markant kleiner ist. Die Wirkung der Elimination zeigt sich erster Linie in den Spektra: für $q=4$ verschwinden die ersten 3 Oberschwingungen und für $q=21$ die ersten 20. Die niedrigste verbleibende Harmonische hat die Ordnung $2 q-1$, d.h. ihre Frequenz liegt gerade oberhalb der zweifachen Schaltfrequenz. Die Amplituden der auftretenden Harmonischen stellen sich auf einen Wert ein, der nicht weiter beeinflussbar ist. Dabei sind diejenigen der niedrigsten Ordnungen unter ihnen dominant.

Verzerrungsstrom: Bild 9.8 zeigt den Effektivwert des Verzerrungsstromes in Funktion des Modulationsgrades wiederum für die Schaltzahlen $q=4$ und $q=21$. Auch hier wird deutlich, dass die höhere Schaltzahl eine Reduktion des Verzerrungsstromes mit sich bringt. Der Effektivwert verläuft näherungsweise umgekehrt proportional zur Schaltzahl. Wie bei den Trägerverfahren geht der Verzerrungsstrom für sehr kleine Aussteuerung gegen null zurück. Anders ist hingegen, dass er im Bereich von $M=0.6$ ein Maximum erreicht und darüber wieder kleiner wird. 

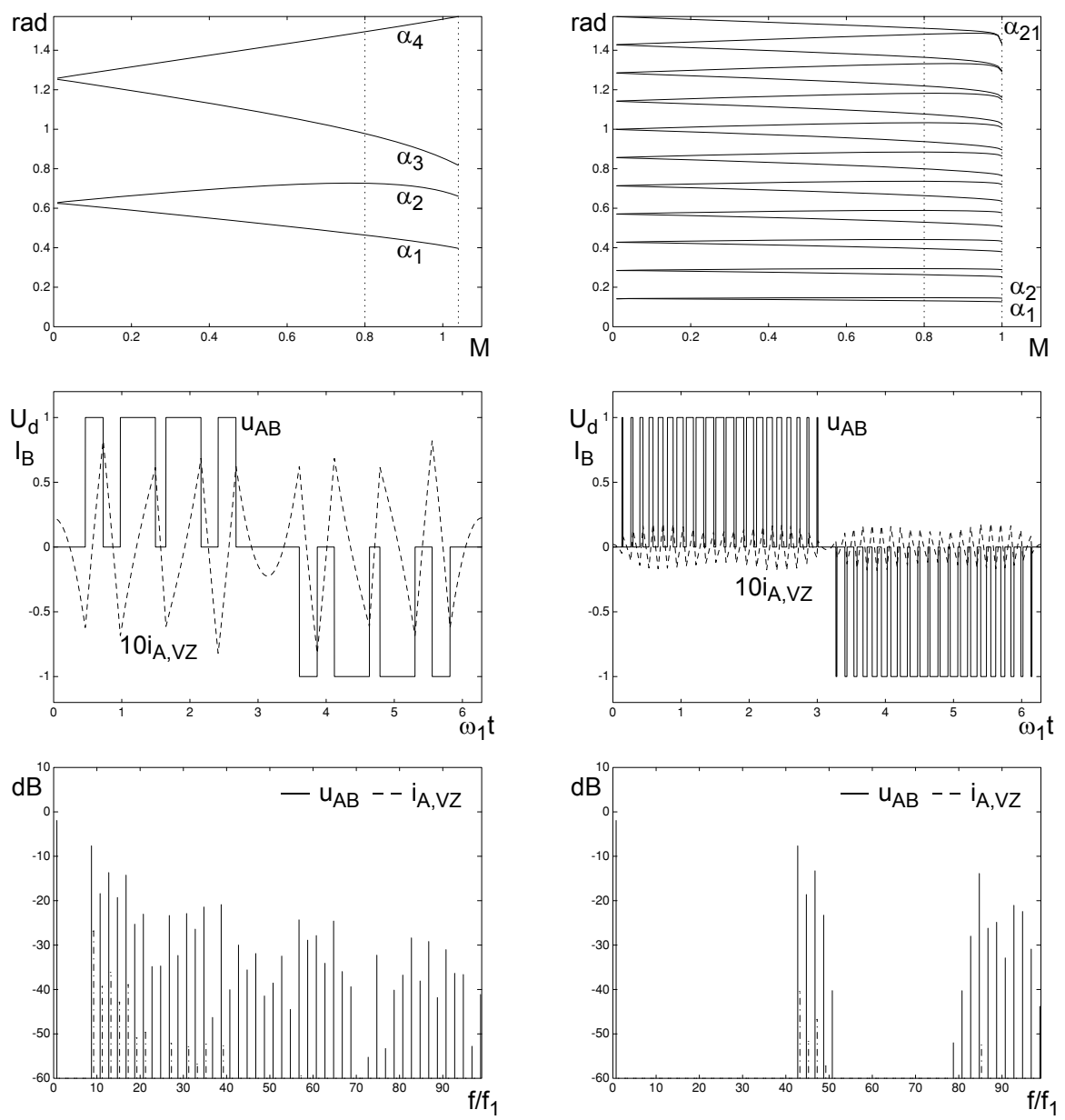

Bild 9.7. Einphasige Pulsmuster mit eliminierten Harmonischen, oben links: Schaltwinkel für $q=4$, oben rechts: Schaltwinkel für $q=21$, mitte: entsprechende Phasenspannungen und Verzerrungsströme, $M=0.8$, unten: zugehörige Spektra, $0 \mathrm{~dB}: U_{d}$ bzw. $I_{B}$

Schaltfunktionen: Die Berechnung der Schaltwinkel wurde ausschliesslich anhand der Phasenspannung durchgeführt. Ihre Aufteilung auf Schaltfunktionen für die beiden Brükkenzweige ist dadurch noch nicht eindeutig definiert. In der Regel gibt es mehrere Möglichkeiten dafür. Normalerweise sind nur diejenigen sinnvoll, welche für beide Umschalter dieselbe Schaltfrequenz ergeben. Bild 9.9 zeigt ein entsprechendes Beispiel mit 3 verschiedenen Aufteilungen. Es ist zu beachten, dass die Schaltfunktionen der einzelnen Brückenzweige nur viertelperiodensymmetrisch sein können, wenn $q$ ungerade ist (Kapitel 5.3.2). Im Bild ist $q$ gerade, d.h. die Schaltfunktionen sind alle höchstens halbperiodensymmetrisch. 


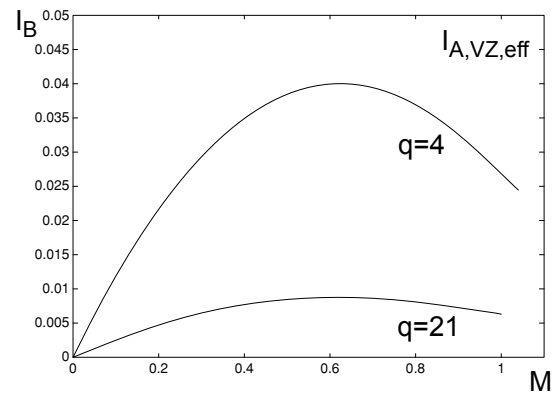

Bild 9.8.

Effektivwert des Verzerrungsstromes in Funktion des Modulationsgrades, einphasige Brücke

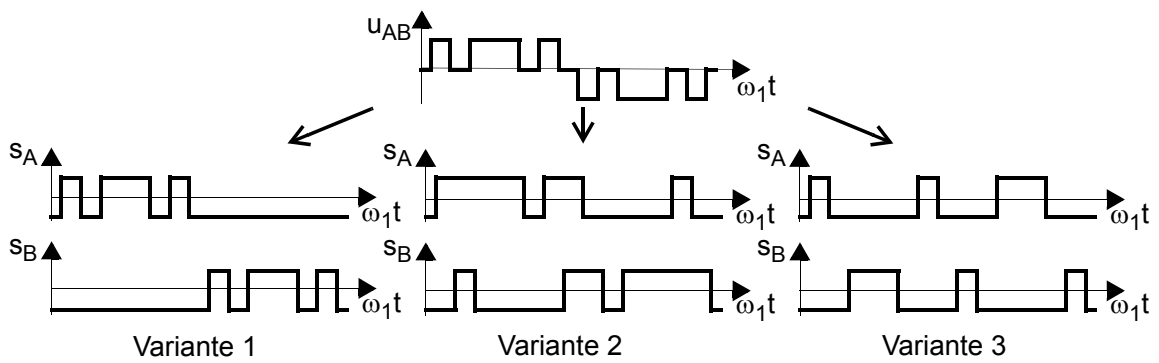

Bild 9.9. Varianten für die Aufteilung der Schaltwinkel auf die beiden Brückenzweige, Beispiel mit $q=3$

\subsubsection{Spezielle Aspekte}

Bisher wurde davon ausgegangen, dass die Oberschwingungen der niedrigsten Ordnung eliminiert werden. Grundsätzlich können jedoch $q-1$ beliebige Oberschwingungen gewählt werden. Dies ist zum Beispiel dann interessant, wenn der Lastkreis Resonanzstellen aufweist, welche durch den Stromrichter nicht angeregt werden dürfen. Enthält das Gleichungssystem (9.4) jedoch andere Harmonische als die angegebenen, so wird das Problem der Anfangswinkel für das numerische Lösungsverfahren sehr komplex. Es sind keine Richtlinien bekannt, welche mit Gewissheit zum Auffinden der Schaltwinkel führen. Ebenso ist nicht absehbar, ob immer noch genau eine oder neu mehrere Lösungen existieren.

Eine weitere Einschränkung wurde bereits bei der Einführung der Phasenspannung in Bild 9.5 gemacht. Prinzipiell könnten in den beiden Halbperioden auch Pulse mit der entgegengesetzten Polarität auftreten. Wie jedoch schon in Kapitel 7.3 erklärt wurde, sind solche Pulsmuster a priori stärker verzerrt. Da mit dem Verlauf nach Bild 9.5 für das behandelte Gleichungssystem (9.4) mit Sicherheit immer eine Lösung existiert, ist die gewählte Einschränkung gerechtfertigt.

Dasselbe gilt für die Vorgabe von viertelperiodischen Verläufen. Es lässt sich zeigen, dass ein verallgemeinerter Ansatz mit nur halbperiodensymmetrischen Pulsmustern oder gar solchen ohne Symmetrie innerhalb der Periode ein Vielzahl von neuen Lösungen bringt. Diese ergeben jedoch durchwegs grössere Verzerrungen als die viertelperiodensymmetrischen. Die grössere Anzahl von freien Schaltwinkeln wird durch die grössere 
Anzahl der zu eliminierenden Komponenten kompensiert $\left(a_{v} \neq 0, v\right.$ nicht mehr nur ungerade). Dabei steigt der Rechenaufwand zur Lösung des Gleichungssystems äusserst stark an.

\subsubsection{Dreiphasige Brücke}

\subsubsection{Berechnung der Schaltwinkel}

Das selektive Eliminieren von Harmonischen in dreiphasigen Pulsmustern basiert auf demselben Prinzip wie in einphasigen. Im Mittelpunkt stehen die Phasenspannungen, da diese über der Last anliegen und für die Phasenströme massgebend sind. Dabei kommt die Eigenschaft zum Tragen, dass beim Anlegen von 3 symmetrischen Schaltfunktionen an einer symmetrischen Last die Spektra der Phasenspannungen keine Vielfachen der 3. Harmonischen aufweisen und dass die restlichen Harmonischen $(v=1,5,7,11,13, \ldots)$ mit denjenigen in den Mittelpunktspannungen übereinstimmen. Zudem sind alle Phasenspannungen abgesehen von den Phasenverschiebungen identisch. Das Problem lässt sich deshalb auf die Elimination der entsprechenden Harmonischen in der für alle 3 Brückenzweige gültigen Schaltfunktion reduzieren.

Es wird wiederum von Viertelperiodensymmetrie ausgegangen. Im dreiphasigen Fall muss dazu $q$ ungerade sein (Kapitel 5.3.2). Die Schaltfunktion und damit die Mittelpunktspannung der Phase $U$ beim dreiphasigen Stromrichter (Schaltung gemäss Bild 5.1) haben

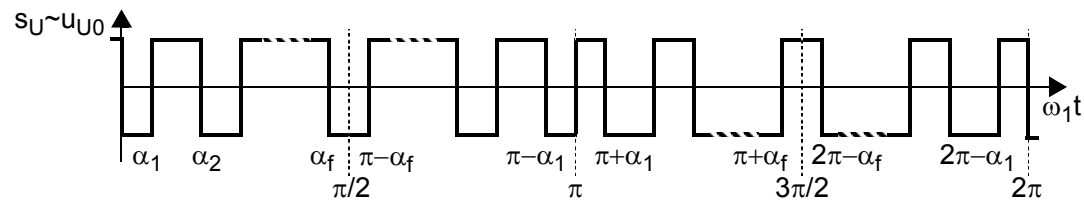

Bild 9.10. Viertelperiodensymmetrische Schaltfunktion bzw. Mittelpunktspannung einer Phase

die allgemeine Form nach Bild 9.10. Es befindet sich immer eine Schaltflanke bei $\omega_{1} t=0$, so dass sich die Anzahl frei wählbarer Winkel $f$ gemäss (9.9) ergibt:

$$
f=\frac{q-1}{2}, \quad q=3,5,7, \ldots
$$

Mit einer Berechnung der Art, wie sie in (9.1) und (9.2) gezeigt wurde, lassen sich die Fourierkoeffizienten der Mittelpunktspannung als Funktion dieser Winkel darstellen:

$$
b_{v}=\frac{8}{v \pi} \frac{U_{d}}{2}\left|\frac{1}{2}+\sum_{i=1}^{J}(-1)^{i} \cos \left(v \alpha_{i}\right)\right|, \quad v=1,3,5 \ldots
$$

Für die Amplituden der Harmonischen in der Phasenspannung der Phase $U$ gilt dann:

$$
\hat{u}_{U, v}=\left|b_{v}\right|, \quad v=1,5,7,11,13, \ldots
$$


Die $f$ Schaltwinkel erlauben es, einerseits einen gewünschten Modulationsgrad $M$ einzustellen und andererseits die $f-1$ Harmonischen niedrigster Ordnung in der Phasenspannung zu eliminieren. Dies wird durch das Gleichungssystem (9.12) zum Ausdruck gebracht. Anstelle der Amplituden der Harmonischen in der Phasenspannung lassen sich direkt die Fourierkoeffizienten der Schaltfunktion einsetzen.

$$
\left.\begin{array}{ccc}
\hat{u}_{U, v=1}-\hat{u}_{U, \text { Soll }}=0 \\
\hat{u}_{U, v=5}=0 \\
\hat{u}_{U, v=7}=0 \\
\ldots
\end{array} \quad \Rightarrow \quad \begin{array}{l}
\left|b_{v=1}\right|-M \frac{U_{d}}{2}=0 \\
b_{v=5}=0 \\
b_{v=7}=0 \\
\ldots
\end{array}\right\} \text { f Gleichungen }
$$

Die 3. Harmonische und ihre (ungeraden) Vielfachen werden nicht berücksichtigt, da sie in der Phasenspannung nicht auftreten. Deshalb lassen sich gegenüber dem einphasigen Fall in einem grösseren Frequenzbereich alle Harmonischen eliminieren. Die Tabelle 9.1 zeigt eine Zusammenstellung der entsprechenden Parameter, wenn jeweils die Harmonischen mit den niedrigsten Ordnungen eliminiert werden.

\begin{tabular}{|c|c|c|c|c|c|}
\hline $\begin{array}{c}\text { Schaltzahl } \\
\mathrm{q}\end{array}$ & $\begin{array}{c}\text { freie } \\
\text { Winkel f }\end{array}$ & eliminierte Harmonische & \begin{tabular}{|} 
niedrigste ver- \\
bleibende Harm.
\end{tabular} & $\begin{array}{c}\text { Anzahl } \\
\text { Lösungen } l\end{array}$ & \multirow{11}{*}{$\begin{array}{l}\text { Tabelle 9.1. } \\
\text { Selektive Eli- } \\
\text { mination von } \\
\text { Harmonischen } \\
\text { in dreipha- } \\
\text { sigen Pulsmus- } \\
\text { tern }\end{array}$} \\
\hline 3 & 1 & - & 5 & 2 & \\
\hline 5 & 2 & 5 & 7 & 3 & \\
\hline 7 & 3 & 5,7 & 11 & 3 & \\
\hline 9 & 4 & $5,7,11$ & 13 & 3 & \\
\hline 11 & 5 & $5,7,11,13$ & 17 & 4 & \\
\hline 13 & 6 & $5,7,11,13,17$ & 19 & 6 & \\
\hline 15 & 7 & $5,7,11,13,17,19$ & 23 & 6 & \\
\hline 17 & 8 & $5,7,11,13,17,19,23$ & 25 & 6 & \\
\hline 19 & 9 & $5,7,11,13,17,19,23,25$ & 29 & 8 & \\
\hline 21 & 10 & $5,7,11,13,17,19,23,25,29$ & 31 & 12 & \\
\hline
\end{tabular}

Auflösung des Gleichungssystems: Bei der Auflösung des Gleichungssystems (9.12) wird im Prinzip gleich vorgegangen wie im einphasigen Fall. Es ist aber zu beachten, dass sich, wie in Bild 9.11 dargestellt, ein bestimmter Modulationsgrad auf zwei verschiedene Arten realisieren lässt. Sie unterscheiden sich in der Schaltflanke bei $\omega_{1} t=0$. Die Formel (9.10) gilt für den Fall, dass diese positiv ist, d.h. für das Pulsmuster vom Typ 1. Bei Pulsmustern nach Typ 2 sind alle Koeffizienten aus (9.10) zusätzlich mit negativem Vorzeichen zu versehen. Um bei der Berechnung der Pulsmuster beide Fälle zu erfassen, muss das Gleichungssystem (9.12) zweimal gelöst werden, wobei (9.10) je einmal mit positiven und mit negativem Vorzeichen eingesetzt wird.

Bereits die Tatsache, dass zwei unterschiedliche Mustertypen existieren, lassen mehr als eine Lösung für das Gleichungssystem (9.12) erwarten. In [Kat1] wird gezeigt, dass 


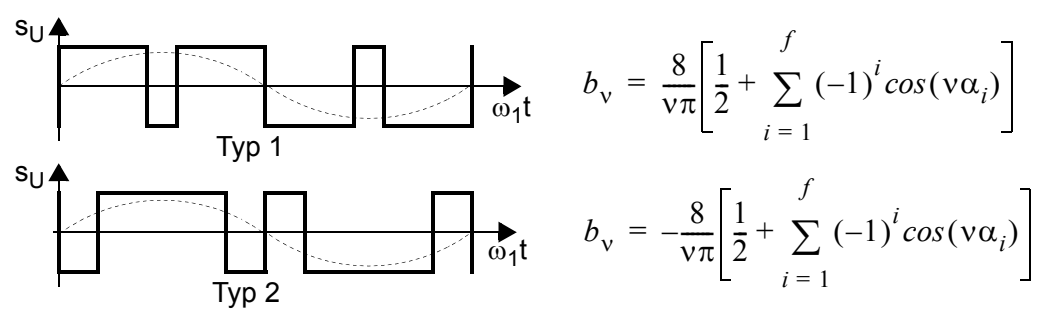

Bild 9.11. Verschiedene Typen von Pulsmustern mit demselben Modulationsgrad

tatsächlich eine Vielzahl von Lösungen existiert, wobei die Anzahl $l$ mit zunehmender Schaltzahl rasch grösser wird. Sie lässt sich in einer Formel ausdrücken:

$$
l=K_{f} 2^{f i x\left(\frac{f+3}{4}\right)}, \quad K_{f}=\left\{\begin{array}{l}
1 \text { für } f=1,5,9,13, \ldots \\
1.5 \text { sonst }
\end{array}\right.
$$

fix $(x)$ bezeichnet die grösste ganze Zahl kleiner als $x$

Die numerische Auswertung von (9.13) ist in der letzten Kolonne der Tabelle 9.1 eingetragen. Nicht alle Lösungen existieren jedoch im Aussteuerbereich von $M=0$ bis zu einem Maximalwert $M_{\text {max }}$. Einige von ihnen gelten nur für einen kleinen Teilbereich, der immer bei sehr grossem Modulationsgrad liegt.

War bereits das Auffinden der Lösung bei einphasigen Pulsmustern nicht unproblematisch, so wird es hier äusserst schwierig. Eine zielgerichtete Methode wird in [Kat1] vorgestellt. Sie basiert auf einem induktiven Prinzip, bei dem die Lösungen aller Schaltzahlen in aufsteigender Reihenfolge mit Hilfe derjenigen der vorangehenden Schaltzahl bestimmt werden.

\subsubsection{Resultate}

Bild 9.12 zeigt die Schaltwinkel für die 3 Lösungen mit der Schaltzahl $q=9$. Die Lösungen 1 und 2 entsprechen Pulsmustern vom Typ 1 (Bild 9.11). Sie existieren im ganzen Aussteuerbereich. Für die Lösung 1 beträgt der maximale Modulationsgrad $M_{\max }=1.178$, für die Lösung 2 1.172. Die Werte sind also nicht identisch. Auffallend ist die sehr starke Sensitivität der Schaltwinkel knapp unterhalb der vollen Aussteuerung. Die Lösung 3 existiert nur in einem sehr kleinen Teilbereich zwischen $M=1.172$ und 1.178.

Bild 9.13 zeigt als Beispiel die zeitlichen Verläufe und die Spektra einer Phasenspannung und des Verzerrungsanteils eines Phasenstromes für die Lösungen 1 und 2 bei $M=0.8$. Obschon beide Pulsmuster Lösungen desselben Gleichungssystems sind, lassen sich deutliche Unterschiede erkennen. Sie äussern sich in unterschiedlichen Amplituden der verbleibenden Harmonischen.

Mit der zur Verfügung stehenden Anzahl freier Schaltwinkel können die Harmonischen über die Schaltfrequenz hinaus eliminiert werden. D.h. diejenigen Harmonischen, welche z.B. bei einem Trägerverfahren im ersten Trägerband dominant herausragen (Kapitel 7.4), sind hier eliminiert. Wie in den einphasigen Pulsmustern sind von den verbleibenden Harmonischen diejenigen der niedrigsten Ordnungen dominant. 

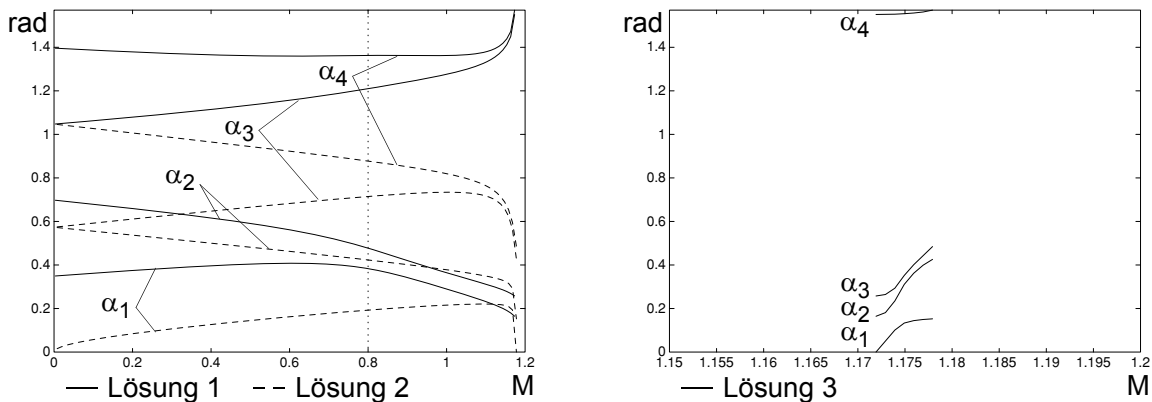

Bild 9.12. Dreiphasige Pulsmuster mit eliminierten Harmonischen, $q=9, f=4$, links: Lösungen 1 und 2 vom Typ 1, rechts: Lösung 3 vom Typ 2
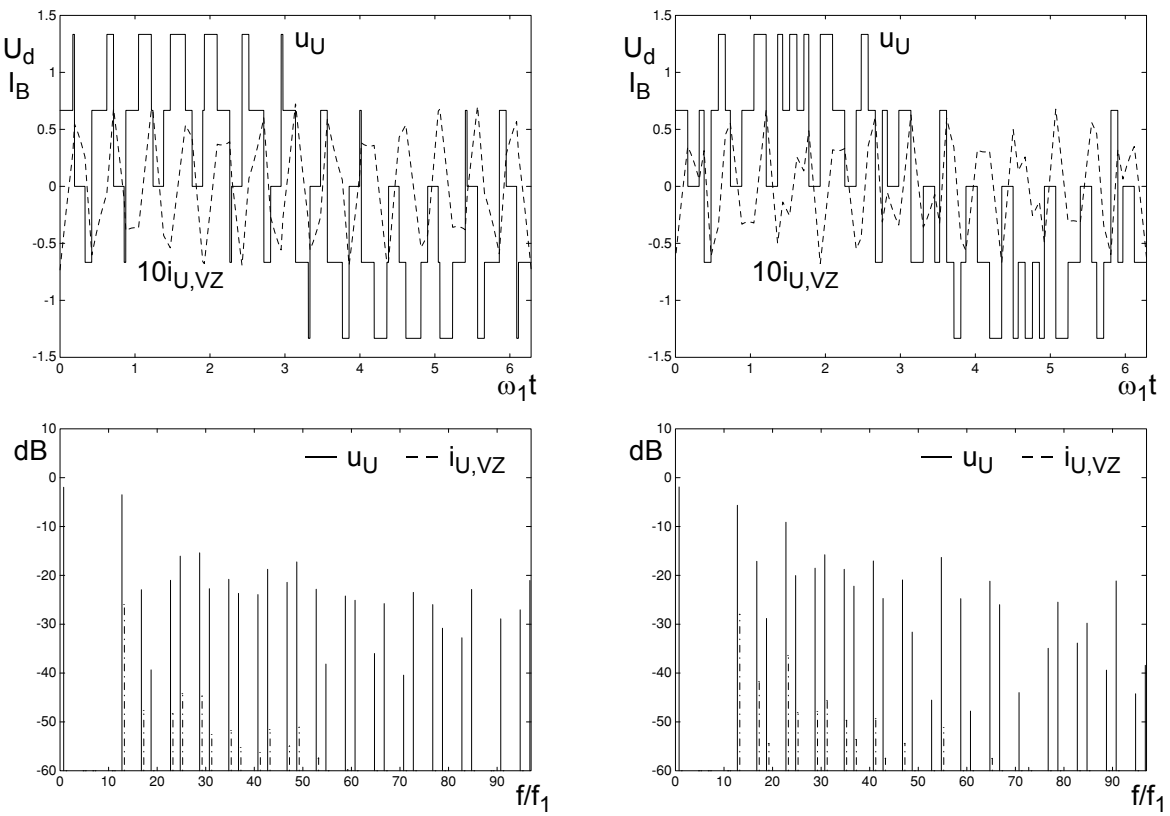

Bild 9.13. Dreiphasige Pulsmuster mit eliminierten Harmonischen, $q=9, f=4$, oben links: Phasenspannung und Verzerrungsstrom für Lösung $1, M=0.8$, oben rechts: Phasenspannung und Verzerrungsstrom für Lösung 2, $M=0.8$, unten: zugehörige Spektra, $0 \mathrm{~dB}: U_{d} / 2$ bzw. $I_{B}$

Verzerrungsstrom: In Bild 9.14 sind die Effektivwerte des Verzerrungsstromes einer Phase in Funktion des Modulationsgrades für alle Lösungen der Schaltzahlen $q=9$ und $q=21$ dargestellt. Wie bereits bei den zeitlichen Verläufen und in den Spektra zeigen sich deutliche Unterschiede zwischen den einzelnen Lösungen. Dabei ergibt eine Lösung für einen grossen Abschnitt des Aussteuerbereiches den Minimalwert. Nur im obersten Teilbereich sind andere Lösungen besser. Die Verläufe der Kennlinien sind teilweise ähnlich 

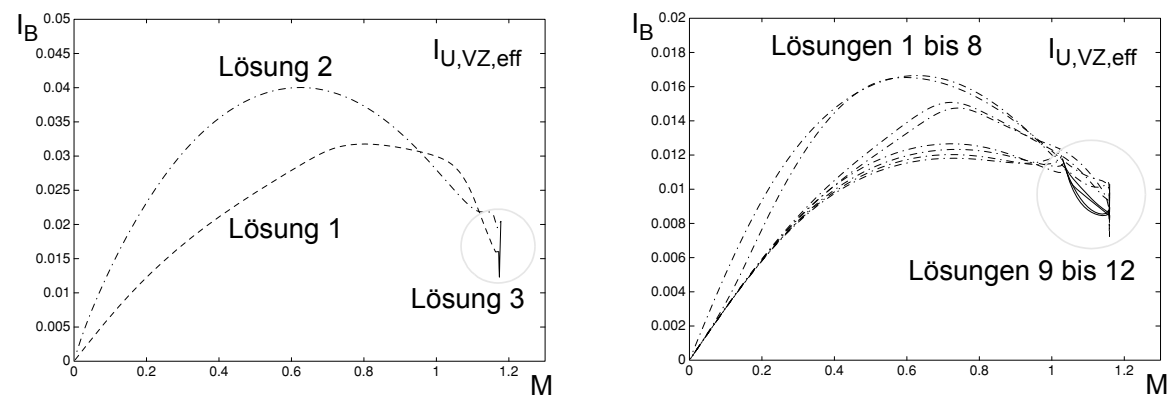

Bild 9.14. Effektivwert des Verzerrungsstromes in einer Phase für alle existierenden Lösungen der entsprechenden Schaltzahl, links: $q=9, f=4, l=3$, rechts: $q=21, f=10, l=12$

zu denjenigen der einphasigen Pulsmuster. Der wichtigste Unterschied ist das ausgeprägte lokale Minimum kurz vor der Maximalaussteuerung. Es tritt dort auf, wo die Position der Schaltwinkel sehr sensitiv auf eine kleine Änderung des Modulationsgrades reagiert (Bild 9.12).

\subsubsection{Spezielle Aspekte}

Bei der Realisierung eines Modulators mit den beschriebenen Pulsmustern stehen grundsätzlich zwei Varianten zur Auswahl: man kann für jeden Modulationsgrad dasjenige Pulsmuster auswählen, das den kleinsten Verzerrungsstrom ergibt. In diesem Fall muss an einer oder an mehreren Stellen im Aussteuerbereich auf eine andere Lösung, also ein Muster mit völlig anderer Schaltwinkelverteilung gewechselt werden. Im Kapitel 9.3.5 wird gezeigt, dass dies dynamisch äusserst unangenehme Auswirkungen haben kann. Beschränkt man sich dagegen darauf, den ganzen Aussteuerbereich mit einer einzigen Lösung abzudecken, so erhält man zwar nicht überall den minimalen Verzerrungsstrom, hat jedoch stetig verlaufende Schaltwinkel.

Wie bereits bei den einphasigen Pulsmustern erwähnt wurde, können anstelle der aufeinanderfolgenden niederfrequenten Harmonischen beliebige Harmonische eliminiert werden. Die Schwierigkeit, das neue Gleichungssystem aufzulösen, nimmt aber noch einmal zu. Auch hier sind keine zielgerichteten Methoden bekannt. Insbesondere ist auch die Anzahl der Lösungen schwer vorhersehbar.

Viertelperiodensymmetrische Pulsmuster für die dreiphasige Brücke können nur mit ungerader Schaltzahl realisiert werden (Kapitel 5.3.2). Schaltfunktionen mit gerader Schaltzahl weisen keine Symmetrie innerhalb der Periode auf. Es ist zwar möglich, für diesen Fall eine Elimination von Harmonischen vorzunehmen, es müssen dazu aber alle $2 q$ Schaltwinkel der Periode mit berücksichtigt werden. Die Anzahl Freiheitsgrade ist dadurch grösser und die Berechnung der Schaltwinkel wird viel aufwendiger. Andererseits sind im allgemeinen alle Fourierkoeffizienten $a_{v}$ und $b_{v}$ der Schaltfunktion ungleich null, so dass die Elimination der Harmonischen wieder in einem vergleichbaren Frequenzbereich wie bei viertelperiodensymmetrischen Pulsmustern möglich ist. Aus diesen Gründen werden gerade Schaltzahlen in der Praxis nicht verwendet. 
Der Rechenaufwand zur Lösung des Gleichungssystems (9.12) nimmt mit wachsender Schaltzahl sehr rasch zu. Da auch die Anzahl der Lösungen näherungsweise exponentionell wächst, bleibt das Verfahren in der Praxis auf sehr niedrige Schaltzahlen beschränkt.

Literatur zu Kapitel 9.2: [Dir1], [Enj1], [Ham1], [Kat1], [Pat1], [Pat2], [Ric2], [Tur1]

\subsection{Optimierte Pulsmuster}

\subsubsection{Das Prinzip der Schaltwinkeloptimierung}

Mit der selektiven Elimination von Harmonischen stellen sich die Amplituden der verbleibenden Harmonischen willkürlich ein. Obschon die entstehenden Verzerrungsströme stets verhältnismässig niedrig sind, entsprechen sie nie dem mit der entsprechenden Schaltzahl erreichbaren Minimum. Bei der Optimierung von Pulsmustern werden die Amplituden aller Harmonischen gezielt beeinflusst. Auf diese Weise kann dasjenige Pulsmuster bestimmt werden, welches bezüglich eines vorgegebenen Gütekriteriums $Z$ optimal ist. Als Gütekriterium wird häufig der Effektivwert des Verzerrungsstromes in Funktion der Schaltwinkel verwendet. Es kann jedoch auch eine beliebige andere Grösse sein, die sich in geschlossener Form durch die Schaltwinkel ausdrücken lässt. Beispiele anderer Gütekriterien werden in Kapitel 9.3.4. betrachtet.

Zur Bestimmung des optimalen Pulsmusters ist das folgende nichtlineare Optimierungsproblem mit Randbedingungen zu lösen:

$$
\begin{aligned}
& Z\left(\alpha_{1}, \alpha_{2}, \ldots, \alpha_{f}\right)=\min \\
& \hat{u}_{v=1}\left(\alpha_{1}, \alpha_{2}, \ldots, \alpha_{f}\right)=\hat{u}_{\text {Soll }} \\
& 0 \leq \alpha_{1} \leq \alpha_{2} \leq \ldots \leq \alpha_{f} \leq \frac{\pi}{2}
\end{aligned}
$$

Die freien Schaltwinkel einer Viertelperiode $\alpha_{1}$ bis $\alpha_{f}$ sind so zu wählen, dass das Gütekriterium $Z$ minimal wird. Die Randbedingung, dass die Grundschwingung der Ausgangsspannung dem vorgegebenen Sollwert entsprechen soll, hat die Form einer Gleichung. Die beiden Funktionen für $Z$ und für $\hat{u}_{v=1}$ sind nichtlinear. Zusätzlich müssen die Schaltwinkel im Intervall $[0, \pi / 2]$ liegen und ihre Reihenfolge ist vorgegeben. Dies entspricht $f+1$ Ungleichungen, welche ebenfalls Randbedingungen darstellen.

Bild 9.15 zeigt das vorliegende Problem in graphischer Form für zwei freie Schaltwinkel $\alpha_{1}$ und $\alpha_{2}$. Über einer dreieckigen Grundfläche, welche durch die Ungleichungen in (9.14) definiert ist (hier: $0 \leq \alpha_{1} \leq \alpha_{2} \leq \pi / 2$ ), ist das Gütekriterium $Z$ als gekrümmte Fläche aufgetragen. Die fett ausgezogenen Linie repräsentiert darauf alle Punkte, welche zusätzlich die Randbedingung der Grundschwingung erfüllen. Die Lösung des Optimierungsproblems findet man, indem man den tiefsten Punkt dieser Linie bestimmt. Das Gütekriterium nimmt dort den Wert $Z_{\text {opt }}$ an und die optimalen Schaltwinkel entsprechen den Koordinaten dieses Punktes. Im allgemeinen Fall mit mehr als zwei freien Schaltwinkeln ist die Problemstellung gleichartig, allerdings in einem $(f+1)$-dimensionalen Raum. 


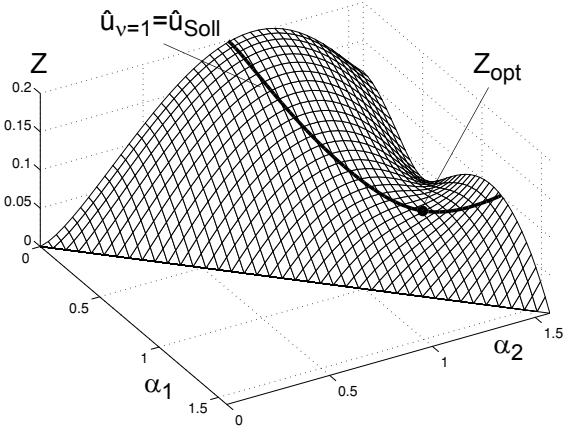

Bild 9.15.

Graphische Interpretation des Optimierungsproblems, Beispiel für zwei freie Schaltwinkel

Die Berechnung der Schaltwinkel kann in den meisten Fällen nur numerisch vorgenommen werden kann. Die zur Verfügung stehenden Lösungsmethoden sind in der Literatur (z.B. [Dir1], [Gil1], [Ham1]) ausführlich beschrieben. Es existieren auch fertige Softwarepakete, die ein Problem der Form (9.14) direkt verarbeiten können. Die gebräuchlichen Verfahren sind, wie das bei der Elimination von Harmonischen gezeigte NewtonVerfahren, gradientenorientiert.

Das Optimierungsproblem hat normalerweise eine eindeutige Lösung, d.h. ein globales Minimum. Daneben existieren jedoch in der Regel eine Vielzahl von lokalen Minima, so dass es äusserst schwierig ist, das globale Minimum zu finden. Wie bei der Elimination von Harmonischen ist es Sache der Erfahrung, mit geeigneten Startwerten für die numerische Lösungsprozedur zum Erfolg zu kommen.

Mit optimierten Pulsmustern lässt sich der Stromrichter generell bis zum Modulations$\operatorname{grad} 4 / \pi$ aussteuern. Dabei nähert sich der Wert des Gütekriteriums gegen Vollaussteuerung hin demjenigen, der mit Grundfrequenzsteuerung erreicht wird.

Die beiden folgenden Kapitel 9.3.2 und 9.3.3 verdeutlichen das Prinzip der Optimierung von Schaltwinkeln. Als Gütekriterium wird der Effektivwert des Verzerrungsstromes in der Standardlast angenommen. Es wird gezeigt, dass die Berechnung des Effektivwertes als Funktion der Schaltwinkel im Zeitbereich oder im Frequenzbereich möglich ist. Es werden auch die Resultate der Optimierungen dargestellt. Auf die Lösungsprozedur wird dagegen nicht eingegangen.

\subsubsection{Effektivwert des Verzerrungsstromes als Gütekriterium bei der einphasigen Brücke}

\subsubsection{Berechnung des Gütekriteriums im Frequenzbereich}

Ausgehend von der Beschreibung der Harmonischen der Ausgangsspannung gemäss (9.2) können leicht auch die Oberschwingungen des Phasenstromes angegeben werden:

$$
\hat{i}_{A, v}=\frac{\hat{u}_{A B, v}}{v \omega_{1} L_{k}}=\left|\frac{4}{v^{2} \pi} \frac{U_{d}}{\omega_{1} L_{k}} \sum_{i=1}^{q}-1^{(i+1)} \cos \left(v \alpha_{i}\right)\right|, \quad v=3,5, \ldots
$$


Der Effektivwert des Verzerrungsanteils wird über die geometrische Summe aller Oberschwingungen gebildet:

$$
I_{A, V Z, \text { eff }}=\sqrt{\sum_{v=3,5, \ldots}^{\infty} \frac{\hat{i}_{A, v}^{2}}{2}}
$$

Damit eine Optimierung praktisch durchführbar ist, kann nur eine endliche Anzahl von Oberschwingungen berücksichtigt werden. Da in (9.15) die Ordnungszahl im Quadrat im Nenner steht, ist eine rasche Abnahme der Amplituden mit wachsender Ordnung garantiert. Zusätzlich beeinflussen Komponenten mit kleinen Amplituden die geometrische Summe nur wenig. Damit kann das Gütekriterium $Z$ gemäss (9.17) formuliert werden. Wenn die Anzahl berücksichtigter Frequenzkomponenten $v_{\text {max }}$ den 5- bis 10-fachen Wert der Schaltzahl $q$ beträgt, so entspricht $Z$ praktisch exakt dem gesamten Effektivwert des Verzerrungsstromes. Durch Einsetzen von (9.15) in (9.17), ergibt sich schliesslich $Z$ gemäss (9.18) als eine reine Funktion der Schaltwinkel.

$$
\begin{aligned}
& Z=\sqrt{\sum_{v=3,5, \ldots}^{v_{\max }} \hat{i}_{A, v}^{2},} \quad v_{\max }=5 \ldots 10 q \\
& Z=\left.\frac{2 \sqrt{2}}{\pi} \frac{U_{d}}{\omega_{1} L_{k}}\right|_{v=3,5, \ldots} ^{v_{\max }}\left[\frac{1}{v_{i=1}^{2}} \sum_{i=1}^{q}-1{ }^{(i+1)} \cos \left(v \alpha_{i}\right)\right]^{2}
\end{aligned}
$$

Zusätzlich ist (9.19) die Beschreibung der Grundschwingung $\hat{u}_{A B, v=1}$ als Funktion der Schaltwinkel, welche für die Randbedingung benötig wird. Sie kann direkt aus (9.2) abgeleitet werden.

$$
\hat{u}_{A B, v=1}=\frac{4}{\pi} U_{d} \sum_{i=1}^{q}(-1)^{i+1} \cos \left(\alpha_{i}\right)=M U_{d}
$$

Damit ist die Beschreibung des Optimierungsproblems vollständig. Im Verlauf der Lösung müssen die Gleichungen (9.17) und (9.19) sehr häufig ausgewertet werden. Dabei ist eine grosse Zahl von trigonometrischen Funktionen zu berechnen, was viel Rechenzeit in Anspruch nimmt. Mit dem nachfolgend beschriebenen Ansatz zur Berechnung des Gütekriteriums im Zeitbereich kann dieses Problem umgangen werden.

\subsubsection{Berechnung des Gütekriteriums im Zeitbereich}

Der Effektivwert des Verzerrungsstromes kann auch aus seinem zeitlichen Verlauf berechnet werden. Dazu wird das Bild 9.16 betrachtet. Es zeigt eine Viertelperiode der Phasenspannung $u_{A B}$ und des zugehörigen Kurzschlussstromes $i_{k}$. Dieser stellt sich ein, wenn die Gegenspannung als null angenommen wird und nur die Induktivität $L_{k}$ im Lastkreis verbleibt. Der Kurzschlussstrom weist ebenfalls Viertelperiodensymmetrie auf. Er ist gegenüber der Phasenspannung um $\pi / 2$ nacheilend, d.h. sein positiver Nulldurchgang liegt bei $\pi / 2$ und sein positiver Scheitelwert bei $\pi$. Es genügt die Berechnung des Verlaufes in 


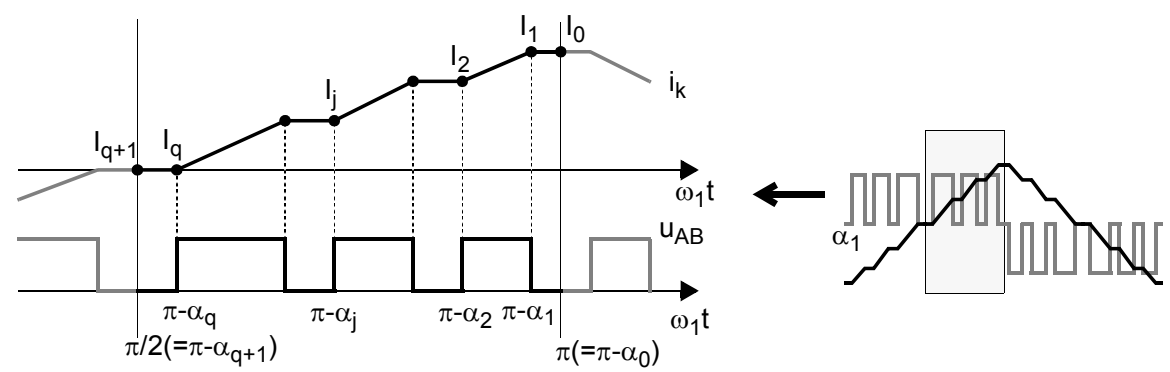

Bild 9.16. Ausschnitt aus den Verläufen der Phasenspannung und des zugehörigen Kurzschlussstromes

diesem Intervall. Im Hinblick auf eine geschlossene Darstellung werden die freien Schaltwinkel $\alpha_{1}$ bis $\alpha_{q}$ einer Viertelperiode durch $\alpha_{0}=0$ und $\alpha_{q+1}=\pi / 2$ ergänzt. Allgemein kann ausgehend vom Strom $I_{j}$ beim Schaltwinkel $\pi-\alpha_{j}$ der Wert $I_{j-1}$ an der Stelle $\pi-\alpha_{j-1}$ berechnet werden:

$$
I_{j-1}=I_{j}+\frac{1}{\omega_{1} L_{k}} \int_{\pi-\alpha_{j}}^{\pi-\alpha_{j-1}} u_{A B} d \omega_{1} t=I_{j}+\frac{\left.u_{A B}\right|_{\left[\alpha_{j}, \alpha_{j-1}\right]}}{\omega_{1} L_{k}}\left(\alpha_{j}-\alpha_{j-1}\right)
$$

$$
\left.u_{A B}\right|_{\left[\alpha_{j}, \alpha_{j-1}\right]} \text { bezeichnet dabei die Spannung im Intervall }\left[\pi-\alpha_{j}, \pi-\alpha_{j-1}\right]
$$

Beginnend mit $I_{q+1}$, das null ist, lässt sich somit jeder Stromwert $I_{j}$ als Funktion der vorangehenden Schaltwinkel ausdrücken:

$$
\begin{aligned}
& I_{q}=I_{q+1}+\frac{\left.u_{A B}\right|_{\left[\alpha_{q+1}, \alpha_{q}\right]}}{\omega_{1} L_{k}}\left(\alpha_{q+1}-\alpha_{q}\right)=\frac{\left.u_{A B}\right|_{\left[\alpha_{q+1}, \alpha_{q}\right]}}{\omega_{1} L_{k}}\left(\alpha_{q+1}-\alpha_{q}\right) \\
& I_{q-1}=I_{q}+\frac{\left.u_{A B}\right|_{\left[\alpha_{q}, \alpha_{q-1}\right]}}{\omega_{1} L_{k}}\left(\alpha_{q}-\alpha_{q-1}\right) \\
& =\frac{\left.u_{A B}\right|_{\left[\alpha_{q+1}, \alpha_{q}\right]}}{\omega_{1} L_{k}}\left(\alpha_{q+1}-\alpha_{q}\right)+\frac{\left.u_{A B}\right|_{\left[\alpha_{q}, \alpha_{q-1}\right]}}{\omega_{1} L_{k}}\left(\alpha_{q}-\alpha_{q-1}\right) \\
& \text { allgemein: } \left.\quad I_{j}=\left.\frac{1}{\omega_{1} L_{k}} \sum_{i=q+1, q, \ldots}^{j} u_{A B}\right|_{\left[\alpha_{i}, \alpha_{i-1}\right]}\left(\alpha_{i}-\alpha_{i-1}\right)\right\rfloor
\end{aligned}
$$

Zwischen den Schaltzeitpunkten verläuft der Strom linear:

$$
\pi-\alpha_{j} \leq \omega_{1} t \leq \pi-\alpha_{j-1}: \quad i_{k}\left(\omega_{1} t\right)=I_{j}+\frac{I_{j-1}-I_{j}}{\alpha_{j}-\alpha_{j-1}}\left(\omega_{1} t-\pi+\alpha_{j}\right)
$$

Die beiden Formeln (9.21) und (9.22) gelten für ein beliebiges konstantes Spannungsniveau zwischen den Schaltzeitpunkten. Im Hinblick darauf, dass sie später auch für die 
dreiphasigen Pulsmuster gebraucht werden können, wurden sie in dieser allgemeinen Form ausgedrückt. Bei der einphasigen Brücke ist jedoch die Phasenspannung $u_{A B}$ abwechslungsweise in einem Intervall null und im nächsten $U_{d}$. Die Formeln könnten deshalb hier noch etwas vereinfacht werden.

Die Berechnung des Effektivwertes erfolgt über eine abschnittweise Integration des Verlaufes über die Viertelperiode:

$$
I_{k, \text { eff }}=\sqrt{\frac{2}{\pi} \int_{\pi / 2}^{\pi} i_{k}^{2} d\left(\omega_{1} t\right)}=\sqrt{\frac{2}{\pi}} \sum_{j=q+1, q, \ldots}^{1}\left[\int_{\pi-\alpha_{j}}^{\pi-\alpha_{j-1}} i_{k}^{2} d\left(\omega_{1} t\right)\right]
$$

Setzt man (9.22) in (9.23) ein, so kann man die Teilintegrale ausrechnen und erhält als Resultat (9.24) bzw. (9.25):

$$
\begin{gathered}
\int_{\pi-\alpha_{j}}^{\pi-\alpha_{j-1}} i_{k}^{2} d\left(\omega_{1} t\right)=\frac{I_{j}^{2}+I_{j} I_{j-1}+I_{j-1}^{2}}{3}\left(\alpha_{j}-\alpha_{j-1}\right) \\
I_{k, \text { eff }}=\sqrt{\frac{2}{\pi} \sum_{j=q+1, q, \ldots}^{1} \frac{I_{j}^{2}+I_{j} I_{j-1}+I_{j-1}^{2}}{3}\left(\alpha_{j}-\alpha_{j-1}\right)}
\end{gathered}
$$

Vom gesamten Kurzschlussstrom kommt man leicht auf den Verzerrungsanteil, das angestrebte Gütekriterium, indem man gemäss (9.26) den Effektivwert der Grundschwingung, die beim vorgegebenen Modulationsgrad entsteht, geometrisch subtrahiert:

$$
I_{A, V Z, e f f}=\sqrt{I_{k, \text { eff }}^{2}-\frac{\hat{i}_{A, v=1}^{2}}{2}}=\sqrt{I_{k, \text { eff }}^{2}-\frac{1}{2}\left(\frac{M U_{d}}{\omega_{1} L_{k}}\right)^{2}}
$$

Berechnet man (9.26) mit Hilfe von (9.25) und (9.21), so erhält man das Gütekriterium als eine Funktion der Schaltwinkel und der jeweiligen Phasenspannung zwischen ihnen. Dabei sind nur Additionen und Multiplikationen auszuführen. Zur Formulierung der Randbedingung für die Grundschwingung muss allerdings weiterhin (9.19), die trigonometrische Ausdrücke enthält, verwendet werden. Trotzdem lässt sich die numerische Optimierung mit erheblich weniger Rechenaufwand durchführen.

Vergleicht man die beiden Berechnungsarten für den Effektivwert des Verzerrungsstromes, so weisen beide ihre Vorteile auf. Die Beschreibung im Zeitbereich erfordert weniger Rechenaufwand bei der Lösung des Optimierungsproblems. Sie ist aber nur für den Fall der Standardlast anwendbar. Ist zum Beispiel ein ohmscher Anteil im Lastkreis vorhanden, so geht der abschnittweise lineare Verlauf des Kurzschlussstromes in einen exponentionellen Verlauf über. Im Frequenzbereich dagegen lässt sich eine beliebige Last berücksichtigen, solange der Frequenzgang ihrer Impedanz bekannt ist. Es ist einfach die Gleichung (9.15) entsprechend abzuändern. Das Prinzip versagt erst dann, wenn die Amplituden der Stromoberschwingungen mit steigender Ordnung nicht mehr genügend abnehmen, so dass die geometrische Summe zur Bildung des Effektivwertes nicht mehr nach 
einer endlichen Anzahl von Gliedern abgebrochen werden darf. Grundsätzlich ergeben aber beide Varianten bei denselben Randbedingungen dieselben Resultate.

\subsubsection{Resultate der Optimierung}

Bild 9.17 zeigt die Resultate der Optimierung für die Schaltzahlen $q=4$ und $q=21$. Die Schaltwinkel nehmen in beiden Fällen stetige Verläufe an. Bei der geraden Schaltzahl $q=4$ trifft der Schaltwinkel $\alpha_{4}$ vorzeitig an den Rand der Viertelperiode bei $\pi / 2$. Dies bedeutet, dass im obersten Teil des Aussteuerbereiches die beiden mittleren Pulse einer Halbperiode in der Phasenspannung zusammenwachsen, so dass sich die Schaltzahl um eins redu-
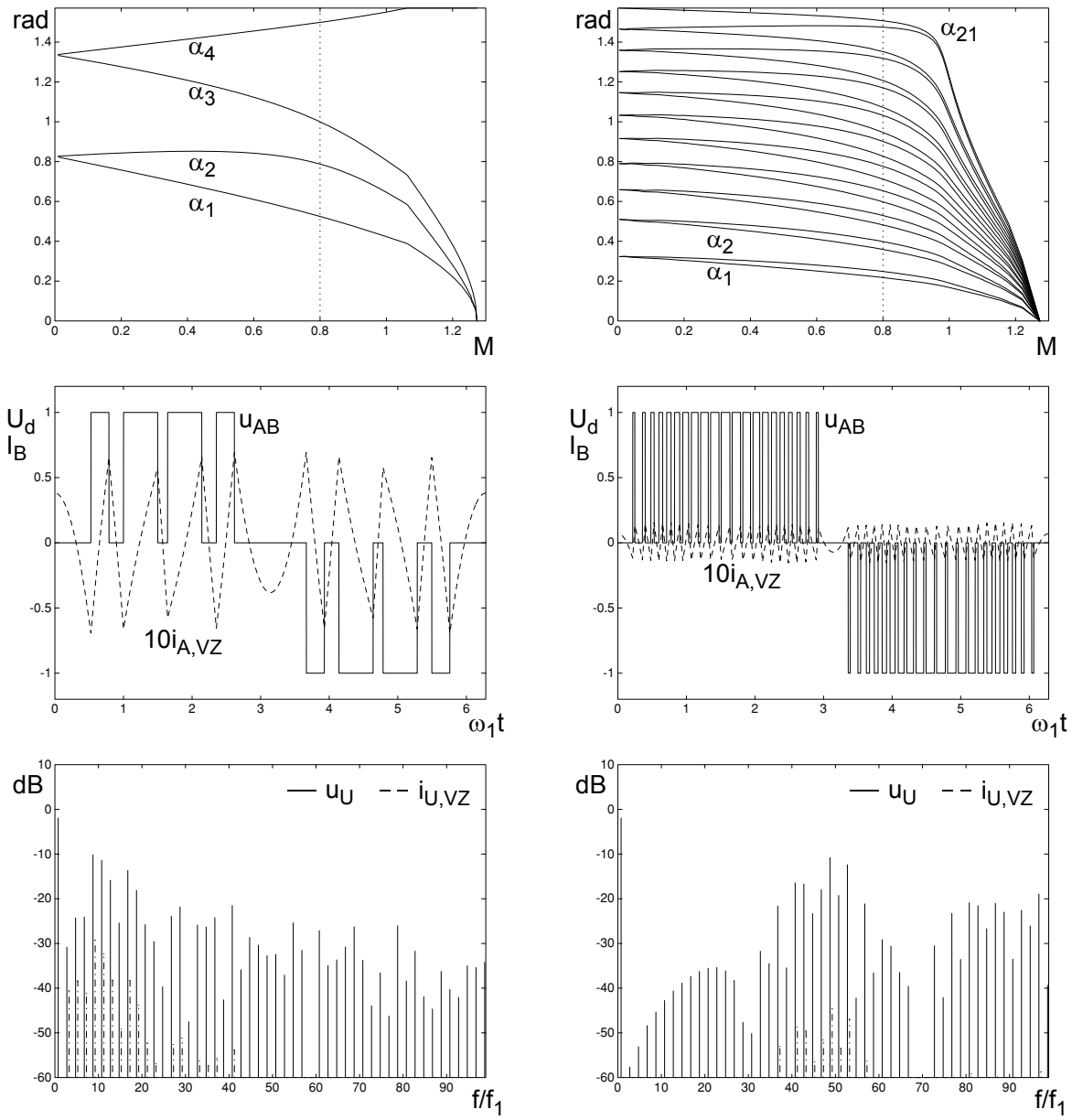

Bild 9.17. Einphasige Pulsmuster für minimalen Effektivwert des Verzerrungsstromes, oben links: Schaltwinkel für $q=4$, oben rechts: Schaltwinkel für $q=21$, mitte: entsprechende Phasenspannungen und Verzerrungsströme, $M=0.8$, unten: zugehörige Spektra, $0 \mathrm{~dB}: U_{d}$ bzw. $I_{B}$ 
ziert. Gegen die Vollaussteuerung hin $(M=4 / \pi)$ verschieben sich in beiden Pulsmustern alle bzw. die verbleibenden Schaltwinkel gegen null. In der Praxis ist es wegen der schaltungsbedingten minimalen Pulsweite notwendig, in diesem Bereich die Schaltzahl sukzessive bis auf Grundfrequenzsteuerung zu reduzieren.

Zusätzlich zu den Schaltwinkeln sind in Bild 9.17 die zeitlichen Verläufe und Spektra der Phasenspannung und des Verzerrungsstromes für $M=0.8$ dargestellt. Vor allem in den Spektra wird deutlich, dass gegenüber den Pulsmustern mit eliminierten Harmonischen hier alle Oberschwingungen vorhanden sind. Dafür sind die dominanten Komponenten kleiner. Die höhere Schaltzahl ergibt erwartungsgemäss deutlich kleinere Verzerrungen.

Verzerrungsstrom: Bild 9.18 zeigt den Effektivwert des Verzerrungsstromes als Funktion des Modulationsgrades für dieselben Schaltzahlen. In beiden Verläufen ist ein Maximum im Bereich $M=0.7$ und ein lokales Minimum bei etwas mehr als $M=1$ zu erkennen. Gegen Vollaussteuerung hin steigt der Effektivwert steil auf den Wert der Grundfrequenzsteuerung an. Wie bei den Pulsmustern mit eliminierten Harmonischen zeigt sich unterhalb von ca. $M=1.1$ näherungsweise eine umgekehrte Proportionalität zwischen Schaltzahl und Verzerrungsstrom. Da der Effektivwert des Verzerrungsstromes als Gütekriterium gewählt wurde, stellen diese Kennlinien die absolut besten erreichbaren Werte aller Steuerverfahren für die entsprechenden Schaltzahlen dar.

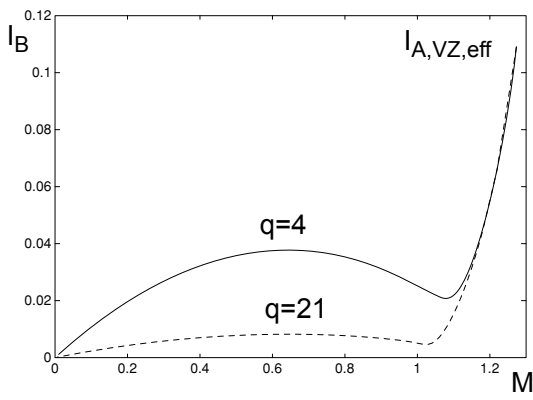

Bild 9.18.

Effektivwert des Verzerrungsstromes in Funktion des Modulationsgrades für zwei verschiedene Schaltzahlen, einphasige Brücke

\subsubsection{Minimaler Effektivwert des Verzerrungsstromes bei der dreiphasigen Brücke}

\subsubsection{Berechnung des Gütekriteriums im Frequenzbereich}

Prinzipiell kann die Optimierung für dreiphasige Schaltungen auf dieselbe Art durchgeführt werden, wie für einphasige. Zur Anwendungen kommen wiederum die Gleichungen (9.15) bis (9.18), wobei jedoch für die Harmonischen der Phasenspannung (9.10) und (9.11) einzusetzen sind. Das Gütekriterium ist hier der Effektivwert des Verzerrungsstromes in einer Phase:

$$
Z=\sqrt{\sum_{v=5,7,11, \ldots}^{v_{\max }} \frac{\hat{i}_{U, v}^{2}}{2},} \quad \hat{i}_{U, v}=\frac{4 U_{d}}{v^{2} \pi \omega_{1} L_{k}}\left|\frac{1}{2}+\sum_{i=1}^{f}(-1)^{i} \cos \left(v \alpha_{i}\right)\right|
$$


Diese Darstellung ist sehr einfach, da sie direkt aus der einphasigen Schaltfunktion abgeleitet werden kann. Es ist jedoch sehr schwierig, das Optimierungsproblem auf diese Weise zu lösen. Wie die später präsentierten Resultate bestätigen werden, sind in verschiedenen Bereichen der Aussteuerung völlig unterschiedliche Pulsmuster optimal. In der Darstellung nach (9.27) sind alle Varianten enthalten. Sie werden jedoch nur gefunden, wenn die Anfangswinkel für die numerische Lösung geeignet gewählt werden können. Dazu fehlen in der Regel die Kriterien.

Anstelle einer Viertelperiode der Schaltfunktion bzw. Mittelpunktspannung einer Phase wird besser eine Zwölftelperiode, d.h. ein Halbsektor aller 3 Mittelpunkt- bzw. Phasenspannungen betrachtet. Das Beispiel in Bild 9.19 zeigt, dass aufgrund der vorhandenen Symmetrien dadurch ebenfalls das gesamte dreiphasige Pulsmuster bestimmt ist. Gleichzeitig tritt so auch die zeitliche Abfolge der Schaltwinkel der einzelnen Brückenzweige innerhalb des betrachteten Halbsektors in Erscheinung. Sie ist zwar bei der Vorgabe einer Schaltfunktion über eine Viertelperiode implizit ebenfalls enthalten, jedoch nur in versteckter Form.
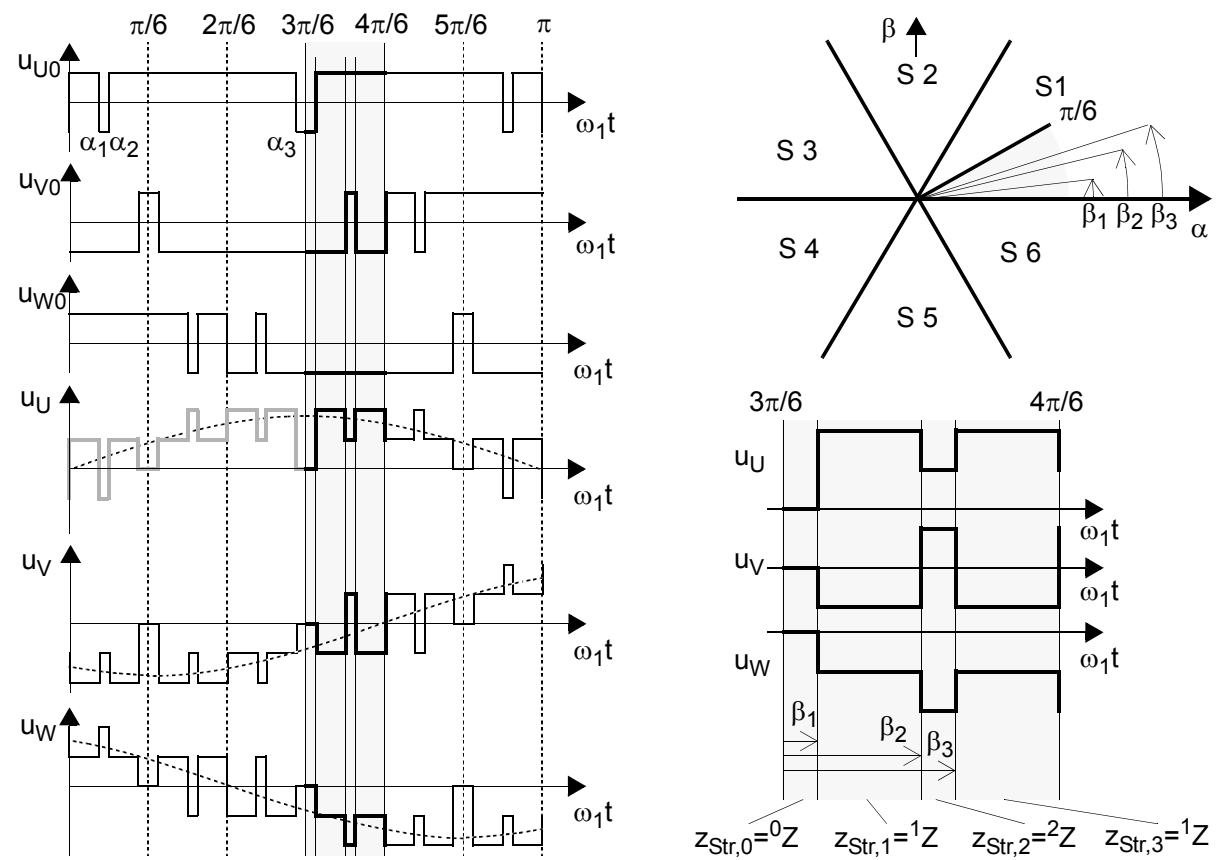

Bild 9.19. Zusammensetzen der Periode aus einem Halbsektor der Phasenspannungen, Beispiel mit $q=7$ und $f=3$

Als Halbsektor für die Beschreibung wird die erste Hälfte des in der Drehzeigerebene definierten Sektors $S 1$ gewählt. Er entspricht in der Darstellung im Bild links dem Intervall $[3 \pi / 6,4 \pi / 6]$. Angegeben werden die im Bild bezeichneten Schaltwinkel $\beta_{1}$ bis $\beta_{f}$ und die Schaltzustände zwischen den Schaltwinkeln $z_{S t r, 0}$ bis $z_{S t r, f}$ (im Bild ist $f=3$, d.h. $\beta_{f}=\beta_{3}$ 
und $z_{S t r . f}=z_{S t r, 3}$. Diese definieren alle 3 Phasenspannungen aufgrund der Schalterstellungen in den Brückenzweigen und können die in Kapitel 3.3.2.3 eingeführten diskreten Zustände ${ }^{0} Z$ und ${ }^{7} Z$ annehmen. Im Gegensatz zur Mittelpunktspannung, wo nur die Schaltwinkel zur Beschreibung notwendig waren (plus die Information, ob die erste Schaltflanke positiv oder negativ ist), sind es hier also zwei Parameter. Neben den Schaltwinkeln, die in beliebiger Weise innerhalb des Halbsektors verteilt sein dürfen, sind zusätzlich verschiedene Sequenzen von Schaltzuständen möglich. Dabei muss eine Sequenz die folgenden Bedingungen erfüllen:

- Um möglichst kleine Verzerrungsströme zu erhalten, dürfen im betrachteten Halbsektor nur die spannungsbildenden Schaltzustände ${ }^{1} Z$ und ${ }^{2} Z$ zusammen mit den beiden Nullzuständen ${ }^{0} Z$ und ${ }^{7} Z$ verwendet werden. Diese Tatsache wurde bereits bei der Drehzeigermodulation in Kapitel 8.2 erläutert.

- Aus der Forderung, dass alle Spannungen viertelperiodensymmetrisch sein sollen, lassen sich mit einiger Überlegung die folgenden Bedingungen für die Ränder des Halbsektors formulieren: an der Anfangsgrenze des Halbsektors (bei $\omega_{1} t=3 \pi / 6$ ) darf sich kein Schaltwinkel befinden, dafür ist an der Abschlussgrenze (bei $\omega_{1} t=4 \pi / 6$ ) einer obligatorisch. Der erste Schaltzustand $z_{S t r, 0}$ muss zudem ${ }^{1} Z$ oder ein Nullzustand sein. Der letzte Zustand darf andererseits kein Nullzustand sein.

- Es sind nur Übergänge zugelassen, bei denen ein einziger Brückenzweig geschaltet werden muss. Alle anderen Übergänge würden Schaltmustern mit höherer Schaltzahl entsprechen, bei denen Schaltwinkel zusammenfallen (Kapitel 8.2).

Mit diesen Bedingungen sind für das Beispiel in Bild 9.19 mit $q=7$ die 7 Sequenzen in Tabelle 9.2 erlaubt. Dargestellt im Bild ist die Sequenz $b$. Die Anzahl der erlaubten Sequenzen anz ist abhängig von der Schaltzahl. Sie nimmt mit wachsender Schaltzahl gemäss der rekursiven Formel (9.28) rasch zu:

$$
\operatorname{anz}(q)=\operatorname{anz}(q-2)+\operatorname{anz}(q-4), \quad \operatorname{anz}(1)=1, \operatorname{anz}(3)=3
$$

\begin{tabular}{c|c|c|c|c} 
Sequenz & ${ }^{\mathrm{Z}_{\text {Str, },}}$ & ${ }^{\mathrm{Z}_{\text {Str, } 1}}$ & ${ }^{\mathrm{Z}_{\text {Str, }}}$ & ${ }^{\mathrm{Z}_{\text {Str, }}}$ \\
\hline $\mathrm{a}$ & ${ }^{0} \mathrm{Z}$ & ${ }^{1} \mathrm{Z}$ & ${ }^{0} \mathrm{Z}$ & ${ }^{1} \mathrm{Z}$ \\
\hline $\mathrm{b}$ & ${ }^{0} \mathrm{Z}$ & ${ }^{1} \mathrm{Z}$ & ${ }^{2} \mathrm{Z}$ & ${ }^{1} \mathrm{Z}$ \\
\hline $\mathrm{c}$ & ${ }^{1} \mathrm{Z}$ & ${ }^{0} \mathrm{Z}$ & ${ }^{1} \mathrm{Z}$ & ${ }^{2} \mathrm{Z}$ \\
\hline $\mathrm{d}$ & ${ }^{1} \mathrm{Z}$ & ${ }^{2} \mathrm{Z}$ & ${ }^{1} \mathrm{Z}$ & ${ }^{2} \mathrm{Z}$ \\
\hline $\mathrm{e}$ & ${ }^{1} \mathrm{Z}$ & ${ }^{2} \mathrm{Z}$ & ${ }^{7} \mathrm{Z}$ & ${ }^{2} \mathrm{Z}$ \\
\hline $\mathrm{f}$ & ${ }^{7} \mathrm{Z}$ & ${ }^{2} \mathrm{Z}$ & ${ }^{1} \mathrm{Z}$ & ${ }^{2} \mathrm{Z}$ \\
\hline $\mathrm{g}$ & ${ }^{7} \mathrm{Z}$ & ${ }^{2} \mathrm{Z}$ & ${ }^{7} \mathrm{Z}$ & ${ }^{2} \mathrm{Z}$
\end{tabular}

Tabelle 9.2.

Erlaubte Schaltsequenzen im betrachteten Halbsektor für das Beispiel $q=7$

Das gewohnte Integral für die Berechnung der Fourierkoeffizienten der Phasenspannung $u_{U}$ über eine Viertelperiode lässt sich in 3 Teilintegrale über je einen Halbsektor aufteilen: 


$$
\begin{aligned}
b_{v}= & \frac{4}{\pi} \int_{0}^{\pi / 2} u_{U} \sin \left(v \omega_{1} t\right) d\left(\omega_{1} t\right)=\frac{4}{\pi}\left[\int_{0}^{\pi / 6} u_{U} \sin \left(v \omega_{1} t\right) d\left(\omega_{1} t\right)+\right. \\
& \left.+\int_{\pi / 6}^{2 \pi / 6} u_{U} \sin \left(v \omega_{1} t\right) d\left(\omega_{1} t\right)+\int_{2 \pi / 6}^{3 \pi / 6} u_{U} \sin \left(v \omega_{1} t\right) d\left(\omega_{1} t\right)\right]
\end{aligned}
$$

In Bild 9.19 lässt sich erkennen, dass der Verlauf von $u_{U}$ in jedem dieser Halbsektoren einer der 3 Phasenspannungen im schraffierten Halbsektor entspricht. Betrachtet man zusätzlich die im Bild skizzierten Grundschwingungen, so lässt sich die folgende Neuformulierung der Teilintegrale vornehmen:

$$
\begin{array}{rl}
\int_{0}^{\pi / 6} u_{U} \sin \left(v \omega_{1} t\right) d\left(\omega_{1} t\right)= & \int_{3 \pi / 6}^{4 \pi / 6} u_{V} \sin \left[v\left(\omega_{1} t-\frac{2 \pi}{3}\right)\right] d\left(\omega_{1} t\right) \\
2 \pi / 6 & 4 \pi / 6 \\
\int_{\pi / 6} u_{U} \sin \left(v \omega_{1} t\right) d\left(\omega_{1} t\right)= & \int_{3 \pi / 6} u_{W} \sin \left[v\left(\omega_{1} t-\frac{4 \pi}{3}\right)\right] d\left(\omega_{1} t\right) \\
3 \pi / 6 & 4 \pi / 6 \\
\int_{2 \pi / 6} u_{U} \sin \left(v \omega_{1} t\right) d\left(\omega_{1} t\right)= & \int_{3 \pi / 6} u_{U} \sin \left[v \omega_{1} t\right] d\left(\omega_{1} t\right)
\end{array}
$$

Durch Einsetzen von (9.30) in (9.29) erhält man das neue Integral für die Fourierkoeffizienten aus den Verläufen aller Phasenspannungen in einem Halbsektor:

$$
\begin{gathered}
b_{v}=\frac{4}{\pi} \int_{3 \pi / 6}^{4 \pi / 6}\left\{u_{U} \sin \left[v \omega_{1} t\right]+u_{V} \sin \left[v\left(\omega_{1} t-\frac{2 \pi}{3}\right)\right]+\right. \\
\left.+u_{W} \sin \left[v\left(\omega_{1} t-\frac{4 \pi}{3}\right)\right]\right\} d\left(\omega_{1} t\right)
\end{gathered}
$$

Durch Einsetzen der Schaltwinkel $\beta_{1}$ bis $\beta_{f}$ und der zugehörigen Schaltzustände bzw. Phasenspannungen in (9.31) lässt sich das Integral ausrechnen. Nach geeigneter Umformung erhält man schliesslich als Resultat (9.32). Die Grössen $u_{U, i}, u_{V, i}$ und $u_{W, i}$ bezeichnen dabei die Phasenspannungen im Intervall $i$, das heisst zwischen $\beta_{i}$ und $\beta_{i+1}$.

$$
\begin{gathered}
b_{v}=\frac{2}{v \pi} \sum_{i=0}^{J} K_{v 1}\left[2 u_{U, i}-u_{V, i}-u_{W, i}\right]\left[\sin \left(v \beta_{i+1}\right)-\sin \left(v \beta_{i}\right)\right] \\
+\sqrt{3} K_{v 2}\left[u_{V, i}-u_{W, i}\right]\left[\cos \left(v \beta_{i+1}\right)-\cos \left(v \beta_{i}\right)\right], \\
K_{v 1}=\left\{\begin{aligned}
1 & \text { rur } v=1, \text { ग } v, 1,1 / \ldots \\
-1 & \text { für } v=7,11,19,23, \ldots
\end{aligned}\right.
\end{gathered}
$$

mit

$$
K_{v 2}=\left\{\begin{array}{r}
1 \text { für } v=1,7,13,19 \ldots \\
-1 \text { für } v=5.11 .17 .23 \ldots
\end{array}\right.
$$


Zur Formulierung des Gütekriteriums für die Optimierung wird erneut auf die Beziehungen (9.15) und (9.16) zurückgegriffen, wobei für die Harmonischen der Phasenspannung jetzt (9.32) zu verwenden ist. Das Gütekriterium wird damit eine Funktion der neuen Schaltwinkel $\beta_{1}$ bis $\beta_{f}$ und der zugehörigen Phasenspannungen (bzw. Schaltzustände). Im weiteren sind auch die Randbedingungen für die Winkel neu zu formulieren, so dass das Optimierungsproblem die Form (9.33) erhält. In der gewählten Darstellung wird $b_{v=1}$ stets positiv.

$$
\begin{aligned}
& Z=\sqrt{\sum_{i=5,7,11 \ldots}^{v_{\max }} \frac{\hat{u}_{U, v}^{2}}{2 v^{2} \omega_{1}^{2} L_{k}^{2}}}=\sqrt{\sum_{i=5,7,11, \ldots}^{v_{\max }} \frac{b_{v}^{2}}{2 v^{2} \omega_{1}^{2} L_{k}^{2}}} \text {, mit } b_{v} \text { nach (9.32), } \\
& \hat{u}_{U, v=1}=b_{v=1}=M \frac{U_{d}}{2}, \\
& 0 \leq \beta_{1} \leq \beta_{2} \leq \ldots \leq \beta_{f} \leq \frac{\pi}{6}
\end{aligned}
$$

Die Optimierung muss für jede erlaubte Schaltsequenz durchgeführt werden. Im Beispiel mit $q=7$ (Tabelle 9.2) sind dies 7 verschiedene Optimierungen. Für jede davon erhält man ein entsprechendes optimales Pulsmuster. Unter dieser Schar von lokalen Minima ist anschliessend das globale Minimum für den betreffenden Modulationsgrad auszuwählen.

Auf den ersten Blick erscheint der Aufwand für die Optimierung auf diese Weise wesentlich grösser als bei der Verwendung der Schaltwinkel eines Brückenzweiges über eine Viertelperiode nach (9.27). Dort sind alle Schaltsequenzen gleichzeitig eingeschlossen. Bei der praktischen Durchführung stellt sich jedoch das Gegenteil heraus: durch die Unterscheidung von Schaltsequenzen werden die grundsätzlich verschiedenen Schaltwinkelverteilungen in einer Schaltfunktion von Beginn weg separiert. Die einzelnen Optimierungen sind dann dafür weitgehend unproblematisch, d.h. nicht mehr sehr stark abhängig von der Wahl der Anfangswinkel. Es wird wesentlich einfacher, das absolute Optimum zu finden.

Beschreibung im Zeitbereich: Wie bei den einphasigen Pulsmustern ist es auch möglich, mit Hilfe der Schaltwinkel den zeitlichen Verlauf des Kurzschlussstromes in einer Phase und daraus den Effektivwert des Verzerrungsanteils zu berechnen. Dies muss zwingend auf der Basis der Phasenspannungen erfolgen, da diese für den Kurzschlussstrom massgebend sind. Verwendet werden wiederum die Schaltwinkel $\beta_{l}$ bis $\beta_{f}$ und die Schaltsequenzen $z_{S t r, 0}$ bis $z_{S t r, f}$ eines Halbsektors. Es wird an dieser Stelle darauf verzichtet, die entsprechende Herleitung zu besprechen. Zur Berechnung des Kurzschlussstromes kommt die allgemeine Formel (9.21) zur Anwendung. Das Resultat für den Effektivwert hat eine ähnliche Form wie (9.25). Der Vorteil dieser Darstellung ist wiederum der, dass sie keine trigonometrischen Funktionen enthält. Zur vollständigen Lösung des Optimierungsproblems sind auch hier sämtliche zulässigen Schaltsequenzen zu berücksichtigen. Die entsprechenden Lösungen sind identisch mit denjenigen, die über die Berechnung im Frequenzbereich gefunden werden. 


\subsubsection{Resultate der Optimierung}

Als Beispiel für die Resultate einer Optimierung sind in Bild 9.20 die Schaltwinkel für $q=9$ als Funktion des Modulationsgrades dargestellt. Gezeigt sind die Winkel $\beta_{1}$ bis $\beta_{f}$ eines Halbsektors der Phasenspannung, die bei der Optimierung direkt berechnet worden sind, und die sich ergebenden Winkel $\alpha_{1}$ bis $\alpha_{f}$ der Schaltfunktion. Die Bereiche, in denen die Schaltwinkel stetig verlaufen, gehören jeweils zur selben Schaltsequenz. An den Sprungstellen erfolgt ein Übergang zu einer anderen Sequenz, die beim entsprechenden Modulationsgrad einen kleineren Verzerrungsstrom ergibt. Die optimalen Sequenzen sind der Tabelle $9.3 \mathrm{zu}$ entnehmen. Mit zunehmender Aussteuerung liegen die Sprungstellen näher beieinander. Gegen den maximalen Modulationsgrad von $4 / \pi$ hin laufen die Schaltwinkel im letzten Teilbereich wie bei den einphasigen Pulsmustern in einem gemeinsamen Punkt zusammen und zwar $\beta_{1}$ bis $\beta_{f}$ bei $\pi / 6$ und $\alpha_{1}$ bis $\alpha_{f}$ bei null.
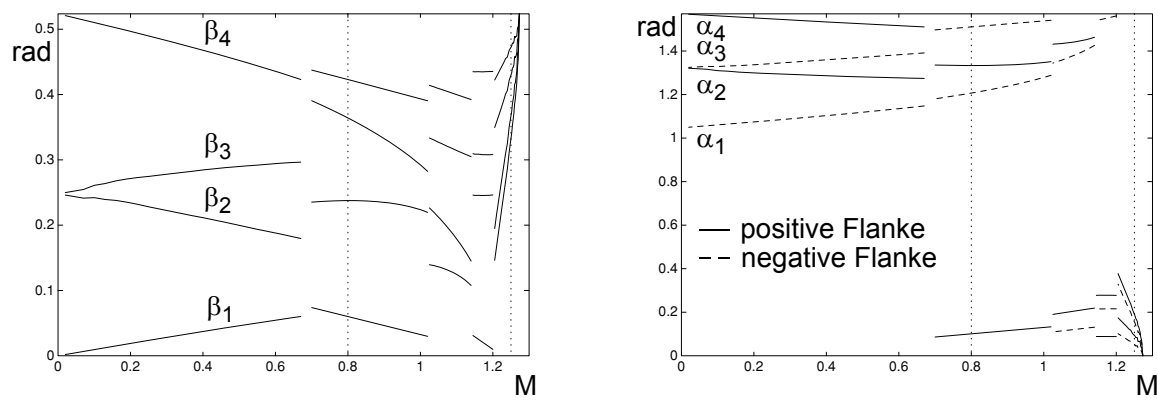

Bild 9.20. Dreiphasige optimierte Pulsmuster für minimalen Effektivwert des Verzerrungsstromes, $q=9, f=4$, links: Schaltwinkel der Phasenspannung, rechts: Schaltwinkel der Mittelpunktspannung bzw. Schaltfunktion

\begin{tabular}{c|c|c|c|c|c} 
Bereich von $M$ & ${ }^{\mathrm{Z}_{\text {Str }, 0}}$ & $\mathrm{Z}_{\text {Str, } 1}$ & $\mathrm{z}_{\text {Str, } 2}$ & $\mathrm{z}_{\text {Str,3 }}$ & ${ }^{\mathrm{Z}}$ Str,4 \\
\hline 0 bis 0.70 & ${ }^{1} \mathrm{Z}$ & ${ }^{0} \mathrm{Z}$ & ${ }^{1} \mathrm{Z}$ & ${ }^{0} \mathrm{Z}$ & ${ }^{1} \mathrm{Z}$ \\
\hline 0.70 bis 1.02 & ${ }^{0} \mathrm{Z}$ & ${ }^{1} \mathrm{Z}$ & ${ }^{0} \mathrm{Z}$ & ${ }^{1} \mathrm{Z}$ & ${ }^{2} \mathrm{Z}$ \\
\hline 1.02 bis 1.14 & ${ }^{1} \mathrm{Z}$ & ${ }^{0} \mathrm{Z}$ & ${ }^{1} \mathrm{Z}$ & ${ }^{2} \mathrm{Z}$ & ${ }^{1} \mathrm{Z}$ \\
\hline 1.14 bis 1.20 & ${ }^{0} \mathrm{Z}$ & ${ }^{1} \mathrm{Z}$ & ${ }^{2} \mathrm{Z}$ & ${ }^{1} \mathrm{Z}$ & ${ }^{2} \mathrm{Z}$ \\
\hline 1.20 bis 1.27 & ${ }^{1} \mathrm{Z}$ & ${ }^{2} \mathrm{Z}$ & ${ }^{1} \mathrm{Z}$ & ${ }^{2} \mathrm{Z}$ & ${ }^{1} \mathrm{Z}$
\end{tabular}

Tabelle 9.3.

Optimale Schaltsequenzen für $q=9$, zugehöriger Verlauf der Schaltwinkel in Bild 9.20

Bild 9.21 zeigt die zeitlichen Verläufe und die Spektra einer Phasenspannung und des Verzerrungsstromes in einer Phase für zwei verschiedene Modulationsgrade. In den Spektra wird deutlich, dass im Gegensatz zu den Pulsmustern mit eliminierten Harmonischen alle Komponenten auftreten. Dafür erscheinen die dominanten Harmonischen mit reduzierten Amplituden. Die Amplitudenverteilung ist sehr stark vom Modulationsgrad abhängig. In den Diagrammen rechts ist zu erkennen, dass für die Erzeugung des hohen Mo- 

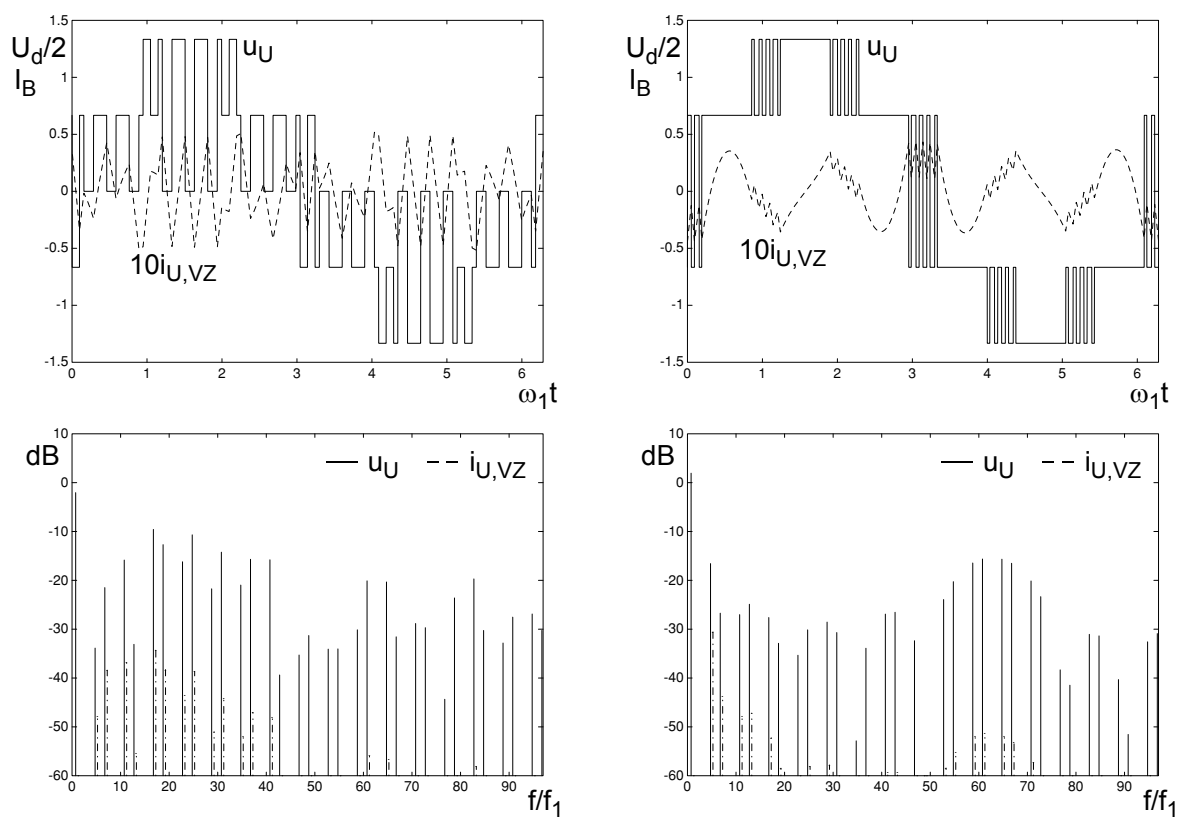

Bild 9.21. Phasenspannungen und Verzerrungsströme für die Pulsmuster in Bild 9.20, links: $M=0.8$, rechts: $M=1.25,0 \mathrm{~dB}: U_{d} / 2$ bzw. $I_{B}$

dulationsgrades von 1.25 ein grosser Anteil 5. Harmonischer zugelassen werden muss. Dieser tritt im Verzerrungsstrom besonders stark in Erscheinung.

Verzerrungsstrom: Bild 9.22 zeigt die Kennlinien für den Effektivwert des Verzerrungsstromes für $q=9$ und $q=21$. Besonders fällt das deutliche lokale Minimum im Bereich $M=1.2$ auf. Darüber steigt der Verzerrungsstrom sehr stark an und erreicht schliesslich den Wert der Grundfrequenzsteuerung. Es ergibt sich auch hier näherungsweise eine umgekehrte Proportionalität zwischen dem Effektivwert des Verzerrungsstromes und der Schaltzahl.

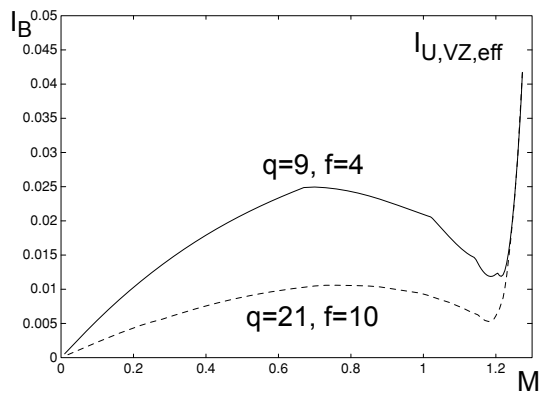

Bild 9.22.

Effektivwert des Verzerrungsstromes in einer Phase für optimierte Pulsmuster mit zwei verschiedenen Schaltzahlen, dreiphasige Brücke 
Da der Verzerrungsstrom das Gütekriterium der Optimierung darstellt, verkörpern die resultierenden Kennlinien die absolut besten erreichbaren Resultate aller Steuerverfahren für die entsprechenden Schaltzahlen.

Zwischenkreisstrom: Als Beispiel eines Zwischenkreisstromes ist in Bild 9.23 der Verlauf von $i_{d}$ für das Pulsmuster in Bild 9.21 links und einem nacheilenden Grundschwingungsstrom der Amplitude $0.2 I_{B}$ dargestellt. Die vollständige dreiphasige Symmetrie bewirkt, dass in seinem Spektrum nur Vielfache der 6. Harmonischen auftreten. Die DCKomponente ist wie gewohnt durch den Wirkstrom bestimmt, während die Amplituden der höheren Harmonischen primär durch das Spektrum der angelegten Schaltfunktionen gegeben sind.
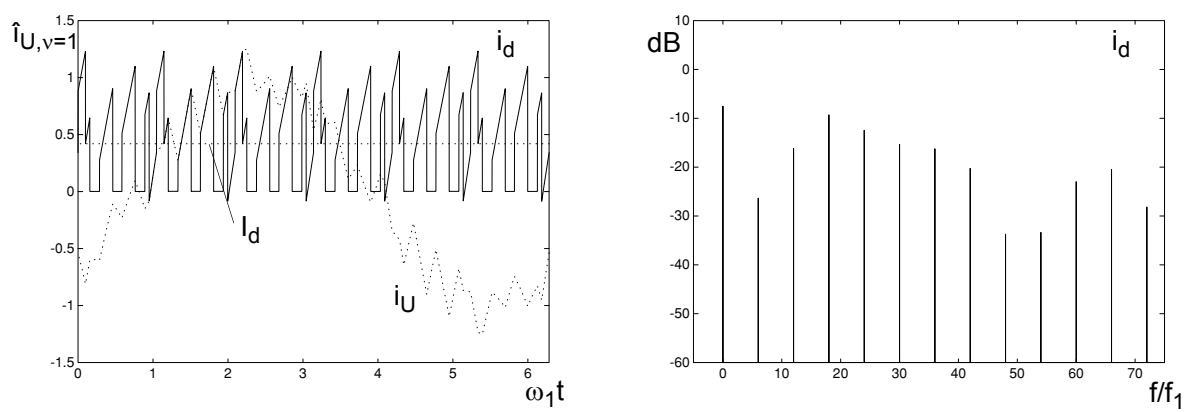

Bild 9.23. Links: Zeitlicher Verlauf und Mittelwert des Zwischenkreisstromes sowie ein Phasenstrom für $q=9, M=0.8, \hat{l}_{U / V / W, v=1}=0.2 I_{B}$, rechts: Spektrum von $i_{d}, 0 \mathrm{~dB}: \hat{l}_{U / V / W, v=1}$

\subsubsection{Optimierung nach weiteren Kriterien}

In der gleichen Art und Weise, wie dies für den Effektivwert des Verzerrungsstromes ausführlich gezeigt worden ist, kann die Optimierung der Pulsmuster nach anderen Kriterien durchgeführt werden. Die wichtigste Voraussetzung dafür ist, dass das Gütekriterium als Funktion der Schaltwinkel ausgedrückt werden kann. Im folgenden werden einige weitere gebräuchliche Gütekriterien kurz vorgestellt.

Individuelle Gewichtung der Harmonischen [Wüe1]: Ausgegangen wird vom folgenden Gütekriterium:

$$
Z=\sqrt{\sum_{v>1}^{v_{\max }} g^{2}(v) \hat{u}_{v}^{2}}, \quad v_{\max } \text { genügend gross }
$$

Die Gleichung stellt die geometrische Summe aller Oberschwingungen dar, wobei jede einzelne Komponente mit einem individuellen Gewicht $g(v)$ versehen ist. Dadurch lässt sich das Spektrum der Ausgangsspannung gezielt formen: eine Harmonische, welche mit einem grossen Gewicht versehen ist, wird gegenüber den anderen im optimierten Pulsmuster entsprechend stark reduziert. 
Die Minimierung des Verzerrungsstromes nach der Gleichung (9.17) ist ein Spezialfall der Gewichtung von Harmonischen. Das Gewicht $g(v)$ ist dort so gewählt, dass die Summanden gerade die Effektivwerte der Stromoberschwingungen sind und dementsprechend das Gütekriterium den Effektivwert des Verzerrungsstromes darstellt:

$$
\begin{aligned}
& g(v)=\frac{1}{\sqrt{2} v \omega_{1} L_{k}} \\
& \Rightarrow \quad Z^{2}=\sum_{v>1}^{v_{\max }} g^{2}(v) \hat{u}_{v}^{2}=\sum_{v>1}^{v_{\max }} \frac{\hat{i}_{v}^{2}}{2}=I_{V Z, e f f}^{2}
\end{aligned}
$$

Bei der Festlegung der Gewichte ist zu beachten, dass die Optimierung nur mit einer endlichen Anzahl Harmonischen durchführbar ist. Wenn die Summanden mit zunehmender Ordnung deutlich kleiner werden, so kann die Summe stets ab einer bestimmten Anzahl Glieder $v_{\max }$ ohne weiteres abgebrochen werden, ohne dass das Resultat der Optimierung merklich verändert wird. Andernfalls muss man sich so behelfen, dass nur einer endlichen Anzahl von Harmonischen ein Gewicht ungleich null gegeben wird.

Alle Optimierungsprobleme, die im Frequenzbereich formuliert werden, stellen eine gewichtete Optimierung von Harmonischen dar.

Verlustminimierung [Zac1], [Tak1]: Häufig steht die Minimierung der durch die Verzerrungsanteile verursachten Zusatzverluste $P_{V, V Z}$ in der Last im Vordergrund. Als Beispiel

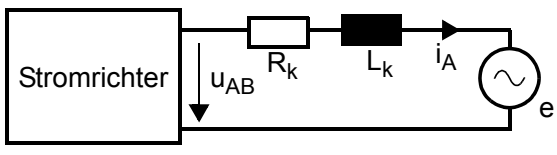

Bild 9.24.

Lastkreis mit ohmschen Anteil

wird von der Schaltung in Bild 9.24 ausgegangen. Die Gegenspannung $e$ verlaufe rein sinusförmig. Die Zusatzverluste fallen in $R_{k}$ an und lassen sich wie folgt ausdrücken:

$$
P_{V, V Z}=R_{k} I_{A, V Z, \text { eff }}^{2}=\frac{R_{k}}{2} \sum_{v>1}^{\infty} \hat{i}_{A, v}^{2}
$$

Die Berechnung der Stromharmonischen ist durch (9.38) gegeben, so dass das Gütekriterium gemäss (9.39) formuliert werden kann:

$$
\begin{aligned}
& \hat{i}_{A, v}=\frac{\hat{u}_{A B, v}}{\sqrt{R_{k}^{2}+v^{2} \omega_{1}^{2} L_{k}^{2}}}, \quad v>1 \\
& Z=P_{V, V Z}=\frac{R_{k}}{2} \sum_{v>1}^{v_{\text {max }}} \hat{i}_{A, v}^{2}=\frac{R_{k}}{2} \sum_{v>1}^{v_{\text {max }}} \frac{\hat{u}_{A B, v}^{2}}{R_{k}^{2}+v^{2} \omega_{1}^{2} L_{k}^{2}}
\end{aligned}
$$


Bei der Optimierung kann leicht auch die Stromverdrängung berücksichtigt werden, indem $R_{k}$ durch den einen variablen Widerstand $R_{k}(v)$ ersetzt wird. Wenn im betrachteten Frequenzbereich der ohmsche Anteil der Lastimpedanz gegenüber dem induktiven sehr klein ist, entsprechen die Resultate den Pulsmustern aus den Kapiteln 9.3.2 und 9.3.3.

Anpassung des Pulsmusters an ein Filter [Wüe1]: Ist zwischen Stromrichter und Last ein Filter geschaltet, wie in Bild 9.25 für den einphasigen Fall dargestellt, so interessiert in erster Linie der Strom $i_{L}$, der in die Last fliesst. Zur Berechnung seiner Harmonischen aus denjenigen der Phasenspannung $u_{A B}$ können die in Kapitel 3.2.6.1 angegebenen Übertragungsfunktionen des Filters auf jede einzelne Komponente angewendet werden:

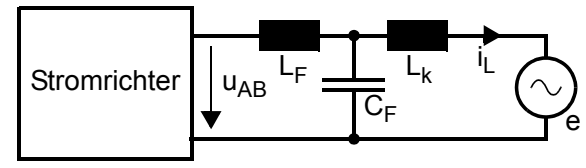

Bild 9.25.

Stromrichter mit Ausgangsfilter

$$
\begin{aligned}
& G(v)=\frac{\hat{i}_{L, v}}{\hat{u}_{A B, v}}=\frac{1}{v \omega_{1}\left(L_{k}+L_{F}\right)-v^{3} \omega_{1}^{3} L_{k} L_{F} C_{F}} \\
& \hat{i}_{L, v}=G(v) \hat{u}_{A B, v}
\end{aligned}
$$

Wie bereits in Kapitel 3.2.6.1 erläutert wurde (Bild 3.17), weist die Filterübertragungsfunktion eine starke Resonanzüberhöhung auf. Wählt man als Gewichtsfunktion $g(v)$ gerade den Filterfrequenzgang $G(v)$, so ist das Gütekriterium proportional zum Effektivwert

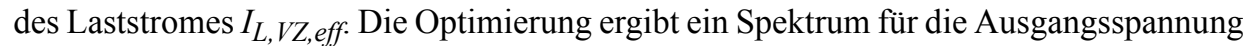
$u_{A B}$, das gut der Filterübertragungsfunktion angepasst ist. Insbesondere ergeben sich im Bereich der Resonanzüberhöhung entsprechend niedrige Harmonische, während die dominanten Frequenzanteile des Verzerrungsanteils hinter die Grenzfrequenz des Filters zu liegen kommen.

Minimale Drehmomentwelligkeit [Zac2]: In einem Antrieb stellt die Drehmomentwelligkeit ein naheliegendes Gütekriterium für die Optimierung dar. In Kapitel 5.2.1.4 wurde gezeigt, dass die Drehmomentwelligkeit im Leerlauf für eine mit synchronen und symmetrischen Pulsmustern gespeiste Maschine (ASM oder SM) aus den Harmonischen der Phasenspannung berechnet werden kann:

$$
M_{e l, V Z}=-\frac{3}{2} \frac{\hat{u}_{v=1}}{\omega_{1}^{2} L_{k}}\left[\sum_{v=6,12,18, \ldots}^{\infty}\left(\frac{\hat{u}_{v-1}}{v-1}-\frac{\hat{u}_{v+1}}{v+1}\right) \sin \left(v \omega_{1} t\right)\right]
$$

Da die Phasenspannungen nur ungerade Harmonische aufweisen, besteht $M_{e l, V Z}$ im Gegensatz zur allgemeinen Formel (5.33) lediglich aus den Vielfachen der sechsten.

Vom mechanischen Gesichtspunkt her gilt es in erster Linie, den Spitzenwert von $M_{e l, V Z}$ zu minimieren. Dieser lässt sich jedoch nicht direkt aus (9.42) bestimmen. An seiner Stelle kann jedoch der Effektivwert $M_{e l, V Z \text {,eff }}$ als Gütekriterium gemäss (9.43) herangezogen 
werden. Es ergeben sich auf diese Weise hinsichtlich des Spitzenwertes zwar nicht optimale, aber immer noch sehr gute Resultate.

$$
Z=M_{e l, V Z, e f f}=\frac{3}{2 \sqrt{2}} \frac{\hat{u}_{U, v=1}}{\omega_{1}^{2} L_{k}} \sqrt{\sum_{v=6,12,18, \ldots}^{v_{\max }}\left(\frac{\hat{u}_{v-1}}{v-1}-\frac{\hat{u}_{v+1}}{v+1}\right)^{2}}
$$

Werden schliesslich die Harmonischen der Phasenspannung mit Hilfe von (9.10) und (9.11) als Funktion der Schaltwinkel ausgedrückt, so liegt das Optimierungsproblem in der gewohnten Form vor.

Es lohnt sich, an dieser Stelle auch einen kurzen Blick auf die Resultate der Optimierung zu werfen. Bild 9.26 zeigt den optimierten zeitlichen Verlauf von $M_{e l, V Z}$ für $q=9$ bei $M=0.8$ und das zugehörige Spektrum des Verzerrungsstromes einer Phase. Zum Vergleich dazu sind dieselben Grössen für das Pulsmuster mit minimalem Verzerrungsstrom gemäss Kapitel 9.3.3 dargestellt. Es zeigen sich deutliche Unterschiede: das auf Drehmomentwelligkeit optimierte Pulsmuster weist starke 5. und 7. Harmonische auf. Ihre Amplituden haben sich so eingestellt, dass beide Komponenten im Phasenstrom praktisch gleich gross sind. Dadurch kompensiert sich ihre Wirkung auf das Drehmoment. Trotz des viel grösseren Verzerrungsstromes ergibt sich eine deutlich kleinere Momentwelligkeit. Das Beispiel demonstriert, dass es nicht in erster Linie darauf ankommt, die Stromverzerrungen klein zu halten. Die Harmonischen müssen für eine kleine Momentwelligkeit vor allem auch in einem günstigen Verhältnis zueinander stehen.
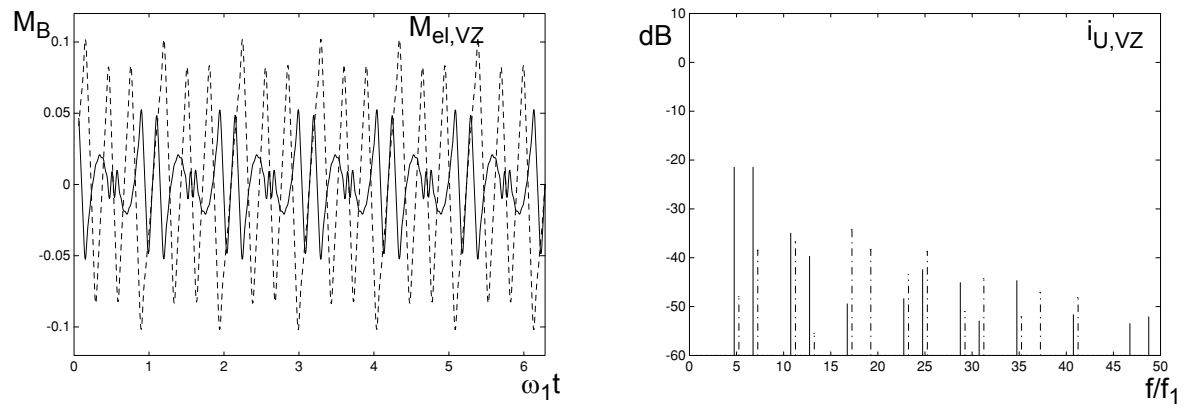

Bild 9.26. Links: zeitliche Verläufe der Drehmomentwelligkeit bei $q=9, M=0.8$, rechts: zugehörige Spektra des Verzerrungsstromes in einer Phase, 0dB: $I_{B}$, ausgezogen: optimiertes Pulsmuster für minimale Drehmomentwelligkeit, gestrichelt: optimiertes Pulsmuster für minimalen Verzerrungsstrom

In der Praxis müssen jedoch auch die ohmschen Anteile in der Maschine berücksichtigt werden. Sie verursachen unterschiedliche Phasenverschiebungen der Stromharmonischen, so dass die Gleichung (9.42) nicht mehr in dieser einfachen Form gilt. Da die Phasenverschiebungen abhängig von $\omega_{1}$ und damit der Drehzahl sind, ist die gezielte gegenseitige Kompensation der Wirkung von Stromoberschwingungen auf das Drehmoment in der Praxis nicht mehr in der gezeigten einfachen Art realisierbar. 
Minimaler Klirrfaktor für den Phasenstrom [Wüe1]: Bei den bisherigen Optimierungen wurde stets davon ausgegangen, dass der Modulationsgrad als Randbedingung vorgegeben ist. Es gibt jedoch Anwendungen, wo nur ein einziges Pulsmuster benötigt wird, z.B. wenn die Amplitude der Grundschwingung durch Variation der Zwischenkreisspannung eingestellt wird. Der Stromrichter kann dann mit Grundfrequenzsteuerung betrieben werden, so dass die Zwischenkreisspannung maximal ausgenützt ist. Es kann jedoch auch ein optimiertes Pulsmuster mit $q>1$ verwendet werden, welches das beste Verhältnis zwischen Grundschwingung und Verzerrungsanteil in den Phasenströmen ergibt. Für die Formulierung des entsprechenden Gütekriteriums kann vom Klirrfaktor des Kurzschlussstromes (9.44) ausgegangen wird. Nach geeigneter Umformung erhält das Gütekriterium schliesslich die Form:

$$
\begin{aligned}
& k_{i}^{2}=\frac{I_{V Z, e f f}^{2}}{I_{k, \text { eff }}^{2}}=\frac{I_{V Z, e f f}^{2}}{I_{V Z, e f f}^{2}+\hat{i}_{k, v=1}^{2} / 2}=\min \\
& \Rightarrow \quad \frac{I_{V Z, e f f}^{2}+\hat{i}_{k, v=1}^{2} / 2}{I_{V Z, e f f}^{2}}=1+\frac{\hat{i}_{k, v=1}^{2}}{2 I_{V Z, e f f}^{2}}=\max \\
& Z=\frac{2 I_{V Z, e f f}^{2}}{\hat{i}_{k, v a x}^{2}}=\frac{\sum_{i>1}^{2} \hat{i}_{v}^{2}}{\hat{i}_{k, v=1}^{2}}=\min
\end{aligned}
$$

Der Kurzschlussstrom kann wie in den Kapiteln 9.3.2.1 und 9.3.2.2 gezeigt, entweder im Zeit- oder im Frequenzbereich berechnet werden. Die Randbedingung für den Modulationsgrad entfällt, da dieser in das Gütekriterium mit einbezogen ist. Die Optimierung ergibt Resultate mit einem Modulationsgrad im Bereich $M=1.05$ für einphasige Pulsmuster und $M=1.2$ für dreiphasige, da dort die Verzerrungsanteile bei sehr grosser Aussteuerung ausgeprägte Minima aufweisen. Diese Tatsache ist in den entsprechenden Bildern 9.18 und 9.22 leicht ersichtlich. Vor allem im dreiphasigen Fall ist die Reduktion der Aussteuerung gegenüber Grundfrequenzsteuerung sehr gering.

\subsubsection{Dynamische Betrachtungen}

\subsubsection{Definition von transienten Zuständen im Modulator}

Grundsätzlich sind die abgespeicherten Pulsmuster für den stationären Betrieb ausgelegt. Es ist naheliegend, Pulsmusterwechsel grundsätzlich nur an ganz bestimmten Stellen, z.B. am Anfang einer Periode bzw. einer Halb- oder Viertelperiode zuzulassen. Ein Wechsel bedeutet einen Sprung von einem stationären Zustand in einen anderen, wenigstens was die Schaltfunktionen angeht. Die Eingangsgrössen werden im entsprechenden zeitlichen Abstand abgetastet. Für die meisten Anwendungen ergeben sich auf diese Weise jedoch unzulässig grosse Reaktionszeiten im Modulator. 
Eine etwas abstraktere Modellierung des Modulators erlaubt jederzeit einen Pulsmusterwechsel. Die Schalttabelle wird als zweidimensionaler Raum betrachtet, der für jeden Phasenwinkel $\alpha$ innerhalb der Grundperiode entsprechend dem vorgegebenen Modulationsgrad $M$ einen Schaltzustand definiert. Bild 9.27 zeigt ein entsprechendes Beispiel. Die Eingangsgrössen des Modulators definieren eine Trajektorie, mit der das Pulsmusterfeld zu durchfahren ist. Im Bild sind zwei Beispiele für solche Trajektorien, zusammen mit den resultierenden Phasenspannungen, dargestellt. Im stationären Zustand wird das Pulsmusterfeld periodisch und mit konstanter Geschwindigkeit auf einer horizontalen Trajektorie durchfahren. Bei dynamischen Vorgängen kann die Trajektorie dagegen einen beliebigen Verlauf annehmen. Im gezeigten Beispiel vergrössert sich der Modulationsgrad stetig. Die Grundfrequenz wird als konstant angenommen, so dass die Geschwindigkeit des Durchlaufs gleich bleibt. Dies ist im Verlauf der Phasenspannung erkennbar. Jeder Schnittpunkt der Trajektorie mit dem Rand einer der eingefärbten Flächen ergibt einen Schaltwinkel. Die Phasenspannung wird jederzeit auf denjenigen Wert eingestellt, welcher durch die aktuelle Position der Trajektorie im Pulsmusterfeld bestimmt ist.
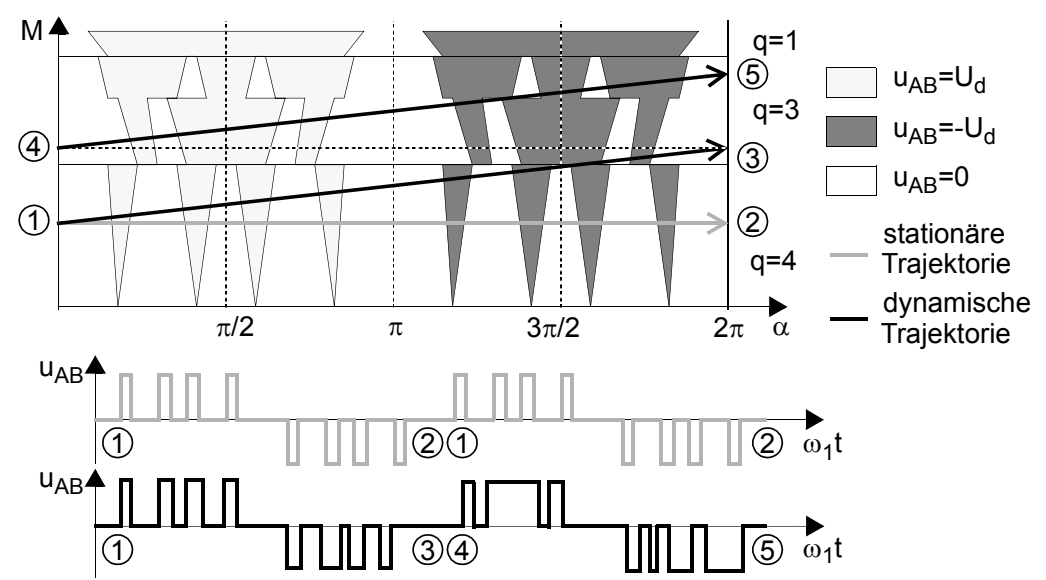

Bild 9.27.

Beispiel eines Pulsmusterfeldes für eine einphasige Brücke

Im allgemeinen Fall kann neben dem Modulationsgrad auch die Grundfrequenz variieren. Dies bedeutet, dass die Geschwindigkeit, mit der das Pulsmuster durchfahren wird, ändert. Zusätzlich können schrittartige Änderungen der Eingangsgrössen Sprünge der Trajektorie verursachen. Durch das Prinzip, nach dem die Phasenspannung stets sofort auf den durch die Trajektorie definierten Zustand eingestellt wird, ist das dynamische Verhalten des Modulators für alle diese Fälle eindeutig definiert.

Es ist bisher nicht berücksichtigt worden, dass der Modulator, der auf einem Rechner implementiert ist, mit quantisierten Grössen arbeitet. In Realität kann die Trajektorie nicht kontinuierlich verlaufen. Sie führt vielmehr kleine Sprünge aus, welche den Quantisierungsschritten in Richtung beider Koordinatenachsen entsprechen. Dies hat jedoch keinen Einfluss auf die prinzipielle Funktionsweise. 


\subsubsection{Realisierung von Übergängen zwischen stationären Zuständen}

Jede Abweichung von einem stationären Betrieb erfordert einigen Aufwand und ist nicht unproblematisch. Dabei ist zu berücksichtigen, dass wie bereits in Bild 9.1 angedeutet, das Pulsmusterfeld in der Regel in Form von Schaltwinkeln und Schaltzuständen abgelegt ist und nicht als direkt adressierbares zweidimensionales Abbild des Pulsmusters gemäss Bild 9.27. Dies würde bei einer genügenden zeitlichen Auflösung zuviel Speicherplatz erfordern.

Pulsmusterwechsel: Vorerst wird der Fall betrachtet, dass die Grundfrequenz gleich bleibt und nur der Modulationsgrad einmalig ändert. Es ist ein Sprung in ein anderes Pulsmuster erforderlich, wobei die angefangene Grundperiode beim selben Phasenwinkel weitergeführt werden soll. Bild 9.28 zeigt zwei unterschiedliche Fälle eines solchen Über-
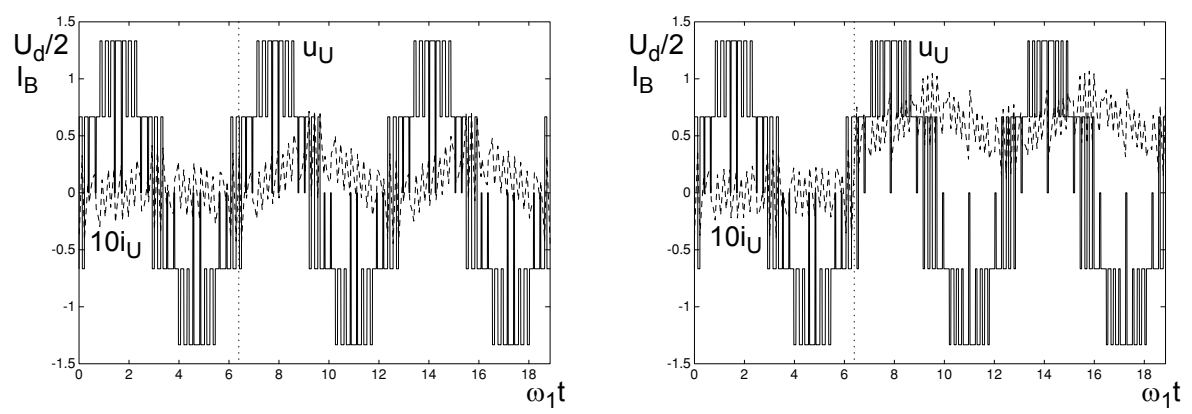

Bild 9.28. Phasenspannung und -strom beim Übergang zwischen zwei Pulsmustern, optimierte Pulsmuster für $q=9$ aus Bild 9.20, links: Wechsel von $M=1.12$ auf 1.14 (gleiche Schaltsequenzen), rechts: Wechsel von $M=1.14$ auf 1.16 (verschiedene Schaltsequenzen)

ganges, die auf den Pulsmustern der Optimierung aus Bild 9.20 mit $q=9$ basieren. Dabei ist im Ausgangszustand die Grundschwingung des Phasenstromes gerade null, d.h. $\hat{e}_{U}$ und $\hat{u}_{U, v=1}$ stimmen überein.

- Im Bild links wird an der punktiert markierten Stelle durch einen Pulsmusterwechsel der Modulationsgrad von 1.12 auf 1.14 erhöht. Die beiden Pulsmuster gleichen sich stark, so dass in der dargestellten Phasenspannung kein Unterschied erkennbar ist. Im Phasenstrom zeigt sich der Wechsel durch eine Veränderung der Grundschwingung. Zusätzlich tritt dort ein kleiner Gleichanteil auf.

- Bild rechts zeigt einen Wechsel von $M=1.14$ auf 1.16. Dabei muss auch die Schaltsequenz geändert werden (Tabelle 9.3). Deshalb sind bereits in der Phasenspannung Unterschiede erkennbar. Der Phasenstrom erhält neben der veränderten Grundschwingung hier vor allem einen wesentlich grösseren Gleichanteil.

Die Gleichanteile in den Phasenströmen klingen in der Praxis wegen der ohmschen Anteile in der Last langsam wieder ab. Sie sind jedoch äusserst unerwünscht, weil sie die 
Schaltung zusätzlich belasten, Sättigungseffekte hervorrufen und das Drehmoment pulsieren lassen. Wie die Beispiele zeigen, sind vor allem Schaltsequenzwechsel problematisch. Weil sich die Verteilung der Schaltwinkel von einer Periode zur anderen völlig verändert, können dort besonders grosse Gleichanteile entstehen.

Weisen die gespeicherten Pulsmuster stetige Schaltwinkelverläufe auf, so sind benachbarte Pulsmuster stets gleichartig. Beispiele dafür sind alle in diesem Kapitel dargestellten einphasigen Pulsmuster. Sie erlauben es, den Modulationsgrad in kleinen Schritten zu variieren, ohne dass im Phasenstrom starke Gleichanteile entstehen. Sind dagegen Unstetigkeiten vorhanden, weil verschiedene Schaltsequenzen oder auch Schaltzahlen verwendet werden, so ist das Überwinden der Sprungstellen problematisch. Im Interesse eines guten dynamischen Verhaltens kann es sinnvoll sein, auf die Verwendung unterschiedlicher Schaltsequenzen zu verzichten und nur mit suboptimalen Pulsmustern einer einzigen Sequenz zu fahren. In der Literatur werden andererseits auch Vorschläge gemacht, wie das Problem der DC-Anregung umgangen werden kann ([Hol2], [Kul1]). Sie basieren darauf, dass bei Pulsmusterwechseln die abgelegten Schaltwinkel vorübergehend in geeigneter Weise modifiziert werden. Ihre Implementierung ist jedoch aufwendig.

Es bleibt anzufügen, dass bei grossen und schnellen Änderungen des Modulationsgrades in jedem Fall DC-Anteile in den Phasenströmen erzeugt werden. Auf dieses Phänomen, von dem alle Verfahren zur Spannungsteuerung betroffen sind, wird in Kapitel 13.5 kurz eingegangen.

Frequenzvariation: Während eine Änderung des Modulationsgrades einen Pulsmusterwechsel bedingt, geschieht dies für die Grundfrequenz über den Proportionalitätsfaktor zwischen den Schaltwinkeln und der Zeitbasis: eine Grundperiode umfasst entsprechend mehr oder weniger Zähleinheiten der Zeitbasis. Die Einstellung einer neuen Grundfrequenz ist deshalb vom Prinzip her einfach.

Praktische Probleme: Das beschriebene dynamische Verhalten basiert auf einer unendlich schnellen Reaktionszeit des Modulators auf Änderungen der Eingangsgrössen. In der Realität ist diese jedoch begrenzt, was zu einer ganzen Reihe von Problemen führt:

- Bis neue Daten bereitstehen muss das vorherige Pulsmuster weitergeführt werden.

- Sobald die Umschaltung auf das neue Pulsmuster möglich ist, müssen bereits vorbereitete Schaltvorgänge des vorangegangenen Pulsmusters bzw. für die vorangegangene Grundfrequenz, die von der Ausgabelogik noch nicht abgearbeitet sind, rechtzeitig wieder gelöscht werden.

- Es ist darauf zu achten, dass die durch die praktische Realisierung des Stromrichters zulässigen minimalen Abstände zwischen zwei Schaltflanken in einem Brückenzweig jederzeit eingehalten werden.

Es stellt sich heraus, dass die praktische Implementierung eines Off-Line Modulators bereits für relativ niedrige Schaltfrequenzen eine sehr leistungsfähige Kontrolleinheit voraussetzt. Wenn zudem die abgespeicherten Pulsmuster sehr ungleichmässige Schaltwinkelverteilungen aufweisen, richtet sich die Verarbeitungskapazität nach den Abschnitten mit der grössten zeitlichen Dichte von Schaltereignissen. Die beschriebene Problematik des dynamischen Verhaltens führt zusätzlich dazu, dass die Off-Line Modulatoren nur für 
vergleichsweise langsame Systeme und niedrige Schaltzahlen einsetzbar sind. Aus diesen Gründen ist die praktische Bedeutung dieser Verfahren eher klein. Im Rahmen einer abgerundeten Theorie über Pulsweitenmodulation sind sie jedoch von grossem Interesse, vor allem weil sie Massstäbe für alle anderen Steuerverfahren setzen.

Literatur zu Kapitel 9.3: [Buj1], [Buj2], [Dir1], [Gil1], [Hol2], [Ham1], [Kul1], [Pit1], [Tak1], [Tak2], [Wüe1], [Zac1], [Zac2] 


\section{Phasenstromregler}

Die bisher beschriebenen Steuerverfahren stellen die Ausgangsspannung des Stromrichters gemäss einem vorgegebenen Sollwert ein. Sie können deshalb unter dem Begriff Verfahren zur Spannungssteuerung zusammengefasst werden. Für sich allein gesehen stellen sie reine Steuerungen dar und sind in der Regel ein Glied in einem Regelkreis für die Phasenströme. Bei der Kategorie von Verfahren, die in diesem Kapitel vorgestellt werden, sind dagegen die Stromregelung und die Erzeugung der Steuersignale für den Stromrichter ein untrennbarer Prozess. Aus diesem Grund werden sie als Verfahren zur direkten Stromregelung bezeichnet.

Phasenstromregler werden in der Literatur auch Hystereseregler oder Toleranzbandregler genannt.

\subsection{Halbbrücke mit Zweipunktregler}

\subsubsection{Funktionsprinzip}

Das Prinzip des Zweipunktreglers ist aus Bild 10.1 ersichtlich: der Phasenstrom des Stromrichters wird in einem vorgegebenen Toleranzband der Breite $I_{\delta}$, das symmetrisch um seinen Sollwert angelegt ist, gehalten. Dies geschieht mit Hilfe eines einfachen Hysterese-Komparators, der den Stromrichter umschaltet, sobald der Strom eine der Bandgrenzen erreicht. Da im regulären Betriebsbereich die Ausgangsspannung betragsmässig grösser als die Gegenspannung der Last ist, wird dadurch mit Sicherheit eine Rückkehr
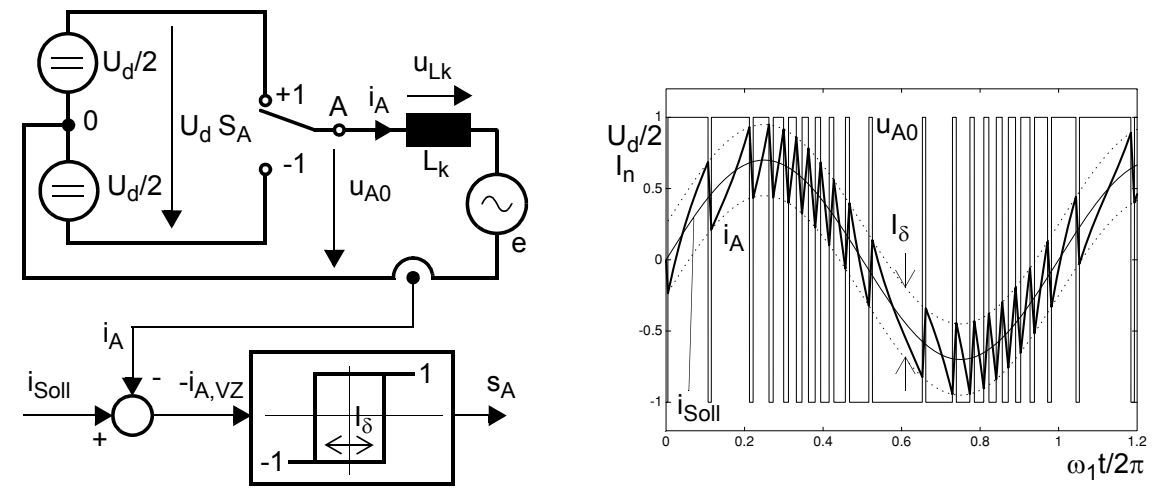

Bild 10.1. Halbbrücke mit Zweipunktregler 
des Stromes ins Innere des Bandes erreicht. Die am Eingang des Komparators anliegende Differenz zwischen Stromsollwert und -istwert stellt gerade den Verzerrungsanteil des Phasenstromes mit umgekehrtem Vorzeichen dar. Er schliesst dabei auch allfällige Abweichungen der Grundschwingung mit ein. Die Grundschwingung der Ausgangsspannung stellt sich betrags- und phasenmässig entsprechend den Lastverhältnissen ein. Sie ist jedoch bei den Phasenstromreglern nur von sekundärem Interesse.

Die grundsätzlichen Eigenschaften des Zweipunktreglers sind sehr leicht abzuleiten:

- Im Gegensatz zu den Verfahren mit Spannungssteuerung ist der Verzerrungsanteil des Phasenstromes praktisch unabhängig vom Betriebspunkt des Stromrichters. Sein Spitzenwert und sein Effektivwert lassen sich allein als Funktion des Toleranzbandes angeben:

$$
\hat{i}_{A, V Z}=\frac{I_{\delta}}{2}, \quad I_{A, V Z, e f f} \approx \frac{I_{\delta}}{2 \sqrt{3}}
$$

Für die Berechnung des Effektivwertes wird angenommen, dass der Verzerrungsstrom zwischen den Berührungspunkten am Toleranzband näherungsweise linear verläuft.

- Bei der Schaltfrequenz verhält es sich umgekehrt. Während sie bei den spannungssteuernden Verfahren jeweils vorgegeben werden konnte, stellt sie sich hier in Abhängigkeit von verschiedenen Parametern von selbst ein und variiert zudem innerhalb einer Grundperiode. Das Verfahren ist grundsätzlich asynchron.

\subsubsection{Schaltfrequenz und Modulationsfunktion}

Im folgenden soll mit Hilfe des in Bild 10.2 dargestellten Ausschnittes aus dem Verlauf des Phasenstromes eine momentane Schaltfrequenz berechnet werden. Dazu werden für

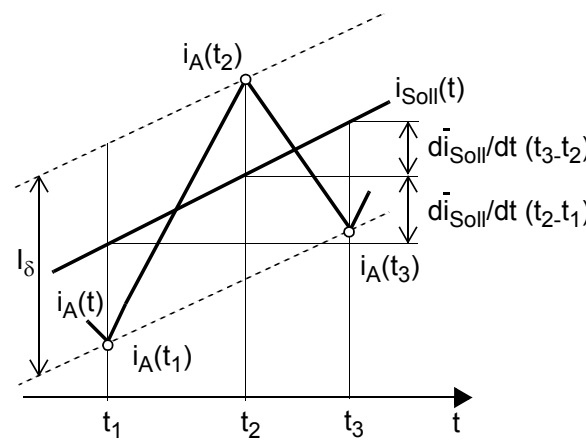

Bild 10.2.

Ausschnitt aus dem Stromverlauf $i_{A}(t)$

die Gegenspannung, für die Spannung über der Induktivität und die Ableitung des Stromsollwertes deren über ein Taktintervall gemittelten Werte $\bar{e}, \bar{u}_{L k}$ sowie $d \bar{i}_{\text {Soll }} / d t$ verwendet. D.h. es wird angenommen, dass diese Grössen kurzzeitig konstant bleiben. Die Differentialgleichung (10.2) für den Phasenstrom vereinfacht sich damit für die Zeitintervalle $\left[t_{1}, t_{2}\right]$ und $\left[t_{2}, t_{3}\right]$ auf die lineare Gleichung (10.3): 


$$
\begin{aligned}
& \frac{d i_{A}}{d t}=\frac{u_{L k}}{L_{k}}, \quad u_{L k}=u_{A 0}-e \\
& \Delta i_{A}=\frac{\bar{u}_{L k}}{L_{k}} \Delta t
\end{aligned}
$$

Setzt man die entsprechenden Werte für die Spannung $\bar{u}_{L k}$ in (10.3) ein, ergibt sich:

$$
\begin{gathered}
t_{1}<t<t_{2}: \quad s_{A}=1, \quad \bar{u}_{L k}=\frac{U_{d}}{2}-\bar{e} \\
\Rightarrow \quad i_{A}\left(t_{2}\right)-i_{A}\left(t_{1}\right)=\left(\frac{U_{d}}{2}-\bar{e}\right) \frac{t_{2}-t_{1}}{L_{k}} \\
t_{2}<t<t_{3}: \quad s_{A}=-1, \quad \bar{u}_{L k}=-\frac{U_{d}}{2}-\bar{e} \\
\Rightarrow \quad i_{A}\left(t_{3}\right)-i_{A}\left(t_{2}\right)=\left(-\frac{U_{d}}{2}-\bar{e}\right) \frac{t_{3}-t_{2}}{L_{k}}
\end{gathered}
$$

Andererseits lässt sich aus Bild 10.2 ablesen:

$$
\begin{aligned}
& i_{A}\left(t_{2}\right)-i_{A}\left(t_{1}\right)=I_{\delta}+\frac{d \bar{i}_{\text {Soll }}}{d t}\left(t_{2}-t_{1}\right) \\
& i_{A}\left(t_{3}\right)-i_{A}\left(t_{2}\right)=-I_{\delta}+\frac{d \bar{i}_{\text {Soll }}}{d t}\left(t_{3}-t_{2}\right)
\end{aligned}
$$

Durch Einsetzen von (10.6) in (10.4) sowie von (10.7) in (10.5) und anschliessendem Auflösen nach $\left(t_{2}-t_{1}\right)$ bzw. $\left(t_{3}-t_{2}\right)$ erhält man:

$$
\left(t_{2}-t_{1}\right)=\frac{I_{\delta}}{\frac{U_{d}}{2 L_{k}}-\frac{\bar{e}}{L_{k}}-\frac{d \dot{i}_{\text {Soll }}}{d t}}, \quad\left(t_{3}-t_{2}\right)=\frac{I_{\delta}}{\frac{U_{d}}{2 L_{k}}+\frac{\bar{e}}{L_{k}}+\frac{d \dot{i}_{\text {Soll }}}{d t}}
$$

Die Summe der beiden Zeitintervalle entspricht einem vollständigen Taktintervall, so dass man daraus auf die momentane Schaltfrequenz $f_{\text {inst }}$ schliessen kann:

$$
f_{\text {inst }}=\frac{1}{t_{3}-t_{1}}=\frac{1}{\left(t_{3}-t_{2}\right)+\left(t_{2}-t_{1}\right)}
$$

Setzt man (10.8) in (10.9) ein, erhält man die momentane Schaltfrequenz als Funktion der Schaltungs- und Steuerungsparameter in diesem Taktintervall:

$$
f_{\text {inst }}=\frac{U_{d}}{4 L_{k} I_{\delta}}\left[1-\frac{4}{U_{d}^{2}}\left(\bar{e}+L_{k} \frac{d \bar{i}_{S o l l}}{d t}\right)^{2}\right]
$$

Die Formel (10.10) kann für beliebige zeitliche Verläufe der Gegenspannung und des 
Stromsollwertes verwendet werden. Im folgenden wird jedoch angenommen, dass beide Grössen sinusförmig verlaufen. Zudem soll das Verhältnis von Schaltfrequenz zu Grundfrequenz so gross sein, dass die über ein Taktintervall gemittelten Werte mit den Momentanwerten näherungsweise übereinstimmen:

$$
\begin{aligned}
& i_{\text {Soll }}=\hat{i}_{\text {Soll }} \sin \left(\omega_{1} t\right) \Rightarrow \frac{d \bar{i}_{\text {Soll }}}{d t} \approx \frac{d i_{\text {Soll }}}{d t}=\omega_{1} \hat{i}_{\text {Soll }} \cos \left(\omega_{1} t\right) \\
& \bar{e} \approx e=\hat{e} \sin \left(\omega_{1} t+\varphi_{e}\right)
\end{aligned}
$$

Da beim Zweipunktregler im Gegensatz zu den spannungssteuernden Verfahren der Strom die Steuergrösse darstellt, ist der Nullpunkt der Zeitachse so gelegt, dass der Phasenstrom zur reinen Sinusfunktion wird. Im allgemeinen weist die Gegenspannung dann eine Phasenverschiebung $\varphi_{e}$ auf. Durch Einsetzen von (10.11) und (10.12) in (10.10) erhält man für die momentane Schaltfrequenz:

$$
f_{\text {inst }}=\frac{U_{d}}{4 L_{k} I_{\delta}}\left\{1-\frac{4}{U_{d}^{2}}\left[\hat{e} \sin \left(\omega_{1} t+\varphi_{e}\right)+\omega_{1} L_{k} \hat{i}_{\operatorname{Soll}} \cos \left(\omega_{1} t\right)\right]^{2}\right\}
$$

An dieser Stelle wird es interessant, die Grundschwingung der Ausgangsspannung des Stromrichters näher zu betrachten. Sie kann allgemein durch die Modulationsfunktion $m$ ausgedrückt werden:

$$
u_{A 0, v=1}=\frac{U_{d}}{2} m, \quad m=\operatorname{Msin}\left(\omega_{1} t+\varphi_{m}\right)
$$

Unter der Voraussetzung, dass der Stromregler die Grundschwingung des Ausgangsstromes genau auf dem Sollwert halten kann, gilt auch:

$$
u_{A 0, v=1}=e+u_{L k, v=1}=\hat{e} \sin \left(\omega_{1} t+\varphi_{e}\right)+\omega_{1} L_{k} \hat{i}_{\operatorname{Soll}} \cos \left(\omega_{1} t\right)
$$

Setzt man (10.15) in (10.14) ein, so erhält man für $m, M$ und $\varphi_{m}$ :

$$
\begin{aligned}
m & =\frac{2}{U_{d}}\left[\hat{e} \sin \left(\omega_{1} t+\varphi_{e}\right)+\omega_{1} L_{k} \hat{i}_{S o l l} \cos \left(\omega_{1} t\right)\right], \\
M & =\frac{2}{U_{d}} \sqrt{\hat{e}^{2}+\omega_{1}^{2} L_{k}^{2} \hat{i}_{\text {Soll }}^{2}+2 \omega_{1} L_{k} \hat{e}^{2} \hat{i}_{\operatorname{Soll}} \sin \varphi_{e}}, \quad \varphi_{m}=\operatorname{atan}\left(\frac{\omega_{1} L_{k} \hat{i}_{\text {Soll }}+\hat{e} \sin \varphi_{e}}{\hat{e} \cos \varphi_{e}}\right)
\end{aligned}
$$

Bild 10.3 ist die graphische Interpretation von (10.16). Es zeigt, wie der Modulationsgrad mit den Parametern Gegenspannung und Phasenstrom zusammenhängt. Insbesondere wird deutlich, dass derselbe Modulationsgrad verschiedene Lastverhältnisse bedeuten kann. Ein möglicher Fall ist immer derjenige mit $\hat{\imath}_{\text {Soll }}=0$ und $M=\hat{e} /\left(U_{d} / 2\right)$ (Kurve c).

Mit Hilfe von (10.16) kann nun (10.13) in eine wesentlich übersichtlichere Form gebracht werden:

$$
f_{\text {inst }}=f_{B}\left[1-m^{2}(t)\right], \quad f_{B}=\frac{U_{d}}{4 L_{k} I_{\delta}}
$$




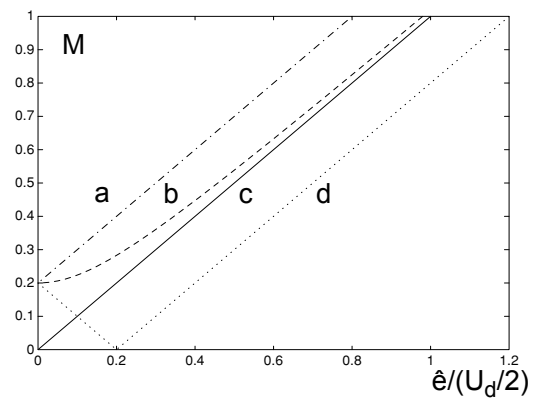

a: î́soll $=I_{n}, \varphi_{e}=-\pi / 2$ (e nacheilend)

b: î́soll $=I_{n}, \varphi_{e}=\pi$ (e gegenphasig)

$\hat{i}_{\text {Soll }}=I_{n}, \varphi_{e}=0$ (e gleichphasig)

c: îsoll $=0$

d: $\hat{I}_{\text {soll }}=I_{n}, \varphi_{\mathrm{e}}=\pi / 2$ (e voreilend)

Bild 10.3.

Modulationsgrad des Stromrichters in Abhängigkeit der Gegenspannung und des Phasenstromes, $I_{n}=0.2 I_{B}$

Die momentane Schaltfrequenz ist allein durch die Modulationsfunktion des Stromrichters bestimmt, und zwar unabhängig davon, welche Stromgrundschwingung sich ergibt. Die Umformung von (10.18) mit Hilfe von (10.2) zeigt, dass auch der Verlauf des Verzerrungsstrom $i_{A, V Z}$ unabhängig von dieser Stromgrundschwingung ist:

$$
\begin{gathered}
i_{A, V Z}=i_{A}-i_{\text {Soll }}, \quad \frac{d i_{A, V Z}}{d t}=\frac{d i_{A}}{d t}-\frac{d i_{\text {Soll }}}{d t} \\
\Rightarrow \quad \frac{d i_{A, V Z}}{d t}=\frac{1}{L_{k}}\left(u_{A 0}-e\right)-\frac{d i_{\text {Soll }}}{d t} \\
\Rightarrow \quad L_{k} \frac{d i_{A, V Z}}{d t}=u_{A 0}-\left(e+L_{k} \frac{d i_{\text {Soll }}}{d t}\right)=u_{A 0}-m \frac{U_{d}}{2} \\
\Rightarrow \quad i_{A, V Z}=\frac{1}{L_{k}} \int\left(u_{A 0}-m \frac{U_{d}}{2}\right) d t
\end{gathered}
$$

Dieses Resultat erlaubt es, alle Betrachtungen bezüglich Schaltfrequenz und Verzerrungsstrom anhand des leicht überblickbaren Spezialfalles mit $\hat{\imath}_{\text {Soll }}=0$ und $M=\hat{e} /\left(U_{d} / 2\right)$ zu machen, wo der Phasenstrom nur aus dem Verzerrungsanteil besteht und die Grundschwingung der Ausgangsspannung mit der Gegenspannung übereinstimmt.

Die mittlere Schaltfrequenz $f_{s}$ des Zweipunktreglers erhält man, wenn man den Mittelwert von $f_{\text {inst }}$ über eine Grundperiode bildet. Sie ist eine Funktion des Modulationsgrades:

$$
f_{s}=f_{B}\left(1-\frac{M^{2}}{2}\right)
$$

Es ist zu beachten, dass $f_{s}$ nicht von der Grundfrequenz $f_{1}$ abhängt. Zum Vergleich mit anderen Steuerverfahren kann das Verhältnis von $f_{s}$ zu $f_{1}$, d.h. die Schaltzahl $q$, gebildet werden, indem in (10.20) die Induktivität $L_{k}$ durch die Reaktanz $X_{k}$ bei $f_{1}$ ersetzt wird:

$$
L_{k}=\frac{X_{k}}{2 \pi f_{1}} \quad \Rightarrow \quad \frac{f_{s}}{f_{1}}=q=\frac{\pi U_{d}}{2 X_{k} I_{\delta}}\left(1-\frac{M^{2}}{2}\right)
$$

In Bild 10.4. ist links der Verlauf der momentanen Schaltfrequenz als Funktion der Zeit und rechts die mittlere Schaltfrequenz als Funktion des Modulationsgrades $M$ aufgetra- 

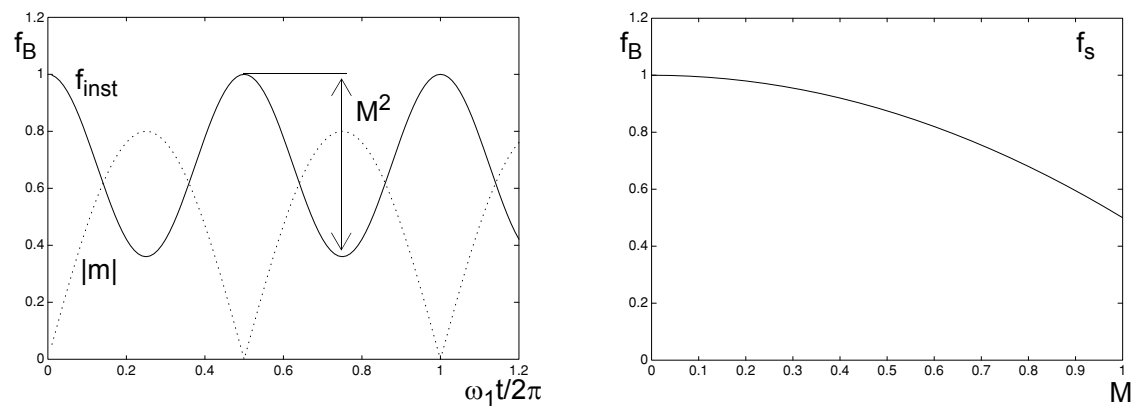

Bild 10.4. Links: Verlauf der momentanen Schaltfrequenz und des Betrages der Modulationsfunktion (Beispiel $M=0.8$ ),

rechts: mittlere Schaltfrequenz in Funktion des Modulationsgrades

gen. Die momentane Schaltfrequenz variiert mit der doppelten Grundfrequenz. Ihr Maximalwert $f_{B}$ tritt jeweils dann auf, wenn die Modulationsfunktion $m$ einen Nulldurchgang aufweist. Der kleinste Wert von $f_{\text {inst }}$ wird dagegen im Scheitelpunkt von $m$ erreicht und ist daher aussteuerungsabhängig. Die Aussteuergrenze des Stromrichters liegt dort, wo die momentane Schaltfrequenz auf null zurück geht. In (10.17) ist ersichtlich, dass dies bei $m=1$ der Fall ist. Solange der Stromrichter mit einem Modulationsgrad von $M<1$ betrieben wird, reicht die Ausgangsspannung jederzeit dazu aus, den Phasenstrom zur Umkehr zu bringen und ihn damit in seinem Toleranzband zu halten. Der Aussteuerbereich, innerhalb der das Verfahren regulär funktioniert, ist also gleich wie bei den einphasigen Trägerverfahren.

Die mittlere Schaltfrequenz $f_{S}$ beträgt bei $M=0$ ebenfalls $f_{B}$ und reduziert sich mit zunehmendem Modulationsgrad bis auf die Hälfte dieses Wertes.

Wird versucht, wie in Bild 10.5 dargestellt, einen Betriebspunkt jenseits der Aussteuergrenze einzustellen, so verharrt der Stromrichter während dieser Zeit in einem Schaltzustand und $f_{\text {inst }}$ bleibt somit auf null stehen. Der Phasenstrom verlässt sein Toleranzband (markierte Fläche in Bild 10.5). Damit nimmt einerseits sein Verzerrungsanteil zu und andererseits wird die geforderte Grundschwingung nicht mehr erreicht.

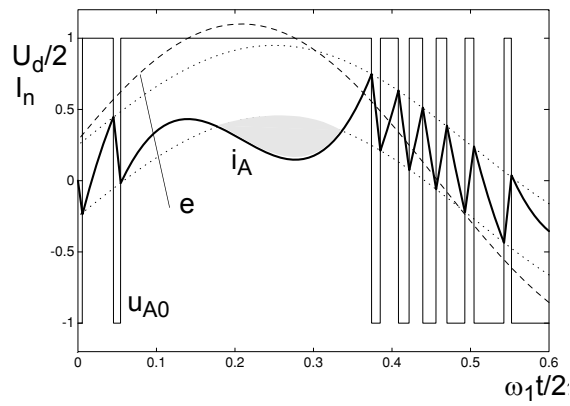

Bild 10.5.

Übersteuerter Zweipunktregler 


\subsubsection{Phasenstrom}

In Bild 10.6. sind die zeitlichen Verläufe der Ausgangsströme und deren Frequenzspektra für je zwei verschiedene Gegenspannungen und Toleranzbänder dargestellt. Die Spektra sind verdichtete Linienspektra, die den asynchronen Charakter des Verfahrens sichtbar machen. Es zeigt sich, dass der Strom bei kleiner Aussteuerung des Stromrichters (Bild 10.6 oben) demjenigen eines Trägerverfahrens gleicht: im Spektrum treten neben der Grundschwingung ausgeprägte Anteile in den Bereichen der mittleren Schaltfrequenz und deren Vielfachen auf. Mit zunehmender Aussteuerung ergibt sich eine Verbreiterung und Abflachung dieser Bänder, bis schliesslich ein Spektrum vorliegt, bei dem die domi-
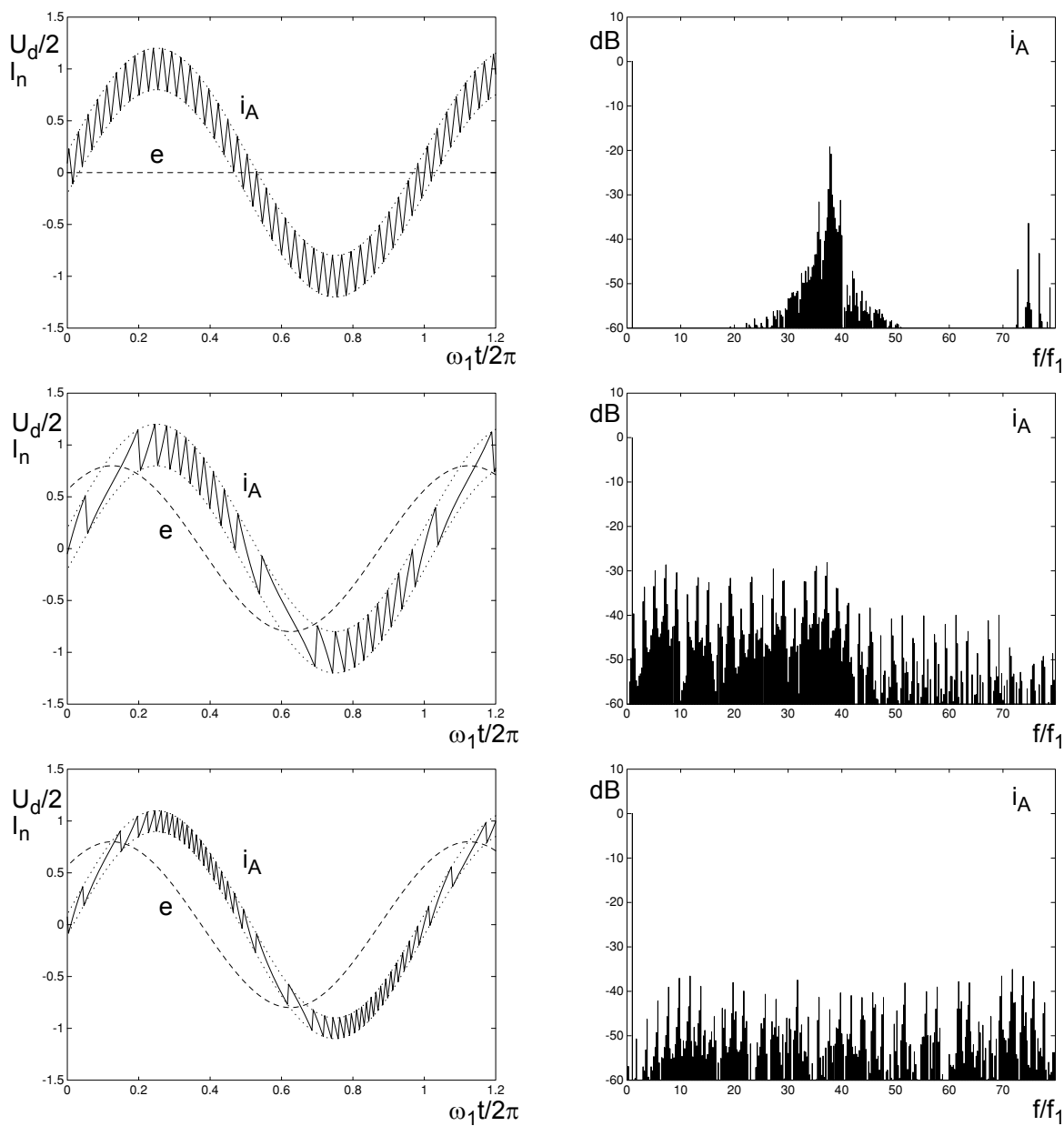

Bild 10.6. Zeitliche Verläufe und Spektra des Phasenstromes bei verschiedenen Gegenspannungen und Toleranzbändern, $I_{n}=0.2 I_{B}, 0 \mathrm{~dB}: I_{n}$, Spektra berechnet über $4 T_{1}$, oben: $\hat{e}=0, M=0.2, q \approx 38.5, I_{\delta}=0.4 I_{n}$, mitte: $\hat{e}=0.8\left(U_{d} / 2\right), M=0.95, q \approx 21.5, I_{\delta}=0.4 I_{n}$, unten: $\hat{e}=0.8\left(U_{d} / 2\right), M=0.95, q \approx 43, I_{\delta}=0.2 I_{n}$ 
nanten Anteile im Bereich von null bis etwa zur zweifachen Schaltfrequenz gleichmässig verteilt sind (Bild 10.6 mitte). Dabei treten auch in geringem Masse Subharmonische auf. Die Verkleinerung des Toleranzbandes (Bild 10.6 unten) bewirkt eine grössere Schaltfrequenz, so dass sich das Band, in dem die dominanten Anteile auftreten, entsprechend verbreitert. Die Amplituden werden dafür kleiner. In allen Fällen ist der Effektivwert des Verzerrungsstromes nach der Näherungsformel (10.1) allein durch das Toleranzband bestimmt.

Die dargestellten Beispiele entsprechen Momentaufnahmen über einen Zeitbereich von einigen Grundperioden. Aufnahmen zu einem anderen Zeitpunkt oder mit einer anderen Beobachtungsdauer würden qualitativ gleiche Spektra mit kleinen Unterschieden im Detail ergeben.

\subsubsection{Dynamisches Verhalten}

Der Zweipunktregler stellt die schnellste Methode zur Regelung des Phasenstromes dar. Sobald dieser sich ausserhalb des Toleranzbandes befindet, wird der Stromrichter unverzüglich so geschaltet, dass er sich mit maximaler Geschwindigkeit dorthin zurückbewegt. Diese Geschwindigkeit ist jedoch nicht in jedem Fall gleich. Sie hängt davon ab, wie gross die Spannung $u_{L k}$ gemacht werden kann. Bild 10.7 zeigt die Reaktion des Zweipunktreglers auf eine schrittartige Änderung des Sollwertes für die Fälle mit einer sehr grossen und einer sehr kleinen Ausregelzeit. Sie treten auf, wenn sich die Gegenspannung gerade im Bereich des positiven oder des negativen Scheitelwertes befindet, so dass die Differenz zwischen Ausgangs- und Gegenspannung besonders klein bzw. gross ist. Demgegenüber würde sich im Bereich der Nulldurchgänge von $m$ eine mittlere Spannungsdifferenz und damit eine mittlere Ausregelzeit ergeben.
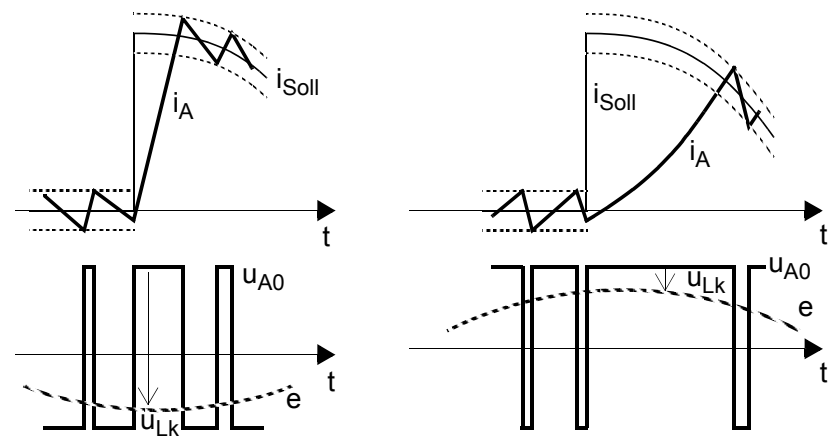

Bild 10.7.

Schrittantwort des Zweipunktreglers bei verschiedenen Phasenlagen der Gegenspannung

Stromrichter mit einem Zweipunktregler sind kurzschlussfest, sofern eine Induktivität im Lastkreis verbleibt und die Reaktionszeit der Regelung genügend klein ist. Der Phasenstrom verläuft weiter in seinem Toleranzband. Eine Verkleinerung der Lastinduktivität hat jedoch den Anstieg der Schaltfrequenz zur Folge.

Literatur zu Kapitel 10.1: [Brod1], [Bos2], [Kli1], [Murr1] 


\subsection{Einphasige Brücke mit Dreipunktregler}

\subsubsection{Funktionsprinzip}

Die einphasige Brücke kann grundsätzlich ebenfalls mit einem Zweipunktregler betrieben werden. Dabei werden die beiden Brückenzweige synchron im Gegentakt geschaltet. Ein solcher Regler macht jedoch keinen Gebrauch vom zusätzlichen Schaltniveau null für die Phasenspannung. Mit einer einfachen Erweiterung des Zweipunkt- auf einen Dreipunktregler lässt sich dies korrigieren. Nach dem in Bild 10.8 dargestellten Prinzip schaltet während einer Halbperiode nur ein Brückenzweig. Auf diese Weise erfolgt jeweils eine Umschaltung der Phasenspannung zwischen $+U_{d}$ und 0 bzw. $-U_{d}$ und 0 . Dies hat zur Folge, dass sich der Phasenstrom im Vergleich zum Zweipunktregler langsamer von einer Toleranzbandgrenze zur anderen bewegt. Die momentane Schaltfrequenz wird generell kleiner. Da jeder Schalter nur während einer Halbperiode aktiv ist, halbiert sich seine mittlere Schaltfrequenz noch einmal. Zweimal pro Periode muss ein Umschalter betätigt werden, der die Schaltsignale vom einen Brückenzweig auf den anderen leitet. Dies geschieht am einfachsten mit Hilfe eines zweiten Komparators mit einer etwas grösseren Hysterese $I_{\delta}+I_{h}$. Der Phasenstrom verlässt dann zweimal das Toleranzband um den Betrag $I_{h} / 2$, wie das in Bild 10.8 mit Pfeilen gekennzeichnet ist. Der Wert von $I_{h}$ kann prinzipiell so klein gemacht werden, wie es die praktische Realisierung erlaubt.
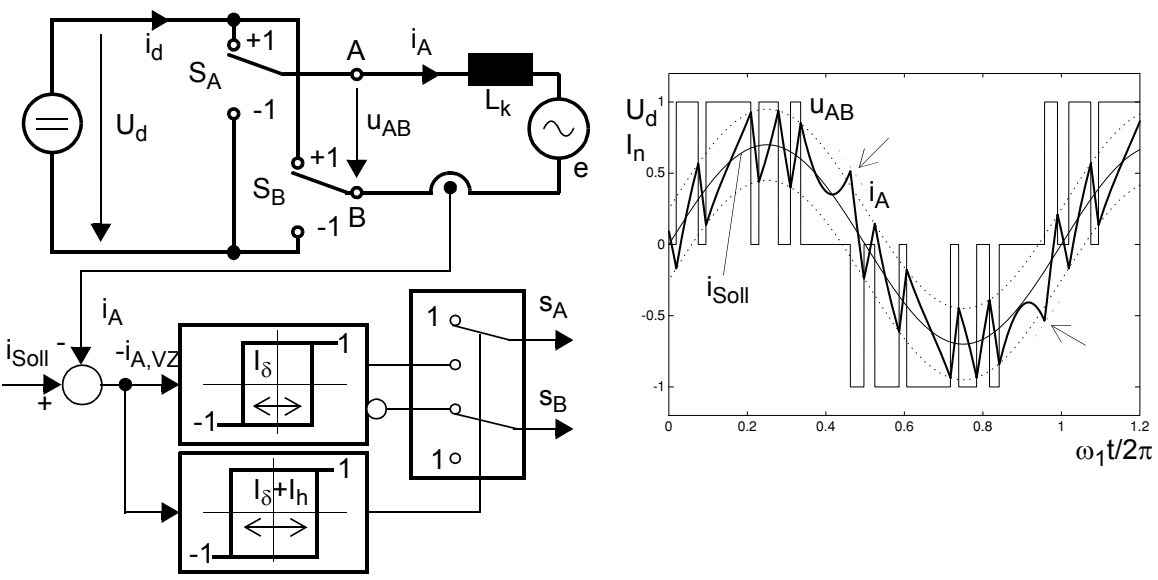

Bild 10.8. Einphasige Brücke mit Dreipunktregler

\subsubsection{Schaltfrequenz}

Für die Berechnung der Schaltfrequenz kann wiederum von der Darstellung in Bild 10.2 und den Beziehungen (10.4) bis (10.7) ausgegangen werden, wobei als Ausgangsspannungen jetzt die Werte $+U_{d}$ bzw. $-U_{d}$ und 0 einzusetzen sind. Als Resultat erhält man für die momentane Schaltfrequenz beim Dreipunktregler: 


$$
f_{\text {inst }}=\frac{U_{d}}{L_{k} I_{\delta}}\left[\frac{1}{U_{d}}\left|\bar{e}+L_{k} \frac{d \bar{i}_{\text {Soll }}}{d t}\right|-\frac{1}{U_{d}^{2}}\left(\bar{e}+L_{k} \frac{d \bar{i}_{\text {Soll }}}{d t}\right)^{2}\right]
$$

In der gleichen Art und Weise, wie dies für den Zweipunktregler in den Gleichungen (10.14) bis (10.17) abgeleitet worden ist, kann $f_{\text {inst }}$ als Funktion des Verlaufes der Modulationsfunktion des Stromrichters $m$ dargestellt werden:

$$
f_{\text {inst }}=2 f_{B}\left(|m|-m^{2}\right), \quad f_{B}=\frac{U_{d}}{2 L_{k} I_{\delta}}
$$

Bei der Definition von $f_{B}$ ist berücksichtigt, dass die Bezugsspannung der Brückenschaltung mit $U_{d}$ gegenüber der Halbbrücke mit $U_{d} / 2$ doppelt so gross ist. Um die mittlere Schaltfrequenz eines Schalters zu erhalten, muss beachtet werden, dass jeder Schalter während einer Halbperiode inaktiv ist:

$$
f_{s}=\frac{1}{T_{1}} \int_{0}^{T_{1} / 2} f_{\text {inst }} d t=f_{B}\left(\frac{2}{\pi} M-\frac{M^{2}}{2}\right)
$$

In Bild 10.9 sind die Verläufe der momentanen Schaltfrequenz als Funktion der Zeit und der mittleren Schaltfrequenz als Funktion des Modulationsgrades wiederum graphisch dargestellt. Die momentane Schaltfrequenz variiert mit der zweifachen Grundfrequenz. Beim Nulldurchgang der Modulationsfunktion reduziert sich $f_{\text {inst }}$ hier bis auf null. Dasselbe gilt bei Erreichen der Aussteuergrenze bei $m=1$ (nicht im Bild). Der Maximalwert von $f_{\text {inst }}$ tritt dagegen bei $|m|=0.5$ auf, wenn die kurzzeitig gemittelte Ausgangsspannung genau in der Mitte zwischen den Schaltniveaus liegt. Die maximale momentane Schaltfrequenz ist nur halb so gross wie beim Zweipunktregler. Bei kleiner Aussteuerung mit $M<0.5$ wird dieser Betriebspunkt zudem gar nicht erreicht, so dass die grösste auftretende Schaltfrequenz dann noch niedriger ist.
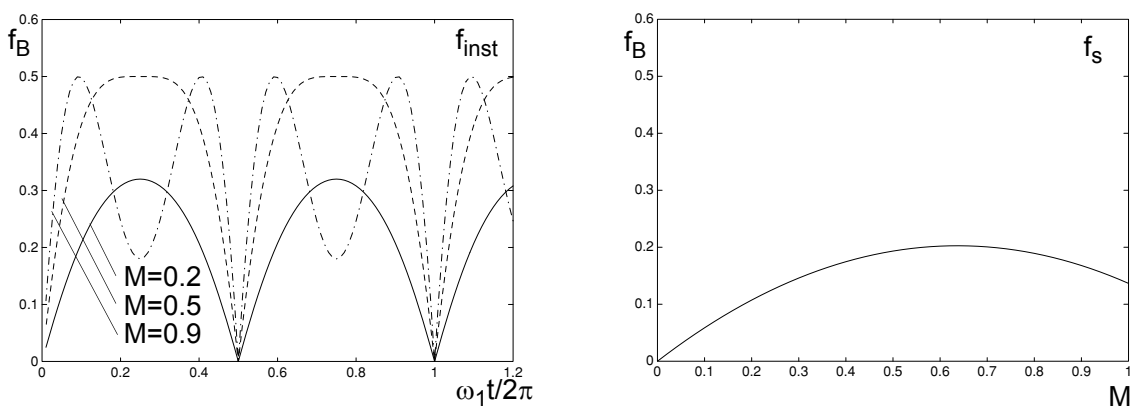

Bild 10.9. Links: Verlauf der momentanen Schaltfrequenz und des Betrages der Modulationsfunktion, rechts: mittlere Schaltfrequenz in Funktion des Modulationsgrades

Die mittlere Schaltfrequenz $f_{S}$ ist gegenüber dem Zweipunktregler drastisch reduziert. Vor allem geht sie bei kleiner Aussteuerung des Stromrichters gegen null zurück. 
Während beim Zweipunktregler die analytisch ermittelten Grössen $f_{\text {inst }}$ und $f_{S}$ die Schaltfrequenz genau beschreiben, stimmen sie hier nur näherungsweise. Es findet im stationären Betrieb eine Synchronisierung des Pulsmusters auf die Grundfrequenz statt. Dieses Phänomen kann anhand von Bild 10.10 erklärt werden: der Verzerrungsstrom $i_{A, V Z}$ verlaufe vor dem Zeitpunkt $t_{0}$ in beliebiger Weise. Da bei $t_{0}$ die Modulationsfunktion einen Nulldurchgang aufweist, berührt er zweimal denselben Rand des Toleranzbandes und schliesst dabei eine bezüglich $t_{0}$ axialsymmetrische Fläche ein. Wegen der Symmetrie von $m$ bezüglich $t_{0}$ stellt auch der weitere Verlauf von $i_{A, V Z}$ nach $t_{0}$, bis zum nächsten Nulldurchgang von $m$ bei $t_{1}$, eine Spiegelung des vorangegangen Abschnittes dar. Auf dieselbe Art und Weise entsteht bei $t_{1}$ die nächste Symmetrieachse, so dass die folgende Halbperiode nach $t_{1}$ mit derjenigen vor $t_{0}$ identisch ist. Damit wiederholt sich der Verlauf einer Halbperiode des Verzerrungsstromes immer wieder, jeweils bezüglich der vorangegangenen gespiegelt. Der Verlauf selbst ist allein durch den Anfangszustand der Schaltung bestimmt. Er sieht für denselben Modulationsgrad bei verschiedenen Anfangsbedingungen anders aus. Die Synchronisation erfolgt, sobald sich die Schaltung im stationären Zustand befindet.

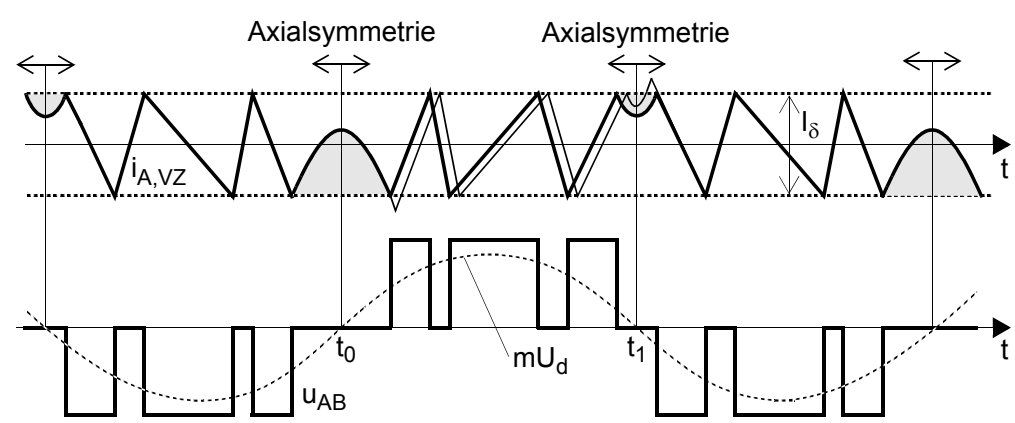

Bild 10.10. Synchronisierung des Pulsmusters auf die Grundfrequenz

Der beschriebene Mechanismus ist im Falle vollständig idealer Kurvenformen leicht verständlich. Es kann aber festgestellt werden, dass selbst bei Abweichungen von der Idealität eine Synchronisation stattfindet. Das Verwenden einer zweiten Schaltschwelle zum Umschalten der Spannungspolarität verursacht eine solche Nichtidealität. Wie in Bild 10.10 durch den dünn eingezeichneten Verlauf angedeutet, geht dadurch zwar die exakte Axialsymmetrie des Verzerrungsstromes bezüglich $t_{0}$ oder $t_{1}$ verloren. Trotzdem stellt sich mit beliebigen Startbedingungen nach wenigen Halbperioden des Einschwingens ein periodischer Verlauf ein. Dasselbe kann auch beobachtet werden, wenn die Last einen ohmschen Anteil aufweist, und selbst wenn in der Gegenspannung, im Stromsollwert oder in der Zwischenkreisspannung Oberschwingungen vorhanden sind. Solange alle von aussen beeinflussten Grössen synchron verlaufen, also keine aperiodischen Störungen aufweisen, stellt sich auch das Pulsmuster synchron ein.

Als Konsequenz der Synchronisation ist die mittlere Schaltfrequenz nach (10.24), die ja im allgemeinen kein ganzzahliges Vielfaches der Grundfrequenz ist, nur als Näherung zu verstehen. Der genaue Wert stellt sich je nach Startbedingung entweder auf das nächst- 
höhere oder das nächsttiefere ganzzahlige Vielfache von $f_{1}$ ein. Die Schaltzahl $q$ wird somit ebenfalls ganzzahlig.

\subsubsection{Phasenstrom und Zwischenkreisstrom}

Bild 10.11 zeigt die zeitlichen Verläufe und die Spektra des Phasenstromes für zwei verschiedene Modulationsgrade und Toleranzbänder. Der synchrone Verlauf hat zur Folge, dass in den Spektra nur echte Oberschwingungen und insbesondere keine Subharmonischen auftreten. Dagegen enthalten die Phasenströme kleine Gleichanteile in der Grössenordnung der Oberschwingungen niedriger Ordnung. Dies ist in den Bildern nicht er-


Bild 10.11. Verläufe und Spektra des Phasenstromes für verschiedene Aussteuerungen und Toleranzbänder, $I_{n}=0.2 I_{B}, 0 \mathrm{~dB}: I_{n}$, oben: $\hat{e}=0, M=0.2, q=2, I_{\delta}=0.4 I_{n}$, mitte: $\hat{e}=0.8 U_{d}, M=0.95, q=6, I_{\delta}=0.4 I_{n}$, unten: $\hat{e}=0.8 U_{d}, M=0.95, q=11, I_{\delta}=0.2 I_{n}$ 
sichtlich. Die Amplitudenverteilung ist im Gegensatz zum Zweipunktregler im ganzen Aussteuerbereich gleichartig: dominant sind stets die niedrigen Harmonischen bis ungefähr zur vierfachen mittleren Schaltfrequenz. Ein kleineres Toleranzband verursacht eine höhere Schaltfrequenz und verbreitert damit das Band der dominanten Harmonischen entsprechend.

Es ist zu beachten, dass andere Anfangsbedingungen andere synchrone Stromverläufe zur Folge haben würden. Die zugehörigen Spektra würden sich jedoch nur geringfügig von den gezeigten Beispielen unterscheiden.

Die Näherungsformel (10.1) für den Effektivwert des Verzerrungsstromes ist auch beim Dreipunktregler anwendbar. Die veränderte Kurvenform von $i_{A}$ im Bereich der Nulldurchgänge von $m$ vergrössert die Ungenauigkeit nur unwesentlich.

Zwischenkreisstrom: Bild 10.12 zeigt den zeitlichen Verlauf und das Spektrum von $i_{d}$ für den Betriebsfall in Bild 10.11 mitte. Es wird deutlich, dass sich die Charakteristik des Spektrums des Phasenstromes auch im Zwischenkreisstrom niederschlägt. Neben den dominanten Gleich- und Pulsationsanteilen $I_{d}$ und $\hat{\imath}_{d, v=2}$ tritt eine Vielzahl von Oberschwingungen auf. Diejenigen mit den grössten Amplituden liegen im Bereich der zweifachen bis vierfachen Schaltfrequenz auf.
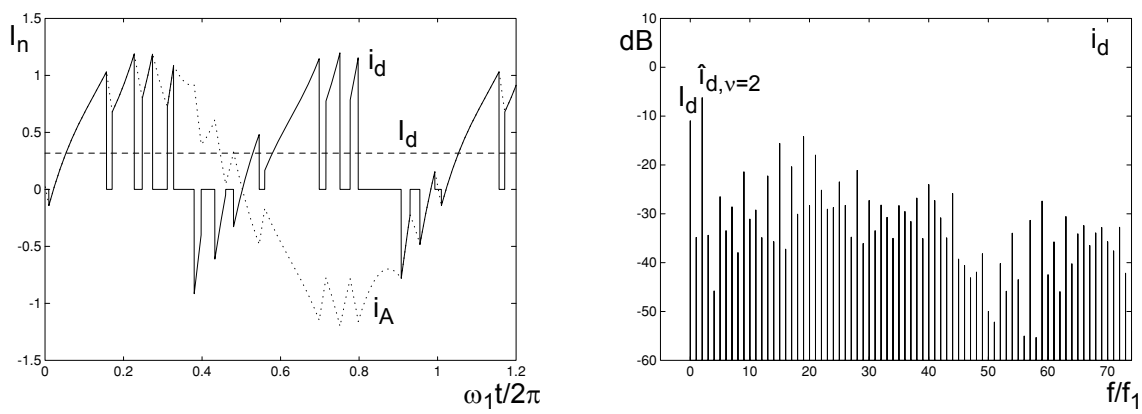

Bild 10.12. Links: Verlauf und Mittelwert des Zwischenkreisstromes sowie Phasenstrom für das Beispiel in Bild 10.11 unten, rechts: Spektrum des Zwischenkreisstromes, 0dB: $I_{n}$

\subsubsection{Dynamisches Verhalten}

Sobald der Phasenstrom bei einem dynamischen Vorgang sowohl das innere wie auch das äussere Toleranzband verlässt, verhält sich der Dreipunktregler gleich wie der Zweipunktregler: beide Komparatorausgänge stehen in derselben Position, was bedeutet, dass die Brückenzweige entgegengesetzte Stellungen annehmen und somit eines der beiden von null verschiedenen Schaltniveaus angelegt ist. Der Phasenstrom wird auf diese Weise mit der maximal möglichen Geschwindigkeit in sein Toleranzband zurückgeholt. 


\subsection{Dreiphasige Brücke mit Zweipunktreglern}

\subsubsection{Funktionsprinzip}

Die 3 Halbbrücken des dreiphasigen Stromrichters können gemäss Bild 10.13 je mit einem Zweipunktregler versehen werden. Wäre der Laststernpunkt $N$ mit dem Mittelpunkt 0 verbunden, so würden sich die 3 Regler wie einphasige Zweipunktregler verhalten. Wegen des offenen Sternpunktes beeinflussen sie sich jedoch gegenseitig, da jede Phasenspannung von allen drei Schalterstellungen abhängt und sich damit jede Schalthandlung auf alle 3 Brückenzweige auswirkt. Dadurch ergibt sich ein deutlich anderes Verhalten. Zur Veranschaulichung sind in Bild 10.14 die zeitlichen Verläufe der Grössen einer Phase dargestellt. Der besseren Übersicht wegen, wurden dabei die Sollwerte $i_{U / V /}$ $W$,Soll $=0$ gewählt. In den Bildern 10.13 und 10.14 lassen sich die folgenden Eigenschaften erkennen:

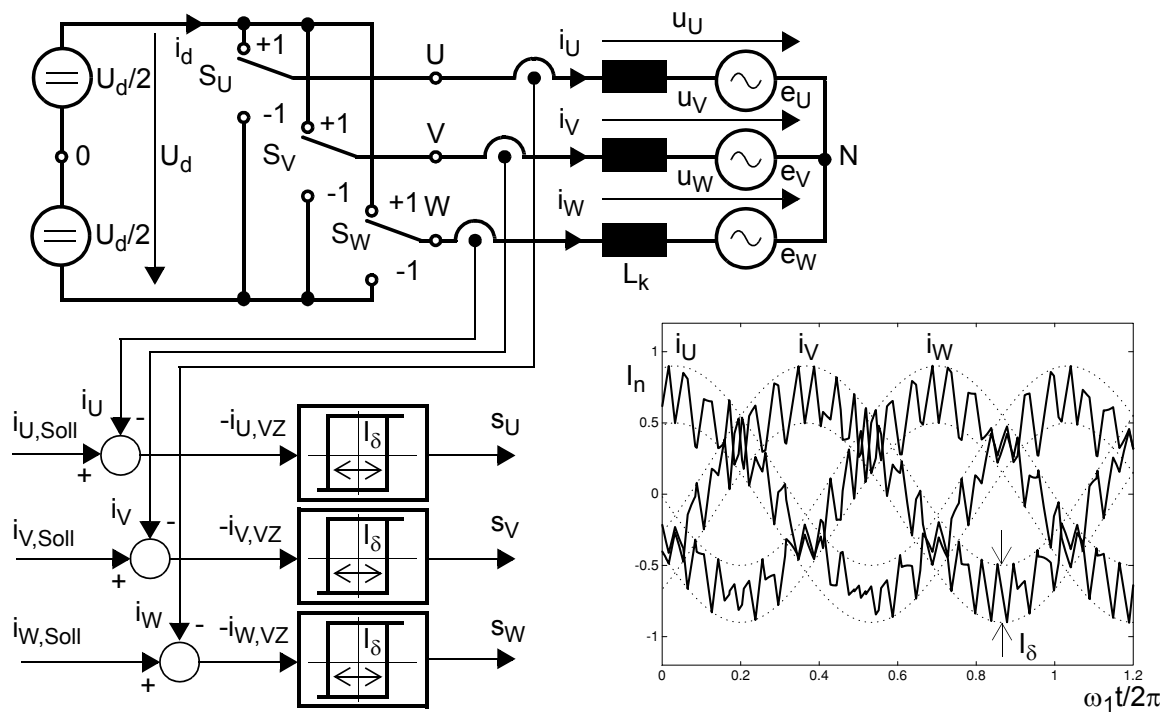

Bild 10.13. Dreiphasige Brücke mit 3 Zweipunktreglern (dreiphasiger Zweipunktregler)

- Der Phasenstrom nützt nicht jederzeit das volle Toleranzband aus. Der Grund dafür sind die Schalthandlungen in den anderen Brückenzweigen, die seinen Verlauf beeinflussen und vor allem das Vorzeichen seiner Ableitung umkehren können.

- Bereits beim einphasigen Zweipunktregler trat die kleinste momentane Schaltfrequenz dort auf, wo die Modulationsfunktion ihren Scheitelwert erreichte. Dieser Effekt wird jetzt noch verstärkt, da die beiden anderen Brückenzweige zur selben Zeit eine grössere Aktivität aufweisen. Die Phase mit dem momentan grössten Wert der Modulationsfunktion kommt vorübergehend kaum mehr zum Schalten.

- Es treten Toleranzbandverletzungen auf. Der Strom kann den Rand seines Toleranzbandes erreichen und überschreiten (in Bild 10.14 mit Pfeil gekennzeichnet), obschon 

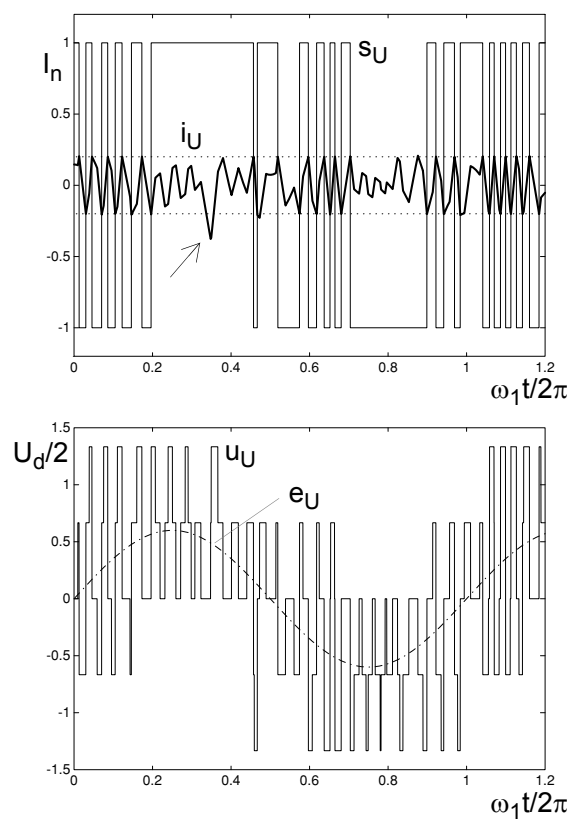

\section{Bild 10.14.}

Zeitliche Verläufe in einer Phase beim dreiphasigen Zweipunktregler

der entsprechende Brückenzweig bereits in der entgegengesetzten Stellung steht. Erst das Schalten eines anderen Brückenzweiges lässt den Stromfehler wieder kleiner werden. Ausgehend von der Annahme, dass sowohl die Summe der Sollwerte der Phasenströme wie die der Istwerte null ist, folgt dasselbe für die Summe der Stromfehler. Da damit der Fehler einer Phase gleich der invertierten Summe der beiden anderen ist, kann im schlimmsten Fall eine Toleranzbandverletzung um das Zweifache auftreten:

$$
i_{U, V Z}+i_{V, V Z}+i_{W, V Z}=0, i_{U, V Z}=-i_{V, V Z}-i_{W, V Z} \Rightarrow\left|i_{U, V Z}\right| \leq 2 \frac{I_{\delta}}{2}
$$

- Der kurzzeitige Mittelwert der Phasenspannung (d.h. die Grundschwingung) stellt sich zwar auf den richtigen Wert ein, es werden aber nicht immer die günstigsten Schaltniveaus verwendet. Damit bewegt sich der Phasenstrom nicht immer mit der kleinstmöglichen Geschwindigkeit im Toleranzband und die Schaltfrequenz wird nicht minimal.

\subsubsection{Schaltfrequenz und Verzerrungsstrom}

Eine analytische Beschreibung der Schaltfrequenz ist beim dreiphasigen Zweipunktregler nicht mehr möglich. Die Verläufe der mittleren Schaltfrequenz und des Effektivwertes des Verzerrungsstromes als Funktion der Aussteuerung des Stromrichters in Bild 10.15. sind Resultate von Simulationen. Dargestellt sind die Werte der Phase $U$. Zum Vergleich ist im Diagramm links auch die Schaltfrequenz des einphasigen Zweipunktreglers nach (10.20) und rechts die Werte für den Effektivwert nach der Näherungsformel (10.1) beigefügt. Es lassen sich die folgenden Aussagen machen: 

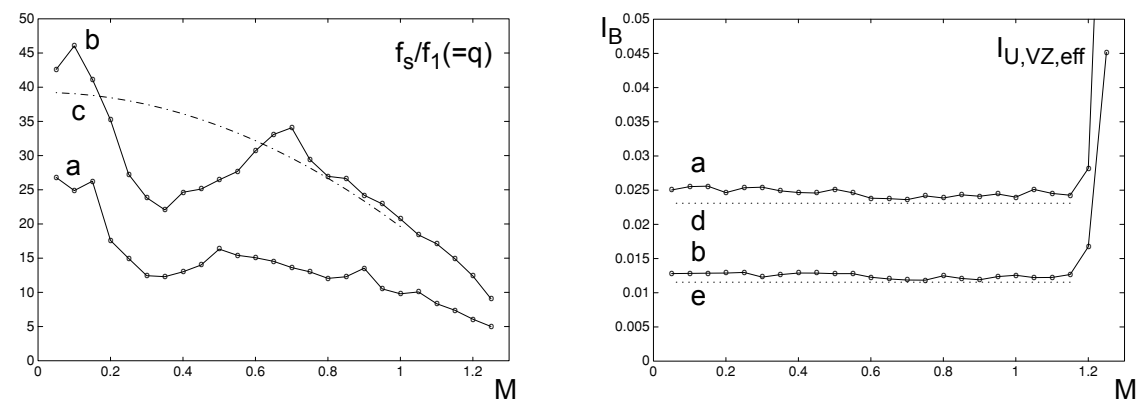

Bild 10.15. Mittlere Schaltfrequenz und Effektivwerte des Verzerrungsstromes beim dreiphasigen Zweipunktregler für zwei verschiedene Toleranzbänder ( $\mathrm{o}=$ simulierte Betriebspunkte), a: dreiphasiger Zweipunktregler, $I_{\delta}=0.4 I_{n}$, b: dreiphasiger Zweipunktregler, $I_{\delta}=0.2 I_{n}$, c: einphasiger Zweipunktregler, $I_{\delta}=0.4 I_{n}$ (zum Vergleich), d und e: Näherungsformel nach (10.1) für $I_{U, V Z, e f f}$ mit $I_{\delta}=0.4 I_{n}$ und $0.2 I_{n}$

- Die dargestellten Resultate sind über einen sehr grossen Zeitraum gemittelt. Bezogen auf den Zeitraum von einer Grundperiode können bei der Schaltfrequenz grosse Variationen festgestellt werden.

- Ein Verdoppelung des Toleranzbandes bringt näherungsweise die Halbierung der Schaltfrequenz sowie die Verdoppelung des Verzerrungsstromes mit sich.

- Die Schaltfrequenz ist im Bereich $0.6<M<1$ sehr ähnlich mit derjenigen des einphasigen Zweipunktreglers mit doppeltem Toleranzband.

- Da der dreiphasige Stromrichter die Fähigkeit hat, alle Phasenspannungen gleichzeitig auf null einzustellen, könnte die Schaltfrequenz wie beim Dreipunktregler im Bereich sehr kleiner Aussteuerung prinzipiell sehr klein werden. Der dreiphasige Zweipunktregler ist jedoch nicht in der Lage, diese Eigenschaft zu nutzen. Aus Gründen, die anschliessend noch genauer erläutert werden, ist die Schaltfrequenz in diesem Bereich sogar am grössten.

- Obschon die Phasenströme nicht mehr streng von einer Bandgrenze zur anderen pendeln, lässt sich die Näherungsformel (10.1) für den Effektivwert des Verzerrungsstromes weiterhin verwenden. Die tatsächlichen Werte sind nur leicht grösser.

- Während bei den einphasigen Schaltungen die Aussteuergrenze bei $M=1$ lag, erreicht sie jetzt analog zu den Trägerverfahren $M=2 / \sqrt{3} \approx 1.155$. Dies zeigt sich in den Diagrammen dadurch, dass darüber der Verzerrungsstrom sofort sehr stark zunimmt. Der Grund dafür ist die bei Übersteuerung auftretende Abweichung der Grundschwingung des Phasenstromes von ihrem Sollwert, die bei den Phasenstromreglern dem Verzerrungsanteil zugerechnet wird.

- Der dreiphasige Zweipunktregler ergibt keine exakt symmetrischen Verhältnisse in den 3 Phasen. Im Mittel stellen sich jedoch für alle Schalter dieselben Schaltfrequenzen und für alle Verzerrungsströme dieselben Effektivwerte ein.

Bei sehr niedriger Aussteuerung ist die Aussage nicht mehr richtig, dass jeweils einer der Regler vorübergehend kaum mehr schaltet. Weil sich die Momentanwerte ihrer Modulationsfunktionen nicht mehr deutlich unterscheiden, arbeiten alle 3 Regler dauernd mit 

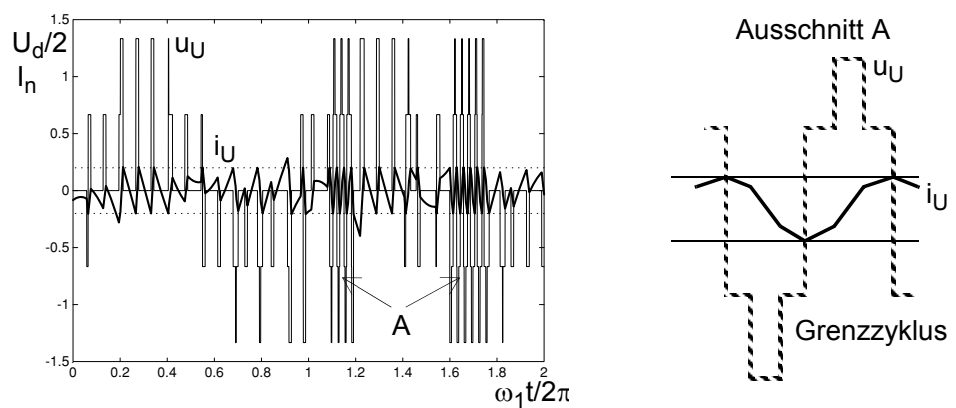

Bild 10.16. Grenzzyklen bei niedriger Aussteuerung, Beispiel mit $\hat{e}_{U / V / W}=0.25 U_{B}$

ungefähr derselben Schaltfrequenz. Sie können so zeitweise in einen sogenannten Grenzzyklus geraten, bei dem die Brückenzweige genau abwechselnd schalten, und zwar so, dass sie nie mehr alle gleichzeitig dieselbe Stellung haben und deshalb die Ausgangspannungen nie zu null werden. In Bild 10.16 ist dieses Phänomen hervorgehoben. Die kurzzeitigen Mittelwerte der eingestellten Phasenspannungen entsprechen dabei durchaus den geforderten Werten nahe bei null. Jedoch tritt innerhalb der Grenzzyklen eine sehr grosse momentane Schaltfrequenz auf. Unter der Annahme, dass der Grenzzyklus gemäss dem Ausschnitt in Bild 10.16 rechts mit äquidistanten Schaltflanken durchlaufen wird, kann die entsprechende momentane Schaltfrequenz eines Schalters auf ähnliche Art, wie es in Kapitel 10.1 beschrieben wurde, bestimmt werden. Das Ergebnis dieser Rechnung lautet:

$$
f_{\text {inst, } \max }=\frac{2 U_{d}}{9 I_{\delta} L_{k}}
$$

$f_{\text {inst,max }}$ ist gleichzeitig die grösste momentane Schaltfrequenz, die überhaupt auftreten kann. Die Grenzzyklen stellen eine Instabilität in der Stromregelung dar. Sowohl die Anzahl Zyklen in einer Folge als auch die Häufigkeit solcher Folgen sind stochastisch. Generell wird ihr Auftreten jedoch mit zunehmender Aussteuerung des Stromrichters seltener. Ab ca. $M>0.4$ verschwinden die Grenzzyklen ganz. Sie bestimmen die mittlere Schaltfrequenz deshalb vor allem im niedrigen Aussteuerbereich massgeblich.

Die unnötig grosse Schaltfrequenz bei niedriger Aussteuerung ist in der Praxis nicht tolerierbar. Zu ihrer Reduktion können mehrere Massnahmen getroffen werden. Zwei davon sollen hier kurz Erwähnung finden:

- Die Zweipunktregler werden jeweils im Bereich ihrer maximalen Aussteuerung während einer Sechstelperiode festgehalten. Damit sind jederzeit nur zwei Regler aktiv. Bild 10.17 zeigt, dass auf diese Weise die Grenzzyklen vermieden werden. Dagegen treten zusätzliche Toleranzbandverletzungen auf, was einen grösseren Effektivwert des Verzerrungsstromes mit sich bringt. Die mittlere Schaltfrequenz geht im Bereich kleiner Aussteuerung deutlich zurück.

- Durch das Einführen einer minimalen Ein- und Ausschaltzeit für die Schalter (Kapitel 19.3) wird die momentane Schaltfrequenz auf einen definierten Maximalwert begrenzt. Liegt dieser unterhalb $f_{\text {inst,max }}$, so werden die Grenzzyklen verhindert. Das Blockieren 

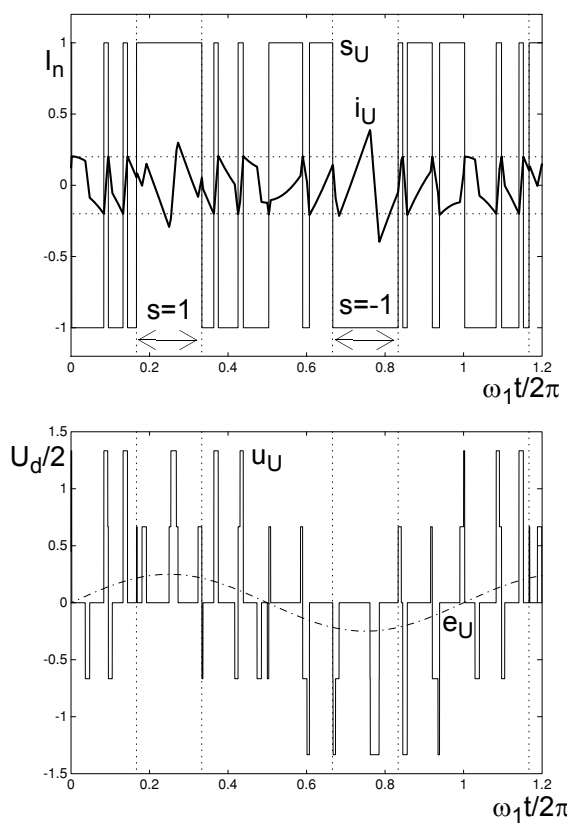

\section{Bild 10.17.}

Zeitliche Verläufe in einer Phase mit sektorweise festgehaltenem Zweipunktregler, Beispiel mit $\hat{e}_{U / V / W}=0.25 U_{B}$

der Schalter während einer gewissen Zeit führt dann jedoch auch zu zusätzlichen Toleranzbandverletzungen und zu einem grösseren Effektivwert des Verzerrungsstromes.

Neben den Phasenstromreglern existieren andere direkte Stromregelverfahren, die vom Prinzip her als dreiphasige Verfahren ausgelegt sind. Sie zeigen die erwähnten Probleme deshalb nicht. Diese Verfahren werden ausführlich in Kapitel 11 behandelt.

\subsubsection{Phasenströme und Zwischenkreisstrom}

Bild 10.18 zeigt die Verläufe und die Spektra eines Phasenstromes mit zwei verschiedenen Toleranzbändern. Der dreiphasige Zweipunktregler ergibt wie der einphasige asynchrone Pulsmuster, so dass die Spektra als verdichtete Linienspektra dargestellt sind. Abgesehen davon, dass nicht nur Harmonische auftreten, ist die Amplitudenverteilung ähnlich wie diejenige des Dreipunktreglers. Die dominanten Komponenten sind in einem Band zu finden, das ungefähr bis zur dreifachen mittleren Schaltfrequenz reicht.

Zwischenkreisstrom: Das Beispiel in Bild 10.19 zeigt, dass auch das Spektrum des Zwischenkreisstromes eine sehr breite und dichte Amplitudenverteilung aufweist. Die dominanten Komponenten treten im Bereich der zweifachen bis dreifachen Schaltfrequenz auf.

\subsubsection{Drehmomentwelligkeit}

Zur Bestimmung der Drehmomentwelligkeit (Kapitel 5.2.1.4) in einem mit Phasenstromreglern betriebenen Antrieb geht man am besten von der Zeigergleichung (10.27) aus: 

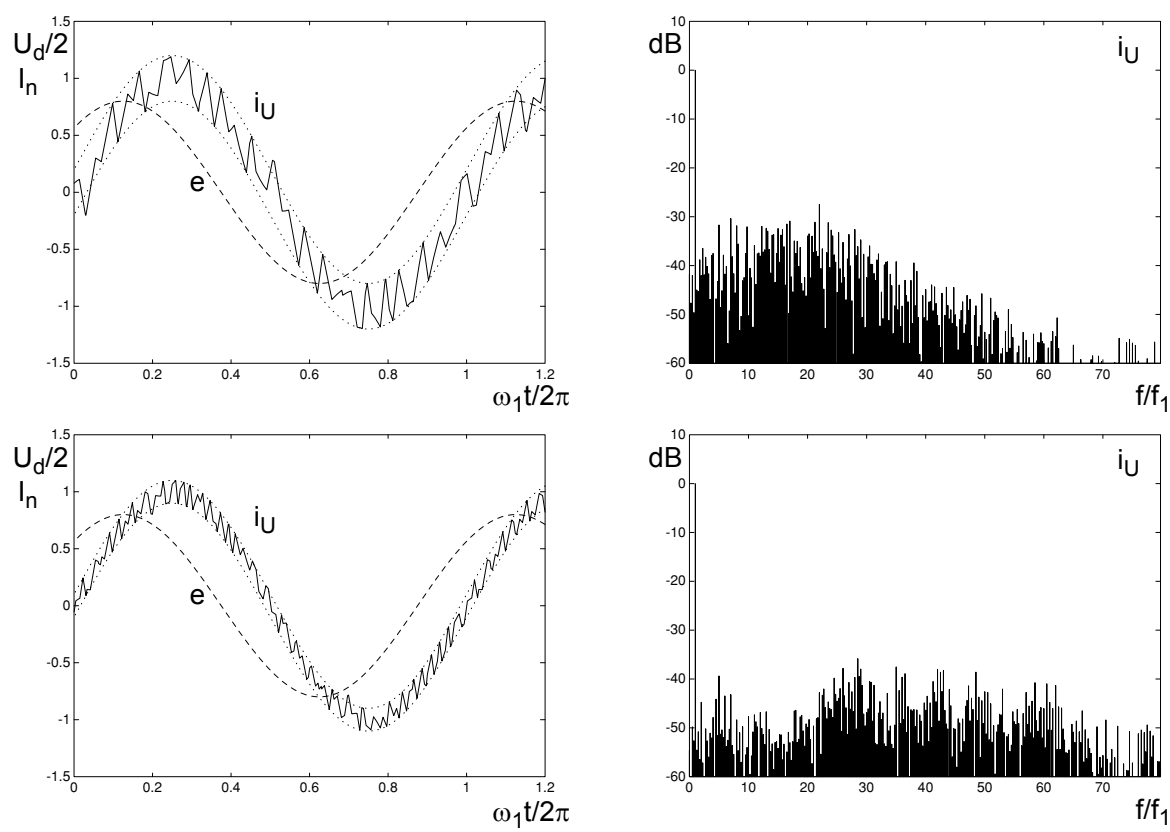

Bild 10.18. Verläufe und Spektra des Phasenstromes in der Phase $U$ für verschiedene Toleranzbänder, $\hat{e}=0.8 U_{d}, M=0.95, I_{n}=0.2 I_{B}, 0 \mathrm{~dB}: I_{n}$, Spektra berechnet über $4 T_{1}$, oben: $q \approx 10.8, I_{\delta}=0.4 I_{n}$, unten: $q \approx 21.7, I_{\delta}=0.2 I_{n}$
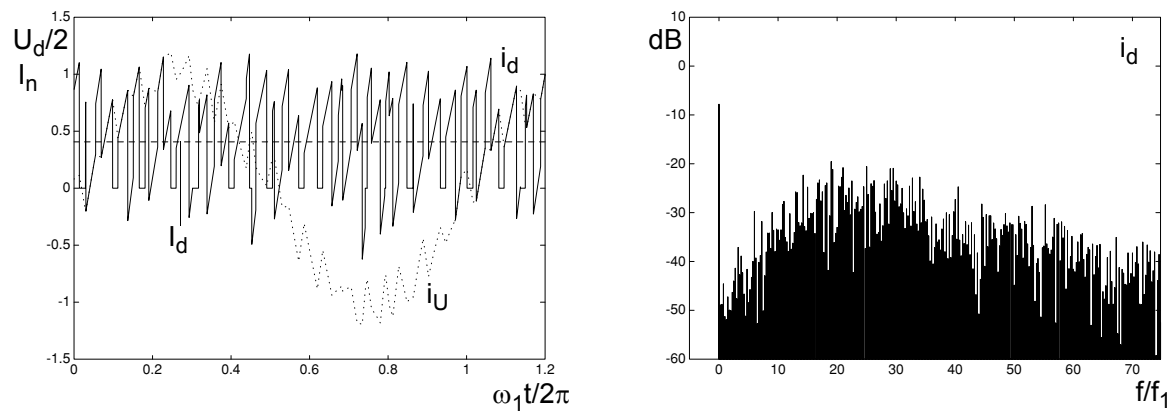

Bild 10.19. Links: Phasenstrom sowie Verlauf und Mittelwert des Zwischenkreisstromes für das Beispiel in Bild 10.18 oben, rechts: Spektrum des Zwischenkreisstromes, 0dB: $I_{n}$

$$
M_{e l, V Z}=\frac{3}{2} \frac{1}{\omega_{1}} \operatorname{Re}\left(e i_{-S t r, V Z}\right)
$$

Der Zeiger $\underline{i}_{S t r, V Z}$ wird durch die Verzerrungsströme in den 3 Phasen gebildet. Jeder Phasenstromregler beschränkt die Lage von $\underline{i}_{S t r}, V Z$ auf einen Streifen von $\pm \pm I_{\delta} / 2$ bezüglich der entsprechenden Projektionsachse $a, b$ oder $c$. Solange alle Phasenströme ihre Toleranzbänder einhalten, liegt $\underline{\underline{i}}_{S t r, V Z}$ innerhalb des in Bild 10.20 dargestellten inneren Sechsecks. 


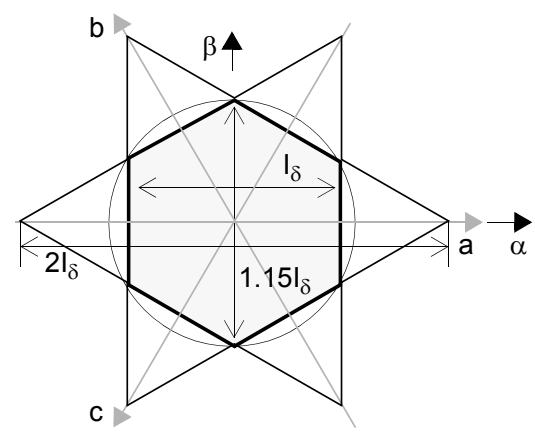

Bild 10.20.

Toleranzfläche des Stromfehlerzeigers $\underline{i}_{S t r, V Z}$ in der $\alpha \beta$-Ebene für Zweipunktregler

Wenn berücksichtigt wird, dass sporadisch einer der Phasenströme sein Toleranzband verletzen kann, so erweitert sich diese Fläche zum eingezeichneten Stern. Ausserhalb des Sterns kommt $\underline{i}_{S t r}, V Z$ im stationären Betrieb nicht zu liegen. Der Betrag des Zeigers $\underline{i}_{S t r}, V Z$ kann deshalb im regulären Bereich $1.15 I_{\delta} / 2$ und bei Toleranzbandverletzungen bis $2 I_{\delta} / 2$ erreichen. Seine Wirkung auf das Drehmoment hängt von der momentanen Lage des mit $\omega_{1}$ rotierenden Gegenspannungszeigers $\underline{e}$ ab. Da das Steuerverfahren asynchron ist, kommt es sporadisch vor, dass die Zeiger $\underline{e}$ und $\underline{i}_{S t r}, V Z$ vorübergehend gleichgerichtet sind. Es ist dann der gesamte Verzerrungsstrom momentbildend und $M_{e l, V Z}$ ist proportional zum Produkt der Beträge der beiden Zeiger. Wenn gleichzeitig $\underline{i}_{S t r}, V Z$ gerade seinen Ma-


zenwerte zu definieren: $M_{e l, V Z, 1}$ kann sich einstellen, wenn die Phasenströme regulär in ihren Toleranzbändern verlaufen und $\hat{M}_{e l, V Z, 2}$ kann auftreten, wenn einer der Phasenströme sein Toleranzband verletzt:

$$
\begin{aligned}
& \hat{M}_{e l, V Z, 1}=\frac{3}{2} \frac{1}{\omega_{1}} \hat{e}_{U / V / W} \frac{2}{\sqrt{3}} \frac{I_{\delta}}{2}=\frac{\sqrt{3}}{2} \frac{\hat{e}_{U / V / W} I_{\delta}}{\omega_{1}}, \\
& \hat{M}_{e l, V Z, 2}=\frac{3}{2} \frac{1}{\omega_{1}} \hat{e}_{U / V / W} \frac{2 I_{\delta}}{2}=\frac{3}{2} \frac{\hat{e}_{U / V / W} I_{\delta}}{\omega_{1}}
\end{aligned}
$$

Unter der Annahme, dass der Antrieb stets mit vollem Fluss betrieben wird, verläuft die Amplitude der Gegenspannung proportional zu $\omega_{1}$. Damit wirkt der Quotient $\hat{e}_{U / V / W} / \omega_{1}$. als Maschinenkonstante. Der Spitzenwert der Drehmomentwelligkeit im Leerlauf ist damit allein durch das Toleranzband für die Phasenströme bestimmt und unabhängig von der Drehzahl.

Bild 10.21 zeigt ein Beispiel für die Verläufe der Phasenströme und der Drehmomentwelligkeit im Leerlauf $\left(i_{U / V / W, v=1}=0\right)$. Die Phasenströme bestehen dabei nur aus ihren Verzerrungsanteilen. Im Bild rechts zeigt sich, dass $M_{e l, V Z}$ grösstenteils im durch $\hat{M}_{e l, V Z, 1}$ begrenzten Band verläuft. Da nicht jederzeit der gesamte Stromfehler momentbildend wirkt, wird dieser Wert nur selten erreicht. Er kann aber auch überschritten werden, nämlich dann, wenn einer der Phasenströme sein Toleranzband verlässt und dieser Stromfehler auch gerade stark auf das Drehmoment wirkt. Der Maximalwert beträgt aber höchstens $\hat{M}_{e l, V Z, 2}$. 



Bild 10.21. Phasenströme und Drehmomentwelligkeit im Leerlauf, $\hat{e}_{U / V / W}=0.8 U_{B}, I_{n}=0.2 I_{B}$, $I_{\delta}=0.4 I_{n}, \omega_{1}=0.8 \omega_{B}$

Literatur zu Kapitel 10.3: [Brod1], [Bos2], [Kaz1], [Mal1], [Wüe2]

\subsection{Phasenstromregler mit konstanter mittlerer Schaltfrequenz}

Die besprochenen Regelverfahren weisen alle eine stark betriebspunktabhängige mittlere Schaltfrequenz auf. In der Praxis ergibt dies Probleme: wird das Toleranzband so gelegt, dass der Stromrichter auch im ungünstigsten Betriebspunkt nicht zu schnell geschaltet wird, so ergibt sich ein entsprechend grosser Verzerrungsstrom. Andererseits geht dann in einem anderen Betriebspunkt die Schaltfrequenz unnötig stark zurück. Zur besseren Ausnutzung des Stromrichters im ganzen Betriebsbereich bietet sich deshalb die Lösung nach Bild 10.22 an. Das Toleranzband ist variabel. Es wird über einen Regelkreis so eingestellt, dass die mittlere Schaltfrequenz konstant bleibt. Die Zeitkonstante dieser Regelung ist deutlich grösser als eine Grundperiode, so dass die momentane Schaltfrequenz weiterhin in der ursprünglichen Art und Weise variieren kann. Die Charakteristik der Stromspektra bleibt erhalten. Jedoch bewirkt die Variation des Toleranzbandes, dass wie bei den spannungssteuernden Verfahren der Effektivwert des Verzerrungsstromes jetzt mit der Aussteuerung des Stromrichters ändert.

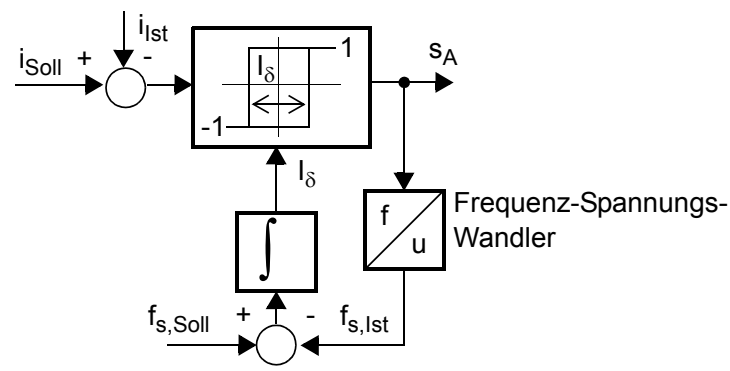

Bild 10.22.

Toleranzband-Regelkreis für eine konstante mittlere Schaltfrequenz 


\subsection{Diskrete Schaltzustandsänderung}

Eine spezielle Variante des Zweipunktreglers stellt das Verfahren der diskreten Schaltzustandsänderung dar. Wie in Bild 10.23 ersichtlich, arbeitet dieser Stromregler abgetastet und der Komparator weist dafür keine Hysterese auf. Die Stellung seines Ausgangs ist nur durch das Vorzeichen der momentanen Differenz zwischen Soll- und Istwert des Stromes definiert und kann in jedem Abtastzeitpunkt wechseln. Damit sind die Schaltfrequenz und der Verzerrungsstrom nicht mehr durch ein Toleranzband, sondern in erster Linie durch die Abtastfrequenz bestimmt. Bild 10.24 zeigt ein Beispiel des Stromverlaufes bei der Halbbrücke. Zum besseren Verständnis der Funktionsweise stellt Bild 10.25 zudem die Verhältnisse bei sehr kleinem und sehr grossem Momentanwert der Gegenspannung $e$ und damit der Modulationsfunktion dar. Die grösste momentane Schaltfrequenz von $f_{\text {Tast }} / 2$ tritt dann auf, wenn in jedem Abtastintervall ein Schaltvorgang stattfindet. Dies ist der Fall, wenn die Modulationsfunktion klein ist. In beiden Schalterstellungen ist dann die Spannung über der Induktivität ungefähr gleich gross, so dass sich der Phasenstrom in beiden Fällen gleich schnell ändert. Gegen den Spitzenwert von $e$ hin nimmt die momentane Schaltfrequenz ab und die Stromverzerrung wird ungefähr doppelt so gross. Dies
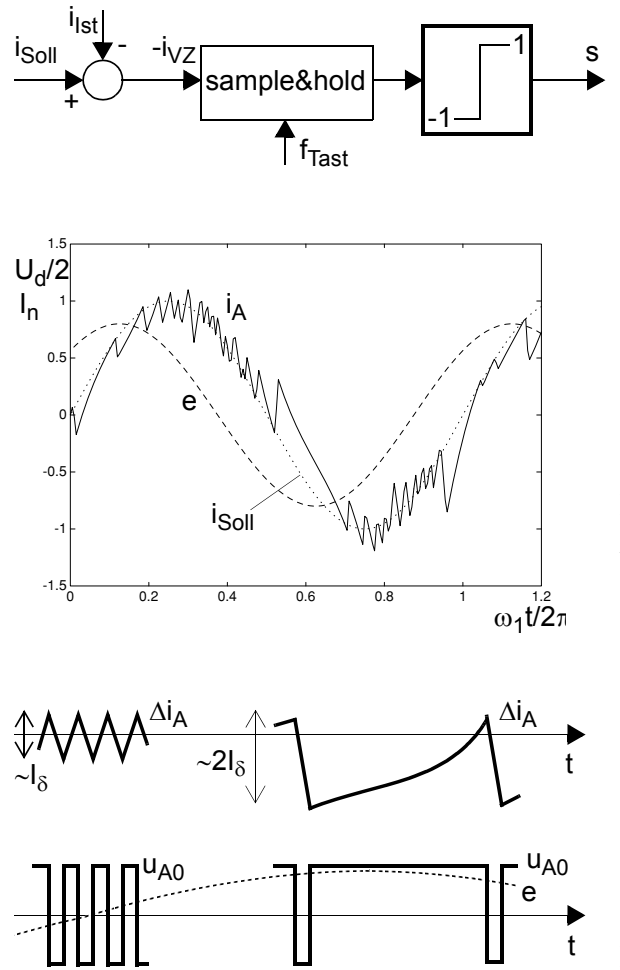

Abtastzeitpunkte
Bild 10.23.

Prinzip des Modulators mit diskreter Schaltzustandsänderung

Bild 10.24.

Zeitlicher Verlauf des Phasenstromes bei der Halbbrücke mit diskreter Schaltzustandsänderung, Beispiel mit $\hat{e}=0.8$

Bild 10.25.

Verzerrungsstrom bei unterschiedlicher Aussteuerung, $\hat{\imath}_{\text {soll }}=0$ 
kommt daher, dass die gegenüber vorher nahezu zweimal so grosse Spannungsdifferenz zwischen Ausgangs- und Gegenspannung in der einen Schalterstellung den Strom innerhalb eines Abtastintervalles auch doppelt so stark ändern lässt. Die Rückkehr des Stromes zu seinem Sollwert dauert dagegen deutlich länger. Weiter ist vor allem in Bild $10.24 \mathrm{zu}$ erkennen, dass der tatsächliche Stromverlauf nicht gleichmässig in positiver und negativer Richtung vom Sollwert abweicht, was zu einem merklichen Fehler in der Grundschwingungsamplitude sowie zu einem DC-Anteil führen kann.

Die mittlere Schaltfrequenz nimmt wie beim Zweipunktregler mit zunehmendem Modulationsgrad ab, wobei die Abnahme wegen der grösser werdenden Stromverzerrung etwas stärker ausfällt.

Der Modulator mit diskreter Schaltzustandsänderung für dreiphasige Schaltungen besteht aus 3 unabhängigen Reglern nach Bild 10.23 für die 3 Phasenströme. Seine mit Hilfe von Simulationen bestimmten Kennlinien für zwei verschiedene Abtastfrequenzen sind in Bild 10.26 dargestellt. Die Schaltfrequenzen können leicht mit denjenigen des dreiphasigen Zweipunktreglers in Bild 10.15 verglichen werden, da die Effektivwerte der Verzerrungsströme bei kleiner Aussteuerung ungefähr übereinstimmen. Es stellt sich heraus, dass auch der dreiphasige Modulator bei kleiner Aussteuerung die maximale Schaltfrequenz von $f_{\text {Tast }} / 2$ erreicht, was bedeutet, dass alle 3 Regler in jedem Abtastzeitpunkt schalten. Im Vergleich mit dem Zweipunktregler ist die mittlere Schaltfrequenz damit ungefähr doppelt so gross. Mit zunehmender Aussteuerung nimmt sie dann stetig ab und erreicht erst bei Vollaussteuerung dieselbe Grössenordnung wie beim Zweipunktregler. Der Verzerrungsstrom steigt dagegen bis zur Vollaussteuerung $(M=1.155)$ auf rund das doppelte an. Darüber wird dieser Anstieg sehr steil, wobei der bei Übersteuerung entstehende Grundschwingungsfehler dem Verzerrungsstrom zugerechnet ist.
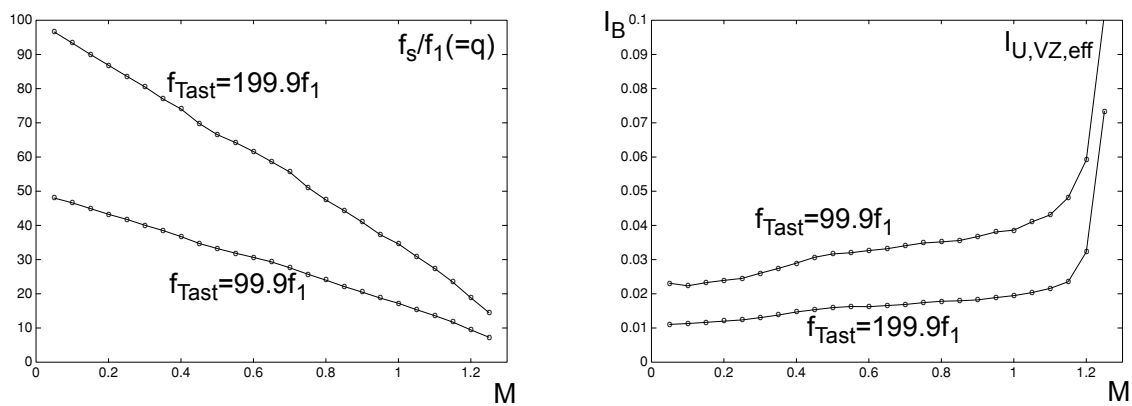

Bild 10.26. Mittlere Schaltfrequenz und Verzerrungsstrom in einer Phase bei der dreiphasigen Brücke mit diskreter Schaltzustandsänderung für zwei verschiedene Abtastfrequenzen (o=simulierte Betriebspunkte)

Das Verfahren der diskreten Schaltzustandsänderung ergibt damit offensichtlich gegenüber dem gewöhnlichen Zweipunktregler deutlich schlechtere Resultate. Trotzdem sprechen vor allem bei Anwendungen kleiner Leistung ohne grosse Ansprüche an den Wirkungsgrad zwei Gründe für eine Verwendung: 
- Das feste Taktintervall garantiert unabhängig vom Betriebspunkt und ohne zusätzlichen Aufwand minimale Ein- und Ausschaltzeiten für die Schalter (Kapitel 19.3).

- Alle Schalthandlungen finden zu definierten Zeitpunkten statt. Damit kann das häufige Problem der Störung der Messwerterfassung im System durch Schalthandlungen im Leistungsteil umgangen werden. Die Messgrössen werden einfach während der ruhigen Phase zwischen den Abtastzeitpunkten des Modulators erfasst.

Neben der beschriebenen Grundausführung des Modulators mit diskreter Schaltzustandsänderung, existiert auch eine Variante, die dem Zweipunktregler ähnlicher ist: der Komparator wird dabei mit einer Hysterese versehen, was der Vorgabe eines Toleranzbandes für den Phasenstrom entspricht. Dieses muss so gross sein, dass sich der Phasenstrom nicht mehr innerhalb eines Abtastintervalles von der einen Bandgrenze zur anderen bewegen kann. Damit wird es massgebend für den Charakter des Pulsmusters.

Literatur zu Kapitel 10.5: [Boe1], [Dzi1], [Rot1] 


\section{Drehzeigerorientierte Stromregelung}

Die in Kapitel 10 vorgestellten Verfahren zur direkten Stromregelung sind alle vom Prinzip her einphasig. Sie lassen sich wie gezeigt wurde auch in dreiphasigen Stromrichtern einsetzen. Da sie jedoch nicht in der Lage sind, die gegenseitige Beeinflussung der einzelnen Phasen in geeigneter Weise zu berücksichtigen, ergeben sie keine optimalen Resultate. Die Schaltfrequenz fällt für einen gegebenen Effektivwert der Phasenströme vor allem bei niedriger Aussteuerung des Stromrichters zu hoch aus. In diesem Kapitel werden Verfahren vorgestellt, welche die Kopplung der 3 Phasen berücksichtigen. Sie arbeiten in der Drehzeigerebene. Jedes von ihnen steht stellvertretend für eine Gruppe von ähnlichen Verfahren, die jeweils gleichartige Resultate liefern.

\subsection{Prinzip der drehzeigerorientierten Stromregelung}

Bild 11.1 zeigt das Prinzip der drehzeigerorientierten Stromregelverfahren. Die Phasenströme werden in den Drehzeiger $\underline{i}_{S t r}$ mit den Komponenten $i_{S t r, \alpha}$ und $i_{S t r, \beta}$ transformiert (Anhang B.1.2). Der durch die Stromsollwerte definierte Sollwertzeiger $i_{\text {Soll }}$ bewegt sich im stationären Zustand auf einem Kreis mit der Kreisfrequenz $\omega_{1}$. Die Aufgabe der Regelung ist es, den Stromzeiger möglichst gut dem Sollwertzeiger nachzuführen. Dies geschieht dadurch, dass aufgrund des Stromfehlers $\underline{i}_{S t r, V Z}$ der Schaltzustand des Stromrich-
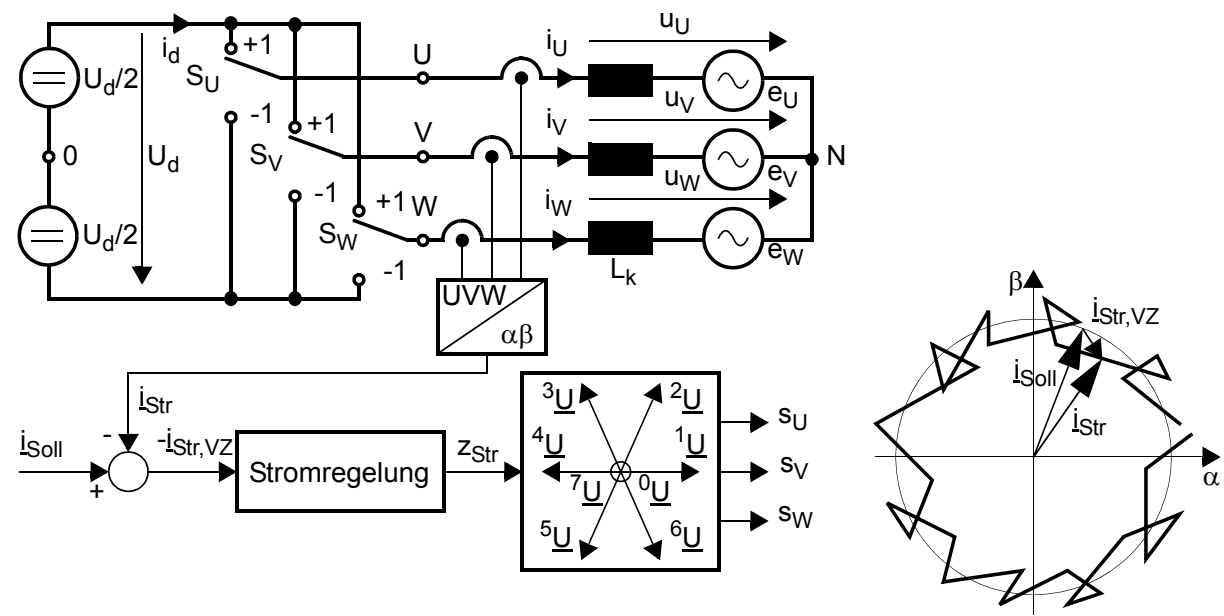

Bild 11.1. Prinzip der drehzeigerorientierten Stromregelung 
ters $z_{S t r}$ bzw. der Spannungszeiger $\underline{u}_{S t r}$ bestimmt wird. Es stehen die diskreten Zustände ${ }^{0} Z$ bis ${ }^{7} Z$ zur Verfügung. Sie entsprechen je einem Tripel von Schaltzuständen für den Stromrichter und bilden die Spannungszeiger ${ }^{0} \underline{U}$ bis ${ }^{7} \underline{U}$ (Kapitel 3.3.2.3). Der Stromfehler $\underline{i}_{S t r, V Z}$ ist derjenige Zeiger, der aus den 3 Phasenstromfehlern gebildet wird. Er bewirkt den in Bild 11.1 skizzierten gezackten Verlauf von $\underline{\underline{i}}_{S t r}$ um den Sollwertzeiger herum und ist identisch mit dem Verzerrungsanteil von $\underline{i}_{S t r}$. Dabei werden, wie bei den Phasenstromreglern, allfällige Abweichungen in den Grundschwingungen als Verzerrungen behandelt. Die Lastseite des Stromrichters nach Bild 11.1 ergibt in der Drehzeigerdarstellung die Ersatzschaltung in Bild 11.2. Die Grundlage für alle drehzeigerorientierten Stromregelverfahren ist die Differentialgleichung (11.1), welche die Bewegung des Stromzeigers in Funktion der angelegten Spannungen beschreibt:

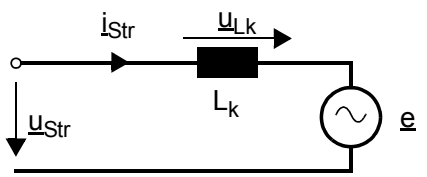

$$
\frac{d i_{-S t r}}{d t}=\frac{1}{L_{k}}{ }_{L}{ }_{L k}=\frac{1}{L_{k}}\left(\underline{u}_{S t r}-\underline{e}\right)
$$

Bild 11.2.

Zeigerbezogenes Ersatzschaltbild des Lastkreises

Wie in Bild 11.3 verdeutlicht ist, bestimmt der Spannungszeiger $\underline{u}_{L k}$ über der Induktivität die Bewegung des Stromzeigers. Sie erfolgt in seiner Richtung mit einer zu seiner Länge proportionalen Geschwindigkeit. Sind die Stromrichterspannung und die Gegenspannung identisch, so ist $\underline{u}_{L k}=0$ und der Stromzeiger steht still.

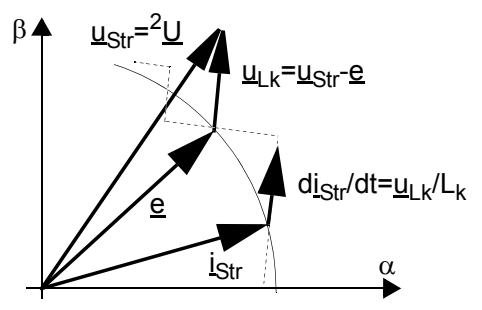

Bild 11.3.

Bewegung des Stromdrehzeigers, Beispiel mit $\underline{u}_{S-}$ $t r={ }^{2} \underline{U}$

\subsection{Stromzeiger-Komponentenregelung}

Eine Möglichkeit zur Führung des Stromzeigers besteht darin, den Stromfehler innerhalb einer vorgegebenen Toleranzfläche zu halten. Diese Fläche bewegt sich zusammen mit dem Sollwertzeiger, so dass dessen Spitze immer in ihrem Zentrum steht. Jedesmal wenn der Stromzeiger den Rand der Fläche berührt, muss ein neuer Schaltzustand eingestellt werden, der ihn wieder ins Innere zurückbewegt. Eine solche Regelung lässt sich sehr einfach realisieren, indem man wie in Bild 11.4 die Toleranzfläche als Quadrat wählt. Die $\alpha$ - 


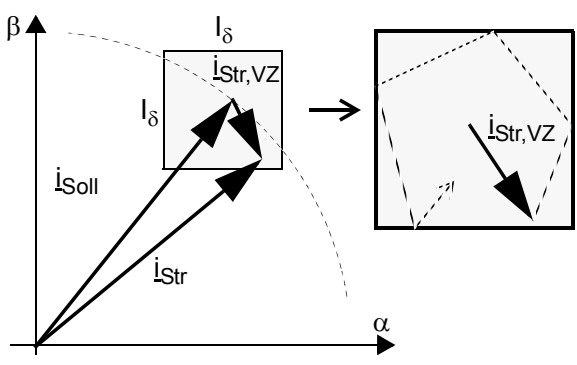

Bild 11.4.

Quadratische Toleranzfläche für den Stromdrehzeiger

und die $\beta$ - Komponente des Stromzeigers müssen dann unabhängig voneinander ein Toleranzband der Breite $I_{\delta}$ einhalten.

Zur Bestimmung der Schaltzeitpunkte können zwei getrennte Regler für die beiden Komponenten eingesetzt werden. Das Prinzip einer solchen Regelung ist in Bild 11.5 dargestellt. Für die $\alpha$-Komponente wird ein Vierstufen- und für die $\beta$-Komponente ein Dreistufenkomparator eingesetzt. Die sich ergebenden 12 Ausgangskombinationen der beiden Komparatoren ermöglichen die gezielte Auswahl des Schaltzustandes mit Hilfe einer Schalttabelle. Es ist zu beachten, dass die Stromfehler, gebildet aus den Differenzen zwischen Soll- und Istwerten, vor der Auswertung in den Komparatoren invertiert werden.
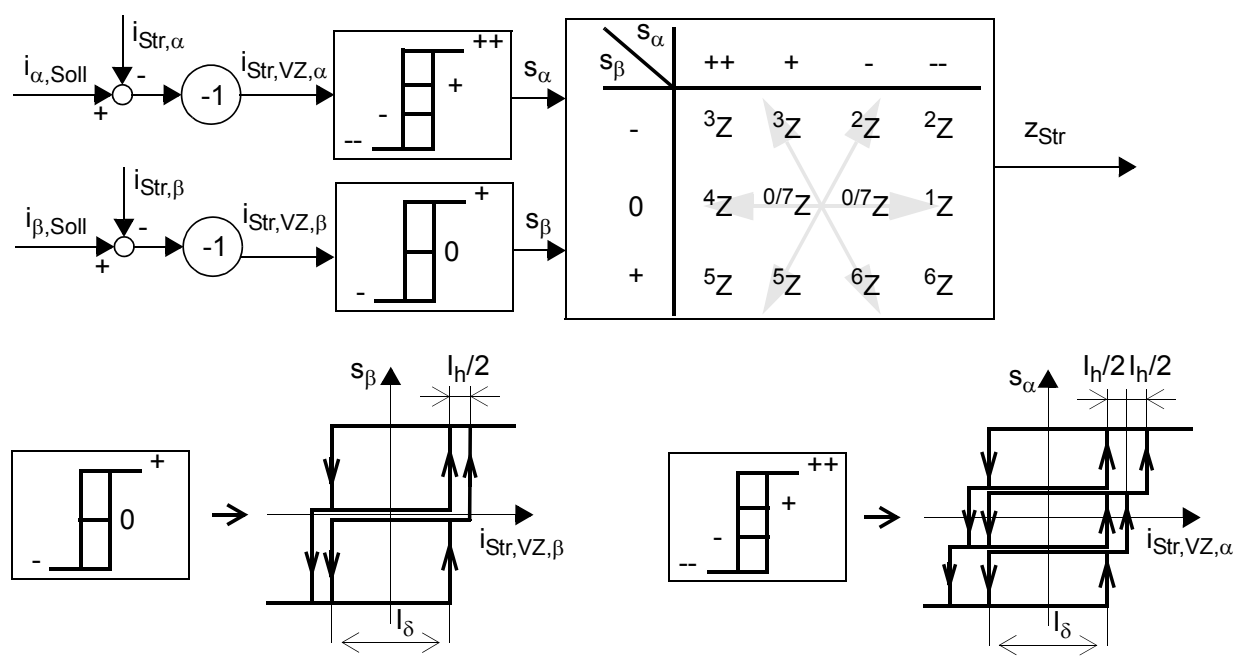

Bild 11.5. oben: Prinzip der Stromzeiger-Komponentenregelung, unten links: Dreistufenkomparator, unten rechts: Vierstufenkomparator

Die Funktionsweise des Verfahrens kann anhand der Beispiele in Bild 11.6 nachvollzogen werden. Solange sich der Stromfehler innerhalb der normalen Toleranzfläche mit der Seitenlänge $I_{\delta}$ bewegt, laufen beide Komparatoren in einer ihrer zwei bzw. 3 inneren Hystereseschleifen. Die Schaltsequenz A in Bild 11.6 zeigt einen solchen Fall. Es werden nur die Felder in der rechten oberen Ecke der Schalttabelle verwendet. Sie entsprechen den beiden untersten Hystereseschleifen für beide Komparatoren. Da in diesem Beispiel der 

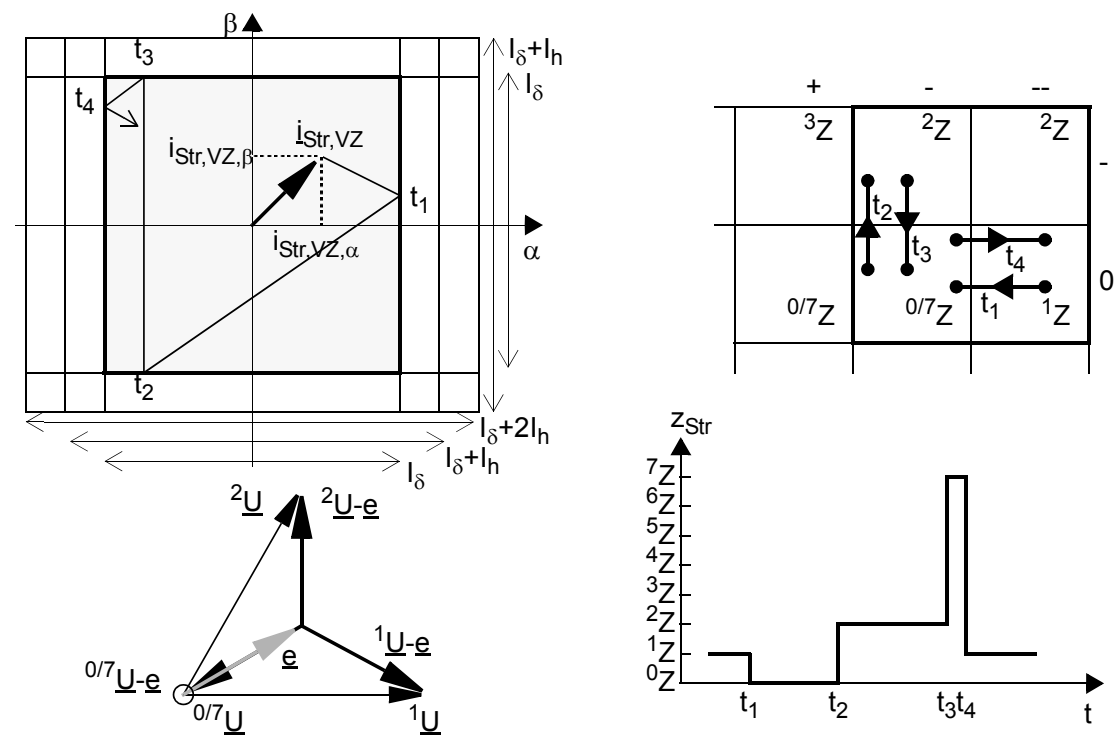

Schaltsequenz A
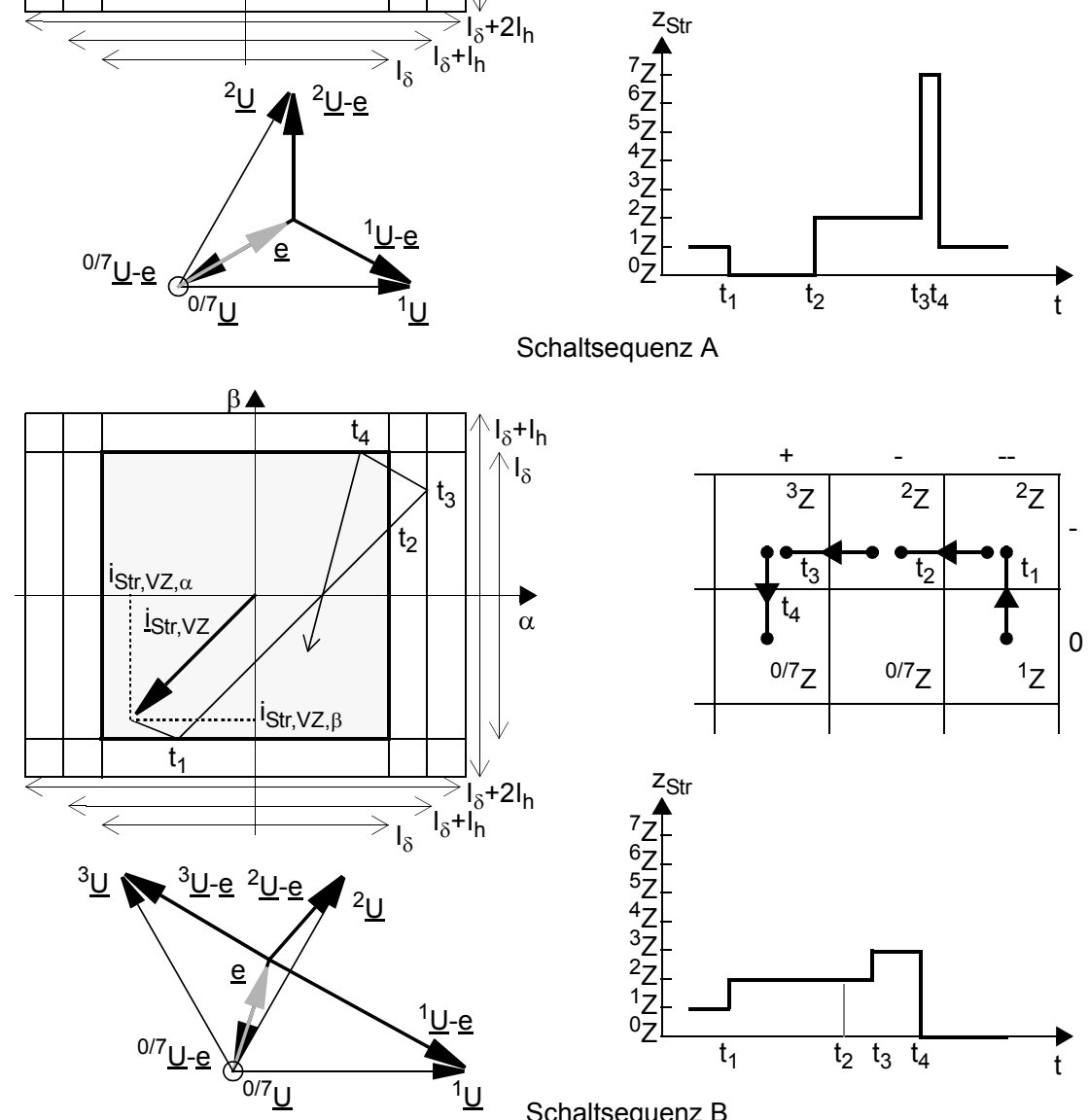

Schaltsequenz B

Bild 11.6. Beispiele von Schaltsequenzen,

Schaltsequenz A: keine Verletzung der inneren Toleranzfläche,

Schaltsequenz B: einmalige Verletzung der inneren Toleranzfläche

Gegenspannungszeiger im Sektor $S 1$ zwischen ${ }^{1} \underline{U}$ und ${ }^{2} \underline{U}$ steht, können beide Komponenten des Stromzeigers, jeweils für sich allein betrachtet, mit den Schaltzuständen dieser 4 Felder in negative oder in positive Richtung bewegt werden. Jedesmal, wenn der Strom- 
fehler den Rand der Toleranzfläche erreicht, wird ein anderer Schaltzustand aktiviert. Die Auswahl erfolgt so, dass in erster Linie diejenige Komponente von $\underline{i}_{S t r}, V Z$ beeinflusst wird, die den Schaltvorgang ausgelöst hat. Die entsprechende Komponente von $\underline{u}_{S t r}$ muss dazu in die entgegengesetzte Richtung verändert werden. Zum Beispiel bei $t_{1}$ muss die $\alpha$-Komponente nach links bewegt werden. Dies erfolgt durch einen Übergang von ${ }^{1} \underline{U}$ auf ${ }^{0} \underline{U}$. Als nächstes ist dann bei $t_{1}$ die $\beta$-Komponente nach oben zu korrigieren. Dazu wird in die Spannung ${ }^{2} \underline{U}$ angelegt. Die Bewegung des Stromzeigers erfolgt dabei stets parallel zu den dargestellten Zeigern der entsprechenden Spannungsdifferenzen.

Speziell zu beachten ist der Wechsel auf einen Nullzustand ${ }^{0} Z,{ }^{7} Z$ entsprechend ${ }^{0} \underline{U},{ }^{7} \underline{U}$. Für die Funktion der Steuerung ist es grundsätzlich gleichgültig, welcher von beiden gewählt wird. Im Hinblick auf eine möglichst kleine Schaltfrequenz wird jedoch von ${ }^{1} Z,{ }^{3} Z$ oder ${ }^{5} Z$ kommend ${ }^{0} Z$ eingestellt, und ausgehend von ${ }^{2} Z,{ }^{4} Z$ oder ${ }^{6} Z$ der komplementäre Zustand ${ }^{7} Z$. Auf diese Weise muss jeweils nur ein Brückenzweig umgeschaltet werden. Im dargestellten Beispiel wird deshalb bei $t_{1}$ auf ${ }^{0} Z$ und bei $t_{3}$ auf ${ }^{7} Z$ geschaltet. Bei der Rückkehr auf einen spannungsbildenden Zustand kann es dagegen vorkommen, dass zwei Umschaltungen erforderlich sind. Dies ist zum Beispiel bei $t_{2}$ der Fall.

Es ist möglich, dass der neu eingestellte Schaltzustand nicht ausreicht, um den Strom in der Toleranzfläche zu halten. In diesem Fall muss der betreffende Komparator in eine andere Hystereseschleife springen. Zur praktischen Realisierung solcher Übergänge ist es notwendig, eine zweite bzw.dritte Schaltschwelle $\left(I_{\delta}+I_{h}\right) / 2$ bzw. $\left(I_{\delta}+2 I_{h}\right) / 2$ einzuführen. Der Mechanismus des Schleifenwechsels kann in der Schaltsequenz B in Bild 11.6 nachvollzogen werden. Er findet bei $t_{3}$ statt, wenn $i_{S t r, V Z, \alpha}$ auf die zweite Schaltschwelle trifft. Es sei nebenbei erwähnt, dass sich der Dreistufenkomparator in Bild 11.5 nur in der Darstellung von demjenigen des Dreipunktreglers in Bild 10.8 unterscheidet. Die Funktion ist identisch.

Übergänge zwischen verschiedenen Schleifen sind immer dann nötig, wenn sich die Lage der Gegenspannung in einen anderen Sektor $S 1$ bis $S 6$ verschiebt. Es werden in der in Bild 11.7 dargestellten Reihenfolge innerhalb einer Grundperiode alle Schleifenkombinationen der beiden Komparatoren durchlaufen. Dazu muss der Stromfehler sechsmal die innere Toleranzfläche verlassen. Zweimal ist die $\beta$-Komponente davon betroffen und viermal die $\alpha$-Komponente, welche je zweimal an die mittlere und an die äussere Schwelle stossen muss. Die bevorzugten Schaltzustände sind jeweils die an den Sektor der Gegenspannung angrenzenden Zeiger.



\section{Bild 11.7.}

Bevorzugte Schaltzustände in Abhängigkeit von der Lage des Gegenspannungszeigers 
Im stationären Betrieb verlaufen die Eingangsgrössen der Komparatoren stetig. In diesem Fall ist sichergestellt, dass zu keiner Zeit einer ihrer Ausgänge um mehr als eine Stufe springt. Demzufolge treten nur Übergänge zwischen Schaltzuständen auf, welche in der Tabelle nebeneinander liegen. Sie sind auch in der Drehzeigerebene benachbart. Auf diese Weise wird die Spannung über der Induktivität stets nur relativ wenig verändert, so dass sich eine möglichst langsame Bewegung des Stromzeigers einstellt. Dies ist im Hinblick auf eine niedrige Schaltfrequenz von grosser Wichtigkeit. Die Nullzustände kommen wegen ihrer zentralen Position in der Schalttabelle sehr häufig zum Zuge. Es ist zu beachten, dass das Verfahren dabei ohne jegliche Information über Betrag und Phase des Gegenspannungszeigers auskommt. Der Wechsel zwischen den Hystereseschleifen der Komparatoren stellt sich von selbst ein.

Spannungen und Ströme: Wie bei den Phasenstromreglern beeinflusst die Aussteuerung des Stromrichters das Verhalten der Komponentenregelung massgeblich. Der Modulationsgrad, der in Zeigerform gemäss (11.2) durch die Gegenspannung und den Stromsollwert gegeben ist, stellt auch hier ein Mass dafür dar.

$$
\left|u_{-S t r, v=1}\right|=M \frac{U_{d}}{2}=\left|e_{-}+j \omega_{1} L_{k-S o l l}\right|
$$

Die Bilder 11.8 und 11.9 zeigen die charakteristischen Resultate des Verfahrens für zwei verschiedene Gegenspannungen. Die Ströme sind auf einen Nennstrom $I_{n}$ des Stromrichters, der $0.2 I_{B}$ entspricht, bezogen. In den einzelnen Bildern lassen sich die folgenden Eigenschaften erkennen:

- Das Verfahren ist wie die Phasenstromregler asynchron.

- Der Regler für die $\beta$-Komponente entspricht einem Dreipunktregler, wie er in Kapitel 10.2 vorgestellt wurde. Beim Vergleich der Schalttabelle (Bild 11.5) mit den in Bild 11.10 herausgehobenen Schaltniveaus für die Komponenten von $\underline{u}_{S t r}$, wird deutlich, dass alle Spannungszustände einer Zeile der Schalttabelle die gleiche $\beta$-Komponente aufweisen. Das bedeutet, das deren Auswahl allein durch den $\beta$-Regler erfolgt. Die Position des $\alpha$-Reglers hat keinen Einfluss darauf. Demzufolge verhält sich $i_{S t r \beta}$ wie der Strom eines einphasigen Stromrichters mit Dreipunktregler und ist unabhängig von der Regelung der $\alpha$-Komponente.

- Für die Regelung der $\alpha$-Komponente stehen gemäss Bild 11.105 Schaltniveaus zur Verfügung. Sie können jedoch nicht unabhängig von der $\beta$-Komponente eingestellt werden, weil die Spalten der Schalttabelle nicht eine einheitliche $\alpha$-Komponente aufweisen. Jeder Übergang, der vom $\beta$-Regler ausgelöst wird, hat gleichzeitig auch eine Veränderung in $\alpha$-Richtung zur Folge. Dies hat auf den zeitlichen Verlauf von $i_{S t r, \alpha}$ grosse Auswirkungen: häufig erreicht der Strom nicht einmal die innere Bandgrenze, weil er vorher bereits durch den $\beta$-Regler zur Umkehr veranlasst wird. Auch der erwartete zyklische Wechsel von einer Komparatorschleife in die nächste ist beeinflusst: die $\alpha$ Komponente verlässt deutlich mehr als viermal pro Grundperiode das innere Toleranzband. Dies bedeutet, dass zusätzliche, willkürlich ausgelöste Schleifenwechsel stattfinden. Und schliesslich treten auch Toleranzbandverletzungen über das äussere Band hin- 

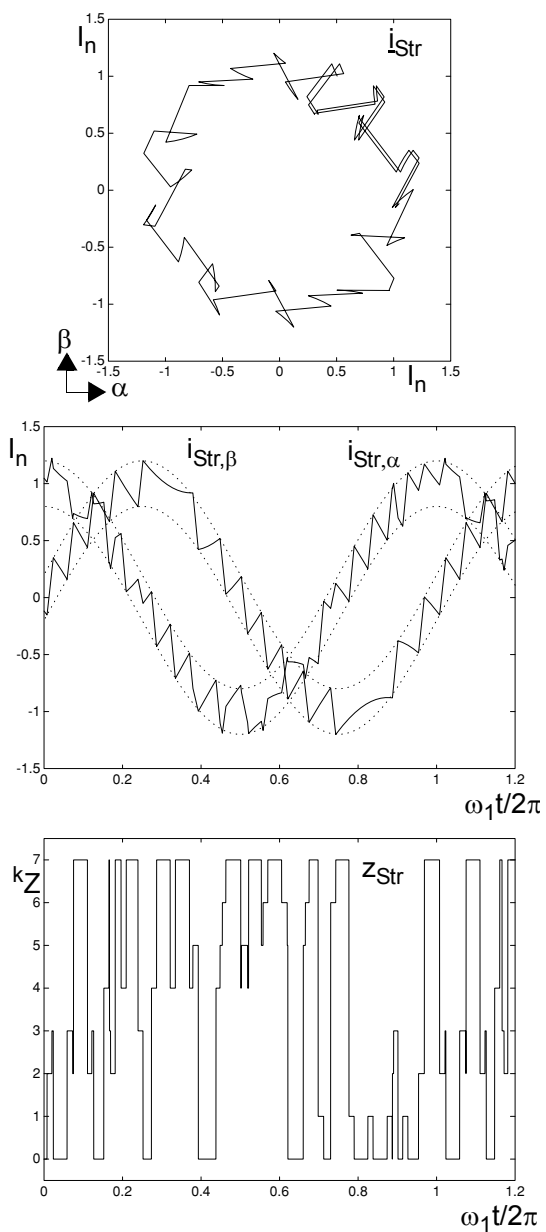

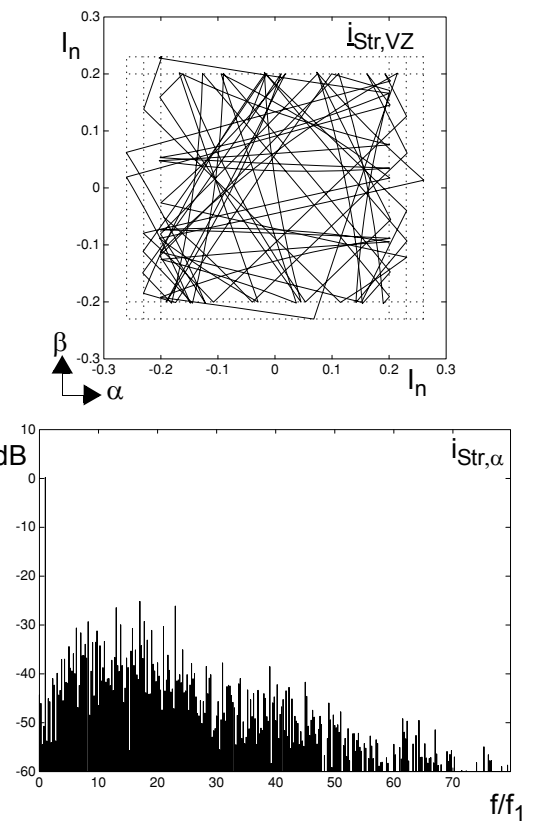

Bild 11.8.

Stromzeiger-Komponentenregelung, $|\underline{e}|=0.2\left(U_{d} / 2\right), M=0.37, I_{n}=0.2 I_{B}, I_{\delta}=0.4 I_{n}$ oben links: Stromdrehzeiger, oben rechts: Drehzeiger des Verzerrungsstromes,

mitte links: Komponenten des Stromzeigers, mitte rechts: Spektrum von $i_{S t r, \alpha}$, berechnet über $4 \mathrm{~T}_{1}, 0 \mathrm{~dB}: I_{n}$, unten: Schaltzustände des Stromrichters

aus auf, sobald die Gegenspannung betragsmässig grösser als der halbe Schaltvektor (d.h. $|\underline{e}|>2 U_{d} / 3$ ) ist. Ein Beispiel dafür ist in Bild 11.9 mit einem Pfeil gekennzeichnet.

- Die dargestellten verdichteten Linienspektra der Komponente $i_{S t r, \alpha}$, die identisch mit dem Phasenstrom $i_{u}$ ist, zeigen die typischen Eigenschaften eines asynchronen Verlaufes. Die Amplitudenverteilungen sind vergleichbar mit denjenigen des dreiphasigen Zweipunktreglers. Die Spektra der beiden anderen Phasenströme sind qualitativ gleich.

- In den Darstellungen der Zustandsabfolge wird deutlich, dass innerhalb einer Periode alle Schaltzustände zur Anwendung gelangen. Im Falle der kleineren Gegenspannung in Bild 11.8 ist dabei die Verweilzeit auf den Nullzuständen deutlich grösser.

Schaltfrequenz und Verzerrungsstrom: Wie bei den Phasenstromreglern ergibt sich bei der Komponentenregelung einerseits eine variable momentane Schaltfrequenz und andererseits auch eine vom eingestellten Betriebspunkt abhängige mittlere Schaltfrequenz für die einzelnen Umschalter. Dabei ist auch hier neben den Toleranzbändern und der Lastin- 

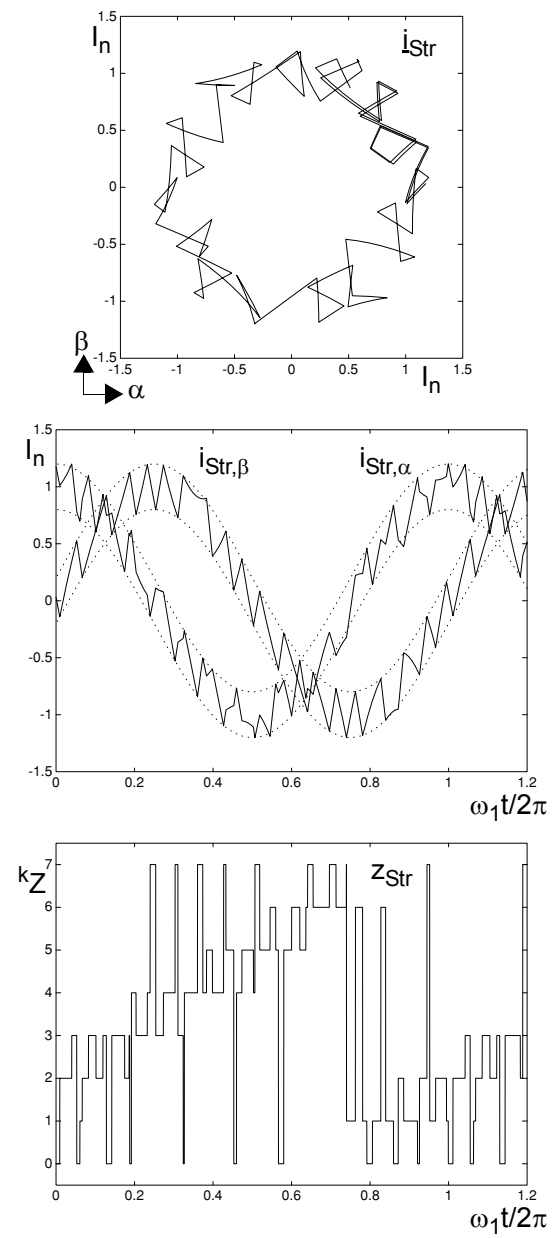
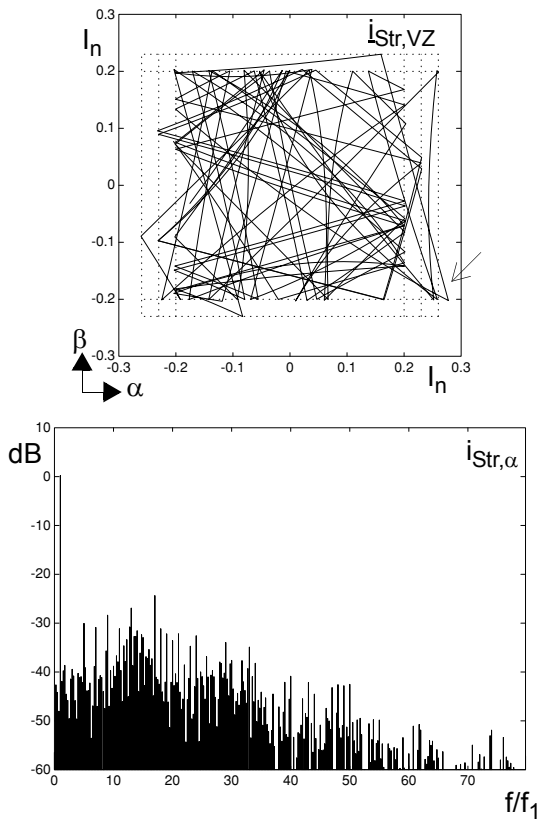

Bild 11.9.

Stromzeiger-Komponentenregelung, $|\underline{e}|=0.8\left(U_{d} / 2\right), M=0.95, I_{n}=0.2 I_{B}, I_{\delta}=0.4 I_{n}$, oben links: Stromdrehzeiger, oben rechts: Drehzeiger des Verzerrungsstromes,

mitte links: Komponenten des Stromzeigers, mitte rechts: Spektrum von $i_{S t r, \alpha}$, berechnet über $4 \mathrm{~T}_{1}, 0 \mathrm{~dB}: I_{n}$, unten: Schaltzustände des Stromrichters

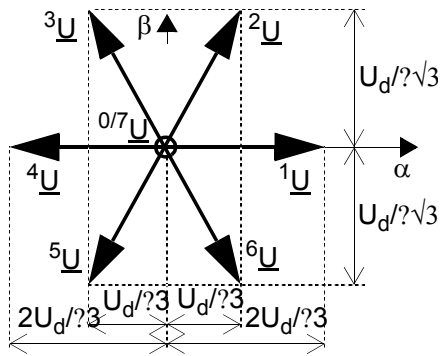

Bild 11.10.

Schaltniveaus der Komponenten von $\underline{u}_{S t r}$

duktivität der Modulationsgrad des Stromrichters der entscheidende Parameter. Es ist nicht möglich, die Schaltfrequenz analytisch zu bestimmen. Die Kennlinien der mittleren Schaltfrequenz und des Effektivwertes des Verzerrungsstromes in Bild 11.11 sind Resultate von Simulationen. Die Diagramme lassen die folgenden Schlüsse zu: 

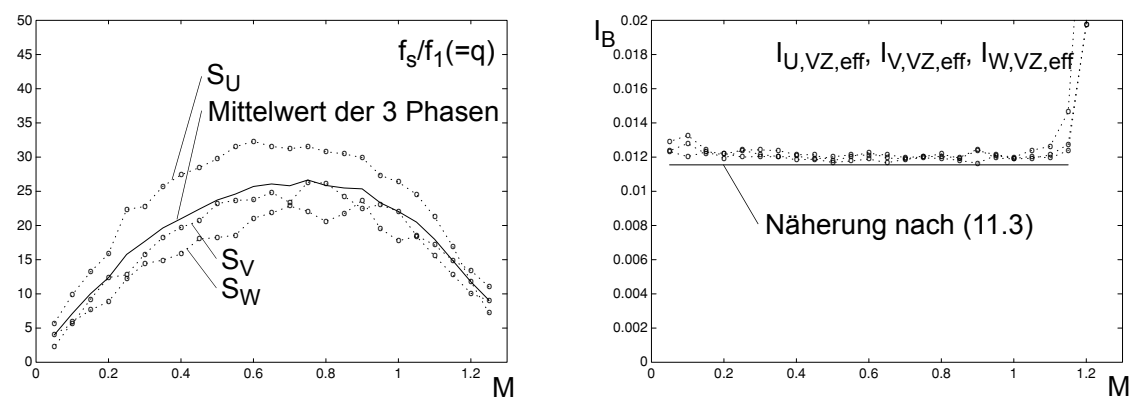

Bild 11.11. Mittlere Schaltfrequenzen und Effektivwert des Verzerrungsstromes in einer Phase für die Stromzeiger-Komponentenregelung, $I_{n}=0.2 I_{B}, I_{\delta}=0.2 I_{n}$ ( $\mathrm{o}=$ simulierte Betriebspunkte)

- Die Schaltfrequenz der 3 Brückenzweige ist nicht gleich gross. Der Umschalter $S_{U}$ führt alle Schalthandlungen des $\alpha$-Reglers allein aus. Obschon dieser wegen der grösseren Anzahl Schaltniveaus weniger häufig schaltet als der $\beta$-Regler, ergibt sich für $S_{U}$ die klar grösste Schaltfrequenz. Sie liegt 20 bis $30 \%$ über dem Mittelwert aller 3 Phasen.

- Von $S_{V}$ und $S_{W}$ würde man aus Gründen der Symmetrie erwarten, dass sie im Mittel gleich häufig schalten. Im dargestellten Beispiel bewirkt aber die Bewegung des Gegenspannungszeigers mit positiver Drehrichtung, dass die Schalfrequenz von $S_{V}$ grösser ist als diejenige von $S_{W}$. Es kann gezeigt werden, dass sich dies bei negativer Drehrichtung umkehrt. Mit zunehmender Schaltzahl, wenn die Bewegung des Gegenspannungszeigers in den Schaltintervallen vernachlässigbar wird, verschwindet diese Asymmetrie und die Schaltfrequenzen von $S_{V}$ und $S_{W}$ werden gleich.

- Das dargestellte Beispiel deutet an, dass sich die genauen Schaltfrequenzen der einzelnen Umschalter nur schwer genau voraussagen lassen. Auch die Mittelwerte über eine grosse Zeitspanne sind relativ grossen Schwankungen unterworfen. Dagegen ist die über alle 3 Phasen gemittelte Schaltfrequenz wesentlich stabiler und gut voraussagbar.

- Die systematische Ausnutzung der Nullzustände bringt im Bereich niedriger Aussteuerung eine entsprechend niedrige Schaltfrequenz. Sie steigt mit zunehmendem Modulationsgrad an und erreicht ihr Maximum ungefähr bei $M=0.8$. Darüber wird die Schaltfrequenz bis zur Vollaussteuerung wieder deutlich kleiner.

- Die Effektivwerte der Verzerrungsströme in den einzelnen Phasen sind im Gegensatz zu den Schaltfrequenzen praktisch gleich gross. Zu ihrer Abschätzung kann wiederum die Näherungsformel verwendet werden, die bereits in Kapitel 10.1 eingeführt wurde:

$I_{U / V / W, V Z, e f f} \approx \frac{I_{\delta}}{2 \sqrt{3}}$

Der entsprechende Wert ist zum Vergleich im Diagramm eingezeichnet. Obschon die Berechnungsgrundlage für die Formel, nämlich dass sich die Verzerrungsströme linear von einer Bandgrenze zur anderen bewegen, nicht mehr vollumfänglich erfüllt ist, ist die Übereinstimmung weiterhin gut. Die realen Effektivwerte sind leicht grösser. 
- Die Aussteuergrenze liegt wie bei dreiphasigen Zweipunktreglern bei $M \approx 1.155$. Darüber wird die geforderte Stromgrundschwingung nicht mehr erreicht und der Verzerrungsstrom steigt sehr stark an.

Es geht aus dem Bild 11.11 nicht hervor, dass sich wie bei den Phasenstromreglern die mittleren Schaltfrequenzen weitgehend umgekehrt proportional zum Verzerrungsstrom und damit auch $\mathrm{zu} I_{\delta}$ verhalten.

Drehmomentwelligkeit: Die Drehmomentwelligkeit in einem Antrieb im Leerlauf ist bekanntlich durch die folgende Zeigergleichung gegeben (Kapitel 5.2.1.4):

$$
M_{e l, V Z}=\frac{3}{2} \frac{1}{\omega_{1}} \operatorname{Re}\left(\underline{e}_{-S t r, V Z}\right)
$$

Der Gegenspannungszeiger $\underline{e}$ rotiert mit der Grundkreisfrequenz $\omega_{1}$. Die Bewegung des Verzerrungsstromes $\underline{i}_{S t r, V Z}$ innerhalb seiner Toleranzfläche ist im Verhältnis dazu relativ schnell. Deshalb ist er in unregelmässig wechselnder Weise mehr oder weniger stark momentbildend. Wenn man nur die innere Toleranzfläche betrachtet, erreicht der Betrag von $\underline{i}_{S t r, V Z}$ in ihrer Ecke den Spitzenwert $\sqrt{2} I_{\delta} / 2$. Dementsprechend kann im Drehmoment der folgende Spitzenwert auftreten:

$$
\hat{M}_{e l, V Z}=\frac{3}{2} \frac{1}{\omega_{1}}|\underset{-}{e}| \sqrt{2} \frac{I_{\delta}}{2}=\frac{3 \sqrt{2}}{4} \frac{|e| I_{\delta}}{\omega_{1}}
$$

Geht man davon aus, dass die Maschine immer mit vollem Fluss betrieben wird, so verläuft die Gegenspannung proportional zu $\omega_{1}$. Der Quotient $|\underline{e}| / \omega_{1}$ ist dann konstant und $\hat{M}_{e l, V Z}$ ist allein durch die Abmessungen der Toleranzfläche bestimmt.

In Bild 11.12 ist ein Beispiel für den Verlauf von $M_{e l, V Z}$ dargestellt. Es zeigt sich, dass der Spitzenwert nach (11.5) nur selten erreicht wird, weil sich einerseits $\underline{\underline{i}}_{S t r, V Z}$ nicht ständig den maximalen Betrag aufweist und andererseits nicht jederzeit stark momentbildend wirkt. Dagegen ist auch ersichtlich, dass der Spitzenwert $M_{e l, V Z}$ gelegentlich überschritten wird (im Bild mit einem Pfeil bezeichnet). Dies ist aber höchstens dann möglich, wenn der Stromfehler die innere Toleranzfläche verlassen hat.

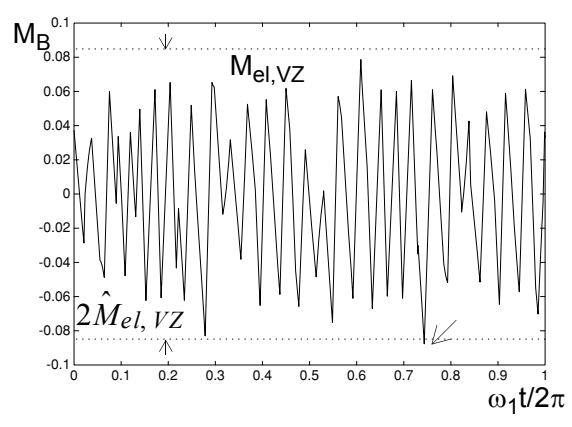

Bild 11.12.

Verlauf der Drehmomentwelligkeit für $|\underline{e}|=0.8$, $\omega_{1}=0.8 \omega_{B}, I_{n}=0.2 I_{B}, I_{\delta}=0.4 I_{n}$ 
Dynamisches Verhalten: Während im stationären Zustand jeweils nur Übergänge zwischen benachbarten Schaltzuständen ausgeführt werden, ist dies bei dynamischen Vorgängen nicht mehr der Fall: eine sprungartige Änderung des Sollwertzeigers hat zur Folge, dass einer oder sogar beide Komparatoren direkt in den oberen bzw. unteren Anschlag springen. Dies bewirkt augenblicklich ein Umschalten auf denjenigen Zustand, der den Stromzeiger raschmöglichst in die durch den Sollwertsprung verschobene Toleranzfläche zurück bewegt. Ein entsprechendes Beispiel ist in Bild 11.13 dargestellt. Im Zeitpunkt des Sprunges bei $t=0$ schaltet der Stromrichter sofort auf den Zustand ${ }^{5} \underline{U}$, um den Betrag des Stromzeigers raschmöglichst zu verkleinern. Die Ausregelzeit ist wie bereits bei den Phasenstromreglern erklärt wurde, weitgehend durch die Gegenspannung bestimmt, da nur die Differenz zwischen ihr und der Ausgangsspannung über der Lastinduktivität anliegt. Das dynamische Verhalten ist jedoch stets für den gegebenen Betriebspunkt optimal. Vor allem ist auch zu betonen, dass das Verfahren nach Bild 11.5 den stationären wie auch den dynamischen Betrieb bewältigen kann.
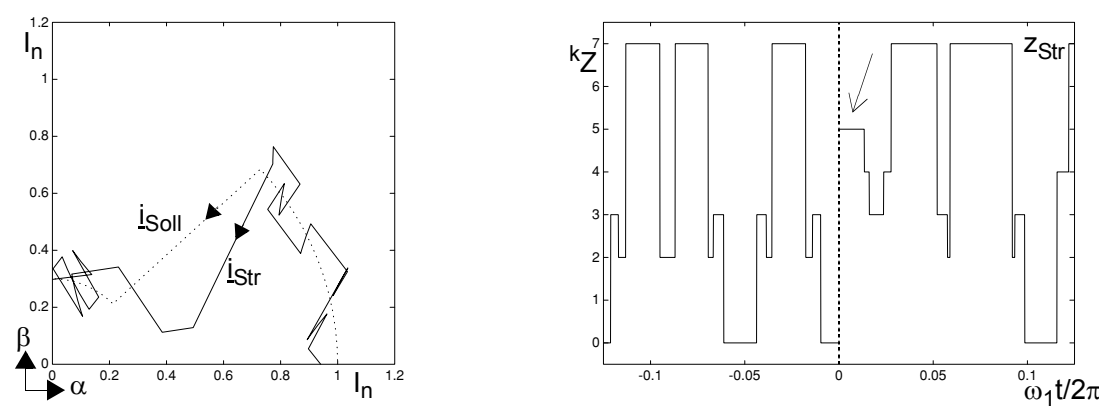

Bild 11.13. Schrittantwort der Drehzeiger-Komponentenregelung

Das vorgestellte Verfahren wird in [Ack1] vorgestellt. Es stellt jedoch nur ein Beispiel zur Stromzeiger-Komponentenregelung dar. Weitere Varianten ergeben sich durch die Verwendung von zwei dreistufigen oder zwei vierstufigen Komparatoren. Andererseits bietet auch die Schalttabelle Freiraum für unterschiedliche Lösungen. Im wesentlichen weisen jedoch alle diese Verfahren die hier gezeigten Eigenschaften auf. Insbesondere ergeben sich stets ungleiche Schaltfrequenzen für die 3 Umschalter.

Literatur zu Kapitel 11.2: [Ack1], [Pfa1], [Kaz1], [Kaz2], [Wüe2]

\subsection{Prädiktive Stromregelung}

Unter den Begriff prädiktive Stromregelung fallen diejenigen Stromregelverfahren, welche Informationen über die Lage oder die Länge des Gegenspannungszeigers verarbeiten. Damit wird es möglich, den Verlauf des Stromzeigers nach dem nächsten Schaltvorgang mehr oder weniger genau vorauszusagen und deshalb gezielt den am besten geeigneten 
Schaltzustand anzulegen. Das Problem bei der Realisierung solcher Verfahren ist die Beschaffung der Information über die Gegenspannung, da diese in vielen Fällen nicht direkt messbar ist. Im Falle einer Asynchronmaschine als Last ist sie, wie in Kap. 3.2 gezeigt wurde, nur eine Ersatzgrösse. Man muss sich deshalb mit Nachbildungen behelfen.

In den Kapiteln 11.3 .1 bis 11.3 .3 werden 3 verschiedene prädiktive Verfahren vorgestellt.

\subsubsection{Prädiktive Stromregelung mit Schalttabelle}

Die Ableitung des Stromfehlers kann ausgedrückt werden durch die Ableitungen des Stromzeigers und seines Sollwertes:

$$
i_{-S t r, V Z}=i_{-S t r}-i_{-S o l l} \quad \Rightarrow \quad \frac{d i_{-S t r, V Z}}{d t}=\frac{d i_{-S t r}}{d t}-\frac{d i_{-S o l l}}{d t}
$$

Durch Einsetzen von (11.6) in (11.1) und nach geeigneter Umformung erhält man die Gleichung (11.7), welche die Ableitung des Stromfehlers in Abhängigkeit der Spannungen im Lastkreis beschreibt:

$$
\frac{d i_{-S t r, V Z}}{d t}=\frac{1}{L_{k}}\left(\underline{u}_{S t r}-\underline{e}\right)-\frac{d i_{-S o l l}}{d t}=\frac{1}{L_{k}}\left(\underline{u}_{S t r}-\underline{u}_{\text {Soll }}\right), \quad \underline{u}_{\text {Soll }}=\underline{e}_{-}+L_{k} \frac{d i_{-S o l l}}{d t}
$$

Der Zeiger $\underline{u}_{\text {Soll }}$ stellt dabei die exakte, kontinuierlich verlaufende Spannung dar, welche der Stromrichter einstellen müsste. Im Hinblick auf eine wirkungsvolle Kontrolle des Stromfehlers ist die Kenntnis der Differenz zwischen dem angelegten Spannungszustand und dem Sollwert $\underline{u}_{S t r} \underline{u}_{S o l l}$ von Bedeutung. Da nur 6 spannungsbildende Schaltzustände und zwei Nullzustände zur Verfügung stehen, genügt jedoch eine reduzierte Information. Das im folgenden beschriebene Regelverfahren basiert darauf, dass nur bekannt ist, in welchem der $\pi / 3$-Sektoren $S 1$ bis $S 6$ sich $\underline{u}_{S o l l}$ befindet. Daraus kann dann für jeden einzelnen Schaltzustand ein Kreissegment vorausgesagt werden, in dem die Differenz $\underline{u}_{S t r}{ }^{-}$ $\underline{u}_{\text {Soll }}$ liegen muss. In Bild 11.14 sind als Beispiel die Segmente dargestellt, die sich ergeben, wenn $\underline{u}_{\text {Soll }}$ im Sektor $S 1$ liegt. Der Radius des Segmentes ist durch die maximale Länge von $\underline{u}_{\text {Soll }}$ gegeben. Unter der Annahme, dass der Stromrichter nicht übersteuert werden soll, berührt das Segment gerade die Sekante, die von den beiden angrenzenden Schaltzuständen gebildet wird. Der Radius beträgt dann $(2 / \sqrt{3})\left(U_{d} / 2\right)$, was einem Modulationsgrad des Stromrichters von $M \approx 1.155$ entspricht.

Die Information über die Lage der Spannungszeiger lässt sich optimal ausnützen, wenn für den Zeiger des Phasenstromes die sechseckige Toleranzfläche in Bild 11.15 vorgegeben wird. Dadurch ist es möglich, jede Berührung des Stromfehlers am Rand dieser Fläche einem der Sektoren SI bis SVI zuzuordnen. Der neu einzustellende Schaltzustand kann dann so bestimmt werden, dass der Stromfehler einerseits mit Sicherheit wieder ins Innere der Toleranzfläche zurückbewegt wird, und dass dies andererseits mit der kleinstmöglichen Geschwindigkeit passiert. Auf diese Weise dauert es entsprechend lange, bis der nächste Schaltvorgang notwendig wird und es ergibt sich eine niedrige Schaltfrequenz. 


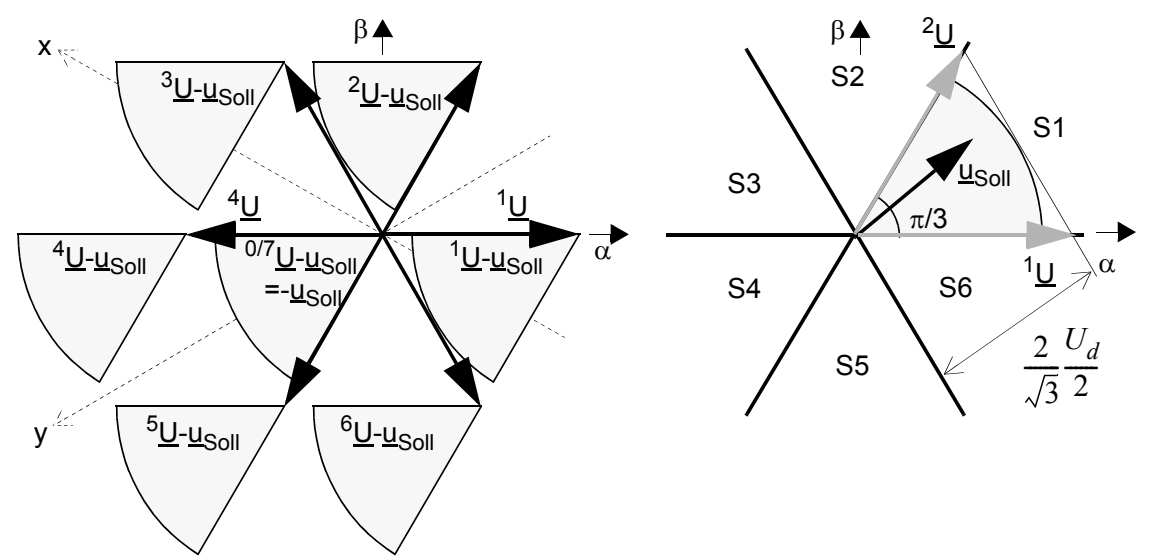

Bild 11.14. Auswertung der Kenntnis über die Gegenspannung links: Bereiche für den Spannungszeiger $\underline{u}_{S t r}-\underline{u}_{\text {Soll }}$ wenn $\underline{u}_{\text {Soll }}$ im Sektor $S 1$ liegt rechts: Sektor für $\underline{u}_{\text {Soll }}$ mit begrenzender Sekante

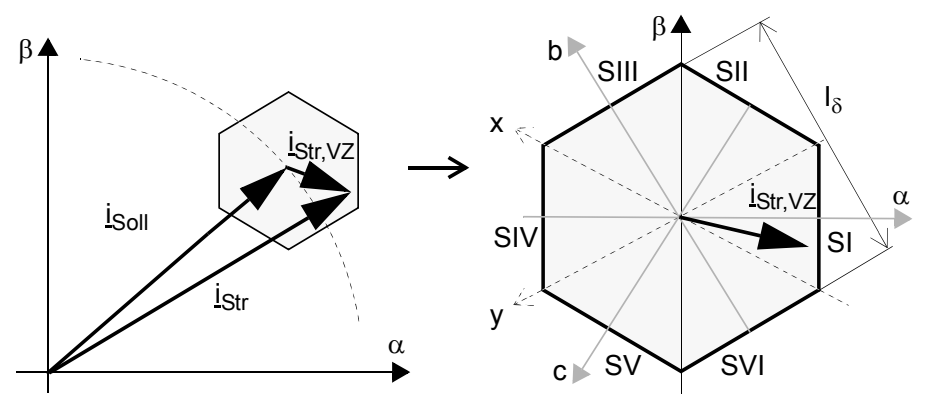

Bild 11.15.

Sechseckige Toleranzfläche für den Stromfehler

Das Auswahlverfahren des Schaltzustandes soll an 3 Beispielen erläutert werden:

- Der Gegenspannungszeiger liege im Sektor $S 1$, so dass die Verhältnisse in Bild 11.14 gelten. Berührt nun $\underline{i}_{S t r, V Z}$ den Rand der Toleranzfläche im Sektor SI, so muss der Stromfehler mit erster Priorität nach links bewegt werden. Dazu muss die Differenz $\underline{u}_{S t r} \underline{u}_{S o l l}$ eine negative $\alpha$-Komponente aufweisen, was bedeutet, dass der ganze Kreissektor für den zu wählenden Spannungszustand links der $\beta$-Achse zu liegen hat. Die Zustände ${ }^{0 /}$ ${ }^{7} \underline{U},{ }^{3} \underline{U},{ }^{4} \underline{U}$ und ${ }^{5} \underline{U}$ erfüllen diese Forderung. Zur Anwendung gelangt ${ }^{0 / 7} \underline{U}$, da der entsprechende Sektor am nächsten am Koordinatenursprung liegt. Dadurch ergibt sich die langsamste Bewegung des Stromfehlers und der nächste Schaltvorgang wird möglichst lange hinausgezögert.

- Berührt $\underline{i}_{S t r, V Z}$ den Rand im Sektor SII, so muss das Kreissegment für $\underline{u}_{S t r} \underline{u}_{S o l l}$ vollständig unterhalb der Hilfsachse $x$ liegen. Nach den selben Überlegungen wie vorher gelangt von den 4 möglichen Zuständen ${ }^{0 / 7} \underline{U},{ }^{4} \underline{U},{ }^{5} \underline{U}$ und ${ }^{6} \underline{U}$ wiederum ${ }^{0 / 7} \underline{U}$ zur Anwendung.

- Erfolgt die Berührung in Sektor SIII, so ist die Hilfsachse y für die Beurteilung massgebend. Es liegen 3 Kreissegmente vollständig unterhalb von ihr und sind prinzipiell 
zulässig. Hier ergibt ${ }^{1} \underline{U}$ die langsamste Bewegung des Stromfehlers und wird deshalb bevorzugt.

In gleicher Art und Weise lassen sich die geeigneten Schaltzustände für eine Berührung in den restlichen 3 Sektoren SIV, SV und SVI bestimmen. Anschliessend sind für die Lage des Gegenspannungszeiger in den Sektoren $S 2$ bis $S 6$ die analoge Graphik zu Bild 11.14 mit den Kreissegmenten für $\underline{u}_{S t r}-\underline{u}_{S o l l}$ zu ermitteln und ebenfalls die geeigneten Schaltzustände für die verschiedenen Fälle von Berührungen der Toleranzflächenränder festzulegen. Zuletzt liegt dann die vollständige Schalttabelle 11.1 vor. Die Felder, welche den oben beschriebenen Beispielen entsprechen, sind darin grau hinterlegt. Die Tabelle enthält alle notwendigen Informationen für die Steuerung des Stromrichters im stationären Betrieb, bzw. solange der Stromfehler ständig innerhalb der Toleranzfläche gehalten werden kann. Auf die dynamischen Aspekte des Verfahrens wird später noch speziell eingegangen. Erwähnenswert sind die Übergänge auf einen Nullzustand. Wie bei der Stromzeiger-Komponentenregelung erfolgt die Auswahl zwischen ${ }^{0} Z$ und ${ }^{7} Z$ derart, dass nur ein Brückenzweig umgeschaltet werden muss. Bei der Rückkehr auf einen spannungsbildenden Zustand ist es aber auch hier möglich, dass zwei Brückenzweige schalten müssen.

\begin{tabular}{c|c|c|c|c|c|c} 
Sektoren & SI & SII & SIII & SIV & SV & SVI \\
\hline S1 & ${ }^{0 / 7} Z$ & ${ }^{0 / 7} Z$ & ${ }^{1} Z$ & ${ }^{1} Z$ & ${ }^{2} Z$ & ${ }^{2} Z$ \\
\hline S2 & ${ }^{3} Z$ & ${ }^{0 / 7} Z$ & ${ }^{0 / 7} Z$ & ${ }^{2} Z$ & ${ }^{2} Z$ & ${ }^{3} Z$ \\
\hline S3 & ${ }^{4} Z$ & ${ }^{4} Z$ & ${ }^{0 / 7} Z$ & ${ }^{0 / 7} Z$ & ${ }^{3} Z$ & ${ }^{3} Z$ \\
\hline S4 & ${ }^{4} Z$ & ${ }^{5} Z$ & ${ }^{5} Z$ & ${ }^{0 / 7} Z$ & ${ }^{0 / 7} Z$ & ${ }^{4} Z$ \\
\hline S5 & ${ }^{5} Z$ & ${ }^{5} Z$ & ${ }^{6} Z$ & ${ }^{6} Z$ & ${ }^{0 / 7} Z$ & ${ }^{0 / 7} Z$ \\
\hline S6 & ${ }^{0 / 7} Z$ & ${ }^{6} Z$ & ${ }^{6} Z$ & ${ }^{1} Z$ & ${ }^{1} Z$ & ${ }^{0 / 7} Z$
\end{tabular}

Tabelle 11.1.

Schalttabelle für prädiktive Stromregelung,

Zeilen: Sektor von $\underline{u}_{\text {Soll }}$, Spalten: Sektor von $\underline{i}_{S t r, V Z}$

In Bild 11.16 ist schliesslich das Prinzipschaltbild des Regelverfahrens dargestellt. Die sechseckige Toleranzfläche lässt sich sehr einfach realisieren, wenn anstelle des Stromzeigers die Phasenströme zur Regelung verwendet werden. Sie sind bekanntlich identisch mit den Projektionen des Stromzeigers auf die in Bild 11.15 eingezeichneten Achsen $a, b$ und $c$. Das Toleranzsechseck ist gerade so ausgerichtet, dass immer die zwei gegenüber-

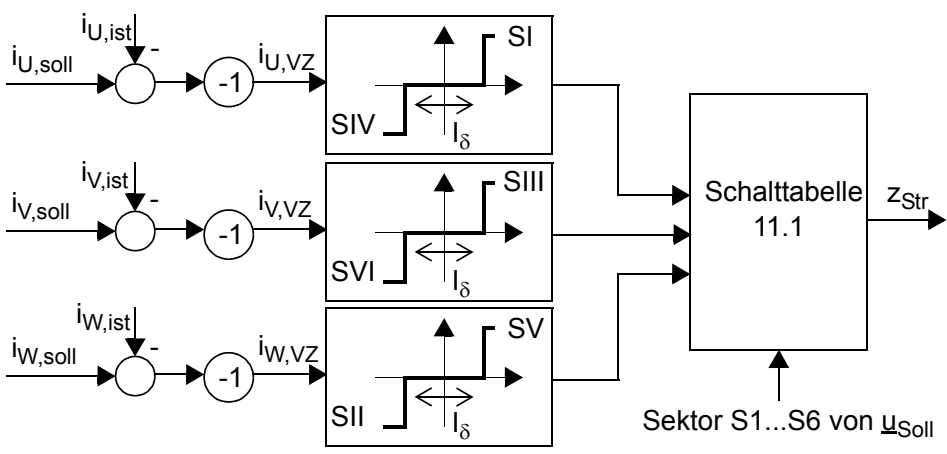

Bild 11.16.

Blockschaltbild der prädiktiven Stromzeigersteuerung 
liegenden Seiten ein Toleranzband für einen Phasenstrom definieren. Jeder Komparator detektiert die Berührung von zwei Sektorgrenzen. Die dargestellte Charakteristik der Komparatoren erlaubt es, dass mehrmals hintereinander dieselbe Schwelle berührt werden kann. Immer derjenige Komparator, der zuletzt angesprochen hat, ist der massgebende für die Schalttabelle.

Spannungen und Ströme: Das Verhalten des Verfahrens, das in den Bildern 11.17 und 11.18 an zwei Beispielen mit verschiedenen Toleranzflächen gezeigt ist, unterscheidet sich in einigen Punkten von demjenigen der Stromzeiger-Komponentenregelung. Einmal bleibt der Stromfehler im stationären Betrieb jederzeit innerhalb der Toleranzfläche. Im weiteren erkennt man im Verlauf der Schaltzustände ein geordneteres Durchlaufen aller Zustände. Dabei wird über einen kurzen Zeitausschnitt betrachtet jeweils nur mit zwei spannungsbildenden Zuständen und einem Nullzustand operiert. Trotz dieser Unterschiede sehen die Stromspektra qualitativ praktisch gleich aus wie diejenigen der Komponentenregelung. Die dargestellten Spektra von $i_{\alpha}$ entsprechen gleichzeitig auch denjenigen der Phasenströme $i_{U}$. Da das Verfahren asynchron ist, sind die Phasenströme zwar nicht exakt symmetrisch, ihre Verläufe und Spektra sind jedoch gleichartig. Es zeigt sich deutlich, dass die Verkleinerung der Toleranzfläche eine Verbreiterung des Frequenzbandes mit sich bringt, in dem die dominanten Verzerrungsanteile auftreten. Diese weisen dafür entsprechend kleinere Amplituden auf.
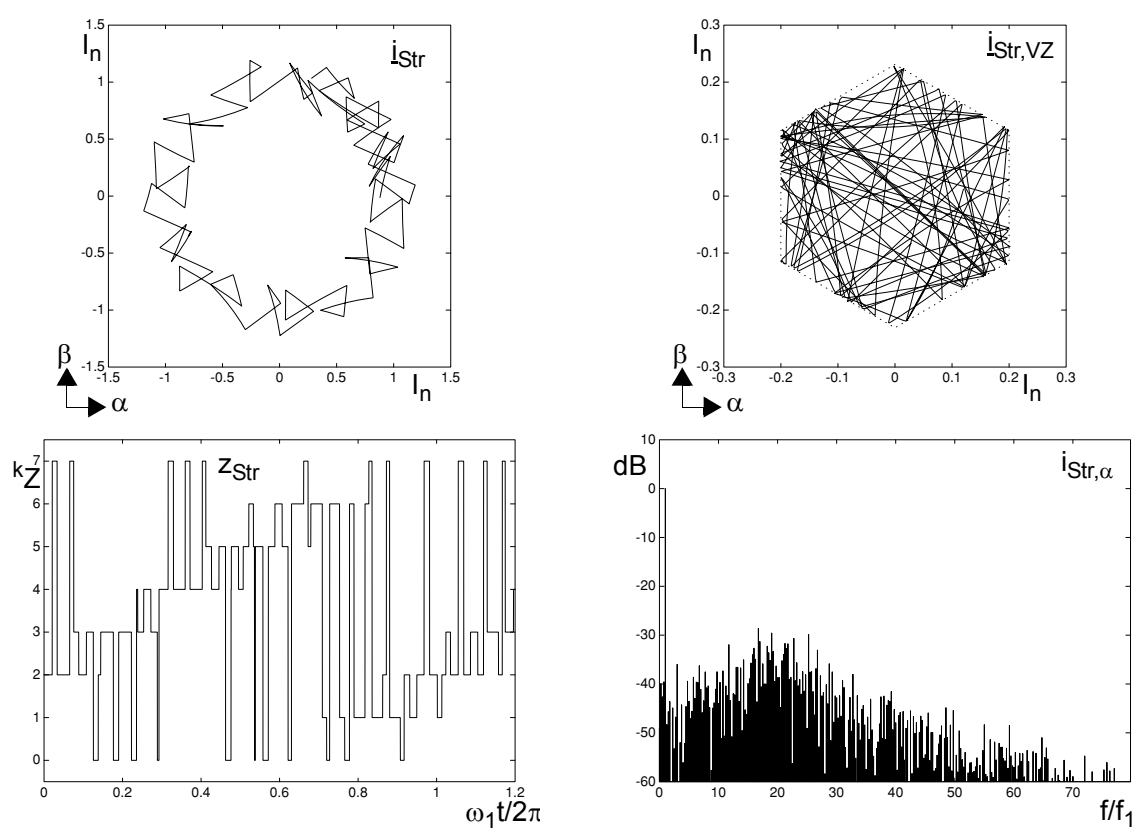

Bild 11.17. Prädiktive Stromzeigerregelung für $|\underline{e}|=0.8\left(U_{d} / 2\right), M=0.95, I_{n}=0.2 I_{B}, I_{\delta}=0.4 I_{n}$, oben links: Stromdrehzeiger, oben rechts: Drehzeiger des Verzerrungsstromes, unten links: Schaltzustände des Stromrichters, unten rechts: Spektrum von $i_{S t r, \alpha}$, berechnet über $4 T_{1}, 0 \mathrm{~dB}: I_{n}$ 

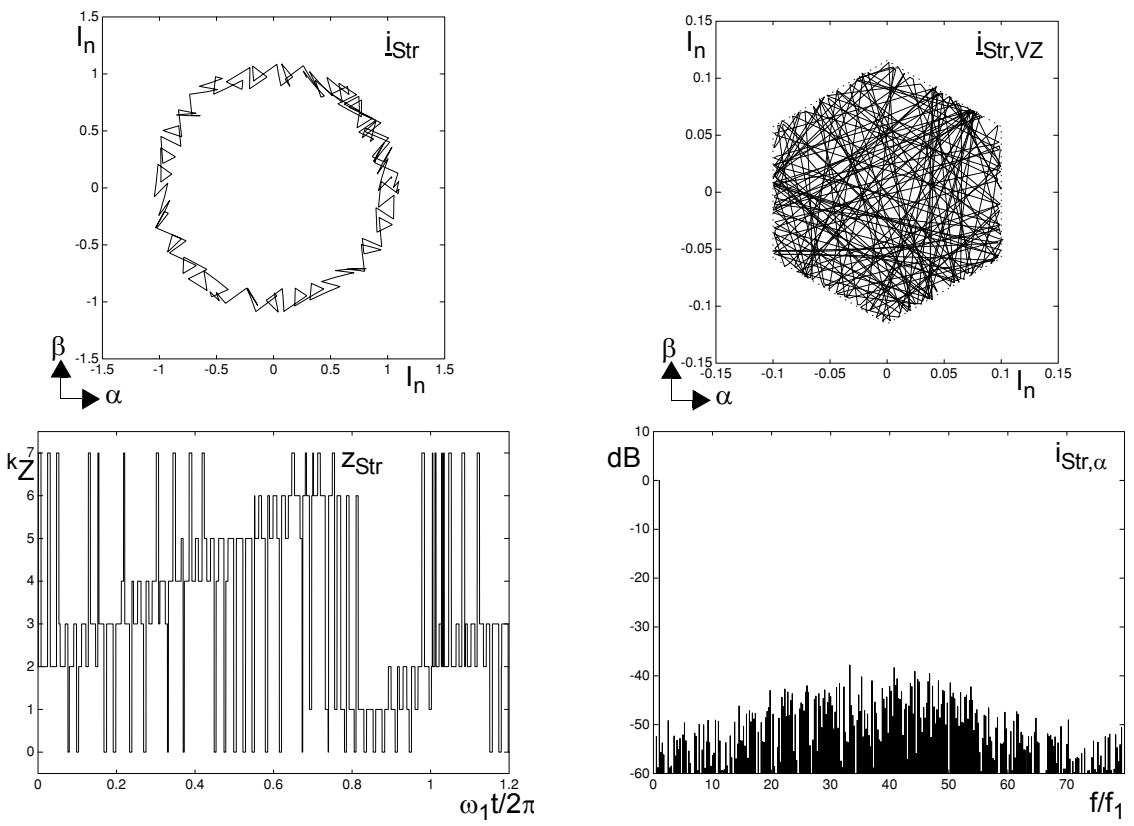

Bild 11.18. Prädiktive Stromzeigerregelung für $|\underline{e}|=0.8\left(U_{d} / 2\right), M=0.95, I_{n}=0.2 I_{B}, I_{\delta}=0.2 I_{n}$, oben links: Stromdrehzeiger, oben rechts: Drehzeiger des Verzerrungsstromes, unten links: Schaltzustände des Stromrichters, unten rechts: Spektrum von $i_{S t r, \alpha}$, berechnet über $4 T_{1}, 0 \mathrm{~dB}: I_{n}$

Schaltfrequenz und Verzerrungsstrom: Bild 11.19 zeigt die mittlere Schaltfrequenzen und den Effektivwerte des Verzerrungsstromes in einer Phase als Funktion des Modulationsgrades für zwei verschiedene Toleranzflächen. Die Kennlinien sind wiederum mit Hilfe von Simulationen berechnet. Die Verläufe weisen prinzipiell dieselbe Charakteristik auf wie diejenigen der Komponentenregelung. Allerdings ist hier die Symmetrie besser: sowohl die mittleren Schaltfrequenzen als auch die Effektivwerte der Verzerrungsströme
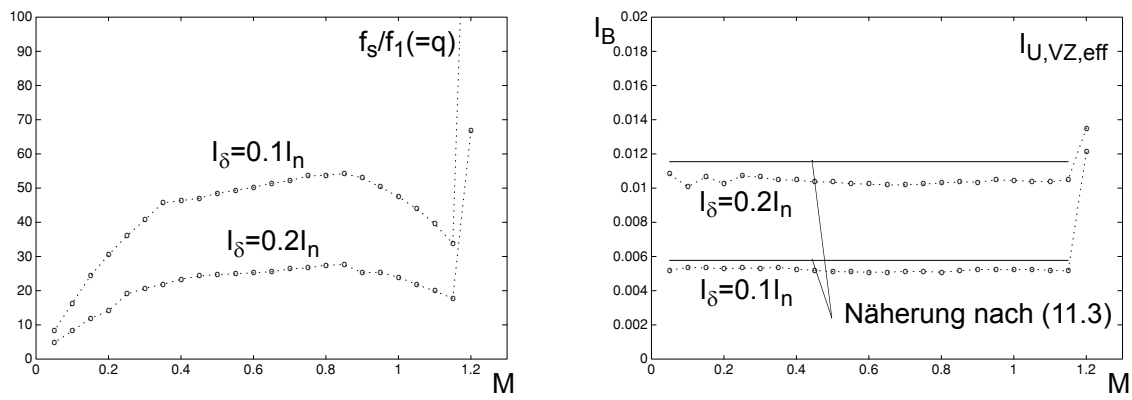

Bild 11.19. Mittlere Schaltfrequenz und Effektivwert des Verzerrungsstromes in einer Phase für die prädiktive Stromregelung mit Schalttabelle, $I_{n}=0.2 I_{B}$ (o=simulierte Betriebspunkte) 
aller 3 Phasen stimmen gut überein. Die maximale Schaltfrequenz tritt ungefähr bei $M=0.85$ auf. Beim Vergleich der Bilder 11.11 und 11.19 lässt sich feststellen, dass sich bei Toleranzflächen mit dem gleichen $\mathrm{I}_{\delta}$ bei beiden Verfahren die über alle 3 Phasen gemittelte Schaltfrequenz auf ungefähr gleiche Werte einstellt. Das prädiktive Verfahren ergibt jedoch einen um rund 15\% reduzierten Verzerrungsstrom. Er liegt generell leicht unterhalb des Wertes, den die Näherungsformel (11.3) ergibt. Weiter zeigen die beiden Beispiele, dass sich der Verzerrungsstrom weitgehend proportional und die Schaltfrequenz umgekehrt proportional zu $I_{\delta}$ verhalten.

Im Bereich der Übersteuerung steigen sowohl die Schaltfrequenz wie auch der Verzerrungsstrom sofort sehr stark an.

Drehmomentwelligkeit: Der Spitzenwert der Drehmomentwelligkeit im Leerlauf bei einem Antrieb ist aus den Abmessungen der Toleranzfläche bestimmt:

$$
\hat{M}_{e l, V Z}=\frac{3}{2} \frac{|\underline{e}|}{\omega_{1}} \frac{2}{\sqrt{3}} \frac{I_{\delta}}{2}=\frac{\sqrt{3}}{2} \frac{|e| I_{\delta}}{\omega_{1}}
$$

Er wird dann erreicht, wenn der Stromfehler gerade vollständig momentbildend ist und die Spitze seines Zeigers in einer Ecke des Toleranz-Sechseckes liegt. Dies kommt relativ selten vor, wie das Beispiel in Bild 11.20 bestätigt. Im Gegensatz zu den Phasenstromreglern und der Komponentenregelung treten hier stationär keine Übertretungen des Spitzenwertes nach (11.8) auf, da der Stromfehler seine Toleranzfläche nicht verlässt.

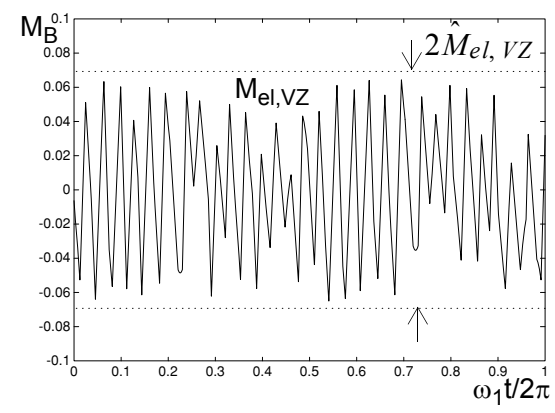

Bild 11.20.

Verlauf der Drehmomentwelligkeit für $|\underline{e}|=0.8\left(U_{d} / 2\right), \omega_{1}=0.8 \omega_{B}, I_{n}=0.2 I_{B}, I_{\delta}=0.4 I_{n}$

Dynamisches Verhalten: Die Schalttabelle 11.1 ist so gewählt, dass im stationären Zustand eine möglichst niedrige Schaltfrequenz auftritt. Bei dynamischen Vorgängen, wenn der Stromfehler die Toleranzfläche verlassen kann, wählt sie jedoch nicht denjenigen Schaltzustand aus, der die schnellste Reaktion des Stromrichters ermöglicht. Deshalb muss für diesen Fall eine zweite Tabelle definiert werden. Sie wird aktiviert, wenn der Stromfehler an den Rand einer zweiten, leicht grösseren Toleranzfläche stösst, bzw. diese sogar verlassen hat. Bild 11.21 zeigt die äussere Fläche mit der zugehörigen Tabelle. Sie ist unabhängig von der Lage der Gegenspannung und besteht deshalb nur aus einer Zeile. Der Wert $h$ kann prinzipiell sehr klein gemacht werden. Das dynamische Verhalten wird auf diese Weise weitgehend gleich wie dasjenige der Komponentenregelung. 


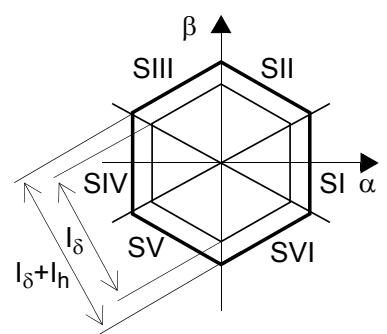

dynamische

Schalttabelle

SI SII SIII SIV SV SVI Bild 11.21.

${ }^{4} \underline{U}{ }^{5} \underline{U}{ }^{6} \underline{U}{ }^{1} \underline{U}{ }^{2} \underline{U}{ }^{3} \underline{U}$
Äussere Toleranzfläche und Schalttabelle zur Verbesserung des dynamischen Verhaltens

Das beschriebene Verfahren ist ziemlich robust auf Fehler in der Bestimmung der Lage des Sollspannungszeigers $\underline{u}_{\text {Soll }}$. Kleine Fehler haben höchstens eine leicht höhere mittlere Schaltfrequenz zur Folge und die zuletzt besprochene dynamische Schalttabelle garantiert, dass sich die Regelung auch bei massiven Störungen sofort wieder fängt. Aus diesem Grund, und weil ohnehin nur eine Sektorbestimmung notwendig ist, kommt das Verfahren mit einer sehr groben und ungenauen Schätzung der Gegenspannung aus. Diese Tatsache ist für die Implementierung in vielen Fällen von grosser Bedeutung.

Literatur zu Kapitel 11.3.1: [Nab1], [Pfa1]

\subsubsection{Prädiktive Stromregelung mit optimaler Auswahl des Schaltzustandes}

Bei vollständiger Kenntnis des Gegenspannungszeigers kann der Verlauf des Stromzeigers im Prinzip exakt berechnet werden. Dadurch ist es möglich, vor jedem Schaltvorgang den optimalen nächsten Schaltzustand zu bestimmen. In Bild 11.22 ist das Prinzip eines solches Verfahrens dargestellt. Der Stromfehler $\underline{i}_{S t r}, V Z$ wird innerhalb einer kreisförmigen Toleranzfläche gehalten. Jedesmal wenn er den Rand dieser Fläche berührt, wird wie bei den vorher beschriebenen Verfahren ein neuer Schaltzustand eingestellt. Dazu wird für jeden zur Verfügung stehenden Zustand ${ }^{k} Z$ die Trajektorie von $\underline{i}_{S t r}, V Z$ bestimmt und der Zeit-

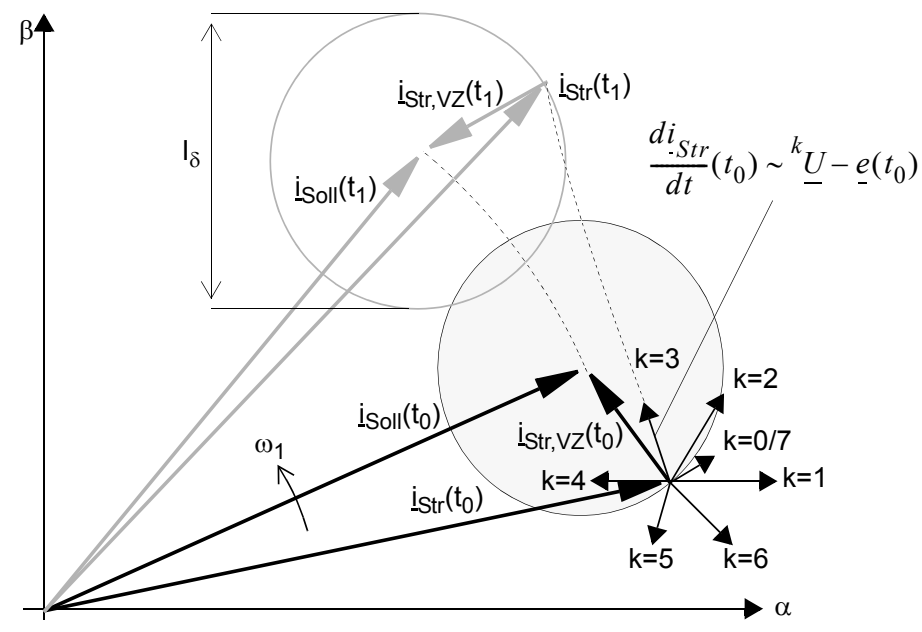

Bild 11.22.

Prinzip der prädiktiven Stromsteuerung mit optimierter Zustandsauswahl 
punkt $t_{1}(k)$ berechnet, zu dem der nächste Schaltvorgang fällig würde. Es kann gezeigt werden, dass das Quadrat des Stromfehlerbetrages $\left|i_{S t r, V Z}\right|^{2}$ zwischen zwei Schaltvorgängen näherungsweise eine Parabel beschreibt. Für das dargestellte Beispiel ergeben sich die Verläufe für in Bild 11.23. Es ist ersichtlich, dass die Zustände ${ }^{1} Z,{ }^{5} Z$ und ${ }^{6} Z$ nicht eingestellt werden können, weil sie den Stromfehler gar nicht in die Toleranzfläche zurückführen würden. ${ }^{0 / 7} Z,{ }^{2} Z$ und ${ }^{4} Z$ wären zwar erlaubt, würden jedoch den Stromfehler so beeinflussen, dass er relativ schnell wieder an den Rand stossen würde. Mit ${ }^{3} Z$ dagegen verbleibt der Stromfehler am längsten innerhalb der Toleranzfläche. Dieser Zustand wird deshalb eingestellt. Bei $t_{1}$ ist der nächste Schaltvorgang fällig. Dort ist für die Auswahl des nächsten Zustandes wieder die gleiche Überlegung anzustellen.

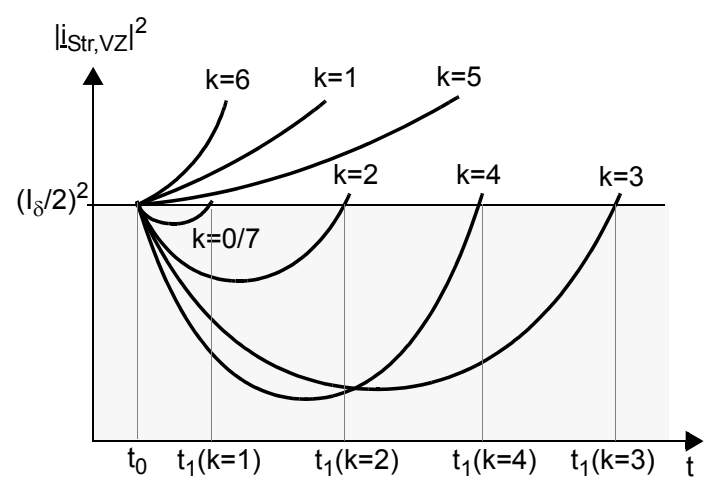

Bild 11.23.

Trajektorien des quadrierten Stromfehlerbetrages für die verschiedenen Schaltzustände

Die Zustandsauswahl nach diesem Verfahren basiert damit auf einer lokalen Optimierung mit dem Zeitintervall $t_{1}(k)-t_{0}$ als Gütekriterium. Sie soll für die gegebene Toleranzfläche eine möglichst niedrige Schaltfrequenz ergeben. Dazu ist allerdings auch zu berücksichtigen, dass für die Einstellung eines Schaltzustandes je nach vorhergehendem Zustand ein, zwei oder sogar alle 3 Brückenzweige umzuschalten sind. Deshalb ist das Gütekriterium wie folgt zu modifizieren:

$$
\frac{t_{1}(k)-t_{0}}{N_{k}}=\min , \quad N_{k}=1,2,3
$$

$N_{k}$ bezeichnet dabei die Anzahl Umschaltungen zum Erreichen des entsprechenden $\mathrm{Zu}$ standes. Es wird an dieser Stelle darauf verzichtet, den Weg der Berechnung des Gütekriteriums aufzuzeigen. Es ist jedoch erwähnenswert, dass die Berechnung mit Hilfe von linearisierten Verläufen nicht sehr aufwendig wird.

Das Blockschaltbild in Bild 11.24 fasst das Prinzip des Verfahrens der optimalen Zustandsauswahl zusammen. Es wird dabei, wie bereits früher erwähnt, nicht darauf eingegangen, auf welche Weise die Gegenspannung $\underline{e}$ bestimmt werden kann. Dies hängt ohnehin sehr stark von der tatsächlichen Last ab.

Das Verfahren der prädiktiven Stromregelung mit optimierter Zustandsauswahl ist vom Prinzip her sehr einleuchtend und elegant. Es kommt aus verschiedenen Gründen aber in der Praxis kaum zur Anwendung: 


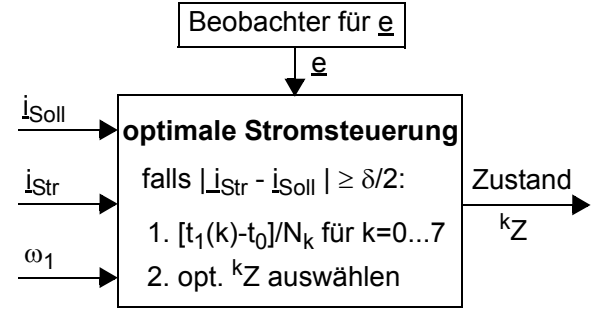

Bild 11.24.

Blockdiagramm der prädiktiven Stromregelung mit optimierter Zustandsauswahl

- Die sich einstellende mittlere Schaltfrequenz ist gegenüber den beiden vorhergehenden einfacheren Verfahren bei vergleichbaren Verzerrungsströmen nicht kleiner. Dies hängt damit zusammen, dass die Optimierung nur lokal, d.h. für den nächsten Schaltvorgang erfolgt. Betrachtet man jedoch eine Sequenz von mehreren Schaltvorgängen, so kann sich eine insgesamt niedrigere Schaltfrequenz ergeben, wenn nicht alle Schaltvorgänge für sich allein gesehen optimal sind. Eine Reduktion der mittleren Schaltfrequenz würde sich nur dann erzielen lassen, wenn die Trajektorie des Stromfehlers für eine ganze Sequenz von Schaltvorgängen vorausbestimmt und optimiert würde. Der Rechenaufwand dazu wäre aber wegen der grossen Anzahl von verschiedenen möglichen Sequenzen riesig.

- Schon bei der beschriebenen Optimierung ist der Rechenaufwand erheblich: die Berechnung des Gütekriteriums für eine kreisförmige Toleranzfläche ist zwar wie erwähnt nicht allzu aufwendig, sie muss aber für alle 6 zur Verfügung stehenden neuen Schaltzustände durchgeführt werden. Bis zum tatsächlichen Schaltzeitpunkt entsteht dadurch eine Verzögerung, die eine Vergrösserung des Stromfehlers mit sich bringt. Damit dies in kontrollierbarem Rahmen bleibt, muss ein sehr schneller Rechner eingesetzt werden.

Auf die Präsentation von Resultaten wird wegen der geringen praktischen Bedeutung des Verfahrens verzichtet. Qualitativ verhalten sich die Spannungen, Ströme und Schaltfrequenzen ähnlich wie bei der prädiktiven Stromregelung mit Schalttabelle.

Literatur zu Kapitel 11.3.2: [Hol3], [Hol4], [Kaz1], [Kol6]

\subsubsection{Prädiktive Stromregelung nach dem Deadbeat-Prinzip}

Bei den bisher vorgestellten stromzeigerorientierten Verfahren bewegt sich der Stromzeiger zwar jederzeit innerhalb einer vorgegebenen Toleranzfläche, er stimmt in der Regel jedoch nie exakt mit seinem Sollwert überein. Zudem ist die Schaltfrequenz variabel. Im folgenden wird ein Verfahren gezeigt, das mit einem festen Abtastintervall $T_{\text {Tast }}$ arbeitet. Am Ende dieses Intervalles stimmt der Istwert des Stromzeigers stets genau mit seinem Sollwert überein. Die Spannung am Ausgang des Stromrichters wird dabei mittels einer Drehzeigermodulation (Kapitel 8) eingestellt, so dass sich für alle Brückenzweige eine gleiche, konstante Schaltfrequenz ergibt. Voraussetzung für diese Art der Stromregelung ist, dass der Gegenspannungszeiger exakt bekannt ist.

Der Verlauf des Stromzeigers innerhalb eines Abtastintervalles $\left[t_{0}, t_{0}+T_{\text {Tast }}\right]$ kann ausgehend von (11.1) linear angenähert werden: 


$$
i_{\text {Str }}\left(t_{0}+T_{\text {Tast }}\right)-\underline{-i}_{-S t r}\left(t_{0}\right) \approx \frac{T_{\text {Tast }}}{L_{k}}\left[\underline{u}_{S t r}\left(t_{0}\right)-\underline{e}\left(t_{0}\right)\right]
$$

Ausgehend von der Forderung, dass der Stromzeiger am Ende des Intervalles mit seinem Sollwert übereinstimmen soll, ergibt sich ein Sollwert $\underline{u}_{\text {Soll }}$ für die Ausgangsspannung am Stromrichter:

$$
\begin{aligned}
& { }_{-S t r}\left(t_{0}+T_{\text {Tast }}\right)--_{-S t r}\left(t_{0}\right)=i_{-S o l l}\left(t_{0}\right)-\underline{-i}_{\text {Str }}\left(t_{0}\right)=\frac{T_{\text {Tast }}}{L_{k}}\left[\underline{u}_{\text {Soll }}\left(t_{0}\right)-\underline{e}_{-}\left(t_{0}\right)\right] \\
& \Rightarrow \quad \underline{u}_{\text {Soll }}\left(t_{0}\right)=\frac{L_{k}}{T_{\text {Tast }}}\left[i_{\text {Soll }}\left(t_{0}\right)-\underline{-i}_{\text {Str }}\left(t_{0}\right)\right]+\underline{e}\left(t_{0}\right)
\end{aligned}
$$

Der geforderte Spannungszeiger wird nach dem Prinzip der Drehzeigermodulation aus den zur Verfügung stehenden Schaltzuständen zusammengesetzt. Das Prinzip ist in Bild 11.25 veranschaulicht Der Stromzeiger beschreibt eine aus 4 geraden Abschnitten zusammengesetzte Bahn, wobei jeder Abschnitt parallel zur jeweiligen Differenz ${ }^{k} \underline{U}-\underline{e}$ verläuft. Im dargestellten Beispiel liegt die Gegenspannung im Sektor $S 1$ und die Schaltsequenz
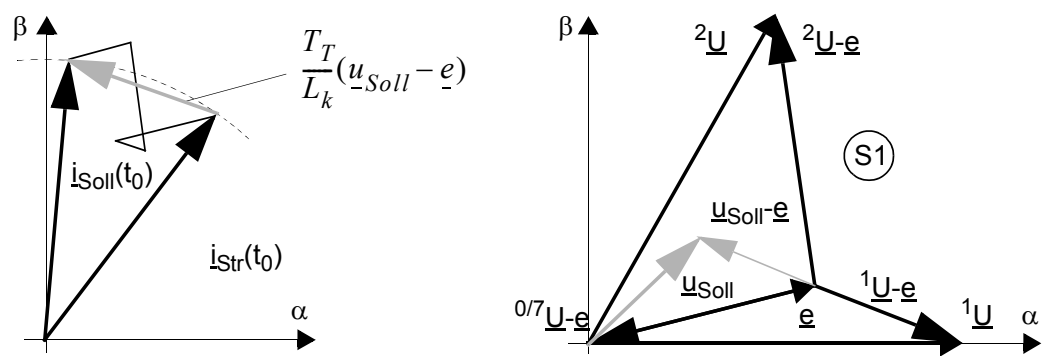

Bild 11.25. Prinzip der prädiktiven Stromregelung mit fester Schaltfrequenz, links: Überführung des Stromzeigers in seinen Sollwert, rechts: eingesetzte Schaltzustände und Spannungszeiger ( $\underline{e}$ im Sektor $S 1)$

durchläuft die Spannungen ${ }^{0} \underline{U}, \underline{\underline{U}},{ }^{2} \underline{U}$ und ${ }^{7} \underline{U}$, wobei die beiden Nullzustände gleich lange eingestellt sind. Dies entspricht der Standardmethode der Drehzeigermodulation, die bereits in Kapitel 8.2 beschrieben wurde. Das nächste Abtastintervall würde dementsprechend in der umgekehrten Folge durchlaufen. Damit der Mittelwert der Ausgangsspannung mit $\underline{u}_{S o l l}$ übereinstimmt, muss die Beziehung (11.12) gelten:

$$
\underline{u}_{\text {soll }}=t_{0}{ }^{0} \underline{U}+t_{1}{ }^{1} \underline{U}+t_{2}{ }^{2} \underline{U}+t_{7}{ }^{7} \underline{U} \quad \text { mit } \quad t_{0}+t_{1}+t_{2}+t_{7}=T_{\text {Tast }}
$$

Unter Verwendung von (11.11) und mit Kenntnis der Gegenspannung lassen sich die Zeitabschnitte $t_{0}, t_{1}, t_{2}$ und $t_{7}$ berechnen. Auch darauf wurde bereits in Kapitel 8.2 eingegangen. Desgleichen sind die dort gemachten Überlegungen betreffend andere Varianten von Schaltsequenzen auch hier gültig. Grundsätzlich ist jede Sequenz zulässig, welche den geforderten Spannungsmittelwert einstellen kann. Bei der Standardsequenz ergibt sich für jeden Brückenzweig genau ein Schaltvorgang pro Abtastintervall. Damit ist die Schaltfre- 
quenz konstant und gemäss (11.13) allein durch die Abtastfrequenz gegeben. Ist diese ein gerades ganzzahliges Vielfaches der Grundfrequenz, so wird das Verfahren synchron.

$$
f_{s}=\frac{f_{\text {Tast }}}{2}
$$

Da der Istwert des Stromzeigers jeweils erst am Ende des Abtastintervalles mit dem zu Beginn des Intervalles abgetasteten Sollwert übereinstimmt, weist die Regelung eine Totzeit von $T_{\text {Tast }}$ auf. Dies kann durch eine Vorsteuerung umgangen werden: anstelle des aktuellen Sollwertes $\underline{i}_{S o l l}\left(t_{0}\right)$ wird der gemäss (11.14) geschätzte Wert des nächsten Abtastzeitpunktes $\underline{i}_{\text {Soll }}\left(t_{0}+T_{\text {Tast }}\right)$ verwendet.

$$
i_{\text {Soll }}\left(t_{0}+T_{\text {Tast }}\right)=i_{\text {Soll }}\left(t_{0}\right) e^{j 2 \pi \frac{T_{\text {Tast }}}{T_{1}}}
$$

Für die Schätzung muss die momentane Grundfrequenz $\omega_{1}=2 \pi / T_{1}$ bekannt sein. Es wird dann einfach angenommen, der Sollwert laufe mit $\omega_{1}$ auf einem Kreis weiter. Die Verhältnisse mit und ohne Vorsteuerung sind in Bild 11.26 veranschaulicht. Es zeigt einen Ausschnitt aus dem stationären Betrieb. Da dort die Positionen der beiden Stromzeiger in
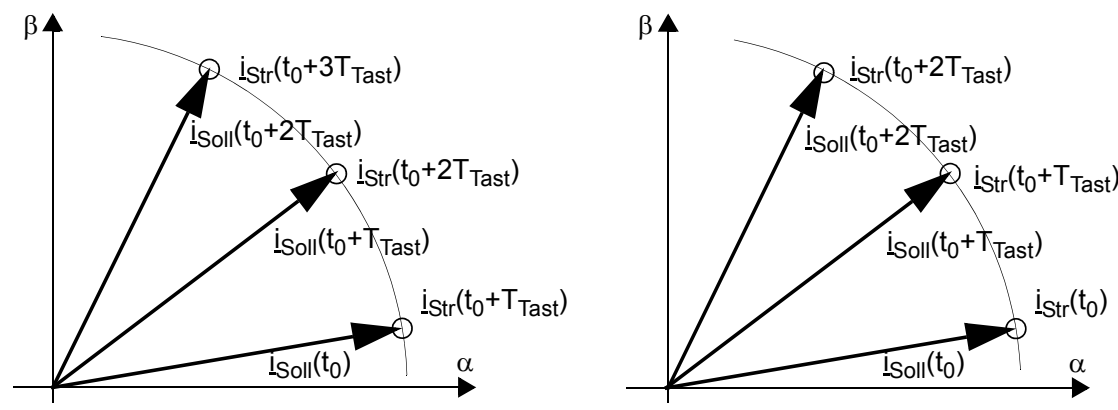

Bild 11.26. Einfluss der Vorsteuerung auf die Totzeit der Regelung im stationären Betrieb, links: ohne Vorsteuerung, rechts: mit Vorsteuerung

den Abtastzeitpunkten übereinstimmen, entweder gleichzeitig oder um $T_{\text {Tast }}$ verschoben, kann der Wert von $\underline{u}_{\text {Soll }}$ ausgehend von (11.11) leicht angegeben werden:

$$
u_{- \text {Soll }}\left(t_{0}\right)=\frac{L_{k}}{T_{\text {Tast }}}\left[i_{\text {Soll }}\left(t_{0}+T_{\text {Tast }}\right)-\underline{-}_{- \text {Soll }}\left(t_{0}\right)\right]+\underline{e}_{-}^{e}\left(t_{0}\right)
$$

Setzt man den Schätzwert nach (11.14), der im stationären Betrieb exakt mit dem tatsächlichen Wert übereinstimmt, in (11.15) ein, so erhält man:

$$
\underline{u}_{\text {Soll }}\left(t_{0}\right)=\frac{L_{k}}{T_{\text {Tast }}{ }_{\text {Soll }}}\left(t_{0}\right)\left[e^{j 2 \pi \frac{T_{\text {Tast }}}{T_{1}}}-1\right]+\underline{e}\left(t_{0}\right)
$$


Geht man davon aus, dass einerseits der Sollwertzeiger und andererseits der Gegenspannungszeiger auf einem Kreis verlaufen, so ist leicht einzusehen, dass die Abtastwerte für $\underline{u}_{\text {Soll }}$ ebenfalls einen Kreis beschreiben. Es liegen deshalb exakt dieselben Verhältnisse vor wie bei der Drehzeigermodulation im stationären Betrieb. Dort wurde einfach davon ausgegangen, dass direkt der Spannungssollwert für die Ausgangsspannung vorgegeben ist. Es ergeben sich dementsprechend dieselben Kurvenformen und Spektra für die Spannungen und Ströme.

Obschon jeweils zu Beginn und am Ende eines Abtastintervalles exakt die richtigen Stromwerte eingestellt sind, bedeutet dies noch nicht unbedingt, dass auch der kurzzeitigen Mittelwert des Stromzeigers, der den Grundschwingungen der Phasenströme entspricht, stimmt. Mit Hilfe von Analogiebetrachtungen zur Drehzeigermodulation wird allerdings klar, dass es sich zumindest im stationären Betrieb so verhält.

Das dynamische Verhalten ist wie bei allen direkten Stromregelverfahren sehr gut. Kleine Störungen werden innerhalb eines Abtastintervalles vollständig ausgeregelt. Bei grossen Abweichungen des Stromzeigers vom Sollwert kann, wie in Bild 11.27 dargestellt, der Spannungszeiger $\underline{u}_{\text {soll }}$ ausserhalb des Sektors, für den die Gleichung (11.12) erfüllt werden kann, liegen. Der Stromsollwert ist dann innerhalb eines Abtastintervalles nicht erreichbar. In diesem Fall kann beispielsweise der grösste mögliche Spannungszeiger $\underline{u}_{\text {soll }}$ in Richtung von $\underline{u}_{\text {soll }}$ eingestellt werden. Diese Massnahme ergibt die bestmögliche Reduktion des Stromfehlers innerhalb des Abtastintervalles. Sie wird wenn nötig solange angewendet, bis der Fehler wieder auf die normale Art und Weise innerhalb eines Intervalles auf null gebracht werden kann.

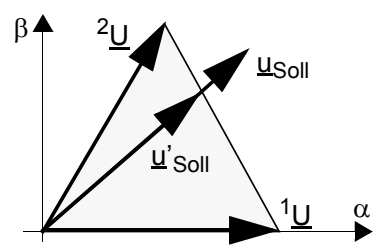

Bild 11.27.

Ersatzsollwert für den Spannungszeiger bei grossen Stromfehlern

Die prädiktive Stromregelung nach dem Deadbeat-Prinzip stellt eine Möglichkeit dar, die Vorteile eines direkten Stromreglers in bezug auf das dynamische Verhalten mit den guten stationären Eigenschaften einer Spannungssteuerung zu kombinieren.

Literatur zu Kapitel 11.3.3: [Pfa1], [Hab1] 


\section{Spezielle Steuerverfahren für Drehstrommaschinen}

\subsection{Flussorientierte Stromregelung}

Die in den Kapiteln 10 und 11 vorgestellten Stromregelverfahren beziehen sich auf die Ströme am Ausgang des Stromrichters. In einem Drehstromantrieb gemäss Bild 12.1 links sind diese identisch mit den Statorströmen in der Maschine. Als Gütekriterium für die Stromregler gelten in erster Linie die Verzerrungsanteile, ohne Rücksicht darauf, wie diese das Drehmoment und den Fluss in der Maschine beeinflussen. In Kapitel 3.2.2 wurde jedoch am Beispiel der Asynchronmaschine (ASM) gezeigt (3.20), dass sich der Statorstrom in Drehzeigerdarstellung $\underline{i}_{S}$ in zwei Komponenten aufspalten lässt, welche sehr verschiedene Wirkungen ausüben: der zur Rotorflussverkettung $\underline{\Psi}_{R}$ senkrechte Anteil $i^{\prime \prime}{ }_{S}$ ist verantwortlich für das Drehmoment, während der parallele Anteil $i^{\prime}{ }_{S}$ flussbildend wirkt. Betrachtet man nur die Verzerrungsanteile, so ergibt $i^{\prime \prime}{ }_{S, V Z}$ die Drehmomentwelligkeit, $i^{\prime} S, V Z$ dagegen verursacht wegen der grossen Rotorzeitkonstanten praktisch keine Welligkeit von $\underline{\Psi}_{R}$. Aus diesem Grund ist $i_{S, V Z}$ für die Maschine höchstens wegen der zusätzlichen Verluste von Bedeutung. Es ist naheliegend, die Stromregelung für einen Antrieb so auszulegen, dass diese Eigenschaft ausgenutzt wird.
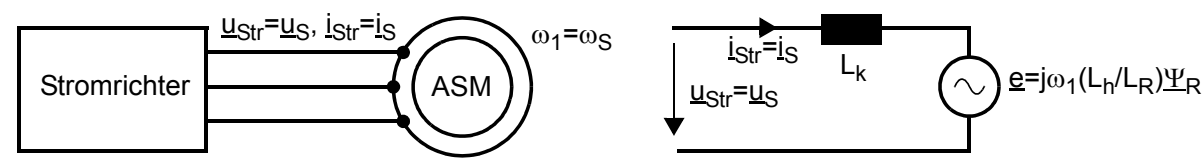

Bild 12.1. Stromrichterantrieb mit ASM,

links: Prinzipschaltung, rechts: zeigerbezogenes Ersatzschaltbild der ASM

In Kapitel 4.4.2 wurde dargelegt, dass Regelungen für Drehstromantriebe häufig in einem mit der Rotorflussverkettung $\underline{\Psi}_{R}$ rotierenden Festzeigersystem mit den Achsen $d$ und $q$ (Anhang B.1.2) implementiert werden. Die Kreisfrequenz des Festzeigersystems entspricht der Statorkreisfrequenz $\omega_{S}$ und ist identisch mit der Grundfrequenz $\omega_{1}$ des Stromrichters. Wie Bild 12.2 zeigt, kommt der Zeiger für die Rotorflussverkettung $\Psi_{R}$ in dieser Darstellung auf die $d$-Achse zu liegen. Die $d$ - und $q$-Komponenten des Strom̃zeigers $i_{S}$ entsprechen den Drehzeigern $i^{\prime}{ }_{S}$ und $i^{\prime \prime}{ }_{S}$. Die Gleichungen (3.16) und (3.20) nehmen die folgende Form an:

$$
\frac{d\left|\Psi_{R}\right|}{d t}=\frac{L_{h}}{T_{R}} i_{S, d}-\frac{1}{T_{R}}\left|\Psi_{R}\right|, \quad T_{R}=\frac{L_{R}}{R_{R}}
$$




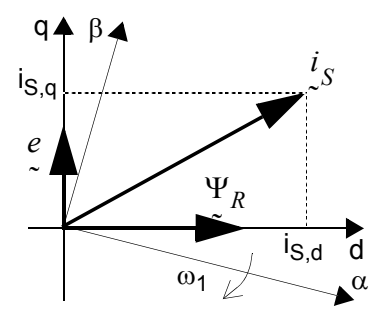

$$
M_{e l}=\frac{3}{2} \frac{L_{h}}{L_{R}}\left|\Psi_{R}\right| i_{S, q}=\frac{3}{2} \frac{|e|}{\omega_{1}} i_{S, q}
$$

Die Gegenspannung $e$ im Ersatzschaltbild der Maschine steht senkrecht auf $\Psi_{R}$ :

$$
\underset{\sim}{e}=j \omega_{1} \frac{L_{h}}{L_{R}} \Psi_{R}
$$

Analog zum Verfahren der Drehzeiger-Komponentenregelung (Kapitel 11.2) kann eine Stromregelung implementiert werden, die sich an den $d$ - und $q$-Komponenten des Stromzeigers orientiert. Konkret wird für $i_{S t r, V Z}$ gemäss Bild 12.3 eine rechteckige Toleranzfläche vorgegeben. Da diese Fläche im Gegensatz zur Regelung in der Drehzeigerdarstel-
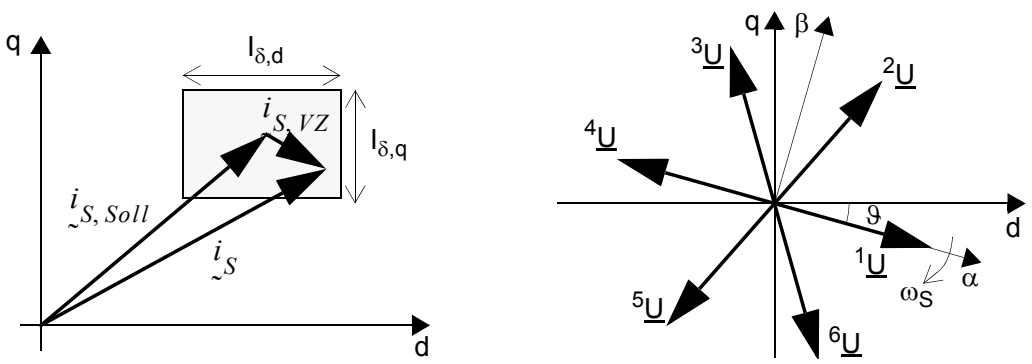

Bild 12.3. Stromregelung im $d q$-System mit rechteckiger Toleranzfläche, links: rechteckige Toleranzfläche für den Stromzeiger, rechts: rotierende Spannungszeiger des Stromrichters im $d q$-System

lung hier ihre Lage bezüglich $\Psi_{R}$ stets beibehält, ist der Spitzenwert der Drehmomentwelligkeit durch die Abmessung $I_{\delta, q}$ bestimmt:

$$
\hat{M}_{e l, V Z}=\frac{3}{2} \frac{|e|}{\omega_{1}} \frac{I_{\delta, q}}{2}=\frac{3}{4} \frac{|e|}{\omega_{1}} I_{\delta, q}
$$

Im Gegensatz zu den Regelverfahren für Drehzeiger, wo die Toleranzfläche möglichst symmetrisch gewählt werden musste, drängt sich hier die Verwendung eines Rechteckes auf. $I_{\delta, q}$ wird so gewählt, dass die Drehmomentwelligkeit den gewünschten Wert nicht überschreitet. Um gleichzeitig die mittlere Schaltfrequenz des Stromrichters klein zu hal- 
ten, wird $I_{\delta, d}$ grösser gewählt, da der Verzerrungsanteil $i_{S, V Z, d}$ einen weniger ungünstigen Einfluss auf den Betrieb der Maschine hat als $i_{S, V Z, q}$.

Das Verfahren weist gegenüber denjenigen für Drehzeiger einen wesentlichen Unterschied auf: die diskreten Spannungszeiger des Stromrichters ${ }^{1} \underline{U}$ bis ${ }^{6} \underline{U}$, welche in der Drehzeigerebene ortsfest waren, rotieren in der $d q$-Ebene mit der Kreisfrequenz - $\omega_{1}$ (Bild 12.3 rechts). Die Stromzeiger $i_{S, \text { Soll }}$ und $i_{S}$ sowie der Gegenspannungszeiger $e$ bleiben dafür im stationären Betrieb fest. Bild 12.4 zeigt das Blockschaltbild der entsprechenden Stromregelung. Wie bei der Stromzeiger-Komponentenregelung (Kapitel 11.2) oder der prädiktiven Regelung mit Schalttabelle (Kapitel 11.3) erfolgt immer dann ein Wechsel auf einen neuen Schaltzustand, wenn der Stromzeiger den Rand der vorgegebenen Fläche erreicht. Die Auswahl des neuen Zustandes hängt von der Stellung der beiden Mehrstufenkomparatoren ab. Zusätzlich wird jedoch hier auch der Winkel $\vartheta$ zwischen dem ortsfesten $d q$ - und dem rotierenden $\alpha \beta$-System benötigt, der die momentane Lage der Schaltspannungen im $d q$-System bezeichnet.

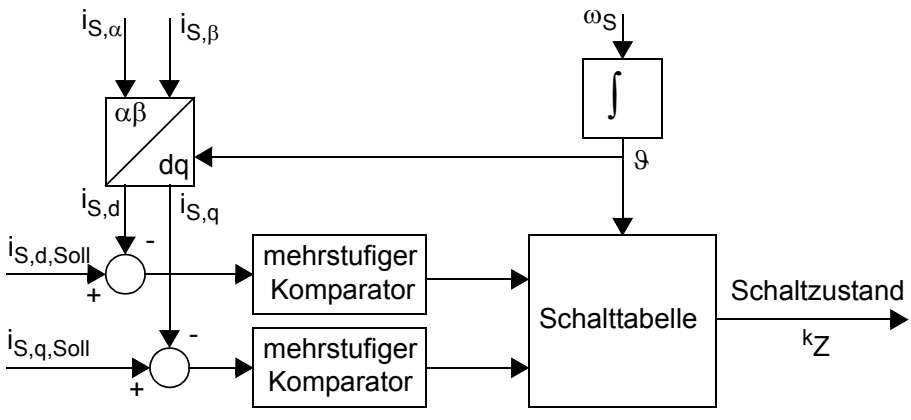

Bild 12.4.

Prinzip der flussorientierten Stromregelung

Es wird an dieser Stelle darauf verzichtet, die genaue Realisierung der Schalttabelle zu beschreiben. Sie erfolgt nach demselben Prinzip, das in Kapitel 11 angewandt wurde: es wird stets derjenige Spannungszustand eingestellt, der den Stromzeiger einerseits ins Innere der Toleranzfläche zurückbewegt und andererseits diese Bewegung möglichst langsam macht, um die Schaltfrequenz niedrig zu halten. Das Verhalten dieser Stromregelung entspricht im wesentlichen demjenigen der drehzeigerorientierten Verfahren, d.h. die Schaltfrequenz ist betriebspunktabhängig und erreicht ihr Maximum im Bereich mittlerer Aussteuerung. Die mittleren Schaltfrequenzen der 3 Brückenzweige sind aber trotz der rechteckigen Toleranzfläche hier gleich, da diese in bezug auf die Schaltzustände rotiert.

Wie schon bei den drehzeigerorientierten Verfahren gezeigt wurde, kann das Verhalten verbessert werden, wenn man auf eine prädiktive Regelung übergeht, d.h. wenn man die Gegenspannung bei der Auswahl des Schaltzustandes berücksichtigt. Bei der flussorientierten Regelung kommt einem dabei entgegen, dass $e$ stets auf der $q$-Achse liegt. Es muss deshalb mit Hilfe von (12.3) nur noch der Betrag berechnet werden.

Das Verfahren der feldorientierten Stromregelung kann in gleicher Weise auch für Synchronmaschinen angewandt werden.

Literatur zu Kapitel 12.1: [Kha1], [Kaz2], [Hol5], [Pfa1] 


\subsection{Direkte Fluss- und Drehmomentregelung}

\subsubsection{Regelung mit Schalttabelle}

Bei den bekannten feldorientierten Regelverfahren für Drehstrommaschinen werden üblicherweise die Regelgrössen Fluss und Drehmoment in getrennten Regelkreisen eingestellt. Sie ergeben, wie auch (12.2) und (12.1) zeigen, zwei zueinander orthogonale Komponenten für den Stromsollwert. Dieser lässt sich zum Beispiel mit Hilfe eines der bisher beschriebenen direkten Stromregelverfahrens einstellen. Bei der direkten Fluss- und Momentregelung werden die beiden Regelkreise zusammen mit dem eigentlichen Modulator auf einfache Weise in eine einzige Funktionseinheit zusammengefasst.

Ausgangspunkt dazu ist die Statorflussverkettung $\underline{\Psi}_{S}$, welche unter Beizug der dynamischen Gleichungen der ASM aus Kapitel 3.2.2.1 aus den Statorgrössen berechnet werden kann.

$$
\underline{\Psi}_{S}=\int\left(\underline{u}_{-}-R_{S_{-}} i\right) d t
$$

Mit Hilfe von $\underline{\Psi}_{S}$ und $\underline{i}_{S}$ kann anschliessend auf das Drehmoment geschlossen werden:

$$
M_{e l}=\frac{3}{2} \operatorname{Im}\left(\underline{\Psi}_{S}{ }^{*} i_{S}\right)=\frac{3}{2}\left(\Psi_{S, \alpha} i_{S, \beta}-\Psi_{S, \beta} i_{S, \alpha}\right)
$$

Damit sind die beiden Regelgrössen durch Klemmengrössen bestimmbar, wobei von den Maschinenparametern nur der Widerstand der Statorwicklung $R_{S}$ bekannt sein muss. Im Bereich genügend grosser Drehzahl kann zudem der Spannungsabfall $R_{S \underline{i}_{S}}$ häufig vernachlässigt werden.

Im weiteren ist die Tatsache von Bedeutung, dass das Drehmoment eine Funktion der Rotorkreisfrequenz $\omega_{R}$ ist. Diese stellt, wie schon in Kapitel 2.2.2 (Bild 2.5) gezeigt wurde, die Differenz zwischen der Drehzahl und der Statorkreisfrequenz dar:

$$
M_{e l}=F\left(\omega_{R}\right) \quad \text { mit } \quad\left\{\begin{array}{l}
\omega_{R}>0 \Rightarrow M_{e l}>0 \\
\omega_{R}<0 \Rightarrow M_{e l}<0
\end{array}\right.
$$

Da bei einem Antrieb das Drehmoment normalerweise die primär zu regelnde Grösse und die Statorkreisfrequenz eine der Stellgrössen darstellt, ist es sinnvoll, die Beziehung (12.7) in die Form (12.8) umzuwandeln:

$$
\omega_{S, \text { Soll }}=F\left(M_{e l, \text { Soll }}, \omega_{\text {mech }}\right) \text { mit }\left\{\begin{array}{l}
M_{e l, \text { Soll }}>0 \Rightarrow \omega_{S}>\omega_{\text {mech }} \\
M_{e l, \text { Soll }}<0 \Rightarrow \omega_{S}<\omega_{\text {mech }}
\end{array}\right.
$$

Die Gleichung zeigt, dass für ein positives Moment die Statorkreisfrequenz grösser als die mit der Polpaarzahl multiplizierte Drehzahl sein muss und für ein negatives Moment kleiner.

Das Regelverfahren besteht darin, dass die Statorflussverkettung auf konstantem Betrag $\underline{\Psi}_{S, \text { Soll }} \mid$ gehalten und mit der dem gewünschten Drehmoment entsprechenden Kreisfrequenz $\omega_{S, \text { Soll }}$ rotiert wird. Da der Stromrichter nur diskrete Ausgangsspannungen einstellen kann, ist dies, wie in Bild 12.5 dargestellt, nur für die kurzzeitig gemittelten Grössen 

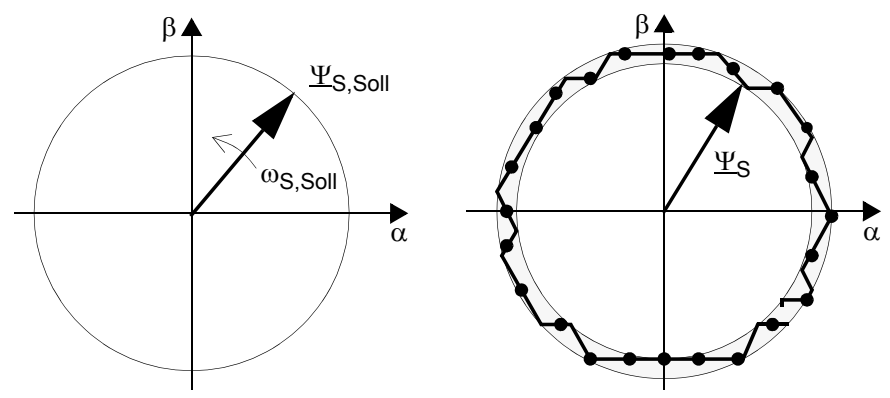

Bild 12.5.

Führung der Statorflussverkettung bei einer Asynchronmaschine, links: ideal, rechts: realer Verlauf bei Stromrichterspeisung

möglich. Für die Länge des Flusszeigers wird deshalb ein Toleranzband um den Sollwert herum vorgegeben. Die momentane Kreisfrequenz des Zeigers und damit das momentane Drehmoment hängt vom eingestellten Schaltzustand ab. Mit (12.5) lässt sich die Bewegungsrichtung und die Geschwindigkeit des Flusszeigers angeben. Geht man davon aus, dass der Spannungsabfall am Statorwiderstand klein ist, so sind diese Grössen allein durch den eingestellten Spannungszeiger bestimmt:

$$
\frac{d \underline{\Psi}_{S}}{d t}=\underline{u}_{S}-R_{S} i_{-S}, \quad R_{S} \rightarrow 0 \quad \Rightarrow \quad \frac{d \underline{\Psi}_{S}}{d t}=\underline{u}_{S}={ }^{k} \underline{U}
$$

Ist ein Nullzustand eingestellt, so bedeutet dies Stillstand des Flusszeigers. Diese Stellen sind in Bild 12.5 rechts durch einen Punkt gekennzeichnet. Da dort die Kreisfrequenz des Flusszeigers momentan null ist, ergibt sich eine von der Drehzahl abhängige negative Rotorkreisfrequenz:

$$
k=0,7 \quad \Rightarrow \quad \omega_{S}=0, \quad \omega_{R}=-\omega_{\text {mech }}
$$

Ist ein spannungsbildender Zustand eingestellt, so wird dieser jeweils so gewählt, dass er eine möglichst tangentiale Bewegung des Flusszeigers verursacht. Dadurch ergibt sich für die entsprechende Kreisfrequenz näherungsweise:

$$
\begin{aligned}
k=1, \ldots, 6 \Rightarrow \omega_{S} & \approx \mid \frac{{ }^{k}}{\underline{\Psi}_{S} \mid} \approx \frac{\left|{ }^{k} \underline{U}\right|}{\left|\underline{\Psi}_{S, \text { Soll }}\right|}, \\
& \omega_{R} \approx \frac{\left|{ }^{k}\right|}{\left|\underline{\Psi}_{S, \text { Soll }}\right|}-\omega_{\text {mech }}>0, \quad\left|{ }^{k}\right|=\frac{2}{3} U_{d}
\end{aligned}
$$

Damit im Mittel wahlweise ein positives oder ein negatives Drehmoment entwickelt werden kann, muss die momentane Kreisfrequenz $\omega_{S}$ hier auf jeden Fall grösser als $\omega_{\text {mech }}$ sein. Dies wird durch eine richtige Auslegung des Stromrichters erreicht (genügend Spannungsreserve). Durch abwechslungsweises Anlegen von Nullzuständen und spannungsbildenden Zuständen wird der kurzzeitig gemittelte Wert von $\omega_{S}$ seinem Sollwert angepasst. Das Drehmoment reagiert dabei nicht unmittelbar auf Sprünge von $\omega_{R}$, sondern mit einer Verzögerung, wie dies in Bild 12.6 skizziert ist. Diese Tatsache kann aus den Gleichungen für die ASM abgeleitet werden. Sie lässt sich aber auch einfach begründen mit der Feststellung, dass der Statorstrom wegen der Induktivitäten in der Maschine auf einen 


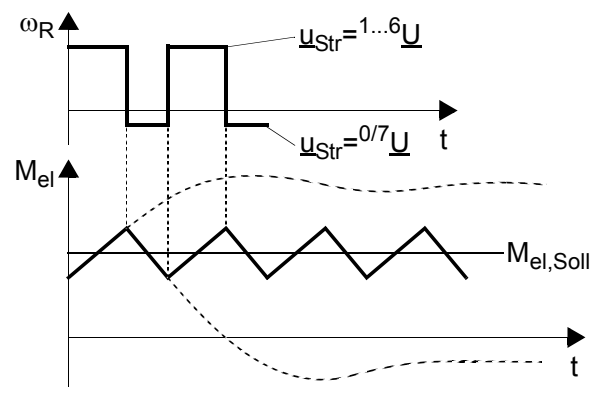

Bild 12.6.

Qualitativer Drehmomentverlauf in einer Asynchronmaschine bei schrittartigen Änderungen der Rotorfrequenz

Spannungssprung nur verzögert reagiert. Da die Schaltzeitintervalle gegenüber den entsprechenden Zeitkonstanten klein sind, ergibt sich der dargestellte sägezahnförmige Drehmomentverlauf.

Das Blockdiagramm in Bild 12.7 zeigt eine Regelstruktur nach dem Prinzip der direkten Fluss- und Momentregelung. Mit Hilfe der Ausgangssignale von zwei Hysteresekomparatoren für Flussbetrag und Drehmoment wird ähnlich wie bei der Stromzeigersteuerung direkt in einer Tabelle der einzustellende Schaltzustand bestimmt. Die entsprechenden Istwerte werden aus den gemessenen und transformierten Statorspannungen und -strömen nach (12.5) und (12.6) berechnet. Dazu wird zusätzlich die Information benötigt, in welchem $\pi / 3$-Sektor $S$ I bis $S$ VI sich $\underline{\Psi}_{S}$ momentan befindet. Die Schaltstrategie für einen Sektor ist in Bild 12.8 veranschaulicht. Es kommen jeweils zwei spannungsbildende Zustände zum Zug, wovon einer den Flussbetrag kleiner und der andere grösser werden lässt. Beide bewegen den Zeiger jedoch in erster Linie mit positivem Drehsinn in tangentialer Richtung. In der ebenfalls abgebildeten Schalttabelle für den grau hinterlegten Sektor ist ersichtlich, dass der Ausgang des Reglers für den Flussbetrag $s_{\Psi}$ bestimmt, welcher der beiden spannungsbildenden Zustände anzulegen ist. Der Momentregler ist dagegen zuständig für den Wechsel zwischen spannungsbildenden Zustand und Nullzuständen. Er kann damit den Flusszeiger vorübergehend zum Stillstand bringen und auf diese Weise die mittlere Kreisfrequenz einstellen. Beim Umschalten auf einen Nullzustand wird, wie schon bei den Stromzeigerregelungen, derjenige gewählt, der durch Umschalten eines Brückenzweiges erreichbar ist, so dass die Schaltfrequenz möglichst klein ausfällt. Die vollständige Schalttabelle für den vollen Kreisumfang besteht aus 6 gleichartigen Teiltabellen nach Bild 12.8, die je für einen Sektor $S$ I bis $S$ VI gelten.

Die direkte Flusssteuerung stellt ein sehr einfaches feldorientiertes Regelverfahren dar. Es stellt über die Momentregelung automatisch die richtige Rotorkreisfrequenz ein, ohne dass die Drehzahl der ASM bekannt sein muss. Das Verfahren ist ausserdem robust und leicht implementierbar, da ausser $R_{S}$ keine der zum Teil betriebsabhängigen Maschinenparameter verwendet werden. Die einzige Information über den Betriebszustand der Maschine liefert der Statorstrom. In Ergänzung zum dargestellten Prinzip sind die folgenden Feststellungen von Bedeutung:

- Die Schaltstrategie in Bild 12.8 gilt für eine Drehrichtung der Maschine. Für eine Umkehr der Drehrichtung ist die Schalttabelle entsprechend zu erweitern.

- Im Bereich sehr kleiner Drehzahlen kann $\omega_{R}$ bei angelegtem Nullzustand nur einen kleinen negativen Wert erreichen, was einen langsamen Abbau des Drehmomentes zur 


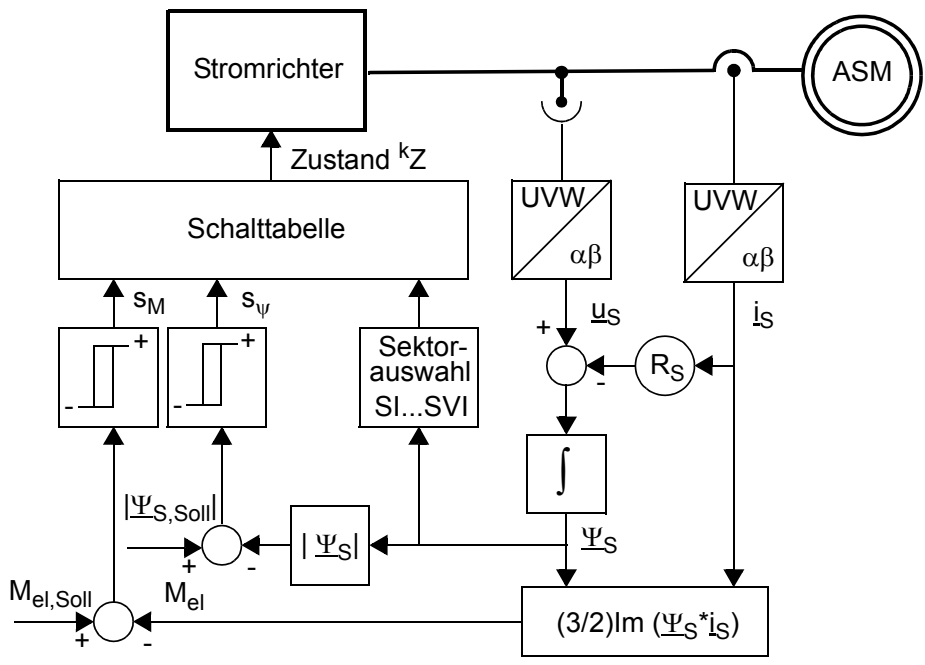

Bild 12.7.

Blockdiagramm einer direkten Fluss- und Momentregelung

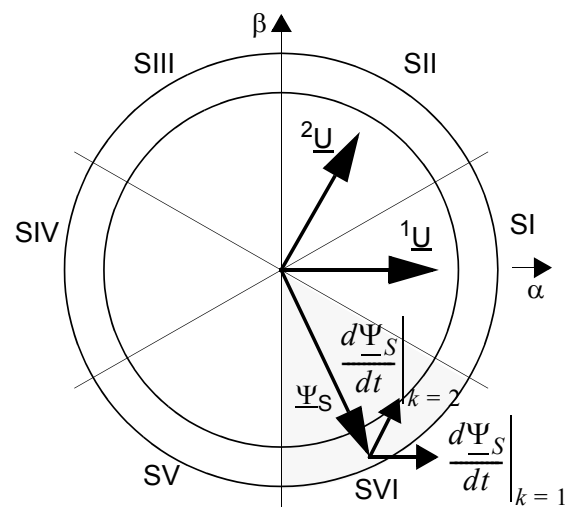

Schalttabelle für den Sektor SVI

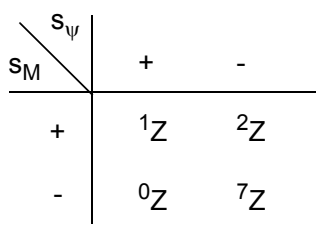

Bild 12.8.

Schaltstrategie für $\underline{\Psi}_{S}$ im Sektor SVI

Folge hat. Um die Dynamik der Regelung zu erhöhen, kann in diesem Betriebsbereich anstelle des Nullzustandes ein anderer spannungsbildender Zustand angelegt werden, der den Flusszeiger nicht nur stillstehen lässt, sondern sogar rückwärts bewegt. Dazu ist ebenfalls eine erweiterte Schalttabelle erforderlich.

- Der Bereich kleiner Drehzahlen ist jedoch in der Praxis ohnehin problematisch. Der Anteil der Statorspannung, der zur Flussbildung beiträgt, ist klein und die Berechnung der Statorflussverkettung über die Integration wird ungenau. Deshalb wird normalerweise in diesem Betriebsbereich ein anderes Regelverfahren verwendet.

- Die Messung der Statorspannungen in Bild 12.7 ist nur der Übersicht wegen in dieser Form dargestellt. Die Statorspannungen bzw. der Spannungszeiger $\underline{u}_{S}$ können mit Hilfe des angelegten Schaltzustandes und der als konstant angenommenen Zwischenkreisspannung berechnet werden. Soll deren Welligkeit in der Regelung mitberücksichtigt werden, so kann dazu auch die gemessene Zwischenkreisspannung verwendet werden.

- Eine Feldschwächung lässt sich einfach durch Reduktion von $\left|\underline{\Psi}_{S, \text { Soll }}\right|$ erreichen. 
- Die direkte Fluss- und Momentregelung kann auch bei Synchronmaschinen eingesetzt werden.

Schaltfrequenz: Ein quantitativer Vergleich der Eigenschaften des vorgestellten Verfahrens mit anderen Steuerverfahren ist schwierig, da andere Kenngrössen im Vordergrund stehen. Aus diesem Grund wird an dieser Stelle auf die Präsentation von Resultaten verzichtet. Qualitativ lässt sich das Verfahren am ehesten mit der prädiktiven Stromregelung mit Schalttabelle vergleichen. Es kommen dieselben Sequenzen von Schaltzuständen zum Zuge. Die Schaltfrequenzen der 3 Brückenzweige stellen sich jedoch auf denselben Mittelwert ein. Dieser ist durch die Breite der Toleranzbänder für Fluss und Drehmoment bestimmt. Wie bei den direkten Stromregelverfahren wird er im Bereich mittlerer Aussteuerung des Stromrichters, was einer mittleren Drehzahl entspricht, maximal.

Literatur zu Kapitel 12.2.1: [Tak3], [Mor1], [Tör1]

\subsubsection{Direkte Selbstregelung}

Die direkte Selbstregelung (DSR) stellt einen Spezialfall der direkten Fluss- und Momentregelung dar. Wegen ihrer Einfachheit und ihrer grossen Verbreitung in der Praxis soll sie hier speziell zur Sprache kommen. Bei Antrieben mit grosser Leistung muss die Schaltfrequenz des Stromrichters möglichst klein gehalten werden. Dies wird durch entsprechend grosse Toleranzbänder erreicht. Dabei ist zu beachten, dass die Drehmomentwelligkeit bei Antrieben die grössere Rolle spielt als die Welligkeit der Statorflussverkettung. Diese beeinflusst den Betrieb eigentlich nur dadurch, dass sie zu einer kleinen Reduktion der maximalen Fluss-Grundschwingungsamplitude zwingt, damit das Eisen zu keinem Zeitpunkt in die Sättigung gerät. Es ist deshalb sinnvoll, mit der zur Verfügung stehenden Schaltfrequenz in erster Linie die Drehmomentwelligkeit klein zu halten.

Vergrössert man das Toleranzband für den Flussbetrag $\left|\underline{\Psi}_{S}\right|$, so geht die Schaltfrequenz des entsprechenden Komparators zurück. Bereits bei einer maximalen Abweichung von $\pm 6.75 \%$ vom Sollwert kann der Verlauf des Flusszeigers, wie in Bild 12.9 dargestellt, zu einem Sechseck degenerieren. In zyklischer Folge wird mit jedem der spannungsbildenden Zustände eine Seite des Sechseckes durchfahren. Die Ecken entsprechen den Übergängen auf den nächsten Zustand. Sie liegen jeweils in der Mitte der Sektoren SI bis SVI.

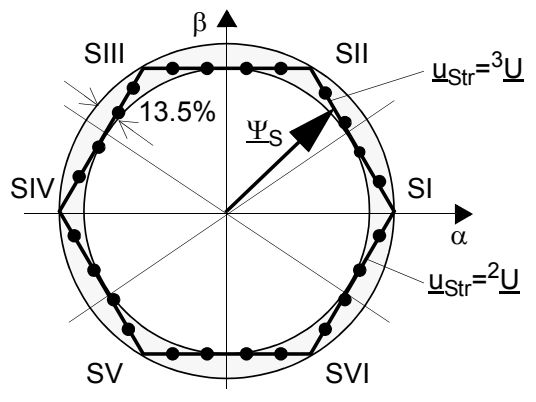

Bild 12.9.

Verlauf des Zeiger für die Statorflussverkettung bei der DSR 
Geht man davon aus, dass $\underline{\Psi}_{S}$ den in Bild 12.9 gezeigten Verlauf annimmt, so kann die entsprechende Regelung sehr einfach realisiert werden: die Sechseckkurve lässt sich gemäss Bild 12.10 unter Verwendung der phasenbezogenen Flusskomponenten erzeugen. Ohne eingeschaltete Stillstände des Flusszeigers entspricht dies der Grundfrequenzsteuerung, d.h. der Durchmesser des Sechsecks und die Geschwindigkeit des Flusszeigers sind fest vorgegeben. Die zusätzliche Drehmomentregelung nach Bild 12.11 erlaubt die Variation der mittleren Kreisfrequenz des Flusszeigers, indem sie zwischendurch nach Bedarf Nullzustände einlegt. Die trapezförmigen Verläufe der phasenbezogenen Flusskomponenten können dadurch bei gleichbleibender Amplitude in der Zeitachse gedehnt werden. Zur Vervollständigung des Blockschaltbildes der DSR im Vergleich zu Bild 12.7 wären in Bild 12.11 die Blöcke zur Erfassung der Statorspannungen und -ströme sowie zur Berechnung des Drehmomentes zu ergänzen.


Bild 12.10. Flussregelung bei der DSR ohne Nullzustände, links: Blockdiagramm, rechts: Verläufe der Phasengrössen
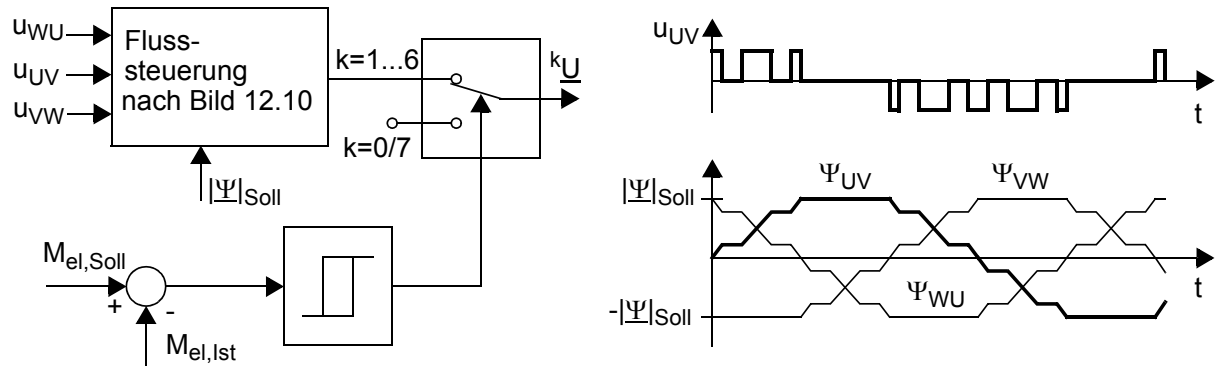

Bild 12.11. Erzeugung der Schaltsignale bei der DSR, links: Blockdiagramm, rechts: Verläufe der Phasengrössen

Schaltfrequenz: Die Schaltfrequenz der DSR wird in erster Linie bestimmt durch das Toleranzband für das Drehmoment. Sie variiert jedoch bei festem Toleranzband in Abhängigkeit des Betriebspunktes der Maschine und erreicht wie das vorher besprochene allgemeine Verfahren im mittleren Drehzahlbereich ihr Maximum. Erwähnenswert ist, dass die DSR eine stetige Steigerung der Aussteuerung des Stromrichters bis zur reinen Grundfre- 
quenzsteuerung ermöglicht. Bei der Fluss- und Momentregelung nach Kapitel 12.2.1 mit Führung des Flusszeigers auf einem Kreis müsste dazu das Toleranzband im obersten Aussteuerbereich (d.h. in der Nähe der Nenndrehzahl) soweit vergrössert werden, dass sich ebenfalls ein sechseckförmiger Verlauf einstellen kann.

Literatur zu Kapitel 12.2.2: [Dep2], [Dep3]

\subsection{Deltamodulation}

Die Deltamodulation ist ein äusserst einfaches Steuerverfahren für anspruchlose Anwendungen von Drehstromantrieben. Sie ist besonders geeignet für die Spannungsführung nach dem Spannungs-Frequenz-Kennlinien Verfahren und stellt eine reine Spannungssteuerung dar.

Das Prinzip der Deltamodulation ist in Bild 12.12 dargestellt. Das Verfahren ist phasenorientiert und besteht aus 3 gleichen Modulatoren für die 3 Phasenspannungen. Sie sind nur für sinusförmige Sollwerte geeignet. Das Schaltsignal wird durch einen Komparator mit Hysterese erzeugt. An dessen Eingang liegt die Differenz zwischen dem Sollwertsignal $x_{\text {Soll }}$ und dem zurückgeführten und integrierten Schaltsignal $x_{I s t}$. Es liegt damit ein Zweipunktregler für $x_{I s t}$ vor. Die Bezugskreisfrequenz $\omega_{B}$ stellt einen Skalierungsfaktor dar, dessen Bedeutung später ersichtlich wird.
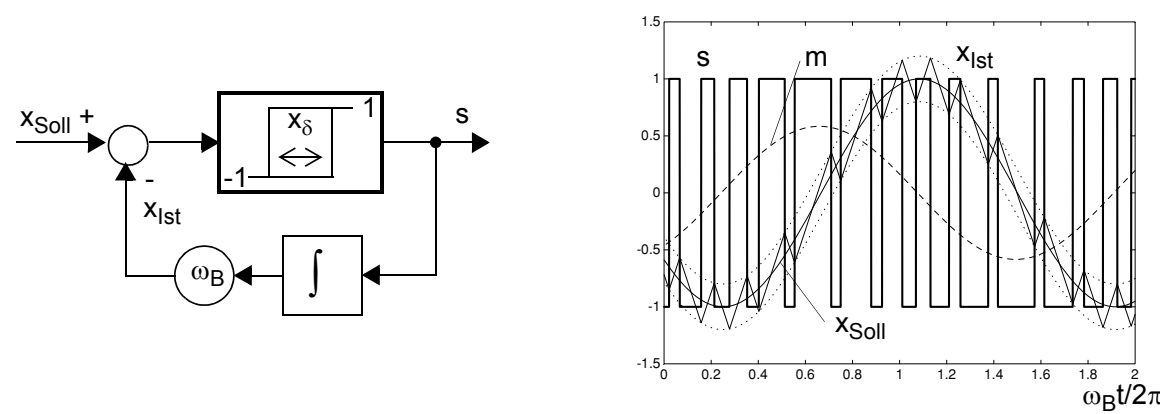

Bild 12.12. Links: Prinzip der Deltamodulation für einen Brückenzweig (eine Phase), rechts: Signalverläufe im Modulator für $\omega_{1}=0.6 \omega_{B}$ (Punkt A in Bild 12.14)

Die Schaltfunktion $s$ ist proportional zur Mittelpunktspannung am Stromrichter. Vernachlässigt man den Widerstand der Statorwicklung $R_{S}$, so ist damit die Grundschwingung von $x_{I s t}$ proportional zu derjenigen der Statorflussverkettung $\Psi_{S}$ in der entsprechenden Phase. Diese Aussage kann aus (12.5) abgeleitet werden, wenn in der Gleichung die Zeiger durch Phasengrössen ersetzt werden. So betrachtet ist die Deltamodulation eine Steuerung, welche die Statorflussverkettung in der Maschine näherungsweise proportional zum angelegten Sollwert $x_{\text {Soll }}$ einstellt. Der Verzerrungsanteil von $x_{I s t}$ stimmt aller- 
dings nicht mit demjenigen der Flussverkettung überein, weil für deren Erzeugung die Phasenspannung und nicht die Mittelpunktspannung massgebend ist.

Die Kurvenverläufe in Bild 12.12 rechts zeigen, dass $x_{I s t}$ mit der für einen Zweipunktregler typischen Charakteristik dem Sollwert folgt. Die Schaltfunktion stellt sich dabei so ein, dass ihre Grundschwingung, entsprechend der Modulationsfunktion $m$, dem Sollwert um $\pi / 2$ voraus eilt.

Für die analytische Behandlung des Verfahrens kann auf das Kapitel 10.1 zurückgegriffen werden. Der Deltamodulator funktioniert analog zu einem Zweipunktregler für den Phasenstrom einer Halbbrücke bei einer Gegenspannung von null. Das rückgeführte Signal $x_{I s t}$ entspricht dabei dem Phasenstrom und $x_{\text {Soll }}$ dessen Sollwert. Der Integrator ist die Nachbildung der Lastinduktivität. Es lassen sich die Gleichungen aus Kapitel 10.1.2 anwenden, wobei stets $\hat{e}=0$ anzunehmen ist. Ersetzt man in (10.16) die Grössen $\hat{\imath}_{\text {Soll }}$ durch $\hat{x}_{\text {Soll, }} L_{k}$ durch $1 / \omega_{B}$ und $U_{d} / 2$ durch 1 , so ergibt sich für den Modulationsgrad des Stromrichters:

$$
M=\frac{\omega_{1}}{\omega_{B}} \hat{x}_{\text {Soll }}
$$

Er ist also proportional zur Grundkreisfrequenz $\omega_{1}$ und zur Amplitude des Sollwertes $\hat{x}_{\text {Soll }}$ im Modulator.

Bild 12.13 zeigt das Blockdiagramm für einen Antrieb, der nach dem Spannungs-Frequenz-Kennlinien Verfahren betrieben wird. Der Drehzahlregler erzeugt den Sollwert für die Statorkreisfrequenz $\omega_{1}=\omega_{S}$. Ein spannungsgesteuerter Dreiphasenoszillator (VCO) generiert daraus die sinusförmigen Eingangssignale $x_{U / V / W \text {, Soll }}$ für die 3 Deltamodulatoren. Sie sind proportional zu den gewünschten Statorflussverkettungen in der Maschine. Ihre Amplituden sind konstant und betragen $\hat{x}_{U / V / W, \text { Soll }}=1$, was dem Nennfluss entsprechen soll. Der Modulationsgrad am Stromrichter stellt sich gemäss (12.12) ein, d.h. er ist proportional zur Grundkreisfrequenz $\omega_{1}$.

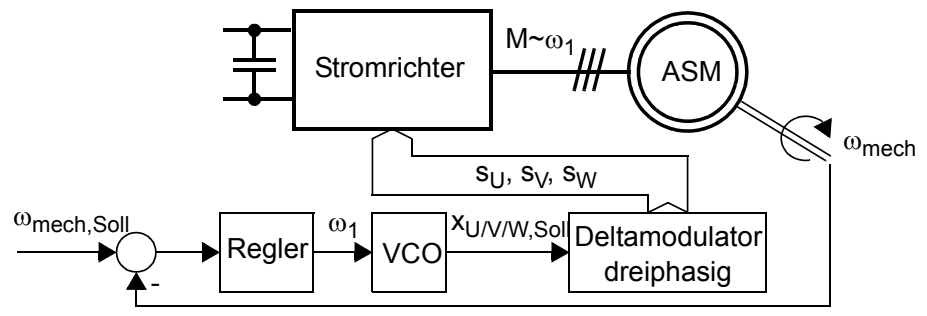

Bild 12.13.

Regelung der ASM nach dem SpannungsFrequenz-Kennlinien Verfahren

In Bild 12.14 links ist die entsprechende Kennlinie für den Modulationsgrad dargestellt. Die Proportionalität nach (12.12) gilt nur unterhalb der Bezugskreisfrequenz $\omega_{B}$. Die Signalverläufe in Bild 12.12 stammen aus diesem Bereich (Punkt A). Bei $\omega_{B}$ wird $M=1$. Oberhalb davon beginnt die Kennlinie abzuflachen und verläuft ab $\omega_{G F}$ schliesslich horizontal. Die Beispiele in Bild 12.15 zeigen entsprechende Kurvenverläufe. Man erkennt, dass $x_{I s t}$ hier nicht mehr jederzeit im Toleranzband gehalten werden kann. Der Modulator ist im Bild links leicht und rechts stark übersteuert. Bei $\omega_{G F}$ geht die Schaltfunktion in 
eine Grundfrequenzsteuerung über und der Modulationsgrad erreicht dementsprechend $M=1.27$. Die Amplitude von $x_{I s t}$ nimmt mit steigender Frequenz ab. Dies bedeutet, dass in der Maschine eine Feldschwächung stattfindet. Der Fluss wird proportional mit der Grundschwingung von $X_{I s t}$ reduziert. Die Amplituden der Sollwerte $x_{U / V / W, \text { Soll }}$ brauchen dazu nicht verändert zu werden.


Bild 12.14. Links: Spannungs-Frequenz-Kennlinie, rechts: mittlere Schaltfrequenz in Funktion der Grundkreisfrequenz
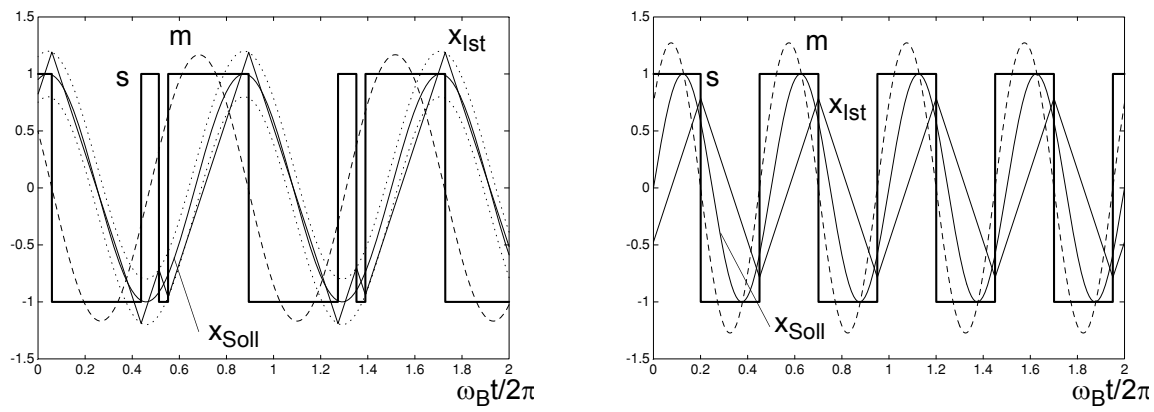

Bild 12.15. Kurvenverläufe eines Deltamodulators bei Übersteuerung, links: $\omega_{1}=1.2 \omega_{B}$ (Punkt B in Bild 12.14), rechts: $\omega_{1}=2 \omega_{B}$ (Punkt C in Bild 12.14)

Die Übergangsstelle $\omega_{G F}$ ist leicht abhängig von der Grösse des Toleranzbandes (Komparatorhysterese). Es kann gezeigt werden, dass das Verhältnis von $\omega_{G F} \mathbf{z u} \omega_{B}$ maximal 1.86 erreichen kann. In den dargestellten Beispielen ist im weiteren zu erkennen, dass die Phasenverschiebung zwischen $m$ und $x_{\text {Soll }}$ im Bereich der Übersteuerung mit zunehmender Kreisfrequenz kleiner wird.

Schaltfrequenz: Für die Berechnung der mittleren Schaltfrequenz des Modulators kann wiederum auf die Formeln in Kapitel 10.1 zurückgegriffen werden. Im linearen Aussteuerbereich unterhalb von $\omega_{B}$ lässt sich aus (10.20) ableiten:

$$
f_{s}=\frac{\omega_{B}}{2 x_{\delta}}\left(1-\frac{M^{2}}{2}\right) \quad \Rightarrow \quad f_{s}=\frac{\omega_{B}}{2 x_{\delta}}\left(1-\frac{\omega_{1}^{2}}{2 \omega_{B}^{2}}\right), \quad \omega_{1} \leq \omega_{B}
$$


Die Schaltfrequenz ist bei $M=0$ maximal und nimmt bis $M=1 \mathrm{um} 50 \%$ ab. Ihr weiterer Verlauf ist in der graphischen Darstellung in Bild 12.14 rechts ersichtlich: im Bereich der Übersteuerung sinkt sie vorerst weiter, bis der Modulator in Grundfrequenzsteuerung übergeht. Da die Schaltfrequenz dort mit der Grundfrequenz gekoppelt ist, steigt sie schliesslich wieder linear an. In Anbetracht dessen, dass eine dreiphasige Schaltung vorliegt, ergibt sich im Bereich niedriger Aussteuerung eine unnötig grosse Schaltfrequenz.

Die Verläufe der Phasenströme, d.h. vor allem die ihrer Verzerrungsanteile, hängen nicht nur von den einzelnen Schaltfunktionen ab, sondern vor allem auch davon, ob und wie die an sich unabhängigen Modulatoren der 3 Phasen miteinander koordiniert werden. Verzichtet man auf eine Koordination, so ist es dem Zufall überlassen, ob die Modulatoren im Gleichtakt oder versetzt arbeiten. Unter Umständen können auf diese Weise unnötig grosse und vor allem stark unsymmetrische Verzerrungsströme auftreten. Aus diesem Grund ist eine Koordination zu empfehlen. Eine einfache Möglichkeit dazu besteht darin, dass die Integratoren jeweils bei den Nulldurchgängen der Sollwerte der entsprechenden Phase auf null zurückgesetzt werden. Die Schaltfunktionen werden dadurch im stationären Fall synchron und exakt symmetrisch. Dasselbe gilt dann auch für die Spannungen und Ströme.

Die Qualität der Ausgangssignale ist bei der Deltamodulation im Vergleich zu anderen Verfahren schlechter. Die Vorzüge liegen in der eleganten Weise der Realisierung einer linearen Frequenz-Spannungs-Kennlinie und in der zusätzlichen Eigenschaft, dass diese Kennlinie eine kontinuierliche Fortsetzung bis zu einer beliebig hohen Grundfrequenz aufweist, so dass sich von selbst eine Feldschwächung ergibt. Die unnötig hohe Schaltfrequenz bei niedrigen Drehzahlen erlaubt jedoch nur Anwendungen im Bereich kleiner Leistungen und nur dann, wenn die Schaltverluste keine wesentliche Rolle spielen. Zudem genügt das beschriebene Regelverfahren dynamisch nur sehr kleinen Ansprüchen.

Literatur zu Kapitel 12.3: [Zio1], [Rah1], [Gre1] 


\section{Vergleich der wichtigsten Steuerverfahren}

Im folgenden werden die wichtigsten Steuerverfahren aus den Kapiteln 6 bis 12 miteinander verglichen. Die genauen Erläuterungen der verwendeten Begriffe, Formeln und Sachverhalte sind in den entsprechenden Kapiteln zu finden.

\subsection{Klassierung der Verfahren}

Bild 13.1 zeigt ein Klassierungsschema, dass die behandelten Steuerverfahren in 4 Grundtypen einteilt. Drei davon sind Verfahren zur Spannungssteuerung, die für sich allein keinen geschlossenen Regelkreis darstellen (Feed-Forward). Sie lassen sich weiter unterteilen in die Steuerungen mit Off-Line bestimmten, synchronen Pulsmustern und die OnLine-Verfahren. Darunter fallen die Trägerverfahren und die Drehzeigermodulation. Sie sind vom Prinzip her asynchron, können jedoch auch synchron eingesetzt werden. Die Grundfrequenzsteuerung wird in diesem Schema den Off-Line-Verfahren zugeordnet, weil sie auf einem vorgegebenen Pulsmuster basiert. Im weiteren gehören die vorausberechneten Pulsmuster mit Schaltzahlen grösser eins zu den Off-Line-Verfahren. Die andere Hauptgruppe von Steuerverfahren sind diejenigen, die direkt eine Grösse (Strom, Fluss, Drehmoment) regeln, so dass zwischen dem Modulator und der Schaltung eine Rückkopplung besteht (Feed-Back). Sie sind zwangsläufig On-Line-Verfahren und durchwegs asynchron. Sie könnten weiter in Untergruppen aufgespaltet werden (z.B. nach ihren Regelgrössen). Da ihre Eigenschaften bezüglich der wichtigsten Kenngrössen eines Steuerverfahrens jedoch alle sehr ähnlich sind, wird hier darauf verzichtet.

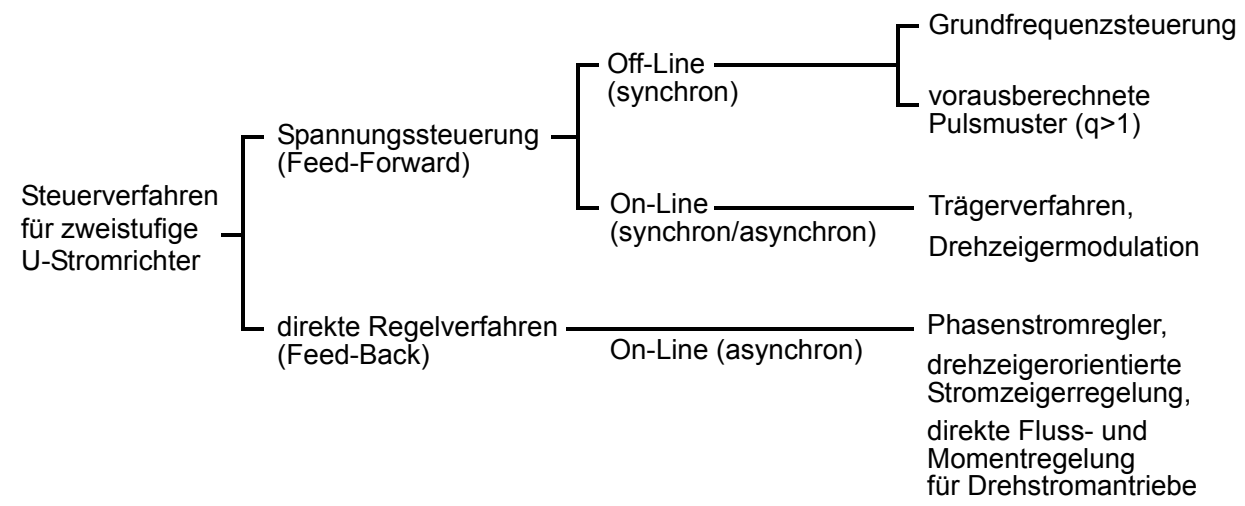

Bild 13.1. Klassierungsschema für Steuerverfahren des zweistufigen U-Stromrichters 


\subsection{Kennlinien}

Als erstes werden die in der Praxis wichtigsten Kenngrössen der AC-Seite des Stromrichters in Funktion des Modulationsgrades betrachtet. Es sind dies: der Effektivwert und der Spitzenwert des Verzerrungsstromes in einer Phase, die Schaltfrequenz bzw. die Schaltzahl und für Antriebe die Drehmomentwelligkeit im Leerlauf. Es werden jeweils 4 Verfahren als Vertreter der 4 im Schema in Bild 13.1 definierten Grundtypen verglichen. Die Vergleiche werden für die ein- und dreiphasigen Brückenschaltungen durchgeführt.

\subsubsection{Einphasige Brücke}

Betrachtet werden die Kennlinien für sinusförmige Sollwerte und konstanter Grundfrequenz der Ausgangsspannungen. Sie sind massgebend für zwei der Hauptanwendungsgebiete der einphasigen, selbstgeführten Stromrichter, nämlich der Netzstromrichter und der Speisung von Inselnetzen.

Bild 13.2 zeigt die Kennlinien der Effektiv- und Spitzenwerte des Verzerrungsanteils im Phasenstrom sowie der zugehörigen Schaltzahlen für die folgenden 4 Verfahren:

Kurven a: Grundfrequenzsteuerung nach Kapitel 6.2.

Kurven $b$ : Trägerverfahren mit dreieckförmigem Trägersignal und versetzter Taktung (doppelte Trägerfrequenz in den Ausgangsgrössen) nach Kapitel 7.3.

Kurven c: optimierte Pulsmuster für minimalen Effektivwert des Verzerrungsstromes nach Kapitel 9.3.2.

Kurven d: Dreipunktregler für den Phasenstrom nach Kapitel 10.2.

Die Kennlinien sind mit den in den entsprechenden Kapiteln vorgestellten analytischen Methoden berechnet. Die verwendeten Formeln sind im Bild angegeben.

\section{Kommentar zu Bild 13.2:}

- Die Aussteuergrenze liegt für die Grundfrequenzsteuerung und die optimierten Pulsmuster bei $M=4 / \pi$, bei den beiden anderen Verfahren dagegen nur bei eins. (Grundsätzlich lässt sich aber beim Trägerfahren der Modulationsgrad durch Übersteuerung ebenfalls bis auf $4 / \pi$ steigern.)

- Die Grundfrequenzsteuerung ergibt in einem weiten Bereich den deutlich grössten Verzerrungsstrom, allerdings bei der minimal möglichen Schaltzahl bzw. Schaltfrequenz.

- Die anderen Verfahren weisen eine umgekehrte Proportionalität zwischen den Kennlinien des Verzerrungsstromes (Effektivwert und Spitzenwert), und derjenigen der Schaltzahl auf. Sie gilt für Trägerverfahren und Phasenstromregler exakt und für die optimierten Pulsmuster näherungsweise. Der Verzerrungsstrom kann also durch Erhöhen der Schaltfrequenz beliebig reduziert werden.

- Im weiteren zeigt sich deutlich die grundsätzlich verschiedene Charakteristik der Verfahren: die spannungssteuernden Verfahren (a bis c) weisen bei einer konstanten Schaltzahl einen aussteuerungsabhängigen Verzerrungsstrom auf. Beim direkten Stromregler (Verfahren d) ist dieser dagegen konstant. Dafür ist die Schaltzahl variabel. 

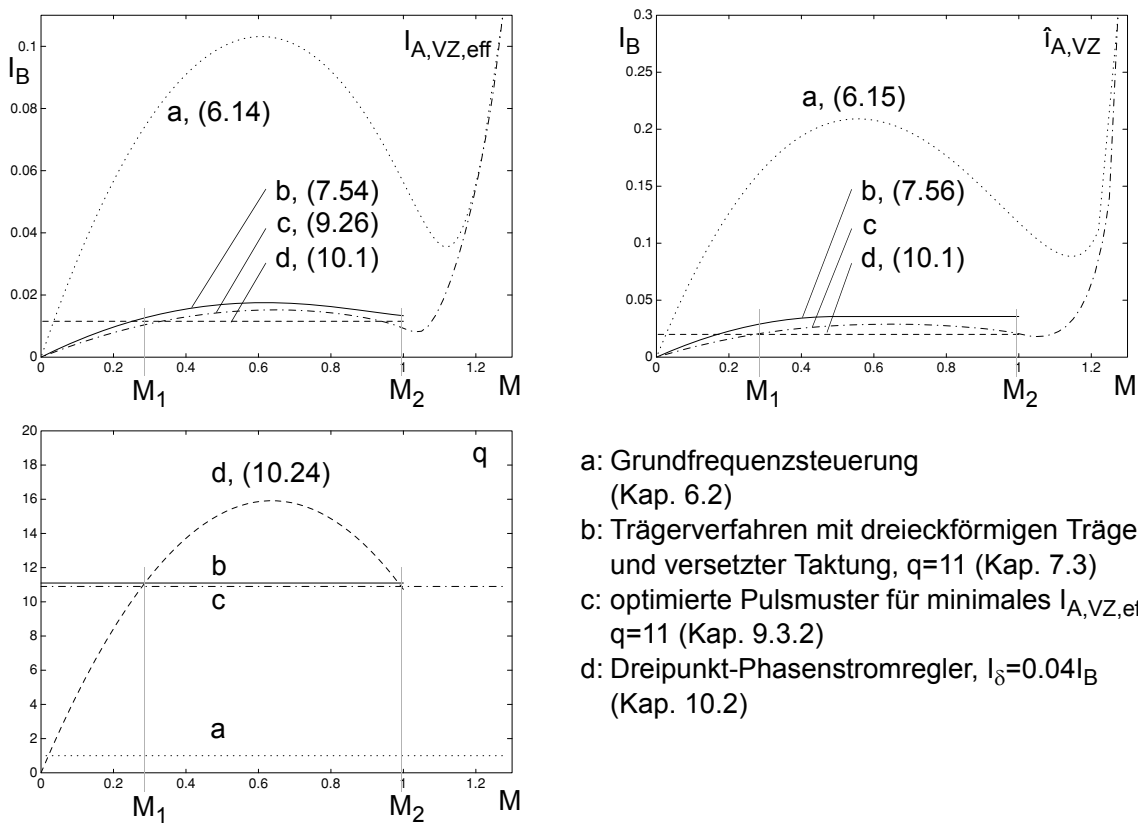

a: Grundfrequenzsteuerung

(Kap. 6.2)

b: Trägerverfahren mit dreieckförmigen Träger und versetzter Taktung, $\mathrm{q}=11$ (Kap. 7.3)

c: optimierte Pulsmuster für minimales $\mathrm{I}_{\mathrm{A}, \mathrm{VZ} \text {,eff, }}$ $\mathrm{q}=11$ (Kap. 9.3.2)

d: Dreipunkt-Phasenstromregler, $I_{\delta}=0.04 I_{B}$ (Kap. 10.2)

Bild 13.2. Kennlinien der verschiedenen Steuerverfahren bei der einphasigen Brücke, oben links: Effektivwert des Verzerrungsstromes, oben rechts: Spitzenwert des Verzerrungsstromes, unten: Schaltzahl (proportional zur Schaltfrequenz)

- Eine genaue Betrachtung der Stellen, wo die Verfahren b, c und d dieselbe Schaltzahl aufweisen (bei $M_{1}$ und $M_{2}$ ) zeigt, dass die Unterschiede in den Verzerrungsströmen relativ klein sind. Die optimierten Pulsmuster ergeben (entsprechend dem Optimierungskriterium) den kleinsten Effektivwert des Verzerrungsstromes. Beim Spitzenwert ist der Phasenstromregler praktisch gleichwertig. Das Trägerverfahren ergibt dagegen in beiden Kennlinien jeweils die grössten Werte.

\subsubsection{Dreiphasige Brücke}

Die Kennlinien für die beiden Hauptanwendungsgebiete der dreiphasigen Brücke werden getrennt betrachtet: diejenigen mit konstanter Grundfrequenz für Netzstromrichter und diejenigen mit einer zum Modulationsgrad proportionalen Grundfrequenz für Antriebsstromrichter.

\subsubsection{Netzstromrichter}

Bild 13.3 zeigt die Kennlinien für einen dreiphasigen Stromrichter mit konstanter Grundfrequenz. Die gewählten Verfahren sind hier:

Kurven a: Grundfrequenzsteuerung nach Kapitel 6.3 mit variabler Zwischenkreisspannung $\left(U_{d} \sim M\right)$. 

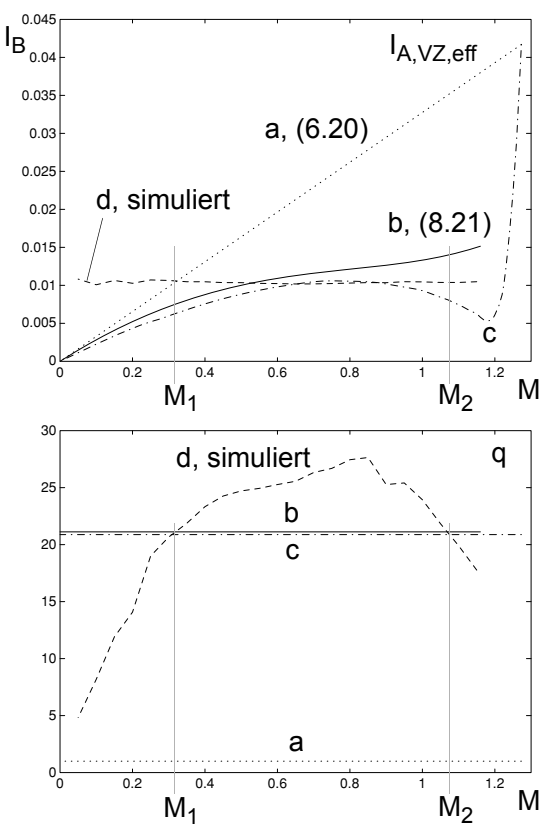

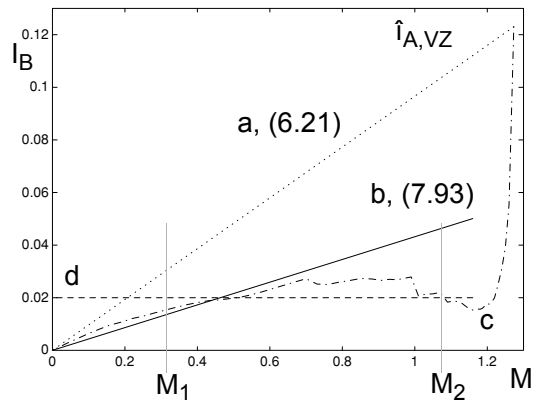

a: Grundfrequenzsteuerung (Kap. 6.3)

b: Standard-Drehzeigermodulation $\left(t_{0}=t_{7}\right), q=21$ (Kap. 8.2)

c: optimierte Pulsmuster für minimales $\mathrm{I}_{\mathrm{A}, \mathrm{VZ} \text {,eff, }}$, $q=21$ (Kap. 9.3.3)

d: Prädiktive Stromzeigerregelung mit Schalttabelle, $\mathrm{I}_{\delta}=0.04 \mathrm{I}_{\mathrm{B}}$ (Kap. 11.3.1)

Bild 13.3. Kennlinien der verschiedenen Steuerverfahren bei der dreiphasigen Brücke mit konstanter Grundfrequenz, oben links: Effektivwert der Verzerrungsstromes einer Phase, oben rechts: Spitzenwert des Verzerrungsstromes, unten: Schaltzahl (proportional zur Schaltfrequenz)

Kurven $b$ : Standard-Drehzeigermodulation $\left(t_{0}=t_{7}\right)$ nach Kapitel 8.2 als Vertreter aller Drehzeigermodulationen und der mit ihnen verwandten Trägerverfahren.

Kurven c: optimierte Pulsmuster für minimalen Effektivwert des Verzerrungsstromes nach Kapitel 9.3.3.

Kurven d: prädiktive Stromzeigerregelung mit Schalttabelle nach Kapitel 11.3.1.

\section{Kommentar zu Bild 13.3:}

- Bei Grundfrequenzsteuerung kann der Modulationsgrad nur durch eine entsprechende Anpassung der Zwischenkreisspannung variiert werden, was ein aufwendigeres Umrichterkonzept bedingt (Kapitel 2). Würde auch bei den anderen Verfahren eine variable Zwischenkreisspannung zugelassen, so würde sich der Verzerrungsstrom proportional dazu reduzieren lassen. Da in den dargestellten Kennlinien der Bezugsstrom $I_{B}$ stets auf die volle Zwischenkreisspannung bezogen ist, schneidet die Grundfrequenzsteuerung deshalb im Vergleich bei Teilaussteuerung im Prinzip zu gut ab.

- Die Aussteuergrenze liegt bei der Grundfrequenzsteuerung und bei den optimierten Pulsmustern bei $M=4 / \pi$, bei den anderen Verfahren bei $2 / \sqrt{3} \approx 1.155$. Die Drehzeigermodulation (oder ein Trägerverfahren) könnte aber mit Übersteuerung $4 / \pi$ erreichen.

- Die Verfahren b, c und d zeigen eine näherungsweise umgekehrte Proportionalität zwischen den Kennlinien des Verzerrungsstromes und derjenigen der Schaltzahl. Der Verzerrungsstrom kann durch Erhöhen der Schaltfrequenz beliebig reduziert werden. 
- Die genaue Betrachtung an den Stellen, an denen die Verfahren dieselbe Schaltzahl aufweisen (bei $M_{1}$ und $M_{2}$ ) zeigt: da die Kennlinien des Verzerrungsstromes für die Drehzeigermodulation mit dem Modulationsgrad ansteigen und diejenigen der Stromzeigerregelung konstant bleiben, fällt der Vergleich der beiden Verfahren sehr unterschiedlich aus. Während bei niedriger Aussteuerung die Drehzeigermodulation klar kleinere Werte ergibt, ist es bei grosser Aussteuerung ebenso deutlich die Stromzeigerregelung. Die optimierten Pulsmuster weisen entsprechend dem Gütekriterium der Optimierung für eine gegebene Schaltzahl überall den kleinsten Effektivwert des Verzerrungsstromes auf. Bei $M_{2}$ ist der Abstand zu den anderen Verfahren ziemlich gross. Der Spitzenwert, der nicht minimiert ist, wird an beiden Stellen ungefähr gleich gross wie das bessere der beiden anderen Verfahren.

\subsubsection{Antriebsstromrichter}

Bild 13.4 zeigt die Kennlinien für einen Antriebsstromrichter, der die Grundfrequenz proportional zum Modulationsgrad einstellt. Neben den bisherigen Grössen sind zusätzlich die Schaltfrequenz, welche hier nicht einfach proportional zur Schaltzahl verläuft, und die Drehmomentwelligkeit im Leerlauf dargestellt. Die Bezugsgrössen der Kurven sind so gewählt, dass bei $M=1$ die Grundkreisfrequenz ihren Bezugswert $\omega_{B}$ erreicht (entsprechend Bild 5.7). Die dargestellten Verfahren sind dieselben wie in Kapitel 13.2.2.1.

Kommentar zu Bild 13.4: Die einzelnen Steuerverfahren zeigen grundsätzlich dieselben Eigenschaften, die bereits beim Netzstromrichter besprochen wurden. Diese Tatsache könnte in jedem einzelnen Betriebspunkt durch eine entsprechende Anpassung der verschiedenen Schaltfrequenzen bzw. Toleranzbänder sichtbar gemacht werden. Hier steht jedoch im Vordergrund, wie die Verfahren den gesamte Betriebsbereich einer Drehstrommaschine mit variabler Grundfrequenz durchfahren können. In dieser Beziehung sind die folgenden Aspekte von Bedeutung:

- Die Grundfrequenzsteuerung und die optimierten Pulsmuster sind synchrone Verfahren. Sie ergeben bei kleiner Grundfrequenz auch eine kleine Schaltfrequenz, was den Verzerrungsstrom gross werden lässt. (Bei der Grundfrequenzsteuerung bleibt dieser nur wegen der reduzierten Zwischenkreisspannung konstant.) Die optimierten Pulsmuster können dem Problem begegnen, indem die Schaltzahl mit kleiner werdendem Modulationsgrad schrittweise erhöht wird. In der Praxis ergibt sich trotzdem stets eine untere Einsatzgrenze für dieses Verfahren.

- Die beiden anderen Verfahren sind dagegen vom Prinzip her asynchron. Unabhängig von der Grundfrequenz kann die Drehzeigermodulation ihre Schaltfrequenz bzw. die Stromzeigerregelung ihre Toleranzbänder beibehalten. Die Schaltzahlen bei niedriger Grundfrequenz werden dann entsprechend gross.

- Die Kennlinien des Spitzenwertes für die Drehmomentwelligkeit zeigen gewisse Abweichungen gegenüber denjenigen des Verzerrungsstromes: die Grundfrequenzsteuerung ergibt einen verblüffend niedrigen Wert, der von den restlichen Verfahren erst mit einer deutlich höheren Schaltfrequenz als dargestellt unterboten wird. Damit zum Beispiel die Drehmomentwelligkeit der Drehzeigermodulation im ganzen Aussteuerbereich kleiner ausfällt, muss die Schaltfrequenz gegenüber dem Bild auf $26 f_{B}$ verdoppelt 

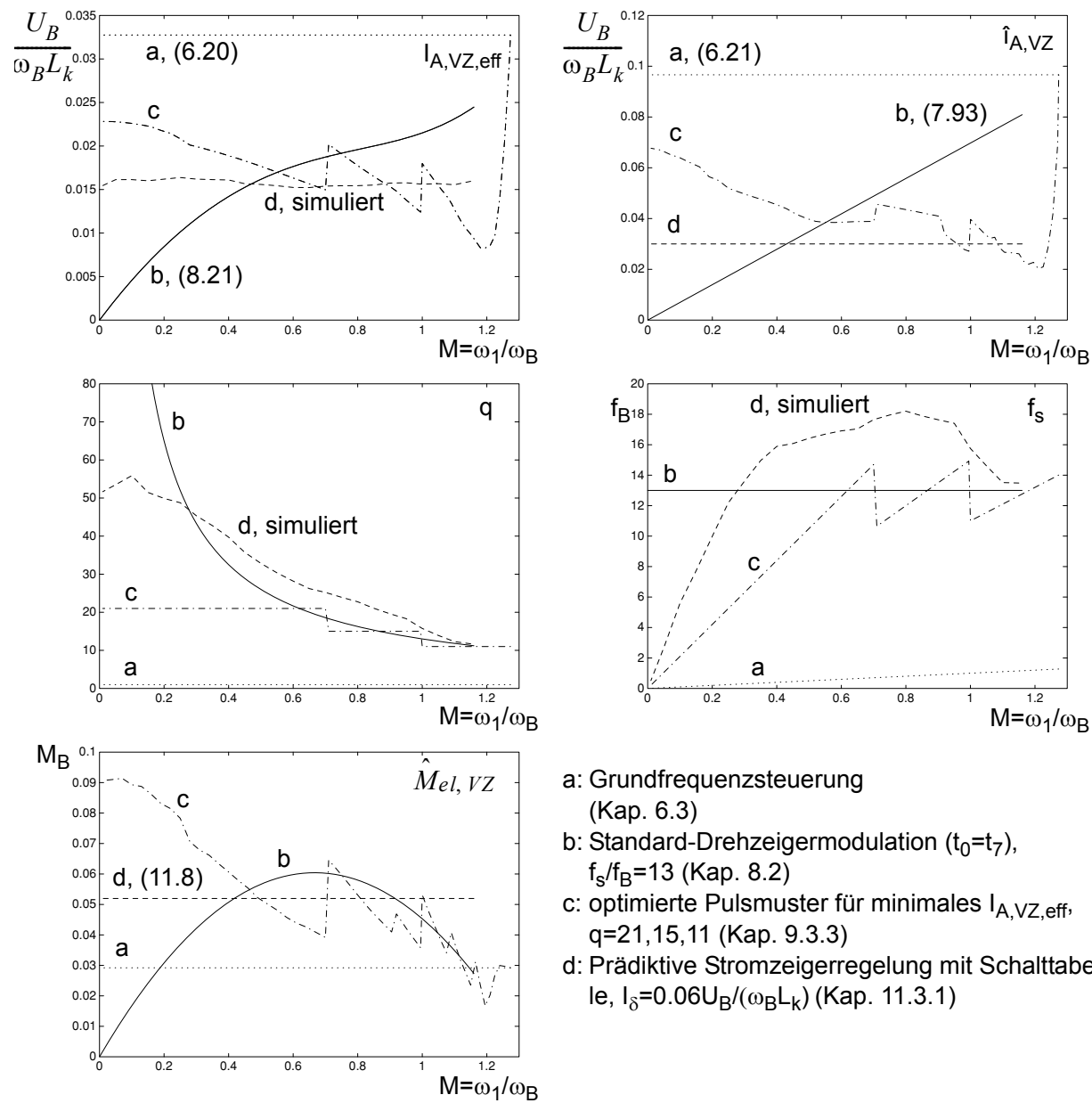

a: Grundfrequenzsteuerung

(Kap. 6.3)

b: Standard-Drehzeigermodulation $\left(\mathrm{t}_{0}=\mathrm{t}_{7}\right)$,

$f_{S} / f_{B}=13$ (Kap. 8.2)

C: optimierte Pulsmuster für minimales $\mathrm{I}_{\mathrm{A}, \mathrm{VZ}, \text { eff }}$, $q=21,15,11$ (Kap. 9.3.3)

d: Prädiktive Stromzeigerregelung mit Schalttabelle, $\mathrm{I}_{\delta}=0.06 \mathrm{U}_{\mathrm{B}} /\left(\omega_{\mathrm{B}} \mathrm{L}_{\mathrm{k}}\right)($ Kap. 11.3.1)

Bild 13.4. Kennlinien der verschiedenen Steuerverfahren bei der dreiphasigen Brücke mit variabler Grundfrequenz,

oben links: Effektivwert der Verzerrungsstromes einer Phase, oben rechts: Spitzenwert des Verzerrungsstromes einer Phase, mitte links: Schaltzahl, mitte rechts: mittlere Schaltfrequenz, unten: Spitzenwert der Drehmomentwelligkeit im Leerlauf

werden. Der Grund dafür ist, dass sich bei der Grundfrequenzsteuerung die Wirkung der Harmonischen des Verzerrungsstromes auf das Drehmoment teilweise gegenseitig kompensiert. Im weiteren zeigt auch die Drehzeigermodulation bei grosser Aussteuerung eine Drehmomentwelligkeit, die kleiner ist als aus den Kennlinien des Verzerrungsstromes zu erwarten wäre. Die entsprechende Kennlinie der optimierten Pulsmuster fällt vor allem durch ihre Sprungstellen auf: sie entstehen dort, wo ein Übergang zwischen zwei verschiedenen Schaltsequenzen stattfindet. Es bleibt anzumerken, dass ein mit dem Gütekriterium Drehmomentwelligkeit optimiertes Pulsmuster diesbezüglich die besten Resultat ergeben würde (Kapitel 9.3.4). 


\subsection{Spektra}

In der Tabelle 13.1 sind die wesentlichen Eigenschaften der Spektra der Phasenspannungen und -ströme für die verschiedenen Grundtypen von Steuerverfahren kurz charakterisiert. Es wird dabei zwischen ein- und dreiphasigen Schaltungen unterschieden.

Die Charakteristiken der Spektra sind vor allem auch massgebend für die entstehenden Geräusche (Kapitel 3.2.2.1):

- Bei Trägerverfahren und Drehzeigermodulationen äussern sich die dominanten Trägerbänder in den Spektra durch einen klaren, anhaltenden Ton, z.B. ein Pfeifen.

- Bei den direkten Regelverfahren, die keine ausgeprägten Komponenten in den Spektra ergeben, entsprechen die Geräusche eher einem Surren.

- Bei der Grundfrequenzsteuerung führen die dominanten niedrigen Harmonischen dagegen zu einem Brummen.

\subsection{Frequenzbereiche}

Bei der Beschreibung der einzelnen Verfahren und auch bei der Darstellung der Kennlinien in Kapitel 13.2 wurden stets relativ niedrige Schaltzahlen gewählt. Dadurch konnten die Funktionsweisen der Verfahren und die Vergleiche untereinander einfach und übersichtlich dargestellt werden. In der Praxis werden jedoch die Verfahren in sehr unterschiedlichen Frequenzbereichen und mit stark voneinander abweichenden Schaltzahlen eingesetzt. Die Gründe dafür sind einerseits die stark variierenden Anforderungen und Randbedingungen der entsprechenden Anwendungen und andererseits die besonderen Qualitäten der Steuerverfahren, die bei unterschiedlichen Schaltfrequenzen in Erscheinung treten.

Bild 13.5 zeigt die Bereiche der Schaltzahlen und absoluten Schaltfrequenzen, in welchen die verschiedenen Verfahren üblicherweise eingesetzt werden. Die angegebenen Grenzen sind als Richtwerte anzusehen, die in Einzelfällen durchaus unter- bzw. überschritten werden können:

- Die Grundfrequenzsteuerung hat den grössten Einsatzbereich. Am unteren Ende der Frequenzskala liegen Netzanwendungen und Antriebe sehr grosser Leistung, wo das Verfahren wegen der minimalen Schaltzahl zur Anwendung kommt. Der mittlere Bereich wird durch Antriebe für sehr hohe Drehzahlen und entsprechend hoher Grundfrequenz abgedeckt. Darüber folgen die DC-AC-DC-Wandler in getakteten Stromversorgungen sowie andere spezielle Anwendungen.

- Die Trägerverfahren und Drehzeigermodulatoren sind diejenigen Verfahren mit dem weitesten Anwendungsgebiet. Sie sollten vorzugsweise mit Schaltzahlen oberhalb von 10 bis 15 eingesetzt werden. Nur dann kommt ihr Vorzug, die deutliche Trennung zwischen Nutzsignal und Verzerrungen im Frequenzbereich zum Tragen. Die typischen Schaltfrequenzen beginnen deshalb im Bereich von einigen 100Hz. Die entsprechende obere Grenze ergibt sich durch die auftretenden Schaltverluste. Häufig wird eine Schaltfrequenz im Bereich von 15 bis 20kHz gewählt, damit die durch die Verzerrungen 
Tabelle 13.1. Eigenschaften der Spektra von Phasenspannungen und -strömen für die verschiedenen Steuerverfahren, sinusförmige Aussteuerung mit Grundfrequenz $f_{1}$

\begin{tabular}{|c|c|c|}
\hline Verfahren & Spektra bei der einphasigen Brücke & Spektra bei der dreiphasigen Brücke \\
\hline $\begin{array}{l}\text { Grundfrequenz- } \\
\text { steuerung }\end{array}$ & alle ungeraden Harmonischen von $f_{1}$ & $\begin{array}{l}\text { - Harmonische von } f_{1} \text { der Ordnungen } 1, \\
\text { 5/7, 11/13 usw. } \\
\text { - Amplituden der Spannungsharmo- } \\
\text { nischen umgekehrt proportional zur Ord- } \\
\text { nungszahl }\end{array}$ \\
\hline $\begin{array}{l}\text { Trägerverfahren } \\
\text { Drehzeiger- } \\
\text { modulation } \\
\text { asynchron }\end{array}$ & $\begin{array}{l}\text { - Trägerbänder um die geradzah- } \\
\text { ligen Vielfachen der Trägerfre- } \\
\text { quenz (bei versetzter Taktung) } \\
\text { - diskretes Linienspektrum, beste- } \\
\text { hend aus Interharmonischen } \\
\text { - bei sehr niedriger Schaltzahl }(q<7) \\
\text { werden Subharmonische wirksam } \\
\text { (Seitenbänder des } 1 \text {. Trägerbandes) }\end{array}$ & $\begin{array}{l}\text { - Trägerbänder um alle Vielfachen der Trä- } \\
\text { gerfrequenz } \\
\text { - diskretes Linienspektrum, bestehend aus } \\
\text { Interharmonischen } \\
\text { - Gleichtaktkomponenten fehlen } \\
\text { - der Anteil bei der Trägerfrequenz ist } \\
\text { Gleichtaktkomponente und tritt nicht auf } \\
\text { - bei sehr niedriger Schaltzahl }(q<7) \text { wer- } \\
\text { den Subharmonische wirksam }\end{array}$ \\
\hline $\begin{array}{l}\text { Trägerverfahren } \\
\text { Drehzeiger- } \\
\text { modulation } \\
\text { synchron }\end{array}$ & $\begin{array}{l}\text { - Trägerbänder um die geradzah- } \\
\text { ligen Vielfachen der Trägerfre- } \\
\text { quenz (bei versetzter Taktung) } \\
\text { - diskretes Linienspektrum beste- } \\
\text { hend aus Harmonischen } \\
\text { - bei sehr niedriger Schaltzahl }(q<7) \\
\text { kann die Grundschwingung ver- } \\
\text { fälscht werden } \\
\text { - es treten keine Subharmonischen } \\
\text { auf, dafür bei sehr kleiner Schalt- } \\
\text { zahl eine Gleichkomponente (Sei- } \\
\text { tenlinie des 1. Trägerbandes) }\end{array}$ & $\begin{array}{l}\text { - Trägerbänder um alle Vielfachen der Trä- } \\
\text { gerfrequenz } \\
\text { - diskretes Linienspektrum bestehend aus } \\
\text { Harmonischen } \\
\text { - Gleichtaktkomponenten fehlen } \\
\text { - der Anteil bei der Trägerfrequenz ist } \\
\text { Gleichtaktkomponente und tritt nicht auf } \\
\text { - wird die Trägerfrequenz auf ein Viel- } \\
\text { faches der 3. Harmonischen gelegt, so } \\
\text { sind die Pulsmuster exakt symmetrisch } \\
\text { - bei sehr niedriger Schaltzahl }(q<7) \text { kann } \\
\text { die Grundschwingung verfälscht werden } \\
\text { - es treten keine Subharmonischen auf, da- } \\
\text { für bei sehr kleiner Schaltzahl eine } \\
\text { Gleichkomponente }\end{array}$ \\
\hline $\begin{array}{l}\text { voraus- } \\
\text { berechnete } \\
\text { Pulsmuster }\end{array}$ & $\begin{array}{l}\text { - im Prinzip treten alle ungeraden } \\
\text { Harmonischen auf (bei Halbperio- } \\
\text { densymmetrie) } \\
\text { - Amplituden der Harmonischen ge- } \\
\text { mäss Optimierung }\end{array}$ & $\begin{array}{l}\text { - im Prinzip treten alle Harmonischen der } \\
\text { Ordnungen } 1,5,7,11,13 \text { usw. auf (bei } \\
\text { Halbperiodensymmetrie) } \\
\text { - Amplituden der Harmonischen gemäss } \\
\text { Optimierung }\end{array}$ \\
\hline $\begin{array}{l}\text { Verfahren zur } \\
\text { direkten Strom- } \\
\text { regelung (Pha- } \\
\text { senstromregler, } \\
\text { Stromzeigerreg- } \\
\text { ler) }\end{array}$ & $\begin{array}{l}\text { - im Prinzip kontinuierliche Spektra } \\
\text { - der Dreipunktregler synchronisiert } \\
\text { mit der Grundfrequenz, so dass nur } \\
\text { noch Harmonische auftreten } \\
\text { - die dominanten Amplituden treten } \\
\text { in einem weiten Frequenzbereich } \\
\text { auf } \\
\text { - bei gross gewählten Toleranzbän- } \\
\text { dern treten Subharmonische und } \\
\text { Gleichanteile in Erscheinung }\end{array}$ & $\begin{array}{l}\text { - kontinuierliche Spektra } \\
\text { - die dominanten Amplituden treten in } \\
\text { einem weiten Frequenzbereich auf } \\
\text { - bei gross gewählten Toleranzbändern tre- } \\
\text { ten Subharmonische und Gleichanteile in } \\
\text { Erscheinung }\end{array}$ \\
\hline
\end{tabular}




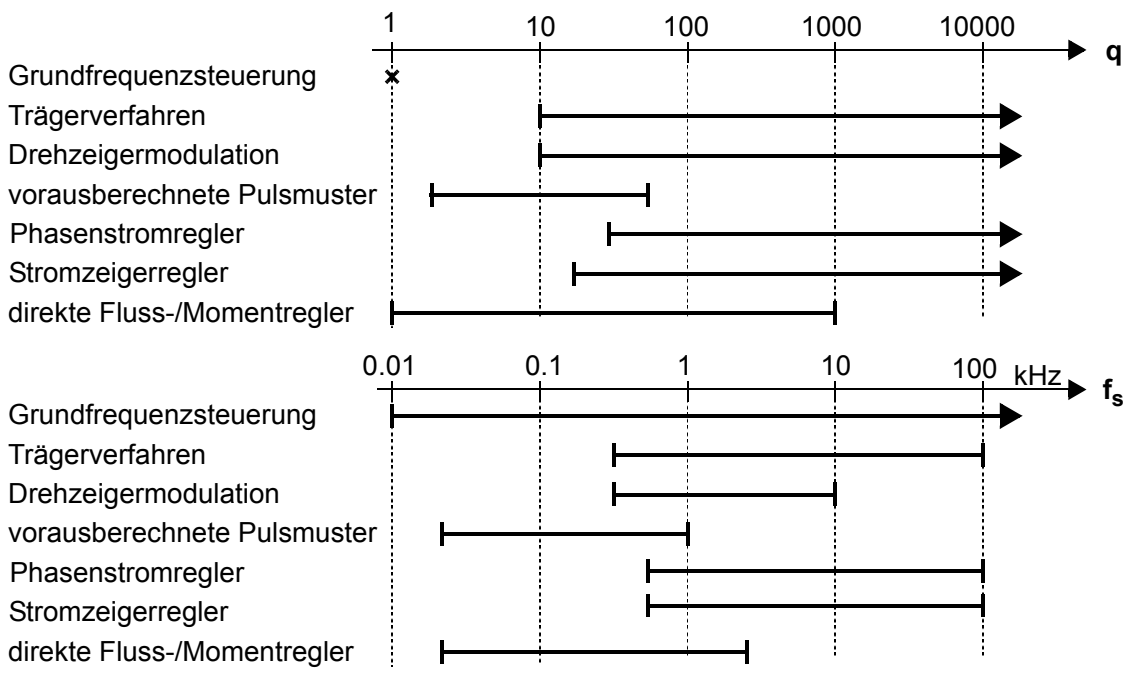

Bild 13.5. Einsatzbereiche der verschiedenen Steuerverfahren, oben: Schaltzahlen, unten: absolute Schaltfrequenzen

verursachten Geräusche oberhalb des Hörbereichs liegen. Bei der Drehzeigermodulation ergibt sich wegen der notwendigen Implementierung auf einem Rechner eine etwas kleinere obere Grenze der Schaltfrequenz als für diskret realisierte Trägerverfahren.

- Die vorausberechneten Pulsmuster werden für Schaltzahlen von 2 bis ca. 50 eingesetzt. Darüber werden die Schalttabellen zu gross und die Implementierung zu aufwendig. Überdies sind die Algorithmen zur Bestimmung der Schaltwinkel für grosse Schaltzahlen fast unlösbar und der Gewinn gegenüber On-Line-Verfahren fällt immer weniger ins Gewicht. Das Anwendungsgebiet ist relativ klein. Es umfasst spezielle Antriebe und Netzstromrichter. Die Schaltfrequenz ist auf einen eher engen Bereich begrenzt.

- Alle direkten Stromregelverfahren werden mit verhältnismässig kleinen Toleranzbändern und -flächen eingesetzt. Nur so sind die niederfrequenten Verzerrungen, vor allem auch die Subharmonischen und Gleichanteile, genügend klein. Es ergeben sich entsprechend hohe Schaltzahlen und Schaltfrequenzen. Die Anwendungen liegen im Bereich kleiner Leistungen, und vor allem dort, wo eine hohe Dynamik und eine robuste Regelung verlangt sind. Direkte Stromregler sind auch geeignet für schwingfähige Lasten, weil sie die Phasenströme jederzeit innerhalb von Toleranzbändern halten können.

- Die direkten Fluss- und Momentregelverfahren erlauben eine kontinuierliche Steigerung der Aussteuerung des Stromrichters bis zur Grundfrequenzsteuerung. Dabei geht die Schaltzahl von einem relativ hohen Wert bei niedriger Drehzahl hinunter bis auf eins bei voller Drehzahl. Die nötige Berechnung von Fluss und Drehmoment aus den Klemmengrössen der Maschine ergibt eine obere Grenze für die Schaltfrequenz. Diese Verfahren ermöglichen eine hochdynamische Regelung des Antriebes auch bei einer niedrigen Schaltfrequenz. Dies gilt vor allem für die direkte Selbstregelung (Kapitel 12.2.2). 


\subsection{Dynamik}

Tabelle 13.2 gibt einen Überblick über die dynamischen Eigenschaften der einzelnen Verfahren. Es wird angegeben, wie die wichtigen Betriebsparameter durch den Modulator variiert werden können. Bei den spannungssteuernden Verfahren sind dies Modulationsgrad, Grundfrequenz und Phasenwinkel der Ausgangsspannungen und bei den direkten Stromreglern sind es die Phasenströme. In beiden Fällen kommt die Schaltfrequenz als Parameter dazu. Die Angaben beziehen sich auf die ein- und dreiphasigen Brücken.

Tabelle 13.2. Übersicht über das dynamische Verhalten der verschiedenen Steuerverfahren

\begin{tabular}{l|l}
\multicolumn{1}{c|}{ Verfahren } & \multicolumn{1}{c}{ Variation der Parameter } \\
\hline \multirow{3}{*}{ Grundfrequenz- } & $\mathrm{M}: \quad$ einphasig über $\alpha$, dreiphasig nur über $\mathrm{U}_{\mathrm{d}}$ \\
steuerung & $\varphi_{\mathrm{m}}: \quad$ durch Verschieben des Pulsmusters \\
& $\mathrm{f}_{\mathrm{f}}: \quad$ über Zeitbasis des Modulators \\
$\mathrm{f}_{\mathrm{s}}: \quad$ identisch mit $\mathrm{f}_{1}$
\end{tabular}

Die Verfahren zur Spannungssteuerung können bei der schnellen Verstellung der Parameter transiente DC-Anteile in den Phasenströmen auslösen. Dieses Phänomen ist in Bild 13.6 am Beispiel einer einphasigen Brücke mit Grundfrequenzsteuerung veranschaulicht. Der dargestellte Sprung des Modulationsgrades soll die Grundschwingung des Phasenstromes verstellen. Zusätzlich überlagert sich dem Strom aber auch eine grosse DC-Komponente, die in einer realen Schaltung wegen der ohmschen Anteile in der Last langsam wieder abklingt. Solche transienten DC-Komponenten entstehen im Prinzip bei allen schnellen Änderungen der Modulator-Parameter. Sie fallen je nach Art, Grösse und Zeit-

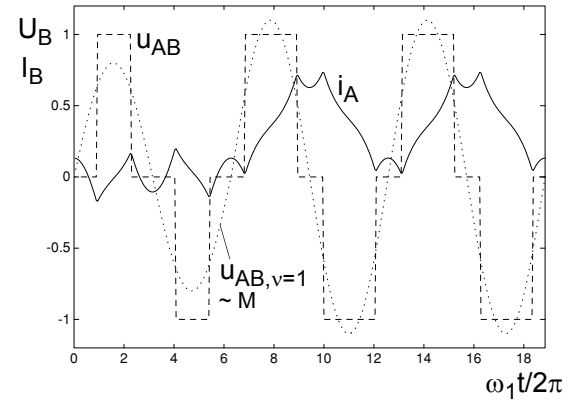

Bild 13.6.

Entstehung eines Gleichanteiles im Phasenstrom durch Sprung des Modulationsgrades von $M=0.8$ auf 1.1 bei der einphasigen Brücke mit Grundfrequenzsteuerung 
punkt des Sprunges verschieden gross aus. Den DC-Komponenten entgegenwirken kann nur der Stromregler. Durch eine geeignete Führung des Spannungssollwertes für den Modulator lassen sich die Gleichanteile vermeiden oder rasch ausregeln. Am besten gelingt dies in Stromregelkreisen mit On-Line Steuerverfahren und hoher Schaltzahl. Bei den direkten Stromreglern besteht das Problem der transienten DC-Erzeugung nicht.

Dagegen ergeben die direkten Stromregler stets kleine Abweichungen der Grundschwingungen von ihren Sollwerten. Die Fehler bewegen sich in einem Streubereich, der proportional zu den gegebenen Toleranzbändern bzw. -flächen ist. Da jedoch praktisch immer eine übergeordnete Regelung vorhanden ist (Kapitel 4), lassen sie sich gewöhnlich leicht ausregeln.

\subsection{Anwendungsgebiete}

Tabelle 13.3 zeigt schliesslich eine kurze Zusammenstellung der Hauptanwendungsgebiete für die einzelnen Steuerverfahren. Sie ergeben sich einerseits aus den Anforderungen der Anwendungen und andererseits aus den Eigenschaften der Steuerverfahren.

Tabelle 13.3. Anwendungsgebiete der verschiedenen Steuerverfahren

\begin{tabular}{l|l}
\multicolumn{1}{c|}{ Verfahren } & \multicolumn{1}{c}{ Anwendungsgebiete } \\
\hline $\begin{array}{l}\text { Grundfrequenz- } \\
\text { steuerung }\end{array}$ & - Antriebe und Netzanwendungen für sehr grosse Leistungen \\
& - Antriebe für sehr hohe Drehzahlen \\
& - - Speisprung von Übertragern in Schaltnetzteilen
\end{tabular}




\section{Teil III}

\section{Anwendung der Steuerverfahren auf weitere Stromrichter und Stromrichtergruppen}

\section{Steuerverfahren für Stromzwischenkreis-Stromrichter}

Bei selbstgeführten Stromrichtern mit Stromzwischenkreis (I-Stromrichter) wird der Last ein Strom aufgeprägt. Entsprechend den sinusförmigen Spannungen bei U-Stromrichtern sind die Sollwerte hier normalerweise sinusförmige Ströme. Selbstgeführte Stromrichter mit Stromzwischenkreis haben im Vergleich zu Schaltungen mit Spannungszwischenkreis bis heute nur eine begrenzte Bedeutung erlangt.

Die einphasige I-Stromrichterbrücke wird in Kapitel 3.3.2.2 und die dreiphasige in Kapitel 3.3.2.3 beschrieben. Die entsprechenden U- und I-Stromrichter sind zumindest teilweise dual. Dank dieser Dualität ist es möglich, die Steuerverfahren für U-Stromrichter weitgehend auf die I-Stromrichter zu übernehmen. Für die Betrachtung der Modulationsverfahren wird, dual zur konstanten Zwischenkreisspannung beim U-Stromrichter, der Zwischenkreisstrom konstant gewählt. Am I-Stromrichter wird üblicherweise ebenfalls die Standardlast, bestehend aus Spannungsquelle $e$ und Induktivität $L_{k}$, angeschlossen. Wie in Bild 14.1 dargestellt, kann die Standardlast durch eine Stromquelle angenähert werden. Die Parallelschaltung der Filterkapazität $C_{F}$ mit dieser Stromquelle ergibt die duale Last zur Standardlast beim U-Stromrichter. Eine kurze Einführung in das Thema Dualität von U- und I-Stromrichtern gibt das Kapitel 3.3.7.

Im vorliegenden Kapitel werden zuerst die Beziehungen zwischen den Steuerverfahren für die beiden Stromrichtertypen hergeleitet. Danach wird ein spezielles Verfahren für dreiphasige I-Stromrichter vorgestellt. Um Verwechslungen zu vermeiden, werden bei den I-Stromrichtern die Schaltfunktionen für die Brückenzweige und der Modulationsgrad zusätzlich mit dem Index $I$ gekennzeichnet $\left(s_{I, A}, s_{I, B}, M_{I}\right)$. Auf die Spannungen an der Last und im Zwischenkreis wird nicht näher eingegangen. Die Lastspannung muss mit Hilfe des Laststromes und der Last bestimmt werden. Bei bekannter Lastspannung kann dann die Zwischenkreisspannung mit den Schaltfunktionen und den in Kapitel 3.3.5 vorgestellten Formeln einfach berechnet werden. 


\subsection{Einphasige Brücke}

Die beiden Schaltungen in Bild 14.1 haben die gleiche Struktur und sind zusätzlich dual zueinander. Dual bedeutet bei der einphasigen Brücke unter anderem, dass die Phasenspannung beim U-Stromrichter dem Phasenstrom beim I-Stromrichter entspricht. Sind die Schaltfunktionen $s_{A}$ und $s_{B}$ für eine bestimmte Spannungsform beim U-Stromrichter bestimmt, so können diese in die Schaltfunktionen $s_{I, A}$ und $s_{I, B}$ des I-Stromrichters transformiert werden, so dass der resultierende Phasenstrom den gleichen Verlauf hat wie die Spannung beim U-Stromrichter.
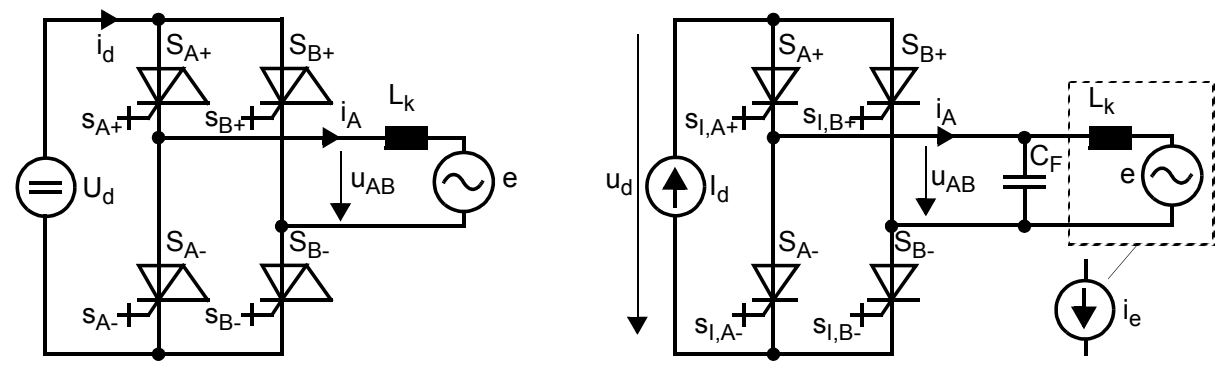

Bild 14.1. Einphasige Brücken, links: U-Stromrichter, rechts: I-Stromrichter

Schalteransteuerung: Bei der Ansteuerung der Schalter des I-Stromrichters müssen zwei Einschränkungen beachtet werden: beim I-Stromrichter dürfen nie beide Schalter $\left(S_{I, A+}, S_{I, B+}\right.$ bzw. $S_{I, A-}, S_{I, B-}$ ) in der oberen bzw. unteren Brückenhälfte gleichzeitig ausgeschaltet werden. Damit der Stromfluss immer definiert ist, darf pro Brückenhälfte aber auch nur je ein Schalter eingeschaltet sein. Diese Forderungen erfasst die Gleichung:

$$
s_{I, A^{+}}+s_{I, B^{+}}=1, \quad s_{I, A-}+s_{I, B-}=1
$$

Unter Berücksichtigung der obigen Einschränkung gilt für den Phasenstrom:

$$
i_{A}=s_{I, A^{i}{ }_{d}=-s_{I, B} i_{d}} \quad \text { mit } \quad\left\{\begin{array}{c}
s_{I, A}=s_{I, A+}-s_{I, A-} \\
s_{I, B}=s_{I, B+}-s_{I, B-}=-s_{I, A}
\end{array}\right.
$$

Die Funktionen $s_{I, A}$ und $s_{I, B}$ können 3 Werte annehmen: -1,0,1. Laststrom null kann mit zwei Schaltzuständen eingestellt werden: entweder leiten beide Schalter im Brückenzweig $A$ oder beide im Zweig $B$. Im nichtleitenden Zweig sind beide Schalter offen. Damit die Schalter gleichmässig belastet werden, ist die gleiche Behandlung beider Zweige anzustreben. Werden nach den Regeln der Dualität die beiden Schaltzustände für Spannung null vom U-Stromrichter übernommen, so ist die gleichmässige Belastung gewährleistet, falls dort beide Nullzustände gleich oft benutzt wurden. Dies ist bei den üblichen Modulationsverfahren erfüllt.

Die dualen Beziehungen zwischen den Spannungen beim U-Stromrichter und den Strömen beim I-Stromrichter sowie den entsprechenden Schaltfunktionen sind in der Tabelle 


\begin{tabular}{c|c|c|c|c|c}
\multicolumn{3}{c|}{ U-Stromrichter } & \multicolumn{3}{c}{ I-Stromrichter } \\
\hline $\mathrm{s}_{\mathrm{A}}$ & $\mathrm{s}_{\mathrm{B}}$ & $\mathrm{u}_{\mathrm{AB}}$ & $\mathrm{s}_{\mathrm{I}+}$ & $\mathrm{s}_{\mathrm{I}-}$ & $\mathrm{i}_{\mathrm{A}}$ \\
\hline-1 & -1 & 0 & $\mathrm{~s}_{\mathrm{I}, \mathrm{B}+}=1$ & $\mathrm{~s}_{\mathrm{I}, \mathrm{B}-}=1$ & 0 \\
\hline 1 & -1 & $\mathrm{U}_{\mathrm{d}}$ & $\mathrm{s}_{\mathrm{I}, \mathrm{A}+}=1$ & $\mathrm{~s}_{\mathrm{I}, \mathrm{B}-}=1$ & $\mathrm{I}_{\mathrm{d}}$ \\
\hline-1 & 1 & $-\mathrm{U}_{\mathrm{d}}$ & $\mathrm{s}_{\mathrm{I}, \mathrm{B}+}=1$ & $\mathrm{~s}_{\mathrm{I}, \mathrm{A}-}=1$ & $-\mathrm{I}_{\mathrm{d}}$ \\
\hline 1 & 1 & 0 & $\mathrm{~s}_{\mathrm{I}, \mathrm{A}+}=1$ & $\mathrm{~s}_{\mathrm{I}, \mathrm{A}-}=1$ & 0
\end{tabular}

Tabelle 14.1.

Schaltfunktionen und Phasenspannung beim U-Stromrichter sowie Schaltfunktionen und Phasenstrom beim I-Stromrichter

14.1 zusammengestellt. In der Tabelle wird dazu mit $s_{I_{+}}$die Schaltfunktion der oberen Brückenhälfte des I-Stromrichters und mit $s_{I-}$ die der unteren Hälfte bezeichnet.

Die Beziehungen zwischen den Schaltfunktionen der beiden Stromrichter können aus der Tabelle hergeleitet werden:

$$
\begin{array}{ll}
s_{I, A^{+}}=\left(s_{A}+1\right) / 2, & s_{I, A-}=\left(s_{B}+1\right) / 2 \\
s_{I, B+}=-\left(s_{A}-1\right) / 2, & s_{I, B-}=-\left(s_{B}-1\right) / 2
\end{array}
$$

Die Gleichungen zeigen, dass die Schaltfunktionen der Zweige beim U-Stromrichter zu Schaltfunktionen der oberen bzw. unteren Brückenhälfte beim I-Stromrichter werden. Die Schaltfunktion des Zweiges $A$ des U-Stromrichters beschreibt die obere, diejenige des Zweiges $B$ die untere Hälfte des I-Stromrichters. Diese Beziehung resultiert auch direkt aus den Dualitäts-Gesetzen. Der so beschriebene I-Stromrichter kann statt mit 4 Einzelschaltern auch mit Umschaltern, entsprechend Bild 14.2, dargestellt werden.
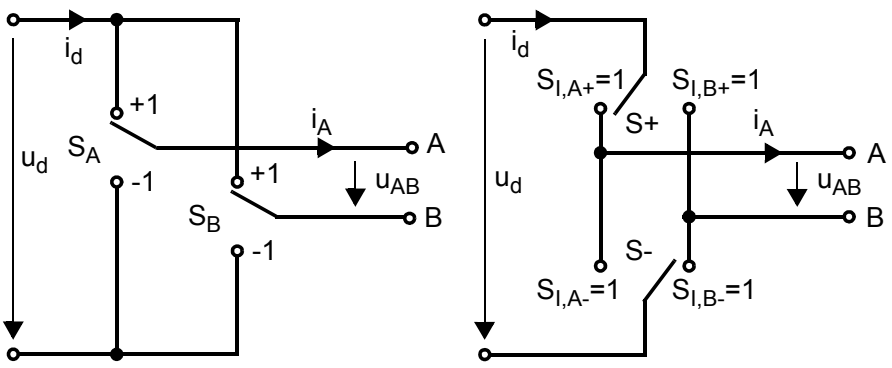

Bild 14.2.

Ersatzschaltbilder für die Brückenschaltungen mit Umschaltern, links: U-Stromrichter, rechts: I-Stromrichter

Für die Beschreibung des Phasenstromes und der Zwischenkreisspannung eignen sich die Funktionen $s_{I, A}$ und $s_{I, B}$ in (14.2) besser als die Schaltfunktionen der Brückenhälften. Das Einsetzen von (14.3) in (14.2) ergibt:

$$
s_{I, A}=-s_{I, B}=\frac{s_{A}-s_{B}}{2}
$$

Aus den hergeleiteten Gleichungen (14.2) und (14.4) sowie (3.85) und (3.86) resultiert für den Phasenstrom und die Zwischenkreisspannung des I-Stromrichters in Funktion der Schaltfunktion des U-Stromrichters (14.5). Für eine einfache Grundfrequenzsteuerung illustriert Bild 14.3 diese Transformation. 


$$
i_{A}=s_{I, A} i_{d}=\frac{s_{A}-s_{B}}{2} i_{d}, \quad u_{d}=s_{I, A} u_{A B}=\frac{s_{A}-s_{B}}{2} u_{A B}
$$

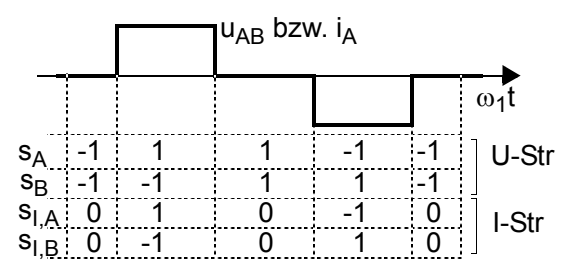

\section{Bild 14.3.}

Phasenspannung beim U-Stromrichter und entsprechender Phasenstrom beim I-Stromrichter mit den zugehörigen Schaltfunktionen

Die Berechnungen der Stromgrundschwingung, des Verzerrungsstromes etc. können nach Vertauschen von Strömen und Spannungen einfach von den einphasigen U-Stromrichtern übernommen werden. Dies gilt auch für die Verfahren zur Generierung der einphasigen Schaltmuster für I-Stromrichter. Dabei muss aber beachtet werden, dass auch Schaltmuster, welche bei den U-Stromrichtern keine kurzen Pulse in den Brückenzweigen ergeben, bei I-Stromrichtern unter Umständen die minimalen Schaltzeiten der Leistungshalbleiter nicht einhalten.

\subsection{Dreiphasige Brücke}

Die Struktur der dreiphasigen I-Stromrichterbrücke ist identisch mit derjenigen des entsprechenden U-Stromrichters. Die Schaltungen sind aber nicht dual zueinander, da zu beiden Topologien keine duale Schaltung existiert. Bei der Modellierung mit Umschaltern kann aber für jeden spannungsbildenden Zustand des U- ein Zustand des I-Stromrichters hergeleitet werden, der an der Last duale Verhältnisse ergibt [Kol7]. Damit der Stromfluss im Zwischenkreis des I-Stromrichters gewährleistet und in der Last eindeutig ist, muss wie bei der einphasigen Brücke immer genau ein Schalter in der oberen und einer in der unteren Brückenhälfte eingeschaltet sein. U- und I-Stromrichter unterscheiden sich in den Zuständen, in denen die Ausgangsgrössen null sind. Beim U-Stromrichter ergeben zwei Zustände (Nullzustände) Spannung null an der Last: $s_{U}=s_{V}=s_{W}=1$ und $s_{U}=s_{V}=s_{W}=-1$. Dagegen wird beim I-Stromrichter die Last in den 3 Zuständen $s_{U+}=s_{U-}=1, s_{V+}=s_{V_{-}}=1$ oder $s_{W+}=s_{W-}=1$ stromlos. Unter Berücksichtigung dieser Eigenschaften lässt sich die Schaltung in Bild 14.4 links durch die Ersatzschaltung rechts modellieren.

Für die Herleitung der Transformationsformeln für beliebige Schaltfunktionen wird folgende duale Eigenschaft der Lastkreise verwendet: die verketteten Spannungen beim UStromrichter entsprechen den Phasenströmen beim I-Stromrichter. Die verketteten Spannungen sind proportional zu den Differenzen $s_{U}-S_{V}, s_{V} s_{W}$ und $s_{W} s_{U}$ der Schaltfunktionen. Damit gelten zwischen den Schaltfunktionen des U-Stromrichters und den entsprechenden Phasenströmen des I-Stromrichters folgende Beziehungen:

$$
\left(s_{U}-s_{V}\right) \sim i_{U}, \quad\left(s_{V}-s_{W}\right) \sim i_{V}, \quad\left(s_{W}-s_{U}\right) \sim i_{W}
$$



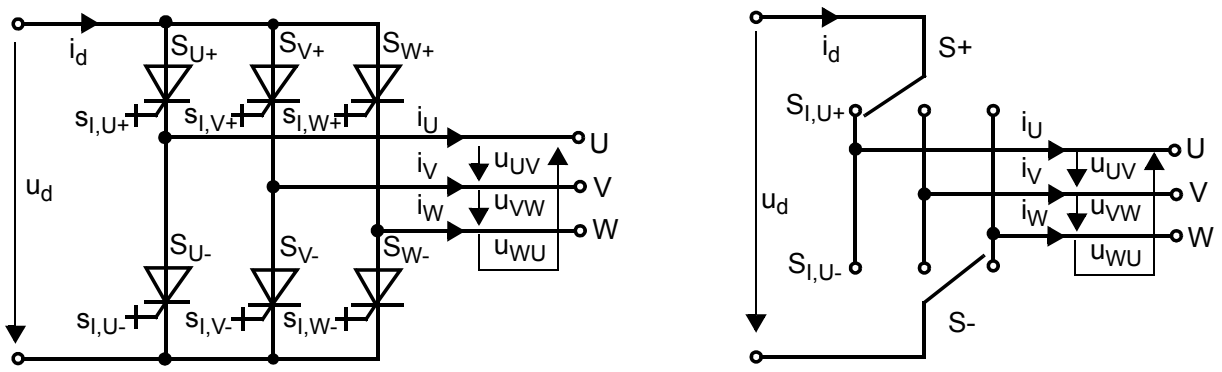

Bild 14.4. Dreiphasiger I-Stromrichter, links: Schaltung, rechts: Ersatzschaltung mit je einem dreipoligen Umschalter pro Brückenhälfte

Die Zustände der Stromrichter, die Schaltfunktionen des U-Stromrichters und die entsprechenden Ströme, Schalterzustände und Schaltfunktionen des I-Stromrichters sind in Tabelle 14.2 zusammengestellt.

Tabelle 14.2. Beziehung zwischen den Schaltfunktionen des U-Stromrichters und den Strömen und Schaltfunktionen des I-Stromrichters

\begin{tabular}{c|c|c|c|c|c|c|c|c|c|c|c} 
& \multicolumn{1}{|c|}{$\mathrm{U}-$ Stromrichter } & \multicolumn{10}{c}{ I-Stromrichter } \\
\hline Zustand & $\mathrm{s}_{\mathrm{U}}$ & $\mathrm{s}_{\mathrm{V}}$ & $\mathrm{s}_{\mathrm{W}}$ & $\mathrm{i}_{\mathrm{U}}$ & $\mathrm{i}_{\mathrm{V}}$ & $\mathrm{i}_{\mathrm{W}}$ & $\mathrm{s}_{\mathrm{I}+}$ & $\mathrm{s}_{\mathrm{I}-}$ & $\mathrm{s}_{\mathrm{I}, \mathrm{U}}$ & $\mathrm{s}_{\mathrm{I}, \mathrm{V}}$ & $\mathrm{s}_{\mathrm{I}, \mathrm{W}}$ \\
\hline${ }^{0} \mathrm{Z}$ & -1 & -1 & -1 & & & & & & & & \\
\hline${ }^{1} \mathrm{Z}$ & 1 & -1 & -1 & $\mathrm{I}_{\mathrm{d}}$ & 0 & $-\mathrm{I}_{\mathrm{d}}$ & $\mathrm{s}_{\mathrm{I}, \mathrm{U}+}=1$ & $\mathrm{~s}_{\mathrm{I}, \mathrm{W}-}=1$ & 1 & 0 & -1 \\
\hline${ }^{2} \mathrm{Z}$ & 1 & 1 & -1 & 0 & $\mathrm{I}_{\mathrm{d}}$ & $-\mathrm{I}_{\mathrm{d}}$ & $\mathrm{s}_{\mathrm{I}, \mathrm{V}+}=1$ & $\mathrm{~s}_{\mathrm{I}, \mathrm{W}-}=1$ & 0 & 1 & -1 \\
\hline${ }^{3} \mathrm{Z}$ & -1 & 1 & -1 & $-\mathrm{I}_{\mathrm{d}}$ & $\mathrm{I}_{\mathrm{d}}$ & 0 & $\mathrm{~s}_{\mathrm{I}, \mathrm{V}+}=1$ & $\mathrm{~s}_{\mathrm{I}, \mathrm{U}-}=1$ & -1 & 1 & 0 \\
\hline${ }^{4} \mathrm{Z}$ & -1 & 1 & 1 & $-\mathrm{I}_{\mathrm{d}}$ & 0 & $\mathrm{I}_{\mathrm{d}}$ & $\mathrm{s}_{\mathrm{I}, \mathrm{W}+}=1$ & $\mathrm{~s}_{\mathrm{I}, \mathrm{U}-}=1$ & -1 & 0 & 1 \\
\hline${ }^{5} \mathrm{Z}$ & -1 & -1 & 1 & 0 & $-\mathrm{I}_{\mathrm{d}}$ & $\mathrm{I}_{\mathrm{d}}$ & $\mathrm{s}_{\mathrm{I}, \mathrm{W}+}=1$ & $\mathrm{~s}_{\mathrm{I}, \mathrm{V}-}=1$ & 0 & -1 & 1 \\
\hline${ }^{6} \mathrm{Z}$ & 1 & -1 & 1 & $\mathrm{I}_{\mathrm{d}}$ & $-\mathrm{I}_{\mathrm{d}}$ & 0 & $\mathrm{~s}_{\mathrm{I}, \mathrm{U}+}=1$ & $\mathrm{~s}_{\mathrm{I}, \mathrm{V}-}=1$ & 1 & -1 & 0 \\
\hline${ }^{7} \mathrm{Z}$ & 1 & 1 & 1 & & & & & & & & \\
\hline${ }^{01} \mathrm{Z}$ & & & & 0 & 0 & 0 & $\mathrm{~s}_{\mathrm{I}, \mathrm{U}+}=1$ & $\mathrm{~s}_{\mathrm{I}, \mathrm{U}-}=1$ & 0 & 0 & 0 \\
\hline${ }^{02} \mathrm{Z}$ & & & & 0 & 0 & 0 & $\mathrm{~s}_{\mathrm{I}, \mathrm{V}+}=1$ & $\mathrm{~s}_{\mathrm{I}, \mathrm{V}-1}=1$ & 0 & 0 & 0 \\
\hline${ }^{03} \mathrm{Z}$ & & & & 0 & 0 & 0 & $\mathrm{~s}_{\mathrm{I}, \mathrm{W}+}=1$ & $\mathrm{~s}_{\mathrm{I}, \mathrm{W}-}=1$ & 0 & 0 & 0
\end{tabular}

Aus der Tabelle können die Schaltfunktionen der einzelnen Schalter für die strombildenden Zustände ${ }^{1} Z$ bis ${ }^{6} Z$ mit hergeleitet werden. Ist der Ausdruck in den rechteckigen Klammern erfüllt, so ist die Funktion gleich eins, andernfalls null:

$$
\begin{array}{ll}
s_{I, U^{+}}=\left[\left(s_{U}-s_{V}\right)>0\right], & s_{I, U_{-}}=\left[\left(s_{U}-s_{V}\right)<0\right] \\
s_{I, V+}=\left[\left(s_{V}-s_{W}\right)>0\right], & s_{I, V_{-}}=\left[\left(s_{V}-s_{W}\right)<0\right] \\
s_{I, W+}=\left[\left(s_{W}-s_{U}\right)>0\right], & s_{I, W-}=\left[\left(s_{W}-s_{U}\right)<0\right]
\end{array}
$$


Die Schaltfunktionen für die einzelnen Schalter können wieder in die dreistufigen Funktionen $s_{I, U / V / W}(=-1,0,1)$ zusammengefasst werden, mit denen sich gemäss (3.90) und (3.93) direkt die Phasenströme und die Zwischenkreisspannung beschreiben lassen:

$$
\begin{aligned}
& s_{I, U}=\frac{1}{2}\left(s_{U}-s_{V}\right), \quad s_{I, V}=\frac{1}{2}\left(s_{V}-s_{W}\right), \quad s_{I, W}=\frac{1}{2}\left(s_{W}-s_{U}\right) \\
& i_{U}=s_{I, U} I_{d}, \quad i_{V}=s_{I, V} I_{d}, \quad i_{W}=s_{I, W} I_{d} \\
& u_{d}=s_{I, U} u_{U}+s_{I, V} u_{V}+s_{I, W} u_{W}
\end{aligned}
$$

Die spannungsbildenden Zustände des U-Stromrichters entsprechen den strombildenden des I-Stromrichters. Der I-Stromrichter verfügt aber über 3 Nullzustände: ${ }^{01} Z,{ }^{02} Z$ und ${ }^{03} Z$. Damit alle Schalter gleichmässig belastet werden, müssen die Nullzustände im Mittel gleich auf die 3 Brückenzweige verteilt werden. Optimal ist, den Kurzschluss in einem Zweig zu machen, in dem vorher ein Schalter Strom führte und in dem nach dem Nullzustand wieder ein Schalter eingeschaltet ist. Damit kann die Anzahl Schaltvorgänge minimal gehalten werden.

Für die Bestimmung der optimalen Nullzustände wird angenommen, dass mittels Drehzeigermodulation (Kapitel 8) für einen U-Stromrichter erzeugte Schaltfunktionen für einen I-Stromrichter umgesetzt werden sollen. Bei positiver Drehrichtung des Zeigers werden die spannungsbildenden Zustände entlang den Pfaden in Bild 14.5 links von ${ }^{1} Z$ bis ${ }^{6} Z$ durchlaufen und bei negativer Drehrichtung von ${ }^{6} Z$ bis ${ }^{1} Z$. Dazwischen treten Nullzustände auf, die aber für die anschliessende Konversion der Schaltfunktionen bedeutungslos sind.

Am Beispiel der folgenden Schaltsequenz ist ersichtlich, wie ausgehend vom U-Stromrichter der Nullzustand beim I-Stromrichter optimal gewählt wird: aus dem Zustand ${ }^{1} Z$ habe der U-Stromrichter den Zustand ${ }^{0} Z$ eingenommen. Bei positiver Drehrichtung des Zeigers können darauf nur die Zustände ${ }^{1} Z$ oder bei einem Sektorwechsel ${ }^{3} Z$ folgen. Diese spannungsbildenden Zustände ergeben beim I-Stromrichter die gleichen, strombildenden Zustände. Wird der Nullzustand des I-Stromrichters mit einem Zweig gemacht, der in den Zuständen ${ }^{1} Z$ und ${ }^{3} Z$ Strom führt, so treten in keinem Fall unnötige Schaltvorgänge auf. Die gleichen Überlegungen können für jeden Zustand angestellt werden. Die Auswertung zeigt, dass die zu verwendenden Nullzustände von der Drehrichtung abhängig werden. Die optimalen Zustandsübergänge beim I-Stromrichter sind für positive und negative Drehrichtung in Bild 14.5 mitte und rechts dargestellt. Die schwarzen Pfeile bezeichnen die Zustandsübergänge innerhalb eines Sektors und die grauen die Übergänge bei einem Sektorwechsel. Bei jedem Zustandswechsel schalten beim I-Stromrichter zwei Schalter bzw. ein Umschalter, gleich wie beim U-Stromrichter.

Die obigen Betrachtungen gelten auch für Trägerverfahren, da dort die gleichen $\mathrm{Zu}-$ standsabfolgen wie bei der Standardvariante der Drehzeigermodulation auftreten.

\subsubsection{Grundfrequenzsteuerung}

Analog zum U-Stromrichter ist das einfachste Steuerverfahren beim I- Stromrichter die Grundfrequenzsteuerung gemäss Bild 14.6. Dabei ist keine Amplitudenvariation mög- 

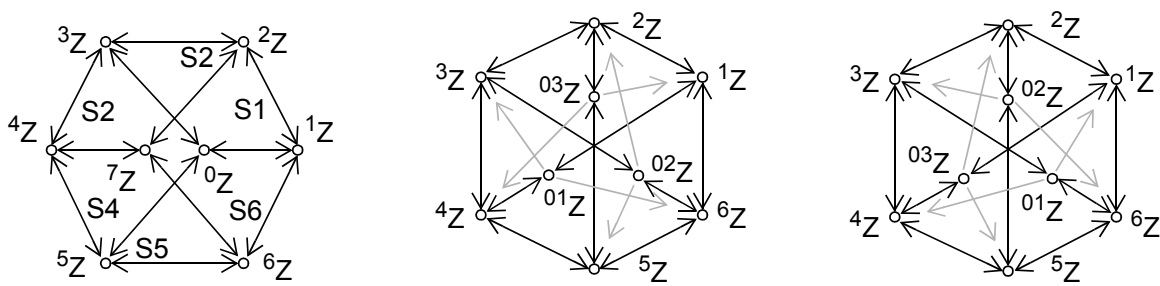

Bild 14.5. Übergänge zwischen den Schaltzuständen bei dreiphasigen U- und I-Stromrichtern, links: U-Stromrichter, mitte: I-Stromrichter mit positiver Drehrichtung des Feldes, rechts: I-Stromrichter mit negativer Drehrichtung des Feldes

lich. Der Stromrichter wird nur in den strombildenden Zuständen ${ }^{1} Z$ bis ${ }^{6} Z$ (Tabelle 14.2) betrieben und die Nullzustände ${ }^{01} Z,{ }^{02} Z$ und ${ }^{03} Z$ werden nicht verwendet. Für die Amplitude der Grundschwingung und damit für den maximal möglichen Modulationsgrad gilt:

$$
\hat{i}_{1, U / V / W}=M_{\text {max }} I_{d}=\frac{2 \sqrt{3}}{\pi} I_{d} \quad \Rightarrow \quad M_{\text {max }}=\frac{2 \sqrt{3}}{\pi} \approx 1.10
$$

Die Erzeugung der Steuersignale für Grundfrequenzsteuerung ist trivial. Sie können aber auch aus der Grundfrequenzsteuerung der entsprechenden Schaltung mit Spannungszwischenkreis hergeleitet werden.



Bild 14.6.

Schaltfunktionen bei Grundfrequenzsteuerung

\subsubsection{Einsatz von Pulsmustern für U-Stromrichter in I-Stromrichtern}

Die Schaltfunktionen des I-Stromrichters werden jeweils aus der Differenz zweier Schaltfunktionen des U-Stromrichters gebildet. Die neuen Funktionen $s_{I, U / V / W}$, und als Folge davon auch die Phasenströme, weisen die gleichen Eigenschaften auf wie die verketteten Spannungen des U-Stromrichters. Aus diesem Grund sollen die Eigenschaften der verketteten Spannungen des U-Stromrichters am Beispiel von $u_{U V}$ genauer betrachtet werden:

$$
u_{U V}=u_{U 0}-u_{V 0}=\left(u_{U}+u_{G}\right)-\left(u_{V}+u_{G}\right)=u_{U}-u_{V}
$$

Diese Gleichung zeigt 3 wichtige Eigenschaften auf:

- In den verketteten Spannungen fallen alle Gleichtaktkomponenten und damit die Vielfachen der 3. Harmonischen weg.

- Es können keine Frequenzen auftreten, die nicht schon in einer der beiden Spannungen enthalten sind. 
- Die Amplituden der Grundschwingungen sind $\sqrt{3}$ mal grösser, als die der Phasenspannungen. Bei synchronen Pulsmustern sind die Amplituden aller verbleibenden Spannungskomponenten entweder null oder ebenfalls $\sqrt{3}$ mal grösser als die entsprechenden Phasenspannungen.

Werden keine speziellen Forderungen an die Schaltfunktionen des U-Stromrichters gestellt, so besteht die Möglichkeit, dass in den verketteten Spannungen sehr kurze Pulse auftreten, d.h. der Stromrichter nur sehr kurze Zeit in einem bestimmten Zustand verweilt. Werden Muster mit solchen Eigenschaften auf I-Stromrichter übertragen, ergibt dies unzulässig kurze Ein- bzw. Ausschaltzeiten einzelner Schalter. Dies muss vermieden werden, indem die Pulslängen kontrolliert und bei Bedarf angepasst werden. Werden die Schaltfunktionen mit einem Drehzeigermodulator erzeugt, so kann das Problem einfach gelöst werden, da dabei die Dauer jedes Umrichterzustandes berechnet wird. Die Resultate müssen lediglich auf- bzw. abgerundet werden.

Beim U-Stromrichter sind die verketteten Spannungen um $\pi / 6$ gegenüber den Phasenspannungen verdreht. Werden die Schaltfunktionen von einem U- auf einen I-Stromrichter übernommen, so tritt diese Phasendrehung zwischen den Phasenspannungen des UStromrichters (Sollwert) und dem Phasenstrom des I-Stromrichters (Istwert) auf.

Modulationsgrad: Werden die Schaltfunktionen des I-Stromrichters aus solchen für einen U-Stromrichter erzeugt, so stimmen die Modulationsgrade aus zwei Gründen nicht überein: zum einen ist der Modulationsgrad $M$ des U-Stromrichters auf $U_{d} / 2$ und $M_{I}$ des I-Stromrichters auf $I_{d}$ bezogen, zum anderen entspricht die verkettete Spannung dem Phasenstrom. Daraus resultiert ein Umrechnungsfaktor von $\sqrt{3} / 2$ :

$$
M_{I}=\frac{\hat{i}_{1, U / V / W}}{I_{d}}=M \frac{\sqrt{3}}{2}
$$

Bei den Modulationsverfahren für U-Stromrichter wurde gezeigt, dass der maximale Modulationsgrad ohne Übersteuerung $2 / \sqrt{3}$ beträgt. Auf I-Stromrichter übertragen bedeutet das, dass für den maximalen Modulationsgrad $M_{I, \max }=1$ gilt.

Konvertierte Schaltmuster: Als Beispiele werden zwei Drehzeigerverfahren aus Kapitel 8.2 betrachtet, die beide einen maximalen Modulationsgrad von $\sqrt{3} / 2$ erreichen: die Standard-Drehzeigermodulation mit identischer Dauer der beiden Nullzustände in Bild 8.7 und eine diskontinuierliche Modulation mit nur einem Nullzustand pro Tastintervall gemäss Bild 8.8. Bild 14.7 zeigt die resultierenden Muster des I-Stromrichters für die beiden Verfahren. Die Funktionen $s_{I, U / V / W}$ sind direkt proportional zu den entsprechenden Phasenströmen. Die dargestellte Funktion der Phase $U$ wird aus den Schaltfunktionen $s_{I, U^{+}}$und $s_{I, U_{-}}$der beiden Schalter $S_{U_{+}}$und $S_{U_{-}}$gebildet. Zur besseren Übersicht werden in den Bildern die beiden Schaltfunktionen verkleinert und $s_{I, U}$ - zusätzlich negativ aufgetragen. Sind die beiden Schaltfunktionen gleichzeitig ungleich null, so ist der Brückenzweig kurzgeschlossen. Die Betrachtung der Schaltfunktionen zeigt, dass jeder Puls des Phasenstromes durch einen vollständigen Umschaltzyklus eines Schalters gebildet wird. Bei gleichem Verhältnis von Grundschwingungs- zu Abtastperiode wird bei diskontinuierlicher Modulation die Schaltzahl $q$ kleiner. Für die gleiche mittlere Anzahl Schaltvor- 
gänge im Stromrichter kann deshalb die Abtastperiode verkleinert werden, wie dies bei der Drehzeigermodulation in Kapitel 8 vorgestellt wurde.
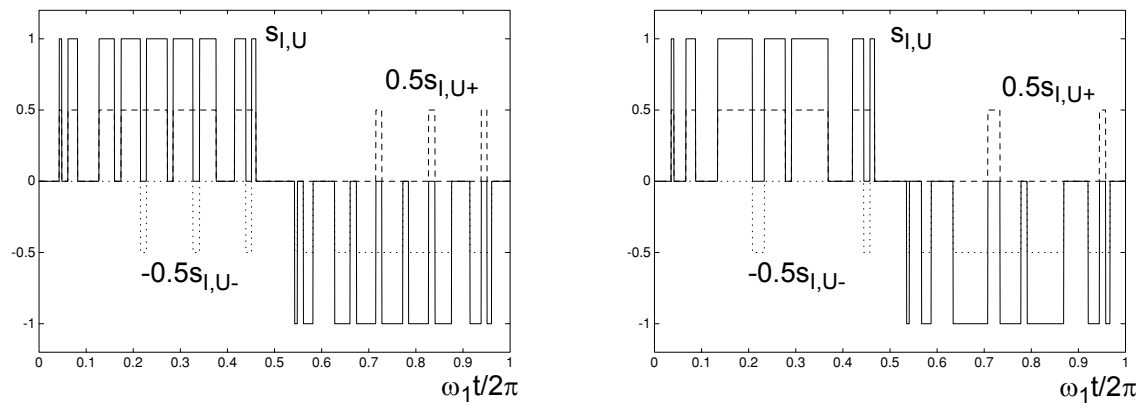

Bild 14.7. Schaltfunktionen für I-Stromrichter hergeleitet aus Drehzeigerverfahren, $T_{1} / T_{\text {Tast }}=18$, links: Standard-Drehzeigermodulation, rechts: diskontinuierliche Modulation

Für beide Verfahren sind in Bild 14.8 die Spektra des Phasenstromes für $q \approx 40$ dargestellt. Um bei der diskontinuierlichen Modulation die gleiche Schaltzahl zu erhalten wie beim Standardverfahren, wurde die Abtastfrequenz um 50\% grösser gewählt. Dies zeigt sich im Spektrum in der Verschiebung des ersten Trägerbandes zu höheren Frequenzen. Leider treten aber prinzipbedingt relativ starke niederfrequente Harmonische auf.

Werden die Schaltfunktionen mittels dem Drehzeigerverfahren gebildet, so kann die Pulsaufbereitung für den I-Stromrichter einfacher gemacht werden: statt zuerst die Schaltfunktionen für den U-Stromrichter zu erzeugen, können die berechneten Zeiten direkt in Schaltfunktionen für den I-Stromrichter umgesetzt werden.
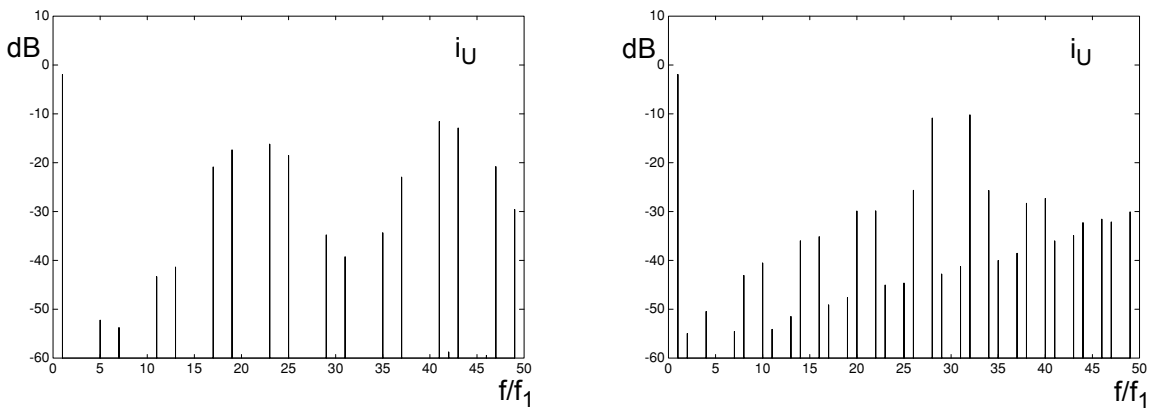

Bild 14.8. Spektra eines Phasenstromes für konvertierte Drehzeiger-Schaltfunktionen, $q \approx 40$, $M_{\Gamma}=0.8,0 \mathrm{~dB}: I_{d}$, links: Standard-Drehzeigermodulation, $T_{1} / T_{\text {Tast }}=21$, rechts: diskontinuierliche Modulation, $T_{1} / T_{\text {Tast }}=30$ 


\subsubsection{Modifiziertes Trägerverfahren für I-Stromrichter}

Für die Modulation von I-Stromrichtern wird häufig ein synchrones Verfahren mit sehr ausgeprägten Symmetrien verwendet [Hom1], [Non1], [Non2], [Ohn1], [Wie1]. Die Pulse werden in $\pi / 3$-Intervallen immer gleichartig erzeugt und anschliessend zu Schaltfunktionen für die 3 Phasen zusammengesetzt. Dabei wird ausgenutzt, dass ein symmetrisches Dreiphasensystem in jedem $\pi / 3$-Intervall aus identischen Kurvenstücken besteht. Sind die Verläufe in einem Ausschnitt bekannt, so können sämtliche Ausschnitte durch Vertauschen und Anpassen der Vorzeichen aus diesen Kurvenstücken aufgebaut werden. Das Prinzip ist in Bild 14.9 dargestellt: aus den zwei Funktionen $x_{1}=M \sin \left(\omega_{1} t\right)$ und $x_{2}=M \sin \left(\omega_{1} t+2 \pi / 3\right)$ werden die Ausschnitte $\omega_{1} t=[0, \pi / 3]$ als Sollwerte für die Modulation verwendet. Die beiden Trägersignale $x_{T 1}$ und $x_{T 2}$ sind gegenphasig. Die resultierenden Schaltfunktionen sind identisch, wenn die eine bei $\pi / 6$ gespiegelt wird. Ihre Summe bildet das Pulsmuster für den Ausschnitt [ $\pi / 3,2 \pi / 3]$, d.h. für den Bereich des Scheitelwertes. Mit diesen 3 Teil-Schaltfunktionen können die Schaltfunktionen für alle 3 Phasen aufgebaut werden. Für die Phase $U$ ist dies in Bild 14.9 rechts dargestellt. Die Schaltfunktion für den Schalter $S_{U+}$ ist dabei positiv und diejenige für $S_{U \text { - }}$ negativ aufgetragen. Im ersten Intervall $[0, \pi / 3]$ wird $s_{I, U^{+}}$aus $S F 1$ gebildet, im zweiten ist $s_{I, U^{+}}$konstant eins und im dritten ist die Funktion durch $S F 2$ bestimmt. Im mittleren Sektor wird die Funktion $s_{I, U}$ gebildet, indem $s_{I, U}$ für die Zeit, in der $S F 3$ null sein soll, eingeschaltet wird.

Das Spektrum für eine Modulation nach diesem Verfahren mit $q=40$ zeigt Bild 14.10.
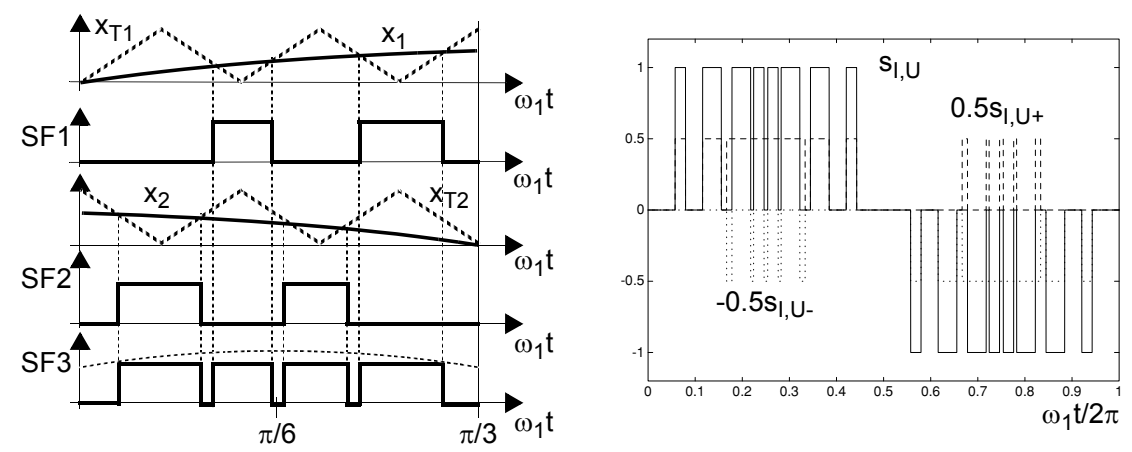

Bild 14.9. Modifiziertes Trägerverfahren für I-Stromrichter,

links: Pulserzeugung in einem $\pi / 3$-Intervall, rechts: Schaltfunktionen in der Phase $U$

Beim vorgestellten, synchronen Musteraufbau sind nur bestimmte Trägerfrequenzen $f_{T}$ und damit Schaltzahlen $q$ möglich:

$$
f_{T}=6(k+0.5), \quad q=8 k, \quad \text { mit } \quad k=1,2,3 \ldots
$$

Grundsätzlich kann bei diesem Verfahren auch die Phasenlage des Trägersignals variiert werden. Dabei wird aber die Schaltzahl grösser. Umgekehrt lassen sich die Seitenlinien des Trägerbandes beeinflussen. Weiter ist es nicht zwingend, das Verfahren synchron auszuführen. Es kann grundsätzlich ein beliebiges Verhältnis von Träger- zu Grundschwin- 


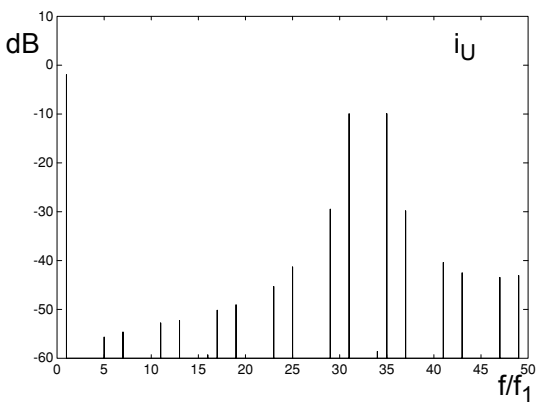

Bild 14.10.

Spektrum eines Phasenstromes beim modifizierten Trägerverfahren für I-Stromrichter $q=40, M_{\Gamma}=0.8,0 \mathrm{~dB}: I_{d}$

gungsfrequenz verwendet werden. Die Resultate sind dann sehr ähnlich zu denjenigen von konvertierten, diskontinuierlichen Träger- oder Drehzeigermustern.

\subsubsection{Weitere Steuerverfahren}

Auch vorausberechnete Pulsmuster können von den U-Stromrichtern (Kapitel 9) übernommen werden. Die Harmonischen der Phasenströme beim I-Stromrichter sind dann proportional zu denjenigen der verketteten Spannungen des U-Stromrichters. Die Berechnung der Schaltwinkel lässt sich aber auch direkt für die Phasenströme des I-Stromrichters durchführen. Die Pulsmuster können dabei auf die zwei in Bild 14.11 dargestellten Arten angesetzt werden: so, dass jeder Puls der $S F 1$ und $S F 2$ einen Puls der $S F 3$ ergibt oder so, dass je ein Puls der $S F 1$ zusammen mit einem der $S F 2$ einen Puls der $S F 3$ ergibt. Für die benötigten Berechnungskriterien sei auf die Literatur verwiesen: [Bow5], [Bet1], [Cam1], [Jen3].
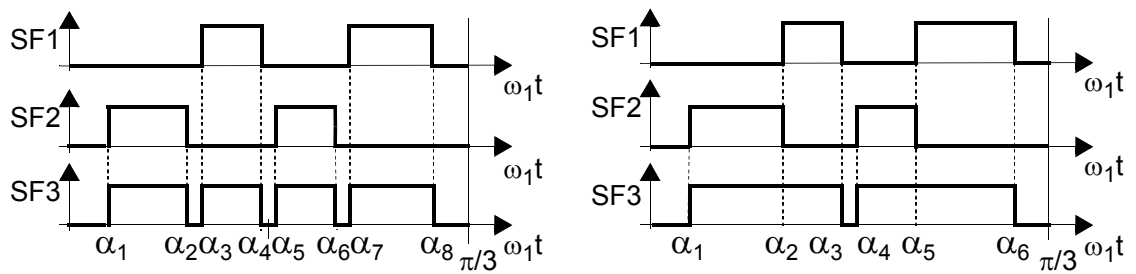

Bild 14.11. Synchrone Pulsmuster für I-Stromrichter, links: alle Pulsflanken von $S F 1$ und $S F 2$ frei, rechts: je eine Pulsflanke von $S F 1$ und $S F 2$ fallen in $S F 3$ zusammen

Bei I-Stromrichtern werden auch Steuerverfahren eingesetzt, die den Ladungsfehler in den Filterkondensatoren (Kapitel 3.2.6.2) als Kriterium für die Pulsmustererzeugung verwenden [Jen3], [Kal1]. Dieser Ladungsfehler entspricht dem Fehler in der Spannungszeitfläche bei U-Stromrichtern bzw. in der Statorflussverkettung in einer angeschlossenen Maschine.

Literatur zu Kapitel 14.2: [Aml1], [Bet1], [Bow5], [Cam1], [Enj2], [Hom1], [Jen3], [Joo1], [Kal1], [Kol7], [Nam1], [Non1], [Non2], [Ohn1], [Wie1], [Wil1], [Zio2] 


\section{Steuerverfahren für zusammengeschaltete Stromrichter}

In Umrichtern für grosse Leistungen können auf der Last- oder auf der Netzseite zwei oder mehr Stromrichter zusammengeschaltet werden. Es gibt zwei Gründe für die Verwendung dieser relativ aufwendigen Schaltungsvariante:

- Die geforderte Leistung ist so gross, dass die Realisierung mit einem einzelnen Stromrichter gar nicht möglich ist.

- Die Realisierung mit einem Stromrichter wäre zwar möglich, jedoch müssten dazu grosse und langsam schaltende Halbleiter eingesetzt werden. Die erlaubte Schaltfrequenz wäre dann zu klein, um die geforderte Qualität der Kurvenformen zu erreichen.

Für die Grundfunktion des Gesamtsystems können die einzelnen Stromrichter im Prinzip unabhängig voneinander nach einem der in Teil II beschriebenen Steuerverfahren betrieben werden. Eine übergeordnete Regelung gibt dabei die Grundschwingungen für die einzelnen Spannungen und Ströme vor. Werden jedoch die Modulatoren der Stromrichter miteinander koordiniert, so lassen sich erheblich bessere Kurvenformen erreichen.

Im folgenden wird ausschliesslich die Zusammenschaltung von zweistufigen U-Stromrichtern betrachtet. Es kommen die idealisierten Modelle mit konstanter Zwischenkreisspannung und Standardlast zur Anwendung. Zudem wird angenommen, dass alle Teilstromrichter für gleiche Leistung ausgelegt sind und mit derselben mittleren Schaltfrequenz betrieben werden.

\subsection{Serieschaltung von Stromrichtern}

\subsubsection{Schaltungen}

Die Serieschaltung von Stromrichtern erfolgt im allgemeinen Fall gemäss Bild 15.1 mit Hilfe eines Transformators, bestehend aus einer entsprechenden Anzahl Teiltransformatoren mit je einem eigenen geschlossenen Kern. Dabei sind die lastseitigen Wicklungen seriegeschaltet und führen alle den Strom $i_{L}$. Die Ausgangsspannung $u_{L}$ ist proportional zur Summe der Ausgangsspannungen der einzelnen Stromrichter. Unter der Annahme, dass alle Teiltransformatoren dasselbe Wicklungsverhältnis $1: n$ aufweisen, gilt:

$$
u_{L}=n\left(u_{a, A B}+u_{b, A B}+u_{c, A B}+\ldots\right), i_{L}=\frac{i_{A}}{n}, i_{d}=i_{a, d}+i_{b, d}+i_{c, d}+\ldots
$$

Die Transformatoren werden dabei als ideal angenommen. Streuinduktivitäten lassen sich berücksichtigen, indem sie vollständig auf die Lastseite verschoben und zur Lastinduktivität $L_{k}$ gezählt werden. Die Gleichungen (15.1) behalten dann ihre Gültigkeit. 

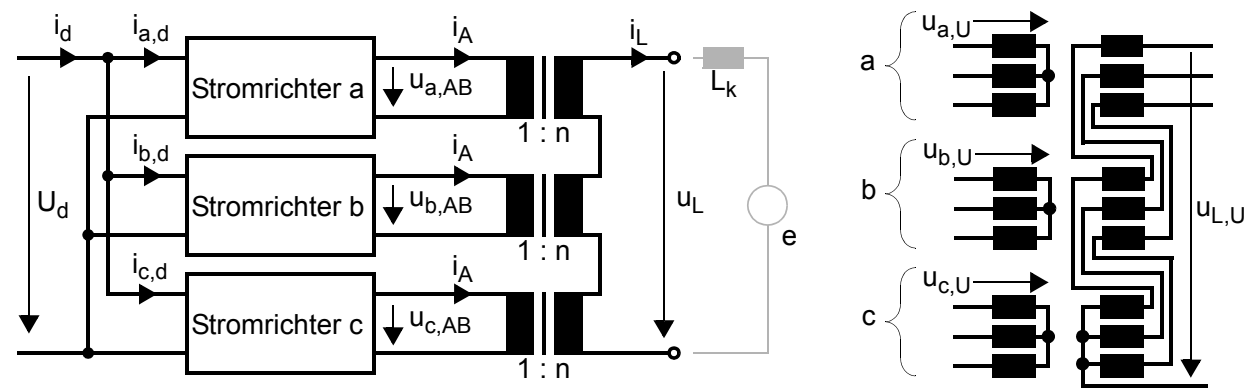

Bild 15.1. Serieschaltung von 3 Stromrichtern

links: Prinzip einphasig, rechts: Transformator für dreiphasige Serieschaltung

Im Normalfall sind die Stromrichter auf der DC-Seite parallel geschaltet. Dadurch ist auf einfache Weise sichergestellt, dass sie alle mit derselben Gleichspannung versorgt werden. Die Aufteilung der Lastspannung auf die einzelnen Stromrichter kann dann mit Hilfe der Modulatoren rein gesteuert erfolgen. Prinzipiell ist es jedoch auch möglich, dass die Zwischenkreise ebenfalls seriegeschaltet sind oder sogar völlig getrennt sind. Ein Beispiel für den ersten Fall ist die Speisung des Zwischenkreises für einen dreistufigen Stromrichter mit zwei zweistufigen Netzstromrichtern (Bild 3.27).

Die Regelung des Gesamtsystems ist besonders einfach: da der Ausgangsstrom für alle Stromrichter identisch ist, kann er durch einen einzigen Regler eingestellt werden. Dasselbe gilt für die Regelung der Zwischenkreisspannung auf der Speiseseite des Umrichters, wenn die Zwischenkreise seriegeschaltet sind.

In dreiphasigen Schaltungen wird der Transformator in Bild 15.1 rechts verwendet. Die Wicklungen für die Teilstromrichter sind unter sich in Stern geschaltet. Lastseitig sind die Teilwicklungen einer Phase in Serie geschaltet und als ganzes in Stern angeordnet. Es ist auch möglich, anstelle von Sternschaltungen überall Dreieckschaltungen zu verwenden.

Bezugsgrössen: Für die folgenden Betrachtungen ist es sinnvoll, neben den bisher verwendeten Bezugsgrössen $U_{B}$ und $I_{B}$ (Kapitel 5.2.1.3), die hier für die Teilstromrichter gelten, für die Lastseite angepasste Bezugsgrössen $U^{\prime}{ }_{B}$ und $I_{B}$ zu definieren:

$$
U_{B}^{\prime}=k n U_{B}, \quad I_{B}=\frac{I_{B}}{n}, \quad k=\text { Anzahl Stromrichter }
$$

Liegt am Ausgang aller Teilstromrichter $U_{B}$ an, so ergibt sich an der Last gerade $U^{\prime}{ }_{B}$. Desgleichen entspricht $I_{B}$ auf der Stromrichterseite $I_{B}$ in der Last.

Spezialfälle von Serieschaltungen: Die in Kapitel 3.3.2.2 eingeführte einphasige Brükkenschaltung ist, wie Bild 15.2 verdeutlicht, im Prinzip eine Serieschaltung von zwei Halbbrücken. Allerdings fehlt der Transformator und die Lastspannung entspricht nicht der Summe, sondern der Differenz der beiden Teilspannungen.

Auch für dreiphasige Schaltungen lässt sich eine Serieschaltung ohne Transformator realisieren. Sie ist in Bild 15.3 dargestellt. Die Last muss dazu pro Phase über zwei Anschlussklemmen verfügen (z.B. beide Klemmen jeder Maschinenwicklung). Dadurch 


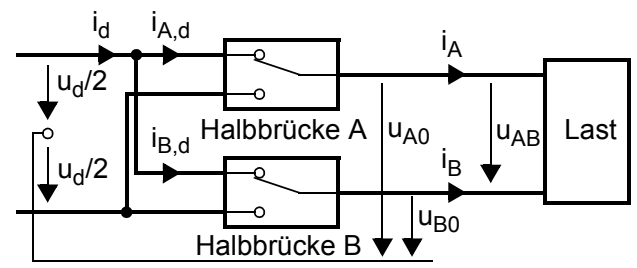

$$
\begin{aligned}
& u_{A B}=u_{A 0}-u_{B 0} \\
& i_{A}=-i_{B} \\
& i_{d}=i_{A, d}+i_{B, d}
\end{aligned}
$$

Bild 15.2.

Einphasige Brücke als Serieschaltung von zwei Halbbrücken

verhält sie sich allerdings wie 3 unabhängige einphasige Brückenschaltungen. Die Vorteile des dreiphasigen Stromrichters gehen verloren: alle Gleichtaktkomponenten in den Mittelpunktspannungen treten auch in den Lastspannungen auf.

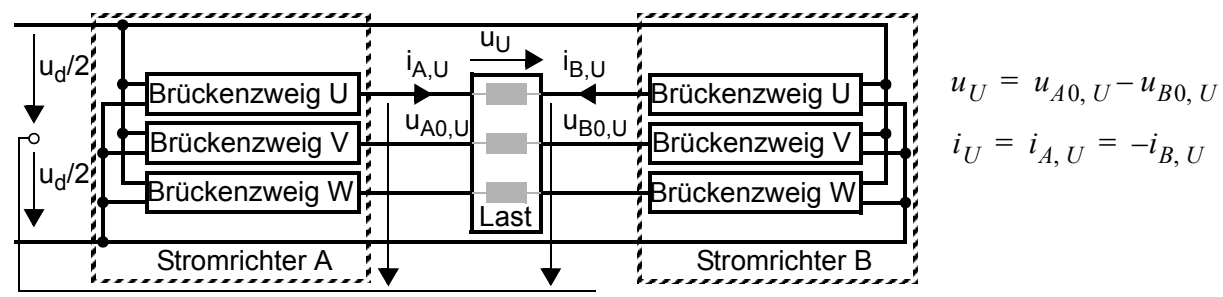

Bild 15.3. 3 einphasige Brücken zur Speisung einer dreiphasigen Last

Im folgenden werden verschiedene Steuerverfahren jeweils am Beispiel der Serieschaltung von 3 Stromrichtern gemäss Bild 15.1 vorgestellt. Die Überlegungen lassen sich jedoch leicht auf $k$ Stromrichter verallgemeinern. Als Teilstromrichter werden nur die in der Praxis verwendeten ein- und dreiphasigen Brückenschaltungen betrachtet. Im einphasigen Fall bedeutet dies im Grunde genommen eine doppelte Serieschaltung: die 3 Brücken sind je aus zwei Halbbrücken zusammengesetzt.

\subsubsection{Grundfrequenzsteuerung}

Zuerst wird die Serieschaltung von 3 dreiphasigen Brücken betrachtet. Wie in Kapitel 6.3 gezeigt, sind die Schaltfunktionen und damit die Mittelpunktspannungen bei Grundfrequenzsteuerung fest gegeben. Der Modulationsgrad beträgt $4 / \pi$. Dies gilt auch für die Phasenspannungen der Teilstromrichter $u_{a, U / V / W}, u_{b, U / V / W}$ und $u_{c, U / V / W}$. Ihre Phasenwinkel sind jedoch frei wählbar. Bild 15.4 zeigt, dass sich durch eine gegenseitige Phasenverschiebung der 3 Teilspannungen eine Gesamtspannung mit deutlich verbesserter Kurvenform erzielen lässt. Es sind die Spannungen der Phase $U$ dargestellt. Die gegenseitigen Phasenverschiebungen sind durch $\varphi_{b}$ und $\varphi_{c}$ bezeichnet, welche prinzipiell verschieden sein können. Möchte man jedoch eine viertelperiodensymmetrische Lastspannung, so muss $\varphi_{b}=-\varphi_{c}$ gelten. Die Grundschwingung $\hat{u}_{L, v=1}$ und der zugehörige Modulationsgrad $M$ sind eine Funktion dieses einen verbleibenden Parameters und werden damit variabel:

$$
\hat{u}_{L, v=1}=\frac{4}{\pi} \frac{1+2 \cos \varphi_{b}}{3} U_{B}^{\prime}=M U_{B}^{\prime}, \quad M=\frac{4}{\pi} \frac{1+2 \cos \varphi_{b}}{3}
$$




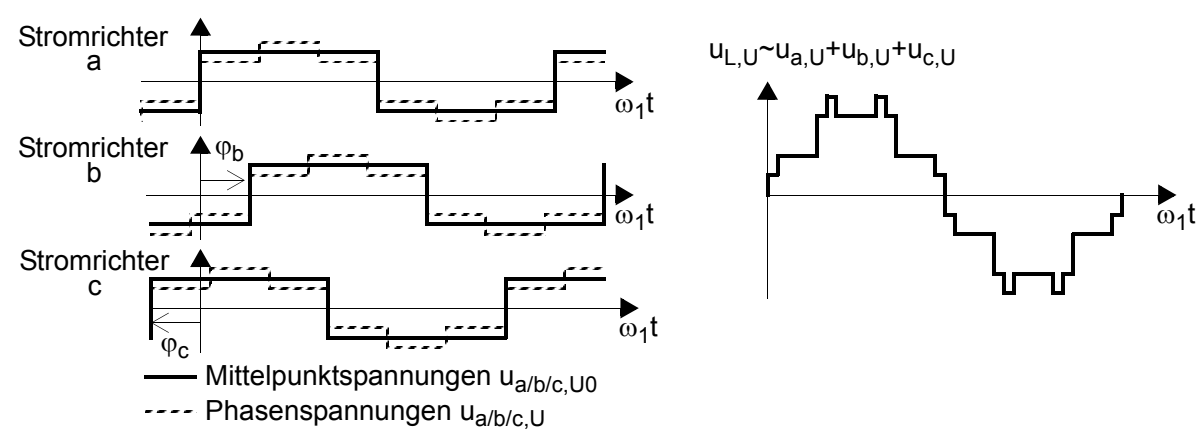

Bild 15.4. Phasenverschiebung der Teilspannungen bei Grundfrequenzsteuerung, links: Mittelpunkt- und Phasenspannungen der Phase $U$ in den Teilstromrichtern, rechts: Lastspannung in der Phase $U$
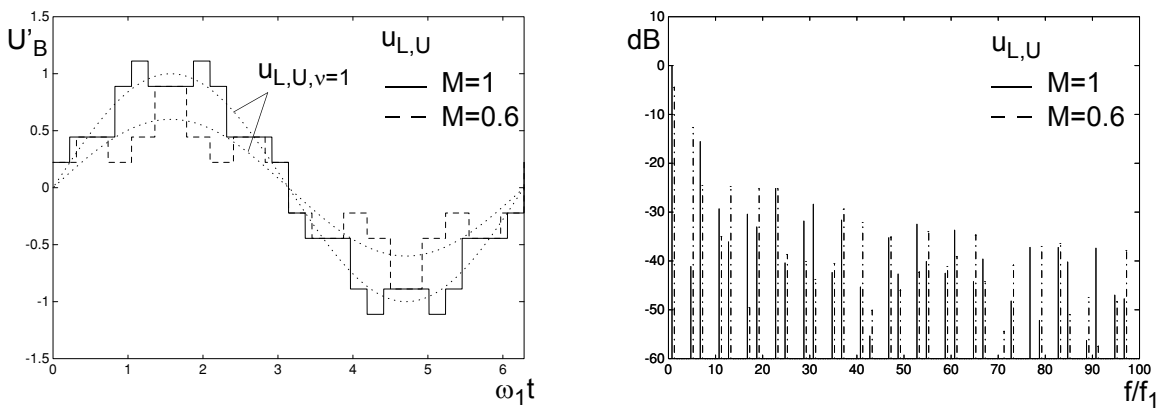

Bild 15.5. Lastspannungen der Phase $U$ für $M=1$ ( $\left.\varphi_{b}=0.83 \mathrm{rad}\right)$ bzw. $M=0.6$ ( $\left.\varphi_{b}=1.36 \mathrm{rad}\right)$, links: zeitliche Verläufe, rechts: zugehörige Spektra, $0 \mathrm{~dB}: U^{\prime}{ }_{B}$

In Bild 15.5 ist als Beispiel die Lastspannung $u_{L, U}$ zusammen mit ihrem Spektrum für $M=1$ und $M=0.6$ dargestellt. Der Verschiebungswinkel $\varphi_{b}$ beträgt dabei 0.83 bzw. 1.36rad. Es muss erwähnt werden, dass bei der beschriebenen Steuerung die Halbleiter der einzelnen Stromrichter nicht dieselbe Durchlassbelastung erfahren. Dies hängt damit zusammen, dass die Phasenlage des gemeinsamen Grundschwingungsstromes bezüglich der Phasenspannungen verschieden ist. Damit sind die Zeitintervalle, während denen die Ströme durch die Dioden fliessen, verschieden.

Bereits bei der Serieschaltung von zwei Stromrichtern ist es möglich, die Grundschwingung der Lastspannung einzustellen. Bei mehr als 3 Stromrichtern entstehen zusätzliche Freiheitsgrade. Sie können dazu benutzt werden, einzelne Oberschwingungen zu eliminieren oder den Verzerrungsanteil nach bestimmten Kriterien zu optimieren. Dabei wird nach den in Kapitel 9 vorgestellten Methoden vorgegangen.

Einphasige Schaltungen: Bei seriegeschalteten einphasigen Brücken mit Grundfrequenzsteuerung lassen sich in gleicher Weise die einzelnen Ausgangsspannungen gegeneinander verschieben. Da jedoch jede Brücke für sich bereits die Serieschaltung von zwei 
Halbbrücken darstellt, erhöht sich die Anzahl Freiheitsgrade gegenüber der dreiphasigen Schaltung um einen Faktor zwei.

\subsubsection{Trägerverfahren mit versetzter Taktung}

Bereits bei der einphasigen Brücke (Kapitel 7.3.3) hat sich gezeigt, dass sich mit Trägerverfahren bei versetzter Taktung besonders gute Kurvenformen an der Last erzielen lassen. Durch die Verwendung von zwei um eine halbe Taktperiode verschobenen Trägersignalen wurden alle ungeraden Trägerbänder, darunter vor allem auch das dominante erste, in der Phasenspannung zum Verschwinden gebracht. Das folgende Beispiel mit dreieckförmigen Trägern und einer ganzzahligen Schaltzahl $q$ zeigt, wie dieses Prinzip auf 3 in Serie geschaltete dreiphasige Stromrichter übertragen werden kann.

Bild 15.6 zeigt die Modulation für die Phase $U$. Für alle 3 Teilstromrichter wird dasselbe Sollwertsignal $x_{U, \text { Soll }}$ verwendet. Die Trägersignale sind dagegen verschieden, nämlich um jeweils einen Drittel der Trägerperiode gegeneinander verschoben. Sie werden je für die Modulation aller 3 Phasen ihres Stromrichters verwendet. Im Bild ist ersichtlich, dass die resultierenden Schaltfunktionen und Phasenspannungen der einzelnen Teilstromrichter leicht verschieden sind. Sie weisen aber alle dieselbe Grundschwingung auf. In Bild 15.7 ist der Mechanismus der versetzten Taktung im Frequenzbereich veranschaulicht. Die Amplitudenspektra aller Schaltfunktionen und Phasenspannungen sind gleich. In den Phasenspannungen fehlen in gewohnter Weise die Vielfachen der 3. Harmonischen, die Gleichtaktkomponenten darstellen. Die Phasenspektra, im Bild rechts sind diejenigen für die Phase $U$ dargestellt, sind dagegen verschieden. Die Phasen der Harmonischen im ersten und zweiten Trägerband unterscheiden sich jeweils um $2 \pi / 3$. Dies bedeutet, dass sich diese Anteile in der Summe kompensieren und in $u_{L}$ fehlen. Die Grundschwingungen und die Harmonischen im 3. Trägerband weisen dagegen in allen Teilstromrichtern dieselbe Phase auf. Sie ergeben deshalb in der Lastspannung die maximalen Amplituden.

Bild 15.8 zeigt die Verläufe und die Spektra der Lastspannung und des zugehörigen Verzerrungsstromes in der Phase $U$. Die Wirkung der versetzten Taktung ist klar ersichtlich: neben der Grundschwingung ist nur noch das 3. Trägerband vorhanden. Eine Betrachtung über den dargestellten Frequenzbereich hinaus würde eine Präzisierung dieser Festestellung erlauben: nur alle Vielfachen des 3. Trägerbandes treten auf. Die Amplituden dieser verbleibenden Anteile sind dabei im Verhältnis zur Bezugsspannung $U^{\prime}{ }_{\mathrm{B}}$ gleich gross wie diejenigen der Teilspannungen bezüglich $U_{B}$. Die wirksame Schaltfrequenz an der Last entspricht der dreifachen Schaltfrequenz in den einzelnen Stromrichtern. Es kommt hin$\mathrm{zu}$, dass die Frequenzanteile im 3. Trägerband gegenüber dem ersten und zweiten bereits deutlich kleinere Amplituden aufweisen. Damit wird der Verzerrungsstrom kleiner als in einem einzelnen Stromrichter mit dreifacher Schaltfrequenz.

Das gezeigte Prinzip der versetzten Taktung lässt sich leicht verallgemeinern. Für eine Serieschaltung von $k$ Stromrichtern sind die einzelnen Trägersignale um jeweils den $k$ ten Teil der Taktperiode gegeneinander zu verschieben. In der Lastspannung treten dann nur noch die Trägerbänder der Ordnungen $k, 2 k, 3 k$ usw. auf. Da die Amplituden der Harmonischen von einem Trägerband zum nächsten generell linear abnehmen, reduziert sich der Effektivwert des Verzerrungsstromes ungefähr quadratisch zu $k$. 

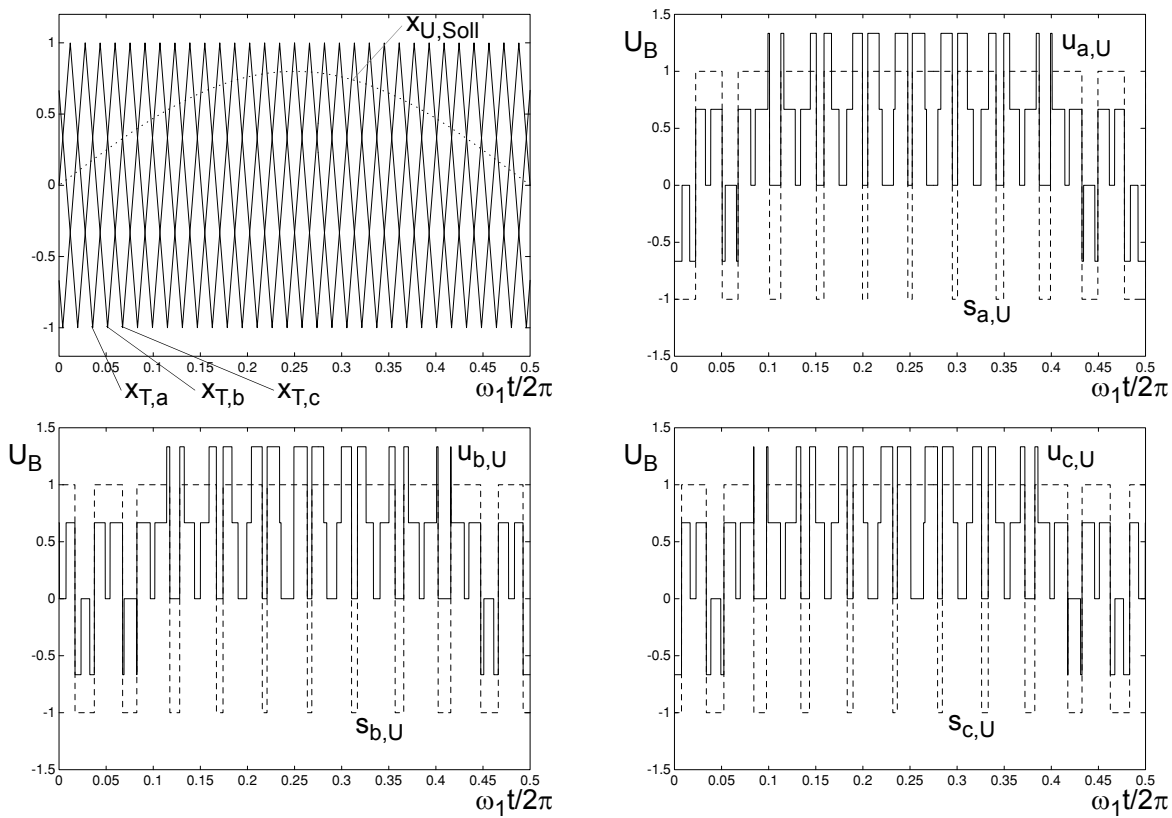

Bild 15.6. Trägerverfahren mit versetzter Taktung, $M=0.8, q=21$, oben links: Sollwert für die Phase $U$ und versetzte Träger für die Teilstromrichter, oben rechts und unten: Schaltfunktionen und Phasenspannungen der Phase $U$
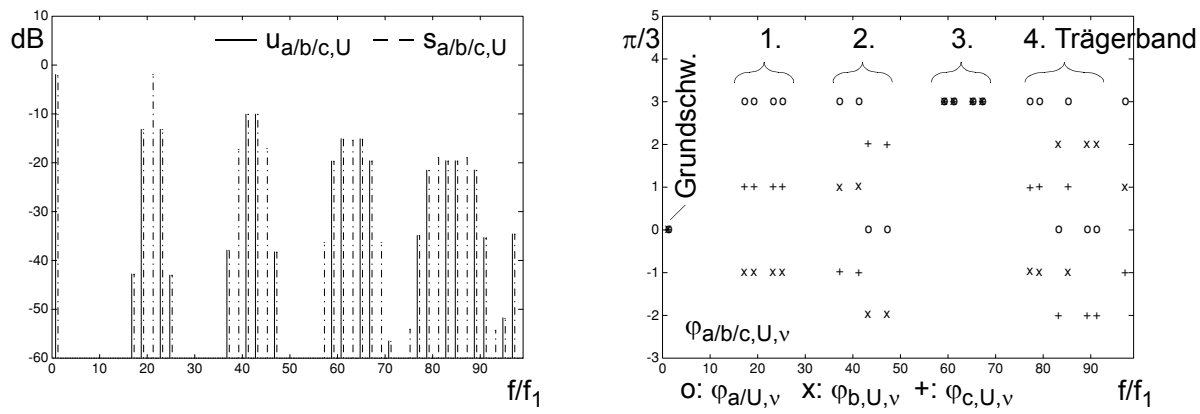

Bild 15.7. Links: Amplitudenspektra aller Phasenspannungen und Schaltfunktionen, $0 \mathrm{~dB}: U_{B}$ bzw. 1, rechts: Phasenspektra der Phasenspannungen in der Phase $U$

Die versetzte Taktung lässt sich mit gleicher Wirkung auch bei nichtsinusförmigen Sollwerten, bei asynchroner Modulation oder mit anderen Trägersignalen einsetzen.

Einphasige Schaltungen: Bei der Serieschaltung von einphasigen Brücken ist zu beachten, dass bereits die einzelne Brücke für sich gesehen mit versetzter Taktung betrieben wird (Kapitel 7.3.3). Für 3 Brücken sind deshalb 6 gegeneinander versetzte Trägersignale zu verwenden. 

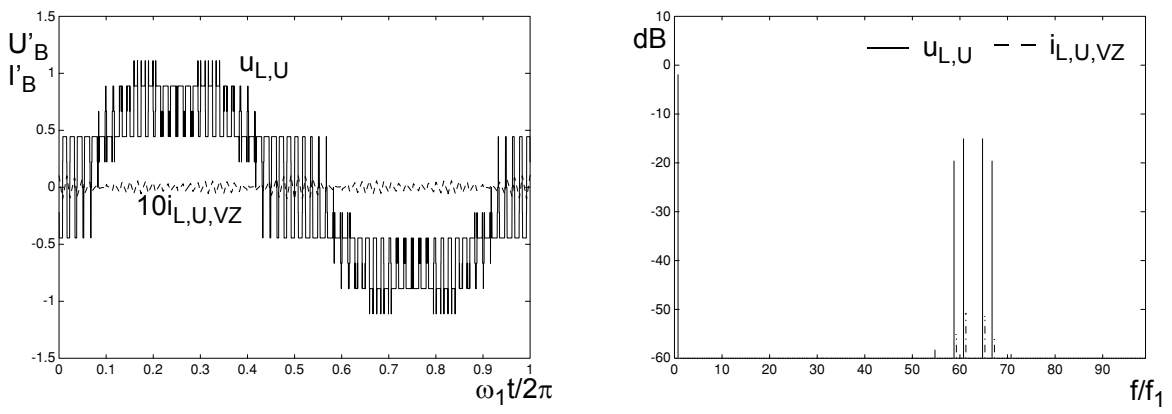

Bild 15.8. Links: Phasenspannung und Verzerrungsstrom in der Phase $U$ der Last, rechts: zugehörige Spektra, $0 \mathrm{~dB}: U^{\prime}{ }_{B}$ bzw. $I_{B}$

\subsubsection{Drehzeigermodulation}

Die lastseitigen Spannungen für 3 seriegeschaltete dreiphasige Stromrichter können in gewohnter Weise als Drehzeiger dargestellt werden. Der entsprechende Zeiger $\underline{u}_{L}$ ist die mit dem Wicklungsverhältnis $n$ multiplizierte Summe der Zeiger $\underline{u}_{a}, \underline{u}_{b}$ und $\underline{u}_{c}$, welche durch die Ausgangsspannungen der Teilstromrichter gebildet werden. Jeder von ihnen kann die bekannten 6 spannungsbildenden Zustände und zwei Nullzustände annehmen. Dies ergibt für $\underline{u}_{L}$ insgesamt $8^{3}(=512)$ mögliche Zustände, die den 37 verschiedenen in Bild 15.9 dargestellten Positionen der Zeigerspitze in der $\alpha \beta$-Ebene entsprechen. Jede dieser Positionen kann durch eine oder durch mehrere verschiedene Kombinationen von Schalterstellungen in den insgesamt 9 Brückenzweigen eingestellt werden. Die meisten Varianten stehen für eine Lastspannung von null zur Verfügung, nämlich 27 Nullzustände.

Bei der Drehzeigermodulation gilt es, den Sollwertzeiger $\underline{u}_{\text {Soll }}$ für ein Abtastintervall durch eine Kombination von angrenzenden Spannungszeigern nachzubilden (Kapitel 8.1). In Kapitel 8.3 wurde andererseits gezeigt, dass jede Drehzeigermodulation stets nur eine andere Darstellung eines Trägerverfahrens ist. Es ist deshalb naheliegend, das Prinzip der versetzten Taktung auch hier zur Anwendung zu bringen. Dies geschieht, indem jeder Teilstromrichter für sich mit einer Drehzeigermodulation gesteuert wird. Da eine Trägerperiode $T_{T}$ bei der Drehzeigermodulation zwei Abtastintervallen $T_{\text {Tast }}$ entspricht,

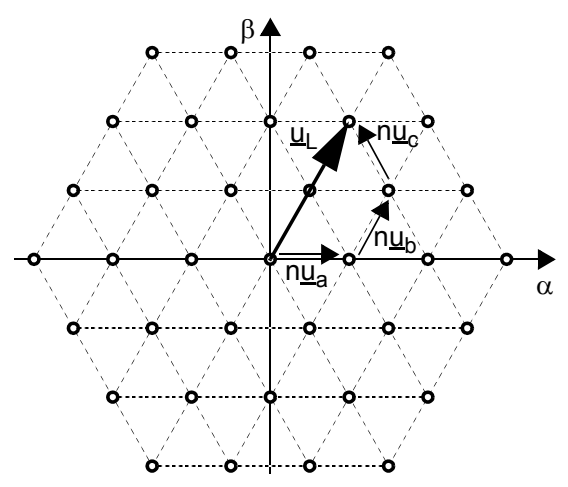

Bild 15.9.

37 verschiedene Schaltzustände für $\underline{u}_{L}$ in der Drehzeigerebene, Zusammensetzung von $\underline{u}_{L}$ aus den Drehzeigern der Teilstromrichter 
sind für die versetzte Taktung die Abtastintervalle um $2 T_{\text {Tast }} / 3$ gegeneinander zu verschieben. Jeder Teilstromrichter führt innerhalb seines Abtastintervalles eine normale Sequenz von 2 Nullzuständen und zwei spannungsbildenden Zuständen aus. Bild 15.10 zeigt ein entsprechendes Beispiel für die Standardvariante der Drehzeigermodulation mit zwei symmetrisch angeordneten Nullzuständen pro Taktintervall. Dies entspricht einem Trägerverfahren mit den abgetasteten und um eine Gleichtaktkomponente erweiterten Sollwerten gemäss Bild 8.7 .

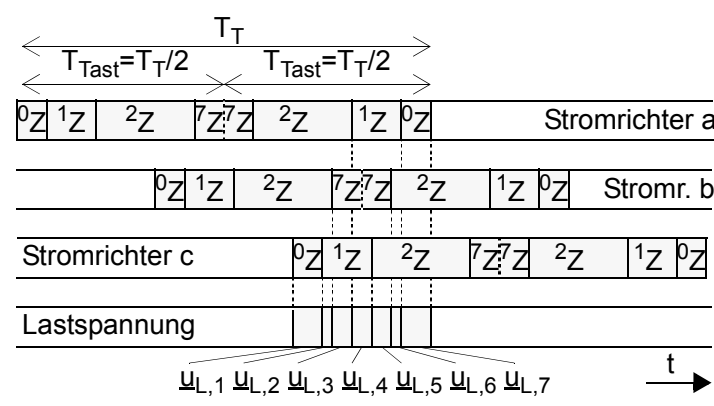

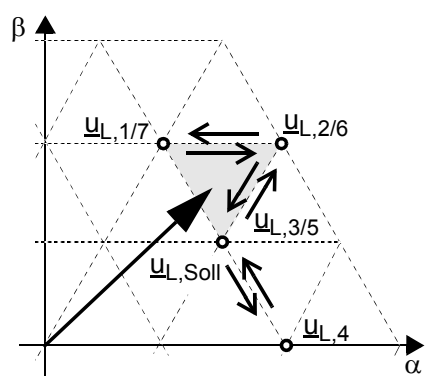

Bild 15.10. Drehzeigermodulation mit versetzten Taktintervallen, links: Schaltsequenzen der Teilstromrichter und des Lastspannungszeigers, rechts: Darstellung einer Trägerperiode in der Drehzeigerebene

Für die Lastspannung entsteht so ein um einen Faktor 3 kürzeres Taktintervall. Die Phasenspannungen an der Last sind ähnlich (andere Sollwertverläufe) wie diejenigen in Bild 15.8. Es treten nur noch die Harmonischen des 3. Trägerbandes und dessen Vielfachen auf.

Bei der Betrachtung der entstehenden Schaltsequenz für $\underline{u}_{L}$ in der Drehzeigerebene (Bild 15.10 rechts) ist zu erkennen, dass diese Art der Steuerung noch nicht das Optimum darstellt: es kommen zwar nur Spannungszustände zum Zuge, die nahe beim Sollwert $\underline{u}_{L, S o l l}$ liegen, die Auswahl ist jedoch nicht auf die unmittelbar nächsten beschränkt $\left(\underline{u}_{L, 4}\right.$ gehört nicht zu diesen). Optimal wäre die ausschliessliche Verwendung der Zustände, die das grau hinterlegte Dreieck umschliessen. Damit würden die Verzerrungen von $\underline{u}_{L}$ am kleinsten. Eine derartige Modulation ist mit einem auf die Gesamtschaltung orientierten Schaltzyklus grundsätzlich realisierbar. Es sind dabei zwei Randbedingungen zu erfüllen:

- Bei der Drehzeigermodulation eines einzelnen Stromrichters hängt die Schaltsequenz für ein Abtastintervall davon ab, in welchem Sektor sich $\underline{u}_{\text {Soll }}$ befindet. Es werden stets diejenigen Zustände verwendet, die das Dreieck des entsprechenden Sektors bilden. Dabei sind die Zustände am Anfang und am Ende jedes Abtastintervalles Nullzustände. Ein Übergang auf eine andere Schaltsequenz, wenn $\underline{u}_{\text {Soll }}$ den Sektor wechselt, ist problemlos möglich, da auch diese mit einem Nullzustand beginnt. Im erweiterten $\mathrm{Zu}$ standsdiagramm von seriegeschalteten Stromrichtern hat jede zu einem Dreieck gehörende Schaltsequenz einen eigenen Anfangszustand, der im allgemeinen kein Nullzustand ist. Der Wechsel von einer Sequenz auf eine andere, wenn sich $\underline{u}_{L, \text { Soll }}$ in ein anderes Dreieck bewegt, erfordert deshalb stets eine Überführungsoperation. 
- Fast jeder Zustandsübergang von $\underline{u}_{L}$ lässt sich auf mehrere Arten realisieren. Es kann ausgewählt werden, welcher Teilstromrichter geschaltet werden soll. Dabei ist aber zu beachten, dass im Mittel alle Schalter mit derselben Schaltfrequenz zu belasten sind.

Die Möglichkeiten für die Entwicklung von Schaltsequenzen sind sehr zahlreich. Sie nehmen mit der Anzahl der verwendeten Stromrichter zudem rasch noch weiter zu. Das Thema der optimalen Drehzeigermodulation für seriegeschaltete Stromrichter wird aber in der Literatur bisher kaum behandelt.

\subsubsection{Vorausberechnete Pulsmuster}

Off-Line-Steuerverfahren lassen sich auch auf seriegeschaltete Stromrichter anwenden. Die abgespeicherten Pulsmuster können dabei nach beliebigen Kriterien optimiert werden (Kapitel 9). Die Optimierung zielt immer auf die Grössen, die an der Last auftreten, d.h. die Lastspannung $u_{L}$, den Verzerrungsstrom $i_{L, V Z}$ usw. Sie wird im allgemeinen viertelperiodensymmetrisch gemacht. Die Teilspannungen sind nur soweit von Interesse, als dass alle Stromrichter dieselbe Schaltfrequenz aufweisen sollen. Es ist jedoch nicht zweckmässig, die Teilspannungen zu optimieren. Durch eine direkte Optimierung von $u_{L}$ unter Verwendung aller Freiheitsgrade lässt sich stets das beste Resultat erzielen. Die Aussteuerung der Teilstromrichter wird dabei meistens unterschiedlich. Dies ist aber nicht weiter von Bedeutung, da die entsprechenden Phasenströme alle gleich sind. Weiter sind die Teilspannungen in der Regel nicht mehr viertelperiodensymmetrisch.

Einphasige Schaltungen: Bei der Optimierung von Pulsmustern für $u_{L}$ stellt sich das Problem, dass die Anzahl Freiheitsgrade schon für sehr kleine Schaltzahlen gross wird. Dies soll am Beispiel von 3 in Serie geschalteten einphasigen Brücken mit der Schaltzahl $q=2$ gezeigt werden. In Bild 15.11 ist eine Viertelperiode von $u_{L}$ dargestellt. Da jede Schaltfunktion eines Brückenzweiges 4 Schaltwinkel pro Periode aufweist, ergibt dies in den Phasenspannungen der Teilstromrichter 8 und in $u_{L} 24$ Schaltwinkel pro Periode. Es stehen für eine viertelperiodensymmetrische Lastspannung 6 freie Schaltwinkel für die Optimierung zur Verfügung. Für die Spannungsniveaus dazwischen lassen sich die folgenden Regeln aufstellen:



\section{Bild 15.11.}

Viertelperiode einer Lastspannung, zusammengesetzt aus 3 Teilspannungen mit $q=2$

- Die Stelle $\omega_{1} t=0$ wird mit $u_{L}=0$ durchfahren.

- Im Bereich der positiven Halbwelle sind nur positive Spannungen sinnvoll. Sie können null, $U_{B}^{\prime}{ }_{B} / 3,2 U^{\prime}{ }_{B} / 3$ oder $U_{B}^{\prime}$ betragen. Diese Niveaus entsprechen einer positiven Phasenspannung an keiner, einer, zwei oder allen 3 Brücken. 
- Bei jedem Schaltwinkel erfolgt ein Sprung auf ein benachbartes Niveau. Andere Übergänge würden den Spezialfall darstellen, dass zwei Schaltereignisse zusammenfallen. Aufgrund dieser Einschränkungen sind die 13 Spannungssequenzen in der Tabelle 15.1 erlaubt. Die gekennzeichnete Sequenz Nr. 10 entspricht dem Beispiel in Bild 15.11. Mit Hilfe der Schaltwinkel und der zugehörigen Spannungen lassen sich die Fourierkoeffizienten von $u_{L}$ berechnen:

\begin{tabular}{|c|c|c|c|}
\hline Sequenz Nr. & $\mathrm{u}_{\mathrm{L}, 0 \ldots \mathrm{G}} /\left(\mathrm{U}^{\prime}{ }_{\mathrm{B}} / 3\right.$ & Sequenz Nr. & $\mathrm{u}_{\mathrm{L}, 0 \ldots \mathrm{G}} /\left(\mathrm{U}^{\prime}{ }_{\mathrm{B}} / 3\right.$ \\
\hline 1 & $\begin{array}{llllllll} & 1 & 0 & 1 & 0 & 1 & 0\end{array}$ & 8 & 0121210 \\
\hline 2 & 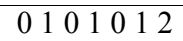 & 9 & 0121212 \\
\hline 3 & $\begin{array}{llllllll}0 & 1 & 0 & 1 & 2 & 1 & 0\end{array}$ & 10 & 0121232 \\
\hline 4 & 010101212 & 11 & 0123210 \\
\hline 5 & 010101232 & 12 & 0123212 \\
\hline 6 & 0121010 & \multirow[t]{2}{*}{13} & \multirow[t]{2}{*}{0123232} \\
\hline 7 & 011210012 & & \\
\hline
\end{tabular}

Tabelle 15.1.

Mögliche Spannungssequenzen für $u_{L}$ mit $q=2$

$$
b_{v}=\frac{4}{v \pi} U_{B} \sum_{i=0}^{0} u_{L, i}\left[\cos \left(v \alpha_{i}\right)-\cos \left(v \alpha_{i+1}\right)\right], \quad \hat{u}_{L, v}=\left|b_{v}\right|
$$

mit

$$
\alpha_{0}=0, \alpha_{7}=\frac{\pi}{2} \quad \text { und } \quad v=1,3,5,7, \ldots
$$

Die Gleichung (15.4) bildet die Grundlage für die Formulierung von Gütekriterien und die Berechnung der Schaltwinkel. Die Optimierung muss, wie schon in Kapitel 9.3.3 für den zweistufigen Stromrichter gezeigt wurde, für jede Sequenz einzeln durchgeführt werden. Die erhaltenen Resultate sind zu vergleichen und die beste Sequenz auszuwählen. Gewöhnlich sind für verschiedene Modulationsgrade andere Sequenzen optimal.

Wenn das Pulsmuster für $u_{L}$ bestimmt ist, muss die Aufteilung der Schaltwinkel auf die einzelnen Stromrichter festgelegt werden. Dazu existieren fast immer mehrere Möglichkeiten. Die wichtigste Randbedingung bei der Auswahl der Aufteilung ist eine gleiche Schaltfrequenz für alle Stromrichter.

Dreiphasige Schaltungen: Bei der Serieschaltung von dreiphasigen Stromrichtern ist die Anzahl der Schaltsequenzen für die Lastspannung noch grösser, da mehr Spannungsniveaus zur Verfügung stehen. Die globale Optimierung von Pulsmustern unter Berücksichtigung aller Freiheitsgrade scheitert deshalb in der Praxis bald einmal am Aufwand. Sie ist z.B. für 3 Stromrichter höchstens mit $q=2$ oder 3 noch durchführbar. Auf die Besprechung des Vorgehens im Detail wird an dieser Stelle verzichtet.

\subsubsection{Direkte Stromregelverfahren}

Grundsätzlich eignet sich die Anordnung nach Bild 15.1 sehr gut für die direkte Stromregelung, sei es für Phasenstromregler (Kapitel 10) oder für drehzeigerorientierte Verfahren 
(Kapitel 11). Da alle AC-seitigen Ströme gekoppelt sind, werden sie durch eine einzige Regelung, die auf den Laststrom wirkt, erfasst. Alle Stromrichter können dazu benutzt werden, die geeignete Lastspannung einzustellen. Die vielen Spannungsniveaus erlauben es, die Phasenströme bzw. ihre Drehzeigerkomponenten mit vergleichsweise wenig Schaltvorgängen innerhalb der gegebenen Toleranzbänder zu halten. Die Schaltfrequenz für die Schalter fällt dabei gegenüber einem einzelnen Stromrichter deutlich kleiner aus. Ähnlich wie bei der Drehzeigermodulation muss jedoch darauf geachtet werden, dass alle Stromrichter im Mittel gleich häufig schalten.

Trotz der vielversprechenden Aussichten werden die direkten Stromregelverfahren für seriegeschaltete Stromrichter bisher nicht verwendet. Der Grund ist, dass diese Art von Steuerverfahren generell für Systeme grosser Leistung eher ungeeignet ist (Kapitel 13).

\subsubsection{Zwischenkreis}

Verwendet man eines der beschriebenen Verfahren zur Steuerung der seriegeschalteten Stromrichter, so ergibt sich auch im Zwischenkreis ein verbesserter Strom. Dies wird am Beispiel der Trägerverfahren mit versetzter Taktung verdeutlicht. Bild 15.12 zeigt den Zwischenkreisstrom $i_{d}$ und den Anteil $i_{a, d}$ des Stromrichters $a$ für den in den Bildern 15.6 bis 15.8 dargestellten Betriebsfall. Dabei ist ein nacheilender Grundschwingungsstrom angenommen. In den Spektra ist zu erkennen, dass $i_{a, d}$ alle Trägerbänder der Schaltfunktion enthält, während in $i_{d}$ nur noch das 3 . Trägerband in Erscheinung tritt. Auch im Zwischenkreis ergibt sich offensichtlich durch die Serieschaltung eine Verdreifachung der wirksamen Schaltfrequenz. Dies kommt dadurch zustande, dass sich die Komponenten im ersten und zweiten Trägerband über alle 3 Stromrichter gesehen gegenseitig kompensieren. Neben dem im Bild sichtbaren 3. Trägerband treten in $i_{d}$ alle dessen Vielfachen auf.

Der Gleichstromanteil $I_{d}$ im Zwischenkreis entspricht dem dreifachen Wert der einzelnen Teilstromrichter $I_{a / b / c, d}$. Dieser Zusammenhang ist durch die Wirkleistungsbilanz bestimmt: die Leistung im Zwischenkreis entspricht der Summe der Wirkleistungen der Teilstromrichter auf der AC-Seite.
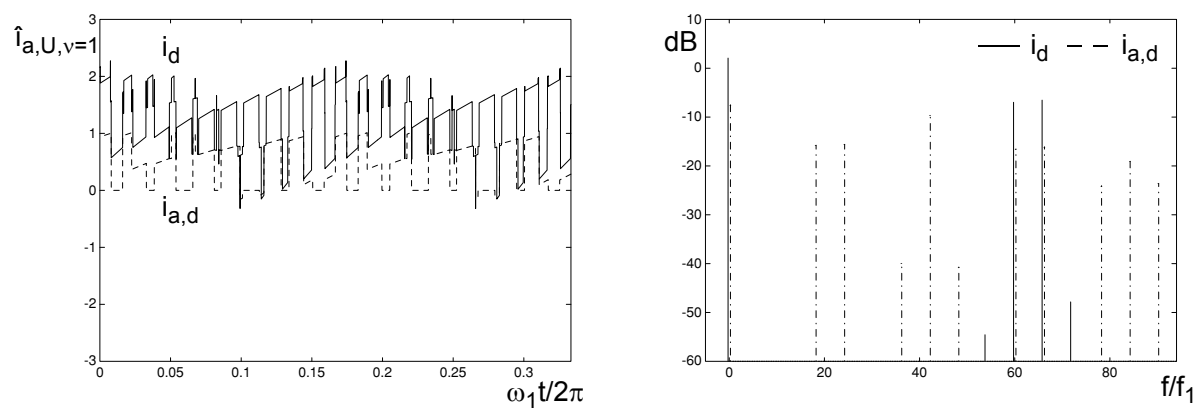

Bild 15.12. Links: Zwischenkreisstrom für Trägerverfahren mit versetzter Taktung, rechts: zugehöriges Spektrum, $0 \mathrm{~dB}: \hat{\imath}_{a, U, v=1}$ 
Die besprochenen Eigenschaften lassen sich analytisch herleiten, wenn man die Transformationsgleichungen für den Zwischenkreis in Kapitel 3.3 verwendet und die einzelnen Harmonischen mit ihren Phasen dort einsetzt.

\subsection{Parallelschaltung von Stromrichtern}

\subsubsection{Schaltungen}

Die AC-seitige Parallelschaltung von Stromrichtern funktioniert nach dem in Bild 15.13 gezeigten Prinzip. Es ist dafür grundsätzlich kein Transformator notwendig, sondern nur die Entkopplungsinduktivitäten $L_{\sigma, a}, L_{\sigma, b}$ und $L_{\sigma, c}$. Häufig erfordern jedoch andere Gründe, z.B. Potentialtrennung oder Spannungsanpassung, den Einsatz eines Transformators. Dieser besteht dann im Gegensatz zu demjenigen für die Serieschaltung nur aus einem Kern und ist deshalb wesentlich einfacher. Wichtig sind seine stromrichterseitigen Streuinduktivitäten: sie müssen die Funktion der Entkopplungsdrosseln übernehmen.
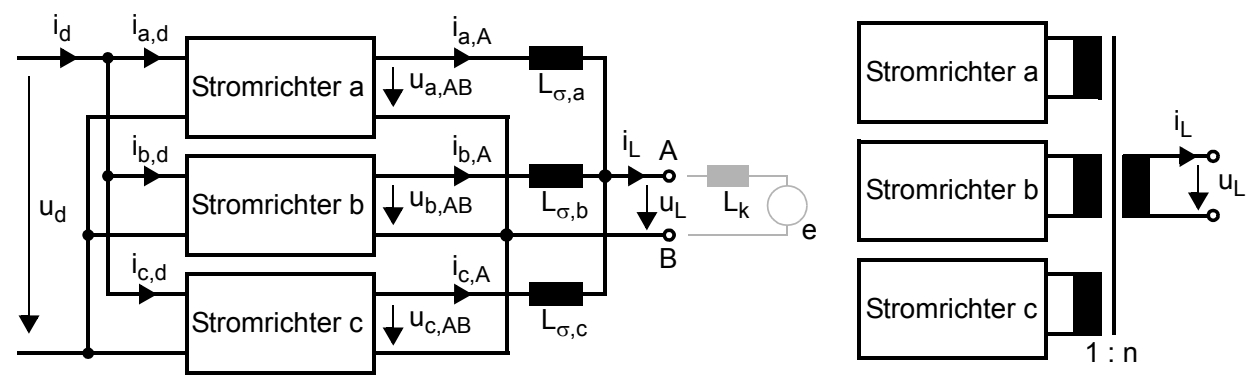

Bild 15.13. Parallelschaltung von Stromrichtern, Beispiel mit 3 einphasigen Stromrichtern, links: mit Entkopplungsdrosseln, rechts: mit Transformator

Die Gleichungen für die Spannungen und Ströme lauten wie folgt:

$$
\begin{aligned}
& u_{L}=u_{a, A B}-L_{\sigma, a} \frac{d i_{a, A}}{d t}=u_{b, A B}-L_{\sigma, b} \frac{d i_{b, A}}{d t}=u_{c, A B}-L_{\sigma, c} \frac{d i_{c, A}}{d t}=\ldots, \\
& i_{L}=i_{a, A}+i_{b, A}+i_{c, A}+\ldots
\end{aligned}
$$

Unter Annahme des üblichen Falles identischer Induktivitäten $L_{\sigma}=L_{\sigma, a / b / c}$ können die Gleichungen (15.5) zusammen in die Form (15.6) gebracht werden:

$$
u_{L}=\frac{u_{a, A B}+u_{b, A B}+u_{c, A B}}{3}-\frac{L_{\sigma}}{3} \frac{d i_{L}}{d t}
$$

Damit gilt für den Lastkreis die Ersatzschaltung in Bild 15.14. Die beiden Induktivitäten $L_{\sigma} / 3$ und $L_{k}$ können in einer gesamten Lastinduktivität $L^{\prime}{ }_{k}$ zusammengefasst werden. Als Eingangsspannung für die Ersatzschaltung wirkt $u_{L}^{\prime}$ : 


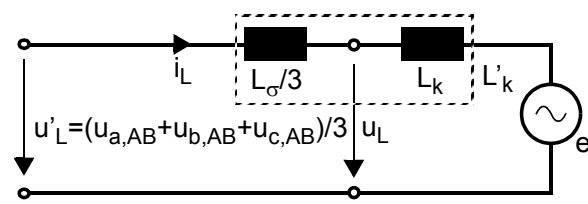

Bild 15.14.

Ersatzschaltung für den Lastkreis

$$
L_{k}^{\prime}=\frac{L_{\sigma}}{3}+L_{k}, \quad u_{L}^{\prime}=\frac{u_{a, A B}+u_{b, A B}+u_{c, A B}}{3}
$$

Betrachtet man die Entkopplungsinduktivitäten als Teil der Last, so ist die Ersatzschaltung gleich wie für seriegeschaltete Stromrichter. Die Spannungen und Ströme in der Last unterscheiden sich gegenüber denjenigen bei einer Serieschaltung nur um einen Proportionalitätsfaktor. In beiden Fällen wirkt im Prinzip die Summe der Teilspannungen auf den Lastkreis. Allerdings ist die Spannung $u^{\prime}{ }_{L}$ nur eine Ersatzgrösse, die in der Schaltung nirgends gemessen werden kann.

Geht man davon aus, dass alle Stromrichter an einen gemeinsamen Zwischenkreis angeschlossen sind und mit exakt demselben Modulationsgrad angesteuert werden, so ergibt sich eine symmetrische Stromaufteilung. Da die Entkopplungsinduktivitäten $L_{\sigma}$ normalerweise eher klein sind, können jedoch bereits kleine Unsymmetrien die Stromaufteilung erheblich verändern. In der Praxis sorgt deshalb meistens eine getrennte Regelung der Stromrichter für symmetrische Ströme.

Dreiphasige Schaltung: In Bild 15.15 ist die Parallelschaltung von 3 dreiphasigen Stromrichtern dargestellt. Für jede Phase sind die Ausgänge der Teilstromrichter über gleiche Entkopplungsinduktivitäten $L_{\sigma}$ zusammengeführt. Damit gilt für jede Phase des Lastkreises die Ersatzschaltung in Bild 15.14. Die Parallelschaltung verhält sich für die Last auch hier gleich wie eine Serieschaltung.

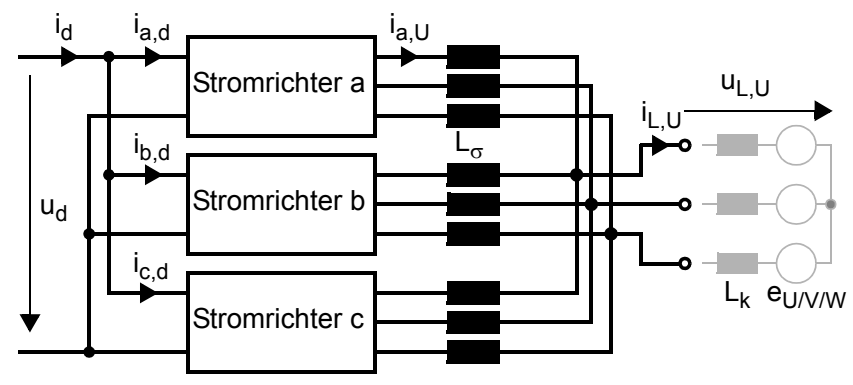

Bild 15.15.

Parallelschaltung von 3 dreiphasigen Stromrichtern

\subsubsection{Steuerverfahren}

Die für die Serieschaltung von Stromrichtern vorgestellten Steuerverfahren können alle auch für die Parallelschaltung verwendet werden. Die Kurvenformen der Lastspannungen und -ströme sind identisch. Aus diesem Grund kann für die Erzeugung der Pulsmuster ganz auf die Kapitel 15.1.2 bis 15.1.5 verwiesen werden. 
Aus der Sicht, dass die Spannungen und Ströme in der Last gleichwertig sind, wären die Parallelschaltungen wegen des geringeren Schaltungsaufwandes gegenüber den Serieschaltungen zu bevorzugen. Für sie spricht zusätzlich ihre redundante Struktur: bei Ausfall eines der Stromrichter ist der Rest der Schaltung in der Regel noch funktionstüchtig und erlaubt zumindest noch einen Betrieb mit reduzierter Leistung. Bei einer Serieschaltung ist dies nicht der Fall.

Wie im nächsten Abschnitt gezeigt wird, weist die Parallelschaltung dagegen auf der Stromrichterseite ein deutlich schlechteres Verhalten auf. Die Verzerrungsströme sind dort bei gleicher Steuerung erheblich grösser.

\subsubsection{Kreisströme zwischen den Stromrichtern}

Die Verhältnisse am Ausgang der Teilstromrichter werden an einem Beispiel betrachtet: die Teilstromrichter werden dazu mit dem Trägerverfahren und versetzter Taktung gemäss Bild 15.6 betrieben. Bild 15.16 zeigt für diesen Fall den Verzerrungsstrom für die Phase $U$ in der Last, $i_{L, U, V Z}$, sowie denjenigen am Ausgang des Teilstromrichters $a$, $i_{a, U, V Z}$. Dabei sind die Induktivitäten $L_{\sigma}=L_{k}=3 L^{\prime}{ }_{k} / 4$ angenommen. $i_{L, U, V Z}$ ist gleich wie für die Serieschaltung in Bild 15.8, enthält also nur die Harmonischen des 3. Trägerbandes und dessen Vielfachen. $i_{a, U, V Z}$ enthält dagegen alle Oberschwingungen der Phasenspannung. Diejenigen Komponenten, die auch im Laststrom zu finden sind, verteilen sich gleichmässig auf die Teilstromrichter. Auf $i_{a, U, V Z}$ entfällt somit ein Drittel davon. Die restlichen Komponenten sind Kreisströme zwischen den Teilstromrichtern. Ihre Amplituden sind im Verhältnis grösser als die Laststromanteile, da zu ihrer Begrenzung nur $L_{\sigma}$ wirksam ist.
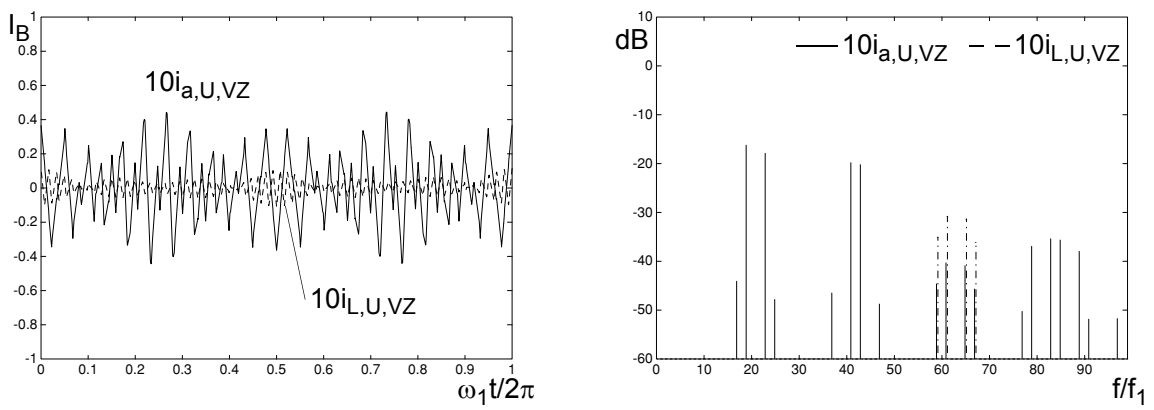

Bild 15.16. Links: Verzerrungsstrom der Phase $U$ im Stromrichter $a$ und in der Last (Trägerverfahren mit versetzter Taktung nach Bild 15.6), rechts: zugehörige Spektra, 0dB: $I_{B}$

Die einzelnen Stromrichter sowie die Entkopplungsdrosseln bzw. die stromrichterseitigen Transformatorwicklungen werden offensichtlich bei der Parallelschaltung mit einem erheblich ungünstigeren Strom belastet als bei der Serieschaltung. Es ergeben sich höhere Verluste und die Komponenten der Schaltung müssen grösser dimensioniert werden. 


\subsubsection{Zwischenkreis}

Wie für die Last spielt es auch für den Zwischenkreis im Prinzip keine Rolle, ob die Stromrichter AC-seitig serie- oder parallelgeschaltet sind. Der resultierende Strom $i_{d}$ sieht gleich aus. Bild 15.17 zeigt als Beispiel die Zwischenkreisströme $i_{d}$ und $i_{a, d}$ wiederum für ein Trägerverfahren mit versetzter Taktung gemäss Bild 15.6. Im Vergleich mit dem Zwischenkreisstrom für die Serieschaltung in Bild 15.8 wird deutlich, dass der Teilstrom $i_{a, d}$ anders aussieht. Dies rührt daher, dass die Phasenströme bei der Parallelschaltung viel stärker verzerrt sind (Bild 15.16). Da die zusätzlichen Verzerrungen jedoch Kreisströme zwischen den Teilstromrichtern sind, heben sie sich auch im Zwischenkreis gegenseitig auf. Der Gesamtstrom $i_{d}$ ist somit in beiden Fällen gleich.


Bild 15.17. Links: Zwischenkreisstrom für Trägerverfahren mit versetzter Taktung gemäss Bild 15.6, rechts: zugehöriges Spektrum, OdB: $\hat{\imath}_{a, U, v=1}$

\subsection{Spezielle Transformatorschaltungen}

Die bisher betrachteten dreiphasigen Transformatoren wiesen primär- und sekundärseitig gleichartige Wicklungssysteme auf, d.h. beidseitig Stern- oder Dreieckschaltungen. Im folgenden wird gezeigt, dass mit Hilfe von geeigneten anderen Schaltungen die Kurvenform der Lastspannung zusätzlich verbessert werden kann. Solche Schaltungen sind von der Anwendung mit fremdgeführten Stromrichtern her bekannt. Sie werden dort als zwölf- und höherpulsige Schaltungen bezeichnet.

\subsubsection{Stern-/Dreieckschaltung}

Bei einer Serieschaltung von zwei Stromrichtern kann gemäss Bild 15.18 stromrichterseitig ein Wicklungssystem in Stern und das andere in Dreieck geschaltet werden. Wie in den dargestellten Zeigerdiagrammen ersichtlich ist, sind die Grundschwingungen der Ausgangsspannungen am Stromrichter $b$ gegenüber Stromrichter $a$ um $\pi / 6$ nacheilend. Dadurch werden die Grundschwingungen der Wicklungsspannungen einer Phase jeweils gleichphasig. Die Schaltung wird in der Regel mit synchronen und symmetrischen Puls- 


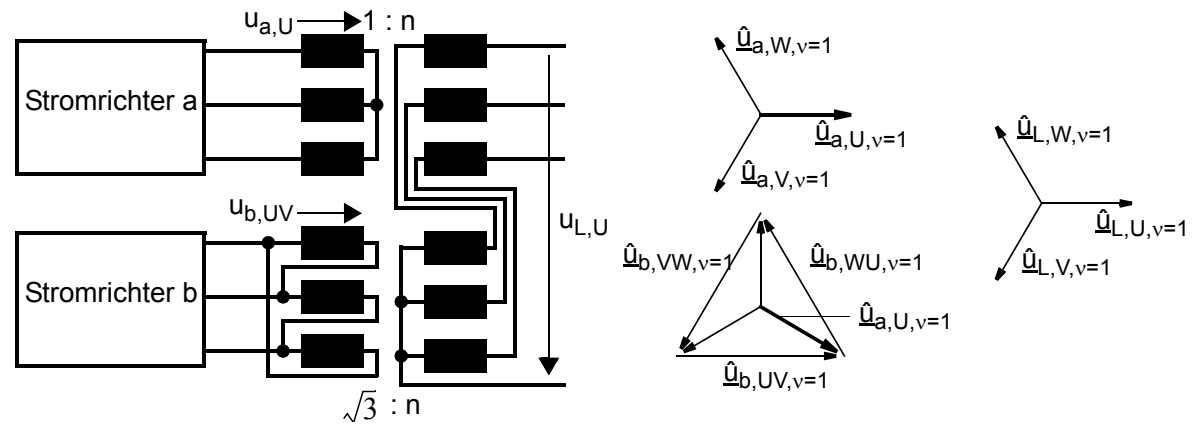

Bild 15.18. Transformator mit Wicklungssystemen Stern-Dreieck-Stern, links: Schaltung, rechts: komplexe Zeiger der Grundschwingungen

mustern betrieben, d.h. die Schaltfunktionen aller Brückenzweige sind abgesehen von den Phasenverschiebungen identisch. Damit sind auch die Fourierkoeffizienten $b_{v}$ aller Phasenspannungen gleich. Die Transformationsgleichung für eine Phase ist durch (15.8) gegeben. Sie lässt sich gemäss (15.9) auf jede einzelne Harmonische anwenden. Setzt man die entsprechenden Zeitfunktionen in (15.9) ein, so erhält man schliesslich (15.10) für die Harmonischen der Lastspannung.

$$
\begin{aligned}
& u_{L, U}= n\left[u_{a, U}+\frac{1}{\sqrt{3}}\left(u_{b, U}-u_{b, V}\right)\right] \\
& u_{L, U, v}= n\left[u_{a, U, v}+\frac{1}{\sqrt{3}}\left(u_{b, U, v}-u_{b, V, v}\right)\right] \\
& u_{L, U, v}= n\left[b_{v} \sin \left(v \omega_{1} t\right)+\frac{b_{v}}{\sqrt{3}}\left(\sin \left\{v \omega_{1} t-v \frac{\pi}{6}\right\}-\sin \left\{v \omega_{1} t-v \frac{\pi}{6}-v \frac{2 \pi}{3}\right\}\right)\right] \\
&= n b_{v}\left\{\left[1+\frac{1}{\sqrt{3}} \cos \left(v \frac{\pi}{6}\right)-\frac{1}{\sqrt{3}} \cos \left(v \frac{5 \pi}{6}\right)\right] \sin \left(v \omega_{1} t\right)-\right. \\
&=\left.-\frac{1}{\sqrt{3}}\left[\sin \left(v \frac{\pi}{6}\right)-\sin \left(v \frac{5 \pi}{6}\right)\right] \cos \left(v \omega_{1} t\right)\right\} \\
& 0 \text { für } v=5,7,17,19, \ldots
\end{aligned}
$$

Es stellt sich heraus, dass sich die Hälfte der Harmonischen in der Lastspannung genau überlagert, während sich die andere Hälfte vollständig kompensiert und dadurch fehlt. Die niedrigste auftretende Oberschwingung ist erst die 11. Dieser Effekt wirkt sich besonders günstig aus bei Grundfrequenzsteuerung, da dort die niedrigsten Harmonischen die grössten Amplituden aufweisen. Bild 15.19 zeigt die resultierenden Spannungen am Transformator für diesen Fall. Es ist zu beachten, dass die Amplitude der Grundschwingung hier nicht variiert werden kann. Die Phasenlage der Teilspannungen ist fest vorgegeben. 

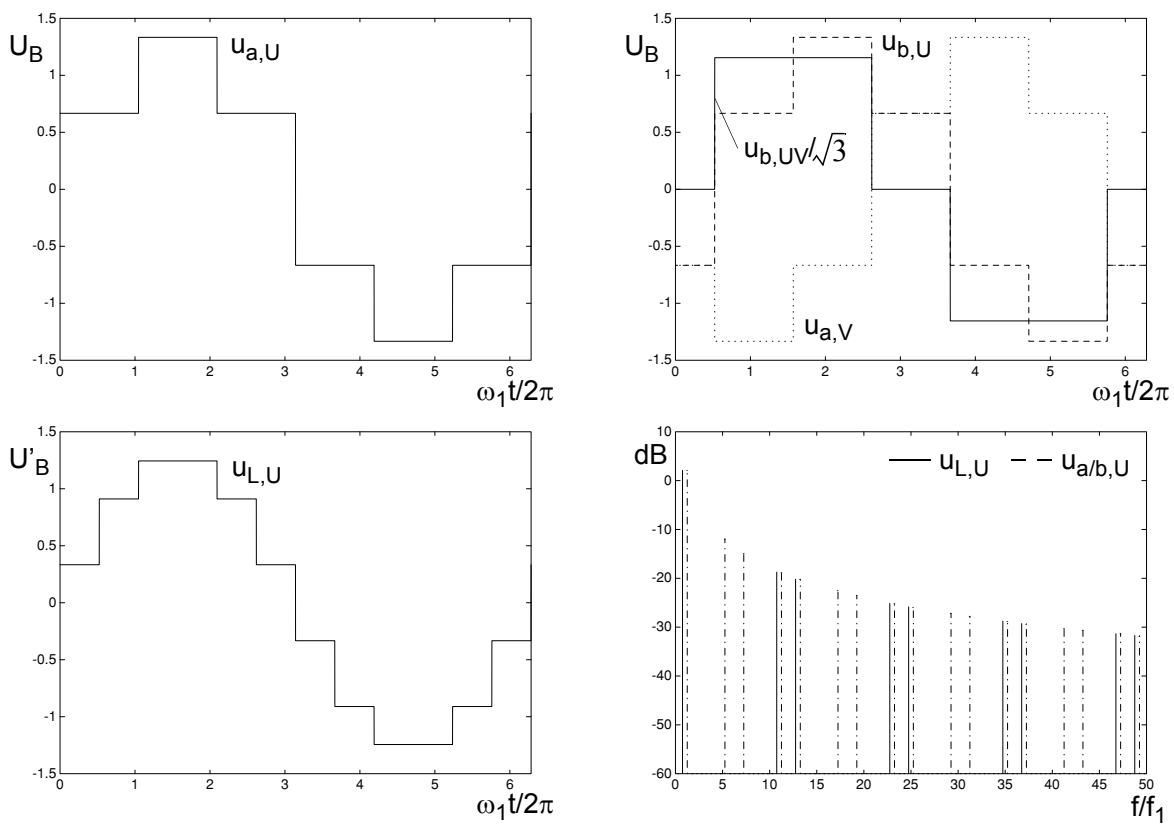

Bild 15.19. Schaltung mit Dreiwicklungstransformator, Spannungen in der Phase $U$, oben: Spannungen auf der Stromrichterseite, unten: Phasenspannung und zugehöriges Spektrum auf der Lastseite, $0 \mathrm{~dB}: U^{\prime}{ }_{B}$

Dieselbe Wirkung in der Last ergibt sich, wenn der Transformator eine Parallelschaltung durchführt. Dies bedeutet, dass beide stromrichterseitigen Wicklungssysteme auf demselben Kern angebracht sind und lastseitig nur ein Wicklungssystem vorhanden ist. Wie schon in Kapitel 15.2.2 gezeigt, weisen dann aber die Phasenströme der Teilstromrichter alle Harmonischen der Teilspannungen auf. Diejenigen, welche in der Last fehlen, fliessen als Kreisströme zwischen den Stromrichtern.

Der Zwischenkreisstrom bei der Stern-/Dreieckschaltung besteht neben der DC-Komponente nur aus Harmonischen der Ordnungen $v=12,24,36, \ldots$

\subsubsection{Transformator mit Schwenkwicklungen}

Die Kompensation von Harmonischen durch eine geeignete Verschaltung der Transformatorwicklungen lässt sich für den Fall von mehr als zwei Stromrichtern erweitern. Dazu werden stromrichterseitig jeweils für jede Phase zwei Teilwicklungen, sogenannte Schwenkwicklungen, angebracht, die auf verschiedenen Schenkeln des Transformatorkerns liegen. Bild 15.20 zeigt eine entsprechende Schaltung für 3 Teilstromrichter. Die Grundschwingungen der Gesamtspannungen pro Phase am Transformator $u_{a, U / V / W}^{\prime}$, $u_{b, U / V / W}^{\prime}$ und $u_{c, U / V / W}^{\prime}$ sind wiederum in allen Wicklungssystemen gleichphasig. Dazu müssen jedoch die Pulsmuster der einzelnen Stromrichter und damit die Spannungen $u_{b, U / V / W}$ um $\pi / 9$ und die Spannungen $u_{c, U / V / W}$ um $2 \pi / 9$ gegenüber $u_{a, U / V / W}$ verschoben 


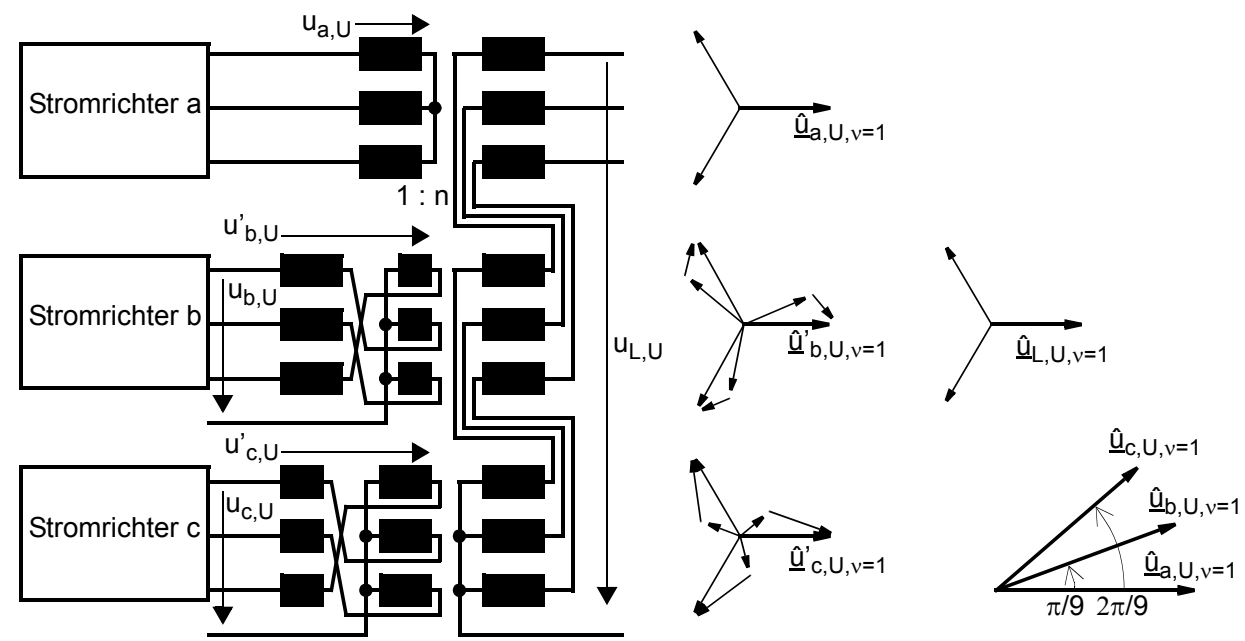

Bild 15.20. Transformator mit Schwenkwicklungen, links: Schaltung, rechts: komplexe Zeiger der Grundschwingungen

sein. Auf dieselbe Weise wie im vorherigen Abschnitt kann gezeigt werden, dass dadurch in der Lastspannung jetzt zwei Drittel der Harmonischen wegfallen. Es verbleiben die Harmonischen der Ordnungen $v=1,17 / 19,35 / 37, \ldots$ Die niedrigste auftretende Oberschwingung ist die 17.:

$$
u_{L, U, v}=\left\{\begin{array}{c}
3 n b_{v} \sin \left(v \omega_{1} t\right) \text { für } v=1,17,19,35,37, \ldots \\
0 \text { für } v=5,7,11,13,23,25,29,31, \ldots
\end{array}\right.
$$

Auch dieses Prinzip kann sowohl für die Serie- als auch für die Parallelschaltung von Stromrichtern angewendet werden. Im Fall der Parallelschaltung treten stromrichterseitig die restlichen Harmonischen als Kreisströme auf. In beiden Fällen reduzieren sich zusätzlich die Harmonischen im Zwischenkreisstrom auf diejenigen der Ordnungen $\mathrm{v}=0,18,36,54, \ldots$

Anstelle der Grundfrequenzsteuerung kann prinzipiell jedes synchrone und symmetrische Pulsmuster verwendet werden. Da die Schaltung mit Schwenkwicklungen aber nur für sehr grosse Leistungen in Frage kommt, hat dies in der Praxis wenig Bedeutung.

Die Schaltung mit Schwenkwicklungen lässt sich verallgemeinern: für $k$ Stromrichter sind die Wicklungen so zu wählen, dass die Pulsmuster jeweils um $\pi /(3 k)$ gegeneinander verschoben sind. In der Lastspannung treten dann nur die Harmonischen der Ordnungen $v=1,6 k \pm 1,12 k \pm 1, \ldots$ auf.

Die in Kapitel 15.3.1 vorgestellte Stern-/Dreieckschaltung kann ersetzt werden durch eine Schaltung mit Schwenkwicklungen. Die beiden Teilwicklungen pro Phase für den Stromrichter $b$ werden dann gleich gross und die Phasenverschiebung der Pulsmuster beträgt auch $\pi / 6$. Weil diese Schaltung jedoch aufwendiger ist als die Dreieckschaltung, wird sie nicht verwendet.

Literatur zu Kapitel 15: [Heu1], [Mey1], [Ste3], [Wüe3] 


\section{Steuerverfahren für dreistufige \\ Spannungszwischenkreis-Stromrichter}

\subsection{Prinzip der Steuerung von dreistufigen Stromrichtern}

Der dreistufige U-Stromrichter wurde in Kapitel 3.3.3 vorgestellt. Bild 16.1 zeigt noch einmal einen entsprechenden Brückenzweig zusammen mit der Ersatzschaltung als Umschalter. Die Zwischenkreisspannung ist dabei als konstant angenommen. Zusätzlich ist ein Beispiel für die Schaltfunktionen und die resultierende Ausgangsspannung mit der Schaltzahl $q=3$ ( $q$ bezieht sich auf die einzelnen Schalter) dargestellt. Es zeigt sich, dass jeweils die Schalter $s_{A^{+}+}$und $s_{A_{-}}$(im Bild schwarz gezeichnet) sowie $s_{A^{+}}$u und $s_{A_{--}}$(grau) im Gegentakt geschaltet werden. Das eine Schalterpaar erzeugt die positiven Spannungs-
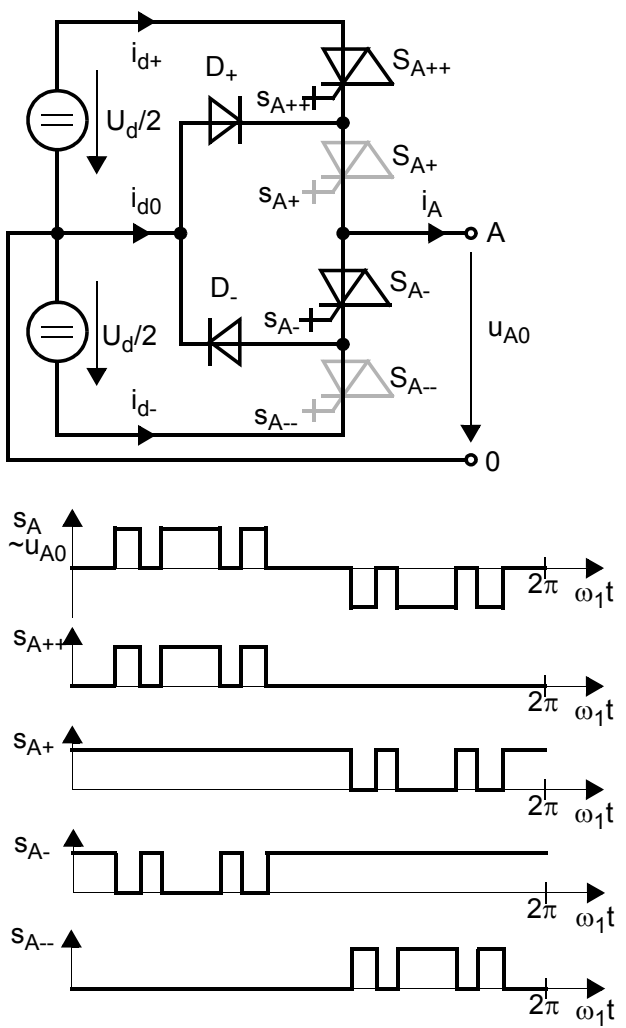

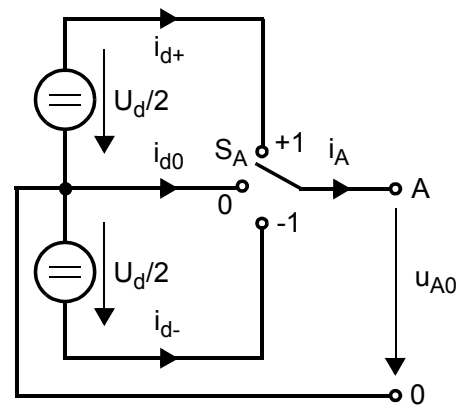

Bild 16.1.

Oben links: Brückenzweig eines dreistufigen Stromrichters,

oben rechts: Ersatzschaltung mit Umschalter, unten: Schaltfunktionen für die einzelnen Schalter bzw. den Umschalter (Beispiel mit $q=3$ ) 
pulse und das andere die negativen. Beide Paare sind jeweils während einer Halbperiode aktiv. In der anderen Halbperiode liegen sie in ihrer 'Ruhestellung', d.h. der innere Schalter $s_{A^{+}}$bzw. $s_{A^{-}}$ist eingeschaltet und der äussere $s_{A^{++}}$bzw. $s_{A_{--}}$ausgeschaltet. Die Ruhestellung ermöglicht dem anderen Paar das Umschalten.

Dies bedeutet auch, dass die beiden inneren Schalter im Mittel eine grössere Stromleitdauer aufweisen. Überhaupt muss an dieser Stelle darauf hingewiesen werden, dass im dreistufigen Stromrichter die Durchlassbelastung der einzelnen Halbleiter sehr unterschiedlich sein kann. Die Unsymmetrie ist dabei vor allem auch betriebsabhängig (vom Modulationsgrad sowie von der Phasenlage des Phasenstromes).

Die Kurvenform der Ausgangsspannung $u_{A 0}$ ist gleich wie bei einer Serieschaltung von zwei zweistufigen Halbbrücken gemäss Kapitel 15.1. Für die Last ist es deshalb gleichbedeutend, ob sie von einem dreistufigen Stromrichter oder von zwei in Serie geschalteten zweistufigen gespeist wird. Es lassen sich mit beiden Varianten gleichartige Pulsmuster generieren. Diese Eigenschaft gilt für ein- und dreiphasige dreistufige Stromrichter.

Die Steuerverfahren, welche im Zusammenhang mit seriegeschalteten Stromrichtern vorgestellt worden sind, können jedoch nicht direkt auf die dreistufigen Stromrichter übertragen werden. Der Grund dafür liegt darin, dass der dreistufige Brückenzweig 3 Schaltzustände kennt, zwei seriegeschaltete zweistufige Zweige dagegen 4 (zwei verschiedene Nullzustände). Es lassen sich jedoch gleichwertige Steuerverfahren finden.

\subsection{Steuerverfahren}

\subsubsection{Grundfrequenzsteuerung}

Bild 16.2 links zeigt die Mittelpunktspannung eines Brückenzweiges mit Grundfrequenzsteuerung. Sie besteht aus je einem positiven und einem negativen Spannungspuls, was einem Umschaltvorgang pro Schalter und Grundperiode entspricht. Damit sich kein DCAnteil ergibt, müssen die beiden Pulse gleich lang sein. Im Prinzip brauchen sie nicht
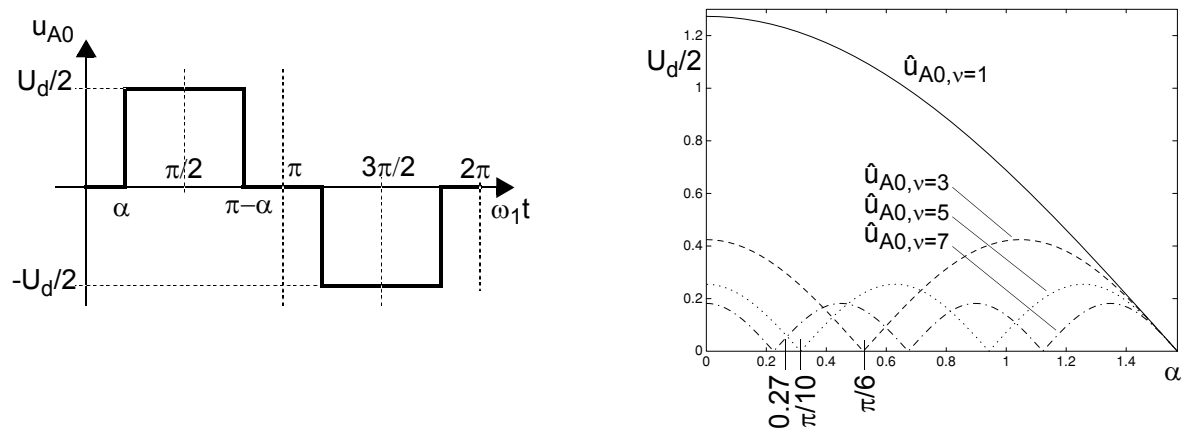

Bild 16.2. Mittelpunktspannung eines dreistufigen Brückenzweiges mit Grundfrequenzsteuerung, links: zeitlicher Verlauf, rechts: Amplituden der Grundschwingung sowie der 3., 5. und 7. Harmonischen als Funktion des Steuerwinkels $\alpha$ 
symmetrisch bezüglich der jeweiligen Halbperiode zu liegen. Es wird jedoch fast ausschliesslich die dargestellte Viertelperiodensymmetrie verwendet. Sie führt wie bei zweistufigen Schaltungen stets zu den kleinsten Verzerrungsanteilen in den Phasengrössen. Als einziger freier Parameter im Pulsmuster verbleibt dann der Steuerwinkel $\alpha$. Die Harmonischen von $u_{A 0}$ lassen sich als Funktion davon ausdrücken:

$$
b_{v}=\frac{4}{v \pi} \cos (v \alpha) \frac{U_{d}}{2}, \quad \hat{u}_{A 0, v}=\left|b_{v}\right|, \quad v=1,3,5,7, \ldots
$$

Im Gegensatz zur zweistufigen Schaltung ist der Modulationsgrad damit variabel:

$$
M=\frac{4}{\pi} \cos \alpha
$$

Der Verlauf der Amplituden der Grundschwingung sowie der 3., 5. und 7. Harmonischen von $u_{A 0}$ sind in Bild 16.2 rechts graphisch dargestellt. Abgesehen von der Bezugsspannung ist die Mittelpunktspannung identisch mit der Phasenspannung einer einphasigen Brücke mit Grundfrequenzsteuerung.

Ausgehend von der Mittelpunktspannung des Brückenzweiges werden nun die beiden in der Praxis verwendeten Brückenschaltungen betrachtet:

Einphasige Brücke: Bei der zweistufigen Schaltung wird der Modulationsgrad über die Phasenverschiebung der Schaltfunktionen für die beiden Brückenzweige eingestellt. Bei der dreistufigen Schaltung kann dieser Freiheitsgrad bereits zur Verbesserung des Spektrums verwendet werden. Bild 16.3 rechts zeigt eine typische Phasenspannung und ihre Zusammensetzung aus zwei Mittelpunktspannungen. In der Praxis werden nur viertelpe-


Bild 16.3. Links: einphasige, dreistufige Brücke,

rechts: Mittelpunktspannungen und Phasenspannung bei Grundfrequenzsteuerung

riodensymmetrische Phasenspannungen verwendet, da diese die kleinsten Verzerrungen aufweisen. Sie lassen sich stets mit zwei gleichen, phasenverschobenen Mittelpunktspannungen bilden, so dass als freie Parameter der Steuerwinkel $\alpha$ und der Verschiebungswinkel $\beta$ verbleiben. Die Harmonischen der Phasenspannung sind damit eine Funktion dieser beiden Parameter: 


$$
b_{v}=\frac{4}{v \pi} \cos (v \alpha) \cos (v \beta) \frac{U_{d}}{2}, \quad \hat{u}_{A 0, v}=\left|b_{v}\right|, \quad v=1,3,5,7, \ldots
$$

Die Steuerung des Stromrichter kann nach verschiedenen Kriterien erfolgen:

- Durch die Wahl von $\alpha=\pi / 6$ wird die dominante 3. Harmonische im Spektrum von $u_{A B}$ eliminiert. Der Modulationsgrad lässt sich dann proportional zu $\cos \beta$ im Bereich von 0 bis ca. 1.10 variieren. Neben der 3. Harmonischen werden gleichzeitig alle ihre Vielfachen, d.h. die 9., 15. usw. eliminiert.

- Soll der Modulationsgrad nicht variabel sein, können $\alpha$ und $\beta$ zur Elimination von zwei Harmonischen oder zur Minimierung von mehreren Harmonischen eingesetzt werden. Beispielsweise eliminieren $\alpha=\pi / 6$ und $\beta=\pi / 10$ die 3 . und die 5 . Harmonische sowie alle Vielfachen davon. Der Modulationsgrad beträgt dabei 1.05. Das resultierende Spektrum ist in Bild 16.4 links dargestellt. Die Elimination könnte auch mit anderen Steuerwinkeln erreicht werden: z.B. mit $\beta=3 \pi / 10$ für die 5. Es ist aber diejenige Lösung zu bevorzugen, die den grössten Modulationsgrad liefert. Der Spannungsklirrfaktor ist dann am kleinsten.

- Die Steuerwinkel $\alpha$ und $\beta$ können auch als freie Parameter für die Optimierung des Pulsmusters nach beliebigen Kriterien benützt werden (Kapitel 9), wobei der gewünschte Modulationsgrad als Randbedingung berücksichtigt wird.
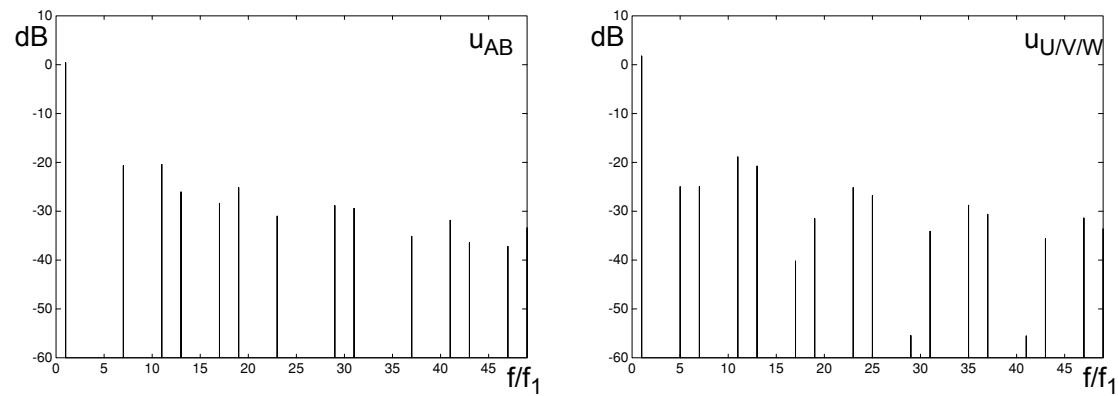

Bild 16.4. Grundfrequenzsteuerung bei dreistufigen Schaltungen, links: Spektrum der Phasenspannung der einphasigen Brücke, $\alpha=\pi / 6, \beta=\pi / 10$, rechts: Spektrum der Phasenspannung der dreiphasigen Brücke, $\alpha=0.27,0 \mathrm{~dB}: U_{B}$

Dreiphasige Brücke: Es wird davon ausgegangen, dass alle 3 Brückenzweige mit demselben Steuerwinkel $\alpha$ betrieben werden, so dass die Phasenspannungen symmetrisch sind. In diesem Fall wirken die Vielfachen der 3. Harmonischen im Spektrum der Mittelpunktspannungen als Gleichtaktkomponenten und fehlen in den Phasenspannungen. Es sind dann die folgenden Steuerstrategien möglich:

- Der Steuerwinkel $\alpha$ wird zur Variation des Modulationsgrades eingesetzt. Die weiteren Harmonischen stellen sich dementsprechend ein.

- Durch die feste Wahl von $\alpha=\pi / 10$ wird die 5. Harmonische, die den grössten Verzerrungsstrom verursacht, eliminiert. Der Modulationsgrad ist dadurch auf 1.21 festgelegt. 
- Mit $\alpha=0.27$ nehmen die 5. und 7. Harmonische denselben, gegenüber der Vollaussteuerung deutlich reduzierten Wert an, was einen kleinen Verzerrungsstrom ergibt. Der Modulationsgrad beträgt dann 1.23. Bild 16.4 rechts zeigt das entsprechende Spektrum.

- Auch hier kann $\alpha$ als freier Parameter für eine beliebige Optimierung des Pulsmusters verwendet werden. Allerdings kann dann keine Randbedingung für den Modulationsgrad vorgegeben werden.

Die besprochenen Spannungsformen weisen ausnahmslos Viertelperiodensymmetrie auf. Verzichtet man auf diese, so ergeben sich sofort weitere Freiheitsgrade bei der Erzeugung von Pulsmustern. Wie schon erwähnt wurde, sind die Verzerrungen aber im Vergleich zu viertelperiodensymmetrischen Pulsmustern immer grösser.

\subsubsection{Trägerverfahren}

Bei der Steuerung von zwei in Serie geschalteten zweistufigen Stromrichtern mit Trägerverfahren kann durch versetzte Taktung eine Verdoppelung der in der Last wirksamen Schaltfrequenz erreicht werden (Kapitel 15.1.3). Derselbe Effekt kann auch in dreistufigen Stromrichtern erreicht werden. Bild 16.5 zeigt die entsprechende Steuerung für einen Brückenzweig. Es werden zwei verschiedene Trägersignale für die Steuerung der bei-
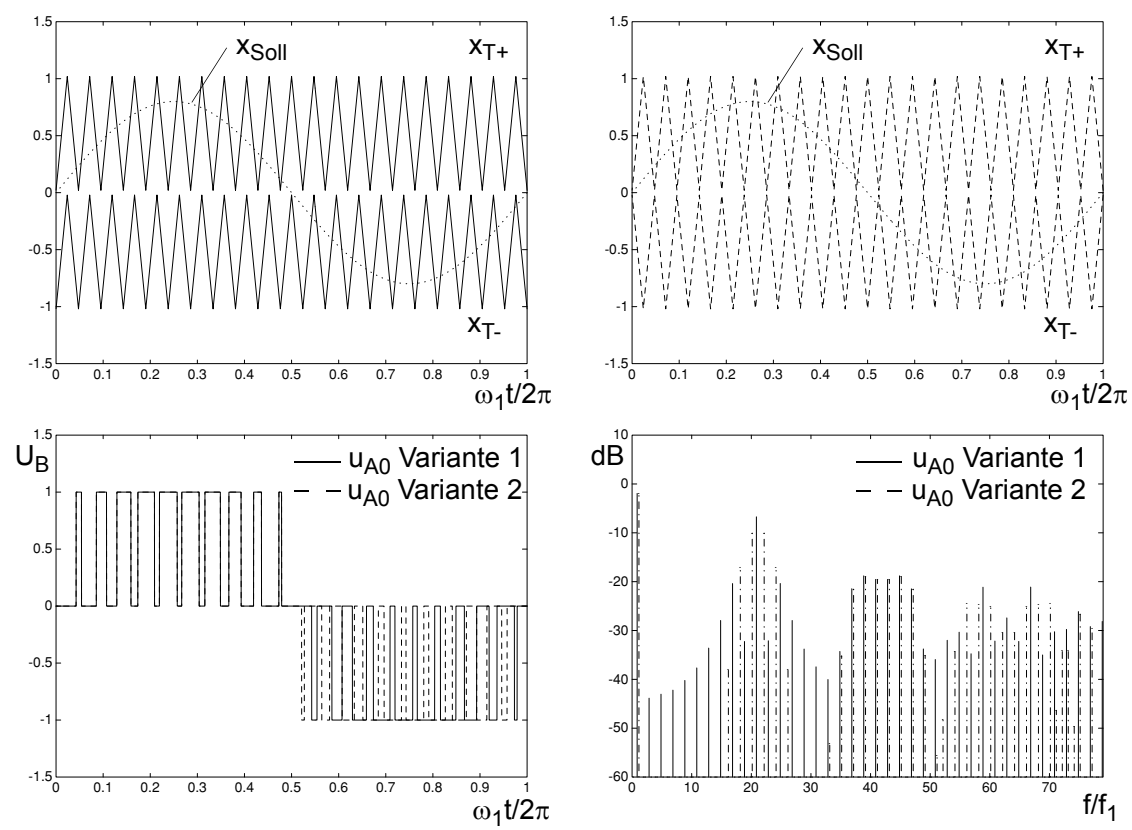

Bild 16.5. Trägerverfahren für dreistufigen Brückenzweig, $T_{1} / T_{T}=21, q=10.5$, oben links: Steuer- und Sollwertsignale für Variante 1 (gleichphasige Träger), oben rechts: Steuer- und Sollwertsignale für Variante 2 (gegenphasige Träger), unten: Verläufe und Spektra der Phasenspannung für beide Varianten, 0dB: $U_{B}$ 
den Schalterpaare verwendet ( $x_{T^{+}}$für $S_{A^{++}}$und $S_{A^{-}}$sowie $x_{T_{-}}$für $S_{A^{+}}$und $S_{A_{-}-\text {). Ihre Ver- }}$ schiebung nach oben bzw. unten bewirkt, dass sich jeweils nur für eine Polarität des gemeinsamen Sollwertsignals $x_{\text {Soll }}$ Schnittpunkte und damit Schaltflanken ergeben. Weil damit die einzelnen Schalter nur während je einer Halbperiode schalten, entspricht ihre mittlere Schaltfrequenz der halben Trägerfrequenz. Im dargestellten Beispiel beträgt die Trägerfrequenz $21 f_{1}$, die Schalter schalten jedoch nur mit 10.5 $f_{1}(q=10.5)$. Im Spektrum der Phasenspannung $u_{A 0}$ ist ersichtlich, dass sich das erste Trägerband um $21 f_{1}$ herum bildet. Damit ist dies auch die für die Last wirksame Schaltfrequenz. Die Steuerung ist von ihren Eigenschaften her also vergleichbar mit einer versetzten Taktung.

Weiter ist im Bild ersichtlich, dass es zwei Varianten zur Steuerung gibt. Sie unterscheiden sich in der Phasenlage des Trägersignals für die negative Halbwelle: in Variante 1 ist dieses gleichphasig mit demjenigen für die positive Halbwelle, in Variante 2 ist es gegenphasig. Im Verlauf der Phasenspannung ergibt sich nur in der negativen Halbwelle ein Unterschied. Dieser hat im Spektrum ein deutlich unterschiedliches erstes Trägerband zur Folge. Es ist für Variante 1 deutlich ausgeprägter.

Die Phasenspannung nach Variante 2 ist identisch mit derjenigen einer zweistufigen Brücke bzw. zwei in Serie geschalteten, zweistufigen Brückenzweigen mit versetzter Taktung. Die Variante 1 ergibt dagegen leicht veränderte Kurvenformen.

Wird der dreistufige Stromrichter mit relativ hoher Schaltzahl betrieben, so ergibt sich für die einzelnen Schalter eine stark wechselnde Belastung zwischen häufiger Schalttätigkeit und Ruhe. Vor allem bei einer niedrigen Grundfrequenz, z.B. im Anlaufbereich eines Antriebes kann dies zur Folge haben, dass die Schaltfrequenz unter den eigentlich zulässigen Mittelwert gesenkt werden muss, weil die thermische Belastung der Schalter während der aktiven Halbperiode sonst zu gross wird.

Einphasige Brücke: Es werden entweder beide Brückenzweige nach Variante 1 oder beide nach Variante 2 gesteuert. Die Trägersignale des Zweiges $B, x_{T, B^{+}}$und $x_{T, B-}$, sind gegenüber denjenigen des Zweiges $A$, $x_{T, A^{+}}$und $x_{T, A_{-}}$, vertauscht, so dass während der positiven Halbperiode von $x_{\text {Soll }}$ die Schalter $S_{B^{+}} / S_{B--}$ schalten und während der negativen Halbperiode $S_{B++} / S_{B-}$. Zusätzlich sind die Trägersignale um eine halbe Taktperiode verschoben, damit sich eine versetzte Taktung ergibt. Die Steuer- und Sollwertsignale sowie die resultierende Phasenspannung für Variante 1 sind in Bild 16.6 dargestellt. Letztere sieht jedoch für beide Varianten der Steuerung gleich aus. Unabhängig davon, welche Variante gewählt wird, erhält man nämlich für die beiden Brückenzweige zusammen stets dieselben 4 Trägersignale. Bild 16.6 oben links gilt deshalb auch für Variante 2, nur müsste die Beschriftung der Trägersignale geändert werden: $x_{T, A-}$ und $x_{T, B+}$ wären vertauscht. Es ist naheliegend, dass deshalb auch in beiden Fällen dieselbe Phasenspannung entsteht. Diese weist ein Spektrum auf, in dem als niedrigstes Trägerband dasjenige um die zweifache Trägerfrequenz bzw. die vierfache Schaltfrequenz der Schalter verbleibt (im Beispiel $42 f_{1}$ ). Sie ist damit gleichwertig wie die Ausgangsspannung von zwei seriegeschalteten zweistufigen Brücken.

Dreiphasige Brücke: Zur Steuerung einer dreiphasigen Brücke werden alle 3 Brückenzweige mit denselben beiden Trägersignalen entweder nach Variante 1 oder 2 gesteuert. Bild 16.7 zeigt die entsprechenden Träger- und Sollwertsignale sowie die entstehenden 

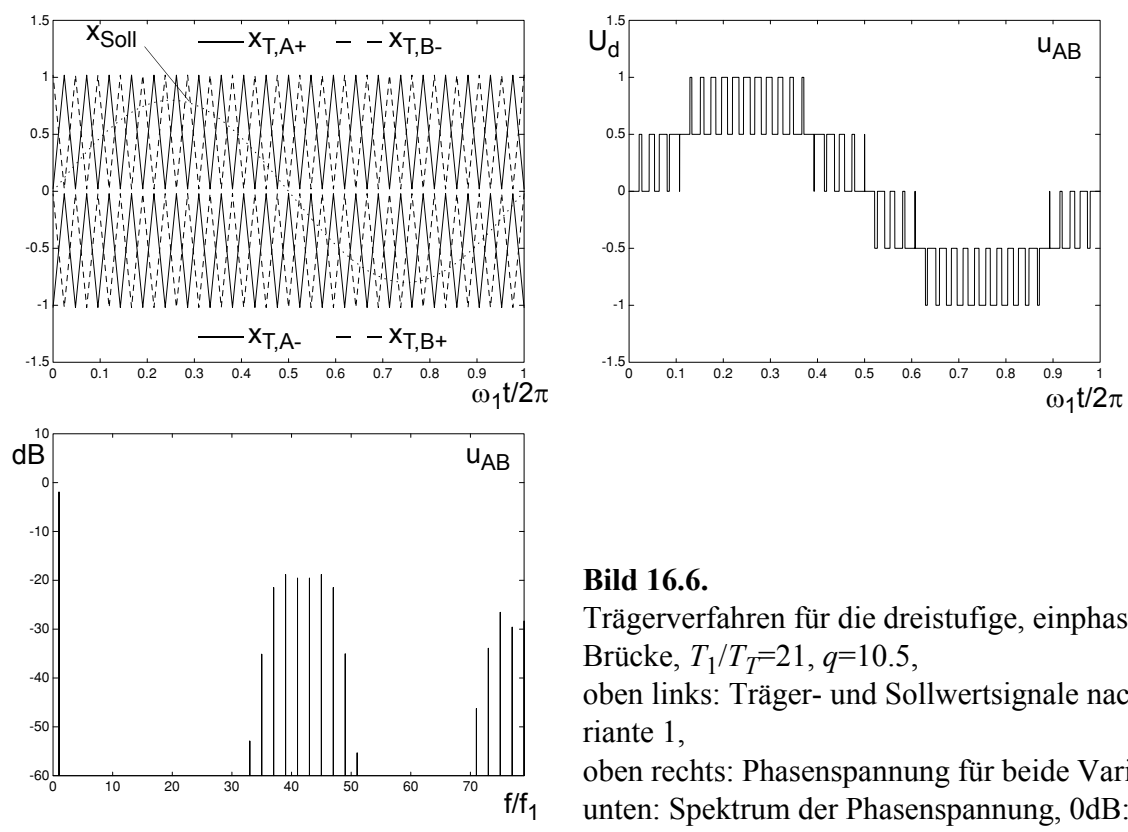

Bild 16.6.

Trägerverfahren für die dreistufige, einphasige Brücke, $T_{1} / T_{T}=21, q=10.5$, oben links: Träger- und Sollwertsignale nach Variante 1 , oben rechts: Phasenspannung für beide Varianten, unten: Spektrum der Phasenspannung, 0dB: $U_{d}$

Phasenspannungen für die Phase $U$. Sowohl in den zeitlichen Verläufen wie auch in den Spektra sind deutlich Unterschiede zwischen den beiden Varianten erkennbar. Bei der Variante 2 erreichen beide Trägersignale jeweils gleichzeitig ihren Extremalwert +1 bzw. -1 . An diesen Stellen, d.h. einmal pro Trägerperiode, stehen jeweils alle Brückenzweige in derselben Position und alle Phasenspannungen sind null. Bei der Variante 1 ist dies nicht überall der Fall. Die Spektra unterscheiden sich vor allem im ersten Trägerband. Dabei fällt auf, dass bei Variante 1 die Komponente der Trägerfrequenz, die im Spektrum der Mittelpunktspannung sehr ausgeprägt aufgetreten ist (Bild 16.5 unten rechts), hier fehlt. Sie wirkt als Gleichtaktkomponente. Obschon diese Variante mehr Seitenlinien im ersten Trägerband ergibt, ist sie wegen der gegenüber Variante 2 deutlich kleineren Amplituden dieser Linien ganz eindeutig vorzuziehen. Der resultierende Verzerrungsstrom ist kleiner.

Es ist anzufügen, dass die Trägerverfahren im Prinzip asynchron sind. Obschon die gezeigten Beispiele auf synchronen Pulsmustern beruhen, gelten die gezeigten Eigenschaften in gleicher Weise auch für asynchrone Modulation.

\subsubsection{Drehzeigermodulation}

Bereits in Kapitel 3.3.2.3 wurde beschrieben, dass die Spitze des Zeigers für die Ausgangsspannung des dreistufigen Stromrichters $\underline{u}_{S t r} 19$ verschiedene Positionen aufweisen kann, die jeweils durch einen bis 3 Schaltzustände eingestellt werden können. In Bild 16.8 sind diese Punkte noch einemal dargestellt. Sie liegen mit Ausnahme des Nullspannungszeigers auf einem inneren und einem äusseren Sechseck. 

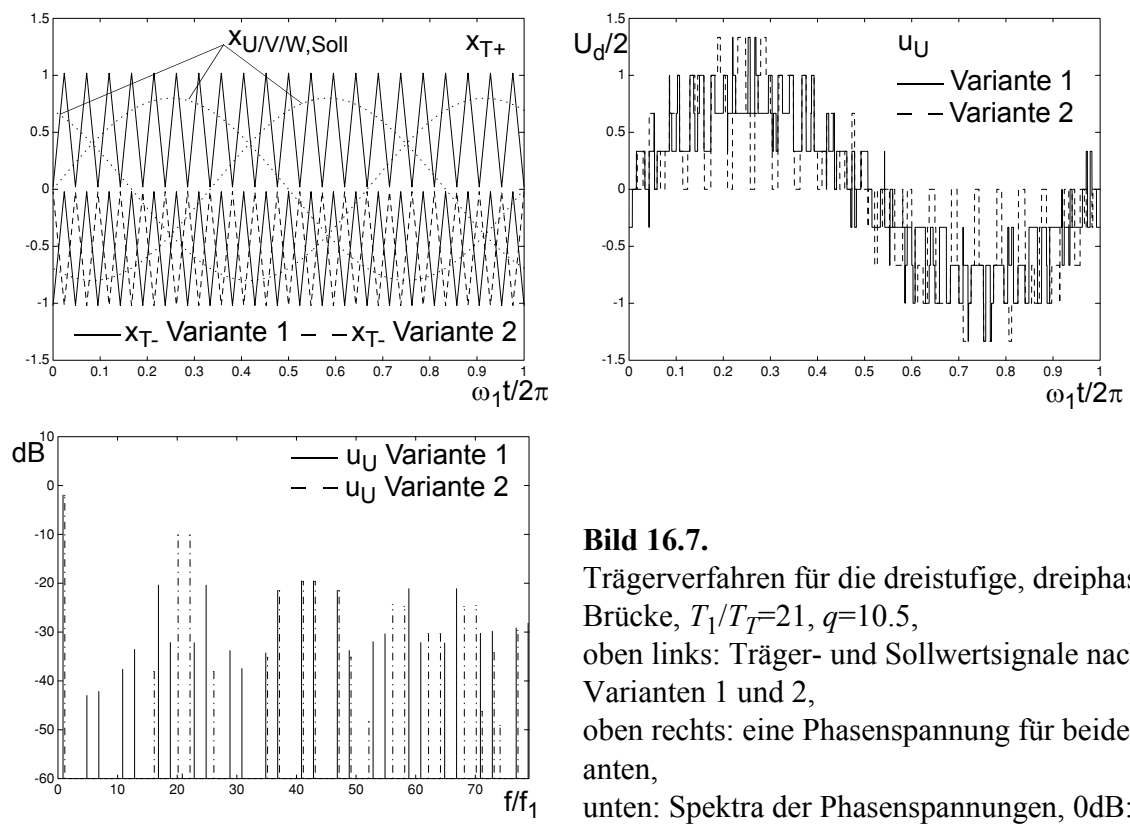

\section{Bild 16.7.}

Trägerverfahren für die dreistufige, dreiphasige Brücke, $T_{1} / T_{T}=21, q=10.5$, oben links: Träger- und Sollwertsignale nach den Varianten 1 und 2, oben rechts: eine Phasenspannung für beide Varianten,

unten: Spektra der Phasenspannungen, 0dB: $U_{d} / 2$

Bei der Drehzeigermodulation wird in gewohnter Weise der Sollwertzeiger der Stromrichterspannung $\underline{u}_{S o l l}$ für ein Abtastintervall $T_{\text {Tast }}$ durch die in der Nähe liegenden Spannungszustände nachgebildet. Für die weiteren Betrachtungen wird die Analogie zu den Trägerverfahren verwendet. Das Abtastintervall entspricht dabei einer halben Trägerperiode $T_{\text {Tast }}=T_{T} / 2$ (Kapitel 8.3).

Betrachtet man die Varianten 1 und 2 der Trägerverfahren für die dreiphasige Brücke in Drehzeigerdarstellung, so ergeben sich die in Bild 16.8 mitte und rechts dargestellten Schaltsequenzen. Eine Trägerperiode entspricht 6 Zustandsübergängen. Es lässt sich zeigen, dass bei der Variante 2 (im Bild rechts), unabhängig von der Länge des Sollwertzeigers $\underline{u}_{\text {Soll }}$, jede Trägerperiode immer in einem Zustand auf dem äusseren Sechseck beginnt. In der Mitte der Trägerperiode tritt stets ein Nullzustand auf. Der Hin- und der Rückweg zwischen dem Anfangszeiger und dem Nullspannungszeiger sind identisch. Bei der Modulation nach Variante 1 (mitte) beginnt dagegen jede Trägerperiode mit einem Spannungszeiger auf dem inneren Sechseck. Von dort wird die Spitze des Sollwertzeigers auf dem durch die umliegenden Spannungszeiger gebildeten Dreieck umfahren. Der Zeiger in der Mitte der Trägerperiode stimmt mit dem Ausgangszeiger überein. Er wird aber durch den anderen möglichen Schaltzustand für diesen Zeiger gebildet. Anschliessend wird auch hier derselbe Weg in umgekehrter Richtung zurückgefahren. Je nach Länge von $\underline{u}_{\text {Soll }}$ wird, wie im Bild ersichtlich ist, eine nach aussen oder eine nach innen gerichtete Schaltsequenz angelegt.

Es ist leicht einzusehen, dass die Modulation nach Variante 1 kleinere Verzerrungen ergibt. Die Nachbildung des Sollwertzeigers erfolgt stets mit Hilfe der 3 am nächsten gelegenen Spannungszeiger. Damit bestätigt sich die Erkenntnis, die bei den Trägerverfahren 

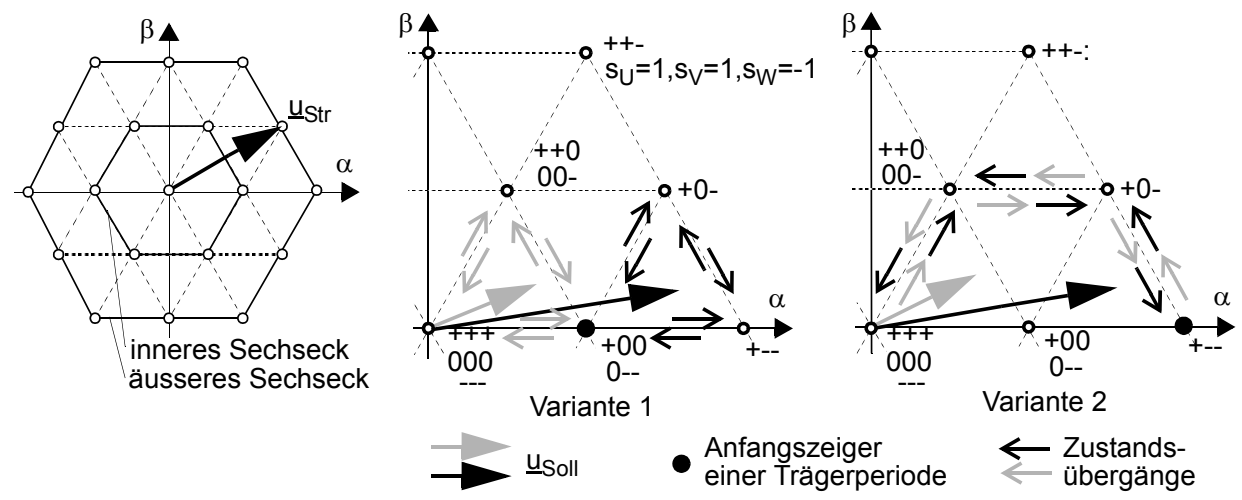

Bild 16.8. Schaltsequenzen bei Drehzeigermodulation, links: mögliche Spannungszeiger des dreistufigen Stromrichters, mitte: analog zu Trägerverfahren nach Variante 1, rechts: analog zu Variante 2

anhand der Spektra gewonnen wurde. Es wird an dieser Stelle darauf verzichtet, die genaue Abfolge der Schaltsequenzen über eine ganze Periode und für verschiedene Längen des Sollwertzeigers nachzuvollziehen. Sie können durch Analogieüberlegungen aus dem entsprechenden Trägerverfahren abgeleitet werden.

Da sich die Schaltsequenz einer Trägerperiode aus zwei Abtastintervallen zusammensetzt, wird $\underline{u}_{\text {Soll }}$ in der Mitte der in Bild 16.8 dargestellten Schaltsequenzen neu abgetastet. Die Einschaltzeit des Schaltzustandes an dieser Stelle setzt sich dabei aus zwei Teilintervallen zusammen, welche je in eines der angrenzenden Abtastintervalle fallen. Bild 16.9 zeigt entsprechendes Beispiel für den Ablauf der beiden Abtastintervalle.

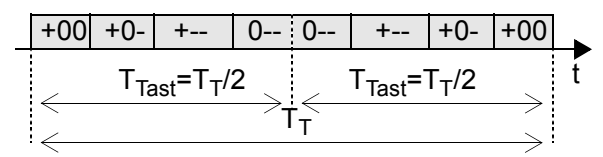

\section{Bild 16.9.}

Aufteilung einer Trägerperiode in zwei Abtastintervalle, Schaltsequenz nach Bild 16.8 mitte (schwarze Pfeile)

Bei der Drehzeigermodulation für den zweistufigen Stromrichter in Kapitel 8 wurde gezeigt, dass die Verteilung der gesamten Nulleitdauer auf die beiden Nullzustände eines Abtastintervalles einen wichtigen Freiheitsgrad darstellt. Dieser ist auch bei der Steuerung des dreistufigen Stromrichters nach Variante 1 vorhanden: da der Anfangs- und der Endzustand jedes Abtastintervalles gleich sind, kann die gesamte Einschaltzeit dieses Zeigers ebenfalls frei auf die beiden Teilintervalle verteilt werden. Die symmetrische Verteilung, gemäss dem Beispiel in Bild 16.9, entspricht wie beim zweistufigen Stromrichter der Standardmethode für eine Drehzeigermodulation. Die Pulsmuster sind dann gleich wie bei einem Trägerverfahren nach Bild 16.7, wobei aber den Sollwerten ein Gleichtaktsignal gemäss Bild 8.7 überlagert ist. 


\subsubsection{Weitere Steuerverfahren}

Die dreistufigen Stromrichter haben ein verhältnismässig kleines Anwendungsgebiet im Bereich grosser Leistungen (Kapitel 3.3.3). Dabei kommen kaum andere als die bereits gezeigten Steuerverfahren zum Zuge. Die sehr guten Eigenschaften der Grundfrequenzsteuerung und der Trägerverfahren beim Einsatz in dreistufigen Schaltungen lassen auch wenig Bedarf nach weiteren Steuerverfahren aufkommen. Trotzdem lassen sich natürlich alle im Teil II vorgestellten Verfahren auf dreistufige Stromrichter übertragen. Im folgenden werden dazu einige kurze Bemerkungen gemacht.

Vorausberechnete Pulsmuster [Hal1], [Nab2]: In gleicher Weise, wie bereits für seriegeschaltete Stromrichter gezeigt wurde, können die Schaltwinkel für den dreistufigen Stromrichter nach beliebigen Gütekriterien bestimmt werden. Wegen der grossen Anzahl von Freiheitsgraden wird allerdings die globale Lösung der Optimierungsprobleme bereits für niedrige Schaltzahlen schwierig.

Direkte Regelverfahren: Das zusätzliche Schaltniveau jedes Brückenzweiges ergibt für alle direkten Regelverfahren (Phasenstromregler, Stromzeigerregler sowie Fluss-und Momentregler) eine gegenüber den zweistufigen Schaltungen deutlich reduzierte Schaltfrequenz bei gegebenen Toleranzbändern. Der sinnvolle Bereich für die Schaltfrequenzen bei den meisten dieser Verfahren liegt aber trotzdem für die bei grosser Leistung angesiedelten dreistufigen Stromrichtern zu hoch. Eine Ausnahme bildet die direkte Selbstregelung, welche in Antrieben mit dreistufigen Stromrichtern eingesetzt wird [Spr1].

\subsubsection{Zwischenkreis}

Während auf der AC-Seite des dreistufigen Stromrichters eine starke Analogie mit seriegeschalteten zweistufigen Schaltungen erkennbar ist, ergeben sich auf der DC-Seite deutliche Unterschiede. In den Gleichungen (3.79), (3.81) und (3.83) für die 3 Leiterströme im Zwischenkreis tritt überall das Produkt aus dem Quadrat der Schaltfunktionen und den zugehörigen Phasenströmen auf. Dies bedeutet, dass in den Spektra dieser Ströme mehr Frequenzlinien auftreten als bei den zweistufigen Schaltungen. Insbesondere ergibt sich durch die genannte Multiplikation ein zusätzlicher Pulsationsanteil der dreifachen Grundfrequenz $3 f_{1}$.

Einphasige Brücke: Bild 16.10 zeigt die Zwischenkreisströme $i_{d^{+}}$und $i_{d 0}$ für die mit dem Trägerverfahren nach Variante 1 gesteuerte Brücke. Nur der Strom $i_{d+}$ (sowie $i_{d-}$ ) weist niederfrequente Anteile auf. Diese bestehen, wie bei der zweistufigen Brücke, aus einer DC-Komponente und einem Pulsationsanteil der Frequenz $2 f_{1}$, während die Frequenzen $f_{1}$ und $3 f_{1}$ fehlen. Der Verzerrungsanteil von $i_{d+}$ wird durch Trägerbänder um die Vielfachen der Trägerfrequenz gebildet. Der Strom im mittleren Leiter $i_{d 0}$ weist dagegen nur einen Verzerrungsanteil auf. Im Gegensatz zum Spektrum der Phasenspannung, wo wegen der versetzten Taktung das erste Trägerband wegfällt, ist dieses in allen Zwischenkreisströmen vorhanden. 

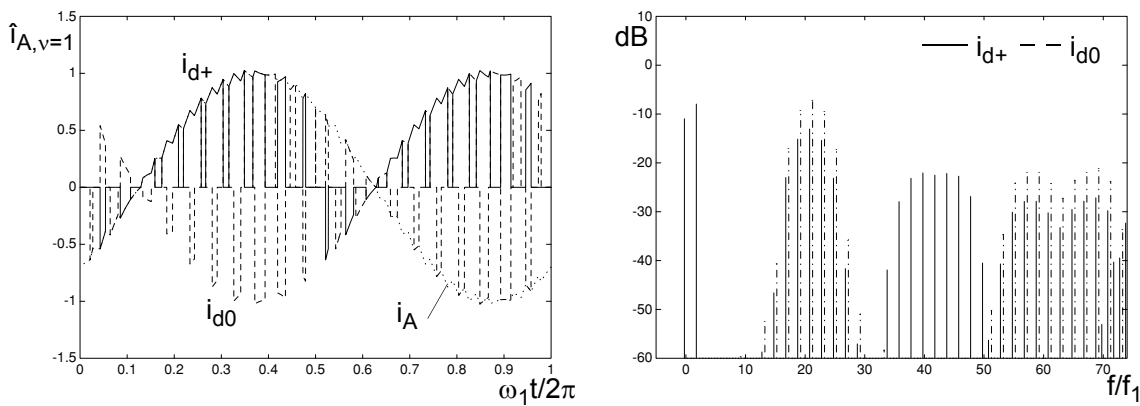

Bild 16.10. Zwischenkreisströme bei der einphasigen Brücke, Steuerung gemäss Bild 16.6, links: Verläufe der Zwischenkreisströme sowie des Phasenstromes, $\hat{\imath}_{A, v=1}=0.2 I_{B}$, rechts: Spektra der Zwischenkreisströme, $0 \mathrm{~dB}: \hat{\imath}_{A, v=1}$

Dreiphasige Brücke: Bild 16.11 zeigt die Zwischenkreisströme für die dreiphasige Brükke, ebenfalls mit Steuerung mittels Trägerfahren nach Variante 1 . Hier weist der Strom $i_{d+}$ (sowie $i_{d-}$ ) neben dem DC-Anteil einen Pulsationsanteil der Frequenz $3 f_{1}$ auf. Der Verzerrungsanteil besteht wiederum aus den Trägerbändern um die Vielfachen der Trägerfrequenz, wobei diese deutlich breiter sind als bei der einphasigen Brücke. Der Strom $i_{d 0}$ zeigt neben dem Verzerrungsanteil hier auch einen Pulsationsanteil mit $3 f_{1}$.


Bild 16.11. Zwischenkreisströme bei der dreiphasigen Brücke, Steuerung gemäss Bild 16.7, links: Verläufe der Zwischenkreisströme sowie eines Phasenstromes, $\hat{\imath}_{U / V / W, v=1}=0.2 I_{B}$, rechts: Spektra der Zwischenkreisströme, $0 \mathrm{~dB}: \hat{\imath}_{U / V / W, v=1}$

In vielen Anwendungen ist der Nullpunkt des Zwischenkreises nur virtuell vorhanden (Bild 3.27 oben). In diesem Fall können kleine Unsymmetrien bei der Umsetzung der Pulsmuster im Stromrichter zu einer langsamen Veränderung der Spannungsaufteilung im Zwischenkreis führen. Als Gegenmassnahme muss eine Regelung für die Spannungsaufteilung implementiert werden. Entsprechende Vorschläge werden z.B. in [Liu1], [Stei1], [Kla1] gemacht.

Literatur zu Kapitel 16: [Car1], [Hal1], [Kla1], [Liu1], [Nab2], [Spr1], [Ste3], [Ste4], [Stei1], [Stei2], [Stei3], [Vel1] 


\section{Teil IV}

\section{Praktischer Einsatz von Steuerverfahren}

\section{Nichtidealitäten im Modulator und im Leistungskreis}

In den Kapiteln 6 bis 12 wurden die Steuerverfahren für zweistufige U-Stromrichter ideal, d.h. ohne irgendwelche praktischen Verfälschungen behandelt. Die realen Pulsmuster am Ausgang des Modulators bzw. die Ausgangsspannungen der Stromrichter weichen aber aus verschiedenen Gründen von ihren idealen Formen ab. In diesem Kapitel werden diese Abweichungen und ihre Einflüsse auf die interessierenden Grössen diskutiert. Da eine exakte Berücksichtigung aller Nichtidealitäten sehr schwierig und in den meisten Fällen auch unnötig ist, werden nur die wichtigsten Einflüsse mit Modellen erfasst und dargestellt. Dabei wird vor allem die Wirkung der einzelnen Phänomene aufgezeigt. Die Entstehungsorte für die Verfälschung der Pulsmuster zeigt symbolisch Bild 17.1: das vom Prinzip her ideale Pulsmuster wird schon bei der Erzeugung im Modulator verändert. Ein Teil der Veränderungen ist durch die Art der Pulserzeugung bedingt und ein weiterer Teil wird gezielt vorgenommen (z.B. Einhalten der minimalen Pulsweiten). Mit den im Modulator erzeugten Schaltfunktionen werden die Leistungshalbleiter angesteuert. Diese verzögern und verzerren das Pulsmuster bei der Umsetzung in Spannungen weiter. Dabei spielen die Lastströme und der Stromrichteraufbau eine nicht zu vernachlässigende Rolle. Auch die Zwischenkreisspannung hat einen Einfluss auf die resultierenden Mittelpunktspannungen. Sie wird unter anderem durch die Speisung und den Laststrom beeinflusst. Letzterer ist abhängig von der Art und den Eigenschaften der Last.

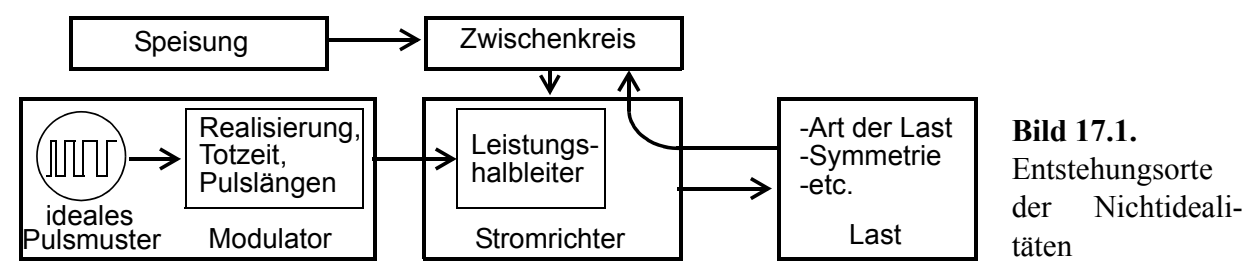

Die verschiedenen Einflüsse werden jeweils am Beispiel eines einfachen, synchronen Trägerverfahrens mit dreieckförmigem Träger gezeigt. Damit tritt im unverzerrten Fall 
ein Spektrum mit nur wenigen dominanten Frequenzlinien pro Trägerband auf. Jede Veränderung des Spektrums ist so leicht erkennbar. Die Betrachtungen werden an einem Brückenzweig gemäss Bild 17.2 durchgeführt.

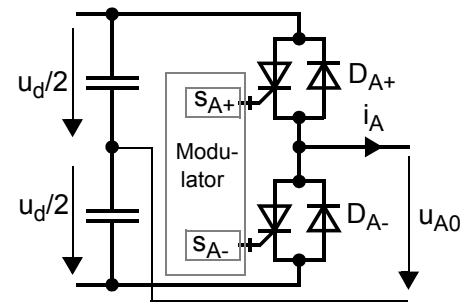

Bild 17.2.

Brückenzweig für die Betrachtung des nichtidealen Umsetzens der Schaltfunktion

\subsection{Modulator}

Im Modulator werden die Schaltfunktionen entsprechend einem bestimmten Steuerverfahren erzeugt. Bedingt durch die physikalische Implementierung des Modulators (Kapitel 19.1) kann dies mehr oder weniger genau geschehen. Bei analogen Schaltungen wird die Genauigkeit durch das Schaltungsprinzip, die eingesetzten Bauelemente und Rauschen bestimmt. Analogschaltungen werden nachfolgend nicht weiter betrachtet, da die Grössenordnung der auftretenden Fehler schwierig zu quantisieren ist. Bei geeigneter Schaltungsauslegung lassen sich aber gute Resultate erreichen.

Digitale Schaltungen arbeiten amplitudenquantisiert und beim Einsatz von sequentiellen Schaltungen und Prozessoren auch zeitquantisiert. Diese Eigenschaften führen zu unerwünschten Abweichungen von der idealen Schaltfunktion.

\subsubsection{Quantisierungsfehler bei digitalen Modulatoren}

Betrachtet werden die Einflüsse von Zeit- und Amplitudenquantisierung des Sollwertes und des Trägersignals. Als erstes wird nur das Sollwertsignal quantisiert, das Trägersignal bleibt ideal. Dieser Fall tritt beispielsweise dann auf, wenn der Sollwert für einen analogen Modulator in einem Rechner erzeugt wird. Den Einfluss der Quantisierung zeigt Bild 17.3. In den Abtastzeitpunkten kann der quantisierte Sollwert um $\pm \Delta x_{\text {Soll }}$ vom kontinuierlichen Sollwert abweichen. Zwischen den Abtastungen können die Abweichungen noch grösser werden. Die resultierenden Pulse der Schaltfunktion variieren in ihrer Weite und Lage gegenüber den idealen Pulsen.

Für die Analyse der auftretenden Verzerrungen wird der Aussteuerbereich von -1 bis +1 des Sollwertsignals in 200 Stufen aufgeteilt. Dies entspricht einem Quantisierungsschritt von $\Delta x_{\text {Soll }}=1 / 100$, wie er sich schon mit einer digitalen Wortbreite von 8 Bit erreichen lässt. Die Dauer einer Grundschwingungsperiode wird in 100 Schritte eingeteilt, d.h. $\Delta t_{1}=T_{1} / 100$. Der Verlauf des Sollwertes und das resultierende Spektrum in Bild 17.4 zeigen, dass diese relativ grobe Quantisierung den Sollwert und das resultierende Spektrum im Vergleich zur idealen Trägermodulation nur geringfügig verfälschen. Es treten zusätz- 

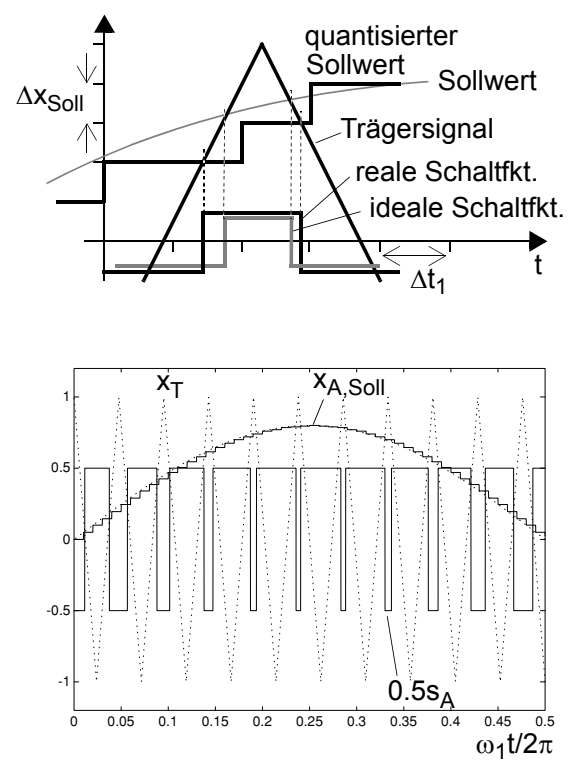

Bild 17.3.

Verfälschung der Schaltfunktion durch die Zeit- und Amplitudenquantisierung des Sollwertsignals



Bild 17.4. Zeit- und amplitudenquantisierte Sollwerte, $M=0.8, q=21, \Delta x_{\text {Soll }}=1 / 100, \Delta t_{1}=T_{1} / 100$, links: ideales und quantisiertes Sollwertsignal, Trägersignal und Schaltfunktion, rechts: Spektra der beiden Schaltfunktionen, 0dB: 1

liche niederfrequente Spannungsanteile auf. Eine weitere Vergrösserung der Amplitudenund Zeitschritte ab diesem Punkt ergibt schnell schlechtere Resultate. Insbesondere ist eine noch geringere Zeitauflösung kritisch.

Den Einfluss eines quantisierten Trägersignals (Sollwert nicht quantisiert) auf das Spektrum der Schaltfunktion zeigt Bild 17.5. Die Quantisierungsstufen für die Amplitude betragen $\Delta x_{T}=1 / 100$. Die Zeitachse ist aber so aufgelöst, dass eine Trägerperiode in 100 Zeiteinheiten aufgeteilt ist: $\Delta t_{T}=T_{T} / 100=T_{1} / 100 q$. Mit diesen Auflösungen sind die auftretenden zusätzlichen Verzerrungen etwa gleich gross wie bei einem mit $\Delta x_{\text {Soll }}=1 / 100$ und $\Delta t_{1}=T_{1} / 100$ quantisierten Sollwert. Es ist aber zu beachten, dass dafür die effektive Zeitauflösung beim Trägersignal $q$-mal besser sein muss als beim Sollwert. Auch hier ist die Zeitauflösung die kritische Grösse.

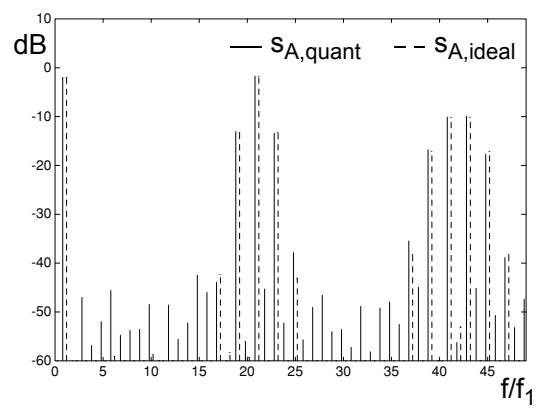

Bild 17.5.

Spektra der Schaltfunktionen bei einem zeitquantisierten Trägersignal, $M=0.8, q=21$, $\Delta x_{T}=1 / 100, \Delta t_{T}=T_{1} / 100 q, 0 \mathrm{~dB}: 1$ 
Es lässt sich zeigen, dass für Sollwert und Trägersignal die Zeitauflösung pro Periode etwa gleich der jeweiligen Amplitudenauflösung sein sollte. Praktische Realisierungen mit 8 Bit Wortbreite ergeben schon sehr geringe Abweichungen der Schaltfunktionen vom idealen Verlauf.

Die Resultate für die zeitliche Auflösung gelten näherungsweise für beliebige Modulationsverfahren (z.B. Off-Line Verfahren), wenn für $\Delta t=T_{1} / 100 q$ eingesetzt wird. D.h. mit steigender Schaltzahl $q$ muss der Modulator eine immer bessere Zeitauflösung aufweisen. Beispiel: bei einer Schaltzahl von $q=20$ und einer Grundfrequenz von $50 \mathrm{~Hz}$ resultiert für die Zeitauflösung $\Delta t=T_{1} / 100 q=10 \mu$ s.

\subsubsection{Minimale Pulsweiten}

Leistungshalbleiter sollten immer während einer minimalen Zeit $T_{\text {min }}$ ein- bzw. ausgeschaltet sein. Wie in Kapitel 3.1.2 besprochen besteht sonst die Gefahr, dass sie durch punktuelle Überhitzung oder weil die Entlastungsnetzwerke noch nicht richtig vorbereitet sind, überlastet werden. In Kapitel 19.3 sind Massnahmen zur Einhaltung dieser Bedingungen beschrieben. Die Minimalzeit kann auf verschiedene Arten gewährleistet werden: eine erste Möglichkeit ist, zu kurze Pulse in den Schaltfunktionen immer auf $T_{\min }$ zu verlängern. Ein zweiter Ansatz ist das Auslassen von Pulsen kürzer als $T_{m i n}$. An dieser Stelle soll der Einfluss der kombinierten Anwendung der beiden Verfahren betrachtet werden: sind die Pulse länger als $T_{\min } / 2$ werden sie auf $T_{\min }$ verlängert und andernfalls ausgelassen. Dies entspricht einem einfachen Auf- bzw. Abrunden der Pulsweiten. Die Anpassung ist nur bei grossem Modulationsgrad nötig, da nur dann kurze Pulse auftreten können.

Bei Trägerverfahren entspricht die Korrektur der Pulsweiten einer Modulation mit dem in Bild 17.6 dargestellten modifizierten Sollwert. Diese Darstellung zeigt direkt, wie die resultierende Mittelpunktspannung nach dem Ausfiltern der hochfrequenten Anteile aussieht. Mittels einfacher geometrischer Überlegungen ist einsehbar, dass die Pulsweiten direkt durch die Sollwertamplitude bestimmt sind. Im Beispiel wird der Sollwert $x_{A \text {,Soll }}$ $\left(\hat{x}_{A, \text { Soll }} \leq 1\right)$ zwischen 0.8 und 0.9 auf 0.8 abgerundet. Über 0.9 wird auf eins aufgerundet. Die resultierenden Pulse haben damit ein minimale Länge von $0.2 T_{T}$ oder aber null. (Die gewählte Minimalzeit ist aus Darstellungsgründen sehr gross gewählt.) Mit diesem neuen, verzerrten Sollwertverlauf wird eine ganz normale Trägermodulation vorgenommen. In der Schaltfunktion ist besonders der Bereich des Aufrundens deutlich; dort fehlen die Pulse, d.h. die Schaltfrequenz wird kleiner. Im Bild rechts ist das Spektrum der Schaltfunktion mit und ohne Korrektur der Pulsweiten dargestellt. Wie zu erwarten war, enthält das gerundete Pulsmuster niederfrequente Verzerrungen, herrührend von den verzerrten Sollwerten.

Die vorgestellte einfache Realisierung funktioniert einwandfrei, solange der Sollwert nach oben begrenzt wird, d.h. gar keine kurzen Pulse auftreten können. Wird aufgerundet, so kann im Bereich des Sollwertsprunges auf eins bei unglücklicher Phasenlage zwischen Sollwert und Träger die minimale Pulsweite unterschritten werden. Dies kann geschehen, wenn ein Puls kurz vor dem Aufrund-Zeitpunkt angefangen wird. Springt der Sollwert als Folge des Rundens auf eins, so wird der Puls sofort beendet wie in Bild 17.7 dargestellt. Die Pulsweitenbegrenzung durch Aufrunden des Sollwertes darf deshalb höchstens bei synchronen Pulsmustern angewendet werden. 

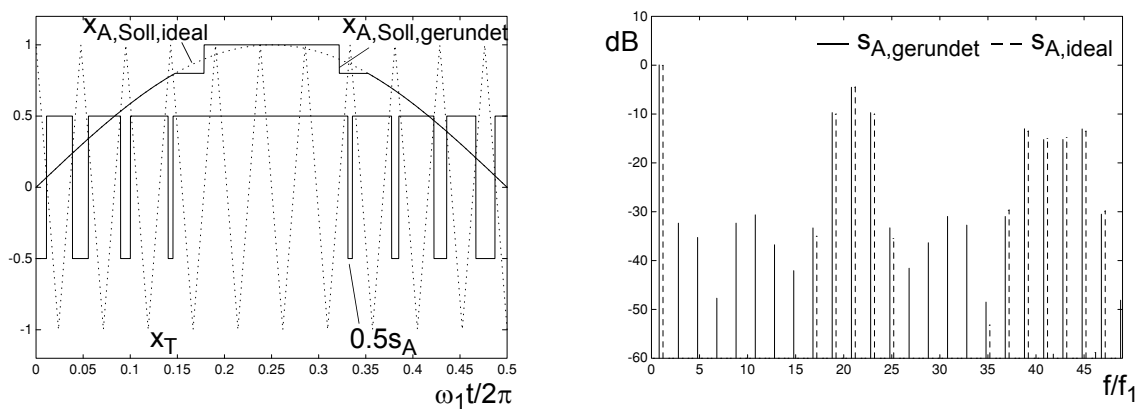

Bild 17.6. Runden der Pulsweiten, $M=1, q=21, T_{\min }=0.2 T_{T}$ resp. 0 , links: idealer und modifizierter Sollwert, Träger und Schaltfunktion, rechts: Spektra der idealen und der Schaltfunktion mit minimalen Pulslängen, 0dB: 1

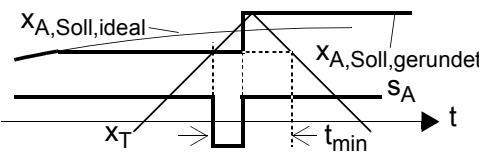

Bild 17.7.

Fehlerhafte Pulserzeugung im Bereich des Sollwertsprunges

\subsubsection{Unsymmetrien}

Bei der Erzeugung und Umsetzung der Schaltfunktionen treten immer kleine Unsymmetrien in Amplituden und Phasenlagen auf. Bei einphasigen Schaltungen können als Folge davon Gleichspannungskomponenten entstehen. Im dreiphasigen Fall können Spannungsanteile, die eigentlich Gleichtaktkomponenten sein müssten, in den Phasenspannungen auftreten. Normalerweise sind die Unsymmetrien so klein, dass sie nicht stören. Eine Ausnahme bilden auch hier die Gleichspannungskomponenten. Sie können in Transformatoren mit sehr kleinen Magnetisierungsströmen zu Sättigungserscheinungen führen. Treten solche Probleme auf, so muss eventuell mit einer zusätzlichen Regelung Abhilfe geschaffen werden.

\subsection{Leistungshalbleiter}

In Kapitel 3.1 wurde gezeigt, wie sich die realen Leistungshalbleiter von den idealen unterscheiden. Die Effekte basieren auf anspruchsvollen, physikalischen Gesetzmässigkeiten. Für die Diskussion ihrer wichtigsten Einflüsse genügt es jedoch, sie mit vereinfachten Modellen darzustellen. Untergeordnete Effekte können vernachlässigt werden.

\subsubsection{Leitspannungsabfall}

Der Leitspannungsabfall über den Halbleitern verfälscht die Amplituden der Stromrichter-Mittelpunktspannungen. Bei U-Stromrichtern liegt immer eine Diode antiparallel zum abschaltbaren Leistungshalbleiter. Diese muss in die Betrachtung mit einbezogen werden. 
Vorzeichen und Betrag des Spannungsabfalls über dem Schalter sind stromabhängig. Verschiedene Modellierungen der Kombination Leistungshalbleiter mit der zugehörigen antiparallelen Diode zeigt Bild 17.8:

- Schaltung a modelliert einen MOSFET. Der Drain-Source-Widerstand $r_{D S}$ beschreibt den FET in Vorwärtsrichtung. Die antiparallele Diode kann relativ genau durch die Spannungsquelle $u_{D F}$ und den differentiellen Widerstand $r_{D d}$ nachgebildet werden. Die idealen Dioden $\left(U_{F}=0\right) D_{1}$ und $D_{2}$ leiten den Strom entsprechend seiner Polarität entweder durch die FET- oder die Diodenersatzschaltung.

- Für die Untersuchung des Einflusses des Leitspannungsabfalls kann das Modell noch etwas vereinfacht werden, indem der differentielle Widerstand der Diode weggelassen wird. Das führt zur Ersatzschaltung gemäss Figur $b$.

- Schaltung $c$ beschreibt bipolare Halbleiter. Diese lassen sich in Vorwärts- und in Rückwärtsrichtung durch Spannungsquellen und differentielle Widerstände, darstellen.

- Auch das Modell für bipolare Halbleiter lässt sich entsprechend Schaltung $d$ vereinfachen, indem die differentiellen Widerstände weggelassen werden.

Die beiden Modelle $b$ und $d$ sind für die Betrachtung des Einflusses, welcher der Leitspannungsabfall auf die Stromrichterspannung hat, genügend genau.


Bild 17.8. Leitspannungsabfall bei Leistungshalbleitern,

Schaltungen $a$ und $b$ : Modelle für einen MOSFET,

Schaltungen $c$ und $d$ : Modelle für bipolare Halbleiter (IGBT, GTO, Bipolartransistor)

Die Verfälschung der Ausgangsspannung durch die Spannungsabfälle über dem Leistungshalbleiter, respektive der antiparallelen Diode, zeigt Bild 17.9 links. Um die Verhältnisse zu illustrieren sind die Spannungsabfälle sehr gross gewählt: $0.15 U_{d} / 2$ für den abschaltbaren Halbleiter und $0.1 U_{d} / 2$ für die Diode. Im dargestellten Beispiel eilt der idealisierte Phasenstrom der Grundschwingung der Mittelpunktspannung um $\pi / 2$ nach. Haben Strom und Schaltfunktion das gleiche Vorzeichen, so leiten die abschaltbaren Elemente. Der Betrag der Ausgangsspannung wird dabei um den Spannungsabfall über diesem Element reduziert. Sind die Vorzeichen von Schaltfunktion und Strom unterschiedlich, so leiten die Dioden und der Betrag der Ausgangsspannung steigt um die Flussspannung der Diode. Dieses Verfälschen der Mittelpunktspannung ergibt niederfrequente Verzerrungen im Spannungsspektrum. Für typische Werte der Spannungsabfälle sind die Verzerrungen aber relativ klein.

Für einen Spannungsabfall von $0.01 U_{d} / 2$ für den abschaltbaren Halbleiter und die Diode, und einer Schaltzahl von $q=21$, ist das resultierende Spektrum in Bild 17.9 rechts dargestellt. 

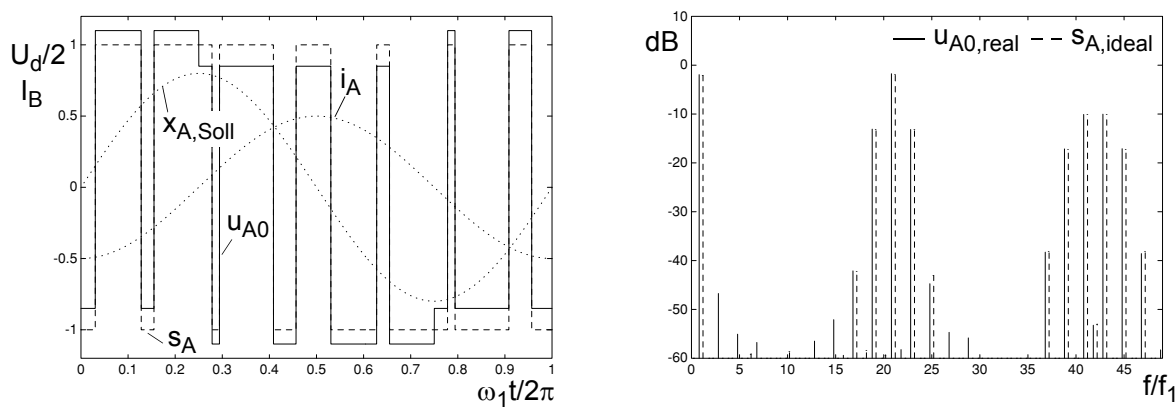

Bild 17.9. Einfluss der Leitspannungsabfälle auf die Mittelpunktspannungen, $M=0.8$, links: Sollwert, Schaltfunktion, Mittelpunktspannung und Phasenstrom, $q=7$, rechts: Spektra der Schaltfunktion und Mittelpunktspannung, $q=21,0 \mathrm{~dB}: U_{d} / 2$ bzw. 1

\subsubsection{Schaltverzögerung und Steilheit der Spannungsflanken}

Wie in Bild 17.10 dargestellt, setzen sich die Umschaltvorgänge in einem Brückenzweig aus einer Reihe von Teilvorgängen zusammen. Im Modulator wird zuerst das Schaltsignal $s_{A}$ erzeugt. Für die Schalteransteuerung wird es anschliessend in die beiden Ansteuersignale $s_{A+}$ und $s_{A \text { - }}$ zerlegt. Dabei muss bei beiden Schaltflanken die Totzeit $T_{\text {tot }}$ eingefügt werden, damit beim Umschalten sicher keine Kurzschlüsse im Brückenzweig auftreten. Bei positiver Phasenstrompolarität fliesst der Strom entweder durch den Schalter $S_{A+}$


$D_{A+}$ führen keinen Strom. Auf die negative Flanke von $s_{A}$ reagiert zuerst die Mittelpunktspannung. Der Spannungsabfall über dem Leistungshalbleiter wird so lange grösser, bis die Mittelpunktspannung den Wert $-U_{d} / 2$ erreicht. Danach beginnt die Diode $D_{A \text { - }}$ zu leiten und der Schalterstrom wird auf null abgebaut. Die Verzögerungszeit $t_{d \text { - }}$ der Spannungsflanke ist somit etwas kürzer als $t_{d, i, a u s}$ des Stromes. Die Ausschaltverzögerungen sind vor allem bei bipolaren Halbleitern stromabhängig: je grösser der Strom ist, desto länger werden sie. Auch die Anstiegs- und die Abfallzeiten $t_{r, i}, t_{r}, t_{f, i}$ und $t_{f}$ von Spannung und Strom variieren in Abhängigkeit des Stromes. Wird $S_{A+}$ mit dem Schaltsignal $s_{A+}$ eingeschaltet, so vergeht die Zeit $t_{d, \text { i,ein }}$ bis der Strom im Schalter ansteigt. Die Mittelpunktspannung selber kann erst ändern, nachdem der Diodenstrom auf null abgebaut ist, da erst dann die Diode sperrt.

Bei negativem Phasenstrom sind, wie im Bild rechts dargestellt, $S_{A \text { - }}$ und $D_{A+}$ aktiv und es gelten die gleichen Abläufe wie vorher wenn Ein- und Ausschaltvorgang vertauscht werden. In diesem Fall sind $S_{A^{+}}$und $D_{A_{-}}$stromlos.

Schaltverzögerung: Alle Leistungshalbleiter reagieren verzögert auf ihre Ansteuersignale. Die Verzögerungszeiten beim Ein- und Ausschalten sind im allgemeinen nicht identisch. Die Einschaltverzögerung $t_{d, i, e i n}$ ist relativ kurz und wenig stromabhängig. Die Ausschaltverzögerung $t_{d, i, a u s}$ dagegen ist vor allem bei stromgesteuerten Halbleitern (Bipolartransistoren und GTOs) stark vom Laststrom abhängig. Die unterschiedlichen Verzögerungen beim Ein- und Ausschalten sind der Grund dafür, dass beim Umschalten im Zweig relativ grosse Totzeiten zwischen dem Ausschalten des einen Schalters und dem 

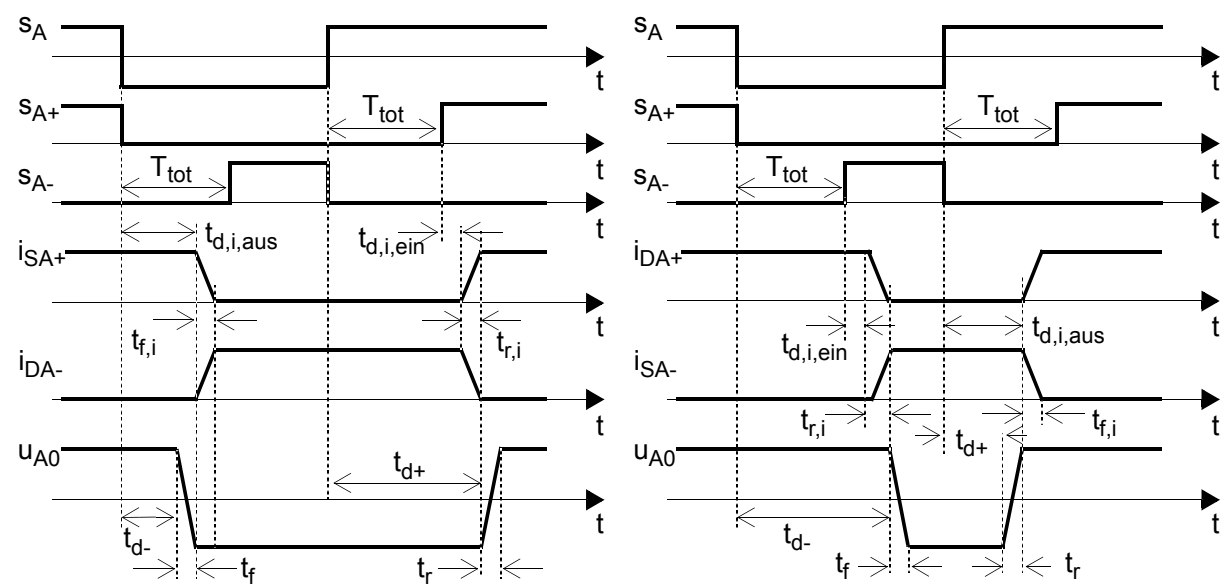

Bild 17.10. Umschaltvorgang in einem Brückenzweig, mit Totzeit und Schaltverzögerung links: bei positivem Phasenstrom, rechts: bei negativem Phasenstrom

Einschalten des anderen eingefügt werden müssen, um transiente Brückenkurzschlüsse zu vermeiden. Die Totzeit $T_{\text {tot }}$ muss so gross gewählt werden, dass der zuletzt leitende Schalter sicher ausgeschaltet ist, bevor der neue einschaltet, d.h. grösser als die maximal mög-

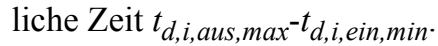

Eine weitere Verfälschung des Schaltvorganges ergibt sich durch den Sperrverzug der Dioden. Die Sperrverzugszeit ist normalerweise aber so kurz, dass sie keine störende Verfälschung des Pulsmusters ergibt.

Stromrichterzustände während der Totzeit $T_{t o t}$ : In dieser Zeit erhält kein Halbleiter des Brückenzweiges ein Ein-Signal. Die Mittelpunktspannung wird durch die Ausschaltverzögerung des zuletzt leitenden Halbleiters, die Freilaufdioden und die Polarität des Phasenstromes bestimmt. Sind die abschaltbaren Halbleiter ausgeschaltet und ist der Strom positiv, so leitet die Diode $D_{A \text { - }}$, die Mittelpunktspannung beträgt im Idealfall $-U_{d} / 2$, andernfalls leitet $D_{A+}$ und es ist $u_{A 0}=U_{d} / 2$. Dieser Zustand gilt anschliessend an die Totzeit solange, bis derjenige Schalter, der neu ein Einschaltsignal erhalten hat, wirklich reagiert. Für die Verzögerung der Mittelpunktspannung gegenüber der Schaltfunktion resultiert so:

$$
\begin{aligned}
i_{A}>0: & t_{d+} \approx T_{\text {tot }}+t_{d, i, \text { ein }}+t_{r, i} & t_{d-} \approx t_{d, i, \text { aus }} \\
i_{A}<0: & t_{d+} \approx t_{d, i, \text { aus }} & t_{d-} \approx T_{\text {tot }}+t_{d, i, \text { ein }}+t_{r, i}
\end{aligned}
$$

Für die Verzerrungen ist also beim einen Schaltvorgang nur die Ausschaltverzögerung des Halbleiters und beim anderen die Summe von Totzeit, Einschaltverzögerung und Anstiegszeit des Stromes massgebend. Bild 17.11 links illustriert diesen Fall. Totzeit und Ausschaltverzögerung plus Anstiegszeit sind mit 5\% $T_{T}$ respektive $3 \% T_{T}$ an der oberen Grenze der praktisch auftretenden Werte gewählt. Das gleiche Bild rechts zeigt die entstehenden beachtlichen, niederfrequenten Verzerrungen. 



Bild 17.11. Mittelpunktspannung bei Ansteuerung mit Totzeit und Schaltverzögerung, links: Schaltfunktion, idealisierter Phasenstrom und Mittelpunktspannung, rechts: Spektra der idealen Schalfunktion und der Mittelpunktspannung, $T_{\text {tot }}=5 \% T_{T}, t_{d, i, e \text { in }}+t_{r, i}=t_{d, i, a u s}+t_{r, i}=3 \% T_{T}, 0 \mathrm{~dB}: U_{d} / 2$ bzw. 1

Kompensation der Ausschaltverzögerung: Mit Vorsteuerungen ist es möglich, den Einfluss der Verzögerung, die durch die Schalter verursacht wird, zu kompensieren. Damit kann die Totzeit kürzer gewählt werden, was zu einer Reduktion der Verzerrungen führt. Obwohl Vorsteuerungen nur selten und auch dann nur für die Ausschaltverzögerungen Anwendung finden, soll eine Variante kurz vorgestellt werden. Sie beruht auf der geregelten Verzögerung des Ansteuersignals für die einzelnen Schalter, wie in Bild 17.12 dargestellt: die Ein- und Ausschaltflanken des Schalteransteuersignals $S_{A+}{ }^{+}$werden so gesteuert, dass beide Flanken des Schalterstroms um die gleiche Zeit $t_{V}$ gegenüber der Schaltfunktion $s_{A+}$ verzögert sind. Die Einschaltverzögerung $t_{V \text {,ein }}$ ist näherungsweise gleich $t_{V}$. Die Ausschaltverzögerungszeit $t_{V \text {,aus }}$ wird der erwarteten Ausschaltverzögerung angepasst. Dies kann nach verschiedenen Kriterien geschehen: eine Variante ist die Korrektur aufgrund der gemessenen Verzögerung $t_{V} t_{V \text {,aus }}$ beim letzten Ausschaltvorgang. Dabei wird angenommen, dass der Phasenstrom von einem Ausschaltvorgang zum nächsten nur wenig ändert, was bei grossen Schaltzahlen $q$ normalerweise erfüllt ist. Eine weitere Möglichkeit ist eine Vorsteuerung aufgrund des gemessenen Schalterstromes $i_{S_{+}}$. Dieses Verfahren funktioniert auch bei kleinen Schaltzahlen. Beide Varianten haben ihre Vor- und Nachteile. Beim Einfügen der Totzeit ist zu beachten, dass die Pulse verkürzt werden können. Die verbleibende Einschaltzeit der Leistungshalbleiter muss aber immer mindestens die minimale Pulsweite betragen. Dies muss bei der Ansteuerung der Halbleiter garantiert werden.

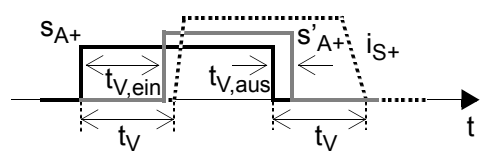

Bild 17.12.

Regelung der Ausschaltverzögerung, $\mathrm{s}_{\mathrm{A}^{+}}$: Schaltfunktion, $\mathrm{s}_{\mathrm{A}^{+}}$: Ansteuersignal für den Leistungshalbleiter

Die Eigenschaft, dass während der Totzeit die Polarität der Mittelpunktspannung durch die Last bestimmt wird, kann bei Antrieben unter speziellen Bedingungen zu Instabilitä- 
ten führen. In [Jeo1] wird ein Ansatz gezeigt, wie die auftretenden Verzerrungen durch eine gesteuerte Modifikation der Sollwerte wieder reduziert werden können.

Flankensteilheit der Mittelpunktspannung: Die Geschwindigkeit der Spannungsänderung $(d u / d t)$ über den Halbleitern ist begrenzt. Die Ursachen dafür sind die effektive Schaltgeschwindigkeit des Halbleitermaterials, parasitäre Kapazitäten (Miller-Kapazität) und eventuell vorhandene Entlastungsnetzwerke. Zum Teil wird der Schaltvorgang auch gezielt über die Ansteuerung verlangsamt, um die hochfrequenten Störungen zu reduzieren (Kapitel 18.1.3). All dies führt dazu, dass die idealen Flanken der Schaltfunktion in den Mittelpunktspannungen endliche Anstiegs- und Abfallzeiten aufweisen und nicht linear sind.

Für die folgende Betrachtung wird angenommen, dass die Ein- und die Ausschaltverzögerungen identisch sind und die Pulslängen nicht verfälscht werden. Um den Einfluss der Flankensteilheit auf die Spannungsverläufe zu betrachten, können die realen Spannungsverläufe in guter Näherung durch lineare Verläufe ersetzt werden. Weisen die ansteigenden und die abfallenden Flanken der Mittelpunktspannung immer die gleiche Steilheit auf, so ergibt sich keine niederfrequente Verfälschung der Mittelpunktspannung. Wie Bild 17.13. zeigt, werden auch bei sehr langsamen Schaltvorgängen (Anstiegszeit $t_{r}=t_{f}=0.1 T_{\mathrm{T}}$ ) nur die höherfrequenten Anteile gedämpft. Dieser Betriebsfall beschreibt in guter Näherung das Verhalten von Leistungshalbleitern ohne Entlastungsnetzwerke.
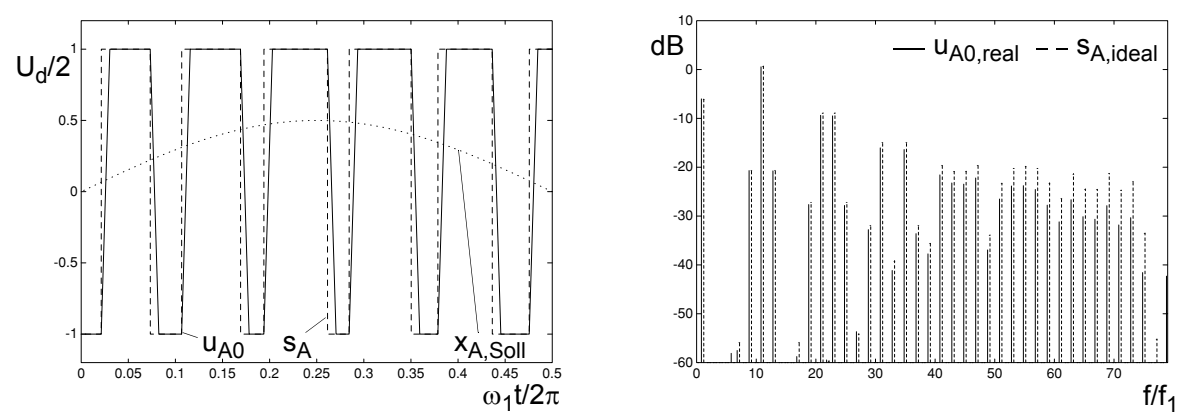

Bild 17.13. Einfluss der begrenzten Steilheit des Spannungsanstiegs, $M=0.5, q=11, t_{r}=t_{f}=0.1 T_{T}$, links: Sollwert, Schaltfunktion und Mittelpunktspannung, rechts: Spektra der Schaltfunktion und Mittelpunktspannung, 0dB: $U_{d} / 2$ bzw. 1

Vor allem bei der Verwendung von Entlastungsnetzwerken sind $t_{r}$ und $t_{f}$ zusätzlich von Betrag und Vorzeichen des Phasenstromes abhängig. Genau genommen sind dabei jeweils nicht beide Flanken der Mittelpunktspannungen gleich steil. Näherungsweise darf aber angenommen werden, dass die beiden Flanken gleich steil und in der gleichen Art stromabhängig sind, da sie durch gleiche Halbleiterpaare erzeugt werden. Die Stromabhängigkeit der Flankensteilheit kann folgendermassen modelliert werden:

$$
t_{r}=t_{f}=\frac{t_{r 0}}{\left(1+K i_{A} / \hat{i}_{A, \max }\right)}
$$


Mit $t_{r 0}$ wird die Anstiegsgeschwindigkeit bei Phasenstrom null bezeichnet. Mit $K=1$ wird $t_{r}$ beim maximalen Phasenstrom auf $0.5 t_{r 0}$ verkürzt. Dies tritt typischerweise bei der Verwendung von Entlastungsnetzwerken auf. Um den Einfluss der variablen Anstiegsgeschwindigkeit deutlich zu machen, wird $t_{r 0}$ sehr gross gewählt: $t_{r 0}=0.1 T_{T}$. Bild 17.14 links zeigt die entstehenden Signalverläufe. Die Verzerrungen in der Mittelpunktspannung werden durch die auftretenden niederfrequenten Verzerrungen etwas grösser.
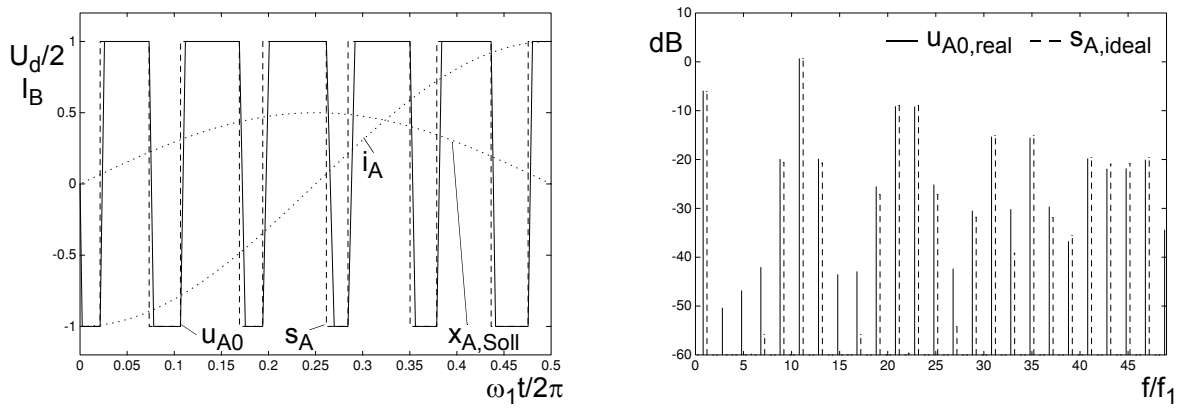

Bild 17.14. Stromabhängige Steilheit des Spannungsanstiegs, $M=0.5, q=11, t_{r 0}=0.1 T_{T}$, links: Sollwert, Schaltfunktion, Mittelpunktspannung und Strom, rechts: Spektra von Schaltfunktion und Mittelpunktspannung, $0 \mathrm{~dB}: U_{d} / 2$ bzw. 1

\subsection{Stromrichteraufbau}

Der konstruktive Aufbau des Stromrichters hat ebenfalls einen Einfluss auf die auftretenden Spannungs- und Stromformen. Leichte Pulsmusterverfälschungen treten durch die Einflüsse parasitärer Streuinduktivitäten auf. Da bei der Verwendung von modernen Schaltelementen immer kompaktere Bauformen der Stromrichter notwendig werden, sind die parasitären Streuinduktivitäten für die Strom- und Spannungsformen nicht mehr sehr wichtig. In vielen Schaltungen werden aber gezielt kleine Induktivitäten zur Stromanstiegsbegrenzung eingesetzt. Deren Einfluss kann nicht immer vernachlässigt werden.

Strommesseinrichtungen wie Shunts, Stromwandler und Transfoshunts werden so ausgelegt, dass möglichst wenig Verluste auftreten. Sie stören deshalb normalerweise nicht.

In jedem Stromrichter treten kapazitive und induktive Kopplungen zwischen den verschiedenen Schaltungsteilen auf. Dadurch resultieren Beeinflussungen von Phase zu Phase. Dieses Übersprechen ist sehr stark vom Aufbau des Stromrichters abhängig. Quantitative Betrachtungen sind sehr schwierig anzustellen.

Viele Stromrichter müssen ausgangsseitig mit Störschutzfiltern versehen werden, damit die EMV-Vorschriften eingehalten werden können (Kapitel 18.2). Je nach Art der Filter ergibt auch dies Verfälschungen der Ausgangsgrössen. 


\subsection{Last}

Bei gegebenen Stromrichter-Mittelpunktspannungen ist nur noch die Last für die Stromverläufe massgebend. Abweichungen von den bei der Besprechung der einzelnen Steuerverfahren angenommenen ein- und dreiphasigen Standardlasten ergeben andere Grundschwingungen und vor allem auch andere Verzerrungsströme. Ist die Last bekannt, so lassen sich diese veränderten Ströme im allgemeinen leicht berechnen.

In dreiphasigen Schaltungen kann die Last unsymmetrisch sein. Dies verursacht auch bei symmetrischen Mittelpunktspannungen unsymmetrische Ströme. Diese haben einen pulsierenden Leistungsfluss in der Last und im Zwischenkreis zur Folge. Aus der Sicht der Steuerverfahren ist in erster Linie von Bedeutung, dass Frequenzanteile in den Mittelpunktspannungen, die im symmetrischen Fall reine Gleichtaktkomponenten wären, bei Unsymmetrien diese Eigenschaft verlieren. Sie sind deshalb in den Phasenspannungen und -strömen ebenfalls zu finden. Der pulsierende Leistungsfluss bei Unsymmetrien führt dazu, dass auch in dreiphasigen Schaltungen der Zwischenkreisstrom einen Pulsationsanteil der zweifachen Grundfrequenz erhält.

In Antrieben sind Unsymmetrien im allgemeinen sehr klein. Sie können aber in aussergewöhnlichen Fällen, z.B. wenn das Eisen gesättigt wird, auftreten. Bei Stromrichtern am Netz ist die Gefahr von Unsymmetrien grösser, weil dort einphasig angeschlossene Verbraucher die Netzimpedanz und die Netzspannung in einer Phase verändern können.

\subsubsection{Einfluss der geschalteten Spannungen auf die Last}

Durch die geschaltete Arbeitsweise der Stromrichter treten an der Last sehr steile Spannungsflanken $d u / d t$ auf. Dies kann insbesondere bei Drehstrommaschinen zu Isolationsproblemen, Teilentladungen und Durchschlägen in den Wicklungen führen. Der Grund dafür sind sehr schnelle Spannungsreflexionen in den Wicklungen, die punktuell zu wesentlich höheren Spannungen als die Stromrichterspannung selbst führen. Für eine ausführliche Behandlung des Themas sei auf die Literatur verwiesen [Per1]. Es existieren Ansätze, dem Problem mit LC-Filtern am Ausgang des Stromrichters zu begegnen. Diese Filter weisen hohe Güten auf. Als Folge davon können sich Resonanzprobleme ergeben.

\subsection{Welligkeit der Zwischenkreisspannung}

Der Verlauf der Zwischenkreisspannung wird durch die Speisung (Gleichrichter), die Zwischenkreiskapazität und die Rückwirkungen der Last bestimmt. Bei ein- und dreiphasigen Schaltungen gilt: je einfacher die Speiseschaltung, desto grösser die Welligkeit. Bei einphasigen Schaltungen wird die Welligkeit zusätzlich durch die Leistungspulsation des Stromrichters selbst, d.h. durch den Pulsationsanteil von $i_{d L}$, stark beeinflusst.

Speisung des Zwischenkreises: Für die Speisung von Spannungszwischenkreisen werden sehr oft ein- oder dreiphasige Dioden- bzw. Thyristorbrücken mit zusätzlicher Glät- 


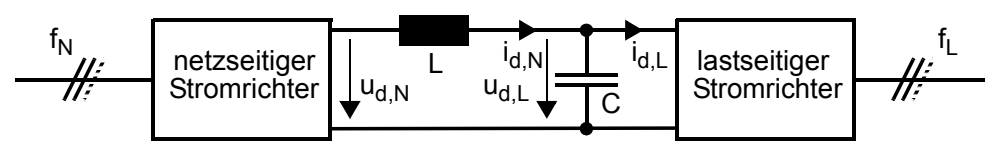

Bild 17.15.

Zwischenkreis für U-Stromrichter

tungsinduktivität entsprechend den Schaltungen 1 und 2 in Kapitel 2.2 bzw. der Blockschaltung in Bild 17.15 verwendet.

Die niedrigste Frequenz der so erzeugten Zwischenkreisspannung entspricht bei einphasiger Speisung der doppelten und bei dreiphasiger Speisung der 6-fachen Netzfrequenz. Weiter können alle Harmonischen der jeweiligen Grundwelligkeit auftreten. Am $50 \mathrm{~Hz}-\mathrm{Netz}$ ergibt das die bekannten $100 \mathrm{~Hz}$ bzw. $300 \mathrm{~Hz}$ Welligkeiten. Die Spannungswelligkeit von $u_{d, N}$ am Stromrichter ist die Ursache für einen nicht konstanten Ladestrom $i_{d, N}$ und dieser wiederum verursacht eine Welligkeit der Kondensatorspannung $u_{d, L}$, wie in Bild 17.16 dargestellt. Der Mittelwert des Gleichstromes ist im betrachteten Fall so gross, dass er in der Drossel $L$ nicht lückt. Die Knickfrequenz des Schwingkreises gebildet durch die Serieinduktivität und den Zwischenkreiskondensator, ist für die einphasige und die dreiphasige Schaltung je auf einen Drittel der entsprechenden tiefsten Zwischenkreisfrequenz ausgelegt. Die Simulationen zeigen, dass die Welligkeit am Zwischenkreiskondensator jeweils gut sinusförmig ist, d.h. alle höherfrequenten Anteile werden sehr stark abgedämpft. Die Kurvenverläufe bestätigen die zu erwartende wesentlich grössere Welligkeit der einphasigen Schaltung. Ohne die Glättungsinduktivität wird sie noch grösser. Zusätzlich ergeben sich dann noch stärker verzerrte Netzströme.

Werden für die Speisung des Zwischenkreises selbstgeführte Stromrichter eingesetzt, die sinusförmigen Wechselstrom beziehen, so pulsiert der Leistungsfluss und damit $u_{d}$ bei einphasigen Schaltungen stark mit der doppelten Speisefrequenz. Bei dreiphasigen Schaltungen dagegen ist ein kontinuierlicher Leistungsfluss ohne Pulsation möglich. Die durch die Schaltfrequenz der Halbleiter verursachte hochfrequente Welligkeit kann normalerweise vernachlässigt werden.



Bild 17.16.

Zwischenkreisspannungen auf der Netzseite des Filters $\left(u_{d, N}\right)$ und am Zwischenkreiskondensator $\left(u_{d, L}\right)$ für eine ein- und eine dreiphasige Speisung mittels Diodenbrücke und LCGlättung.

Rückwirkung des Stromrichters: Bei einphasigen Stromrichtern pulsiert der Leistungsfluss. Dies beeinflusst den Zwischenkreis gleich wie eine einphasige Speiseschaltung. Bei dreiphasiger, symmetrischer Last dagegen ist der Leistungsfluss konstant und es treten keine niederfrequenten Rückwirkungen auf. Sobald aber die Ansteuerung der Schalter 
oder die Last unsymmetrisch sind, enthält der Zwischenkreisstrom einen Pulsationsanteil, der eine Spannungswelligkeit ergibt.

Einfluss der Zwischenkreisspannungs-Welligkeit auf die Ausgangsspannungen: Multipliziert mit der Schaltfunktion ergibt sich aus der Zwischenkreisspannung die Mittelpunktspannung:

$$
u_{A 0}=u_{d}(t) s_{A}
$$

Bis anhin wurde immer angenommen, dass $u_{d}=U_{d}=k o n s t a n t$ gilt. Aus den besprochenen Gründen ist dies nicht exakt. Auch im stationären Betrieb weist $u_{d}$ immer eine Welligkeit auf. Die Zwischenkreisspannung kann als Summe von Teilspannungen beschrieben werden. Die tiefste auftretende Frequenzkomponente werde mit $\omega_{d}$ bezeichnet. Allgemein gilt so für die Zwischenkreisspannung und die Schaltfunktion:

$$
u_{d}=U_{d}+\sum_{i=1}^{\infty} \hat{u}_{d, i} \sin \left(\omega_{d, i} t+\varphi_{d, i}\right), s_{A}=\sum_{v=1}^{\infty}\left(\sqrt{a_{v}^{2}+b_{v}^{2}}\right) \sin \left(\omega_{v} t+\varphi_{v}\right)
$$

Durch die grossen Zwischenkreiskondensatoren werden die höherfrequenten Anteile sehr stark gedämpft. In guter Näherung kann die Zwischenkreisspannung deshalb durch den Mittelwert $U_{d}$ und die tiefste auftretende Frequenzkomponente $u_{d, 1}$ der Welligkeit dargestellt werden. Den Verlauf der so resultierenden Mittelpunktspannung für eine Zwischenkreis-Spannungswelligkeit mit der fünffachen Grundfrequenz zeigt Bild 17.17 links. Nach dem Einsetzen von (17.4) in (17.3) folgt nach einer Zwischenrechnung:

$$
\begin{aligned}
& u_{A 0}=U_{d} \sum_{v=1}^{\infty}\left(\sqrt{a_{v}^{2}+b_{v}^{2}}\right) \sin \left(\omega_{v} t+\varphi_{v}\right) \\
&+ \frac{\hat{u}_{d, 1}}{2} \sum_{v=1}^{\infty}\left(\sqrt{a_{v}^{2}+b_{v}^{2}}\right) \cos \left[\left(\omega_{v}-\omega_{d, 1}\right) t+\varphi_{v}-\varphi_{d, 1}\right] \\
&-\frac{\hat{u}_{d, 1}}{2} \sum_{\substack{\infty \\
\text { ind }}}\left(\sqrt{a_{v}^{2}+b_{v}^{2}}\right) \cos \left[\left(\omega_{v}+\omega_{d, 1}\right) t+\varphi_{v}+\varphi_{d, 1}\right]
\end{aligned}
$$

Es zeigt sich, dass im Spektrum der Mittelpunktspannungen wie im idealen Fall die Frequenzlinien der Schaltfunktion multipliziert mit $U_{d}$ auftreten. $\mathrm{Zu}$ jeder Linie treten aber zusätzlich zwei neue Linien mit den Frequenzen $\omega_{v} \pm \omega_{d, 1}$ auf, wie Bild 17.17 rechts bestätigt. (Man beachte, dass 'negative' Frequenzen in dieser Darstellung auf der positiven Frequenzachse auftreten.)

Sollen weitere Frequenzkomponenten der Zwischenkreisspannung berücksichtigt werden, so kann dies in der gleichen Art geschehen, wie für die Grundschwingung gezeigt wurde.

Berechnung der vom Lastkreis verursachten Spannungswelligkeit: Die Zwischenkreisspannung kann immer exakt bestimmt werden, wenn der netz- und der lastseitige 

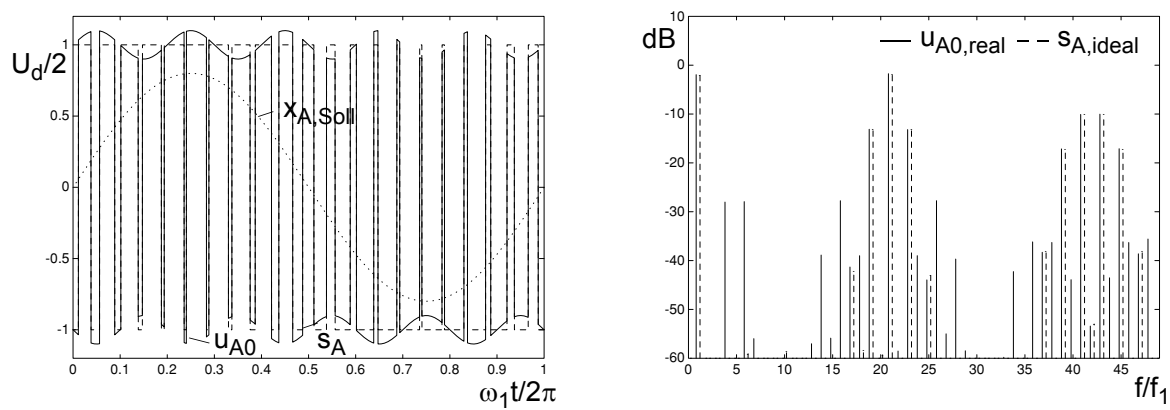

Bild 17.17. Einfluss der Zwischenkreisspannungs-Welligkeit, $q=21, \hat{u}_{d, 1}=0.1 U_{d}, M=0.8$, links: Sollwert, Schaltfunktion und Mittelpunktspannung, rechts: Spektra der Schaltfunktion und der Mittelpunktspannung, 0dB: $U_{d} / 2$ bzw. 1

Zwischenkreisstrom bekannt sind. Aus der Differenz der beiden Ströme kann die Zwischenkreisspannung bestimmt werden:

$$
u_{d}=\frac{1}{C_{d}} \int_{0}^{t}\left(i_{d, N}-i_{d, L}\right) d \tau+u_{d}(t=0)
$$

Für die Welligkeit allein sind im stationären Betrieb nur die Wechselanteile der beiden Ströme massgebend. Die Gleichanteile müssen identisch sein. Um den Einfluss des Lastkreises zu betrachten, wird angenommen, dass von der Netzseite ein konstanter Gleichstrom eingespeist wird. Mit der Schaltfunktion des lastseitigen Stromrichters und den Phasenströmen kann der Zwischenkreisstrom bestimmt werden. Der Wechselanteil davon bestimmt die Spannungswelligkeit.

Normalerweise ist die Zwischenkreiskapazität so gross, dass die hochfrequenten Anteile von $i_{d, L}$ vernachlässigt werden können. Weiter ist die Zwischenkreisspannung so gut konstant, dass der Phasenstrom kaum verfälscht wird. Damit kann die niederfrequente Rückwirkung der Last auf den Zwischenkreis folgendermassen bestimmt werden:

1. Die Phasenströme werden für eine ideale, d.h. konstante Zwischenkreisspannung berechnet.

2. Sind die Phasenströme nicht zu stark verzerrt, so können sie durch ihre Grundschwingungen nachgebildet werden.

3. Mit den Schaltfunktionen und den Phasenströmen wird der Zwischenkreisstrom bestimmt.

4. Mit dem Wechselanteil des Zwischenkreisstromes kann nun die Spannungswelligkeit am Kondensator berechnet werden.

Ist die durch die Schaltvorgänge bestimmte hochfrequente Welligkeit der Zwischenkreisspannung nicht von Interesse, so kann der Zwischenkreisstrom anstatt mit den Schaltfunktionen auch mit den zeitkontinuierlichen Modulationsfunktionen bestimmt werden.

Literatur zu Kapitel 17: [Dun1], [Gra2], [Jeo1], [Per1] 


\section{Elektromagnetische Verträglichkeit}

\subsection{Umgebung von selbstgeführten Stromrichtern}

Elektromagnetische Veträglichkeit (EMV, englisch: EMC) ist eine Eigenschaft von elektrischen Geräten und Einrichtungen. Sie bedeutet, dass diese in einer vorgegebenen Umgebung betrieben werden können, ohne dass sie andere Geräte in dieser Umgebung unzulässig beeinflussen bzw. von diesen beeinflusst werden. Ungewollte Beeinflussungen werden generell Störungen genannt. Das störende Gerät wird als Störquelle und das gestörte als Störsenke bezeichnet. Dazwischen liegt der Störweg.

\subsubsection{Selbstgeführte Stromrichter als Störquellen}

Leistungselektronische Schaltungen stellen im allgemeinen starke Störquellen dar. Die Ursache dafür liegt in den verzerrten Spannungen und Strömen und vor allem in den überaus steilen Schaltflanken mit grossen Amplituden.

Bild 18.1 zeigt einen Spannungspuls, wie er typischerweise in einem selbstgeführten Stromrichter erzeugt wird, zusammen mit seinem Frequenzdichtespektrum $u(f)$ (Anhang B). Dieses beschreibt den Frequenzgehalt des Pulses. Sein Verlauf wird in der doppelt logarithmischen Darstellung durch eine Hüllkurve begrenzt, die sich durch lineare Abschnitte approximieren lässt. Die entsprechenden Knickpunkte sind durch die Pulsdauer $T_{\text {ein }}$ und die Anstiegszeit $T_{r}$ bestimmt. Je kleiner diese Zeiten sind, desto mehr verschieben sich die Knickpunkte auf der Frequenzachse nach rechts. Das Beispiel zeigt, dass die Pulse eines Stromrichters relevante Frequenzanteile bis in den $\mathrm{MHz}-$ Bereich aufweisen.
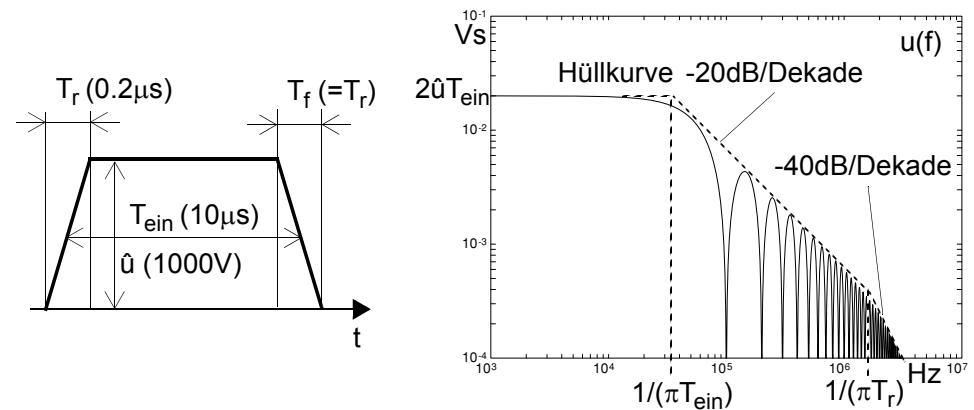

Bild 18.1.

Links: Spannungspuls, rechts: zugehöriges Frequenzdichtespektrum

Pulse der gezeigten Art treten sowohl in Spannungen als auch in Strömen von Stromrichtern auf, und zwar an den äusseren Klemmen (z.B. Ausgangsspannungen) und intern 
(z.B. Schalterströme). Sie stellen die eigentlichen Störquellen dar. Es ist leicht ersichtlich, dass die Störungen besonders breitbandig werden, wenn kurze Pulse mit steilen Schaltflanken auftreten. Diese Breitbandigkeit öffnet eine ganze Reihe von Wegen für die Ausbreitung der Störungen.

Es ist zu anzufügen, dass der Schaltpuls in Bild 18.1 eine starke Idealisierung der tatsächlichen Vorgänge in den Stromrichtern repräsentiert. In der Realität sind den Schaltpulsen häufig kurze Spitzen oder auch hochfrequente Schwingungen überlagert, die den Gehalt an hochfrequenten Anteilen zusätzlich erhöhen. Der Grund für diese Erscheinungen sind Nichtidealitäten in den Stromkreisen (Streuinduktivitäten bzw. -kapazitäten) und in den Schaltelementen (Sperrschichtkapazitäten, Sperrverzug bei Dioden usw.).

Das eingesetzte Steuerverfahren legt die Breite der einzelnen Spannungs- bzw. Strompulse fest. Die Steilheit der Flanken ist dagegen allein durch den Aufbau des Stromrichters und die verwendeten Halbleiter gegeben. Demzufolge bestimmt das Steuerverfahren in erster Linie die Störaussendung im niedrigen und mittleren Frequenzbereich, während der Gehalt an sehr hohen Frequenzen nur wenig beeinflusst werden kann. Die Entwicklung von Leistungshalbleitern mit immer grösserer Schaltgeschwindigkeit bringt zwar Vorteile im Bereich der Schaltverluste, vergrössert aber die Störaussendung im Bereich hoher Frequenzen massiv. Den EMV-Aspekten kommt deshalb eine immer grössere Bedeutung zu.

\subsubsection{Störwege}

Es gibt 5 Grundtypen von Störwegen, auf denen sich Störungen eines selbstgeführten Stromrichters bzw. eines Umrichters ausbreiten können. Sie sind in Bild 18.2 schematisch dargestellt. Gemäss der Klassierung in Bild 18.3 lassen sie sich in leitungsgebundene und leitungsungebundene (gestrahlte Störungen) Störwege unterteilen.

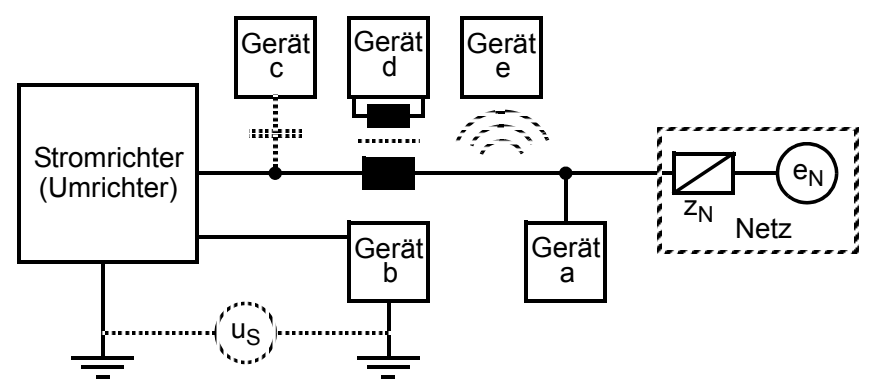

Bild 18.2. Typische Störwege bei einem Stromrichter bzw. Umrichter

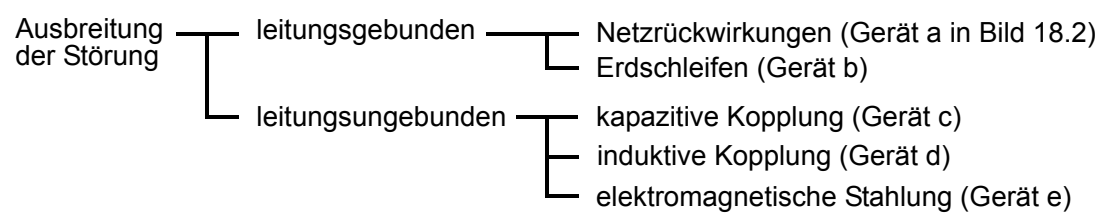

Bild 18.3. Klassierung der Störwege in leitungsgebundene und leitungsungebundene 
Netzrückwirkungen (leitungsgebunden): ins Netz eingespeiste nichtsinusförmige Ströme beeinflussen vor allem die in der Nähe angeschlossenen Geräte. Die Störströme werden zum Teil direkt durch diese Geräte aufgenommen. Der restliche Teil fliesst über die Innenimpedanz des Netzes $z_{N}$ und verursacht dort Spannungsabfälle. Dadurch ergibt sich in der Nähe des Anschlusspunktes eine verzerrte Netzspannung. Weiter können sich höherfrequente Störungen im Netz leicht auf leitungsungebundene Art weiter verbreiten. Netzkabel sowie Verteil- und Übertragungsleitungen wirken als Induktionsschleifen und Antennen.

Erdschleifen (leitungsgebunden): dieser Störweg ergibt sich vor allem zwischen dem Stromrichter und seinen direkt angeschlossenen Geräten, z.B. seiner Steuerung oder Messgeräten. Bei getrennter Erdung kann eine Störspannung zwischen den Erdungspunkten entstehen, welche einen Störstrom in den Verbindungsleitern hervorruft. Erdschleifen übertragen sowohl niederfrequente (z.B. 'Netzbrumm') als auch hochfrequente Störungen.

Kapazitive Kopplung (elektrische Abstrahlung): bei steilen Spannungsflanken im Stromrichter fliessen Verschiebungsströme über die stets vorhandenen Koppelkapazitäten zwischen dem Leistungsteil und seiner Umgebung. Diese Verschiebungsströme werden mit zunehmendem hochfrequenten Anteil in den Störungen grösser. $\mathrm{Zu}$ beachten sind sie in der Regel ab einigen $\mathrm{kHz}$.

Induktive Kopplung (magnetische Abstrahlung): analog zur kapazitiven Kopplung induzieren steile Stromflanken Störspannungen in Stromkreisen in der Umgebung, sofern diese eine magnetische Kopplung mit dem verursachenden Stromkreis im Stromrichter aufweisen. Auch dieses Phänomen ist ab einigen $\mathrm{kHz}$ zunehmend zu beachten.

Elektromagnetische Strahlung: eine Abstrahlung von Störungen über elektromagnetische Wechselfelder findet statt, wenn die Abmessungen der Stromkreise in der Grössenordnung der entsprechenden Wellenlänge liegt. Für das Mass der Abstrahlung in den verschiedenen Frequenzbereichen ist deshalb vor allem auch wichtig, in welcher Weise sich die Störungen in die Netz- und Lastkreise ausbreiten, da diese Teile der Schaltung wie schon erwähnt als Antennen wirken. Im allgemeinen tritt die elektromagnetische Störstrahlung erst im MHz-Bereich in Erscheinung.

\subsubsection{Massnahmen zur elektromagnetischen Verträglichkeit}

Die Theorie über Massnahmen zur Verbesserung der EMV ist sehr komplex und vielschichtig. Es ist nicht der Zweck dieses Buches, näher darauf einzugehen. Dazu sei auf die entsprechende Fachliteratur verwiesen, die am Ende dieses Kapitels aufgeführt ist. Im Sinne einer Abrundung der bisherigen Ausführungen werden in Tabelle 18.1 aber stichwortartig einige Massnahmen erwähnt. Sie sind unterteilt in Massnahmen zur Reduktion der Störspannungen und -ströme (Ursachenbekämpfung) und solche zur Verhinderung der Ausbreitung (Symptombekämpfung). 
Tabelle 18.1. Massnahmen zur Verbesserung der EMV bei selbstgeführten Stromrichtern bzw. Umrichtern

\begin{tabular}{|c|c|c|}
\hline Störweg & Reduktion der Störquelle & Verhinderung der Ausbreitung \\
\hline $\begin{array}{l}\text { Netzrück- } \\
\text { wirkungen }\end{array}$ & $\begin{array}{l}\text { - Einsatz von geeigneten Steuerverfah- } \\
\text { ren }\end{array}$ & $\begin{array}{l}\text { - kleine Netzimpedanz } \\
\text { - Filter zur Reduktion der Verzerrungen } \\
\text { in den Netzströmen: Niederfrequenzfil- } \\
\text { ter (Kapitel 3.2.6), Saugkreise, Stör- } \\
\text { schutzfilter }\end{array}$ \\
\hline Erdschleifen & - gemeinsame sternförmige Erdung & - potentialgetrennte Signalübertragung \\
\hline $\begin{array}{l}\text { kapazitive } \\
\text { Kopplung }\end{array}$ & $\begin{array}{l}\text { - Spannungsflanken weniger steil (führt } \\
\text { zu erhöhten Schaltverlusten) }\end{array}$ & $\begin{array}{l}\text { - kompakter Schaltungsaufbau } \\
\text { - Abschirmung gegen elektrisches Feld } \\
\text { (metallischer Schirm) } \\
\text { - Abstand zu gefährdeten Geräten }\end{array}$ \\
\hline $\begin{array}{l}\text { induktive } \\
\text { Kopplung }\end{array}$ & $\begin{array}{l}\text { - Stromflanken weniger steil (führt zu } \\
\text { erhöhten Schaltverlusten) }\end{array}$ & $\begin{array}{l}\text { - induktionsarmer Schaltungsaufbau, } \\
\text { kurze Leiter, kleine Schleifen } \\
\text { - Abschirmung gegen magnetisches Feld } \\
\text { (magnetischer Schirm) } \\
\text { - Abstand zu gefährdeten Geräten }\end{array}$ \\
\hline $\begin{array}{l}\text { elektromag. } \\
\text { Strahlung }\end{array}$ & $\begin{array}{l}\text { - Spannungs- und Stromflanken weniger } \\
\text { steil (führt zu erhöhten Schaltverlusten) }\end{array}$ & $\begin{array}{l}\text { - kompakter Schaltungsaufbau } \\
\text { - Abschirmung } \\
\text { - Abstand zu gefährdeten Geräten } \\
\text { - Störschutzfilter auf Netz- und evtl. } \\
\text { auch auf Lastseite }\end{array}$ \\
\hline
\end{tabular}

\subsection{Normen und Vorschriften}

Die Normierung im Bereich der EMV hat eine grosse Bedeutung. Sie basiert darauf, dass in einer vorgegebenen Umgebung bestimmte, maximal zu erwartende Störpegel, sogenannte Verträglichkeitspegel, definiert werden. Grenzwerte für die Emission von Störungen sollen dann sicherstellen, dass die Summe der Störungen aller in dieser Umgebung eingesetzten Geräte und Anlagen die Verträglichkeitspegel nicht überschreiten. Andererseits sollen genormte Prüfverfahren für die Immunität gegen Störungen dafür sorgen, dass alle Geräte den Verträglichkeitspegeln standhalten können. Ein weiterer wichtiger Gegenstand der Normierung ist die Festlegung von Mess- und Prüfverfahren sowohl im Bereich der Immunität als auch der Emission.

Die rasch wachsende Anzahl verschiedenartiger Störquellen in allen Bereichen der Elektrotechnik, insbesondere auch in der Leistungselektronik, schafft ständig Bedarf nach Anpassung und Erweiterung der bestehenden Normen. Auf internationaler Ebene wird zur Zeit (d.h. in den neunziger Jahren) eine vollständige Neugestaltung des EMV-Normenwerks vorgenommen. Ein grosser Teil der vorgesehenen Normen, die unter den Sammelnummern IEC 1000-x bzw. EN 61000-x erscheinen, ist jedoch erst in Vorbereitung. Zukünftige Normen bzw. ihre Unternormen sollen dabei eindeutig einer der folgenden 3 Kategorien zugeordnet werden können: 
Basisnormen ('basic standards') beschreiben in erster Linie die relevanten EMV-Phänomene sowie die Messverfahren und -geräte zur Bestimmung der Emission von Störungen und der Immunität gegen Störungen. Im weiteren definieren sie Umgebungen und legen die zugehörigen Verträglichkeitspegel fest. Sie enthalten aber keine Grenzwerte.

Grundnormen ('generic standards') beziehen sich auf eine bestimmte Anwendungsumgebung und legen dafür eine auf das Notwendigste reduzierte Anzahl Grenzwerte betreffend Emission und Immunität fest. Für die entsprechenden Messverfahren beziehen sie sich auf die Basisnormen. Grundnormen gelten dort, wo keine spezifischen Produktnormen existieren. Zusätzlich stellen sie das Koordinationswerkzeug für die Schaffung von Produktenormen dar.

Produktenormen ('product familiy standards') legen für bestimmte Produkte bzw. Produktegruppen spezifische Grenzwerte betreffend Emission und Immunität fest. Sie greifen in der Regel auf die Messverfahren aus den Basisnormen zurück. Produktenormen sollten gut koordiniert sein mit den Grundnormen. Sie haben gegenüber allen anderen Normen Vorrang.

Die meisten der älteren, aber zur Zeit noch gültigen Normen auf internationaler und nationaler Ebene lassen sich allerdings noch nicht eindeutig in eine dieser Kategorien einteilen. Sie haben einen gemischten Charakter, indem sie sowohl Verträglichkeitspegel, als auch Messverfahren und Grenzwerte enthalten.

Tabelle 18.2 gibt eine Übersicht über die wichtigsten existierenden und geplanten EMV-Normen, welche selbstgeführte Stromrichter und ihre Steuerelektronik betreffen. Es wird dabei angegeben, welcher der erwähnten Kategorien sie angehören und welche Untergebiete der EMV (Emission oder Immunität bzw. leitungsgebundene oder gestrahlte Störungen) sie behandeln. Aufgeführt sind Normen der internationalen Institutionen IEC ('International Electrotechnical Comission'), CENELEC ('Comité Européen de Normalisation Electrotechnique') und CISPR ('Comité International Spécial des Perturbations Radioélectriques') sowie des deutschen VDE ('Verein deutscher Elektrotechniker') und des schweizerischen SEV ('Schweizerischer Elektrotechnischer Verein'). Die meisten von ihnen sind Sammelwerke, die aus mehreren Einzelnormen bestehen. Nur ein Teil davon ist jeweils im Zusammenhang mit Stromrichtern von Bedeutung. Die Normen verschiedener Institutionen in derselben Zeile der Tabelle stimmen zumindest teilweise überein.

Im Hinblick auf die Steuerverfahren für selbstgeführte Stromrichter sind vor allem die Normen zur Emission von leitungsgebundenen Störungen im niedrigen Frequenzbereich wichtig. Diese sind praktisch ausschliesslich durch die Charakteristik der verwendeten Pulsmuster bestimmt und können deshalb durch das Steuerverfahren stark beeinflusst werden. Die obere Grenze des betreffenden Frequenzbereiches liegt je nach Verfahren und Schaltfrequenz zwischen 1 und 100kHz. Die entsprechenden Normen sind gegenwärtig noch sehr unvollständig. Verbindliche, wenn auch recht hohe Grenzwerte für Netzrückwirkungen existieren nur in der EN 60555. Sie gelten lediglich für Niederspannungsnetze und Ströme $<16$ A, d.h. für Stromrichter kleiner Leistung (jedoch über 200W). Eine Erweiterung auf grössere Ströme steht allerdings unmittelbar bevor. Stromrichter, die direkt an ein Mittelspannungsnetz angeschlossen sind, werden dagegen nur von der Schwei- 





zer Norm SEV3600 erfasst. Ein weiterer Mangel der Grenzwerte nach EN 60555 ist ihre Beschränkung auf den Frequenzbereich 0 bis $2 \mathrm{kHz}$ (bis zur 40. Harmonischen). Darüber existieren nur die Funkstör-Grenzwerte aus den EN 55011 bzw. 55014, die aber erst oberhalb von $9 \mathrm{kHz}$ gelten. Der Zwischenbereich von 2 bis $9 \mathrm{kHz}$, in dem die dominanten Verzerrungsanteile vieler selbstgeführter Stromrichter liegen, ist zur Zeit überhaupt nicht abgedeckt. Es ist jedoch geplant, in Zukunft diese Lücke in der Normierung zu schliessen und gleichzeitig die bestehenden Grenzwerte zu verschärfen. Zusätzlich gilt auch ausserhalb der Normen der Grundsatz, dass die Funktion anderer Geräte nicht gestört werden darf. In diesem Sinn ist auch die EN 50065 zu beachten, welche die Signalpegel für die Informationsübertragung in Netzen festlegt. Solche Anlagen dürfen nicht gestört werden.

Die bisherige relativ grosse Freiheit bezüglich Netzrückwirkungen erlaubte es, in Umrichtern und anderen Schaltungen mit Gleichrichtung der Netzspannung ungesteuerte Gleichrichter oder netzgeführte Thyristorschaltungen einzusetzen (Kapitel 2). Diese kostengünstigen Lösungen werden mit der kommenden Verschärfung und Erweiterung der bestehenden Grenzwerte vermehrt durch selbstgeführte Schaltungen ersetzt werden müssen.

Literatur zu Kapitel 18: [DIN1], [DIN2], [Lap2], [Schw1], [Wilh1] 


\section{Implementierung von Modulatoren}

\subsection{Schaltungen}

Für die meisten Steuerverfahren gibt es eine ganze Reihe von Möglichkeiten zur Implementierung. Aus diesem Grund ist die Vielfalt von Modulator-Schaltungen ausserordentlich gross. Die folgenden Beispiele zeigen häufig verwendete Grundstrukturen, ohne dabei ins Detail zu gehen. Die vorgestellten Schaltungen werden in zahlreichen Varianten eingesetzt.

Für die Beschreibung der implementierten Verfahren und die Definition der verwendeten Begriffe wird auf die entsprechenden Kapitel verwiesen.

\subsubsection{Analoge Schaltungen}

Die klassischen Steuerverfahren für selbstgeführte Stromrichter, d.h. die Grundfrequenzsteuerung (Kapitel 6), die Trägerverfahren (Kapitel 7) und die Phasenstromregler (Kapitel 10), lassen sich alle auf sehr einfache Weise in analoger Schaltungstechnik realisieren. Bild 19.1 zeigt die Schaltungen für die Steuerung eines Brückenzweiges. Sie bestehen alle im wesentlichen aus einem Komparator, der die Schaltsignale erzeugt. Dies geschieht in den 3 Modulatoren wie folgt:


Bild 19.1. Grundausführungen für die analoge Steuerung eines Brückenzweiges, links: Grundfrequenzsteuerung, mitte: Trägerverfahren, rechts: Phasenstromregler

- Bei der Grundfrequenzsteuerung bestimmt das Eingangssignal $u_{\text {Ein }}$ die Grundfrequenz $f_{1}$. Bei jedem Nulldurchgang des Signals wird eine Schaltflanke ausgelöst.

- Der Modulator mit Trägerverfahren enthält intern einen Generator für das Trägersignal $x_{T}$. Dieses wird zusammen mit dem Eingangssignal, das dem Sollwert $x_{\text {Soll }}$ entspricht, auf den Komparator geführt. Die Schnittpunkte der beiden Signale ergeben die Schaltzeitpunkte. 
- Beim Phasenstromregler ist $u_{\text {Ein }}$ proportional zum Sollwert des Phasenstromes. Zusätzlich ist ein zweites Eingangssignal $u_{I s t}$ vorhanden, das dem Stromistwert entspricht. Beide Signale werden direkt auf den Komparator geführt. Dieser weist im Gegensatz zu den beiden anderen Modulatoren eine Hysterese auf. Sie bestimmt die maximale Differenz zwischen Soll- und Istwert und damit die Grösse des Toleranzbandes für den Phasenstrom.

Die dargestellten Modulatoren zeigen die bekannten Probleme analoger Schaltungen: eine beschränkte Genauigkeit wegen der Toleranzen der verwendeten Komponenten, Rauschen, Offset und Temperaturdrift, eine geringe Störfestigkeit sowie wenig Flexibilität bezüglich Parametervariationen (Trägerfrequenz, Toleranzband usw.). Trotz ihrer Einfachheit kommen sie deswegen heute nur noch beschränkt zum Einsatz:

- in 'Low-Cost'-Anwendungen, wo die Digitalisierung der Signale zu aufwendig ist

- in Anwendungen mit sehr hoher Schaltfrequenz, wo die Abtastrate für die Digitalisierung zu gross würde

- in älteren Generationen von Geräten, die zu einer Zeit entwickelt wurden, als die digitalen Steuerungen und Regelungen noch nicht im heutigen Masse eingesetzt wurden.

In zunehmendem Masse werden jedoch heute digitale Schaltungen für die Modulation eingesetzt. Sie sind robuster und bieten mehr Flexibilität. Weil auch die Regelung digital implementiert ist, stehen die Eingangsgrössen des Modulators meistens bereits in digitaler Form zur Verfügung

\subsubsection{Digitale Schaltungen}

Bild 19.2 zeigt eine mögliche digitale Realisierung eines Trägerverfahrens (Kapitel 7). Die Kurvenform des Sollwertes ist in Form von Stützwerten in einem PROM abgespeichert. Durch Anlegen eines Zählers an den Adresseingang werden diese Stützwerte zyklisch ausgelesen. Die Grundfrequenz $f_{1}$ wird über die Clockfrequenz des Adresszählers (am Clockeingang C) eingestellt. Sie beträgt $k_{1} f_{1}$, wobei $k_{1}$ die Anzahl Stützwerte pro Periode bezeichnet. Die Multiplikation der Stützwerte mit dem Modulationsgrad $M$ in einem Hardware-Multiplizierer ergibt schliesslich den digitalisierten Sollwert. Das Trägersignal wird durch einen Auf/Ab-Zähler (bzw. Auf- oder Ab-Zähler für sägezahnförmige Träger) erzeugt. Die Clockfrequenz dieses Zählers beträgt $2 k_{T} f_{T}$, wobei $k_{T}$ der Stufenzahl des Trägerdreiecks und $f_{T}$ der Trägerfrequenz entsprechen. Sie ist deutlich grösser als die Clockfrequenz des Adresszählers. Die Wortbreite der digitalisierten Signale $x_{S o l l}$ und $x_{T}$ ist



Bild 19.2. Digitale Implementierung eines Trägerverfahrens, links: Blockdiagramm, rechts: Prinzip der Modulation durch Zeitfunktionen dargestellt 
durch die Stufenzahl gegeben und für beide gleich. Diese Signale werden auf einen digitalen Komparator geführt, der die Schaltfunktion $s$ als Digitalsignal erzeugt.

Die beiden Clocksignale sind im allgemeinen nicht aufeinander synchronisiert. Für eine einwandfreie Funktion der Schaltung empfiehlt es sich deshalb, den langsameren Clock am Eingang mit Hilfe eines Synchronisations-Flipflops (mit Eingang D und Ausgang Q) auf den schnelleren zu synchronisieren.

Die dargestellte Schaltung ist wesentlich robuster als die entsprechende analoge. Sie erlaubt zudem mit Hilfe der abgespeicherten Stützwerte beliebige periodische Sollwertverläufe zu implementieren. Dies ist für dreiphasige Modulatoren von Bedeutung, da dort den Sollwerten mit Vorteil spezielle Gleichtaktkomponenten überlagert werden (Kapitel 7.4.2). Die zeitliche Auflösung der Schaltflanken ist auf eine Clockperiode des Trägersignals begrenzt. Diese wird klein, wenn eine grosse Wortbreite für die digitalisierten Signale gewählt wird. Dabei wirkt jedoch der Multiplizierer, der in diesem Zeitintervall eine Multiplikation durchführen muss, als begrenzender Faktor.

Die Verfälschung der Pulsmuster, die sich durch die Quantisierung der Signale im Modulator ergibt, wurde in Kapitel 17.1.1 betrachtet.

\subsubsection{Modulatoren mit logischen Schaltwerken}

Ein gutes Hilfsmittel für die Implementierung von Modulatoren sind logische Schaltwerke (auch: sequentielle Logikschaltungen). Wie die beiden folgenden Beispiele zeigen, lassen sich mit ihnen leicht Ablaufsteuerungen, z.B. für Schaltsequenzen oder für die Kontrolle von Einschaltzeiten, realisieren. Es kommt hinzu, dass logische Schaltwerke sehr einfach in speziellen programmierbaren Bausteinen untergebracht werden können.

Drehzeigermodulator: Bild 19.3 links zeigt das Blockdiagramm für einen Drehzeigermodulator (Kapitel 8). Am Eingang liegen der Modulationsgrad $M$ und der aktuelle Phasenwinkel $\varepsilon$ des Sollwertzeigers $\underline{u}_{S o l l}$ an. In einer Sektorlogik wird zuerst der Sektor $S 1 \ldots 6$
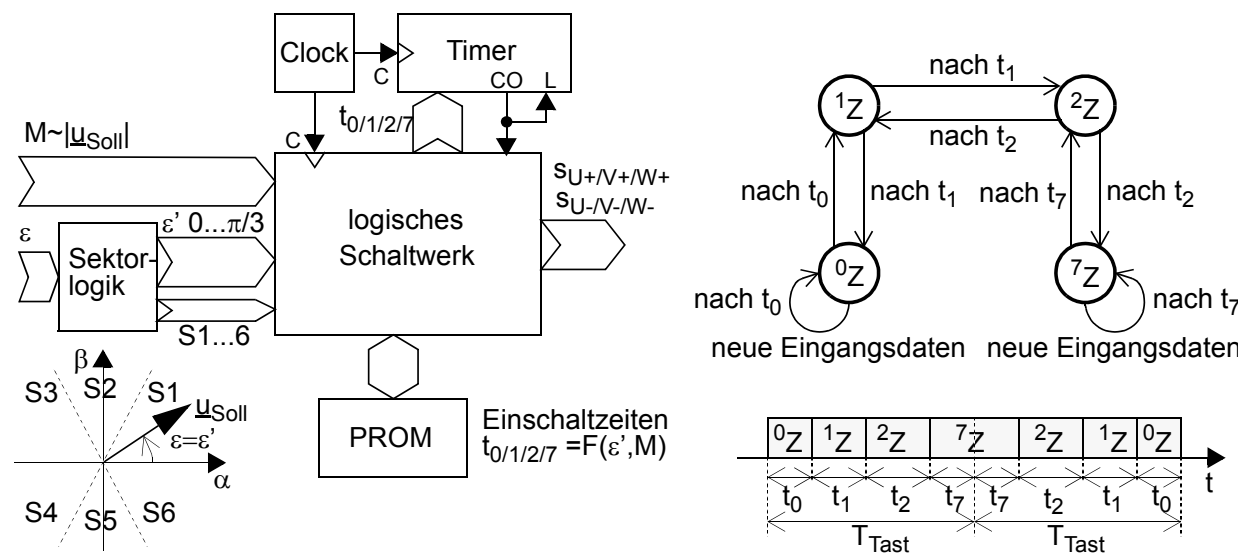

Bild 19.3. Drehzeigermodulator mit logischem Schaltwerk, links: Blockdiagramm, rechts: Zustandsdiagramm und Schaltsequenz für den Sektor $S 1$ 
des Sollwertzeigers und der Restwinkel $\varepsilon$ ' innerhalb dieses Sektors bestimmt. Der Sektor legt die Schaltsequenz, d.h. die Abfolge der Schaltzustände fest. Im Beispiel im Bild rechts für den Sektor $S 1$ sind dies die Zustände ${ }^{0} Z,{ }^{1} Z,{ }^{2} Z,{ }^{7} Z$ und in umgekehrter Reihenfolge zurück auf ${ }^{0} Z$. Die Parameter $M$ und $\varepsilon$ ' ergeben die zugehörigen Einschaltzeiten $t_{0 / 1 / 2 / 7}$. Diese sind in einem PROM tabellarisch abgelegt. Das Schaltwerk liest sie in der richtigen Reihenfolge aus und lädt sie einzeln in einen Timer. Jeweils nach Ablauf einer Einschaltzeit sendet dieser ein Carry-Output-Signal (CO) an das Schaltwerk zurück. Darauf werden die 6 Schaltsignale am Ausgang des Modulators entsprechend dem nächsten Zustand ${ }^{0 \ldots .7} Z$ umgeschaltet. Der Carry-Output startet über den Load-Eingang (L) auch gleich den Timer wieder, bei dem in der Zwischenzeit die nächste Einschaltzeit programmiert worden ist. Die Summe der gespeicherten Schaltzeiten $t_{0}$ bis $t_{7}$ bzw. $t_{7}$ bis $t_{0}$ ist stets gleich gross und entspricht dem Abtastintervall $T_{\text {Tast }}$ des Modulators. In jedem Nullzustand $\left({ }^{0} Z\right.$ bzw. $\left.{ }^{7} Z\right)$ werden die Eingangsdaten aktualisiert.

Die zeitliche Auflösung der Schaltflanken ist primär durch den Clock des Timers bestimmt. Es ist jedoch zu beachten, dass die tabellarisch abgelegten Einschaltzeiten auf quantisierten Werten für den Modulationsgrad und den Phasenwinkel des Sollwertzeigers beruhen. Diese Quantisierung sollte mit der Clockfrequenz und mit der Wortbreite für die Einschaltzeiten abgestimmt sein.

Drehzeigerorientierte Stromregelung: Auch das in Kapitel 11.2 vorgestellte Verfahren kann mit einem Schaltwerk realisiert werden. Wie in Bild 19.4 dargestellt, weist die einfachste Ausführung des Modulators allerdings einen analogen Eingangsteil auf: der Dreiund der Vierstufenkomparator können am einfachsten mit zwei bzw. 3 einfachen Komparatoren mit verschiedenen Hysteresen implementiert werden. Eine digitale Version kommt nur in Frage, wenn die Stromkomponenten für die Regelung schon in digitaler Form vorliegen. Allerdings müssen dann die Abtastfrequenz und die Auflösung genügend gross sein, da die Toleranzbänder des Modulators nur einige Prozent der maximalen Stromamplituden betragen. Die Transformation der Phasenströme in Drehzeiger kann mit

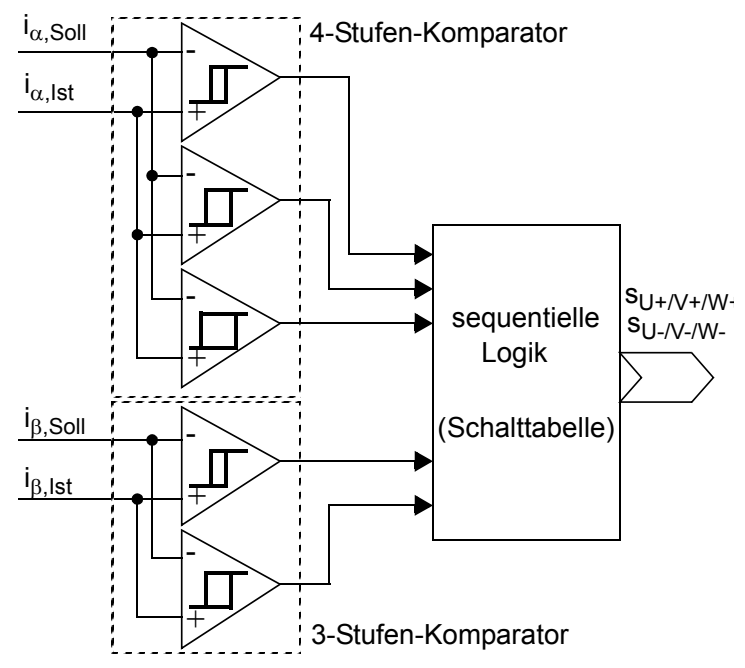

Bild 19.4.

Implementierung der drehzeigerorientierten Stromregelung mit sequentieller Logik 
Hilfe von Operationsverstärkerschaltungen andererseits auch analog aufgebaut werden. Sie besteht nur aus Multiplikationen mit konstanten Koeffizienten und Additionen.

Das Schaltwerk übernimmt die Auswertung der Ausgangssignale von den Komparatoren und stellt die Schaltsignale aufgrund der Schalttabelle nach Bild 11.5 ein.

\subsubsection{Implementierungen auf einem Mikroprozessor}

Die Verwendung eines Rechners (Mikroprozessor oder Signalprozessor) als zentrale Einheit des Modulators schafft ein Maximum an Flexibilität. Es wird möglich, verschiedene Steuerverfahren bzw. verschiedene Varianten eines Verfahrens mit derselben Hardware zu implementieren.

Drehzeigermodulator: Bild 19.5 zeigt das Blockdiagramm der Hardware und die Flussdiagramme der zwei Interruptroutinen für einen Drehzeigermodulator (Kapitel 8). Die Hardware besteht aus dem Mikroprozessor, einem Timer, einem Schreib-/Lesespeicher (RAM) und einer speziellen Ausgabeeinheit. Diese übernimmt die Steuerung der Modulatorausgänge, welche mit möglichst grosser zeitlicher Präzision erfolgen soll. Die nächste Schaltzeit und der zugehörige Schaltzustand werden in den Registern (Reg.) der Ausgabeeinheit programmiert. Diese vergleicht die Schaltzeit fortlaufend mit dem Timerstand. Bei Gleichheit der Zeiten wird der Schaltzustand bzw. die 6 Schaltsignale für die Brückenzweige an den Ausgang durchgeschaltet. Eine Rückmeldung der Ausgabeeinheit löst im Prozessor nach jedem Schaltvorgang die Interruptroutine 2 aus, die ihre Register neu lädt.

Ein Timerzyklus entspricht dem Abtastintervall $T_{\text {Tast }}$ der Modulation. Dies wird erreicht, indem der Endwert, bei dem der Timer sein Carry-Output-Signal (CO) ausgibt, auf den entsprechenden Wert programmiert wird. Der Timerstand ist auf diese Weise ein Mass für den Zeitpunkt innerhalb des Abtastintervalles. Der Carry-Output stellt jeweils
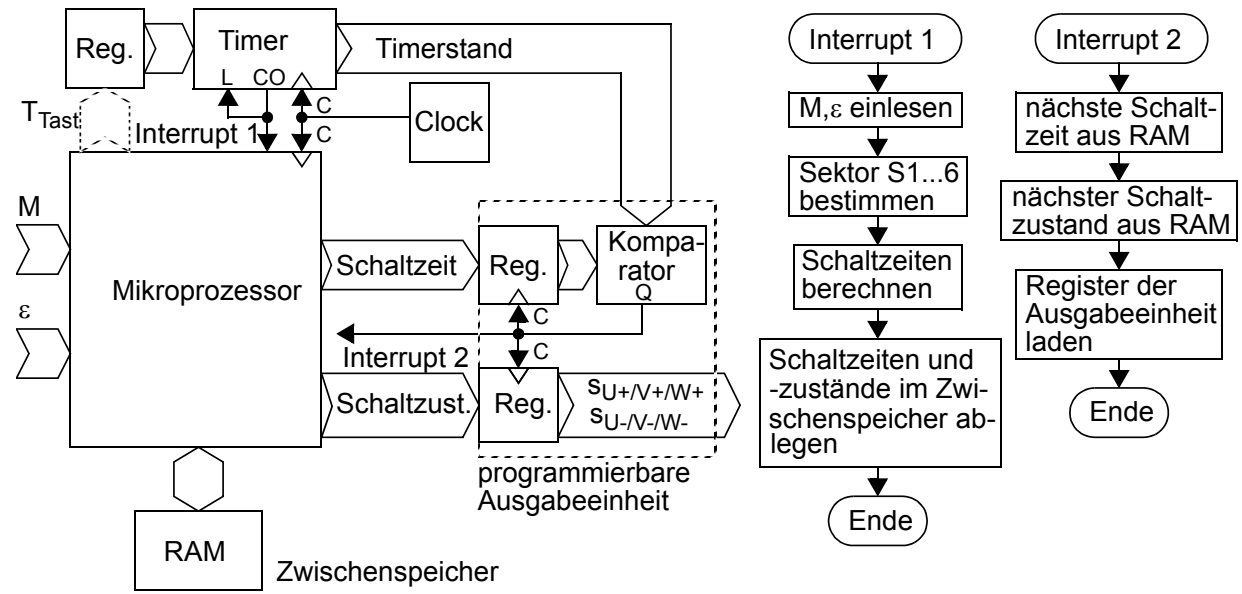

Bild 19.5. Implementierung der Drehzeigermodulation mit einem Mikroprozessor, links: Blockdiagramm, rechts: Flussdiagramme der Interruptroutinen 
gleich wieder das Startsignal für den Timer dar, der damit das nächste Abtastintervall beginnt. Zusätzlich löst er die Interruptroutine 1 aus. Diese liest die Eingangsgrössen ein, bestimmt den Sektor des Sollwertzeigers, legt die Schaltsequenz für das nächste Taktintervall fest und berechnet die zugehörigen Schaltzeiten. Diese Daten werden im RAM abgelegt, so dass die Interruptroutine 2 darauf zurückgreifen kann.

Es ist zu beachten, dass nicht die Einschaltzeiten der einzelnen Zustände programmiert werden, sondern der Timerstand, bei dem das Schaltereignis stattfinden soll. Weiter muss sichergestellt sein, dass die nächsten Daten stets rechtzeitig im Zwischenspeicher bereit liegen. Dies lässt sich zum Beispiel erreichen, indem die Daten jeweils ein Abtastintervall im voraus bearbeitet werden.

Bei der Realisierung eines derartigen Drehzeigermodulators existieren zahlreiche Optionen, von denen hier einige kurz erwähnt werden sollen:

- Der Mikroprozessor kann neben der Modulation weitere Aufgaben übernehmen. Meistens verbleibt zwischen der Abarbeitung der Interruptroutinen genug freie Zeit, um z.B. auch eine einfache Regelung durchzuführen. In diesem Fall sehen die Schnittstellen am Eingang der Schaltung entsprechend anders aus.

- Grundsätzlich können, wie bei der Schaltung nach Bild 19.3, die Schaltzeiten auch aus einer Tabelle entnommen werden. Diese ist dann in einem PROM unterzubringen.

- Das Abtastintervall $T_{\text {Tast }}$ und damit die Schaltfrequenz für den Stromrichter kann durch den Mikroprozessor durch Überschreiben des entsprechenden Registers für den Timer variiert werden.

- Die Ausgabeeinheit kann so ausgeführt sein, dass sie in der Lage ist, mehrere Schaltereignisse zu speichern. Unter Umständen kann dadurch auf die Zwischenspeicherung der Daten im RAM verzichtet werden.

Off-Line-Modulator: In Bild 19.6 sind das Blockdiagramm und das Flussdiagramm für einen Off-Line-Modulator (Kapitel 9) dargestellt. Die Hardware ist sehr ähnlich wie diejenige des Drehzeigermodulators. Wie erwähnt wurde, ist es ohne grossen Aufwand möglich, eine der beiden Schaltungen so zu erweitern, dass beide Steuerverfahren auf ihr implementiert werden könnten.

Beim Off-Line-Modulator dient der Timer als Zeitbasis (Kapitel 9.1). Die Länge der Grundperiode $T_{1}$ (bzw. je nach Symmetrie der gespeicherten Pulsmuster einer Halb- oder Sechstelperiode) wird als Zykluszeit programmiert. Der Timerstand ist ein Mass für den Zeitpunkt innerhalb dieser Periode. Die Schaltwinkel und -zustände sind als Funktion des Modulationsgrades in einer Tabelle (PROM) gespeichert. Der Prozessor wählt aus der Tabelle die richtigen Daten aus und stellt sie in dem als Zwischenspeicher dienenden RAM bereit. Ihre Programmierung in die Ausgabeeinheit erfolgt durch eine gleiche Interruptroutine wie beim Drehzeigermodulator (Bild 19.5).

Die Aufbereitung der Pulsmuster ist relativ aufwendig. Da zudem die einzelnen Schaltereignisse sehr unterschiedliche zeitliche Abstände aufweisen können, ergibt sich zeitweise eine grosse Auslastung der Rechenkapazität. In der Regel wird deshalb bei OffLine-Modulatoren der Prozessor allein für die Modulation eingesetzt. Die Aufbereitung des Pulsmusters stellt das Hauptprogramm dar. Es beginnt mit dem Einlesen der Eingangsgrössen. Falls sich die Grundfrequenz geändert hat, wird anschliessend der Start- 


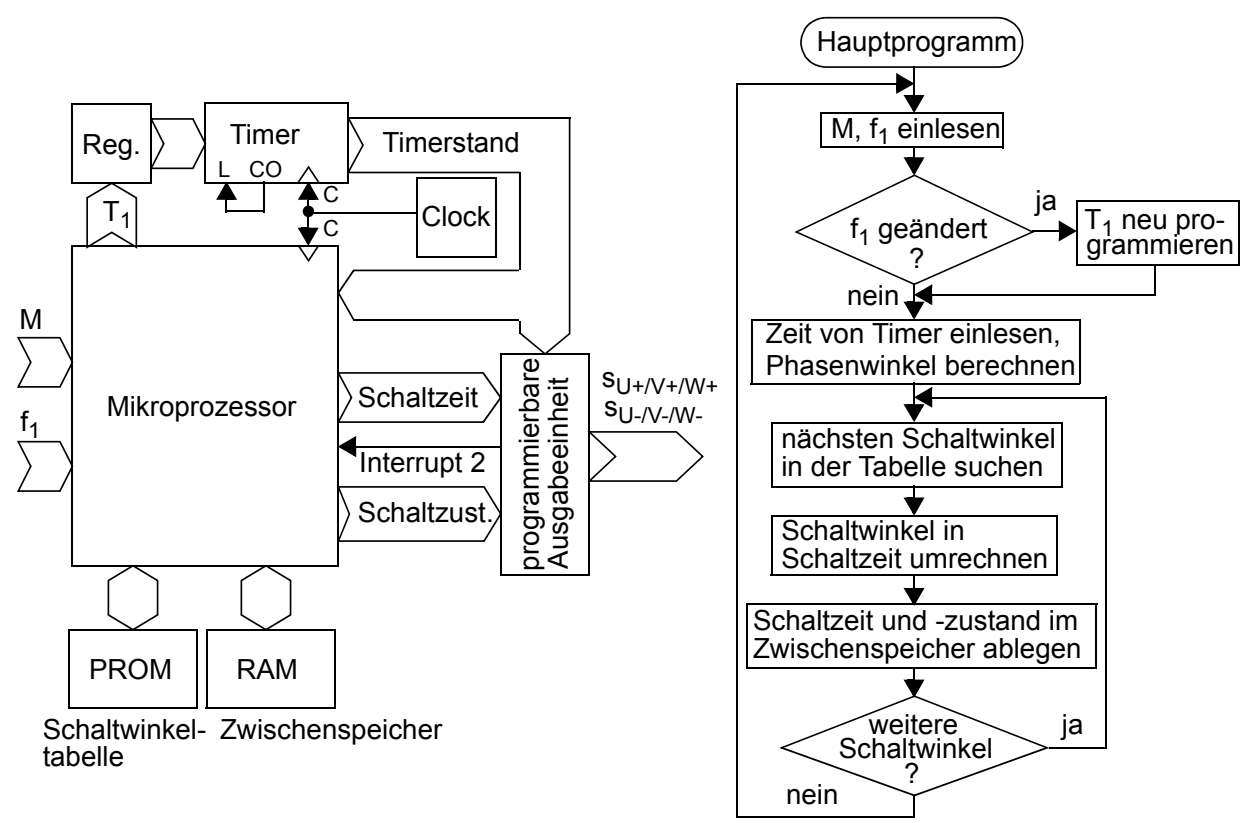

Bild 19.6. Implementierung eines Off-Line-Modulators mit Mikroprozessor, links: Blockdiagramm, rechts: Flussdiagramm des Hauptprogrammes

wert der Zeitbasis neu programmiert. Dann wird in der Tabelle der nächste Schaltwinkel gesucht und zusammen mit dem zugehörigen Schaltereignis im RAM abgelegt. Bei diesen Operationen ist zu beachten, dass der Timerstand einer Zeit entspricht, während die abgelegten Daten als Winkel relativ zur Grundperiode zur Verfügung stehen. Es sind deshalb Umrechnungen mit $T_{1}$ als Parameter vorzunehmen. Am Ende der Routine kann gewählt werden, ob weitere Schaltwinkel desselben Pulsmusters programmiert werden sollen oder ob zum Start zurückgesprungen wird, um die Eingangsdaten zu aktualisieren.

Der erwähnte, relativ grosse Aufwand ergibt sich bei der Realisierung der einzelnen Blöcke des Flussdiagrammes: zum einen ist bei allen Operationen mit Zeiten und Winkeln zu berücksichtigen, dass der Timer periodisch auf seinen Startwert zurückschaltet. Es sind deshalb stets Übertragsberechnungen vorzunehmen. Zum anderen muss stets garantiert sein, dass die Daten rechtzeitig im Zwischenspeicher abgelegt werden. Die Schaltzeit darf zum Zeitpunkt ihrer Programmierung auf keinen Fall schon vorbei sein. Sonst würde das Schaltereignis erst eine Periode später ausgelöst und das Pulsmuster würde unzulässig verfälscht. Vor allem bei dynamischen Vorgängen ist dies speziell zu beachten (Kapitel 9.3.5). 


\subsection{Integrierte Bausteine}

Der allgemeinen Trend bei der Implementierung von Modulatoren geht in Richtung Modularisierung und Integration. Es werden mehr und mehr spezielle Bausteine entwickelt und eingesetzt, welche die gesamte Funktion von Modulatoren oder zumindest Teile davon erfüllen. Neben der Verringerung des Schaltungsaufwandes wird dadurch die Zuverlässigkeit und die Störfestigkeit der Schaltungen entscheidend erhöht. Die folgende Übersicht über verschiedene Bausteinkategorien enthält ganz bewusst keine Beispiele. Die rasche Entwicklung auf diesem Gebiet bewirkt einen schnellen Wechsel im Angebot.

Die überwiegende Zahl von Bausteinen unterstützt spannnungssteuernde Verfahren, insbesondere Trägerverfahren bzw. Drehzeigermodulationen. Der Grund dafür ist die grosse Verbreitung dieser Verfahren, welche eine Produktion in grosser Stückzahl ermöglicht. Die Bausteine lassen sich grob in die folgenden Kategorien einteilen:

- Analoge PWM-Bausteine (mit Trägerverfahren) existieren seit langem in grosser Zahl. Ihr Anwendungsgebiet liegt in erster Linie bei Schaltnetzteilen. In Umrichtern finden sie höchstens Anwendung, wenn die ganze Regelung analog realisiert ist. In einzelnen Fällen ist die PWM-Funktion direkt auf speziellen Ansteuerbausteinen für Leistungshalbleiter oder Brückenzweigen integriert.

- ASICs (Application Specific Integrated Circuits) sind in der Regel Peripheriebausteine für Mikroprozessoren, die für spezifische Aufgaben ausgelegt sind. Ein Modulator oder Teilfunktionen davon stellen derartige Blöcke dar. Die Realisierung als ASIC stellt in Anbetracht der heute zumeist digitalen Regelungen den eindeutigen Trend der Entwicklung dar. Die Bausteine sind so ausgelegt, dass sie sich über die externe Beschaltung oder durch eine spezifische Programmierung auf bestimmte Anwendungen anpassen lassen. ASICs können in analoger und digitaler Schaltungstechnik ausgeführt sein, sowie auch ein Rechenwerk umfassen. Es sind deshalb im Prinzip alle in Kapitel 19.1 vorgestellten Schaltungen als ASIC realisierbar.

- Microcontroller eignen sich ausgezeichnet für die Realisierung von rechnerbasierten Modulatoren nach Kapitel 19.1.4. Sie haben neben dem eigentlichen Rechenwerk auch die benötigte Peripherie, d.h. Speicher, Timer, Schnittstellen etc. auf dem Baustein mitintegriert. Es existieren auch Microcontroller mit einer Ausgabeeinheit gemäss Bild 19.5 .

- Für Anwendungen mit hohen Stückzahlen, bzw. für universell einsetzbare Steuer- und Regelsysteme werden vereinzelt auch integrierte Steuer- und Regelbausteine ('OneChip-Controller') angeboten. Sie basieren auf einem Mikroprozessorsystem mit einem oder zwei Prozessoren sowie der notwendigen Peripherie und übernehmen die Regelung des Stromrichters sowie die Modulation. 


\subsection{Praktische Probleme}

In Ergänzung zu den bisher betrachteten Realisierungen von prinzipiellen Funktionen von Modulatoren werden im folgenden einige ausgewählte praktische Probleme kurz beleuchtet.

Pulsverriegelung: Bereits in Kapitel 17.2 wurde erläutert, dass bei der Ausführung der Schaltflanken im Stromrichter eine Verzögerungszeit sowie eine endliche Flankensteilheit auftritt. Deshalb dürfen bei der Umschaltung der Brückenzweige eines U-Stromrichters die beiden Halbleiter nicht mit exakt entgegengesetzten Signalen gesteuert werden. Da in der Regel die Abschaltverzugszeit grösser ist als die Einschaltverzugszeit, würde dabei der Zweig kurzzeitig kurzgeschlossen. Ein sehr schnell ansteigender Kurzschlussstrom über beide Schalter wäre die Folge. Um diesen Fall mit Sicherheit ausschliessen zu können, müssen die Steuersignale, wie in Bild 19.7 dargestellt, nachbearbeitet werden: jeweils die Einschaltflanke wird um eine gegebene Totzeit $T_{\text {tot }}$ verzögert. Die Schaltverzögerungen sind unter anderem abhängig von den Schalterströmen, d.h. variabel. Der Verlauf der Mittelpunktspannung $u_{A 0}$ am Stromrichter im Bereich des Umschaltens ist deshalb nicht immer gleich, was im Bild durch die eingefärbte Fläche angedeutet ist. Die Zeit $T_{\text {tot }}$ ist so zu wählen, dass auch im schlechtesten Fall kein Kurzschluss auftreten kann.

Das Einfügen der Totzeit bewirkt eine kleine Phasenverschiebung von $u_{A 0}$ gegenüber der Schaltfunktion $s_{A}$. Diese kann normalerweise durch die übergeordnete Regelung kompensiert werden. Problematischer ist dagegen die zusätzliche Verzerrung der Ausgangsspannung, welche durch die nichtidealen und in Abhängigkeit des Schalterstromes verzögerten Schaltflanken entsteht. Sie wurde in Kapitel 17.2 behandelt.


Bild 19.7. Verriegelung der Steuersignale für einen Brückenzweig, links: Blockschaltbild, rechts: Signalverläufe

Auch beim I-Stromrichter müssen die Steuersignale für die einzelnen Schalter individuell korrigiert werden. Es muss sichergestellt sein, dass der Zwischenkreisstrom beim Umschalten zwischen zwei Brückenzweigen nie unterbrochen wird. Dazu sind die Schaltpulse für den eingeschalteten Zustand der beteiligten Schalter leicht zu überlappen. 
Einhalten von minimalen Pulsweiten: Im Kapitel 3.1 wurde erklärt, dass aus Gründen der auftretenden Verlustspitzen zwischen zwei Schalthandlungen eines Halbleiters eine minimale Zeit liegen muss. Diese minimale Pulsweite $T_{\text {min }}$ gilt für positive und negative Pulse. Sie kann bei den meisten Steuerverfahren bereits bei der Bildung des Pulsmusters berücksichtigt werden:

- Bei Trägerverfahren treten die kürzesten Pulse auf, wenn der Sollwert seinen Spitzenwert durchläuft. Die Beschränkung der Sollwertamplitude auf einen Wert, der kleiner ist als die Trägeramplitude, garantiert die Einhaltung einer minimalen Pulsweite. Diese ist dann wie folgt bestimmt:

$$
T_{\text {min }}=T_{T} \frac{\hat{x}_{T}-\hat{x}_{\text {Soll, } \text { max }}}{\hat{x}_{T}}
$$

Diese Einschränkung des Aussteuerbereichs ist allerdings in der Regel unerwünscht. An ihrer Stelle wird deshalb meistens die Methode angewandt, die in Kapitel 17.1.2 vorgestellt wurde: im Bereich grosser Aussteuerung, wo zu kurze Pulse entstehen würden, wird der Sollwert der Modulation gemäss Bild 17.6 modifiziert, so dass nur erlaubte Pulse oder gar keine entstehen. Dadurch bleibt der Aussteuerbereich unverändert.

- Bei Off-Line-Modulatoren ist der kürzeste Puls durch das kleinste auftretende Winkelintervall $\Delta \alpha_{\text {min }}$ in den abgelegten Pulsmustern und die zugehörige Grundkreisfrequenz $\omega_{1}$ bestimmt:

$$
T_{\text {min }}=\frac{\Delta \alpha_{\text {min }}}{\omega_{1}}
$$

Bei der Berechnung der Pulsmuster, z.B. einer Optimierung, kann die minimale Pulsweite als Randbedingung mit einbezogen werden.

- In direkten Stromreglern treten die kürzesten Pulse auf, wenn die maximale Spannungsdifferenz $\hat{u}_{L k \text {,max }}$ über der Lastinduktivität anliegt. Die Zeit zwischen zwei Schaltvorgängen ist dann bestimmt durch diese Spannung, die Lastinduktivität und das Toleranzband. Für die Phasenstromregler gilt:

$$
T_{\text {min }}=\frac{L_{k} I_{\delta}}{\hat{u}_{L k, \text { max }}}
$$

Bei gegebener Schaltung muss das Toleranzband $\mathrm{I}_{\delta}$ so gewählt werden, dass die minimale Pulsweite nicht unterschritten wird.

Die gezeigten Massnahmen zur Einhaltung der minimalen Pulsweite genügen nur für den stationären Betrieb. Dynamisch können vereinzelt oder auch mehrfach trotzdem unzulässig kurze Pulse auftreten. Aus diesem Grund wird in der Regel im Modulator eine nachträgliche Kontrollstufe für die Pulsweite eingesetzt. Bild 19.8 zeigt zwei entsprechende Varianten. Sie basieren darauf, dass die Schaltfunktion über ein Flipflop geführt wird, das mit einem Monoflop über den Enable-Eingang (En) aktiviert bzw. deaktiviert wird. Zu kurze Pulse werden entweder ausgeblendet (Variante im Bild links) oder auf die minimale zulässige Länge $T_{\text {min }}$ erweitert (Variante rechts). Eine Ausblendung reduziert 
die Schaltfrequenz. Deshalb ist diese Methode generell zu bevorzugen. Sie ist aber nur realisierbar, wenn das gesamte Pulsmuster um $T_{\text {min }}$ verzögert weitergegeben wird. Damit ist sie ungeeignet für die direkten Stromregler. Dort ist nur die Pulsverlängerung möglich, die verzögerungsfrei implementiert werden kann.
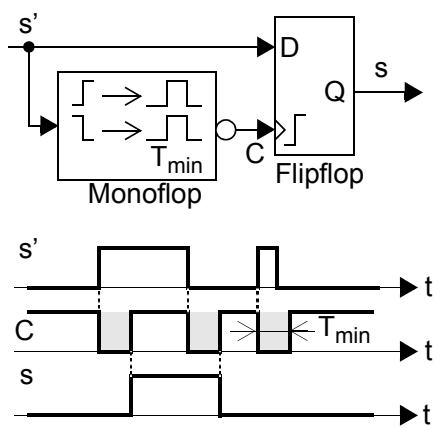
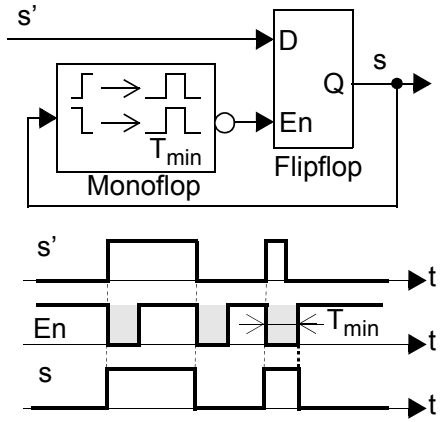

Bild 19.8.

Korrektur von zu kurzen Pulsen,

links: durch Ausblenden,

rechts: durch Verlängern auf die minimale Pulsweite

Beide Varianten bringen im Bereich, in dem sie in die Pulsmuster eingreifen müssen, eine Veränderung der Spektra mit sich. Die Verzerrungsanteile der Schaltfunktionen werden grösser, und zwar vor allem im niederfrequenten Bereich (Kapitel 17). Die Veränderung tritt in erster Linie in Stromrichtern grosser Leistung in Erscheinung, weil dort die

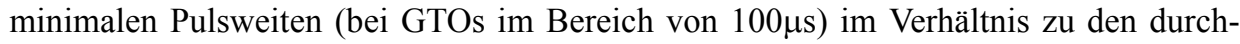
schnittlichen Abständen zwischen den Schaltflanken relativ gross sind.

Häufig ist auch eine gemischte Version der Pulskorrektur realisiert: Pulse, die kürzer sind als $T_{\text {min }} / 2$ werden ausgelassen und solche die grösser sind, werden auf $T_{\text {min }}$ verlängert.

Vermeiden von Mehrfachpulsen: Die im Modulator verwendeten Signale sind immer störungsbehaftet. Dies kann die Funktion beeinträchtigen. Bild 19.9 rechts zeigt am Beispiel einer analogen Trägermodulation, wie durch Störungen, die durch einen Schaltvorgang im Stromrichter eingestreut werden, Mehrfachpulse entstehen. Die Abhilfe dagegen

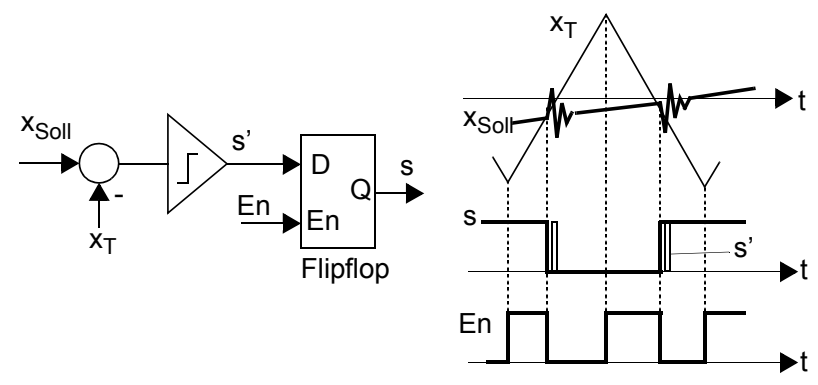

\section{Bild 19.9.}

Unterdrückung der Mehrfachpulse, links: Blockdiagramm, rechts: Signalverläufe

ist im Bild links dargestellt: die Schaltfunktion wird durch ein Flipflop geführt. Dieses wird über seinen Enable-Eingang (En) nach jeder Schaltflanke bis zum Beginn der nächs- 
ten Trägerhalbperiode blockiert. Dadurch werden die Mehrfachpulse vom Stromrichter ferngehalten. Es ist nur eine Schaltflanke pro Trägerhalbperiode möglich. Die Erzeugung des Enable-Signals ist im Bild nicht dargestellt.

In einem klassischen Stromregelkreis drückt der Verzerrungsanteil des Stromistwertes auf den Reglerausgang und damit auf das Eingangsignal des Modulators $x_{\text {Soll }}$ durch. Auch dies kann zu Mehrfachpulsen führen.

Mehrfachpulse sind bei allen Steuerverfahren möglich. Sie lassen sich stets auf ähnliche Weise unterbinden. Häufig genügt es, nach jeder Schaltflanke eine Pulsblockierung für ein festes Zeitintervall vorzunehmen. Dieses Zeitintervall ist so klein zu wählen, dass die normale Funktion des Modulators nicht gestört wird. Es besteht die Möglichkeit, die Überwachungsschaltung für die minimale Pulsweite für diese Aufgabe zu verwenden.

Synchronisation: Bei gewissen Anwendungen, z.B. bei Netzstromrichtern muss die Grundfrequenz des Modulators auf eine externe Frequenz synchronisiert sein. Dies wird in der Regel durch einen Phase-Locked-Loop (PLL) gemäss Bild 19.10 erreicht. Das Signal $x_{E i n}$ ist ein periodisches Signal mit der Grundfrequenz $f_{1}$, z.B. von der Netzspannung abgeleitet. Die Schaltung regelt das rechteckförmige Ausgangssignal $x_{A u s}$ ebenfalls auf diese Frequenz und stellt die Phase zwischen den beiden Signalen auf $\pi / 2$ ein. Zudem entsteht ein hochfrequentes, synchrones Rechtecksignal $x_{C l o c k}$, das z.B. als Clock für den Adresszähler in der Schaltung in Bild 19.2 benützt werden kann. Das Frequenzverhältnis $k$ zwischen der Clock- und der Grundfrequenz ist durch den Frequenzteiler in der Rückführung des PLL einstellbar.

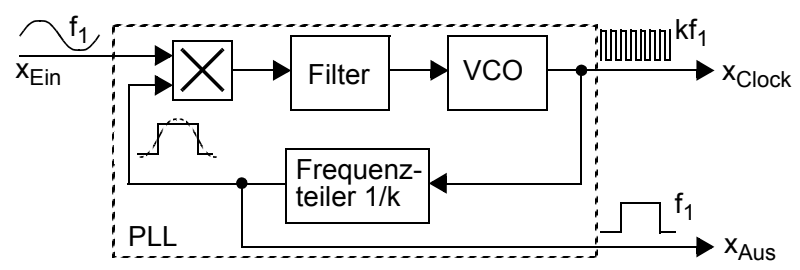

Bild 19.10.

Phase-Locked-Loop (PLL) zur Synchronisation der Grundfrequenz und eines Clocksignals

Ist neben der Synchronisation der Grundfrequenz auch ein synchrones Steuerverfahren verlangt, so müssen alle im Modulator verwendeten Timer mit dem synchronisierten Clock $x_{\text {Clock }}$ versorgt werden.

Ein PLL kann auch zur Implementierung eines Trägerverfahrens mit synchronem Träger verwendet werden. Der Modulatorsollwert $x_{\text {Soll }}$ stellt dann das Eingangssignal dar, so dass mit $x_{\text {Clock }}$ ein synchrones Signal für die Erzeugung des Trägersignals (z.B. mit der Schaltung in Bild 19.2) zur Verfügung steht.

Literatur zu Kapitel 19: [Bos1], [Bow1], [Gilb1], [Mat1], [Schu1], [Tie1], [Zac3] 


\section{Teil V}

\section{Anhänge}

\section{A Literatur}

\section{Bücher}

[Ach1] Achilles D. (1978): Die Fourier-Transformation in der Signalverarbeitung. Springer-Verlag, Berlin Heidelberg

[Bro1] Brosch P.F. (1992): Moderne Stromrichterantriebe. Vogel Verlag (Kamprath-Reihe), Würzburg (2. Auflage)

[Büh1] Bühler H. (1977): Einführung in die Theorie geregelter Drehstromantriebe. Birkhäuser Verlag, Basel und Stuttgart

[Büh2] Bühler H. (1991): Convertisseurs statiques. Presses polytechniques et universitaires romandes, Lausanne

[DIN1] DIN-VDE-Taschenbuch (1991): Elektromagnetische Verträglichkeit 1. VDE-Verlag, Berlin

[DIN2] DIN-VDE-Taschenbuch (1992): Funkentstörung 1. VDE-Verlag, Berlin

[Dir1] Dirkschmid H.J. (1990): Mathematische Grundlagen der Elektrotechnik. Vieweg Verlag, Braunschweig (4. Auflage)

[Fis1] Fischer R. (1986): Elektrische Maschinen. Carl Hanser Verlag, München Wien (6., völlig überarbeitete Auflage)

[Fö11] Föllinger O. (1985): Regelungstechnik. Dr. Alfred Hüthig Verlag, Heidelberg (5., verbesserte Auflage)

[Fö12] Föllinger O. (1982): Laplace- und Fouriertransformation. AEG Telefunken, Berlin und Frankfurt (3., um Aufgaben und Lösungen erweiterte Auflage)

[Gil1] Gill P.E., Murray W. (1974): Numerical Methods for Constrained Optimization. Academic Press Inc., London

[Hag1] Hagmann G. (1993): Leistungselektronik Grundlagen und Anwendungen. AULA-Verlag, Wiesbaden

[Ham1] Hamming R.W. (1973): Numerical Methods for Scientists and Engineers. Mc Graw-Hill Inc. (Second Edition)

[Heu1] Heumann K. (1989): Grundlagen der Leistungselektronik. B.G.Teubner, Stuttgart (4., überarbeitete und erweiterte Auflage) 
[Kle1] Kleinrath H. (1980): Stromrichtergespeiste Drehfeldmaschinen. Springer-Verlag, Wien

[Lap1] Lappe R. und Mitautoren (1994): Handbuch Leistungselektronik. Verlag Technik GmbH, Berlin München

[Lap2] Lappe R., Fischer F. (1993): Leistungselektronik - Messtechnik. Verlag Technik GmbH, Berlin München (2., stark bearbeitete Auflage)

[Leo1] Leonhard W. (1974): Regelung in der elektrischen Antriebstechnik. B.G. Teubner, Stuttgart

[Mey1] Meyer M. (1990): Leistungselektronik. Springer-Verlag, Berlin Heidelberg ]

[Mey2] Meyer M. (1987): Elektrische Antriebstechnik Band 2. Springer-Verlag, Berlin Heidelberg

[Mic1] Michel M. (1992): Leistungselektronik, Springer-Verlag, Berlin Heidelberg

[Mül1] Müller R. (1987): Halbleiterelektronik 2, Bauelemente der Halbleiter -Elektronik. Springer-Verlag, Berlin Heidelberg (3., völlig neubearbeitete und erweiterte Auflage)

[Schw1] Schwab A. J. (1990): Elektromagnetische Verträglichkeit. Springer-Verlag, Berlin Heidelberg

[Spä1] Späth H. (1983): Steuerverfahren für Drehstrommaschinen. Springer-Verlag, Berlin Heidelberg

[Sto1] Stoll R.L. (1974): The analysis of eddy currents. Clarendon Press, Oxford

[Tie1] Tietze U., Schenk Ch. (1989): Halbleiter-Schaltungstechnik. Springer-Verlag, Berlin Heidelberg (9. , neu bearbeitete und erweiterte Auflage)

[Wilh1] Wilhelm J. und 8 Mitautoren (1989): Elektromagnetische Verträglichkeit (EMV). Expert Verlag Ehningen (4., völlig neubearbeitete und erweiterte Auflage).

\section{Übersichtsartikel für Steuerverfahren von selbstgeführten Stromrichtern}

[Boo1] Boost M., Ziogas P. (1988): State-of-the-art carrier PWM techniques: a critical evaluation. IEEE Transactions on Industry Applications, Vol. 21, Nr. 2, S. 271-280

[Bow1] Bowes S.R., Eng C., Mount M.J. (1981): Microprocessor control of PWM inverters. IEE Proceedings B, Vol. 128, Nr. 6, S. 293-305

[Brod1] Brod D.M., Novotny D.W. (1985): Current control of VSI-PWM inverter. IEEE Transactions on Industry Applications, Vol. 21, Nr. 4, S. 562-570

[Hol1] Holtz J. (1992): Pulsewidth modulation - a survey. IEEE Transactions on Industrial Electronics, Vol. 39, Nr. 5, S. 410-420

[Kaz1] Kazmierkowski M.P., Dzieniakowski M.A. (1994): Rewiew of current regulation techniques for three-phase PWM inverters. IEEE Conference on Industrial Electronics, Control and Instrumentation, Record, S. 567-575

[Mal1] Malesani L., Tomasin P. (1993): PWM current control techniques of voltage source converters - a survey. IEEE Conference on Industrial Electronics, Control and Instrumentation, Record, S. 670-673

[Tre1] Trenner A. (1990): Optimale Pulsverfahren für Spannungszwischenkreisumrichter. ELINZeitschrift, Heft 1/2, S. 39-49

\section{Weitere Fachartikel}

[Ack1] Ackva A., Reinold H., Olesinski R. (1992): A simple and self-adapting high-performance current control scheme for three phase voltage source inverters. IEEE Power Electronics Specialists Conference, Record, S. 435-442 
[Aml1] Amler G. (1991): A PWM current-source inverter for high quality drives. EPE Journal, Vol. 1, Nr. 1, S. 21-31

[Bet1] Betz R., Evans R., Cook B. (1986): Optimal pulsewidth modulation for current source inverters. IEEE Transactions on Industrial Electronics, Vol. 33, Nr. 3, S. 318-324

[Boe1] Boehringer A., Stute G., Ruppmann C., Würslin R. (1979): Entwicklung eines drehzahlgesteuerten Asynchronmaschinenantriebs für Werkzeugmaschinen. WT-Zeitschrift für industrielle Fertigung, Nr. 69, S. 463-473

[Bos1] Bose B.K., Sutherland H.A. (1983): A high-performance pulsewidth modulator for an inverter-fed drive system using a microcomputer. IEEE Transactions on Industry Applications, Vol. 19, Nr. 2, S. 235-243

[Bos2] Bose B.K. (1990): An adaptive hysteresis-band current control technique of a voltage-fed PWM inverter for machine drive system. IEEE Transactions on Industrial Electronics, Vol. 37, Nr. 5, S. 402-408

[Bow2] Bowes S.R., Eng C., Bird B.M. (1975): Novel approach to the analysis and synthesis of modulation processes in power converters. IEE Proceedings B, Vol. 122, Nr. 5, S. 507-513

[Bow3] Bowes S.R. (1975): New sinusoidal pulsewidth-modulated invertor. IEE Proceedings B, Vol. 122, Nr. 11, S. 1279-1285

[Bow4] Bowes S.R. (1993): Advanced regular-sampled PWM control techniques for drives and static power converters. IEEE Conference on Industrial Electronics, Control and Instrumentation, Record, S. 662-669

[Bow5] Bowes S.R., Bullough R.I. (1987): Harmonic minimisation in microprocessor controlled current fed PWM inverter drives. IEE Proceedings B, Vol. 134, Nr. 1, S. 25-41

[Boy1] Boys J.T., Handley P.G. (1990): Harmonic analysis of space vector modulated PWM waveforms. IEE Proceedings B, Vol. 137, Nr. 4, S. 197-204

[Boy2] Boys J.T., Andrews M. (1993): Random PWM inverter drive systems: theory and practice. IEEE Conference on Industrial Electronics, Control and Instrumentation, Record, S. 695-700

[Broe1] van der Broeck H. (1991): Analysis of the harmonics in voltage fed inverter drives caused by PWM schemes with discontinuous switching operation. EPE European Conference on Power Electronics and Applications, Proceedings Volume 3, S. 261-266

[Broe2] van der Broeck H.W., Skudelny H.C., Stanke G. (1986): Analysis and realization of a pulse width modulator based on voltage space vectors. IEEE Industry Application Society Annual Meeting, Record, S. 244-251

[Buj1] Buja G.S., Indri G.B. (1977): Optimal pulsewidth modulation for feeding ac motors. IEEE Transactions on Industry Applications, Vol. 13, Nr. 1, S. 38-44

[Buj2] Buja G.S. (1980): Optimum output waveforms in PWM inverters. IEEE Transactions on Industry Applications, Vol.16, Nr. 6, S. 830-836

[Cam1] Cambronne J.P., Rombaut C., Courault J. (1990): An optimal pulsewidth modulation for GTO current source inverter fed induction motor drive system. IEE Conference on Power Electronics and Variable-Speed Drives, Publication Nr. 324, S. 110-114

[Car1] Carrera G., Gardella S., Marchesoni M., Salutari R., Sciutto G. (1992): A new multilevel PWM method: a theoretical analysis. IEEE Transactions on Power Electronics, Vol. 7, Nr. 3, S. 497-505

[Dep1] Depenbrock M. (1977): Pulsewidth control of a 3-phase inverter with non-sinusoidal phase voltages. IEEE Industry Applications Society Meeting, Record, S. 399-403 
[Dep2] Depenbrock M. (1988): Direct self-control (DSC) of inverter-fed induction machine. IEEE Transactions on Power Electronics, Vol. 3, Nr. 4, S. 420-429

[Dep3] Depenbrock M. (1985): Direkte Selbstregelung (DSR) für hochdynamische Drehfeldantriebe mit Stromrichterspeisung. ETZ-Archiv, Band 7, Heft 7, S. 211-218

[Dow1] Dowell P.L. (1966): Effects of eddy currents in transformer windings. IEE Proceedings, Vol. 113, No. 8, S. 1387-1394

[Dun1] Dunford W.G., van Wyk J.D. (1991): Harmonic imbalance in asynchronous PWM schemes. IEEE Power Electronics Specialists Conference, Record, S. 397-401

[Dzi1] Dzieniakowski M.A., Kazmierkowski M.P. (1992): Microprocessor-based novel current regulator for VSI-PWM inverters. IEEE Power Electronics Specialists Conference, Record, S. 459-464

[Enj1] Enjeti P.N., Ziogas P.D. Lindsay J.F. (1990): Programmed PWM techniques to eliminate harmonics: a critical evaluation. IEEE Transactions on Industry Applications, Vol. 26, Nr. 2, S. 302-316

[Enj2] Enjeti P., Choudhury S.A. (1991): A new control strategy to improve the performance of a PWM ac to dc converter under unbalanced operating Conditions. IEEE Power Electronics Specialists Conference, Record, S. 382-389

[Fuk1] Fukuda S., Iwaji Y. (1991): A pulse frequency modulated PWM scheme for sinusoidal inverters. IEEE Power Electronics Specialists Conference, Record, S.390-396

[Gilb1] Gilbert D. (1992): PWM-IC kontrolliert Wechselstrommotor. Elektroniker, Nr. 8, S. 23-26

[Gra1] Grant T.L., Barton T.H. (1979): Control strategies for PWM drives. IEEE Industry Applications Society Annual Meeting, Record, S. 780-784

[Gra2] Grant D.A., Stevens M., Houldsworth J.A. (1985): The effect of word length on the harmonic content of microprocessor-based PWM waveform generators. IEEE Transactions on Industry Applications, Vol. 21, Nr. 1, S. 218-225

[Gre1] Green T.C., Williams B.W. (1992): Spectra of delta-sigma modulated inverters: an analytical treatment. IEEE Transactions on Power Electronics, Vol. 7, Nr. 4, S. 644-653

[Hab1] Habetler T.G. (1993): A space vector-based rectifier regulator for AC/DC/AC converters. IEEE Transactions on Power Electronics, Vol. 8, Nr. 1, S. 30-36

[Hal1] Halazs S., Hassan A., Huu B. (1993): Optimal control of three level PWM inverters. IEEE Conference on Industrial Electronics, Control and Instrumentation, Record, S. 1228-1233

[Han1] Handley P.G, Boys J.T. (1990): Space vector modulation: an engineering review. IEE Conference on Power Electronics and Variable-Speed Drives, Publication Nr. 324, S. 87-91

[Hol2] Holtz J., Beyer B. (1991): Off-line optimized synchronous pulsewidth modulation with on-line control during transients. EPE Journal, Vol. 1, Nr. 3, S. 193-200

[Hol3] Holtz J., Stadtfeld S. (1983): A predictive controller for the stator current vector of ac machines fed from a switched voltage source. IPEC International Power Electronics Conference Tokyo, Record, S. 1665-75

[Hol4] Holtz J. (1993): On the performance of optimal pulsewidth modulation techniques. EPE Journal, Vol. 3, Nr. 1, S.17-26

[Hol5 Holtz J., Bube E. (1988): Field-orientated asynchronous pulsewidth modulation for high performance ac machine drives operating at low switching frequencies. IEEE Industry Applications Society Annual Meeting, Record, S. 412-417

[Hol6] Holtz J., Lotzkat W., Khambadkone A.M. (1993): On continuous control of PWM inverters in the overmodulation range including the six-step mode. IEEE Transactions on Power 
Electronics, Vol. 8, Nr. 4, S. 546-533

[Hom1] Hombu M., Ueda S., Ueda A., Matsuda Y. (1984): A new current source GTO inverter with sinusoidal output voltage and current. IEEE Transactions on Industry Applications, Vol. 21, Nr. 5, S. 1192-1198

[Hou1] Houldsworth J.A., Grant D.A (1984): The use of harmonic distortion to increase the output voltage of a three-phase PWM inverter. IEEE Transactions on Industry Applications, Vol. 20, Nr. 5, S. 1224-1228

[Jen1] Jenni F., Zwicky R. (1988): Speed and flux control of a pulse width modulated current source inverter drive. IEE Conference on Power Electronics and Variable-Speed Drives, Publication Nr. 291, S. 374-377

[Jen2] Jenni F., Wüest D. (1993): The optimization parameters of space vector modulation. EPE European Conference on Power Electronics and Applications, Proceedings Volume 4, S. 376-381

[Jen3] Jenni F., Zwicky R. (1987): Pulsewidth Modulations for Current Source Inverters. EPE European Conference on Power Electronics and Applications, Proceedings, S. 755-760

[Jeo1] Jeong S.-G., Park M.-H. (1991): The analysis and compensation of dead-time effects in PWM inverters. IEEE Transactions on Industrial Electronics, Vol. 38, Nr. 2, S. 108-114

[Joo1] Joos G., Espinoza J. (1993): PWM control techniques in current source rectifiers. IEEE Conference on Industrial Electronics, Control and Instrumentation, Record, S. 1210-1214

[Kal1] Kaltenbach K. (1988): Modulationsverfahren mit Raumzeigern für StromzwischenkreisPulswechselrichter. ETZ-Archiv, Band 10, Heft 9, S. 303-306

[Kat1] Kato T. (1986): Precise PWM waveform analysis of inverter for selected harmonic elimination. IEEE Industry Applications Society Annual Meeting, Record, S. 611-616

[Kaz2] Kazmierkowski M.P., Dzieniakowski M.A., Sulkowski W. (1991): Novel space vector based current controllers for PWM-inverters. IEEE Transactions on Power Electronics, Vol. 6, Nr. 1, S. 158-165

[Kha1] Khambadkone A., Holtz J. (1991): Low switching frequency high-power inverter drive based on field-oriented pulsewidth modulation. EPE European Power Electronics Conference, Proceedings Volume 4, S. 672-677

[Kla1] Klaver H.L. (1991): Control of the neutral point of a three-level-inverter. EPE European Conference on Power Electronics and Applications, Proceedings Volume 3, S. 278-281

[Kli1] Kliman G., Plunkett A.B. (1979): Development of a modulation strategy for a PWM inverter drive. IEEE Transactions on Industry Applications, Vol.15, Nr. 1, S. $72-79$

[Kol1] Kolar J.W., Ertl H., Zach F.C. (1990): Minimization of the harmonic rms content of the mains current of a PWM converter system based on the solution of an extreme value problem. ICHPS IV Conference Budapest, Proceedings

[Kol2] Kolar J.W., Ertl H., Zach F.C. (1990): Analytically closed optimization of the modulation method of a PWM rectifier system with high pulse rate. Conference Power Conversion and Intelligent Motion München, Proceedings

[Kol3] Kolar J.W., Ertl H., Zach F. (1991): Minimizing the current harmonics rms value of threephase PWM converter systems by optimal and suboptimal transition between continuous and discontinuous modulation. IEEE Power Electronics Specialists Conference, Record, S. $372-378$

[Kol4] Kolar J., Ertl H. (1991): Analyse und Vergleich von Steuerverfahren für Spannungszwischenkreis-Pulsumrichtersysteme hoher Taktzahl. ELIN-Zeitschrift, Heft 3/4, S. 96-109 
[Kol5] Kolar J.W., Ertl H., Zach F.C. (1991): Influence of the modulation method on the conduction and switching losses of a PWM converter system. IEEE Transactions on Industry Applications, Vol. 27, Nr. 6, S. 1063-1075

[Kol6] Kolar J.W., Ertl H., Zach F.C. (1991): Analysis of on- and off-line optimized predicitive current controllers for PWM converter systems. IEEE Transactions on Power Electronics, Vol. 6, Nr. 3, S. 451-462

[Kol7] Kolar J.W., Ertl H., Zach F.C. (1993): Quasi-dual modulation of three-phase PWM converters. IEEE Transactions on Industry Applications, Vol. 29, Nr. 2, S. 313-319

[Kul1] Kulka D., Gens W., Berger G. (1993): Direct torque controlling technique with synchronous optimized pulse pattern. IEEE Power Electronics Specialists Conference, Record, S. 245-250

[Liu1] Liu H.L., Cho G.H. (1993): Three-level space vector PWM in low index modulation region avoiding narrow pulse problem. IEEE Power Electronics Specialists Conference, Record, S. 257-262

[Mat1] Mathys P., Van Eck J.L. (1991): Specific integrated circuits for power electronics. EPE Journal, Vol.1, Nr. 1, S. 61-65

[Mor1] Morimoto M., Sato S., Sumito K., Oshitani K. (1989): Single-chip microcomputer control of the inverter by the magnetic flux control PWM method. IEEE Transactions on Industrial Electronics, Vol. 36, Nr.1, S. 42-47

[Mur1] Murai Y., Ohashi K., Hosono I. (1985): New PWM method for fully digitized inverters. IEEE Industry Applications Society Annual Meeting, Record, S. 448-453

[Murr1] McMurray W. (1984): Modulation of the chopping frequency in dc choppers and PWM inverters having current-hysteresis controllers. IEEE Transactions on Industry Applications, Vol. 20, Nr.4, S. 763-768

[Nab1] Nabae A., Ogasawara S., Akagi H. (1985): A novel control scheme of current-controlled PWM inverter. IEEE Industry Applications Society Annual Meeting, Record, S. 473-478

[Nab2] Nabae A., Takahashi I., Akagi H. (1980): A new neutral point clamped PWM inverter. IEEE Industry Applications Society Annual Meeting, Record, S. 518-523

[Nam1] Namuduri C., Sen P. (1986): Optimal pulsewidth modulation for current source inverters. IEEE Transactions on Industry Applications, Vol. 22, Nr. 6, S. 1052-1072

[Non1] Nonaka S., Neba Y.(1985): New GTO current source inverter with pulsewidth modulation control techniques. IEEE Industry Applications Society Annual Meeting, S. 442-447

[Non2] Nonaka S., X. Jin (1989): A PWM control method and steady-state characteristics of a new current output-type GTO converter. Electrical Engineering in Japan, Vol. 109, Nr. 2, S. $126-134$

[Oga1] Ogasawara S., Akagi H., Nabae A. (1989): A novel PWM scheme of voltage source inverters based on space vector theory. EPE European Power Electronics Conference, Proceedings, S. 1197-1202

[Ohn1] Ohnishi T., Okitsu H. (1984): A novel PWM technique for three-phase inverter/converter. Electrical Engineering in Japan, Vol. 104, Nr. 9/10, S. 193-200

[Pat1] Patel H.S., Hoft R.G. (1973): Generalized techniques of harmonic elimination and voltage control in thyristor inverters: part I - harmonic elimination. IEEE Transactions on IndustrY Applications, Vol. 9, Nr. 3, S. 310-317

[Pat2] Patel H.S., Hoft R.G. (1974): Generalized techniques of harmonic elimination and voltage control in thyristor inverters: part II - voltage control techniques. IEEE Transactions on Industry Applications, Vol.10, Nr. 5, S. 666-673 
[Per1] Persson E. (1992): Transient effects in application of PWM inverters to induction motors. IEEE Transactions on Industrial Applications, Vol. 28, Nr. 5, S. 1095-1101

[Pfa1] Pfaff G., Weschta A., Wick A. (1982): Design and experimental results of a brushless ac servo-drive. IEEE Industry Applications Society Annual Meeting, Record, S. 692-697

[Pit1] Pitel I.J., Talukdar S.N., Wood P. (1980): Characterization of programmed-waveform pulsewidth modulation. IEEE Transactions on Industry Applications, Vol.16, Nr. 5, S. 707715

[Rah1] Rahman M.A., Quaicoe J.E., Choudhury M.A. (1987): Performance analysis of delta modulated PWM inverters. IEEE Transactions on Power Electronics, Vol. 2, Nr. 3, S. 227-233

[Ric1] Richardson J., Kukrer O.T. (1991): Implementation of a PWM regular sampling strategy for ac drives. IEEE Transactions on Power Electronics, Vol. 6, Nr. 4, S. 645-655

[Ric2] Richardson J., Bezanov (1991): A PWM 3-phase on-line controller for ac drives. EPE European Conference on Power Electronics and Applications, Proceedings 3, S. 299-304

[Rot1] Roth-Stielow J., Zimmermann W., Boehringer A., Schwarz B. (1989): Zwei zeitdiskrete Steuerverfahren für Pulsumrichter im Vergleich. ETZ-Archiv, Band 11, Heft 12, S. 389396

[Schö1] Schönung A., Stemmler H. (1964): Geregelter Drehstrom-Umkehrantrieb mit gesteuertem Umrichter nach dem Unterschwingungsverfahren. BBC-Mitteilungen, Aug./Sept.

[Schu1] Schumacher W., Kiel E. (1993): Elektrische Antriebe in den neunziger Jahren. Elektronik, Heft 7, S. 94-98

[Spr1] Springmeier F., Steinke J.K. (1991): Field-weakening with direct self-control (DSC) as a control-scheme for high power three-level GTO inverters. EPE European Conference on Power Electronics and Applications, Proceedings Volume 4, S. 666-671

[Ste1] Stemmler H. (1974): Ein- und mehrpulsige Unterschwingungswechselrichter, Steuerverfahren, Strom- und Spannungsverhältnisse. IFAC Symposium Control in Power Electronics an Electrical Drives, Proceedings, Volume 4, S. 457-471

[Ste2] Stemmler H., Eilinger T. (1994): Spectral analysis of the sinusoidal PWM with variable switching frequency for noise reduction in inverter-fed induction motors. IEEE Power Electronics Specialists Conference, Record, S. 269-277

[Ste3] Stemmler, H. (1993): Power electronics in electric traction applications. IEEE Conference on Industrial Electronics, Control and Instrumentation, Record

[Ste4] Stemmler H., Guggenbach P. (1993): Configurations for high power voltage source inverter drives. EPE European Conference on Power Electronics and Applications, Proceedings 5, S. 7-14

[Stei1] Steinke J.K. (1992): Switching frequency optimal PWM control of a three-level inverter. IEEE Transactions on Power Electronics, Vol. 7, Nr. 3, S. 487-496

[Stei2] Steinke J.K. (1990): PWM control of a three-level inverter - principles and practical experience. IEE Conference on Power Electronics and Variable-Speed Drives, Publication Nr. 324, S. 98-103

[Stei3] Steinke J. K. (1988): Grundlagen für die Entwicklung eines Steuerverfahrens für GTODreipunktwechselrichter für Traktionsantriebe. ETZ-Archiv, Band 10, Heft 7, S. 215-220

[Tak1] Takahashi I., Mochikawa H. (1985): A new control of PWM inverter waveform for minimum loss operation of an induction motor drive. IEEE Transactions on Industry Applications, Vol. 21, Nr. 4, S. 580-587

[Tak2] Takahashi I., Mochikawa H. (1986): Optimum waveforms of an inverter for decreasing 
acoustic noise of an induction motor. IEEE Transactions on Industry Applications, Vol. 22, Nr. 5, S. 828-834

[Tak3] Takahashi I., Noguchi T. (1985): A new quick response and high efficiency control strategy of an induction motor. IEEE Industry Applications Society Annual Meeting, Record, S. 496-502

[Tör1] Török V.G. (1983): Near-optimum on-line modulation of PWM inverters. IFAC Symposium Control in Power Electronics an Electrical Drives, Proceedings, S. 247-254

[Trz1] Trzynadlowski A.M., Legowski S., Kirlin R.L. (1987): Random pulse width modulation technique for voltage-controlled power inverters. IEEE Industry Applications Society Annual Meeting, Record, S. 863-868

[Tur1] Turnbull F.G. (1964): Selected harmonic reduction in static dc-ac inverters. IEEE Transactions on Communication Electronics, Vol. 83, S. 374-378

[Vel1] Velaerts B., Mathys P. (1991): Microcontroller-based, multi-mode 3-level pulse-width modulator. EPE Journal, Vol. 1, Nr. 2, S. 133-138

[Web1] Webster M., Diana G., Harley R.G., Levy D.C. (1989): Space vector modulation - a real time application. Transactions of the South-African Institution of Electrical Engineers, September, S. 38-42

[Wei1] Weinhold M. (1991): Appropriate pulse width modulation for a three-phase PWM ac- todc converter. EPE Journal, Vol. 1, Nr. 2, S. 139-148

[Wie1] Wiechmann E.P., Ziogas P.D., Stefanovic V. R. (1985): A novel bilateral power conversion scheme for variable frequency static power supplies. IEEE Transactions on Industry Applications, Vol. 21, Nr. 5, S. 1226-1233

[Will] Williams S.M., Hoft R.G. (1990): Adaptive frequency domain control of PWM switched power line conditioner. IEEE Industry Applications Society Annual Meeting, Record, S. 505-511

[Wüe1] Wüest, D. (1991): An improved PWM optimization technique for a static reactive power compensator with self-commutatded inverter. IEEE Power Electronics Specialists Conference, Record, S. 763-768

[Wüe2] Wüest D., Jenni F. (1993): Space vector based current control schemes for voltage source inverters. IEEE Power Electronics Specialists Conference, Record, S. 986-992

[Wüe3] Wüest D., Stemmler H., Scheuer G. (1992): A comparison of different circuit configurations for an advanced static Var compensator (ASVC). IEEE Power Electronics Specialists Conference, Record, S. 521-529

[Zac1] Zach F.C., Ertl H. (1985): Efficiency optimal control for ac drives with PWM inverters. IEEE Transactions on Industry Applications, Vol. 21, Nr. 4, S. 987-1000

[Zac2] Zach F.C., Martinez R., Keplinger S., Seiser A. (1985): Dynamically optimal switching patterns for PWM inverter drives (for minimization fo the torque and speed ripples). IEEE Transactions on Industry Applications, Vol. 21, Nr. 4, S. 975-986

[Zac3] Zach F.C., Berthold R.J., Kaiser K.H. (1982): General purpose microprocessor modulator for a wide range of PWM techniques for ac motor control. IEEE Industry Applications Society Annual Meeting, Record, S. 446-51

[Zio1] Ziogas P.D. (1981): The delta modulation technique in static PWM inverters. IEEE Transactions on Industry Applications, Vol.17, Nr. 2, S.199-204

[Zio2] Ziogas P., Kang Y.-G., Stefanovic V.R. (1985): PWM control techniques for rectifier filter minimization. IEEE Transactions on Industry Applications, Vol. 21, Nr. 5, S. 1206-1214 


\section{B Verwendete Grundlagen}

\section{B.1 Das Dreiphasensystem}

\section{B.1.1 Spannungen und Ströme}

Betrachtet wird die Schaltung in Bild B.1. Sie ist ein Modell für die Verhältnisse, wie sie typischerweise bei einem selbstgeführten U-Stromrichter mit Last auftreten. Für die bezeichneten Spannungen und Ströme gelten die Knotengleichung für den Sternpunkt (B.1), die Maschengleichungen (B.2) sowie für die Lastkreise (B.3).
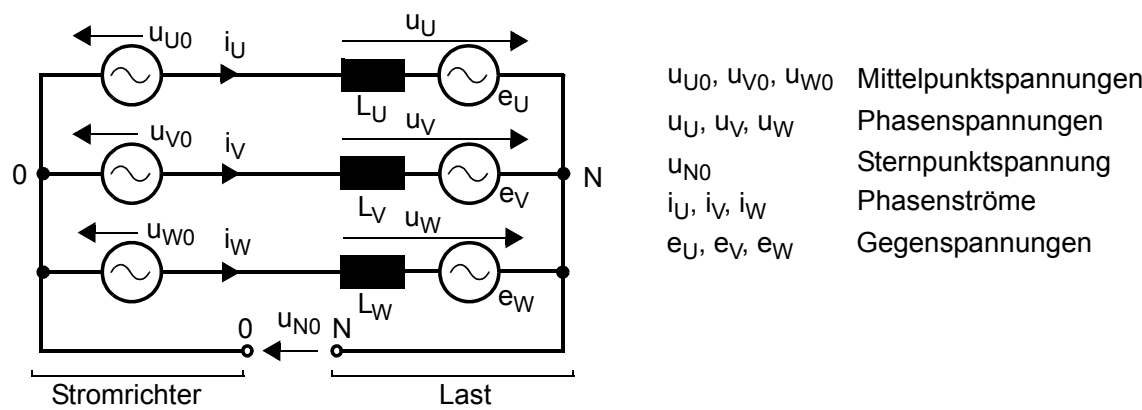

Bild B.1. Ersatzschaltung eines dreiphasigen selbstgeführten Stromrichters mit Last

$$
\begin{aligned}
& i_{U}+i_{V}+i_{W}=0 \\
& u_{U 0}=u_{U}+u_{N 0}, \quad \text { und } \quad \frac{d i_{U}}{d t}+\frac{d i_{V}}{d t}+\frac{d i_{W}}{d t}=0 \\
& u_{U 0}=u_{U}=u_{V}+u_{N 0}, \quad u_{W 0}=u_{W}+u_{N 0} \\
& d t \\
& e_{U}+u_{N 0}, \quad u_{V 0}=L_{V} \frac{d i_{V}}{d t}+e_{V}+u_{N 0}, u_{W 0}=L_{W} \frac{d i_{W}}{d t}+e_{W}+u_{N 0}
\end{aligned}
$$

Das Zusammenfassen von (B.1), (B.2) und (B.3) und Auflösen nach $u_{N 0}$ ergibt:

$$
u_{N 0}=\frac{1}{3}\left(u_{U 0}+u_{V 0}+u_{W 0}\right)-\frac{1}{3}\left(e_{U}+e_{V}+e_{W}\right)-\frac{1}{3}\left(L_{U} \frac{d i_{U}}{d t}+L_{V} \frac{d i_{V}}{d t}+L_{W} \frac{d i_{W}}{d t}\right)
$$


Symmetrisches Dreiphasensystem: Als symmetrisch mit der Grundkreisfrequenz $\omega_{1}=2 \pi f_{1}$ werden in einem Dreiphasensystem Spannungs- bzw. Stromtripel, Quellen und Lasten mit folgenden Eigenschaften bezeichnet:

- Die Spannungs- bzw. Stromverläufe in den 3 Phasen sind identisch, aber je um 1/3 Grundperiode $(2 \pi / 3)$ gegeneinander phasenverschoben:

$$
\begin{aligned}
& x_{V}(t)=x_{U}\left[\omega_{1} t-\frac{2 \pi}{3}\right]=x_{U}\left[\omega_{1}\left(t-\frac{T_{1}}{3}\right)\right], \\
& x_{W}(t)=x_{U}\left[\omega_{1} t-\frac{4 \pi}{3}\right]=x_{U}\left[\omega_{1}\left(t-\frac{2 T_{1}}{3}\right)\right], \quad T_{1}=\frac{1}{f_{1}}=\frac{2 \pi}{\omega_{1}}
\end{aligned}
$$

- Symmetrische Quellen geben symmetrische Spannungen (Spannungsquellen) bzw. symmetrische Ströme (Stromquellen) ab.

- Symmetrische Lasten bestehen aus identischen Impedanzen in allen drei Phasen und symmetrischen Quellen (bzw. Senken).

Die Gleichung (B.5) entspricht der Phasenfolge $U, V, W$. Diese kann entweder durch Vertauschen zweier beliebiger Phasen oder durch Ändern der Grundkreisfrequenz auf $-\omega_{1}$ umgekehrt werden. Dies ist vor allem bei Antrieben von Bedeutung (Umkehr der Drehrichtung).

Gleichtaktkomponenten $u_{G}$ : Gleichtaktkomponenten sind Anteile, die in allen 3 Phasen identisch verlaufen, d.h. auch phasengleich sind. Jedes Tripel von Spannungen und Strömen lässt sich aufspalten in gleichtaktfreie Anteile, deren Summe null ist, und den gemeinsamen Gleichtaktanteil. Für die Mittelpunktspannungen in der Schaltung nach Bild B.1 sind dies die gleichtaktfreien $u_{U 0}^{\prime}, u_{V 0}^{\prime}, u_{W 0}^{\prime}$ sowie die Gleichtaktkomponente $u_{G}$ Für sie gilt:

$$
\begin{aligned}
& u_{U 0}=u_{U 0}^{\prime}+u_{G}, \quad u_{V 0}=u_{V 0}^{\prime}+u_{G}, \quad u_{W 0}=u_{W 0}^{\prime}+u_{G} \\
& u_{U 0}^{\prime}+u_{V 0}^{\prime}+u_{W 0}^{\prime}=0
\end{aligned}
$$

Spezielle Gleichtaktkomponenten sind harmonische Schwingungen mit einer Frequenz der Form $3 v \omega_{1}$ (3. Harmonische und deren Vielfache, bzw. DC-Komponente). Sie bilden zusätzlich auch ein symmetrisches Dreiphasensystem:

$$
\begin{aligned}
& u_{G}=A_{G} \cos \left[3 v\left(\omega_{1} t\right)\right], \quad v=0,1,2, \ldots \\
& A_{G} \cos \left[3 v\left(\omega_{1} t\right)\right]=A_{G} \cos \left[3 v\left(\omega_{1} t-\frac{2 \pi}{3}\right)\right]=A_{G} \cos \left[3 v\left(\omega_{1} t-\frac{4 \pi}{3}\right)\right]
\end{aligned}
$$

Die Phasenströme weisen wegen der Knotenregel (B.1) keine Gleichtaktkomponenten auf.

Symmetrische, gleichtaktkomponentenfreie Last: In den üblichen Stromrichteranwendungen bildet die Last ein symmetrisches System und die Gegenspannungen enthalten keine Gleichtaktkomponenten. Dies bedeutet, dass die Summe der 3 Spannungen $e_{U}, e_{V}$ 
und $e_{W}$ null ist und die 3 Induktivitäten $L_{U}, L_{V}$ und $L_{W}$ identisch sind. Damit verschwinden in (B.4) die beiden letzten Summanden und für die Sternpunktspannung $u_{N O}$ resultiert:

$$
u_{N 0}=\frac{1}{3}\left(u_{U 0}+u_{V 0}+u_{W 0}\right)
$$

Durch Einsetzen von (B.10) in (B.2) erhält man für die Phasenspannungen:

$$
\begin{aligned}
& u_{U}=u_{U 0}-u_{N 0}=\frac{1}{3}\left(2 u_{U 0}-u_{V 0}-u_{W 0}\right), \\
& u_{V}=u_{V 0}-u_{N 0}=\frac{1}{3}\left(2 u_{V 0}-u_{U 0}-u_{W 0}\right), \\
& u_{W}=u_{W 0}-u_{N 0}=\frac{1}{3}\left(2 u_{W 0}-u_{U 0}-u_{V 0}\right)
\end{aligned}
$$

Eine wichtige Eigenschaft der symmetrischen und gleichtaktfreien Last zeigt sich, wenn man annimmt, dass die Mittelpunktspannungen gemäss (B.6) und (B.7) eine Gleichtaktkomponente aufweisen. Setzt man diese Gleichungen in (B.10) und (B.11) ein, so erhält man für die Sternpunktspannung (B.12) und für die Phasenspannungen (B.13).

$$
\begin{aligned}
& u_{N 0}=\frac{1}{3}\left(u_{U 0}+u_{V 0}+u_{W 0}\right)=u_{G} \\
& u_{U}=u_{U 0}-u_{N 0}=u_{U 0}^{\prime}, \quad u_{V}=u_{V 0}^{\prime}, \quad u_{W}=u_{W 0}^{\prime}
\end{aligned}
$$

Die Phasenspannungen entsprechen den Mittelpunktspannungen ohne Gleichtaktanteil. Die Gleichtaktkomponente $u_{G}$ ist identisch mit der Sternpunktspannung und fällt damit vollständig zwischen Stern- und Nullpunkt ab.

Für die Last stehen die Phasenspannungen $u_{U}, u_{V}$ und $u_{W}$ im Vordergrund. Sie bestimmen die Phasenströme $i_{U}, i_{V}$ und $i_{W}$. In der Regel ist gefordert, dass diese Spannungen und Ströme symmetrisch und möglichst unverzerrt sind. Die Mittelpunktspannungen sind durch die Phasenspannungen bis auf den Gleichtaktanteil eindeutig bestimmt. Dieser ist frei wählbar und stellt deshalb einen zusätzlichen Freiheitsgrad bei der Erzeugung von Schaltmustern dar: ein Teil der Verzerrungen in den Mittelpunktspannungen kann als Gleichtaktanteil ausgebildet und damit in die Sternpunktspannung verschoben werden. Er wirkt sich damit nicht auf die Ströme aus. Dies wird bei guten Steuerverfahren ausgenützt. Es ist aber z.T. zweckmässig, die Freiheit insofern einzuschränken, als nur Vielfache der 3. Harmonischen als Gleichtaktanteil verwendet werden. Auf diese Weise werden, wie durch (B.9) gezeigt wurde, auch die Mittelpunktspannungen symmetrisch (synchrone Steuerverfahren).

\section{B.1.2 Beschreibung von Dreiphasensystemen mittels Zeigern}

Die Beschreibung von Dreiphasensystemen kann in vielen Fällen durch die Verwendung von komplexen Zeigern wesentlich vereinfacht werden.

Drehzeiger, $\alpha \beta$-Darstellung: Bild B.2 zeigt die Darstellung der Spannungen $u_{U}, u_{V}$ und $u_{W}$ als Phasengrössen und als Drehzeiger. Mittels der Transformation (B.14) können aus 

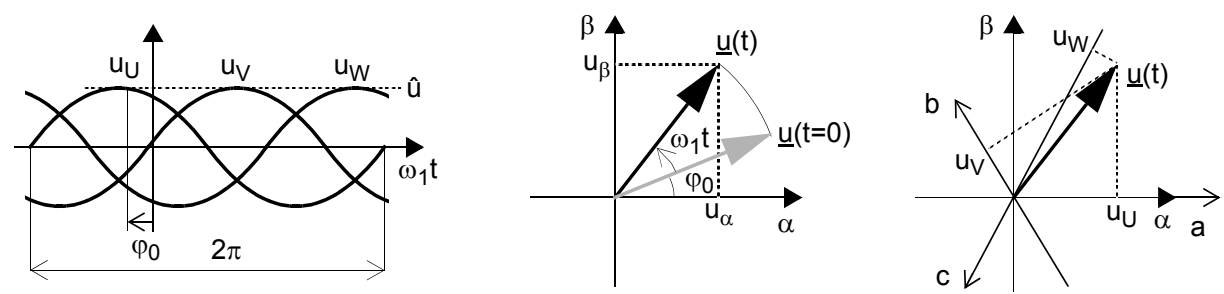

Bild B.2. Darstellung von dreiphasigen Grössen als Phasengrössen und als Drehzeiger, links: Phasengrössen, mitte: Drehzeiger, rechts: Beziehung zwischen dem Drehzeiger und den Phasengrössen

den Phasengrössen die $\alpha$ - und $\beta$-Komponenten des Zeigers $\underline{u}$ bestimmt werden. Mit dem Faktor 2/3 wird die Zeigerlänge so skaliert, dass sie bei einem symmetrischen System der Spannungsamplitude entspricht $\left(|\underline{u}|=\hat{u}_{U / V / W}\right)$. Für symmetrische und sinusförmige Spannungen im stationären Zustand weist der Drehzeiger eine konstante Länge auf und rotiert in der komplexen $\alpha \beta$-Ebene gleichförmig mit der Kreisfrequenz $\omega_{1}$.

$$
\underline{u}=\left[\begin{array}{l}
u_{\alpha} \\
u_{\beta}
\end{array}\right]=\frac{2}{3}\left[\begin{array}{ccc}
1 & -\frac{1}{2} & -\frac{1}{2} \\
0 & \frac{\sqrt{3}}{2} & -\frac{\sqrt{3}}{2}
\end{array}\right]\left[\begin{array}{l}
u_{U} \\
u_{V} \\
u_{W}
\end{array}\right]
$$

Zwischen dem Zeiger in Bild B.2 mitte und seinen Komponenten gelten die folgenden Beziehungen:

$$
\begin{aligned}
& u_{\alpha}=|\underline{u}| \cos \left(\omega_{1} t+\varphi_{0}\right), \quad u_{\beta}=|\underline{u}| \sin \left(\omega_{1} t+\varphi_{0}\right) \\
& |\underline{u}|=\sqrt{u_{\alpha}^{2}+u_{\beta}^{2}}, \quad \omega_{1} t+\varphi_{0}=\operatorname{atan}\left(\frac{u_{\beta}}{u_{\alpha}}\right)
\end{aligned}
$$

Mit (B.17) können aus den Zeigerkomponenten wieder die Phasengrössen bestimmt werden. Bild B.2 rechts illustriert die Rücktransformation aus der Zeigerdarstellung ins zugehörige Dreiphasensystem als Projektion des Zeigers auf die 3 um $2 \pi / 3$ versetzten Achsen $a, b$ und $c$.

$$
\left[\begin{array}{l}
u_{U} \\
u_{V} \\
u_{W}
\end{array}\right]=|\underline{u}|\left|\begin{array}{c}
\cos \left(\omega_{1} t+\varphi_{0}\right) \\
\cos \left\{\left(\omega_{1} t+\varphi_{0}\right)-\frac{2 \pi}{3}\right\} \\
\cos \left\{\left(\omega_{1} t+\varphi_{0}\right)-\frac{4 \pi}{3}\right\}
\end{array}\right|=\frac{3}{2}\left[\begin{array}{cc}
\frac{2}{3} & 0 \\
-\frac{1}{3} & \frac{1}{\sqrt{3}} \\
-\frac{1}{3} & -\frac{1}{\sqrt{3}}
\end{array}\right]\left[\begin{array}{l}
u_{\alpha} \\
u_{\beta}
\end{array}\right]
$$

Die Transformation von den Phasengrössen auf Drehzeiger ist eindeutig. Allfällige Gleichtaktkomponenten gehen jedoch dabei verloren. Die Rücktransformation ist abgesehen von den Gleichtaktkomponenten ebenfalls eindeutig. Die rücktransformierten Pha- 
sengrössen sind gleichtaktfrei und mit den ursprünglichen Funktionen identisch, wenn diese ebenfalls gleichtaktfrei waren. Da sich die Mittelpunkt- und die Phasenspannungen bei symmetrischer Last nur in der Gleichtaktkomponente unterscheiden, werden sie in diesem Fall durch den gleichen Drehzeiger repräsentiert.

Festzeiger, dq-Darstellung: Der Drehzeiger in $\alpha \beta$-Darstellung wird gemäss Bild B.3 in ein mit der Kreisfrequenz $\omega_{1}$ drehendes Koordinatensystem mit den Achsen $d$ und $q$ trans-
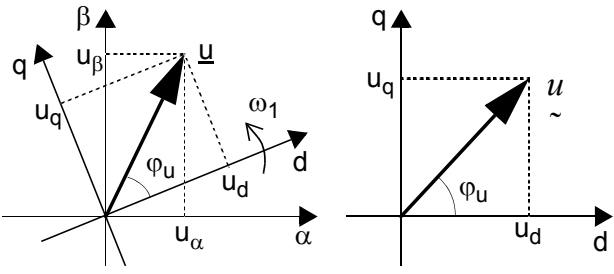

\section{Bild B.3.}

Festzeiger in $d q$-Darstellung, links: mit $\omega_{1}$ drehendes $d q$-Koordinatensystem in $\alpha \beta$-Darstellung,

rechts: Spannungszeiger in $d q$-Darstellung

formiert. Den neuen Festzeiger $u$ erhält man mit den folgenden Transformationsgleichungen aus dem Drehzeiger $\underline{u}$ :

$$
\underset{\sim}{u}=\left[\begin{array}{l}
u_{d} \\
u_{q}
\end{array}\right]=\left[\begin{array}{cc}
\cos \left(\omega_{1} t\right) & \sin \left(\omega_{1} t\right) \\
-\sin \left(\omega_{1} t\right) & \cos \left(\omega_{1} t\right)
\end{array}\right] \underline{u}=\left[\begin{array}{cc}
\cos \left(\omega_{1} t\right) & \sin \left(\omega_{1} t\right) \\
-\sin \left(\omega_{1} t\right) & \cos \left(\omega_{1} t\right)
\end{array}\right]\left[\begin{array}{l}
u_{\alpha} \\
u_{\beta}
\end{array}\right]
$$

Da $\omega_{1}$ der Kreisfrequenz der Spannungen $u_{U}, u_{V}$ und $u_{W}$ bzw. der Drehgeschwindigkeit des Drehzeigers $\underline{u}$ entspricht, ist $u$ für symmetrische und sinusförmige Spannungen ein stillstehender Zeiger konstanter Länge. Die Festzeigerdarstellung eignet sich besonders gut für komplexe Regelalgorithmen. Anwendungsbeispiele sind die feldorientierte Regelung einer Maschine oder die getrennte Regelung der Wirk- und Blindleistung eines Stromrichters am Netz.

\section{B.2 Fourierreihe}

\section{B.2.1 Periodische Signale}

Ein periodisches Signal $x(t)$ mit der Grundperiode $T_{1}=1 / f_{1}=2 \pi / \omega_{1}$ kann als Fourierreihe dargestellt werden. Die Fourierreihe ist eine unendliche Summe von Cosinus- und Sinusfunktionen, deren Kreisfrequenzen geradzahlige Vielfache von $\omega_{1}$ sind:

$$
x(t)=a_{0}+\sum_{v=1}^{\infty}\left[a_{v} \cos \left(v \omega_{1} t\right)+b_{v} \sin \left(v \omega_{1} t\right)\right], \quad \omega_{1}=2 \pi f_{1}=\frac{2 \pi}{T_{1}}
$$

Der zusätzliche Summand $a_{0}$ beschreibt den linearen Mittelwert von $x(t)$, d.h. die DCKomponente. Die Fourierkoeffizienten $a_{0}, a_{v}$ und $b_{v}$ lassen sich mit (B.20), (B.21) und 
(B.22) in Funktion der Zeit $t$ oder des Winkels $\left(\omega_{1} t\right)$ berechnen. Sie haben die gleiche Dimension wie die Grösse $x(t)$.

$$
\begin{aligned}
& a_{0}=\frac{1}{T_{1}} \int_{0}^{T_{1}} x(t) d t=\frac{1}{2 \pi} \int_{0}^{2 \pi} x\left(\omega_{1} t\right) d\left(\omega_{1} t\right) \\
& a_{v}=\frac{2}{T_{1}} \int_{0}^{T_{1}}\left[x(t) \cos \left(v \omega_{1} t\right)\right] d t=\frac{1}{\pi} \int_{0}^{2 \pi}\left[x\left(\omega_{1} t\right) \cos \left(v \omega_{1} t\right)\right] d\left(\omega_{1} t\right) \\
& b_{v}=\frac{2}{T_{1}} \int_{0}^{T_{1}}\left[x(t) \sin \left(v \omega_{1} t\right)\right] d t=\frac{1}{\pi} \int_{0}^{2 \pi}\left[x\left(\omega_{1} t\right) \sin \left(v \omega_{1} t\right)\right] d\left(\omega_{1} t\right)
\end{aligned}
$$

Die Teilschwingungen jeder Frequenz, auch als harmonische Schwingungen oder Harmonische bezeichnet, lassen sich statt durch eine Cosinus- und eine Sinusfunktion auch nur durch eine Sinusfunktion mit Phasenverschiebung beschreiben:

$$
x_{v}(t)=\hat{x}_{v} \sin \left(v \omega_{1} t+\varphi_{v}\right) \text { mit } \quad \hat{x}_{v}=\sqrt{a_{v}^{2}+b_{v}^{2}}, \quad \varphi_{v}=\operatorname{atan}\left(\frac{a_{v}}{b_{v}}\right)+\left\{\begin{array}{l}
0 \\
\pi
\end{array}\right.
$$

Sehr gebräuchlich ist die graphische Darstellung als Linienspektrum, getrennt nach Amplituden- und Phasenspektrum, wobei meistens das Amplitudenspektrum (Beispiel in Bild B.5) allein betrachtet wird. Die Amplituden werden dabei üblicherweise logarith-

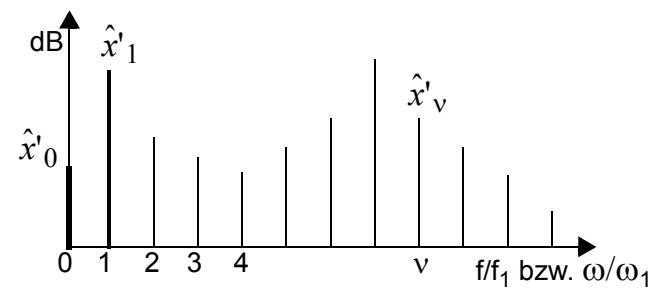

\section{Bild B.4.}

Amplitudenspektrum des periodischen Signals $x(t), \hat{x}_{v}^{\prime}=20 \log \left(\hat{x}_{v} / x_{B}\right)$

misch in dB dargestellt: $\hat{x}_{v}{ }_{v}=20 \log \left(\hat{x}_{v} / x_{B}\right)$ mit $x_{B}$ als beliebigem Bezugswert bzw. der Einheit von $x$. Im Beispiel in Bild B.4 repräsentiert die dick gezeichnete Frequenzlinie bei $f=0$ die Amplitude der DC-Komponente und diejenige bei $f / f_{1}=1$ die der Grundschwingung des Signals $x(t)$. Sind die Fourierkoeffizienten von $x(t)$ bekannt, so lässt sich mit (B.24) auch ihr Effektivwert berechnen:

$$
X_{\text {eff }}=\sqrt{\hat{x}_{0}^{2}+\frac{\hat{x}_{1}^{2}}{2}+\frac{\hat{x}_{2}^{2}}{2}+\ldots}=\sqrt{\hat{x}_{0}^{2}+\sum_{v=1}^{\infty} \frac{\hat{x}_{v}^{2}}{2}}
$$

Die Darstellung von Signalen als Fourierreihe eignet sich sehr gut zur Beschreibung von nichtsinusförmigen periodischen Grössen. 


\section{B.2.2 Nicht exakt periodische Signale}

Im Zusammenhang mit Steuerverfahren für selbstgeführte Stromrichter liegen häufig Signale vor, welche eine stationäre Grundschwingung, und einen nichtperiodischen Verzerrungsanteil aufweisen. Es sind dies zum Beispiel alle asynchronen Pulsmuster. Theoretisch ist die Periodendauer solcher Signale unendlich. Ihr Frequenzspektrum ist deshalb nicht exakt beschreibar. Es bieten sich jedoch zwei Ersatzbetrachtungen an, welche beide eine gute Charakterisierung der frequenzmässigen Zusammensetzung des Signals ergeben. Sie basieren beide darauf, dass nur ein endliches, jedoch gegenüber der Grundperiode $T_{1}$ sehr grosses Zeitintervall $T$ betrachtet wird:

- Unter der Annahme, dass das Signal ausserhalb von $T$ null ist, d.h. der betrachtete Ausschnitt einmalig ist, kann eine Fouriertransformation durchgeführt werden. Es resultiert ein kontinuierliches Frequenzdichtespektrum $X(\omega)$ gemäss dem Beispiel in Bild B.5 links, das die Verteilung der insgesamt begrenzten Energie des Signals auf die einzelnen Frequenzbereiche beschreibt.

- Es kann jedoch auch angenommen werden, das Signal sei in beide Richtungen der Zeitachse durch identische Abschnitte fortgesetzt. Es wird damit als periodisch mit der Periodendauer $T$ betrachtet. In diesem Fall lässt sich eine Fourierreihe bilden und es entsteht ein Linienspektrum gemäss Bild B.5 rechts. Die auftretenden Frequenzen sind die ganzzahligen Vielfachen von $1 / T$. Es ist vorteilhaft, $T$ als ganzzahliges Vielfaches von $T_{1}$ zu wählen. Dadurch ist die Grundfrequenz $f_{1}$ gerade eine der auftretenden Linien. Gegenüber dem Spektrum eines periodischen Signals wird hier von einem verdichteten Linienspektrum gesprochen. Die Linien, welche nicht Harmonische der Grundfrequenz sind, werden auch als Zwischenharmonische (für $f_{v}>f_{1}$ ) bzw. Subharmonische (für $f_{v}<f_{1}$ ) bezeichnet.
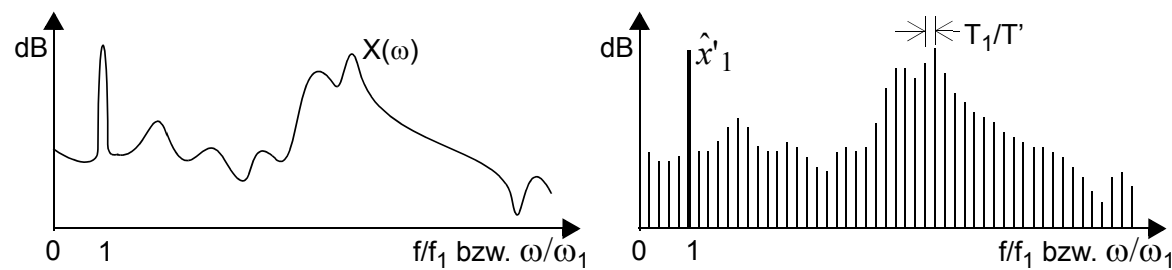

Bild B.5. Frequenzspektrum (nur Amplitudenspektrum) eines nicht exakt periodischen Signals $x(t)$ für ein Zeitintervall $T=T_{1}$,

links: Frequenzdichtespektrum (Fouriertransformierte), rechts: Fourierreihe

Der Vergleich zeigt, dass das Frequenzdichtespektrum proportional zur Hüllkurve des verdichteten Linienspektrums verläuft. Beide Methoden ergeben deshalb eine gleichwertige Information. Allerdings hat das Frequenzdichtespektrum im Gegensatz zum Linienspektrum nicht dieselbe Dimension wie das ursprüngliche Zeitsignal: ist $x(t)$ eine Spannung, so weist $X(\omega)$ die Einheit $\mathrm{V} / \mathrm{Hz}$ auf.

Wird $T$ vergrössert, so bleibt unter Voraussetzung eines in seiner Charakteristik unveränderten Signals in beiden Spektra die relative Amplitudenverteilung des 'Rauschbandes' gleich. Allerdings wird in beiden Spektra der Abstand zwischen der Amplitude der 
Grundschwingung und diesem 'Rauschband' grösser. Dieser Zusammenhang kann qualitativ leicht erklärt werden: für das Frequenzdichtespektrum ergibt sich bei einem grösseren $T$ eine bessere Herausfilterung der Grundfrequenz, so dass dort eine schmalere und höhere Spitze erscheint. Bei der Fourierreihe verteilt sich dagegen die ungefähr konstante Rauschleistung im stärker verdichteten Spektrum auf mehr Frequenzlinien, so dass deren Amplituden kleiner werden. Die Grundschwingung verändert sich dagegen nicht. Wird $T$ genügend gross gewählt, so lässt sich auf beide Arten die frequenzmässige Zusammensetzung des ursprünglichen Signals $x(t)$ näherungsweise beschreiben.

In der Regel wird wegen der einfacheren Handhabung mit dem verdichteten Linienspektrum gearbeitet. Vor allem basieren auch die digitalen Frequenzanalysatoren auf dieser Methode, wobei sie allerdings in der Regel noch spezielle Fensterfunktionen einsetzen (Windowing).

Literatur zu Anhang B.2: [Ach1], [Fö12] 


\section{Sachverzeichnis}

$\alpha \beta$-Darstellung 359

Abtastfrequenz, -intervall, -periode 115, 152, 225,250

Abschirmung 333

Abstrahlung, elektromagnetische 331

Amplitudenspektrum 87, 362

ASM im stationären Betrieb 41

Ausschaltverzögerung, Kompensation 323

Aussteuerungsgrad 86

Aussteuergrenze 267

Beschreibung von Stromrichtern, zeitkontinuierliche 56

Beschreibung von Stromrichtern, mit Drehzeigern 61, 66

Besselfunktion 118

Bezugskreisfrequenz 92

Bezugsgrössen für zusammengeschaltete Stromrichter 287

Bezugsmoment 92

Bezugsspannung, -strom, -reaktanz 91

Blindleistung 27

Bipolartransistor 34, 320

Brückenzweig 54

Dioden 33, 37

Direkte Regelverfahren, Feed-Back 264

Direkte Regelverfahren bei dreistufigen Stromrichtern 313

Drehmomentwelligkeit 42, 44, 91

Drehmomentwelligkeit, minimale 197

Drehzahlbereich 23, 92

Drehzeigerdarstellung 152, 229, 359

Drehzeigermodulation, Standardvariante 158, 166

Drehzeigermodulation für I-Stromrichter 283

Dreiphasensystem, symmetrisches 358

Dualität 275

dq-Darstellung 361

Energieflussrichtung 28

Entlastungsnetzwerke 35, 324

Erdschlaufen 332

Ersatzschaltung mit Umschaltern 54
Feldorientiertes Regelverfahren 253, 257

Festzeigerdarstellung 361

Filterresonanz 50, 176, 197

Filter, Störschutz 325, 335

Filter im Zwischenkreis 327

Flankensteilheit 323, 330

Freiheitsgrade 173, 178, 182, 294, 306

fremdgeführte Stromrichter 21, 26

Frequenzdichtespektrum 330, 363

Frequenzkomponente 84, 88, 90, 361

Frequenzmodulation des Trägersignals 112

Frequenzvariation bei Off-Line Modulatoren 202

Fourierreihe, -koeffizienten 84, 87, 90, 361

Funkstörungen 334

Gate-Turn-Off Thyristor GTO 34, 320, 347

Gegenspannung 45, 357

Gleichanteil des Zwischenkreisstromes 88

Gleichanteile, transiente 202, 273

Gleichrichter 15, 27, 326

Gleichtaktkomponente 136, 146, 358

Grenzzyklen 220

Grundfrequenz, -periode 86

Grundschwingung 56, 84, 85, 362

Gütekriterium 182, 246, 295

Halbperiodensymmetrie 96, 176, 181

Harmonische 84, 362

Harmonische, Gewichtung 195

Harmonische, Injektion 136

Harmonische, Zwischen-, Subharmonische 97, 362

Hystereseregler 204

IGBT 34, 320

Induktivität, Lastinduktivität 45

Induktivität, Streuinduktivität 45, 325

Induktivität, Entkopplungsinduktivität 297

Kenngrössen der AC-Seite 265

Komparator, mehrstufig 230, 340

Kontrolleinheit für Modulatoren 168

Kopplung, kapazitive, induktive 325, 332

Klirrfaktor 87 
Klirrfaktor, minimaler 97, 199

Last, symmetrische, gleichtaktkomponentenfreie 358

Last, unsymmetrische 326

Leistungshalbleiter, abschaltbare 33

Leistungshalbleiter als ideale Schalter 33

Leistungshalbleiter, Ansteuerung 36, 73

Leistungshalbleiter, Einsatzbereiche 35

Leistungshalbleiter, Ersatzschaltung 320

Leistungshalbleiter, Spannungsbelastung 54

Leistungshalbleiter, Verzögerungszeit 35

Linienspektrum 362

Linienspektrum, verdichtetes 97, 363

Maschinentyp, andere als ASM und SM 25

Mehrfachpulse 347

Microcontroller 344

Mittelpunktspannung 54, 59, 357

Modulationsgrad bei I-Stromrichtern 282

Modulation, asynchron 110, 205, 233, 363

Modulation, diskontinuierliche 158, 162, 166

Modulation, synchron 110, 136, 168

Modulation, symmetrische 111, 120, 290

Modulation, Rückflanken-, Vorderflanken- 111

Modulator 73

Modulator, analog, digital 316

Modulationsfunktion 86

Modulationsgrad 56, 86

MOSFET 34, 320

netzgeführte Stromrichter 26

Netzimpedanz 37, 333

Netzrückwirkung 37, 331

Niederfrequenter Anteil 55

Phasenspannungen, -ströme 57, 59, 357

Phasenspektrum 293, 362

Proximity-Effekt 53

Pulsationsanteil des Zwischenkreisstromes 88, 313

Pulsmusterwechsel 201

Pulsverriegelung 345

Pulsweite, minimale 34, 220, 346

Pulsweite, runden 318, 347

Quantisierte Signale 338

Rückwirkung des Stromrichters auf den Zwischenkreis 327

Sampling, Natural 115

Sampling, Regular, Uniform, Single-Edge, Double-Edge 115, 164

Schaltfrequenz 35, 87

Schaltfrequenz, momentane 205, 225
Schaltfunktion, Aufteilung auf die Brückenzweige 96,176

Schaltfunktion, konvertierte für I-Stromrichter 276, 281

Schaltsequenzen 190, 202, 295

Schaltwinkeltabelle 168, 342

Schaltverluste 35

Schaltwinkel 168

Schaltzahl 87

Schaltzustand 54, 60

Seitenbänder 111, 119

Sektoren 61, 241

selbstgeführte Stromrichter 21, 26

Skineffekt 52

Sollwerte, nichtsinusförmige 121, 161, 339

Sollwerte, diskontinuierliche 138, 161

Spannung, verkettete 58

Spannungs-Frequenz-Kennlinien-Verfahren 169,260

Spannungssteuerung, Feed-Forward 264

Sprungstellen von Pulsmustern 193, 202

Sternpunktspannung 59, 159, 357

Steuerung von Wirk- und Blindstrom 75

Steuerwinkel 27, 100, 305

Störungen, Emmission, Immunität 334

Stromanstiegsbegrenzung 325

Stromregelkreis 73, 205

Stromverdrängung 197

Synchronisation 348

SM im stationären Betrieb 44

Synchronisierung des Pulsmusters 214, 348

Taktung, versetzte 129, 296, 308

Toleranzband 204, 230, 260

Toleranzbandregler 204

Toleranzfläche 223, 230, 239, 244, 252

Totzeit 322, 345

Transformatoren 286, 300, 319

Transformationsgleichungen für Stromrichter 53,67

Trägerbänder 111, 119

Übersteuerung 114, 209, 262, 265

Umgebung von Stromrichtern 331

Umrichter 15, 21

Umrichter mit Spannungs-, Stromzwischenkreis 21,31

Verluste, Minimierung 196

Verträglichkeitspegel 334

Verzerrungsanteil 85, 86, 88

Verzerrungsanteil, Effektivwert 90 
Verzerrungsstrom, minimaler 149

Viertelperiodensymmetrie 96, 176, 177, 181, 288

Vorsteuerung 249

Wortbreite, digitale 316

Wechselrichter 15

Wirkleistung 28

Wicklungen, Spannungsbelastung 326

Zeitauflösung von Modulatoren 317, 338

Zeitbasis 168, 342

Zustände, Nullzustände 60, 278

Zustände, spannungsbildende 60

Zustände, strombildende 280

Zwischenkreis beim dreistufigen Stromrichter 63

Zwischenkreisgrössen, Welligkeit 47, 89 